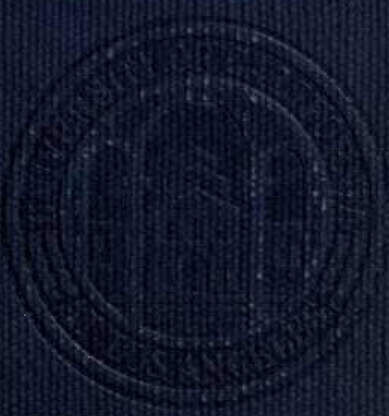




\section{UNIVERSITY OF CALIFORNIA}

AT LOS ANGELES
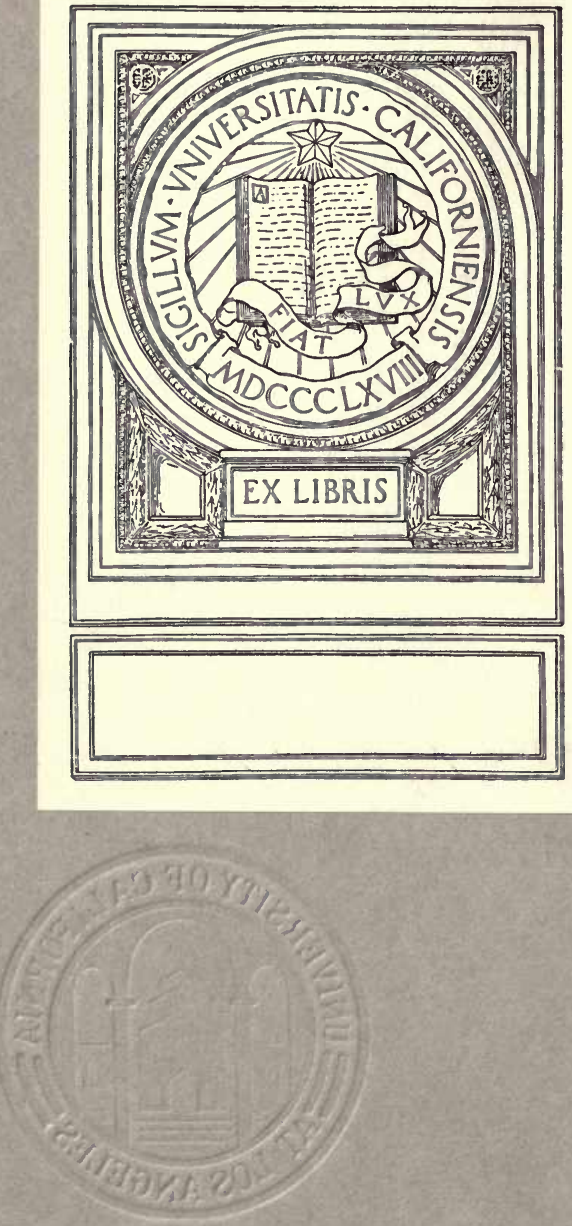





\title{
CONTRIBUTIONS TO A SCIENCE OF
}

NEMATOLOGY

\author{
N. A. СОB
}

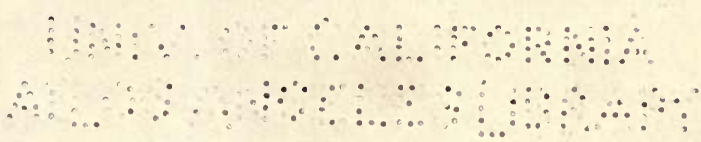




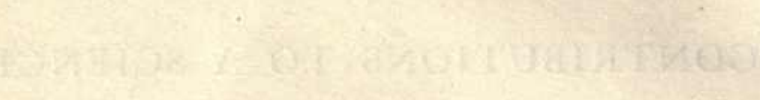

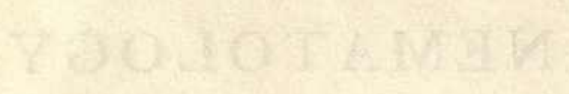

and

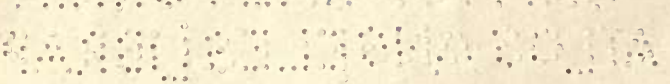


I. Antarctic Marine Free-Living Nematodes of the Shackleton Expedition . . . . . . . .

II. North American Free-Living Fresh-Water Nematodes ................

III. The Asymmetry of the Nematode Bunonema inequale, n. sp. ............

IV. Selachinema, a New Nematode Genus with Remarkable Mandibles

V. Notes on Nemas ............

VI. The Mononchs, a Genus of Free-Living Predatory Nematodes ...............

VII. Filter-Bed Nemas: Nematodes of the Slow Sand Filter-Beds of American Cities ...... 189

VIII. The Orders and Classes of Nemas ....... 213

IX. One Hundred New Nemas

(Type Species of 100 New Genera). . . . . 217

X. Howardula benigna, a Nemic Parasite of the Cucumber-Beetle . . . . . . . . 345

XI. Marionella . . . . . . . . . . 353

XII. Greeffiella ............. 359

XIII. An Amendation of Hoplolaimus Daday 1905 nec auctores .............. 363

XIV. Notes on Paratylenchus, a Genus of Nemas . . 367

XV. Blological Relationships of the Mathematical Series 1, 2, 4, etc. .........

XVI. Nemic Spermatogenesis . . . . . . . . 375

XVII. The Screw Nemas, Parasites of Codfish, Haddock and Other Fishes ............

XVIII. Ungella secta n. gen. n. sp., a Nemic Parasite of the Burmese Oligochaete (Earthworm) . . .

XIX. A New Species of the Nemic Genus Syringolaimus.

XX. The Chromatropism of Mermis subnigrescens . . . 398

XXI. Survey of Nemas in Marine Beach Sand .....

XXII. The Ambulatory Tubes and Other Features of the Nema Draconema cephalatum .........

XXIII. Observations on the Morphology and Physiology of Nemas . . . . . . . . . . . .

XXIV. The Demanian Vessels in Nemas of the Genus Oncholaimus ............... 423

XXV. Metoncholaimus pristiurus (Zur Strassen)... 439 XXVI. A Key to the Genera of Free-Living Nemas 



\section{AN'TARC'TIC MARINE \\ FREE-LIVING NEMATODES \\ OF THE}

\section{SHACKLETON EXPEDITION}

Contributions to a Science of Nematology

I

BY

N. A. $\mathrm{COBB}$

(With fifty illustrations in the text)

BALTIMORE

WILLIAMS \& WILKINS CO.

1914 

To Shackleton

And his Men,

Who to bring these stranger forms

Braved the ice and cold

Of the Southern Main,

This tribute from a comber of milder shores. 



\title{
ANTARCTIC MARINE \\ FREE-LIVING NEMATODES
}

\author{
OF THE
}

\section{SHACKLETON EXPEDITION}

\author{
By N. A. СовB \\ Contributions to a Science of Nematology ${ }^{1}$
}

I

Nematodes are so frequent in the Shackleton collections as to prove the seabottoms of the farthest south to swarm with these little beings. Hundreds of them, male, female and young, were taken from a mere thimbleful of the dredgings. The same tale comes from stations wide apart. Countless myriads find sustenance in these cold dark depths, and must in their turn be devoured by larger forms, until the series culminates in herds of seal and schools of whale. "All that in them is," takes on added meaning!

Whence do these nematodes derive their sustenance? The stomachs of a number of the species contain diatoms with such regularity as to leave no doubt that these microscopic plants constitute a main food supply. The undigested frustules of the diatoms are voided and go to make up the permanent sea floor, so that the interesting little creatures whose portraits follow, or at least a part of them, assist in building what some future epoch may disclose as dry land formations of diatomaceous earth as remarkable as those of the United States or those of Tripoli. Some of the other species appear to be predacious, though none belong to the truly carnivorous group of the Enoplidae. No doubt the greater part of the species are vegetarian.

These antarctic species are on the whole somewhat smaller than those of warmer seas, but one of them, that mentioned last, is a veritable

${ }^{1}$ Nematology-a contraction of Nematodology. The founding of this branch of science, on a par with Entomology for example, is fully justified by the fact that the Nematodes constitute such a distinct and highly characteristic group of organisms, containing an enormous number of species readily susceptible of division into definite Orders, some of which are of great economic importance. 
giant of its kind. Seven tropical Monhysteras taken at random from the writer's collections prove to average hardly 50 per cent longer than the average of the seven polar Monhysteras here described.

There is little evidence that these polar species are less fecund than those found elsewhere. It is hardly conceivable that the body temperature of the marine polar species is higher than that of the water in which they live, namely, near the freezing point of fresh water, and yet, in spite of the freezing temperature, and the long polar night, nematode protoplasm seems to glide on through its mitosis dance to much the same purpose as if bathed in equatorial light and ensconced in the warm pools of tropical reefs.

Through long residence and much travel in Pacific regions the writer has had unusual opportunity to become acquainted with their charac-

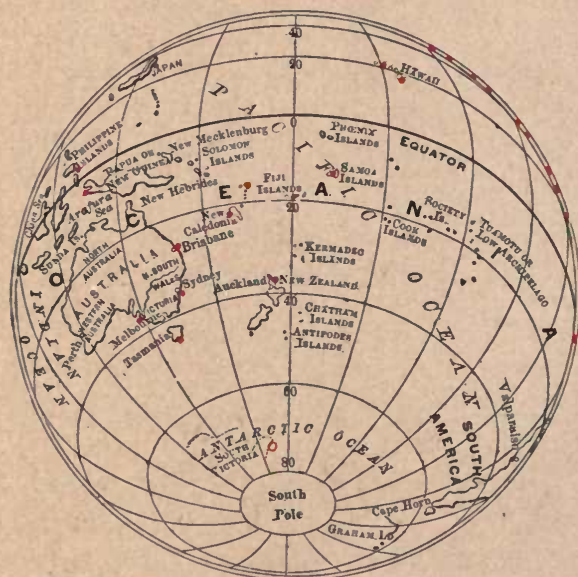

Fig. 1. Marks indicate about twenty of the author's Nematode Stations-North American, Asiatic, Australasian and Oceanic - at the great majority of which he has made personal examinations, and with the nematode fauna of which the Shackleton nematode collections are compared. America. Nearly all of
the Shackleton species belong to known genera, and the two new genera are nearly related to genera already known, Aplectus to the known Plectus and Austronema to the known Monhystera. times paid particular attention to the marine nematodes of these regions (map), is able from personal observation to say concerning the nematodes Lieutenant Shackleton's expedition brought from the far South, that in nothing are they more remarkable than in the striking resemblance they bear to forms found in the warmer parts of the water hemisphere.

Their nearest known relatives are found in New Zealand and the islands off the coast of the south end of South teristies, and having at 
The spermatogenesis of Terschellingia polaris, n. sp. presents some very interesting features which are noted in connection with the description. Recently it has been suggested that of the two kinds of spermatozoa Boveri and Schleip have shown to exist in the males of the free-living generation of Rhabdias bufonis, ${ }^{2}$ one kind, supposedly that producing males, becomes functionless, thus accounting for the succeeding generation consisting solely of "parthenogenetic females," found in frogs' lungs. The spermatozoa of Ascaris equorum ${ }^{3}$ and (?) Cystidicola farionis, ${ }^{4}$ have been shown also to be of two sorts. In all these cases the two kinds of spermatozoa are very similar to each other, so much so that the differences were long overlooked by keen observers. In Terschellingia polaris the differences in the male generative cells are of an extremely striking character, and seem to lend countenance to the earlier suggestion of the writer, that spermatocytes may in their reduction division produce polar bodies or their true homologues, abortive spermatozoa. We realize now that there has been too large a degree of assumption in taking it for granted that all four of the spermatozoa quartette are equivalent simply because they are similar in form and size. A careful examination of their structure is revealing important differences.

Very possibly it is a comparatively minor phenomenon that the polar bodies of eggs remain attached to their larger functional companion cell. Taking this view, three of the four companion-spermatozoa may, without violence, be regarded as homologues of the "polar bodies." Whether they are functional or not is a matter that may be considered quite apart from their history or structure. Generally speaking we know little or nothing about their relative "potency." That all, or any definite fraction of them are functional is more or less pure supposition. What we know is that some of them are functional-perhaps all, perhaps not.

Though these things are thus far outside our field of experience, they constitute problems that seem certain soon to be attacked from the experimental side, by following the history of specific members of the spermatid quartette; and one object of this note is to call attention to the fact that the free-living nematodes offer an attractive field for such work.

${ }^{2}$ Rhabdias bufonis (Schrank 1788) S. and H. 1905 = "Rhabditis nigrovenosa."

"Ascaris equorum Goeze 1782 = "Ascaris megalocephala."

"(?) Cystidicola farionis Fischer 1798 = "Ancryacanthus cystidicola." 
Incidentally there is raised the very interesting question whether Terschellingia polaris may not be the free-living form of a dimorphic species having a parasitic stage in some higher antarctic form. It is the writer's impression, based on a very considerable amount of observation, that numerous free-living nematode forms, marine as well as land and fresh water, belong to such dimorphic species.

The following diagram illustrates the nature of the formula used in the tabulation of the various necessary measurements:

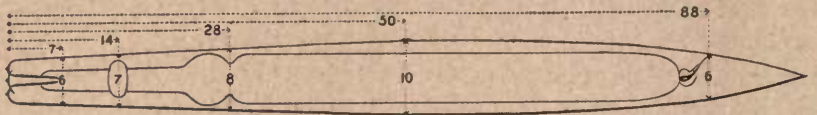

Fig. 2. Diagram of the descriptive decimal formula used for nematodes; 6,7 , $8,10,6$ are the transverse measurements, while $7,14,28,50,88$ are the corresponding longitudinal measurements. The formula in this case is:

7. 14. 28.50 .88 .

6. 7. 8.10 .6 .

The unit of measurement is the hundredth part of the length of the body, whatever that may be. The measurements become, therefore, percentages of the length. The absolute length is given in millimeters as a final non-paired term.

The measurements are taken with the animal viewed in profile; the first are taken at the base of the pharynx, the second at the nerve-ring, the third at the cardiac constriction or end of the neck, the fourth at the vulva in females and at the middle $(M)$ in males, the fifth at the anus. The formulae represent the average of several specimens.

When the specimens were received from Mr. James Murray, the biologist of the Shackleton Expedition, they were in formalin. They were next treated with cold concentrated solution of mercuric chloride, then stained with Mayer's acid carmine and finally examined in balsam. It is well to bear these facts in mind in reading the measurements, as both the relative and absolute measurements vary somewhat with various methods of fixation and preservation. The number of specimens and their state of preservation is noted at the end of each description, and from the data a rough guess may often be made as to the abundance of the species and the relative frequency of the sexes.

By the use of suggestive conventional signs the formulae are made to convey considerable additional information. Thus the formula on $\mathrm{p}$. 7 indicates that the cuticle is traversed by rather coarse transverse striae, ${ }^{5}$

- Formula line of short dashes. See table, p. 7. 
which are resolvable into rows of dot-like markings ${ }^{6}$ modified on the lateral fields, ${ }^{7}$ where there are distinct wings to the cuticle, ${ }^{8}$ one on each side of the lateral lines. The excretory pore is located near the lips, ${ }^{9}$ and the tail end is armed with caudal glands and a spin-

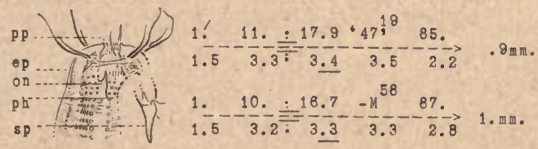
neret. ${ }^{10}$ The oesophagus has a posterior or cardiac bulb two-thirds as wide as the base of the neck. ${ }^{11}$ The two ovaries are symmetrically arranged, reflexed, and occupy 19 per cent of the length of the body. ${ }^{12}$ And, similarly, the male internal sexual organ is single and outstretched, and occupies 56 per cent of the length of the body.

Among the dredgings at Ross Island were three containing nematodes which furnished material for this report. These three were marked: (1) "Bay, Cape Royds, in 10 to 20 fathoms of water, May 31, 1908;" (2) "Cape Royds, in 25 to 50 fathoms of water, July, 1908;" (3) "Bay, in 13 fathoms of water, April 30, 1908." These are referred to in the following descriptions as, (1), Bay, Cape Royds; (2), Cape Royds; and (3), Bay.

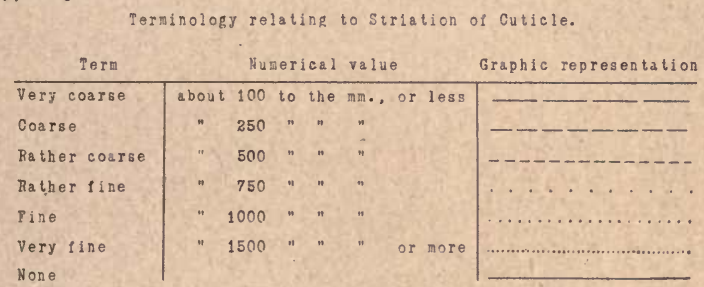

The camera lucida sketches are all made to the same scale, namely, a magnification of 750 diameters, except in the case of Thoracostoma, which was drawn at 400 diameters. The sketches may be relied upon as accurate. Information in the keys is not repeated elsewhere.

B Dots above and below the line between the second and third terms.

7 Modified dots outside those just mentioned in 6 .

- Lines above and below the formula line between the second and third terms.

${ }^{9}$ Oblique line near the pharyngeal terms of the formula.

${ }^{10}$ Angular mark at right-hand end of formula.

11 Underscoring the third diametral measurement, thus indicating the presence of a bulb. Length of mark indicates the size of the bulb.

${ }_{12}$ Curved marks before and after 47 , and 19 used as an exponent figure.

NoTE: Absence of any particular mark indicates the absence of that particular feature so far as at present known. 
TAIL CONOID, THEN CYLINDROID, AT LEAST IN PEMALBS

Posterior(cardiac)oesophageal bulb present

Butb oblate $\ldots \ldots \ldots \ldots \ldots \ldots \ldots \ldots \ldots \ldots \ldots \ldots \ldots \ldots \ldots \ldots$ - Terschellingia polaris

Bulb pyriform

Nusculature of bulb broken into 2 parts.;

striae resolvable into elongate markings

Wings (interrupted striae) inconspicuous .... ' $f$ ' Chromadora meridiana 14

Wings " " pronounced ......m 'spilophora edentata 18

Nusculature of bulb unbroken; striae resolve

into dotlike markings, at least on head

Strise difficult of resolution............. Spira septentrionalis 1

Striae easy to resolve

Cardiac bulb three-fourths as wide as neck .-m' Spilophora antarctica 17

Cardiac bulb one-hall as wide as neck ......-m- 'f' Aplectus antarcticus ' 3

Posterior(cardiacloesophageal bulb none

kmphide almost invisible transverse slits ...... in Anticoma subsinilis 2

Anphids circular, usually easy to see

Pbarynx Plectoid, long, resembling oesophagus ...-m-f' Aplectus antarcticus

Pharynx short, often small

Intestinal cells clearly reticulated ........ Sabateria antarctica

Intestinal cells not clearly reticulated

Oesoph. trifle larger fm.behind nervering ...-m-f Austronema spirurum

Oesophagus plain

Renette distinctly developed

Somatic setae as long as body is wide ....-m- Monbystera pilosa

Somatic setae none or inconsplcuous .......-1 Monhystera uniformis

Renette inconspicuous or none

Chromatin bodies, 1 in front of each amph'd-m-1 Monhystera frigida \&

Chromatin bodies n'r.amphids none or falnt

Contour crenate,esp.ventral side of tail $-\mathbb{m}-f$ Monbystera polaris 10

Contour entire ..................... Konhys. septentrionalis 11

TAIL CONOID, NO PART CYLINDROID

Posterior(cardiac) oesophageal bulb present

Bulb elongated, not clearly subdivided; renette

cell probably ellipsoidal .............. -lll-f- Laxus septentrionalis 23

Bulb pyriform, musculature broken into unequal

parts; renette cell elongated

Contour on ventral side of male tail serrate. $-m$ ' $f$ ' Spilophora serrata 18

Contour entire

Renette cell $1 / 4$ wide as long;Phar.bulb faint.- $\pi$ Chromadora polaris 15

Renette cell $1 / 8$ " " " " "pronounced-m ' $\mathrm{f}$ ' Euchr. septentrionalis 19

Posterior bulb none $(12,13)$, or a mere swelling

Striae of minute more or less elongate elenents

Dorsal tooth with no minute dental opponents.-m $\varphi^{\prime}$ Euchromad. antarctica 20

Dorsal "with 1 (22) or 2 (21) Subr. " "

Tail regular, terminus about $1 / 2$ wide as base. ' $p$ ' Euchromad. denticulata 21

Tarl 1 suddenly narrowed on Vent.side n'r.anus $-\pi$ ' $f$ ' Euchromadora meridiana 22

Striae appear impossible of further resolution

Lips strong, chltinous, acute, conoid, eversible. Axonolainu's polaris 24

Lips not acute, \pm mobile but not eversible

Cardiac region conspicuousiy non-staining....-m-1 Monhystera meridiana 12

Cardiac region of average nature.

Unicellular lateral glands and pores absent $-\pi-1$ Monnystera antarctica 13

Unicellular lateral glands and pores present-m-'f' Thoracostoma polare 25 
IEY, BASED ON MALE CHARACTERS

PRE-ANAL SUPPLEMENTARY ORGANS PRESENT

19 New Species and 2 New Genera

Suppl.organs 10 , submedian, in 2 rows of 5 each ...-19 Thoracostoma polare 25

Supplementary organs in a single ventral row

Organs 3 (or 1 in Anticoma), faint in Sp.serrata

Form tubular, $1 / 5$ as wide as long

Length $2 / 5$ body Diam.; just in front of spicula

a $-\pi$ Anticoma subsinilis 2

length $4 / 5$ "; Ant.Org. 1 tail-Igth. Irm.anus-m-' $f$ " Aplectus antarcticus a

Form companulate, very minute, deep as wide ....- - ' $f$ ' Spilophora serrata 18

Organs 7, equidistant, faint in Sp.edentata

Form cup-like, as deep as body wall is thick

Cardiac bulb present ..................... Chromadora polaris 15

Cardiac bulb reduced to a faint swelling .....-m ' $f$ ' Euchromad. antarctica 20

Form papilloid, row 3 body-widths long ......... ' $f$ ' Spilophora edentata 18

PRE-ANAL SUPPLEMENTARY ORGANS ABSENT

Accessory oieces to spicula absent or faint

Spicula suddenly hookshaped at proximal end ...- -1 -1 Austronema

Spicula of usual form, cephalated by expansion

Form arcuate,proximae ventral to body axis ....- ( $f$ ' Spilophora

Form somewhat L-shaped, Prox.dorsal to B'dy.axis $-m-1$ Monhystera

Accessory piece or pieces to spicula present

Apophysis to accessory piece bending backward

Proximal ends of spicula not cephalated .......-1in-terschellingis polaris 5

Proximal ends of spicula cephalated

Cephalated by constriction .............. - Monhystera antarctica 13

Cephalated by more or less expansion

Form more or less L-shaped

Structure rather robust .............. Monhystera polaris 10

Structure " frail ................... - Nonhystera frigida 9

Forn nearly straight, or arcuate

Spicula nearly straight ................m- Monhystera pilosa 7

Spicula arcuate

Ligth $21 / 2$ times anal body diameter ........ - Monhystera uniformis 8

Ligth $1 \frac{1}{3}$ " " ",not very slender-m--f-Laxus septentrionalis 23

Apophys is to accessory pieces absent

Spicula cephalated by contraction ..........m ' $f$ ' Iuchromadora meridiana 22

Spicula cephalated by expansion

Cephalum set off by a constriction .......... ' $f$ ' Buchr. septentrionalis 19

Cephalum not set of f by a constriction ....... (m) Spilophora antarctica 17

\section{KEY TO SIGNS}

4 ', ovaries 2 , symmetrical, reflexed. - - -, testes 2 , one extending each way.

$-1-$ " 2 " outstretched. ' $m, " 2$, one only outstretched.

-1 , ovary 1 , outstretched forward. $-\mathbb{n}$, testis 1 , outstretched forward.

\section{Abbreviations}

1b, lip or lipregion

on, pharyngeal tooth

an, amphid

1c. locule of cuirasse pp , labial paplllae

ph, pharynx

oe, oesophagus st, cephallc setae

sp, spinneret

ep, excretory pore

pc, cordiform piece 


\section{DESCRIPTIONS OF THE SPECIES}

\section{CHARACTERS COMMON TO ALL THE SPECIES}

Disregarding Thoracostoma, which is exceptional in the large size of the caudal glands, the possession of eye-spots, of distinct dermal pores and of oesophageal glands, and also in the possession of relatively very strong spicula with a compound framework, it may be said that the following characters are common to all the species here described:

There is no median oesophageal bulb, and no pre-rectum. The tail is of approximately the same form in both sexes, and in all cases is supplied with a rather simple spinneret, and with caudal glands - the latter confined to the tail. The eggs, so far as known, are smooth and comparatively thin-shelled, and are deposited before segmentation begins.

All known males have equal spicula of simple framework, and all are without bursa. There are no eye-spots. The cuticle is colorless, or nearly so, is without distinct pores, and is destitute of longitudinal striae except obscure indications in Spilophora serrata and Chromadora meridiana, where the secondary elements of the cuticle arrange themselves also to a certain extent in longitudinal lines.

The renette, when present, has its cell behind the cardiac constriction, except in Anticoma. Glands in the interior of the oesophagus have been seen only in Thoracostoma, and possibly, though these latter are of another character, in Monhystera frigida and polaris. The intestinal granules give rise to a tessellation only in Anticoma and Laxus. The spicula are arcuate, except in some Monhysteras; and cephalated except in Anticoma, Terschellingia and Euchromadora antarctica. There are no male papillae except in Anticoma and Monhystera antarctica. Male supplementary organs occur only in Anticoma, Chromodora polaris, Euchromadora antarctica, Aplectus, Thoracostoma, and in Spilophora serrata and edentata. The musculature of the oesophagus is fine except in Thoracostoma and Monhystera polaris, frigida and pilosa, though it is somewhat coarse in the bulb of Laxus.

If the reader will add these characters to those given under each species heading, and will utilize in a similar way the common characters given in the keys and generic descriptions, he will find himself in possession of a very detailed description of each species, covering a number of new and interesting anatomical features. 


\section{SPIRA, Bastian, $1865^{13}$}

1. Spira septentrionalis, n. sp. The striae, invisible except near the head, are resolvable into rather irregular dots. The three lips are without papillae. The

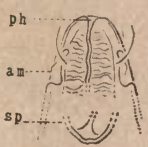
\begin{tabular}{llllll}
1 & 7.4 & 10.6 & $y$ & 92.3 \\
\hdashline & 1.8 & 2. & 1.8 & 1.7 & $1.4 \mathrm{~mm}$.
\end{tabular}

neck is cylindroid posteriorly, convex-conoid anteriorly. The amphids, seen thus far only in dorso-ventral view, have a central elevation, probably appearing as a "fleck" in the surface view, and are about half as wide as the corresponding diameter of the head. The oesophagus is half as wide as the neck, and ends in a rather obscure bulb containing an indistinct valve, two-thirds as wide as itself. No distinct cardia was seen. The intestine, separated from the oesophagus by a collum about half as wide as the neck, becomes at once three-fourths as wide as the body; it is but a few cells in girth, and the cells contain few or no granules. The rectum is conspicuous, the posterior lip of the anus prominently elevated. From the anus the tail tapers for two-thirds of its length, then becomes cylindroid to the swollen terminus, which is one-fifth as wide as the base. The caudal glands were not clearly seen, but are apparently arranged in a loose tandem in the anterior third of the tail. The female reproductive organs are probably double and reflexed.

Habitat; remarks. Cape Royds. Described from a single somewhat shrunken specimen.

\section{ANTICOMA, Bastian, 1865}

2. Anticoma subsimilis, n. sp. The thin cuticle is almost invisibly striated. On each lateral line there is a row of five somewhat curved cervical setae,

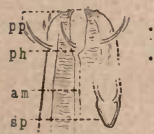

$$
\begin{array}{c|ccccc}
.5 & 12.5 & 26 . & 6 \mu^{51} & 88 . & \\
.8 & 2.2 & 2.6 & 2.7 & 2.5 & 1.5 \mathrm{~mm} .
\end{array}
$$
each having a length equal to onefourth the corresponding width of the neck. These two rows begin at a distance from the anterior end equal to nearly three times the width of the head, and have a length equal to the corresponding diameter of the neck. The cuticle becomes thicker at the lips, which bear a circlet of six papillae. The oesophagus is cylindroid in the anterior part, but becomes conoid posteriorly. The mobility of the lips is proved by the nature of the ingested food. Seen dorso-ventrally the pharynx appears more nearly cylindroid than when seen laterally. Seen laterally it tapers so that finally it is one-fifth as wide as the corresponding part of the head. The lining is rather thin, but refractive. The cardia, one-half as wide as the neck, is conoid with a short cylindroid extension. The rather thick-walled intestine, separated from the oesophagus by a collum three-fifths as wide as the neck, becomes at once three-fourths as wide as the body, and is about six cells in girth. The intestinal cells contain numerous small, uniform, distinct granules. The posterior lip of the anus is elevated. The caudal glands are packed together

${ }^{13}$ The abbreviations used in lettering the sketches, which represent the anterior and posterior extremities respectively, are explained near the foot of page 9 . As a rule the information given in the sketches is not repeated elsewhere. As far as possible the sketches are derived from typical specimens. 
in the anterior two-fifths of the tail. The elongated renette-cell, two and onehalf times as long as the body-diameter, and one-fourth as wide as long, lies a little in front of the cardia, and is not reflexed. The excretory pore is somewhat behind the pharynx. Distinct nerve-cells, more numerous behind the ring, are arranged (at least in front of the ring), in six longitudinal groups. The cylindrical part of the tail is about one-tenth as wide as the base.

The slender, uniform, but rather strong spicula, as wide as the narrowest part of the tail, are twice as long as the anal body-diameter, and lie with their proximal ends dorsal to the body axis. The proximal eighth of each spiculum is separately more or less arcuate, so that one may speak of a curved cephalum or proximal end. Two separate accessory pieces, rather frail, bent at the distal end, one-sixth as long as the spicula and parallel to them, form a rather close collar round the distal ends of the spicula. On each side of the body there are four equidistant, ventro-submedian, pre-anal, papilla-like setae, one-sixth as long as the body-diameter, extending in a row from near the anus to near the supplementary organ. There are about three ventro-submedian post-anal setae on each side of the middle part of the tail.

Habitat; remarks. Cape Royds; Bay, Cape Royds. One adult and several immature somewhat shrunken specimens. This species differs from A. similis in having five pectoral hairs, longer setae, a sub-cylindroid pharynx. and an oesophagus without expansion behind the nerve-ring

\section{APLECTUS, new genus}

Species of this newly proposed genus have the general form of Plectus, but have the pharynx less definitely developed, and the renette-cell farther back and not reflexed. They differ also in having nearly obsolete lips, and a nearly cylindroid oesophagus whose small cardiac bulb is without a three-fold striated valve. The spinneret also differs from that of Plectus, as shown in the sketch. The male supplementary organs in the two genera also differ materially. These facts together with the marine habitat and the relative abundance of the males of Aplectus, appear to make necessary a separate genus for the reception of this antarctic species. The following is the type species.

3. Aplectus antarcticus, n.g., n. sp. The rather thin cuticle is traversed by about 700 duplex striae, which give the contour an obscurely doubly crenate

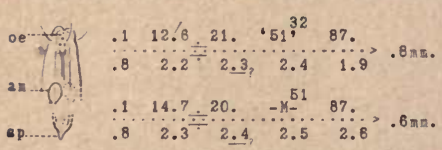
appearance. Two wings, separated by a distance about equal to the width of an annule, begin near the middle of the neck and end near the middle of the tail. Cervical setae, similar to the cephalic setae, occur one or two on each lateral line; and on the male tail there are setae, one-third to one-eighth as long as the body is wide. The lips are very small and difficult to observe. The pharynx, very inconspicuous and hard to distinguish, is probably double, the narrow, tubular, anterior part extending to a little behind the amphids, the posterior part, which closely resembles the oesophagus, extending nearly balfway to the nerve-ring where there is a break in the musculature. In the lateral view there are seen close behind the cephalic setae, under the cuticle, very minute 
bodies that stain, of which those most clearly seen were the dorsal and ventral ones. Probably there is a circlet of these bodies. Just behind these stained bodies, that is, a little behind the setae, there is an appearance as if of a small quadrate cavity, one-third as wide as the head. This is not a cavity, however. Its posterior limits are a trifle more than a head-width from the anterior end. The amphids are variable in size and form, being considerably larger and slightly more elongated in the male. The posterior border appears interrupted. The slender cylindroid oesophagus, is probably very obscurely Rhabditoid, and ends posteriorly in an obscurely rhomboidal to pyriform bulb or swelling, three-fifths to two-thirds as wide as the neck. The cylindroid cardia, one-fourth as wide as the body, and twice as long as wide, is really a modified part of the intestine, and is composed of small strongly staining cells. The thick walled intestine, separated from the oesophagus by a collum one-fourth to two-fifths as wide as the neck, is few, probably four, cells in girth. These cells contain numerous, indistinct, rather fine and uniform granules.

The tail, tapering from the anus, is cylindroid in the posterior two-fifths and ends in an almost imperceptibly swollen apiculate terminus one-third to one-half as wide as the base. The caudal glands are arranged in loose tandem in the anterior half of the tail; their ampullae are distinct and no wider than the ducts, but stain more strongly. The elongated renette-cell, which is difficult to observe, is as long as the body is wide, and one-fifth as wide as long, and occurs at a distance behind the neck equal to eight times the width of the body. The very slender duct, invisible except where stained, empties through a pore apparently opposite the nerve-ring. The nerve-ring surrounds the oesophagus rather squarely and is accompanied by somewhat distinct nerve-cells rather definitely grouped. From the inconspicuous, small continuous vulva the small vagina leads inward halfway across the body to the straight uteri. The rather elongated eggs are two to two and one-half times as long as the body is wide, and fully one-third as wide as long. The broad ovaries, cylindroid, but tapering near the extremities, reach three-fourths of the way back to the vulva, and contain about twelve ova, arranged rather irregularly. The spermatozoa in the uterus are of such a size that it would take at least seven or eight side by side to equal the body-diameter.

The arcuate, rather strong, sub-slender, sub-acute spicula, one and one-fourth times as long as the anal body-diameter, are slightly cephalated by expansion, the cephalum being set off by a broad shallow constriction, and lie with their proximae dorsal to the body-axis. There are two rather strong, sub-slender, slightly bent accessory pieces, parallel to the spicula then bending away, the applied part being one-fourth as long as the spicula. The receding part is arcuate in the same direction as the spicula, and from its end there passes a strand of muscle to the ventral body-wall behind the anus. The ejaculatory duct is onefourth, the vas deferens and cylindroid testes one-half as wide as the body. The blunt blind ends of the testes are two-thirds of the neck's length from the cardia and one and one-half times the tail's length from the anus, respectively.

The protrudable, sub-equidistant supplementary organs are separated by a distance equal to nearly one and one-fourth times the body-diameter, and the posterior one is located at a distance in front of the anus equal to twice the anal body diameter. They are rather straight but have the distal third bent ventrally, and the distal end flattened and pulled out posteriorly into a spur, or 
toe, which always remains outside the body. The entire profile contour is like that of a high boot with its leg bent backward. The projecting part is twice as long as the diameter of the tube, and is roughened at the end with ten or twelve exceedingly minute striae or warts. The proximal ends of the organs are rounded and not cephalated.

Habitat; remarks. Bay, Cape Royds. About fourteen females and six males, in good condition. The sketch is that of a female.

\section{SABATIERIA, de Rouville, 1903}

4. Sabatieria antarctica, n. sp. Striae about 800 , resolvable with great difficulty into dot-like markings. There are no lips. The tubular pharynx, ex-

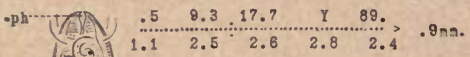
tending to opposite the posterior borders of the amphids, as shown by a slight accentuation of the lining, is surrounded by an almost imperceptible, slightly unsymsp..... (U) metrical pharyngeal bulb three-fourths as wide as the head. At the somewhat oblique nerve-ring the oesophagus has a diameter equal to one-third the width of the corresponding part of the neck, but swells posteriorly to two-thirds the width of the base of the neck. The rather cylindroid cardia is one-third as wide as the neck, and one and one-half times as long as wide. The thick-walled intestine, separated from the oesophagus by a collum two-thirds as wide as the body-diameter, soon becomes three-fourths as wide as the body and is about two cells in girth. From the elevated posferior lip of the anus the rather prominent rectum extends inward and forward a distance equal to the anal body-diameter. From the anus the tail tapers for three-fourths of its length, then becomes cylindroid to the slightly swollen terminus. The caudal glands are probably small and near the anus.

Habitat; remarks. Cape Royds. Described from a single young specimen in fair condition.

\section{TERSCHELLINGIA, de Man, 1888}

5. Terschellingia polaris, n. sp. The cuticle is traversed by about 700 striae, plainly visible near the extremities only. Narrow double wings, having a width about equal to that of two annules of the cuticle, begin near the middle of the neck. The central raised body, or "fleck," of the amphid stains about as strongly as the nuclei else-

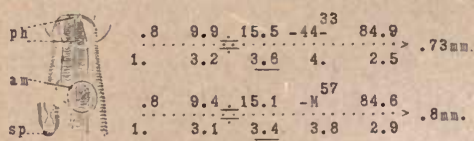

where in the body. Very minute, inconspicuous papillae, probably six in number, occur on the confluent lips. The vestibule is very minutely longitudinally striated. About halfway to the amphids there is a break in the musculature of the oesophagus; the pharynx probably extends to this point. The conoid neck contains a cylindroid oesophagus. which, near the nerve-ring, is one-third as wide as the neck, but ends in a bulb five-sixths as wide as the base of the neck. The lining of the oesophagus is distinct, its most prominent optical expression being a single refractive line. The cardiac valve causes a slight, simple modification in the lining, one-eighth as wide as the bulb. The more or less thick-walled in- 
testine is separated from the oesophagus by a cardiac collum one-sixth as wide as the neck, and soon becomes two-thirds as wide as the body. The first few cells of the intestine, at the cardiac region, are very small, with relatively large nuclei that stain strongly. The intestine is separated from the rectum by a pyloric collum one-fourth as wide as the corresponding portion of the body. From the anus, whose posterior lip is elevated, the chitinized rectum leads inward and forward a distance equal to the length of the anal body-diameter. The granules of the intestinal cells are small and scarce.

The tail tapers from the anus and ends in a slightly swollen terminus. A few small, stiff, cylindroid, blunt caudal setae are to be seen, mostly about one-fourth as long as the terminus is wide. The broadly saccate caudal glands, arranged in a loose tandem in the anterior fourth of the tail, empty through distinct ducts and elongated, narrow ampullae. What appears to be an irregularly ellipsoidal rentte-cell is located at a distance behind the neck equal to the width of the body; it is half as long as the body is wide, and three-fourths as wide as long. The nerve-ring, which surrounds the oesophagus more or less obliquely, is accompanied by distinct nerve-cells definitely grouped, both in front of it and behind, and extending backward to near the cardiac bulb. From the small and inconspicuous, but more or less elevated vulva, the conoid, non-chitinized vagina leads inward at right angles to the ventral surface two-fifths the way across the body. The eggs have a length nearly one and three-fourths times that of the body-diameter, appear about half as wide as long, and have been seen in the uterus one at a time. The medium sized, more or less tapering ovaries contain fifteen to twenty developing ova, for the most part flattened and arranged single file.

The more or less slender, sub-acute, uniform, slightly yellowish spicula have a simple and rather strong framework, and are one and one-fourth times as long as the anal body-diameter. The proximal ends appear to lie opposite the body axis. The triangular, blunt accessory pieces have a simple and rather frail framework; the part applied to the spicula is one-fourth as long as they, while the tapering apophyses lie a little backward and end opposite the body-axis. The ejaculatory duct is one-fourth, and the testis one-half, as wide as the body. The comparatively few (about a dozen) primary spermatocytes occur in the testis in single file, then come three pairs double file-these latter relatively huge. That is to say, the primary spermatocytes increase much in size and then divide transversely into very unequal parts, a small distal part and a large proximal part, and these two unequal parts divide almost simultaneously in the longitudinal direction. Thus there appear four cells arranged in two pairs side by side, a small strongly staining pair with inconspicuous nuclei, and a large pair which do not stain except in their relatively small nuclei which show about seven small more or less globular chromosomes. These two divisions represent the usual reduction divisions, and give rise to spermatozoa of very different size and appearance. The phenomenon is reminiscent of the formation of the polar bodies. In some specimens the smaller cells, those that in their appearance remind one of polar bodies, appear as if divided a second time, but there is uncertainty about this. It is the writer's intention to prepare a separate report on this species and its spermatogenesis.

Habitat; remarks. Cape Royds; Bay, Cape Royds. Numerous specimens, mostly somewhat shrunken. 


\section{AUSTRONEMA, new genus}

The single species for which this new genus is proposed has many of the characters of Monhystera, but differs in so many important respects as to call for separate classification. The principal differences of generic value are the possession by Austronema of an oesophagus altered in the posterior half, a well developed ventral gland, special lateral cells, hamate spicula without accessory pieces, and glandular (?) organs associated with the spicula. Other minor differences exist, such as the occurrence of the special group of setae near the middle of the tail. The following is the type species.

6. Austronema spirurum, n. g., n. sp. The cuticle is traversed by exceedingly minute transverse striae. The lips are confluent. The conoid neck contains a

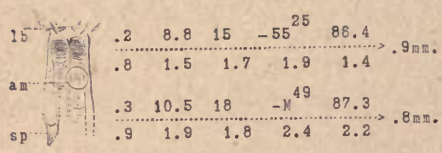
cylindroid oesophagua, whose diameter measured near the nervering is one-half as great as that of the corresponding portion of the neck, but which finally becomes two-thirds as wide as the base of

the neck. There is a break in the musculature of the oesophagus just behind the nerve-ring, and behind this break the refractive nature of the lining is somewhat different, and, moreover, in many of the preserved specimens the diameter of the oesophagus from this point onward is suddenly somewhat greater. There is a conoid to cylindroid cardia about one-half as wide as the base of the neck. The very thick-walled intestine, two to four cells in girth, becomes at once threefourths as wide as the body. For a short distance behind the cardiac collum, namely, for a distance about equal to two-thirds the body width, the tissues of the intestine do not stain. The lining of the intestine is refractive and distinct, so that the almost imperceptibly zigzagged lumen can be readilv followed. The intestine is separated from the oesophagus by a collum two-fifths as wide as the base of the neck. The size of the numerous, yellowish, uniform granules contained in the intestinal cells varies in the different parts of the intestine; they are much coarser posteriorly than anteriorly. From the anus, the posterior lip of which is elevated, the chitinized rectum extends inward and forward a distance equal to the anal body diameter.

The tail tapers in such a manner that at the middle its diameter is about half as great as at the base. Its terminus is about one-fifth as wide as its base. The ellipsoidal caudal glands are arranged in a loose tandem in the anterior third of the tail. Very short, stiff, inconspicuous, ventrally submedian caudal setae occur on cach side of the tail, two on the anterior half of the tail, and three near the middle and close together. and finally, one or two on the cylindroid, narrow, posterior half. The pyriform to ellipsoidal granular renette-cell is located at a distance behind the base of the neck equal to one and one-half body-diameters. It is one and one-half times as long as the body is wide, and one-third as wide as long, and presses the intestine considerably to one side. It does not appear to have any companion cell. The medium sized nerve-ring surrounds the oesophagus somewhat squarely, and is accompanied by distinct nerve-cells extending well back toward the base of the neck. From the rather small, but rather con- 
spicuous, elevated vulva, the well developed, tubular, muscular vagina extends inward and obliquely forward. It is about twice as long as the corresponding body-diameter, and about two-thirds as long as the uterus. The eggs are about as long as the body is wide, and three-fourths as wide as long, and have been seen two or three at a time in the uterus. The ovary is of medium size, and tapers so as to become narrow. It contains many ova, arranged single file-somewhat irregularly so near the uterus.

The slender, more or less uniform spicula taper from the middle toward the proximal ends, and present the striking peculiarity of being strongly curved at the distal extremity, forming a hook across which there is a thin, transparent membrane. They are one and three-fourths times as long as the anal body-diameter, and arc so arranged that their proximal ends appear as if lying to the dorsal side of the body axis. Their yellowish framework is rather strong, and the proximal two-thirds may sometimes be seen to be nearly straight. At the extreme end the spicula have a very minute recurved apiculum or point. Two (?) pairs of ellipsoidal granular unicellular glands (?) occur some distance in front of the spicula. This species has the head of a Monhystera but differs. in having the oesophagus altered in the posterior half, a well developed ventral gland, special lateral cells, hamate spicula without accessory pieces, and the tail with a group of small setae near the middle.

Habitat; remarks. Cape Royds. The five females and five males examined were in fair condition.

MONHYSTERA, Bastian, 1865

The following are characters common to all the species of Monhystera here described.

Cervical and somatic setae none or scattered and short, except in $M$. pilosa. Neck conoid, but cylindroid in the posterior half in $M$. pilosa, and $M$. meridiana. Tail tapering from the anus or a little in front of it. Oesophagus somewhat cylindroid without swellings of any kind, for the most part about half as wide as the neck, but finally three-fifths to three-fourths as wide as the base of the neck. Cardia present, except in $M$. frigida, hemispherical to cylindroid, and one-fourth to onehalf as wide as the base of the neck. Intestine separated from the oesophagus by a collum one-third to one-half as wide as the neck, becoming almost at once about three-fourths as wide as the body, its walls thick and two to four cells in girth, and the lining usually so refractive that the lumen is a rather distinct feature. Granules of the intestinal cells numerous, fine and uniform. Rectum of about the same length as the anal body diameter. Caudal setae none or inconspicuous except in $M$. pilosa. Lateral fields one-third, more rarely one-half, as wide as the body. Renette unknown except in $M$. pilosa and $M$. uniformis. Nerve-ring of medium width, surrounding the oesophagus rather squarely, the nerve cells in its vicinity usually rather diffusely arranged. Vulva small or of medium size and in these species not very conspicuously elevated except in $M$. uniformis. Vagina one to two times as long as the body is wide, and extending inward and forward.

7. Monhystera pilosa, n. sp. Striae about 1700 . There are numerous long, very slender, flexible cervical and somatic setae, of ten arranged in pairs, one member of 


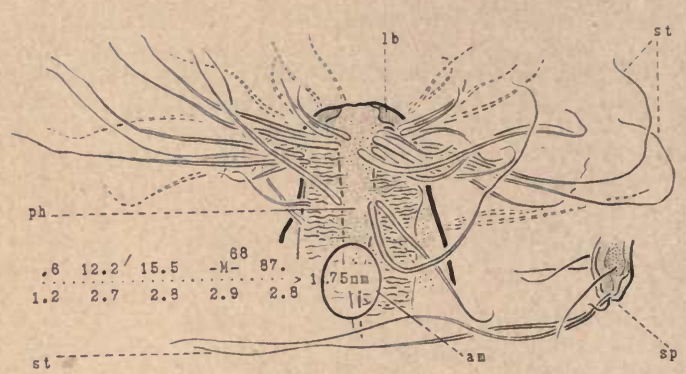

the pair in front of the other, on the sub-median lines. There are probably three lips, bearing six minute setose papillae ar ranged in a single circlet. The lining of the oesophagus is very "distinct, and the musculature

rather coarse. The granules of the intestine are yellowish brown. Toward the posterior extremity, for some distance in front of the anus, there is a strong development of lateral cells. which do not appear to exist elsewhere in the body. These cells extend forward toward the middle of the body, and have been traced no farther. Their size, which is variable, is such that two or three occur side by side in the lateral fields. The tail is cylindroid in its posterior threefifths, with a width there one-eighth as great as that of its base, and has a slightly swollen terminus. The caudal ducts, of which two were seen, are narrow and distinct. From the excretory pore at the end of the third fifth of the neck there leads inward a very narrow duct whose length is nearly equal to the thickness of the cuticle. The duct leading thence back to the renette cell is, however, of considerable width-about one-fourth as wide as the oesophagus-and is readily traced back to a point opposite the anterior part of the intestine, where the renette cell pushes the intestine to one side. The spicula are uniform, with a width near the distal extremity one-sixth as great as the corresponding body width. The single accessory piece, parallel to and close to the distal thirds of the spicula, has an apophysis tapering to an obscure and slightly recurved point. This apophysis lies at right angles to the spicula, and then curves forward a little. The ejaculatory duct is one-third as wide as the body, the vas deferens considerably wider. The blind end of the anterior, larger testis is disposed in one or two coils.

Habitat; remarks. Bay, Cape Royds. The single specimen examined was in good condition.

8. Monhystera uniformis, n. sp. Apparently there are three very obscure confluent lips. The amphids when seen in the dorso-ventral view seem to be well

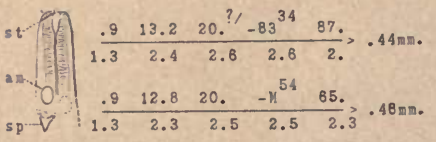
chitinized, and appear deepest in the anterior part, where they are one fifth as deep as the head is wide. The cardia is twice as long as wide. The cylindroid part of the tail is one-third to one-fourth as wide as the base. Broadly saccate caudal glands are found in the anterior half of the tail. At a distance behind the neck equal to three to four body-widths there is an ellipsodial renette cell with a large nucleus. This gland-cell is one and one-half times as long as the body is wide and half as wide as long. The location of the excretory pore has not been made out with certainty; 
possibly it is just behind the nerve-ring. The small, weak, tubular, non-chitinized vagina leads into a uterus which is five times as long as the body is wide. The eggs are about four times as long as the body is wide, and one-fifth as wide as long. The medium sized cylindroid ovary contains about twenty-five ova, arranged somewhat irregularly. The tail of the male is like that of his mate except that it is conoid nearly to the terminus. The very slender uniform spicula are two and one-half times as long as the anal body diameter. Their framework is simple, and the proximal ends lie somewhat dorsal to the body axis. The single accessory piece is frail, its framework simple; the applied part being one eighth as long as the spicula, the blunt, backward pointing apophysis being onefifth as long as the anal body diameter and having its proximal end opposite the body axis. The ejaculatory duct is one-third, the testis two thirds, as wide as the body.

Habitat; remarks. Cape Royds. Six females and three males, in fairly good condition.

9. Monhystera frigida, n. sp. About 1400 striae give to the margin a crenate contour. The very inconspicuous lips are probably three in number. The amphids appear circular, but are really regular spirals of one and one-half winds, having a raised transverse ridge extending part way across. A deeply staining nucleus, of the same size and character as the

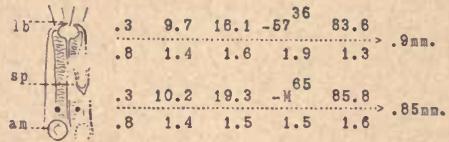
nuclei of the nerve cells near the ring, occurs immerliately in front of each amphid. In the posterior end of the oesophagus there are two, possibly three, elongated, granular gland-cells, quite as long as the body is wide. The rectum is prominent. The vagina is small and weak. The eggs are about four times as long as the body is wide. The rather narrow, cylindroid ovary contains twenty or more ova, arranged mostly in single file. The uniform slender, frail, sub-acute spicula are one and one-half times as long as the anal body diameter and lie with their proximae opposite the body axis. The very inconspicuous, very slender and frail accessory piece is bent so that the applied part is one-third to one-fourth as long as the spicula, while the uniform backward bending part is one-fifth as long as the anal body diameter. Its proximal end lies to the ventral side of the body axis. The ejaculatory duct is one-third, the testis two-thirds, as wide as the body.

Habitat; remarks. Cape Royds. The single female and the two males examined were in fair condition.

10. Monhystera polaris, n. sp. The 600 striae give to the margin a minutely crenate contour, most plainly to be seen just behind the anus. The three double,

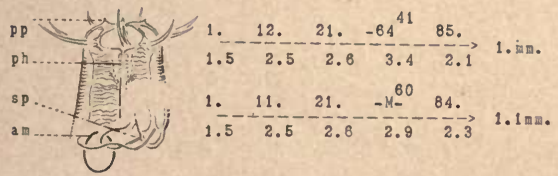

more or less distinct lips are fairly developed. Two innervated papillae occur on each of the lips, six papillae in all. The amphids are sunken, but usually show a well stained projecting margin twothirds as wide as the amphid. A little in front of each amphid is a single somewhat spherical nucleus, one-fourth as wide as the oesophagus, containing granules, 
as do the ganglion cells near the nerve-ring. These two neuclei probably indicate the presence of two nerve cells. They are best seen in the dorso-ventral view. The refractive elements of the lining of the oesophagus, especially in certain aspeets of the head, appear to extend into the pharynx in a peculiar way, as shown in the sketch. The rectum is more or less prominent, and in the male the anus is raised. The tail tapers in such a manner that at the middle it is one-third as wide as at the anus. Its terminus is slightly swollen. Caudal setae appear on the base of the tail.

The vagina extends inward nearly at right angles to the body wall, about half way across the body. The uterus, three to four times as long as the body is wide, contains spermatozoa one fifth as wide as the body. The posterior, rudinentary branch of the uterus, nearly twice as long as the width of the body, also contains spermatoloa. The eggs are nearly twice as long as the body is wide, and one-third as wide as long. The broad tapering ovary contains about twenty ova arranged single file, and as many more packed irregularly in the distal fourth.

The brownish, rather slender, uniform, acute spicula are one and one-third times as long as the anal body diameter. The framework of the spicula is more or less strong, and the proximal ends lie opposite or a little dorsal to the body axis. The single, rather straight and rather frail accessory piece is of a simple character, and has a backward pointing apophysis one-sixth as long as the anal body diameter. The male presents the peculiarity of possessing two testes, an uncommon thing in Monhystera. They are rather wide and of unequal size, the anterior, more or less cylindroid one being considerably the wider. The blind end of one testis is at the cardia, that of the other about one tail-length in front of the anus.

Habitat; remarks. Cape Royds; Bay, Cape Royds; Bay. Seven females and six males, somewhat shrunken. The specimens from the various localities differ slightly in (1) the size and form of the cephalic setae, (2) the length and width of the spicula, (3) the prominence of the amphids, (4) the prominence of the striae on the tail. Diatoms and other unicellular organisms were seen in the intestine.

11. Monhystera septentrionalis, $n$. sp. The lips are confluent. The lining of the oesophagus is rather prominent, and one-fifth as wide as the oesophagus itself. 16 The small and inconspicuous, somewhat ellipsoidal caudal glands are arranged in a loose tandem in the rather muscular vagina is one and one-half times as long as the body diameter. The eggs are elongated, twice as long as the body is wide, and less than half as wide as long. About thirty ova oceur in single file in the gently tapering ovary.

Habitat; remarks. Bay, Cape Royds. Seven good female specimens. The amphids are often a little farther back than illustrated.

12. Monhystera meridiana, n. sp. The striae, about 1000 in number, are responsible for a somewhat crenate contour, more noticeable near the tail. The strongly developed cardiac region, set off by a eonstriction on each side, is as wide as the intestine, and forms a small but distinct segment of the alimentary canal. The vulva is elevated. Eggs twice as long as the body

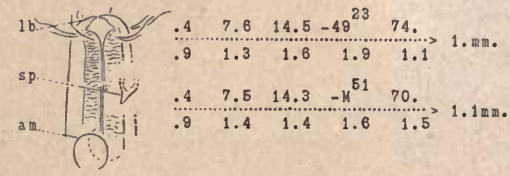


is wide, and one-third as wide as long, occur in the straight uterus. Toward its blind end the narrow, tapering ovary is one-fourth as wide as the body. The tail of the male is conoid in such a fashion that at a distance from the anus equal to four times the anal body diameter it has a width equal to one-fourth the anal body width; thence it tapers very gradually to the terminus, whose width is considerably less than that of the spicula. The slender, uniform, acute spicula are one and three-fourths times as long as the anal body diameter. The ejaculatory duct and vas deferens are one-fourth as wide as the body. Apparently there are two tapering testes, but the end of the posterior one was not definitely seen; it appeared, however, to be eight to twelve body widths in front of the anus. Spermatocytes occur in single file near the end of the anterior testicle.

Habitat; remarks. Bay, Cape Royds. One female and three males in a somewhat shrunken state.

13. Monhystera antarctica, n. sp. The cephalic setae are very minute and diffcult to see. Six excessively minute forward pointing papillae occur, one on each lip. Apparently the pharynx is traversed near its middle by a transverse ridge, and this is the reason why it appears in optical section as if armed with two teeth. From the anus, the posterior lip of which is elevated, the rather prominent, chitinized rectum leads inward. Anal glands are present. The rather large and conspicuous nuclei of the intestinal cells are arranged about a body-width apart. The terminus of the tail is one-fourth as wide as the base. The broadly saccate caudal glands, of which two are larger than the third, are arranged in close tandem in the anterior half of the tail; their ampullae are distinct. The lateral fields are one-half as wide as the body. At a distance behind the base of the neck equal to four times the width of the body there is frequently to be seen in the female a large cell, one-half as wide as the body, with a prominent nucleus. This cell occurs in females only. Though its connections have not been definitely made out, it sometimes seems to empty through a pore a little in its rear. The straight uterus, five to six times as long as the body is wide, contains elongated eggs, as long as the body diameter, and half as wide as long. The cylindroid ovary contains ova arranged in double fileirregularly so toward the blind end.

The tail of the male is a little stouter than that of his mate. The rather frail, slender, uniform, sub-acute spicula, one and one-half to one and three-fourths times as long as the anal body-diameter. lie with their proximae dorsal to the body axis. The single accessory piece, parallel to the distal third of the spicula, and then bending back in a thumb-shaped apophysis, lies with its proximal end opposite the body axis. On the tail there are very faint inconspicuous setose papillaeone ventro-submedian on each side, at the end of the anterior fourth, and two or three sub-ventral just behind the middle of the tail. The ejaculatory duct is onefourth to one-third, the vas deferens and tapering testis one-half, as wide as the body.

Habitat; remarks. Bay, Cape Royds. Numerous slightly shrunken specimens. 


\section{CHROMADORA, Bastian, 1865}

14. Chromadora meridiana, n. sp. Among the secondary elements into which the 600 transverse striae are resolvable there are two longitudinal rows that stand ep is

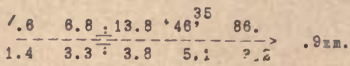
out along the lateral fields a little more prominently than the others. Outside these, on either side, is another row almost imperceptibly emphasized. The annules are retrorse posteriorly, and the reverse anteriorly, the change taking place opposite the vulva on the female. The few very short cervical and somatic setae to be seen scattered here and there are one-half to onethird as long as the body is wide, though a few nearer the head are considerably longer than the cephalic setae. There appear to be twelve subdistinct lips, each bearing a single papilla. The conoid neck contains a cylindroid oesophagus, which, measured at the nerve-ring, is one-third as wide as the corresponding portion of the neck. The oesophagus ends posteriorly in a bulb three-fourths as wide as the base of the neck, containing an inconspicuous elongated valve onethird as wide as itself. The musculature of the bulb is broken into two very unequal parts. There is no cardia. The wall of the intestine varies from thick to somewhat thin, and is six to eight cells in girth. The intestine becomes at once about three-fourths as wide as the body. Its lumen is distinct, and about onefourth as wide as itself. The rather numerous granules to be seen in the cells of the intestines are uniform and small. From the anus, which is depressed, the chitinized rectum leads inward and forward a distance equal to the length of the anal body-diameter.

The arcuate tail tapers from in front of the anus, but is usually cylindroid in the posterior fifth, and has a terminus one-sixth as wide as its base. The broadly saccate caudal glands are packed together in the anterior fourth of the tail, and empty through distinct narrow ducts. The length of the few, scattered, straight caudal setae is about equal to the width of two annules of the cuticle. The lateral fields are one-third as wide as the body. The granular renette cell, which is somewhat longer than the body is wide, and one fourth as wide as long, is located at a distance from the base of the neck equal to the width of the body, and empties by means of a slender duct, through the excretory pore located at the lips. It has a smaller companion cell in its rear. The nerve-ring surrounds the oesophagus somewhat squarely. The cells in its vicinity are distinct in character, and many of them have narrow connections directed forward. From the obscurely depressed somewhat conspicuous vulva, the small tubular vagina leads inward at right angles to the ventral surface one-third the distance across the body. The eggs are about as long as the body is wide, and usually about three-fourths as wide as long, and have been seen in the uterus one to three at a time. The spermatozoa seen in the females are one-eighth as wide as the body. The tapering ovaries reach about threefourths the distance back to the vulva, and contain each about fifteen mostly discoid ova, arranged more or less single file.

Habitat; remarks. Bay, Cape Royds. Five females in fair condition.

15. Chromadora polaris, n. sp. This species closely resembles Chromadora me-ridiana from the same region, but differs in having narrower dimensions, more 
slender oesophagus, bulb and intestine, a more strictly conoid tail, and a somewhat thinner cuticule, the striations of which are

$$
\frac{19}{1.4}-\frac{10 .}{2.6}-\frac{15.2}{2.9}-\frac{-u^{60}}{3.8}-\frac{85.5}{2.9}>1.20
$$
even less modified on the lateral fields. There are about four hundred and fifty transverse striae. The cuticle is hardly perceptibly modified on the lateral fields in the anterior half of the body, but in the posterior half, especially opposite the copulatory muscles, there is a distinct modification, which at its widest part is nearly twice as wide as one of the corresponding striae. The modification becomes less pronounced behind the anus, and disappears near the terminus. The renette cell occurs at a distance behind the neck equal to twice the diameter of the body.

The stoutish, somewhat tapering, rather blunt, yellowish spicula are one and one-fourth times as long as the anal body diameter, and at their widest point onesixth as wide as the body. They are almost imperceptibly cephalated by expansion and lie with their proximae a little ventral to the body axis. The frame has a median piece from near the middle onward. The two arcuate, slender, rather strong accessory pieces are three-fifths as long as the spicula and lie parallel to them. The seven chitinized supplementary organs, separated from each other by a distance equal to the diameter of one of the organs, occupy a space about equal to one and one-half times the body diameter, the posterior one occurring about opposite the proximal ends of the spicula. These organs are very much like those of Chromadora minor. The ejaculatory duct and vas deferens are onefourth, the broad cylindroid testis one-half as wide as the body. Copulatory muscles extend forward somewhat beyond the supplementary organs.

Habitat; remarks. Bay, Cape Royds. Two good specimens, both male.

\section{SPILOPHORA, Bastian, 1865}

The following are characters common to all the species of Spilophora here described.

Cuticle with two lateral wings, beginning near the head and ending on the tail. Neck conoid. Tail tapering from somewhat in front of the anus. The lips are small and often indistinct, but when decipherable can usually be seen to be twelve in number, each with a single forward-pointing papilla. Throughout most of its length the oesophagus is more or less cylindroid, but ends posteriorly in a pyriform bulb two-thirds to four-fifths as wide as the base of the neck, and containing a fusiform valve (sometimes obscure) one-fourth to one-half as wide as itself. There is no distinct cardia. The intestine is separated from the oesophagus by a distinct broad constriction about one-third as wide as the corresponding part of the body. The more or less prominent chitinized rectum, about as long as the anal body diameter, leads inward and forward. The caudal setae are small and scattered. The nerve-ring is of medium size and surrounds the oesophagus rather squarely, and is accompanied by distinct nerve cells. The vulva is more or less elevated and conspicuous and from it the vagina leads inward at right angles to the ventral surface about half way across the body. So far as known the eggs are ellipsoidal. The rather slender, blunt spicula are about one and one-fourth times as long as the anal body diameter. The arcuate accessory piece is parallel to, and half to three-fourths as long as, the spicula. The testis is relatively wide. 
16. Spilophora edentata, n. sp. Striae about 600 , producing a crenate contour, interrupted by the two lateral wings which are so formed as to appear somewhat

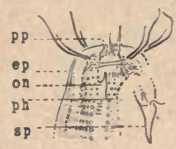

$$
\begin{aligned}
& 1 ! \\
& 1.5 \\
& 1.3 \frac{11 .}{3.3} \frac{17.9}{3.4} \cdot \frac{47^{19}}{3.5} \frac{85 .}{2.2} \quad .9 \mathrm{~mm} . \\
& 1.5-\frac{10 .}{3.2} \frac{16.7}{3.3}-\frac{11^{56}}{3.3}-\frac{87 . \rightarrow}{2.6} \quad 1 . \mathrm{mm} .
\end{aligned}
$$

like the keel and ribs of a boat. Four sub-cephalic setae occur opposite the base of the pharynx. There are few submedian somatic setae, each about one-third as long as the body is wide. There appears to be no distinct dorsal pharyngeal tooth, but pussibly an exceedingly minute more or less ventral one,-unless indeed this appearance be due to the optical effect of one of the striae that exist separately in the posterior part of the pharynx. The masculature of the bulb is broken into two very unequal parts. The rather thin-walled intestine becomes at once twothirds as wide as the body and is about six cells in girth. The cells contain numerous very small, uniform colorless granules. The cylindrical part of the tail of the female is about one-fourth as wide as the base. The broadly saccate caudal glands are arranged in a loose tandem in the anterior half of the tail, and are connected with the spinneret by distinct but very narrow ducts with no clearly visible ampullae. The lateral fields, one-third as wide as the body, contain small scattered nuclei, as well as others less numerous and twice as large. The granular ellipsoidal renette cell, which presses the intestine to one side, a little behind the neck, is about half as long as the body is wide, and one half as wide as long. The duct is hardly half as wide as one of the annules, and the ampulla, opposite the base of the pharynx is almost invisible. The excretory pore is at the lips. The nerve cells are arranged in rather indistinct groups, better seen behind the ring.

The male tail appears to be more nearly conoid throughout, and has a spinneret only about one-eighth as wide as its base. The tapering spicula in their widest part are one-sixth as wide as the corresponding part of the body, and are cephalated by a very inconspicupous constriction. The accessory pieces are slender and rather frail. The testis is about half as wide as the body. Rather distinct, well spaced, oblique copulatory muscles are present in front of the anus for a distance equal to one and one-half tail-lengths. Apparently pairs of male glands, emptying into the cloaca, are present as in Euchromadora and Chromadora, but the details remain unknown.

Habitat; remarks. Bay, Cape Royds. A single female and four males, all in fair condition.

17. Spilophora antarctica, n. sp. Striae about 400 , producing a somewhat crenate contour, and interrupted by two lateral wings separated by a distance

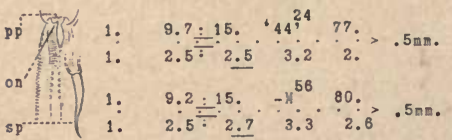
equal to one-fifth the body diameter. Cervical setae occur-at least near the head. The cardiac valve is simple in structure. The relatively somewhat thick-walled intestine soon becomes two-thirds as wide as the body, and is about two cells in girth, these latter containing scattered, rather uniform, colorless granules. The posterior lip of the anus is more or less elevated. The cylindroid part of the tail is one-fifth as wide as the base, or somewhat less. The renette cell 
has not been clearly seen, but the intestine is pushed to one side as if a small one were present, a little behind the neck. The nerve-cells extend past the base of the neck, especially on the ventral side. The eggs occur one at a time in either uterus. The rather broad, tapering ovaries, which extend two-thirds of the way back to the vulva, contain few ova, arranged single file. The testis is onc-half to three-fifths as wide as the body.

Habitat; remarks. Cape Royds; Bay, Cape Royds. Six females and three males, in a somewhat shrunken state.

18. Spilophora serrata, n. sp. Striae about 500 , interrupted by the lateral wings, which occupy a space equal to one-fourth the body diameter. The secondary elements of the cuticle appear as dots on the head, and produce a punctate appearance there. Four rather irregular pairs of subcephalic setae. like the ceph-

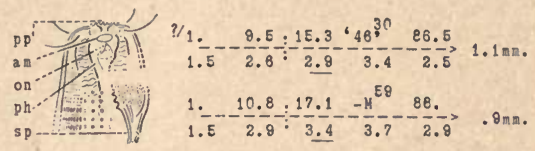
alic setae, but shorter, mated one in front of the other, occur at a distance from the lips equal to twice the width of the head. There are few cervical setae. The vestibule of the pharynx is longitudinally striated. This is one of the few species of Spilophora in which the amphids can be plainly scen. The rather thickwalled intestine soon becomes three-fourths as wide as the body, and is ten to twelve cells in girth, the cells containing numerous uniform, colorless granules nearly equal in diameter to the width of one of the annules of the cuticle. The posterior lip of the anus is elevated. The terminus of the tail is about one-sixth as wide as its base. The two broadly saccate caudal glands are arranged in close tandem opposite the anus and in the anterior fourth of the tail. Their ampullae are not distinct. The third eaudal gland (?), just behind the anus, is a strongly staining cell, with a duct, or connection, that reaches to near the terminus. The non-granular renette cell, one body-width behind the neck, twice as long as the body is wide, has a small companion-cell in its rear. The excretory pore is probably near the lips. Opposite the renette cell there are two, and probably three, ellipsoidal cells in each lateral field, each cell being one-third as long as the body is wide, and one-third as wide as long. These cells constitute two pairs, - or three. The eggs are about one and one-half times as long as the body is wide, and two thirds as wide as long, and occur in the uteri one at a time. Each of the medium sized, tapering ovaries reaches half way back to the vulva, and contains about fifteen ova arranged single file.

The strong, rather uniform, colorless spicula are cephalated by a constriction and are obliquely truncated at the distal end, where there are two or three exceedingly minute teeth. The subslender, rather strong and simple accessory pieces recede a little from the spicula. Of the slightly elevated, somewhat protrudable supplementary organs, whose width is about equal to that of two annules, the hindermost is near the anus. They are farther apart anteriorly, the distance between the first and second equaling one-third of the body width, that between the second and third, one and one-half times the body width. The ejaculatory duct is one-third as wide as the body-the vas deferens and testis one-half. At least one pair of glands of the kind seen in the males of Euchromadora occurs nearly twice as far in front of the anus as the terminus is behind it; each gland-cell is 
fusiform, one-half as long as the body is wide, and one-half as wide as long. Both of the males examined had a ventral swelling near the center of the tail, as long as the corresponding body diameter.

Habitat; remarks. Bay, Cape Royds. The two females and two males studied were in good condition.

\section{EUCHROMADORA, De Man, 1886}

The following are characters common to all the species of Euchromadora here described.

The neck is more or less cylindroid in the posterior part, but usually slightly conoid anteriorly. The tail tapers from in front of the anus, and has no terminal swelling. Cervical setae none or small and scattered, except in E. septentrionalis, somatic setae none or very inconspicuous. Caudal setae none or inconspicuous, except in $E$. denticulata. Each of the six lips is double, so that there appear to be twelve more or less alike. These are usually distinct when the mouth is open, but so folded together when the mouth is closed as to become less distinct, and to impart to the then narrow vestibule a longitudinally striated appearance. Labial papillae twelve, in a single circlet, forming the apices of the lobes of the lips. The pharyngeal region of the oesophagus is swollen, so as to form a faint pharyngeal "bulb," rather obscurely pyriform or elongated in form. The oesophagus is cylindroid in the anterior half, and conoid or perhaps clavate in the posterior part, but is without a true cardiac bulb except in $E$. septentrionalis. Oesophageal lining distinct, often increased posteriorly. There is no distinct cardia. The rather thin walled intestine becomes almost at once three-fifths to threefourths as wide as the body and is from six to nine cells in girth. It is separated from the oesophagus by a distinct collum one-fifth to one-third as wide as the corresponding part of the body. The chitinous rectum, as long as the anal body diameter, leads inward and forward from the more or less elevated anus. The caudal glands are found in the anterior fourth of the tail (and in E. meridiana also a short distance in front of anus), and empty through ducts devoid of ampullae, except in the case of $E$. denticulata. The lateral fields are about one-third as wide as the body. The elongated, granular renette cell, one to two body-widths behind the neck, has one or two smaller companion cells in its rear. The medium sized nerve-ring surrounds the oesophagus rather squarely. From the somewhat elevated but rather inconspicuous vulva the vagina leads inward at right angles to the ventral surface about halfway across the body. The reflexed, tapering ovaries reach half to two-thirds the way back to the vulva, and contain a dozen or more developing ova, arranged more or less irregularly, especially toward the blind end. The more or less slender spicula are sub-acute, and accompanied by arcuate parallel accessory pieces half as long as themselves. The single testis is cylindroid and about half as wide as the body.

19. Euchromadora septentrionalis, n. sp. The 600 striae, very difficult to see except at the extremities, are resolvable into minute elongated elements, which

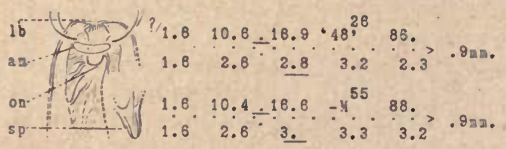

are interrupted by very narrow and inconspicuous lateral wings, beginning near the head and ceasing near the end of the tail. Two sub-cephalic setae, nearly as long as the cephalic 
setae, occur one in front of the other on each sub-median line, at a distance from the head end equal to one and one-fourth times the head width. The cervical setae, more numerous on the anterior part of the neck; are sometimes longer than the cephalic setae. The lips, otherwise typical, have successive rings of elements surrounding the interior of the vestibule. The oesophagus is one-third as wide as the neck, but swells posteriorly into a true cardiac bulb, containing a fusiform valve one-third as wide as itself. The intestinal granules are scarce and colorless. The elongated caudal glands are arranged in a close tandem, and have distinct ducts. The renette cell, three times as long as the body is wide, empties through a very inconspicuous pore at the base of the lips. Strongly staining, elongated, distinctly granular bodies are found in the lateral fields behind the neck. They are one-half as long as the body is wide, and two-thirds as wide as long, and they occur as rightsand lefts, i. e. are paired. The first pair is one body-width behind the neck, and the successive pairs, continuing to the anus, are separated from each other by a distance three times as great as the radius of the body.

The tail of the male is a little stouter than that of his mate. The uniform, slender, frail spicula are as long as the anal body diameter, and one-twelfth as wide as long. There are two strong grooved accessory pieces, twice as wide as the spicula, bearing excessively minute teeth at their blunt distal ends. There are at least five unicellular clavate glands on the dorsal side, extending in front of the anus for a distance equal to the length of the tail. First, anteriorly there is a pair, then a second pair, then apparently a single one, all with slender ducts They were not very well seen, and this enumeration may not be exact.

Habitat; remarks. Cape Royds; Bay, Cape Royds. Numerous specimens, somewhat shrunken.

20. Euchromadora antarctica, n. sp. The cuticle is traversed by about 550 transverse striae, resolvable into dots near the head, and into basketwork-like markings on the neck and elsewhere. These striae are of such a size as to give rise to an obscurely crenate contour line. There is a simple, very obscure cardiac valve, one-third as

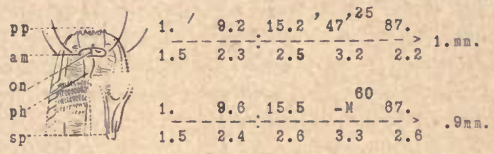

wide as the base of the oesophagus. The cells of the intestine contain numerous rather small, colorless, uniform granules. The ellipsoidal caudal glands are arranged in a loose tandem. and empty through very narrow ducts. The renette cell is about as long as the body is wide, and one-third as wide as long, and presses the intestine somewhat to one side. The nerve-cells around the oesophagus are of a distinct character, but are scattered so as to exhibit no very systematic arrangement. The prolate to ellipsoidal eggs are considerably longer than the body is wide, and three-fourths as wide as long. They have been seen in the uterus one at a time. The tapering ovaries contain upwards of a dozen ova.

The slender, uniform spicula have a more or less frail framework, and are about twice as long as the anal body-diameter. They are not cephalated, but the proximal parts are somewhat wider. This wider part lies a little to the ventral side of the body-axis, that is, it appears to do so when the animal is viewed in profile. The single, slender accessory piece has a framework that is more or less frail, and has its proximal end opposite the body-axis. The supplementary organs are 
spread along a distance about equal to five or six body-diameters, the distance between the adjacent organs being about equal to the radius of the body. These organs.are of such a nature that when the body is stretched out they hardly disturb the ventral contour, but when the tail end is incurved, as is of ten the case, they become slightly raised, so that the altitude is about equal to one-half the width of one of the annules of the cuticle. In this contracted condition of the ventral surface of the body the distance between the organs is about equal to their own diameter. The ejaculatory duct is one-fourth, the vas deferens threefourths, and the testis two-fifths as wide as the corresponding portions of the body. The testis is cylindroid. Rather distinct, oblique copulatory muscles are found coextensive with the supplementary organs.

Habitat; remarks. Cape Royds; Bay, Cape Royds. Numerous specimens, somewhat shrunken.

21. Euchromadora denticulata, n. sp. Striae about 400 , resolvable into basketwork-like markings of a rather obscure character on the anterior part of the

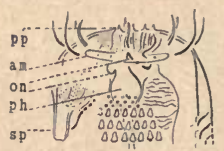

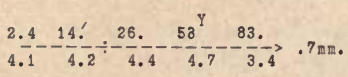

neck. The lips are subdistinct. At the nervering the oesophagus has

a diameter equal to two-

fifths the corresponding diameter of the neck, but swells posteriorly to three-fourths the corresponding diameter. In the posterior swelling the lining is somewhat expanded.

The dimly seen pyloric collum is one-third as wide as the body. Intestinal granules are scarce. The ellipsoidal caudal glands occur in a close tandem opposite the anus and in the anterior fourth of the rather massive tail. The caudal setae, six ventro-submedian, and six dorso-submedian, are slender, stiff, somewhat cylindroid, sub-acute, and one-sixth as long as the anal body-width. The renette-cell, two-thirds as long as the body is wide and one-third as wide as long, empties through a pore opposite the nerve-ring. In both the specimens examined there appears a slender seta-like appendage (?) at the mouth of the excretory pore. The female reproductive organs are double and symmetrical, and probably reflexed.

Habitat; remarks. Cape Royds. Two females, in fair condition.

22. Euchromadora meridiana, n. sp. The cuticle is much like that of Euchromadora vulgaris, (Bast.) de Man. 400 striae, retrorse posteriorly and the reverse anteriorly, make the contour obscurely crenate. The oesophagus, cylindroid anteriorly, conoid posteriorly, has several breaks in its muscula-

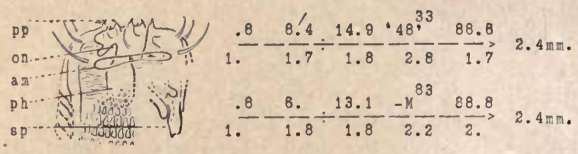
ture, the most prominent being at the beginning of the final fifth of the neck. The numerous intestinal granules are small, uniform, and indistinct. The rather ellipsoidal caudal glands are arranged in an open tandem. Immediately behind the anus the tail has a diameter equal to three-fourths the anal body-diameter; thence onward it tapers but very slightly to the middle, where it begins to taper more rapidly to the terminus which is one-third as wide as the anal body-diameter. The lateral fields are somewhat distinctly cellular. The renette-cell, which pushes 
the intestine to one side, is twice as long as the body is wide, one-fourth to onefifth as wide as long. Just behind the nerve-ring there is a large ampulla as long as the body is wide, and one-third as wide as long. In all the specimens examined there appears a slender seta-like appendage at the mouth of the excretory pore somewhat longer than the cuticle is thick. The ovaries are broad.

The male tail is like that of the female, but more uniformly tapering, with less of a diminution just behind the anus. The strongly arcuate, sub-slender, rather strong and tapering spicula, somewhat cephalated at the tapered distal ends, are one and two-thirds times as long as the anal body-diameter, and lie with their proximae slightly ventral to the body-axis. There are two, more or less separate accessory pieces, each in two parts, one behind the spicula; the other in front or alongside, the front part being like a reduced spiculum. The ejaculatory duct is one-fifth, the vas deferens one-half as wide as the body.

Habitat; remarks. Cape Royds; Bay. About a dozen somewhat shrunken specimens, the two sexes occurring in about equal numbers.

\section{LAXUS, Cobb, 1894}

23. Laxus septentrionalis, $\mathrm{n}$. sp. There are upwards of 1000 plain, transverse striae so small as to be difficult of resolution. The three subdistinct, more or less bluntly conoid lips are united by a membrane, and form a circlet around the mouth, very difficult to see, except when the mouth is open.

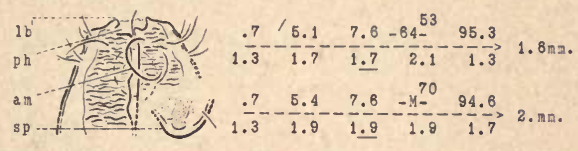

Only when the mouth is open can the connecting membrane be seen. It is probable that the pharynx contains an obscure dorsal tooth. The structure which gives rise to this supposition is of fair size, and thinly covered with chitin. The cylindroid to conoid neck contains a cylindroid oesophagus, which, measured near the nerve-ring, is one-half as wide as the corresponding portion of the neck. Though the amphids appear circular, they are in reality spiroidal. The pharynx is situated in the midst of an almost imperceptible elongated pharyngeal swelling, two-thirds as wide as the head. The thick walled intestine, six cells in girth, soon becomes five-sixths as wide as the body. It has a distinct lumen, and is separated from the oesophagus by a collum hardly one-third as wide as the base of the neck. For a distance about equal to the length of the body-diameter the cells of the intestine are comparatively free from granules. Thereafter they are uniformly packed with yellowish granules having a width a little greater than that of one of the striae of the cuticle. From the continuous anus the prominent, chitinized rectum extends inward and forward a distance three-fourths as great as the length of the anal body-diameter.

The tail tapers from the anus to a terminus one-third as wide as its base. Apparently the ellipsoidal caudal glands are packed together somewhat behind the middle of the tail. Their ampullae, near the terminus, are more or less distinct. The lateral fields, which are one-third as wide as the body; are margined by refractive lines, at least in balsam specimens. The renette has not been clearly seen, but is supposed to be located at a distance behind the neck equal to the 
radius of the body, and to be about one-half as long as the body is wide, and onehalf as wide as long. It empties by means of a short duct, and a rather structureless ampulla half as wide as the oesophagus, through the exeretory pore near the nerve-ring. The vulva is more or less continuous, and rather ineonspieuous. The elongated eggs are one and one-half times as long as the body is wide, and one-third as wide as the body. They have conspicuous nuclei. Of the narrow ovaries the posterior one is only one-third to one-half as long as the anterior, which contains twenty developing ova, arranged single file, while the posterior one contains only about fifteen.

The slender, uniform spicula have a more or less frail framework. The single accessory piece, also more or less frail, presents a stoutish, uniform, blunt apophysis one-half as long as the anal body-diameter, arranged at right angles to the part which is applied to the spieula. This latter is one-third as long as the spicula. The vas deferens is one-third, the tapering testes about one-half as wide as the body. These latter, however, finally become very narrow. The blind end of the posterior testis lies in front of the anus a distance about equal to six times the length of the tail.

Habitat; remarks. Bay, Cape Royds; Bay. Four females and two males, slightly shrunken.

\section{AXONOLAIMUS, de Man, 1889}

24. Axonolaimus polaris, $\mathrm{n}$. sp. The striae of the thin cuticle are very difficult to resolve and are best seen on and near the tail. One is left in doubt as to whether

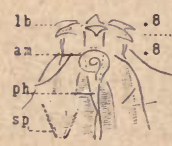

the contour is plain, or very minutely crenate. Apparently there are six subcephalic setae, each half as long as the diameter of the head measured at the labial constriction. Though there are no somatic setae there are a few scattered setae on the neck, generally somewhat shorter than the subcephalic setae. The conoid neck contains a conoid oesophagus, whose diameter near the nerve-ring is two-fifths as great as that of the corresponding part of the neck, and which finally becomes two-thirds as wide as the base of the neck. There is no distinct cardia. The thick walled intestine is two to three eells in girth, and becomes at once three-fourths as wide as the body, being separated from the oesophagus by a collum three-fifths as wide as the base of the neek. On account of the food contained in it the lumen of the intestine is distinct. The intestinal cells contain very fine, scattered, colorless granules in the middle of which the nucleus appears as a distinct feature in stained speeimens. From the raised anus the chitinized rectum extends inward and forward a distance equal to the anal body-diameter. The tail tapers from the anus, bears very minute bairs near the end, and contains broadly saccate eaudal glands packed in its anterior third. The nerve-ring surrounds the oesophagus squarely, is of medium size, and is aecompanied by obscure nerve-cells, which are not very distinctly grouped.

Habitat; remarks. Bay, Cape Royds. Single young specimen, in fair condition. In the sketch the lips are shown partly everted. 
THORACOSTOMA, Marion, 1870

25. Thoracostoma polare, n. sp. The thick yellow cuticle is traversed by minute, transverse striae difficult of resolution, and in its deeper layers, at least near the head, by fine oblique striae erossing each other at an angle of about forty-five degrees. Subcephalic setae, like the cephalic in form and size, oc-

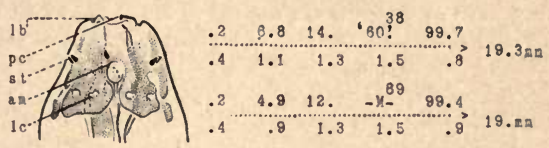
cur in groups of three or four together, near the border of the "cuirasse," and a little farther back. Similar cervical setae occur on the anterior part of the neck singly and separated by a distance one-fourth to one-third as great as the diameter of the neck measured at the middle. The remaining cervical setae as well as the somatic setae are reduced to papillae. All these setae are arranged in more or less irregular lateral and submedian lines. The head is furnished with a chitinous, faintly yellowish "cuirasse," extending back to opposite the base of the pharynx, and divided longitudinally into six lobes by as many sutures. Each of these lobes is perforated near the posterior margin by two oblique, irregular locules, each a little more than half as long as the amphids are wide. The three amalgamated lips are nearly obsolete. Six forward pointing, inconspicuous, innervated papillae form a single circlet on the front of the head. The simple, rather narrow, more or less irregularly pyramidal, well chitinized pharynx is as long as the head is wide, and is somewhat narrowed posteriorly, where the two ventrally submedian oesophageal glands empty into it. The third oesophageal gland, ventral in position, empties into the lumen of the oesophagus a little more than halfway back to the eye-spots. When shut the pharynx rather elosely resembles the lumen of the oesophagus, which possesses a well developed chitinous lining. The only armature of the pharynx is the dorsal, labial, two-lobed cordiform piece of chitin just at the mouth opening, arranged much as described by Dr. de Man for Thoracostoma antarcticum, v. Linst. Two faint refractive lines close together lead backward from the posterior part of each amphid. The two rather widely separated eye-spots are located at a distance from the anterior extremity equal to four times the width of the head, and are one-sixth as wide as the corresponding part of the neck, and about as long as wide. These are somewhat heart shaped, and are composed of compactly arranged brownish granules. There are obscure indications of a refractive body, or "lens," in the hollowed out front part of each eye-spot. The conoid oesophagus is about half as wide as the neck, and presents a faint, elongated pharyngeal swelling. The cylindroid cardia is one-fifth to one-fourth as wide as the base of the neck. The thick-walled intestine, separated from the oesophagus by a collum one-third as wide as the base of the neck, becomes at once three-fifths as wide as the body, and is about six cells in girth. The intestinal cells contain numerous uniform, colorless granules. The posterior lip of the anus is slightly elevated.

The hemispherical-conoid to convex-conoid tail tapers from the anus and ends in a very broad, bluntly rounded terminus. The spinneret is a very slightly depressed pore with a suggestion of yellowish color, arranged a little unsymmetrically, so that it empties somewhat toward the ventral side, and not axially. It 
is unarmed, though a little in front of it there are a very few papilla-like setae. The three more or less elongated saccate caudal glands extend in a loose tandem in front of the anus a distance five times as great as the corresponding bodydiameter. Their distinct and somewhat tortuous ducts widen to three separate ampullae so as to fill the middle of the tail. The vulva is slightly depressed and is rather inconspicuous. The vagina extends inward at right angles to the ventral surface about halfway across the body. Both before and behind the vulva there are well developed unicellular glands. Each of the two straight uteri is about seven times as long as the body is wide. and contains a row of five or six eggs in the specimens examined. The rather thick-shelled eggs are three-fourths as long as the body is wide, and nearly as wide as long. Owing to their pressure on each other the eggs take on a more or less rectangular contour. The broad ovaries reach two-thirds the way back to the vulva, and contain scores of discoid ova, arranged more or less in single file.

The anus of the male is broadly elevated. The tail of the male has a complicated sexual armature. Near the anus on the anterior fourth of the tail there are six stout, stiff, conoid, somewhat blunt, ventrally submedian setae on each side, the longest of which, those nearest the anus, are nearly as long as the cuticle is thick, and the smallest of which, the hindermost, are very small. The preanal setae are arranged in ventrally submedian rows, of which the individual setae are much nearer together posteriorly than anteriorly. Between the anus and the single ventral median supplementary organ there are ten of these setae; between the latter and the posterior member of the ventrally submedian rows of supplementary organs there are two of these setae; between the ultimate and the penultimate members of the submedian series there are also two. Thence forward between any two members of the submedian series there is but a single seta. In all, therefore, there are about seventeen of these setae on each submedian line in front of the anus. In addition there are two very inconspicuous, papilloid, tapering, acute, lateral setae near the terminus. The lateral fields, one-third to two-fifths as wide as the body, are composed of several rows of cells among which are placed glandular, saccate cells, of two or three times as great diameter, connected with the exterior by means of pores in the cuticle. The relative number and position of these glandular cells may be judged to a certain extent from the fact that in the female near the vulva three of these large saccate cells are separated from each other by spaces about equal to their own diameter. These cells are entirely similar to those described by Jaegerskiöld, de Man, and other authors, who have made observations on species of Thoracostoma. The distinct cells connected with the more or less broad and oblique nerve-ring are arranged into rather distinct grours, and are a prominent feature of the middle half of the neck.

The stout, blunt, yellowish spicula, cephalated by a very slight constriction, are arcuate in the distal half, and are one and three-fourths times as long as the anal body-diameter. At the widest part, a little in front of the middle, they are about one-fourth as wide as the corresponding part of the body. From the widest part they taper gradually to the distal ends, but taper more suddenly toward the proximal ends, which lie opposite the body-axis and have a width when viewed in profile about twice as great as the thickness of the adjacent cuticle on the dorsal side of the body. The difference between the anterior and pos- 
terior parts of the spicula is greater than in Thoracostoma setosum, Linst. The strong, chitinous framework is strengthened by a middle piece in the distal threefifths. The two separate, strong, rather stout accessory pieces are bent a very little, and are half as long as the spicula. They are of somewhat irregular width, but appear when seen in profile about twice as wide as the dorsal cuticle is thick. They lie parallel to the spicula, but their cephalated proximal ends, a little wider than those of the spicula, are bent away a little and lie somewhat to the dorsal side of the body-axis.

The elevated, mammiform supplementary organs, about one-sixth as wide as the body and half as high as wide, are arranged in ventrally submedian rows one and two-thirds times as long as the body is wide, the posterior members being located a distance in front of the anus equal to one and two-thirds times the length of the spicula. They are a little farther apart posteriorly than anteriorly, the longest space being about equal to the length of the radius of the body as seen in profile at the corresponding part. In addition to these two rows of five organs each there is a single ventral organ a little in front of the middle of the spicula, similar in size and form to the others, but manifestly of a different structure. Sometimes there is an appearance as if a rudimentary sixth ventrally submedian organ is present in front of the fifth. Oblique copulatory muscles are arranged close together coextensive with the supplementary organs. The ejaculatory duct is about one-fifth as wide as the corresponding part of the body.

Habitat; remarks. Bay, Cape Royds; Cape Royds. Three females and two males, in good condition. 

NORTH AMERICAN

\title{
FREE-LIVING FRESH-WATER NEMATODES
}

\author{
Contributions to a Science of Nematology
}

II

\author{
By N. A. Совв \\ (With eighty illustrations)
}

INTRODUCTION.

The little creatures described in these pages belong to a very important but as yet comparatively little known class of animal organisms, the nematodes.

Something more than a mere reconnaissance leads to the conclusion that over nine-tenths of the nematode species still remain unknown, a greater disproportion between the known and the unknown than exists in almost any other class of organisms.

Nematodes are distributed far and wide in inconceivable numbers, and without doubt constitute a group in the animal kingdom comparable with insects both in number of species and economic importance. They vary in length from one two-hundredth of an inch to several feet, though the great majority are less than half an inch long. They differ from most other slender, wormlike forms in their lack of locomotive appendages, - the outer surface, except for the presence of setae, is usually quite smooth. Being more or less cylindrical in form, they are often called "round-worms." They live free in the soil, in fresh and salt water, and are found parasitic in a great variety of animals and plants.

\section{NEMATODES AS CAUSES OF DISEASE}

The parasitic species often cause fatal diseases of plants and of animals, including mankind. The dreaded hookworm is a nematode. So is that scourge of the tropics, the guinea worm. Trichina,*

*Trichinella spiralis.

Reprinted from Trans. American Microscopical Society. Vol. XXXIII, April, 1914. 
costing civilized nations hundreds of thousands of dollars yearly for the inspection of pork, is a nematode. If trichina-infested or measly pork be eaten by human beings, the result is a serious, oftentimes fatal, sickness, called trichinosis, epidemics of which have claimed victims by the hundred. Nematodes have recently been suspected, with good show of reason, of being carriers of cancer. So the list of serious human nematode diseases might be increased until practically half a hundred had been enumerated.

No less serious are the nematode diseases of plants and of the lower animals. The common gall-worm has been found infesting the roots of several hundred different species of plants, among them most of our cultivated crops, and causes an annual loss amounting to millions of dollars. There is another nematode that has at times completely checked the growing of sugar beets in certain regions. The list of serious plant diseases of this character could easily be increased to scores. The same is true of animals. Every domestic, doubtless every wild, species has a number of specific nematode parasites sapping its vitality.

\section{WONDERFUL VARIETY OF HABITAT.}

Not the least interesting thing about nematodes is the astounding variety of their habitats. They occur in arid deserts and at the bottoms of lakes and rivers, in the waters of hot springs and in polar seas where the temperature is constantly below the freezing point of pure water. They were thawed out alive from Antarctic ice in the far south by members of the Shackelton expedition. They occur at enormous depths in Alpine lakes and in the ocean. As parasites of fishes they traverse the seas; as parasites of birds they float across continents and over high mountain ranges. Their eggs and larvae, invariably of microscopic size, are carried from place to place by an exceedingly great variety of agencies. Almost any visible thing that moves is capable of transporting nematode eggs or larvae. Sometimes the eggs and larvae are so resistant to dryness that if converted to dust they revive when moistened. This revival of mummified nematodes may take place after as long a period as a quarter of a century.

Nematodes are found in queer places. The wildest imagination could hardly outpicture the facts. One species is found prac- 
tically only in the vermiform appendix of man; another has its adult form only in the seeds of wheat. A third form occurs in the felt mats on which the Germans are accustomed to set their mugs of beer, and has been found in no other habitat. On the feet of birds and insects the eggs, larvae, and adults of certain nematodes are carried to the tops of the tallest trees. The sour sap issuing from the wounds of a tree, often many feet above the ground, net infrequently contains nematodes that are specific to the wounds of that particular kind of tree. The tap water of even well-conciucted cities often contains nematodes.

Nematodes are inconceivably abundant. A thimbleful of mud from the bottom of the ocean may contain hundreds of specimens. The number of nematodes in the top six inches of an acre of ordinary arable soil amounts to thousands of millions. Statistical calculations relative to the number of nematodes in a single acre of soil near San Antonio, Texas, U. S. A., disclosed that if they could start in a procession for Washington, D. C., two thousand miles away, each close on the tail of the one in front, the head of the procession would reach Washington before the rear had left San Antonio. As nematodes are usually very prolific, a single female sometimes producing thousands of eggs, the number of eggs vastly exceeds that of the adults.

We must therefore conceive of nematodes and their eggs as being carried by the wind, and by flying birds and running animals; as floating from place to place in nearly all the waters of the earth; and as shipped from point to point throughout the civilized world in vehicles of traffic.

There are beneficial nematodes, though knowledge of this phase of the subject is in its earliest infancy. Some nematodes feed exclusively on their injurious brethren. Others devour baneful microorganisms. Their adaptations and relationships appear to be similar to those of insects.

NORTH AMERICAN FRESH-WATER SPECIES.

The nematodes here described are the main portion of those found during a rather casual search for aquatic species that would serve as the basis of a special chapter in a zoological textbook devoted to fresh water organisms. The object of the textbook was 
to characterize each known genus by describing and illustrating a typical species.

When the work on the nematode chapter was begun there was hardly a single adequately described North American species, so that the initial task was one of pure discovery. This was followed by the work of research and definition, and this finally by that of selection and presentation of typical species in a manner suitable to a college textbook.

The famous Philadelphian, Dr. Joseph Leidy, appears to be the only naturalist who had previously attempted to describe any of our fresh water nematodes, and even he did but little. I have attempted to rehabilitate his Anguillula longa, which, as Bastian surmised, belongs to the more modern genus Trilobus. I have had to assume that the variety Leidy had before him was that most common in the ditches, brooks and rivers of the Chesapeake region, and have given to this form the name Trilobus longus (Leidy) Bastian.

All the other species proved to be new, some of them representatives of new genera. Most of the new genera have already been described in my contribution to the Proceedings of the Washington Academy of Sciences, October, 1913.

An aquatic species as here defined is any species inhabiting either fresh water, or non-brackish swampy soil below the water table; hence a species that will not drown in fresh water; a species fitted to utilize oxygen dissolved in fresh water.

Including those of the present article, the fresh water North American species so far described are as follows:

Achromadora minima

Actinolaimus radiatus

Alaimus simplex

Anonchus monhystera

Aphanolaimus minor

Aphanolaimus spirurus

Bastiana exilis

Cephalobus setosus

Cephalobus subelongatus

Chronogaster gracilis

Cryptonchus nudus
Cyatholaimus truncatus

Diplogaster fictor, Bastian

Dolichodorus heterocephalus

Dorylaimus fecundus

Ethmolaimus americanus

Iota octangulare

Ironus americanus

Mesomermis virginiana

Microlaimus fluviatilis

Monhystera sentiens

Mononchus similis 
Oncholaimus punctatus

Plectus tubifer

Prismatolaimus stenurus

Rhabditis punctatus

Rhabdolaimus minor
Spilophora canadensis

Teratocephalus cornutus

Trilobus longus (Leidy), Båstian

Tripyla lata

Tylenchus symmetricus

There has been little time or opportunity to search specially for these fresh water forms, and the fact that it has been possible in so short a time to assemble so many genera and species is proof of the enormous abundance and multifarious nature of these organisms. No doubt there is a horde of species awaiting discovery in North American waters.

THE NEMATODE FORMULA.

The following diagram illustrates the nature of the formula used in the tabulation of the various necessary measurements:

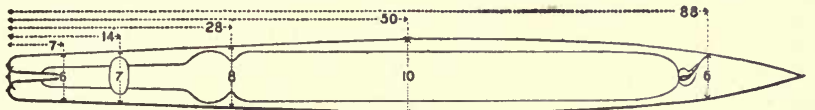

Fig. 1. Diagram of the descriptive decimal formula used for nematodes; $6,7,8,10$, 6 are the transverse measurements, while $7,14,28,50,88$ are the corresponding longitudinal measurements. The formula in this case is:

$$
\begin{array}{ccccc}
7 . & 14 . & 28 . & 50 . & 88 . \\
\hline 6 . & 7 . & 8 . & 10 . & 6 .
\end{array}
$$

The unit of measurement is the hundreth part of the length of the body, whatever that may be. The measurements become, therefore, percentages of the length. The absolute length is given in millimeters as a final non-paired term. This decimal method of presenting the measurements is the most compact and convenient method, and is being used more and more year by year.

The measurements are taken with the animal viewed in profile; the first are taken at the base of the pharynx, the second at the nerve-ring, the third at the cardiac constriction or end of the neck, the fourth at the vulva in females and at the middle (M) in males, the fifth at the anus. The formulae represent the average of several specimens whenever possible.

There are several methods of securing the measurements, one of the most convenient being the preparation of a camera lucida "skeleton" to scale, consisting of a line following the body axis 
from one end to the other, and diametral lines at the base of the pharynx, the nerve-ring, the end of the neck, at the vulva in females and at the middle in males, and finally at the anus. This skeleton is then measured with a metric scale and map measure, and the percentages divided out with the aid of a slide rule.

IOTA, Cobb, 1913.

Fig. 1, Plate II.

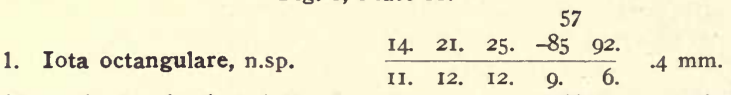

Along the longitudinal fields the strix are so modified as to give the worm somewhat the appearance of being covered by eight rows of relatively large scales arranged in pairs. Six small, simple, flattish lips surround the vestibule. The lips are supplied with six small, innervated papillae. These lie in the midst of a plate-shaped disk, the first annule. There are no recognized traces of amphids, but the recessive nature of the second annule is suggestive, and it is possible that this peculiarity of the second annule is in some way connected with the amphids. There are no eye-spots. The narrow vestibule leads to the equally narrow pharynx, which consists essentially of a muscular tube closely surrounding the spear. This latter is of relatively very large size, and strictly Tylenchoid in structure. Its length is about equivalent to the first twelve annules of the cuticle. It is divisible into two parts: a long cylindrical hollow shaft tapering to a point near its apex, and having a length about equivalent to the first nine annules; behind this shaft a basal portion or "hilt," which in its distal part has a structure like that of the shaft, but with a slightly greater diameter. This hilt terminates in a threefold, flattish bulb about one-fourth as wide as the corresponding portion of the neck. The lumen of the spear is continuous with that of the oesophagus. This latter consists mainly of a narrow, chitinous tube, which is usually somewhat coiled when the spear is at rest. That portion of the oesophagus containing the hilt of the spear and the coiled oesophageal tube just mentioned constitutes a somewhat obscure elongated "bulb." This bulb, however, is not the morphological equivalent of the bulb usually seen in the oesophagus of Tylenchus and Aphelenchus, as it has no radial muscles, and contains no valvular apparatus. The remaining short portion of the oesophagus is tubular and narrow, though it swells slightly at the terminus, where it is about one-eighth to one-ninth as wide as the base of the neck. The excretory pore empties through the exterior margin of the nineteenth annule, or thereabouts. It may be distinctly seen when the worm is viewed from the ventral side, and less distinctly seen in profile.

Habitat: Dismal Swamp, Va. Flemming solution to glycerine. 
RHABDOLAIMUS, de Man, 1880.

Fig. 2, Plate II.

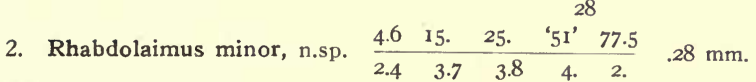

The thin layers of the transparent, naked, colorless cuticle appear to be destitute of any but very fine transverse striations, most clearly visible near the head. The conoid neck ends in a rounded head which is not set off in any way. There are no cephalic setae. Careful focussing appears to indicate on the outer margin of the head the presence of almost invisible papilla-like organs which may perhaps be representatives of cephalic setae. There are no lips. The amphids are located at a distance from the anterior extremity about three times as great as the width of the lip-region, and a little more than twice as great as the width of the head near where they occur. Seen in dorso-ventral view they have the appearance of obscure openings from which there leads inward and backward slight refractive elements which soon become indefinite. Immediately behind the month opening there is at least one obscure refractive element of small size, apparently very much as figured by Dr. de Man in his description of the type species. The tubular pharynx is long and slender, extending backward for a considerable distance behind the amphids. Its total length is probably a little less than one-third the distance to the nerve-ring, though the limits are not definite in the specimens so far examined. The oesophagus is at first about three-fourths as wide as the head and continues to have this diameter, or a slightly greater diameter, until near the end, where it expands to form the pyriform cardiac bulb which contains an obscure valvular apparatus. This bulb is threefourths as wide as the base of the neck. The lining of the oesophagus may be faintly seen throughout its length. The rather thin-walled intestine which is separated from the oesophagus by a slight constriction becomes at once three-fourths as wide as the body. It appears to be composed of cells of such size that probably only four to six are required to build a circumference. From the nearly continuous anus, the rectum, which is somewhat longer than the anal body diameter, extends inward and forward. The tail begins to taper from a little in front of the anus and tapers regularly to the terminus which is armed with a striking, elongated, unarmed, slightly tapering, acute spinneret. Where the tail joins the spinneret the diameter is about one-fifth as great as at the anus. There is a sudden and very slight diminution of diameter of the terminus where it joins the spinneret. From the inconspicuous vulva, the vagina leads inward and forward. The symmetrically reflexed ovaries reach about half way back to the vulva. The thin-shelled, smooth eggs are relatively large and elongated and have been seen in the uterus one at a time. They are about four to five times as long as the body is wide and about one-fifth as wide as long. They appear to be deposited before segmentation begins.

Habitat: Mud, Beach pool, Pine Point, Douglas Lake, Michigan. Sublimate to balsam. 
DIPLOGASTER, Max Schultz, 1857.

Fig. 3, Plate II.

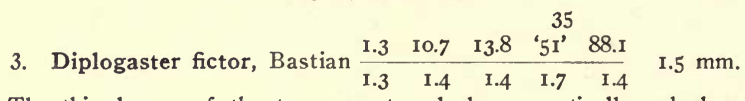

The thin layers of the transparent, colorless, practically naked cuticle are traversed by exceedingly fine transverse striae, resolvable with high powers under favorable conditions, which become considerably coarser toward the head, where they are resolvable into rows of refractive dots arranged in longitudinal, as well as transverse lines. A short distance behind the head the longitudinal rows arrange themselves in pairs. These pairs indicate the locus of about twenty-four cuticular ribs or wings, which extend from the middle of the neck to near the anus. On the tail these ribs again resolve themselves into double rows of dots, and gradually disappear as the tail grows narrower. Very little is to be seen of them behind the middle of the tail. The cylindroid neck ends in a somewhat rounded head, which bears near its outer margin a circlet of six somewhat forward-pointing, tapering cephalic setae, each about one-third as long as the head is wide. There is one of these setae on each submedian line and one on each lateral line. Just in front of the base of each lateral seta a minute obscure pore or papilla was observed, which did not appear to exist in connection with the other setae. The amphids, though only faintly visible, are of relatively large size. Their form is partly indicated by a lateral area on which the punctations of the cuticle are absent. This is a somewhat elliptical area placed transversely on the side of the head, with its long axis at right angles to the lateral line. Its length is equal to half that of the corresponding diameter of the head. Its anterior contour is more definite than the posterior, although it is only by the most caerful focusing that the defintie line of contour can be distinguished. Through the middle of the area, however, there is quite a distinct arcuate line, with its convex side toward the lips. When seen dorso-ventrally the amphids have the appearance of distinct oblique openings, leading inward and backward. When the mouth is opened and the lips recurved the amphids are moved forward somewhat and the transverse line mentioned becomes more strongly curved and forms a semi-circumference. The circular mouth opening is very finely striated on the inner surface of the lip region. These striae begin on the inner face of the lips near where the cuticular wall of the pharynx first appears. They extend backward nearly to the base of the pharynx and end indefinitely. In front of this finely longitudinally striated area the transverse marginal portion of the lips is more coarsely divided into fourteen parts. The lips are capable of being opened, so that the oral aperture is two-thirds as wide as the head. Under such circumstances it is seen that the lip region is flower-like in form with fourteen recurved segments, the distal tapering portion of each of which is plain and 
transparent, while the basal portion is traversed longitudinally by four minute, refractive, somewhat beaded longitudinal striae. The pharynx is abont as deep as it is wide, and in its widest part is a little more than half as wide as the front of the head. It is well filled at the base with the two crescent-shaped, pointed, pharyngeal teeth. These extend inward and then forward, their points being close to the body axis. The front contours of the teeth are incurved, and at one point on their front surface they are exceedingly finely striated. Each of these teeth occupies about one-fourth of the width of the head; measured in the longitudinal direction its extreme limits are less than when measured transversely. The entire length of the pharynx is probably somewhat greater than the width of the head. though it joins the lumen of the oesophagus in such an indefinite way that it is sometimes rather difficult to say where the pharynx ceases and the true oesophagus begins. The portion of the oesophagus surrounding the pharynx is, however, distinctly, though slightly, swollen, so that it is proper to speak of an elongated pharyngeal bulb. Just at the base of the phraynx this bulb is two-thirds as wide as the corresponding portion of the head. At a point two or three body diameters farther back it is only about half as wide as the corresponding portion of the neck; thence onward it expands a very little. Near the middle of the neck the oesophagus expands to form the elongated muscular median bulb, which is three-fourths as wide as the middle of the neck. Here the lining is more strongly developed, and occupies about one-fourth of the optical longitudinal section, while the lining of the preceding portion of the oesophagus occupies not more than one-fifth to onesixth of the diameter. Behind the median bulb the oesophagus is less strongly developed. The lining is much less conspicuous and the radial muscular fibers are less abundant. At first this posterior portion of the oesophagus is only about one-fourth as wide as the middle of the neck. It expands gradually until near the end, then more rapidly so as to form an elongatedclavate swelling, three-fourths as wide as the base of the neck. The lining of this portion of the oesophagus is a distinct feature, but is by no means so strongly developed as that of the preceding portions of the eosophagus. There is a well developed flattish cardia, half as wide as the base of the neck. The intestine, which is separated from the oesophagus by a distinct constriction, becomes at once about three-fourths as wide as the body. Its cells contain scattered, nearly colorless granules of variable size, the largest of which have a diameter nearly equal to the distance between two of the adjacent longitudinal striations. From the slightly depressed anus the rectum, which is about one and one-half times as long as the anal body diameter, extends inward and forward. The tail is conoid from the anus and tapers rather regularly to the extremely fine, hair-like terminus. There are no caudal glands.

From the rather prominently elevated vulva the chitinized vagina leads inward at right angles to the ventral surface half way across the body, where it joins the two symmetrically-placed uteri. The reflexed ovaries reach 
atout half way back to the vulva and contain six to ten developing ova arranged single file. The thin-shelled eggs, which are deposited before segmentation begins, are fully twice as long as the body is wide and about one-third as wide as long, and occur in the uteri one at a time. Full-grown ova are ellipsoidal, and about one and one-half times as long as the body is wide and about half as wide as long.

55

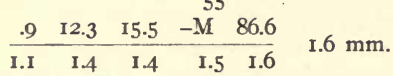

The tail of the male is like that of his mate in form. There are no supplementary organs in front of the anus. The special male papillae characteristic of this genus are located as follows: A single tapering, ventrally submedian papilla a little in front of the proximal ends of the spicula, and slightly farther back a lateral pre-anal papilla; a ventrally submedian papilla as far behind the anus as that first described is in front of it; a lateral papilla twice as far behind the anus as that just described; a ventrally submedian papilla as far behind that just described as the latter is behind the previous post-anal papilla; between the two ventrally submedian papillae just described a group of minute subventral papillae; near the middle of the tail, on the slender part, two sublateral papillae. Most of these papillae are slender, tapering, and one-third to one-half as long as the anal body diameter. The two equal, yellowish, arcuate, acute spicula are about one and onefourth times as long as the anal body diameter. They taper pretty regularly from near the proximal ends, where they are one-fourth as wide as the corresponding portion of the body. The proximal ends diminish suddenly in diameter, and are then cephalated by expansion. Each expansion is a rather strongly refractive, more or less bulbous piece of chitin. The accessory piece is arcuate and about one-third as long as the spicula. It is parallel to and applied closely to the spicula. Its framework consists of two distinct, nearly parallel parts. The ejaculatory duct is about one-fourth as wide as the body. The blind end of the single outstretched testicle lies a little farther behind the base of the neck than this latter is behind the anterior extremity.

Habitat: Spring, Washington Country Club, Chevy Chase, Md. Sublimate to balsam.

PRISMATOLAIMUS, de Man, 1880.

Fig. 4, Plate III.

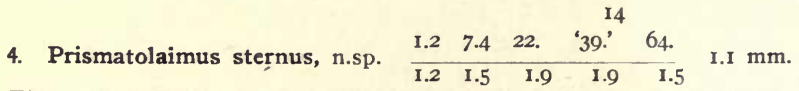

The moderately thin layers of the transparent, colorless cuticle are traversed by about 550 transverse striae, resolvable with high powers into rows of minute elements. There are no longitudinal striations. Scattered on 
the neck and body there are a few hairs arranged at right angles to the surface. These generally have a length equal to the width of two to three annules of the cuticle. The cylindroid neck ends in a somewhat truncated head, which is not set off in any way. On the outer margin of the head at the base of the lips there is a circlet of ten widely spreading, straight, tapering cephalic setae, two of somewhat unequal size on each submedian line, and one on each lateral line. The longest of these setae are a little more than half as long as the head is wide. For two-thirds of their length they are finger-shaped; the remaining third is a much narrower bristle. The very thin lips are arched over the prismoid pharynx, which is nearly as deep as the head is wide, and about three-fifths as wide as the head. It attains its full width immediately behind the lips, and continues to have the same width to the end, so that the bottom of the pharynx, though not even, extends across the base of the head approximately at right angles to the body axis. Where the lumen of the eesophagus joins the pharynx there is, however, a slight backward extension of the pharyngeal cavity. There are no eye-spots. The amphids occur in the form of somewhat elongated transverse markings placed transversely on the neck somewhat farther behind the base of the pharynx than this latter is behind the anterior extremity. Each amphid has a length about one-third as great as the corresponding width of the neck, and is about half as wide as long. Behind each amphid, at a distance equal to the width of two to three annules of the cuticle, there is a short seta. The oesophagus receives the base of the pharynx, and is at once nearly three-fourths as wide as the head. It narrows very slightly in diameter as it passes backward, so that where it passes through the nervering it is about two-thirds as wide as the neck. It continues to have practically the same diameter to near the end, where it is two-thirds as wide as the base of the neck. Immediately in front of the intestine there is a small segment of the oesophagus which is set off by a constriction. This portion is somewhat spheroidal in form. The lining of the oesophagus is a distinct feature throughout its length, and comes into view in the optical section as a single refractive line. This line is nowhere materially altered. There is no distinct cardia, unless the above mentioned spheroidal segment of the oesophagus may be so considered. The rather thick walled intestine, which is set off from the oesophagus by a distinct constriction, becomes at once about two-thirds as wide as the body, and is composed of cells of such a size that probably about four are required to build a circumference. These cells contain numerous granules of rather uniform size, which are not disposed so as to give rise to a tessellation. From the somewhat continuous anus the rectum, which is about as long as the anal body diameter, extends inward and forward. The tail end begins to taper from some distance in front of the anus, and tapers regularly thereafter throughout the anterior portion of the tail. The posterior portion tapers very slightly, and has a diameter one to two times as great as the width of one of the annules of the cuticle, measured near the anus. Though the terminus has considerable 
width there is no spinneret. The lateral fields are about one-third as wide as the body. The nerve-ring surrounds the oesophagus somewhat squarely. On the dorsal side, a little behind the cardiac constriction there is a body which presses the intestine a little to one side, which may perhaps be the renette cell. This body has a length as great as the corresponding body diameter, and is about one-fourth to one-third as wide as long. The vulva is somewhat elevated, and from it the vagina extends inward at right angles to the ventral surface about one-third the distance across the body. There are two symmetrically disposed, reflexed ovaries, the blind ends of which lie near the vulva, the position depending to a considerable extent upon the development of the much elongated eggs. The ovaries are moved backward and forward to a certain extent in accordance with the stage of development of the eggs. The eggs, which have been seen in the uteri one at a time, are much elongated-four or five times as long as the body is wideand about one-fifth as wide as long. They appear to be deposited before segmentation begins. balsam.

Habitat: Roadside pool, Douglas Lake, Michigan. Sublimate to

\section{SPILOPHORA, Bastian, 1865.}

Fig. 5, Plate III.

\section{Spilophora canadensis, n.sp.}

The thin, transparent layers of the colorless, practically naked cuticle are traversed by 750 transverse striae, resolvable with high powers into rows of dots, which at the lateral wings are so modified as to give rise to two distinct longitudinal rows of dots of larger size. Along the middle of the body one of these rows is more pronounced than the other. Opposite the base of the neck the distance between these two longitudinal rows is about four times as great as the distance between two of the dot-like elements. There are no eye-spots, nor have any distinct traces of amphids been seen. If the latter are present they must be very inconspicuous. The lips are so small that it is very difficult to count them, but there appear to be twelve, each one obscurely conical. There are no very marked subdivisions between these lips, so that the shallow cyathiform anterior portion of the pharynx, (a, Fig. 5) which is about half as wide as the head and about one-third to one-half as deep as wide, is not prominently striated radially as is sometimes the case in this and related genera. From the slightly depressed vulva, the vagina leads inward at right angles to the ventral surface about half way across the body, where it joins the two symmetrically-placed uteri. The reflexed ovaries reach fully half way back to the vulva, at least in young specimens such as have been examined, and contain a dozen to twenty developing ova arranged in more or less double file. The eggs appear to be elongated, somewhat longer than the body is wide and about one-third as wide as long. It is possible, however, that there is some error here as the description is 
derived from a single specimen in which these features are rather obscure. The reversal in the striations of the cuticle is plainly to be seen in the region of the vulva, and its location is indicated by a slight rise in the contour. This slight elevation extends also to the subcuticle.

60

$\begin{array}{rrrrrrr}.5 & 8.3 & 14.8 & -\mathrm{M} & 88.4 & .7 & \mathrm{~mm} .\end{array}$

The wing markings on the anterior part of the tail are pronounced, but on the posterior part much less so. A little in front of the anus they are as pronounced as they are at the base of the neck, and both are equally visihle, and the structure not infrequently resembles that of a ladder with the rungs placed close together, just as it does on the neck. The lateral fields appear to be about one-third as wide as the body. Near the middle of the body may plainly be seen the location where the cuticle reverses its structure. Although the striations are minute they present the same character as in species where the striations are coarse, and, upon careful examination, can be seen to be retrorse in opposite directions on the two halves of the body.

Habitat: Fresh water ponds, Cape Breton Island, Dominion of Canada.

MICROLAIMUS, de Man, 1880.

Fig. 6, Plate III.

\section{Microlaimus fluviatilis, n.sp. \begin{tabular}{rrrrrr} 
I.6 & 8.5 & 15.5 & ' $44^{2}$ & 85. \\
\cline { 2 - 5 } & I.9 & 3.5 & 4.3 & 4.7 & 2.6
\end{tabular} $.83 \mathrm{~mm}$.}

The thin layers of the transparent, colorless, naked cuticle are traversed by exceedingly fine transverse striae, which are resolvable with great diffculty into rows of dots, and are not modified on the lateral fields. The conoid neck ends in a rounded head set off by an almost imperceptible constriction. On the outer margin of the head there is a circlet of four submedian, slightly tapering cephalic setae, each about one-third as long as the head is wide. The vestibule is considerably shorter than any of the cephalic setae, and is longitudinally striated, indicating, no doubt, that the lips can be opened outward in receiving food. These longitudinal striations of the vestibule are too minute to be counted, hut there are apparently about twelve. The circular amphids are about one-fourth as wide as the corresponding portion of the head, and are located at a distance from the anterior extremity equal to one and one-half times the diameter of the front of the head. There are no eye-spots. The limits of the pharynx are indicated by a slight constriction in the oesophagus. The base of the pharynx is somewhat more than halfway back to the amphids. The general form of the pharynx is cylindroid or prismoid. It is armed with minute and very inconspicuous, somewhat inward pointing and apparently not very regular teeth or projections. The most conspicuous of these projections are two just behind the base of the lips, one dorsal and the other apparently ventral, and 
a third at the base of the pharynx on the dorsal side. The oesophagus begins as a tube about half as wide as the corresponding portion of the neck, and continues to have this diameter until it swells to form the pyriform cardiac bulb, which is about three-fourths as wide as the base of the neck, and contains a distinct chitinized threefold, rather simple, valvular apparatus, occupying two-fifths of the diameter as seen in longitudinal section. The musculature of the bulb is divided into three sections, a small anterior section, a middle and a posterior section. Of these two latter the posterior is slightly the smaller. The lining of the oesophagus is a fairly distinct feature throughout its length. There is no very distinct cardia. The intestine joins the middle of the posterior surface of the cardiac bulb, and is there onefourth as wide as the base of the neck. It soon expands so as to become about two-thirds as wide as the body. It is rather thick walled, and is composed of rather thick cells which are of such a size that about four are required to build a circumference. These cells contain minute granules. From the anus the rectum, which is considerably longer than the anal body diameter, extends inward and forward. The tail is conoid, and tapers from the anus toward the terminus, which is conoid and has a diameter one-third as great as that of the base of the tail, and bears a blunt convex-conoid unarmed spinneret. The rather small caudal glands appear to be located in the anterior third of the tail. The lateral fields have not been seen. From the inconspicuous vulva the vagina leads inward at right angles to the ventral surface to the single or double uterus, which when single extends forward. There sometimes appears to be a small posterior rudimentary branch to the sexual organs, which is about equal in length to the corresponding body diameter. The reflexed ovary reaches about halfway back to the vulva, and contains about half a dozen or more ova arranged somewhat irregularly. The eggs appear in the uterus one at a time. They are smooth, somewhat thin-shelled, and are about twice as long as the body is wide, and about one-third as wide as long. They appear to be deposited before segmentation begins. Specimens with one ovary, and those with two, appear to be about equally numerous; as no other difference has been detected, they are included for the present under the same name and description. The renette cell is an elongated cell located a short distance behind the base of the neck. It is fully as long as the body is wide, and about one-fourth as wide as the body. The excretory pore appears to be located opposite the nerve-ring. The nerve-ring surrounds the oesophagus somewhat squarely.

Habitat: Maple River, Michigan. Sublimate to balsam.

TRIPYLA, Bastian, 1865.

Fig. 7, Plate III.

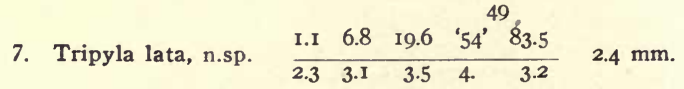

The thick layers of the transparent, colorless, naked cuticle are apparently traversed by about 250 transverse striae, which are not further re- 
solvable. In the lumen of the pharynx at a point removed from the anterior extremity a distance a little greater than the radius of the head, there is a minute inward-pointing dorsal tooth, having a length about equal to the width of one of the annules of the cuticle. This and other matters prove that the pharynx, although not readily distinguished, extends backward a distance about equal to the width of the head. In some specimens it is possible to see a distinct transverse fold in the lining of the oesophageal tube. This is located at a distance from the anterior extremity about equal to the corresponding diameter of the head, and undoubtedly indicates the posterior limits of the pharynx. It is possible that the tooth just mentioned may be connected with internal glandular structures in the oesophagus, as the interior of the dorsal segment of the oesophagus at this point is usually more or less different in structure from the other portions and does not seem to be made up entirely of muscular fibers. The oesophagus is separated from the intestine by a flattish, pseudo-bulb. The bulb is separated from the oesophagus on one side and the intestine on the other by deep and distinct constrictions. In the anterior constriction there are at least two granular, elongated cells of considerable sizc. The nerve-ring surrounds the oesophagus rather squarely. Nothing definite is known concerning the renette or the excretory pore. From the inconspicuous vulva, the vagina leads inward at right angles to the ventral surface fully half way across the body, where it joins the two symmetrically-placed uteri. The reflexed ovaries reach about half way back to the vulva, and contain a dozen to twenty developing ova arranged somewhat irregularly. Both in front of and behind the vulva there are unicellular glands, having a diameter about one-sixth as great as that of the body.

$$
\begin{array}{rrrrrr}
.7 & 6.8 & 19.3 & -M_{-}^{50} & 82.8 & \\
\hline 2.1 & 3.2 & 3.9 & 4.8 & 4.1 & \mathrm{~mm} .
\end{array}
$$

The tail of the male is like that of the female except that the anus is slightly raised. In front of the anus there is a ventral row of about twenty innervated supplementary organs, reaching to near the head. The distance between two consecutive organs is about equal to the radius of the body. Each of these appears to consist of a nerve-ending which penetrates the cuticle and forms a papilla, around which there is an almost imperceptible elevation having a width about equal to two of the annules of the cuticle. The visibility of this small organ is quite as much due to the alteration in the body wall and subcuticle as to that in the cuticle. There are no supplementary setae or papillae either in front of the anus or behind it. The two equal, slightly arcuate, uniformly tapering, acute spicula are about one and one-fourth times as long as the anal body diameter. Their proximal ends are not cephalated, though the wall of the cuticle is very slightly thickened at that point. The spicula taper regularly from the proximal ends to the rather 
truncated points. There are obscure accessory pieces lying close to the surface of the spicula and forming a double groove in which these latter slide. The ejaculatory duct, though narrow at first, soon becomes nearly half as wide as the body. There are two slender, outstretched testicles extending in opposite directions from near the middle of the body. The anterior one has its blind end about as far behind the base of the neck as the nerve-ring is in front of it.

Habitat: Alpine lakes, Bald Mountain, Colorado. Formalin to glycerine.

TERATOCEPHALUS, de Man, 1876.

Fig. 8, Plate IV.

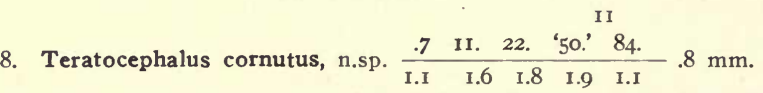

The thin, transparent layers of the colorless naked cuticle are traversed by about 1500 transverse striae, resolvable into rows of minute dots, which are modified on the lateral fields. These modifications have been seen in all parts of the body, and near the tail consist of two to three irregular longitudinal rows of enlarged dots. The posterior portion of the neck tapers but little. The anterior portion becomes convex-conoid toward the head, which bears a lip region more or less set off by a constriction opposite the base of the spreading cephalic setae, which are four in number and submedian in position. Each seta is a little more than half as long as the head. These are connected with a refractive six-ribbed chitinous dome-like framework, which extends back to opposite the middle of the pharynx. The ribs start at the lip region, extend backward, and then join each other opposite the base of the vestibule. The chitinous refractive elements at this latter region form a rather distinctly crenate contour around the head. No other setae than the cephalic setae have been seen in any part of the body. The lips appear to be rather thin, and are arched together over the unarmed pharynx, which is somewhat elongated and about half as wide as the base of the head. While it is somewhat irregular in form it is approximately prismoidal or cylindroid and nearly as deep as the head is wide. Its walls are not very strongly chitinized, but more strongly so anteriorly than posteriorly. The oesophagus receives the base of the pharynx, and soon becomes half as wide as the base of the head. In the anterior portion of the oesophagus, a short distance behind the pharynx are three slightly arcuate, refractive elements, corresponding somewhat in curvature with the contour of the front end of the oesophagus. These resemble to a certain extent the refractive elements in the posterior chamber of the pharynx of Plectus. In the present species they are about as long as the pharynx, and are separated a distance about equal to one body width. They are a little wider and more plainly visible anteriorly than posteriorly. The obscure amphids, as 
far behind the base of the pharynx as this latter is behind the anterior extremity, are one third as wide as the corresponding part of the head, and consist of obscure spirals of one wind. There are no eye-spots. The oesophagus begins as a tube about half as wide as the corresponding portion of the head, but expands very slightly as it passes backward, so that at the middle it is about two-thirds as wide as the middle of the neck. Behind this point it diminishes. There is a break in the musculature somewhat similar to that occurring in Cephalobus. At the break, which occurs at the beginning of the fourth fifth, the oesophagus is somewhat narrow, about half as wide as the corresponding portion of the neck. It finally expands to form the ellipsoidal cardiac bulb, which has a relatively large, chitinous, striated, complicated, three-fold valvular apparatus half as wide as itself. There is a truncateconoid cardia about one-third as wide as the base of the neck. The lining of the oesophagus is a fairly distinct feature. The thick-walled intestine, which is separated from the oesophagus by a constriction, soon becomes twothirds as wide as the body. It is composed of cells of such a size that four are required to build a circumference. The walls of these cells are refractive, so that the cells are readily distinguished from each other, though there is no distinct tessellation. These cells contain minute very uniform granules. The lining of the intestine is refractive, so that the lumen is distinctly to be seen. From the rather inconspicuous anus the rectum, which is about as long as the anal body diameter, extends inward and forward. The internal sexual organs are double and symmetrically reflexed. The tail is conoid from the anus to the subacute terminus. This species is related to those described by Dr. de Man under the name of Teratocephalus. It is doubtful whether these forms will not have to be subdivided at some future time, after the accumulation of the necessary data.

Habitat: Maple River, Michigan. Sublimate to balsam.

ALAIMUS, de Man, 1880.

Fig. 9, Plate IV.

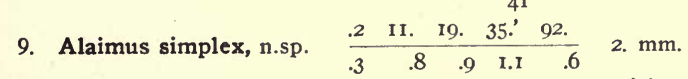

The thin, transparent layers of the colorless, naked cuticle would ordinarily be described as destitute of striations. Very minute striations may be observed in the subcuticle at the extremities, when the specimens are examined under favorable conditions with the highest powers. There are obscure traces of lateral wings. The conoid neck ends in a rounded head not set off in any way. There are no distinct lips, and only the faintest possible indications of labial papillae. These are so obscure that it has been found impossible to enumerate them. There is no pharynx and the mouth opening is exceedingly minute. No amphids or eye-spots have been seen. 
The oesophagus begins at the lip region as a tube two-thirds as wide as the head, and expands gradually and rather regularly to the end, where it has a diameter three-fourths as great as that of the neck. A short distance behind the head end, a distance six times as great as the diameter of the head, there is a slight alteration in the structure of the oesophagus the significance of which remains doubtful. The lining of the oesohpagus is not a very distinct feature. There is a very obscure, small cardia. The intestine, which is separated from the oesophagus by a constriction, becomes at once about two-thirds to three-fourths as wide as the body. At first its cells contain but few scattered granules, but soon they are characterized by the presence in them of loosely scattered yellowish-brown granules of variable size, the largest of which have a diameter nearly one-eighth as great as that of the body itself, and the smallest of which are very much smaller. These are not arranged so as to give rise to any distinct tessellation. Toward the end of the intestine the granules again become less abundant and smaller, so that for some distance in front of the rectum there is only here and there a granule. From the depressed and conspicuous anus the chitinized rectum, which is a little longer than the anal body diameter, extends inward and forward. There is no distinct pre-rectum. The tail end begins to taper from a long distance in front of the anus, but tapers very gradually to behind the anus. Thence onward it is inclined to be arcuate and tapers regularly to near the terminus. Near the end it tapers more rapidly than elsewhere, and finally comes to an almost acute point at the terminus. A short distance in front of the terminus, however, the diameter is about half as great as it is at the anus. There does not appear to be any spinneret, and therefore, of course, caudal glands are absent. The lateral fields appear to be about one-third as wide as the body. Nothing is known concerning the excretory pore. From the slightly elevated vulva the vagina leads inward nearly at right angles to the surface more than halfway across the body, where it joins the single uterus, which extends backward. The ovary is reflexed, and the blind end is about halfway back to the vulva. The eggs occur in the uterus one or two at a time, and are very much elongated. They are about six times as long as the body is wide, and about one-eighth as wide as long, and are covered by a distinct, smooth shell. They are apparently deposited before segmentation begins. The spermatozoa appear to be elongated-ellipsoidal, and a little more pointed at one end than at the other. They have a length one-fifth that of the body diameter.

68

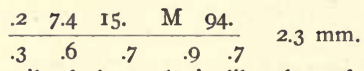

The tail of the male is like that of the female in form and size, but the anus is somewhat elevated. In front of the anus there appears to be a ventral row of very obscure, broad, flattish supplementary organs. Perhaps these ought to be described as locations of nerve endings rather than as distinct organs. Their number remains doubtful, but there are about 
three or four occupying a distance equal to half the length of the tail. The two equal, straight spicula are rather obscure. Their length is no greater than the length of the anal body diameter. Oblique copulatory muscles are seen in the vicinity of the spicula, and there is a refractive element passing backward from the accessory piece toward the ventral side of the tail near the anus, which may be muscular, or possibly chitinous and serving for the attachment of muscles. The ejaculatory duct is about one-third as wide as the body, the vas deferens somewhat wider. Whether there are two testes or only one has not been determined. There is a broad, rounded, blunt end of a testis located as far behind the base of the neck as this latter is behind the anterior extremity.

Habitat: Big Lake, Fla. Bouin solution to glycerine.

PLECTUS, Bastian, 1865.

Fig. 10, Plate IV.

\section{Plectus tubifer, n.sp.}

\begin{tabular}{lrrrr} 
& & \multicolumn{3}{c}{35} \\
2. & 10.7 & 19. & 49 & 92.6 \\
\hline 2.1 & 3.4 & 3.5 & 3.2 & 2.3
\end{tabular}

$.74 \mathrm{~mm}$.

The skin is of medium thickness, colorless, and traversed transversely by fine, plain striae. The striae are of such a size that about twenty of them occupy a space equivalent to the body diameter. There are no hairs on the surface of the body, but throughout the length of the body, more particularly on the submedian lines, may be seen very faint markings in the cuticle, which indicate the location of four rows of pores connecting with interior organs. Immediately behind the lateral organs one sees the first of the series of pores located in the lateral line. This first pore, though much smaller than the lateral organs, is yet somewhat larger than the succeeding ones, which gradually spread out to form two submedian rows. The total number of these pores on one side (of the male) is about one hundred; that is, there are about two hundred in all. The six low, rounded lips are rather massive, and are arched together over the pharynx. These lips appear to be armed with chitinous processes on the inner surface, these processes surrounding a short napiform vestibule leading to the pharynx proper. If any labial papillae are present they are exceedingly minute; what appear to be traces of papillae may occasionally be seen. The lateral organs are well forward, being located only slightly behind the cephalic setae. They present transversely elongated contour markings open on the posterior margin. The lateral fields appear to attain a considerable width and two chitinous, lateral markings close together with a third between them, appear to indicate the existence of a wing on each side of the body. 


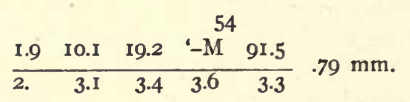

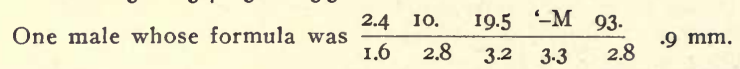

possessed four supplementary organs, the additional one being some little distance in front of the others, which were arranged as illustrated. Sometimes there are only two of these organs, one smaller than the other. The cuticle of the ventral surface is slightly thickened and elevated where it is pierced by these tubular organs, which appear in some instances to be slightly exserted. Apart from these preanal supplementary organs, there are ventrally submedian papillae on the tail; - one pair near the middle, another pair half way between these and the anus, and a third pair part way from that first mentioned to the terminus. There is a fourth pair indistinctly to be seen just behind the ants; these are closer together than the members of the other pairs. Furthermore there are one or two very small pairs near the terminus, but perhaps these may partake more of the nature of hairs than of papillae. Just in front of the anus there is a single ventral almost setose papilla. There occur in front of the anus at least two pairs of ventrally submedian papillae. These are located one just in front of the posterior supplementary organ and the other just behind it. Most of these features are not easy to decipher, very careful observation being necessary to enumerate the papillae as above. There are two testicles, one anterior to the other, the anterior being outstretched and extending forward, the posterior being reflexed near its middle, so that its blind end lies somewhere near the junction of the two. The anterior one of this pair is connected with the seminal vesicle by a tubular portion, which lies alongside the reflexed posterior member.

Habitat: Arlington, Va. Flemming solution to glycerine.

DORYLAIMUS, Dujardin, 1845.

Fig. 12, Plate V.
11. Dorylaimus fecundus, n.sp.

$$
\begin{array}{cccccc}
2 . & 4.4 & 16 . & 43 & 43 & 96 . \\
\cline { 1 - 5 } .5 & 1.1 & 1.6 & 1.9 & 1.2 & 3.4 \mathrm{~mm} .
\end{array}
$$

The thin layers of the transparent, colorless, naked cuticle are traversed by exceedingly fine, plain, transverse striations resolvable with the highest powers of the microscope under favorable conditions. Longitudinal striations are visible throughout the length of the body. The presence of amphids is indicated by a transverse marking immediately behind the lip region, and somewhat in front of the guiding ring of the spear. The anterior contour of each organ is a transverse line about three-fifths as long as the corresponding portion of the head is wide. Extending backward from this are lateral contours which approach each other slightly and end indefinitely. The 
oesophagus begins as a tube about half as wide as the corresponding portion of the neck, and continues to have this diameter until near the middle of the neck, where it first begins to expand. A little behind the middle of the neck it expands rather suddenly, so that the remainder of the organ is about two-thirds as wide as the base of the neck. There is a much elongated conoid cardia nearly as long as the body is wide. The intestine, which becomes at once three-fourths as wide as the body, is separated from the oesophagus by a distinct though slight constriction. The cells forming the intestine are of such a size that about four or five are required to build a circumference. They contain granules of variable size, the larger of which have a diameter equal to the thickness of the outer transparent cuticle, and the smaller a diameter only about one-fourth as great as that of the larger. These granules are so arranged as to give rise to a very obscure tesselation. The pre-rectum is about three to four times as long as the corresponding body diameter, and is separated from the intestine by a difference in structure, the granules contained in its cells being smaller, and not darkening under the influence of osmic acid, as do those of the intestine. Nothing is known concerning the salivary glands or the renette. The longitudinal fields are well developed, being about half as wide as the body. The nerve-ring surrounds the oesophagus somewhat obliquely. Each of the two elongated uteri may contain three to five eggs at a time. These, as they appear in the uteri, are elongated, finely granular, rather thin-shelled, and about one and one-half times as long as the body is wide, and about half as wide as long. The shells are flexible, so that owing to the pressure of one egg on another in the uterus the surfaces are often indented.

$$
\begin{array}{ccccccc}
.3 & 4.8 & 17 . & -\mathrm{M}- & 99 . & & \\
\hline .6 & 1.2 & 1.4 & \text { I. } 4 & \text { I. } 4 & 3.5 &
\end{array}
$$

In addition to the ventral row of supplementary male organs shown in the illustration, there are two rows of ventrally submedian, innervated papillae, arranged in a somewhat equidistant manner. Where these reach the surface of the body they give rise to almost imperceptible elevations which, however, are of a different character from those at the ends of the ventral organ shown in p, Fig. 12. In other words, these papillae are more nearly the homologues of the ordinary tactile hairs or papillae so common on the surface of nematode worms. These submedian papillae are located at a distance from each other equal to about two-thirds the body diameter. They are very nearly coextensive with the oblique copulatory muscles.

Habitat: Algae, Potomac River, Washington, D. C., 1911. Flemming solution to glycerine. 
IRONUS, Bastian, 1865.

Fig. 13, Plate V.

\section{Ironus americanus, n.sp. \\ \begin{tabular}{rrrrrr} 
& \multicolumn{5}{c}{30} \\
3.7 & 9.2 & 21.3 & $522^{\prime}$ & 92.5 & \\
\cline { 1 - 5 } & 2.3 & $\mathrm{~mm}$.
\end{tabular}}

The moderately thin layers of the transparent, colorless, naked cuticle appear to be destitute of striations. There are three somewhat conoid lips, which when open, give to the head a somewhat truncated appearance, when folded a somewhat rounded appearance. Each lip bears internally a slightly arcuate, conical tooth, whose altitude is somewhat greater than the width of its base. When the mouth is so opened that the apices of these refractive teeth are about on a level with the anterior extremity their bases lie a little in front of the bases of the cephalic setae. The apices of these teeth are slightly blunt and they have a slight outward curvature. The walls of the pharynx are strongly refractive, and have about the same thickness as the walls of the cuticle. The chitin of the walls of the pharynx, however, is more refractive than that of the cuticle. In this respect it resembles the chitin of which the teeth are composed. Except for the teeth at the mouth opening the pharynx is unarmed, but presents on the dorsal side about half way between the head and its posterior extremity three or four exceedingly minute projections with corresponding depressions. The oesophagus begins near the base of the pharynx, at least it is at this point that the radial structure becomes pronounced. At first it is about two-thirds as wide as the corresponding portion of the neck. It enlarges a little and very gradually, so that finally it is about half as wide as the base of the neck. The lining of the oesophagus is an exceedingly distinct feature throughout its length. 1t generally has the appearance of three refractive lines occupying a space nearly one-fourth as wide as the oesophagus itself. There is a large cylindroid or hemispherical cardia, one-third as wide as the base of the neck. The intestine, which is separated from the oesophagns by a deep, narrow and distinct constriction, becomes at once about three-fifths as wide as the body. Its cells contain scattered granules of variable size, the largest of which have a diameter nearly equal to the width of the refractive portion of the lining of the oesophagus, and the smallest of which have a diameter not more than one-tenth as great. The body wall is thick, generally occupying about threefifths of the diameter of the body. The lateral fields are a little more than one-third as wide as the body. Nothing is known concerning the renette or the excretory pore. There is no spinneret. The tail, nevertheless, contains small cells near the anus which bear a certain resemblance to the ordinary caudal glands. From the slightly elevated vulva, the vagina leads inward at right angles to the ventral surface half way across the body where it joins the two symmetrically-placed uteri. The reflexed ovaries reach three-fourths the distance back to the vulva, at least in specimens which do not contain eggs. The ovaries contain about a dozen ova arranged for the 
most part single file. Nothing very definite is known concerning the form and size of the eggs, but from the size of the apparently matured ova it is assumed that the eggs are considerably elongated, perhaps two to two and one-half times as long as the body is wide, and if so, it is unlikely that more than one is contained in the uterus at a time.

Habitat: Deer Bottom, Pikes Peak region, Colorado. Formalin to glycerinc.

\section{ONCHOLAIMUS, Dujardin, 1845.}

\section{Fig. 14, Plate V.}

\section{Oncholaimus punctatus, n.sp.}

The rather thin, transparent, colorless layers of the naked cuticle appear to be destitute of striations. It is rather difficult to observe the cuticle on account of the presence in it of numerous dot-like elements, which near the head are arranged in longitudinal groups, of which the widest is the lateral group. The longitudinal arrangement of the granules, is continuous throughout the body, but it is most marked on the lateral fields where there is a definite band of them, having a width about two-fifths as great as the width of the body. Each margin of this band is made up of a distinct longitudinal row of granules arranged single file. The neck is slightly conoid, becoming slightly convex-conoid toward the rounded head, which is not set off in any way, or at most by an exceedingly obscure, broad constriction opposite the pharynx. The six lips are distinct and well developed and have a distinct, somewhat chitinous framework. They are arched together over the pharynx in such a way as to produce a dome-like effect, the very thin and movable flaps at the ends of the lips meeting together at the middle of the front of the head. Opposite the middle of the amphids there is a transverse marking or line which extends around the middle of the head. It is characterized by staining somewhat more strongly with carmine than the other portions of the cuticle. There are no eye-spots. The oesophagus begins at the base of the pharynx with a diameter three-fourths as great as that of the base of the head, and it continues to have this diameter until some distance behind the nerve-ring. It then begins to expand gradually, so that finally it is about three-fourths to four-fifths as wide as the base of the neck. The lining is a distinct feature throughout the length of the oesophagus. There is a distinct conoid cardia, whose base is about half as wide as the corresponding portion of the body. The intestine, which is separated from the oesophagus by a deep and distinct constriction, appears to have rather thin walls and to be composed of cells of such a size that probably six or more would be required to build a circumference. These cells contain numerous granules of rather uniform size.

The lateral fields are about two-fifths as wide as the body. The location of the ventral gland has not been made out. The excretory pore is lo- 
cated just behind the base of the pharynx. The duct which leads to it seems to be destitute of an ampulla.

$$
\begin{array}{ccccc}
\text { I.9 } & 10.2 & 21.4 & -\mathrm{M}- & 92 .
\end{array} \quad \text { I.1 mm. }
$$

In front of the anus there is a series of about fifty pairs of oblique copulatory muscles, whose presence is indicated by the oblique groups of granules in the cuticle. The ejaculatory duct is about one-third as wide as the body. The blind end of the anterior testicle is about twice as far behind the base of the neck as this latter is behind the anterior extremity.

Habitat: Fresh water ponds, Cape Breton Island, Dominion of Canada. Sublimate to balsam.

\section{DOLICHODORUS, n.g.}

Fig. 16, Plate VI.

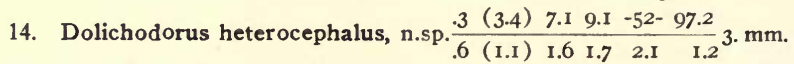

The thin layers of the transparent, naked, colorless cuticle are traversed by transverse striae resolvable with high powers under favorable conditions into rows of exceedingly minute, somewhat irregular elements. This resolution is not at all difficult in specimens which are moulting. Under these conditions scales of the outer cuticle sometimes survive, readily resolvable into refractive transverse markings between which are the small elements mentioned. Ordinarily at first glance, the cuticle appears to be merely resolvable into transverse striae which are somewhat roughened on the edges of the dividing lines. The conoid neck ends in a head which has a very prominent lip-region set off by a very prominent and deep constriction. The front of the head is rounded, and the constriction separating the lip-region from the remainder of the head is of such a nature that the contour of the lip-region, when seen in optical longitudinal section is somewhat elliptical, so that the lip-region has the form of an ellipsoid of rotation. The depth of this figure is about equal to half its width. No papillae have been seen on the lips, but minute papillae might readily be present without having been observed. No traces of amphids have been seen. There are no eye-spots. The vestibule is exceedingly narrow and closely surrounds the apex of the very slender narrow spear. This latter extends nearly half-way to the anterior border of the median bulb and is long and slender like that of Trichodorus. The oesophagus at the base of the spear is a tube about onethird as wide as the corresponding portion of the neck, and continues to have this diameter until it expands suddenly to form the elongated median bulb, which is three-fourths as wide as the corresponding portion of the neck and fully twice as long as wide and is armed internally with a conspicuous, ellipsoidal, rather simple valvular apparatus nearly half as wide as itself. When 
seen in optical longitudinal section the bulb is a little wider posteriorly than anteriorly, so that its sides are not parallel, but approach each other a trifle as they pass forward. The bulb ends more abruptly posteriorly than anteriorly, and is continued by a narrow section of the oesophagus only oneeighth as wide as the corresponding portion of the neck and about as long as the corresponding portion of the neck is wide. This is succeeded by a broadly clavate swelling which does not have any distinct chitinous lining, and is of granular structure, rather than muscular. This swelling is about two-thirds as wide as the base of the neck. The lining of the oesophagus is a fairly distinct feature in all parts in front of the posterior swelling. The intestine joins the middle of the rounded surface of the posterior oesophageal swelling and is at this point only one-tenth to one-eighth as wide as the corresponding portion of the body, but soon expands to be two-fifths as wide as the body. It is, therefore, relatively narrow. On the other hand, the body walls are correspondingly thick. Nothing is known concerning the renette cell. It is probable that there is an excretory pore located near the nerve-ring.

$$
\begin{array}{rrrrrrr}
.3 & (2.3) & 7.9 & 10.9 & -M^{60} & 99.3 & 2.4 \mathrm{~mm} . \\
\hline 4 & (.8) & 1.2 & 1.4 & 1.7 & 1.8 &
\end{array}
$$

The tail of the male is conoid and extends beyond the anus a distance hardly as great as the length of the anal body diameter. It seems likely that the tail is also flattish. When seen dorso-ventrally it appears to be about onehalf as long as the spicula, and ends in a dentate terminus one-third as wide as the base of the tail. The cuticle of the tail is very thick, and at first sight seems rather closely to resemble the structure of the two bursal flaps. The distinctly two-parted hursa springs from opposite the middle of the spicula and extends backward and is completely furcated at a point about opposite the base of the tail. The flaps extend backward beyond the terminus of the tail and give to the posterior extremity somewhat the appearance of ending in three thin transparent, colorless flaps, one of which is soon seen to be the true caudal extension. The flaps of the bursa are striated in much the same manner as the cuticle, and the margins of the flaps are distinctly thickened. No distinct ribs have been seen in the two broad backward pointing flaps of the bursa. The two equal, rather strongly built, tapering spicula have a length about one and one-half times as great as that of the diameter measured opposite their proximal ends. They are accompanied by a rather simple accessory piece one-half as long as themselves, judging from its appearance when seen dorso-ventrally. The proximal ends of the spicula can hardly be said to be cephalated, but they are obliquely trancated and wider than elsewhere. When seen dorso-ventrally this width appears to be one-fourth as great as that of the corresponding portion of the body. No special setae are present and no special papillae have been seen. The single outstretched testicle extends forward and has its blind end located about three to four times as far behind the base of the neck 
as this latter is behind the anterior extremity. Near its blind end its diameter is about one-half as great as that of the body. The spermatozoa are small and numerous and it appears that the reduction divisions take place in a short segment of the testis not far from the blind end.

Habitat: Freshwater, Douglas Lake, Michigan. Silver Spring, Florida. Female, Flemming solution to glycerine; from Florida. Male, from Michigan, sublimate to balsam.

CYATHOLAIMUS, Bastian, 1865.

Fig. 17, Plate VI.

26.

15. Cyatholaimus truncatus, n.sp. \begin{tabular}{cccccc} 
I.6 & 7.1 & I4. ' $49 . '$ & 88. \\
\hline I.8 & 2. & 2.1 & 3.3 & 2.1
\end{tabular} 1.6 mm.

The moderately thick layers of the transparent, colorless, naked cuticle are traversed by about fifteen hundred transverse striae, resolvable into transverse rows of distinct dots, which are not very materially modified in the lateral fields. The slightly conoid neck ends in a truncated head, whose lip region is set off by an almost imperceptible expansion. The cuticle becomes somewhat thicker toward the head, and the dotlike markings more distinct and refractive. Beginning near the head and ending near the spinneret there are circular markings arranged in somewhat irregular rows along the lateral fields. The longitudinal distance between these markings near the middle of the body is a little greater than the radius of the body, and the diameter of the circles is somewhat greater than the width of one of the annules, sometimes twice as great. These markings are not all of the same size, some being nearly twice as large as others.* The lip region is twelve-parted, and there are twelve longitudinal chitinous structures surrounding the vestibule. These no doubt indicate the infolding of the lips when closed. Probably the best conception of the lip region is expressed by saying that there are six lips, each two-parted, but that the divisions are all very similar to each other. On the front of the lips there are six forward pointing innervated papillae arranged in the usual position. Along the outer margin of the head there are ten acute, tapering, cephalic setae, two lateral, and four submedian pairs. The longest of these setae are about equal to the radius of the head. According to the position of the lips these setae vary in attitude. They may be folded together on the front of the head so as to be in the position of the spokes of a wheel, but they may point forward when the mouth is partly open, and are in a somewhat spreading position when the mouth is wide open. The pharynx is on the whole conoid, and about three-fourths as deep as the head is wide. Springing from a little behind its middle is a strong, conical, acute, forward pointing dorsal tooth, the ventral contour of which lies approximately in the axis of the

*The two most pronounced rows are nearly opposite the edges of the lateral fields, but there is an irregular median row. 
head. Surrounding the pharynx the muscles are somewhat more powerful than in the oesophagus immediately behind, so that there is a very faint pharyngeal bulb. The oesophagus is at first about two-thirds as wide as the corresponding portion of the neck, but gradually enlarges after passing through the nerve-ring, so that finally it is two-thirds as wide as the base of the neck. The lining of the oesophagus is a distinct feature throughout its length. There is no very distinct cardia. There are no eye-spots. The amphids, which are about one-third as wide as the head, are spirals of about two and one-half winds, and are placed opposite the apex of the dorsal tooth. The intestine, which is separated from the oesophagus by a distinct constriction, is at first one-half to two-thirds as wide as the body, but in the greater part of its length is about two-thirds as wide as the body. It is thick walled, and is composed of cells of such a size that comparatively few are required to build a circumference, probably about six. The cells contain rather numerous, conspicuous, brownish granules of variable size, the largest of which have a diameter about equal to double the width of one of the annules of the cuticle, the smallest of which are very much smaller. These granules are arranged irregularly, and can hardly be said to suggest any tessellation. From the broad, raised anus the rectum, which is refractive and nearly as long as the anal body diameter, extends inward and forward. The tail is conoid to the subacute conoid spinneret, which has a diameter about one-eighth to one-tenth as great as that of the base of the tail. There do not appear to be any caudal setae. The lateral fields appear to be fully one-third as wide as the body. The nerve-ring surrounds the oesophagus somewhat obliquely. The excretory pore appears to be situated at a distance from the anterior extremity about two and one-half times as great as the width of the head. The renette cell appears to be small and located some distance behind the base of the neck. From the elevated vulva the vagina leads inward at right angles to the ventral surface about half way across the body, where it joins the two-parted uterus. The reflexed, tapering ovaries reach about two-thirds the way back to the vulva, and contain a dozen or more developing ova arranged somewhat irregularly, especially toward the blind end. The rather thin shelled ovate to ellipsoidal eggs are a little longer than the body is wide, and about two-thirds as wide as long. They have been seen to occur in each uterus one at a time, and are apparently deposited before segmentation begins.

59 .

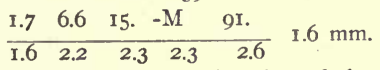

The tail of the male is like that of the female in form and size, but the anus is more prominently elevated. There is a row of four small pre-anal, ventral, tubular, protrudable supplementary organs. The final one of these is opposite the middle of the spicula; the penultimate nearly opposite the proximal ends of the spicula. The foremost is about three times as far from the anus as the penultimate. From this it will be seen that the organs 
are slightly more nearly approximated to each other posteriorly. These organs are not conspicuous features, and scarcely disturb the ventral contour. It is possible that each is connected with a unicellular glandular structure nearby. The two equal, tapering, somewhat arcuate spicula are one and one-third times as long as the anal body diameter. Their proximal ends are cephalated by expansion. The accessory pieces alongside are four-fifths as long as the spicula, and somewhat broader than the spicula. In their widest part they are one-sixth as wide as the corresponding portion of the body. They are of rather uniform width throughout. There is a slight constriction near each end, and the distal extremity appears to be very minutely dentate. The spicula are about half as wide as the accessory pieces. The ejaculatory duct is two-thirds as wide as the corresponding portion of the body, as is also the testis. The blind end is as far behind the base of the neck as this latter is behind the anterior extremity.

Habitat: Silver Springs, Fla. Flemming solution to glycerine.

ETHMOLAIMUS, de Man, 1880.

Fig. 19, Plate VII.

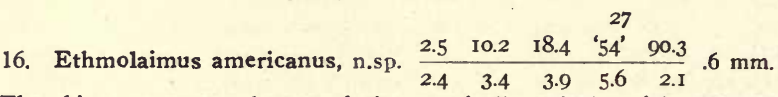

The thin, transparent layers of the practically naked cuticle are traversed by very fine transverse striae, resolvable with high powers into rows of closely set dots, which are not modified on the lateral fields. Near the head the dots are a little coarser than elsewhere on the anterior portion of the body. On the tail the dots are much farther apart and, though really arranged in transverse rows, at first glance appear to be scattered irregularly. The conoid neck ends in a somewhat truncated head, which bears on its outer margin a circlet of four slender, spreading submedian cephalic setae, each about half as long as the head is wide. There appear to be twelve minute papillae in the lip region, which when folded together forms a rather small, cyathiform cavity, in the midst of which stands the thumbshaped, forward-pointing dorsal tooth. This tooth is attached to a distinctly thickened rib of chitin, which extends from the lip region back to the base of the pharynx, and is thicker anteriorly than it is posteriorly. This thickened dorsal rib causes the pharynx to appear somewhat more strongly built on the dorsal side than on the ventral side. The tooth tapers slightly to a blunt point, and has a length about one-fourth as great as the width of the front of the head. Behind it the pharynx is somewhat prismoid and approximately one-fifth as wide as the head. It ends abruptly at the base. The pharynx is surrounded by a pharyngeal bulb, the musculature of which is set off distinctly from that of the oesophagus, in addition to which there is a constriction between the bulb and the oesophagus. The somewhat obscure, slender, apparently spiral amphids consist of about one and one-half winds. They are located opposite the posterior portion of the pharynx, and are one- 
third as wide as the corresponding portion of the head. There are no eyespots. The oesophagus begins as a tube three-fifths as wide as the base of the head, and it continues to have this diameter until it expands rather suddenly to form a somewhat ellipsoidal or obscurely pyriform cardiac bulb, which is four-fifths as wide as the base of the neck, and contains an obscure, elongated, relatively narrow valvular apparatus. The lining of the oesophagus is a distinct feature throughout its length. There is no very distinct cardia, but the preliminary cells of the intestine are different from those which immediately follow. The rather thick-walled intestine is separated from the oesophagus by a deep and distinct constriction, and becomes at once about half as wide as the body. Thereafter it widens out gradually so as to become three-fourths as wide as the body. It is composed of cells of such a size that probably about six to eight are required to build a circumference. 'The cells contain granules of variable size, the largest of which have a diameter half as great as that of the terminus of the tail. From the rather inconspicuous but slightly depressed anus the rectum, which is about as long as the anal body diameter, extends inward and forward. The body begins to taper slightly from some distance in front of the anus, but tapers more rapidly behind the anus in such fashion that at the beginning of the posterior fifth the diameter is about one-sixth as great as at the anus; thence onward the tail is cylindroid, and ends in a tubular spinneret, less than half as wide as the terminus. The spinneret tapers slightly to a blunt point, and is armed at its base with one or more setae as long as itself. The caudal glands appear to be located in the base of the tail. The lateral fields are well-developed and fully one-third as wide as the body. The renette cell appears to be located a short distance behind the cardiac bulb. The position of the excretory pore is unknown,--possibly it is at the base of the lips. The nerve-ring surrounds the oesophagus somewhat obliquely. From the elevated, rather broad vulva the vagina leads inward at right angles to the ventral surface fully half way across the body, where it joins the symmetrically-placed uteri. The reflexed ovaries reach as far back as the vulva, -at least in specimens in which the uteri contain no eggs. The fully developed ova are nearly twice as long as the body is wide and about onethird as wide as long. The forn, size and number of the eggs remains undetermined, but it seems probable that they occur in the titeri one at a time.

Habitat: Spring, Washington Country Club, (Khoy Chase, Md. Sublimate to balsam.

MONHYSTERA, Bastian, 1865.

Fig. 20, Plate VII.

62.

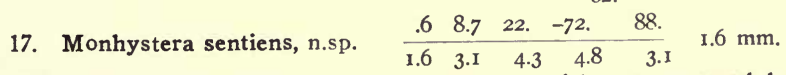

The thin, transparent layers of the colorless cuticle are traversed by about eight hundred transverse striae, resolvable with difficulty into rows of 
somewhat dotlike elements. These striae are more readily visible toward the extremities, especially the posterior extremity. The conoid neck becomes convex-conoid toward the head, which is somewhat rounded. Throughout the body there are rather numerous, long, slender somatic setae having a length equal to the radius of the body. These setae are a little longer toward the extremities than they are near the middle of the body. On the outer margin of the head, a trifle in front of the base of the pharynx, there are ten spreading, tapering cephalic setae, one on each lateral line, and two of somewhat unequal size on each submedian line. The longest of these setae are more than half as long as the head is wide. A little more than twice as far back as the amphids there is a rather definite circlet of cervical or subcephalic setae arranged in submedian positions. The lips appear to be three in number, and are longitudinally striated or fluted. The thin flap-like, striated portions are capable of assuming a revolute position. The basal portion of the lip region is considerably thicker than the flaps, and is arched over the margin of the pharynx. This latter is wide and shallow. Just beneath the lips it is two-thirds as wide as the front of the head. It closes together rapidly, so that its base is located at a distance from the anterior extremity about equal to the radius of the head. In other words, the posterior portion of the pharynx is about half way back to the amphids. These latter are circular, and one-third to one-fourth as wide as the corresponding portion of the head. Their distance from the anterior extremity is about equal to half the width of the front of the head. A short distance in front of each amphid there is a granular nucleus, which closely resembles the nerve cells that are found in the neck, and seems doubtless to be the nucleus of the nerve cell which is connected in some way with the function of the amphids. The oesophagus reaches to the base of the lips, and into it is set the posterior portion of the pharynx. At first the oesophagus is only twothirds as wide as the base of the head. It continues to have this diameter, or a little more, until after it passes through the nerve-ring. Thence onward it gradually increases so that finally it is about two-thirds as wide as the base of the neck. The lining of the oesophagus is a fairly distinct feature. The intestine, which is separated from the oesophagus by a distinct constriction, is composed of cells of such a size that probably six to eight are required to build a circumference. The anterior portion of the intestine is somewhat bulbous in form, and is separated from the oesophagus on the one side and the true intestine on the other, by a pair of constrictions. This portion may be looked upon as a strongly developed cardia. The intestine is moderately thick walled, and ends posteriorly in a short, wide rectum, hardly as long as the anal body diameter. The posterior extremity of the female begins to taper from near the vulva, and continues to taper at about the same rate behind the tail as in front of it. The tail itself is conoid in the anterior fourfifths, and cylindroid in the posterior fifth. The terminus is hardly swollen. and is about one-sixth as wide as the base of the tail. The spinneret is rounded and bears a couple of submedian setae twice as long as its own 
diameter. The three caudal glands are located in the anterior third of the tail. The lateral fields vary in width in different parts of the body. A little in front of the anus they are about two-fifths as wide as the corresponding portion of the body, and contain rather numerous scattered nuclei of such a size that about eight would be required to reach across the field. Farther forward the field is narrower, about one-fourth as wide as the middle of the body. Anteriorly it is wider, about one-third as wide as the base of the neck. Nothing is known concerning the renette. The nervering surrounds the oesophagus somewhat obliquely a little in front of the middle of the neck. From the elevated and rather large and conspicuous vulva the vagina leads inward at right angles nearly half way across the body, where it joins the single uterus, which extends forward, and is of such a size as to contain apparently but one egg at a time. The ovary, which at first is three-fourths as wide as the body, extends forward and tapers gradually, so that at the blind end, considerably in front of the cardia and a short distance behind the nerve-ring, it is one-fourth as wide as the corresponding portion of the body. It sometimes happens that the blind end of the ovary is disposed in a single coil. The rather thick shelled eggs are as long as the body is wide, and about three-fourths as wide as long, and appear to be deposited before segmentation begins. The small spermatozoa found in the uterus are of such a size that at least ten placed side by side would be required to span the diameter of one of the eggs. There is a very small, posterior rudimentary branch to the uterus, which extends backward from the vulva a distance about equal to two-thirds the radius of the body. Around the vulva are four distinct, unicellular vaginal glands.

$7 \mathrm{I}$.

$\begin{array}{ccccc}.7 & 9.4 & 22 . & -\mathrm{M}- & 87 .\end{array} \quad$ I.5 mm.

The tail of the male resembles that of the female in form and size. As in the females, the three caudal glands are found in the anterior third of the tail, and do not extend materially farther forward than the anus. The two equal, L-shaped, slender, slightly tapering brownish spicula are a little longer than the anal body diameter. The anterior three-fifths lie nearly parallel to the body axis, while the posterior two-fifths are arranged nearly at right angles to the body axis. The distal extremities of the spicula are slightly widened. At their widest part, namely near the proximal ends, the spicula are about one-eighth as wide as the corresponding portion of the body. They are very slightly narrower distally than proximally, where they are barely cephalated by expansion. The accessory piece is not strongly developed. The main portion of it lies parallel to the distal two-fifths of the spicula, and is connected with the dorsal surface of the body by means of muscles. The ejaculatory duct is one-half as wide as the corresponding portion of the body. There are two outstretched testes. The blind end of the anterior testis is located a short distance behind the nerve-ring, while the blind end of the posterior testis is located about as far in front of the 
anus as the terminus is behind it. The testes are broad and some parts appear to fill up the main portion of the body cavity.

Habitat: Sand bar off Plummer's Island, Potomac River. Sublimate to balsam.

ONCHOLAIMELLUS, de Man, 1886.

Fig. 21, Plate VII.

18. Oncholaimellus heterurus, n.sp. \begin{tabular}{cccccc}
.9 & 8.5 & 18. & $-\mathrm{M}_{-}^{53}$ & $9 \mathrm{I}$. \\
\hline 8 & I.5 & I.8 & 1.8 & 1.4 & $1.2 \mathrm{~mm}$
\end{tabular} .

The moderately thick layers of the transparent, naked, colorless cuticle seem to be destitute of markings of any kind. If there are any striations they must be exceedingly minute. The conoid neck becomes a little convexconoid toward the head, which is subtruncated, and has a lip region almost imperceptibly expanded. There are ten long, curved, tapering, cephalic setae, located a trifle in front of the middle of the pharynx, two lateral, and eight submedian. The members of the submedian pairs are placed one in front of the other, the hinder members being only about two-thirds as long as the forward members. It is the forward members that are in the same circlet with the lateral setae. These latter are about as long as the longest submedian setae. The bases of the lips are nearly as thick as the wall of the head, but they gradually become quite thin, though the flaps are not so marked as they usually are in Oncholaimus. There are six lips, and each bears on its anterior surface, near the margin of the head, a somewhat outward pointing, minute, innervated papilla, which does not very markedly interfere with the contour of the front of the head. The somewhat cylindroid pharynx is about twice as long as the front of the head is wide, and the average diameter of the cavity is a little more than one-third of its length. At the middle the pharynx is about half as wide as the corresponding portion of the head. Its walls are fairly well chitinized, and appear to be destitute of onchi. The description is derived from a single specimen, in which the mouth is filled with detritus, and it is possible that very minute teeth might have escaped observation, but this does not seem at all probable. The bottom of the pharynx where it is set on the end of the oesophagus, is in the shape of a broad, shallow, hollow cone. At a distance from the anterior extremity twice as great as the length of the pharynx the amphids are seen. These are somewhat elongated, roughly ovate in contour, and more distinctly marked anteriorly than posteriorly. The anterior border is a curved, chitinous structure about one-third as wide as the corresponding portion of the head. Slightly behind the anterior border the amphid has its greatest diameter. It is about as long as the corresponding radius of the head, and is extended backward by an apparently tubular structure, which soon becomes indefinite. Viewed dorso-ventrally the amphids appear like rather deep invaginations of the cuticle, and each occupies fully half of the 
corresponding radius of the neck, and opposite them the oesophagus is a trifle narrower than it is either in front of or behind them. The oesophagus, which at first is about half as wide as the base of the head, continues to have approximately the same diameter until after it passes through the nerve-ring, which is somewhat in front of the middle of the neck. Behind the nerve-ring the oesophagus becomes somewhat wider, and is finally about two-thirds as wide as the base of the neck. The lining of the oesophagus is a fairly distinct feature. The intestine, which becomes at once nearly three-fourths as wide as the body, is set off from the oesophagus by a distinct constriction. There does not appear to be any well developed cardia. The cells composing the intestine contain scattered granules, which give rise to a very obscure tessellation, and also contain doubly refractive granules, which, however are not spherical in form, though their diameter in different directions is not very variable. The tail of the male tapers rapidly from the anus, so that at the end of the anterior fourth it has a diameter but little greater than that of the terminal spinneret. Near the anterior end of the cylindrical part therc occur a pair of subventral arcuate, tapering setae, which are about as long as the corresponding diameter of the tail. In front of these there is a ventrally submedian pair of papillae, and also there is a dorsally strbmedian pair a little in front of these latter. The spinneret is of a simple character, and has the terminus almost imperceptibly swollen. There do not appear to be any setae on the spinneret. A trifle in front of the proximal ends of the spicula there springs from a ventrally submedian lines a somewhat weakly developed bursa, which extends slightly beyond the body contour, but only does this in front of the anus. It recedes into the cuticle at a point slightly in front of the caudal setae. This bursa does not have any ribs and is not striated in any way. The two equal, elongated, rather uniform, slightly cephalated spicula are a little more than twice as long as the anal body diameter, and in their anterior halves are nearly straight. In their posterior halves they are slightly arcuate. At the point where they attain their greatest width they are about one-fourth as wide as the corresponding portion of the body. Seen dorsoventrally they are somewhat narrower and straighter than when seen in profile. The accessory piece appears to be compounded of a number of small chitinous elements, the whole structure being a little less than onethird as long as the spicula. It is not very strongly developed, and is not a very conspicuous feature. The ejaculatory duct is about half as wide as the corresponding portion of the body. There are two testes outstretched in opposite directions. The blind end of the anterior testis is about as far behind the base of the neck as this latter is behind the anterior extremity. The posterior testis is smaller, but just how much smaller is unknown, as its blind end could not be located in the single specimen examined. Little or nothing is known concerning the lateral fields and the renette.

Habitat: Fresh water pond, near Ocala, Fla. Bouin solution to glycerine. 
Note: There is some doubt as to whether this species should be assigned to the genus Oncholaimellus for the following reasons: There are no pharyngeal teeth. The amphid varies somewhat from that of the type species. The spicula are of equal size, whereas in the type species orie of the spicula is only half as long as the other.

CEPHALOBUS, Bastian, 1865.

Fig. 22, Plate VIII.

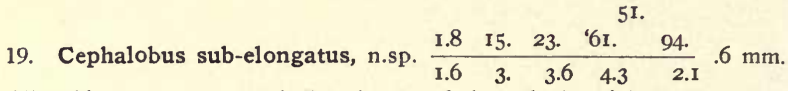

The thin, transparent, colorless layers of the naked cuticle are traversed by about seven hundred plain, transverse striae, resolvable with high powers without very much difficulty. The conoid neck becomes convex-conoid toward the obscurely lobed head, which is rounded in front, and is set off by an almost imperceptible, broad, exceedingly shallow constriction. There are no cephalic setae, and no eye-spots; no amphids have been seen. There are three rather distinct, bluntly conoid lips, which are rounded in front, each of which apparently has two inconspicuous innervated papillae. The open vestibule leads to a conoid pharynx, which is about one and one-half times as long as the lip region is wide, and tapers from front to back in a comparatively uniform way. Between the lips it has a width about one-third as great as that of the lip region. The posterior half of the pharynx rests in the anterior end of the oesophagus, which enlarges very perceptibly, so that a little behind the base of the pharynx it is more than half as wide as the corresponding portion of the head. It continues to have relatively much the same width until near the middle, where it is about three-fifths as wide as the middle of the neck. There it suddenly diminishes in size so as to be only one-fourth to one-fifth as wide as the corresponding portion of the neck. Passing through the nerve-ring with this narrow diameter it finally enlarges to form an ellipsoidal cardiac bulb about two-thirds as wide as the base of the neck, containing a rather distinct triplex but not very complex valvular apparatus of the form usually seen in this genus. There is no very definite cardia. The intestine, which is separated from the oesophagus by a rather broad and shallow constriction, is at first only half as wide as the corresponding portion of the body. It soon becomes thicker walled, and enlarges so as to be three-fourths as wide as the corresponding portion of the body. It is composed of cells of such a size that probably only about two are required to build a circumference. The contents of the cells are not distributed so as to give rise to anything distinct in the way of tessellation. The posterior lip of the anus is very slightly elevated. From the anus the rectum, which is somewhat longer than the anal body diameter, leads inward and forward. The rectum is separated from the intestine by a rather distinct constriction. The tail is at first convex-conoid, the convexity existing 
almost entirely on the dorsal surface. Through the middle third the tail tapers much more rapidly than elsewhere, so that at the beginning of the posterior fourth it has a diameter no more than one-fifth as great as at the anus. Thence onward it is conoid to the acute terminus. There is no spinneret. Very little is known concerning the lateral fields, but at the middle of the body they appear to be one-third as wide as the corresponding portion of the body. There do not appear to be any distinct wings. The excretory pore is located opposite the nerve-ring. This latter surrounds the oesophagus obliquely, and is accompanied by nerve cells, of which the greater number are behind the nerve-ring and in front of the cardiac bulb. From the massive elevated vulva the rather strongly chitinized, slightly colored vagina leads inward at right angles to the ventral surface nearly half way across the body, where it joins the single uterus, which extends forward. In specimens which do not yet contain fully developed eggs, and in which the uterus is occupied by spermatozoa, the flexure in the sexual apparatus occurs about half way between the vulva and the base of the neck. The ovary extends straight backward, and finally tapers to a blunt end a short distance in front of the anus. At the rear end the ovary is one-half as wide as the corresponding portion of the body. The ova appear to be arranged more or less single file in the greater part of the ovary, whose walls contain distinct nuclei of relatively large size. These nuclei are of such a size that about six to eight placed side by side would reach across the body, and are removed from each other in a longitudinal direction a distance equal to about two-thirds of the body diameter. None of these cells could be seen in that portion of the sexual tube in front of the vulva, and it is surmised that the cells composing the ovarian tube are markedly different in their character from those composing the tube between the ovary and the uterus and those composing the wall of the uterus. The spermatozoa have been seen packed in the uterus somewhat like a roll of coin, though each one is thinner at the margins than at the center where the nucleus exists. The spermatozoa are of such a size that the uterus may contain about two dozen, arranged single file.

Habitat: Moss, Bog. W. End of Douglas Lake, Mich. This species closely resembles $C$. elongatus de Man, and possibly may prove to be the same. At present, judging from a single specimen it appears to differ in the following points: (I) The tail of the female is of different form, since that of elongatus is nearly conical; (2) No wings have been seen on sub-elongatus, while they appear to be a marked feature of elongatus; (3) There is no expansion of the lip region in elongatus as in sub-elongatus; (4) The vulva in sub-elongatus is far more massive; (5) the pharynx in elongatus is more elongated, and longer as compared with the length of the head; (6) oesophagus is relatively slenderer in elongatus. Sublimate to balsam.

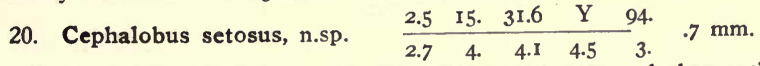

The moderately thick layers of the naked, transparent, colorless cuticle are traversed by 425 transverse striae, which are not further resolvable. 
The striae are more or less interrupted on the lateral fields by two wings, or by a single wing indicated by two longitudinal lines. The width of the wing near the middle of the body is about equivalent to the width of four of the annules of the cuticle. Toward the tail end the width of the interrupted space is about equivalent to the width of three of the annules, and near the middle of the neck the width is also about equivalent to that of three of the corresponding annules. Through the middle of the lateral space on which the annules are interrupted there passes a longitudinal refractive line. The posterior portion of the neck is cylindroid; the anterior half conoid to the head, which is not set off in any way. The lips are arranged in two series: an outer series of six two-parted lips, and an inner series of three obscurely two-parted projecting flaps, each armed at the anterior rounded corners. with short, arcuate, tapering, acute, forward-pointing bristles. Each of the two parts of the six outer lips or appendages is conoid and acute, and the spaces separating them are much narrower than those separating the lips themselves. At the base of each of the lateral two-parted lips there is a transverse mark, which may possibly be the outer expression of the amphid. This marking is very minute and about four times as wide as it is long, and is placed transversely on the base of the lip, iust in front of the foremost cervical annule. It spans about one-sixth of the width of the head at this point. The three inner appendages, which may more properly be termed the lips, are about as long as they are wide, and seem to be flexible. They rather closely surround the mouth opening. Each is half as wide again at its free extremity as it is at the base, and the total length is about equal to one-fourth of the diameter of the front of the head. The setae with which these lips or flaps are armed are about two-thirds as long as the flaps themselves. The rather obscure, narrow, cylindroid pharynx is about as long as the base of the head is wide, and its lumen is hardly wider than the thickness of the cuticle. The oesophagus surrounds the pharynx, but is narrower in this region than it is immediately behind the pharynx. Behind the pharynx it commences as a tube about three-fifths as wide as the base of the head, and continues to have about the same diameter until after it passes through the nerve-ring. It does increase a trifle in diameter, however, so that at the nerve-ring it is about one-half as wide as the corresponding portion of the neck. Some distance behind the nerve-ring, namely, at a distance about equal to one and one-half diameters of the neck, there is a slight break in the musculature of the oesophagus. Behind this break the oesophagus begins to taper very gradually and continues to decrease in diameter until it finally expands to form the somewhat ellipsoidal or pyriform cardiac bulb, which is about two-thirds as wide as the base of the neck, and contains a distinct, rather simple, triple, chitinized valvular apparatus. That portion of the oesophagus immediately in front of the cardiac bulb has a diameter about one-fourth as great as that of the corresponding portion of the neck. The lining of the oesophagus can be seen throughout its length, and is a fairly distinct feature. The intestine which at first is thin- 
walled is separated from the oesophagus by a distinct and deep constriction, and becomes at once about three-fourths as wide as the body. The anterior portion of the intestine is characterized by the presence of a considerable cavity, which, however, soon narrows, and, as the internal wall of the intestine is rather strongly refractive, the narrow, sinuous lumen is a very conspicuous feature. From the rather conspicuous depressed anus the rectum, which is rather strongly chitinized and soniewhat longer than the anal body diameter, extends inward and forward. The tail is somewhat concave conoid from the anus to the very acute terminus. There are no caudal glands. The lateral fields appear to be about one-third as wide as the body. The nervering surrounds the oesophagus somewhat squarely. The excretory pore appears to be located near the nerve-ring. The nature of the internal sexual organs remains uncertain, but the vulva is evidently located near the middle of the body. The description is derived from an immature specimen.

Habitat: Cranberry bog, Arlington Farm, Virginia. Flemming solution to glycerine.

BASTIANA, de Man, 1876.

Fig. 23, Plate VIII.

\section{Bastiana exilis, n.sp.

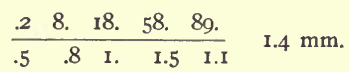

The moderately thick layers of the transparent, colorless, naked cuticle are traversed by about eight hundred forty transverse striae, which do not appear to be further resolvable. These striae exist in the outer as well as inner cuticle, so that the entire contour of the body is crenate. Rather conspicuous lateral wings are present, the optical expression of which is two distinctly refractive longitudinal lines opposite the lateral fields, separated from each other by a distance somewhat greater than the width of one of the annules of the cuticle. The gradually tapering conoid neck ends in a somewhat truncate head, which is not set off in any way, and which bears a circlet of at least six cephalic setae, of which four submedian are the longer, and somewhat longer than the head is wide. Possibly each of these latter is accompanied by a shorter seta, thus making ten in all. Apparently labial papillae are present, but they have not been sufficiently clearly seen to permit of enumeration. There is no distinct pharynx. The amphids, located as in B. gracilis deMan, are somewhat in the form of the end of a shepherd's crook. They are perhaps to be regarded as broad spiral bands of about one turn. So regarded, the band may be conceived to begin on the ventral side where its end is rounded and its contour distinct, pass forward, and then curve backward and end indefinitely at a point somewhat in the rear of the beginning. The amphids are half to two-thirds as wide as the corresponding portion of the neck, and somewhat longer than wide. There are no eye-spots. The oesophagus begins as a tube fully two-thirds as wide as the head. It gradually increases in diameter as it passes backward, and at 
its posterior extremity is three-fourths as wide as the base of the neck. There is no definite cardia. The lining of the oesophagus is indistinct. The intestine, which is separated from the oesophagus by an obscure constriction, becomes at once three-fourths as wide as the body, is rather thick walled, and its cells contain rather uniform, colorless granules, not arranged in any definite manner. From the rather raised anus the conspicuous rectum, which is twice as long as the anal body diameter, extends inward and forward. The tail is conoid, but tapers more rapidly near the acute terminus. A short distance in front of the terminus it is nearly half as wide as at the base. Nothing is known concerning the renette. The lateral fields have not been distinctly seen, but they appear to be about one-third as wide as the body. Nothing definite is known concerning the internal sexual organs, as the specimens examined were not fully developed.

60.

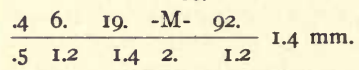

The tail of the male is like that of the female in form and size, except that the anus is more prominently raised. In front of the anus and extending forward to the basc of the neck there is a ventral row of ninety supplementary organs. These are of small size, and each appears when seen in profile to be a slight conical, innervated elevation having an altitude about one-third as great as the width of its base. These organs are removed from each other a distance on an average about equal to the radius of the body or a little more. They are somewhat nearer together posteriorly than anteriorly. Near the base of the neck the distance between two adjacent organs is about equal to the body diameter. The two equal, tapering, acute, nearly straight spicula are about one and one-third times as long as the anal body diameter. They are slightly cephalated by expansion, the cephalum occupying about one-third of the length. At the widest part, namely in the cephala, the spicula are one-fourth to one-third as wide as the corresponding portion of the body. They do not appear to have any accessory pieces. The spicula appear, when seen in profile, to be arranged at an angle of forty-five degrees to the body axis. Beginning at the anus and extending obliquely backward there is a strand of muscle which is attached to the dorsal side of the body. The two testes are outstretched in opposite directions. The blind end of the anterior testis is about as far behind the base of the neck as the nerve-ring is in front of it, while the blind end of the posterior testis is about twice as far in front of the anus as this latter is in front of the terminus. cerine.

Habitat: Fresh water, Tynne Station, Fla. Bouin solution to gly- 
APHANOLAIMUS, de Man, 1880.

Fig. 25, Plate VIII.

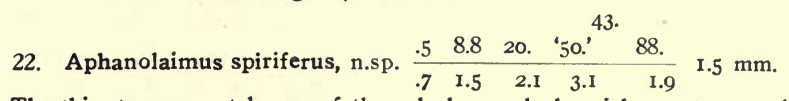

The thin, transparent layers of the colorless, naked cuticle are traversed by about one thousand transverse striae, which do not appear to be further resolvable, or at any rate are resolvable with difficulty. The cuticle is also traversed by longitudinal striations, manifestly due to subcuticular structures,- - the submedian fields-and the attachment of the muscle cells. The contour of the body is minutely crenate. There is a distinct wing extending throughout the length of the body, whose presence is indicated by two refractive lines occupying a space hardly greater than the width of one of the annules of the cuticle. On being carefully examined these lines show traces of the striations of the cuticle. The conoid neck ends in a somewhat rounded head, whose lip region is set off by a minute constriction just in front of the amphids. The contour of the front of the head is rounded, or, like that of a very flat truncated cone. In the midst of the truncation there is an exceedingly minute depression, the mouth pore. There does not appear to be any very distinct pharynx, but the lining of the oesophagus is slightly altered in the region of the head back as far as the posterior margins of the amphids. It is not unlikely that this represents a distinct, though rudimentary pharynx. The species, however, would naturally at first sight be classed as being without a pharynx. The oesophagus begins as a tube about half as wide as the base of the head, and expands very gradually until after it passes through the nerve-ring. At the nerve-ring its width is about onethird that of the corresponding portion of the neck. Behind the nerve-ring the oesophagus begins to expand a little more rapidly, though still slowly. When it reaches the end of the neck it is half as wide as the base of the neck. For a distance equal to one body diameter, however, it decreases in size, so that where it joins the intestine it is very narrow, only about onefifth as wide as the corresponding body diameter. Nevertheless, there appears to be a distinct, though exceedingly minute cardia. 'The lining of the oesophagus is a fairly distinct feature throughout its length. The intestine, which is at first thin-walled, gradually becomes half as wide as the body, and is composed of cells of such a size that only two or three are required to build a circumference. For the most part the wall of the intestine is moderately thick. From the very slightly raised, rather inconspicuous anus the slender rectum, which is about one and one-half times as long as the anal body diameter, extends inward and forward. It is separated from the intestine by a very distinct and deep constriction. Near the middle the lateral fields are about one-third as wide as the body. The ventral gland is a rather elongated, saccate cell a little longer than the base of the neck is wide, and about one-third as wide as long. It contains a large spherical nucleus, 
with a large, fairly distinct nucleolus. There extend backward from this cell two finger-shaped, submedian extensions nearly as long as the cell itself. Notwithstanding the distinctness of the organ and appendages the position of the excretory pore remains to be discovered. The tail tapers from in front of the anus in such fashion that at the beginning of the final fourth it has a diameter about one-fourth as great as at the anus. Thence onward the tail is cylindroid to the terminus, which bears a truncated, conical, unarmed spinneret. The three caudal glands are minute, close together, opposite to and a little behind the anus. From the slightly depressed vulva the chitinized vagina leads inward at right angles to the ventral surface half way across the body, where it joins the two symmetrically placed uteri. The reflexed ovaries reach about one-third the distance back to the vulva and contain a few developing ova arranged single file. The species is viviparous. Two cmbryos and a developing egg have been seen in each uterus at the same time. The eggs are somewhat elongated, about as long as the body is wide, and a little less than half as wide as they are long.

\begin{tabular}{|c|c|c|c|c|}
\hline & & & 6 & \\
\hline .2 & 10. & 14. (?) & $\mathrm{M}$ & 82. \\
\hline
\end{tabular}

The tail of the male is like that of the female in form and size. In front of the anus there is a ventral series of seven tubular, protrudable, supplementary organs, occupying a space nearly equal to the length of the tail. The hindermost is opposite the proximal ends of the spicula. Each of these organs is about half as long as the body is wide, has its proximal end slightly cephalated by expansion, and its distal end slightly curved. The circular apertures through which the organs are protruded disturb the ventral contour distinctly, each of them occupying a space about equal to the width of four annules of the cuticle. There are no special setae or papillae either in front of the anus or behind it. The two equal, uniform, slender, very strongly arcuate spicula are about one and one-half times as long as the anal body diameter, and their proximal ends are cephalated by expansion. The proximal ends lie nearly opposite the body axis. There is a simple accessory piece, applied for a short distance to the distal ends of the spicula, having a backward pointing process two-thirds as long as the anal body diameter.

Habitat: Potomac River, Washington, D. C. Sublimate to balsam.

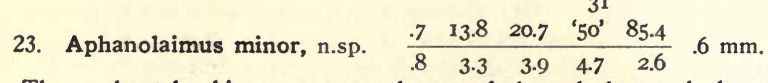

The moderately thin, transparent layers of the colorless, naked cuticle are traversed by fine transverse striae, resolvable with moderate powers, and not modified on the lateral fields. The presence of wings is indicated by two lines extending from near the middle of the neck well onto the tail. 
These occupy a space a little more than the width of one of the annules of the cuticle, but sometimes one of these lines is more prominent than the other. The conoid neck ends in a rounded head, which is not set off in any way. There are no cephalic setae. There does not appear to be any distinct pharynx. The lining of the oesophagus is slightly modified back twice as far as the posterior margins of the amphids, and very likely this portion of the tube may be regarded as a narrow rudimentary pharynx. Twice as far from the anterior extremity as the base of this supposed pharynx the oesophagus is distinctly interrupted by a break in the musculature, although there is no change in size, and just behind this break there are three distinct, elongated nuclei, one in each segment of the oesophagus. These appearances are reminiscent of the arrangement in Plectus, to which Aphanolaimus is doubtless related. There do not appear to be any distinct lips, though possibly there may be three exceedingly minute, well amalgamated, flattish ones. Although at first glance the amphids appear to be circular, they are really spiral in form. They are about one-fifth as wide as the corresponding portion of the neck. Their distance from the anterior extremity is about equal to the diameter of the head; or in other words, their centers are removed from the anterior extremity a distance about twice as great as their diameter. The amphids appear to be slightly larger in the male than in the female. There are no eye-spots. The oesophagus begins at the base of the above described pharynx, as a tube about half as wide as the corresponding portion of the neck. It widens very gradually, so that where it passes through the nerve-ring it is nearly one-third as wide as the corresponding portion of the neck. At the middle, nearly opposite the nerve-ring, there is an almost imperceptible increase in diameter, indicative of a deteriorated median swelling. Finally, it expands into the narrowly pyriform cardiac swelling destitute of any distinct valvular apparatus. This swelling is half to two-thirds as wide as the base of the neck. The lining of the oesophagus is a distinct feature throughout its length. There is no distinct cardia. The intestine, which is at first only one-fifth as wide as the base of the neck, joins the middle of the posterior surface of the cardiac swelling. It is moderately thick-walled, and gradually becomes about two-thirds as wide as the body. It appears to be made up of cells of such a size that few, probably two or three, or possibly four, are required to build a circumference. The body tapers gradually from a long distance in front of the anus. This latter is very slightly raised, more particularly on the anterior margin. From it the rectum, which is about as long as the anal body diameter, extends inward and forward. The tail is conoid in the anterior three-fourths. The posterior fourth is cylindrical and has a diameter nearly one-third as great as that of the base of the tail. It ends in a rounded, unarmed spinneret, bearing an apiculum. The lateral fields appear to be about one-third as wide as the body. The nerve-ring surrounds the oesophagus somewhat obliquely. There appears to be a small renette cell opposite the anterior portion of the intestine, but the position of the excretory pore has not been discovered. 
From the inconspicuous vulva the vagina leads inward at right angles to the ventral surface about half way across the body, where it joins the two symmetrically-placed uteri. The reflexed ovaries reach about half way back to the vulva and contain half a dozen or more developing ova arranged somewhat irregularly. The thin-shelled, ellipsoidal eggs are about as long as the body is wide and about three-fifths as wide as long. They have been seen in the uterus one at a time, and apparently are deposited before segmentation begins.

$$
\begin{array}{llllll}
\text { 3. } & 15 . & 23 . & -\mathrm{M}- & 85 . \\
\hline \mathrm{I} .8 & 3.4 & 3.8 & 4.2 & 3.4
\end{array} .5 \mathrm{~mm} .
$$

The tail of the male is conoid in the anterior three-fourths in such fashion that the beginning of the final fourth has a diameter of about oneeighth that of the base of the tail; thence onward the tail is nearly cylindrical or expands slightly, and ends in a rounded terminus bearing an apiculum forming an unarmed spinneret. The caudal glands are located in the base of the tail. In front of the anus in the male there is a ventral row of eight or nine protrudable, obscurely S-shaped, yellowish supplementary organs. These appear to be of rather uniform size and are equally spaced. Their length is somewhat greater than that of the radius of the body, and they are placed at an angle of about thirty degrees to the body axis. They are of rather uniform diameter throughout their length, but taper a little toward the blunt distal extremity, and are obscurely cephalated at the proximal ends. The distal extremity of the hindermost of these organs is located nearly opposite the proximal ends of the spicula. The entire distance occupied by the series is about one and one-half times as great as the length of the tail. The two equal, tapering, strongly arcuate, brownish yellow spicula are about one and one-half times as long as the anal body diameter. Their proximal ends are cephalated by expansion, and extending from the proximal ends toward the distal extremities there are straight, refractive lines indicating possibly that there is more to the framework of the spicula than appears at first glance. The spicula curve inward and backward to near the dorsal side of the tail, and then pass forward so that their proximal extremities lie opposite the body axis. The spicula are accompanied by a rather straight, slender, slightly tapering, blunt accessory piece applied to their distal fifths. From this point the accessory piece extends inward and backward at an angle of sixty degrees, and ends a little to the dorsal side of the body axis. At their widest part, just behind the cephala, the spicula have a width about one-fifth as great as that of the corresponding portion of the body. A little behind the middle of the tail there are two ventrally submedian setae, about half as long as the corresponding diameter of the tail. The ejaculatory duct is about one-third as wide as the body. There are two testicles, one extending forward and the other backward. The blind end of the anterior testicle is a little farther behind the base of the neck than the 
nerve-ring is in front of it. The blind end of the posterior testicle lies just in front of the anterior supplementary organ.

Habitat: Mud, Potomac River. Sublimate to balsam.

TYLENCHUS, Bastian, 1865.

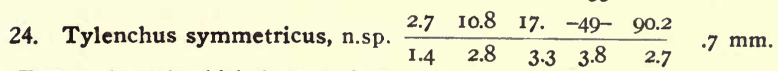

The moderately thick layers of the transparent, colorless, naked cuticle are traversed by fine plain transverse striae, which are not further resolvable. There are two wings on the lateral fields, removed from each other a distance about equal to one-fourth to one-fifth the body diameter. The space between these wings is very faintly marked with longitudinal lines, of which two near the middle are more prominent than the others. The wings themselves bear traces of transverse striation. These wings begin near the middle of the neck and end on the tail. The conoid neck becomes convexconoid toward the somewhat rounded head, which is not set off, or at least only by an almost imperceptible constriction a short distance behind the lip region. There are no distinct lips. What appear to be the faintest possible traces of papillae may be seen on the lips, but these do not disturb the rounded contour of the head. Were it not for the innervations it would be practically impossible to see them. The pharynx is tubular and just wide enough to make a passage for the spear. This latter is slender, nearly one and onehalf times as long as the base of the head, and has a three-bulbed base about one-fifth to one-fourth as wide as the corresponding portion of the head. Near the middle the spear is closely surrounded by a rather inconspicuous guiding-ring or cylinder, about one-fourth as long as the spear. At its widest part, namely, in the proximal half, the diameter of the spear is less than the width of two of the adjacent annules of the cuticle. The oesophagus begins as a tube about one-third as wide as the base of the head, and continues to have this diameter until it expands to form the ellipsoidal or prolate median bulb. This latter is about three-fifths to two-thirds as wide as the middle of the neck, and contains a distinct ellipsoidal valvular apparatus about one-fifth as wide as the bulb itself. Behind the median bulb the oesophagus is smaller than elsewhere. For a distance equal to twice the width of the neck it has a width only about one-eighth to one-sixth as great as that of the corresponding portion of the neck. Thereafter, it expands to form the elongated-pyriform non-muscular cardiac swelling, which is half as wide as the base of the neck, and contains a rather conspicuous nucleus. The lining of the oesophagus is a fairly distinct feature in its anterior part; but posteriorly it is much less distinct, and is very diffcult to observe in the cardiac swelling. The intestine, which is separated from the oesophagus by an indistinct constriction, becomes at once fully twothirds as wide as the body. Its cells are packed with large granules of somewhat variable size, the largest of which have a diameter about one-eighth as great as that of the body, the smallest being considerably smaller. These 
granules are so prominent as to obscure the cellular structure of the intestine. From the very slightly elevated and rather inconspicuous anus the rectum, which is hardly as long as the anal body diameter, extends inward and forward. The tail is conoid to the terminus, where it rather suddenly tapers much more rapidly to a very acute point. This rapidly tapering portion has a length about equal to one-fourth the anal body diameter, and the diameter at the point where the change in taper begins is about equal to onefifth of the anal body diameter. The lateral fields appear to be a little more than one-third as wide as the body. The nerve-ring surrounds the oesophagus obliquely. The excretory pore is located a little behind the nerve-ring, a little more than half way from the base of the neck to the middle of the median bulb. From the slightly elevated, rather massive vulva the vagina leads inward at right angles to the ventral surface nearly half way across the body, where it joins the two uteri. Apparently the ovaries are outstretched. A single egg has been seen in one of the uteri. It is about twice as long as the body is wide and about one-third as wide as long. It is thinshelled and apparently undergoes segmentation before being deposited.

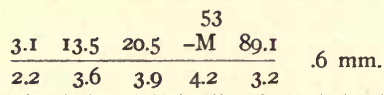

The tail of the male is like that of the female in form. From the raised anus it is arcuate-conoid to the terminus, which is concave-conoid and ends in an exceedingly fine slender point. The length of the terminus is about one-third as great as the anal body diameter, while its diameter is about one-fourth as great as the anal body diameter. There are no supplementary organs, nor have any special papillae been seen either in front of the anus or behind it. There is a fairly well developed, but not very prominent bursa, which extends but little beyond the ventral contour at any point. It springs from the submedian lines at a distance as far in front of the anus as the middle of the tail is behind it. The bursa is striated like the cuticle, and its margin is entire; it extends to near the end of the tail. Near the middle of the tail there appears to be a bursal rib on each side, which does not reach to the margin of the bursa. The ejaculatory duct is about one-third as wide as the body. The blind end of the single outstretched testicle lies a short distance behind the base of the neck.

Habitat: Marsh in black clay with much decayed vegetation, near Impatiens, Arlington Farm, Virginia. Flemming solution to glycerine.

RHABDITIS, Dujardin, 1845.
25. Rhabditis punctata, n.sp.$$
\begin{array}{rrrrr} 
& & \multicolumn{3}{c}{71} \\
1.2 & 10.8 & 16.8 & \text { '55' } & 93.8 \\
\hline 1.2 & 3.3 & 4.3 & 4.9 & 1.6
\end{array}
$$
$1.8 \mathrm{~mm}$.

The thin layers of the transparent, naked, colorless cuticle are traversed by transverse striae, which are resolvable with high powers and appear to be resolvable into rows of dots, but there is some doubt about this. The cuticle 
is longitudinally striated. Near the middle of the male there is a lateral area nearly half as wide as the body, which is nearly destitute of striations. On either side of this field the cuticle may be seen with high powers to be distinctly longitudinally striated. This lateral field narrows toward both extremities so as to be hardly wider than the spicula, so that the main portion of the cuticle of these regions appears there to be longitudinally striated. The neck is conoid, becoming almost imperceptibly convex-conoid toward the head, which is somewhat rounded and bears six lips, which are fairly well separated from each other, and each of which appears to be papillate. The simple, unarmed pharyn $x$ is cylindrical and one-third as wide as the base of the head. Its cavity is faur to five times as long as wide. No amphids have been seen, and there are no eye-spots. The oesophagus receives the base of the pharynx and becomes at once about two-thirds as wide as the base of the head. It continues to have this diameter until it expands to form the ellipsoidal median swelling, which has a distinctly radiated fibrous structure. This swelling is about two-thirds as wide as the corresponding portion of the neck, and is separated from the portion of the oesophagus preceding it, as well as the portion succeeding it, by a refractive division in the internal musculature. Behind the median swelling the oesophagus has a diameter less than half as great as that of the corresponding portion of the neck, and it narrows continuously until it reaches the pyriform cardiac bulb, which is three-fourths as wide as the base of the neck and contains a well developed, complicated, chitinous valvular apparatus half as wide as the bulb itself. There is no distinct cardia. The somewhat transparent and colorless intestine, which is separated from the oesophagus by a distinct constriction, is composed of cells containing scattered granules of variable size, the largest of which have a diameter somewhat less than the thickness of the cuticle, and the smallest of which are very much smaller. The anus is slightly raised, and from it the rectum, which is one and one-half times as long as the anal body diameter, leads almost directly forward. The body tapers for some distance in front of the anus; from the anus onward it is conical to the acute terminus. There is no spinneret, and there are no caudal glands. Nothing definite is known concerning the renette cell. The excretory pore is located about half way between the median and posterior bulbs, just behind the oblique nerve-ring. The two uteri extend in opposite directions, and the ovaries are reflexed, the flexures occurring the one not far behind the base of the neck and the other not far in front of the anus. The mature females contain scores of eggs in various stages of development, the most advanced containing well-developed embryos.

63

\begin{tabular}{rrrrrr}
2.3 & 16.2 & 25.9 & $-\mathrm{M}$ & 96.3 & 1. $\mathrm{mm}$. \\
\hline 1.9 & 3.4 & 3.5 & 3.7 & 2.6 &
\end{tabular}

The tail of the male is conoid from the anus in such fashion that at the beginning of the posterior third it has become reduced to a mere striated nearly straight spine, which is no wider than one of the ribs of the bursa. 
The dorsal contour of the tail continues as a more or less regular extension of that of the body. The ventral contour, however, is elevated at the anus; at any rate, when the spicula are partly extruded. This elevation appears to be largely median, and there is a distinct groove on either side between it and the bursa. When this groove is brought into optical section and the anal elevation is disregarded the tail appears to be asymmetrically conoid. The two equal, brownish, nearly straight spicula are fully twice as long as the tail, or one and one-half times as long as the body diameter, measured opposite the proximal ends of the spicula. The spicula in the distal three-fourths taper to a blunt point. At their widest part, considerably in front of the middle, they are one-fourth to one-fifth as wide as the corresponding portion of the body. In the proximal fourths the spicula are rather suddenly narrowed and terminate in bulbs or cephala, which have a diameter twice as great as the portion of which they are expansions. Parallel to the spicula there are accessory pieces. These are two-thirds to three-fourths as long as the spicula, which they partially envelop. They are about as strongly chitinized as the spicula themselves. The transparent, colorless bursa springs from the submedian lines, nearly opposite the proximal ends of the spicula, and when seen in profile only slightly exceeds the ventral contour in front of the anus, but behind the anus exceeds the ventral contour of the tail proper so much that nearly the entire length of the ribs comes plainly into view. The ribs are arranged in three groups: (I) Two ribs close together opposite the middle of the spicula. (2) Four ribs close together opposite to, and a trifle behind the anus. (3) Four ribs of which three are very close together, and one a little farther forward, the whole group being opposite the middle of the tail. Most of these ribs reach to the margin of the bursa, which is entire and is not distinctly striated. The outer members of the middle and posterior groups do not reach quite to the margin. The ejaculatory duct is nearly one-half as wide as the body. The single, outstretched testicle has its blind end located about half as far behind the base of the neck as this latter is behind the anterior extremity. At its blind end the testicle is about one-third as wide as the body. It gradually increases in width, so that it occupies four-fifths of the diameter of the middle of the body. The spermatocytes are of large size, one-third to one-half as wide as the body.

Habitat: About the roots of aquatic plants, Potomac River, Washington, D. C.

TRILOBUS, Bastian, 1865.

Fig. 15, Plate VI. 40

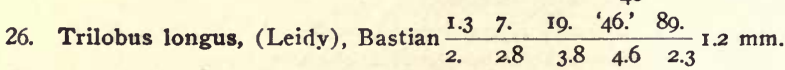

The thin, transparent layers of the colorless cuticle are traversed by exceedingly fine plain transverse striae, more particularly visible in the 
lower layers with high powers. There are short hairs to be found here and there on the surface of the body, more particularly toward the extremities. The conoid neck ends in a somewhat rounded head not set off by any constriction. The cephalic setae appear to be ten in number (eight submedian and two lateral), outward pointing, each about onesixth as long as the head is wide, and placed on the outer margin of the front of the head opposite the posterior portion of the pharynx. The members of the submedian pairs are, however, of unequal size. There are six small, pointed lips surrounding the mouth opening. It is certain that these lips bear papillae, but the number is not known. Lateral organs in the form of somewhat stirrup shaped markings onefourth as broad as the corresponding portion of the head, occur at a short distance behind the base of the rudimentary pharyngeal cavity. This latter is a more or less triquetrous cyathiform cavity having its walls distinctly chitinized and bearing at its base one or more protuberances near the point where the lumen of the oesophagus begins. One of these is a ridge, the others minute teeth. The average width of the main pharyngeal cavity is about one-third that of the head. The oesophagus is slightly expanded to receive the pharynx, but otherwise begins as a tube about three-fifths as wide as the anterior part of the neck, and expands very gradually to the end, where it is a little more than half as wide as the base of the neck. The lining of the oesophagus is a distinct feature throughout its length. There is a distinct rounded cardia. The intestine, which becomes at once about two-fifths as wide as the body, is separated from the oesophagus by a distinct constriction, in which lie the three granular bodies whose presence gave origin to the name of the genus. The intestine is relatively thick walled, its small cells containing scattered granules of small size, which are not arranged in any very definite manner. The intestine frequently contains diatoms in large numbers, thus indicating that these are a common source of nourishment for this species. From the slightly depressed anus the rectum, which is about as long as the anal body diameter, extends inward and forward to join the intestine, which is narrowed at its extremity so as to be about two-fifths as wide as the body. Nothing is known concerning the ventral gland. The longitudinal fields are distinctly developed, being about one-fourth as wide as the worm itself. The nerve-ring surrounds the oesophagus somewhat obliquely. The tail is conoid in the anterior third in such fashion that at the beginning of the middle third it has a diameter about onefourth as great as at the anus. Thence onward it tapers gradually to the slightly swollen spinneret or outlet for the caudal glands. These latter, three in number, are located tandem in the tail opposite to and slightly behind the anus. From the slightly elevated vulva the vagina leads inward at right angles to the ventral surface fully half way across the body. The vagina is bifurcated and extends in opposite di- 
rections, the reflexed ovaries passing about two-thirds the way back to the vulva. Two or three eggs may occur in each uterus at one time. These are somewhat ellipsoidal and thin shelled, being about two-thirds as long as the body is wide and about two-thirds as wide as long. The eggs appear to indicate at least the early stages of segmentation before being deposited. The walls of the vagina present the peculiarity of being very thick, and composed of concentric layers to the number of six or seven, so that the organ is considerably broader than it is deep. Its internal wall presents the peculiarity of staining strongly with carmine.

$$
\begin{array}{lrrrr}
1.3 & 8.3 & 13.7 & -\mathrm{M}- & 92 . \\
\hline 2 . & 2.6 & 3.2 & 3.8 & 2.7
\end{array} 1.2 \mathrm{~mm} .
$$

The tail of the male is very much like that of the female in form and size. Supplementary organs are found in front of the anus only. These are six in number, of which three are very prominent. The anterior member of this group of three is about twice as far in front of the anus as the spinneret is behind it. The members of this group are equidistant, the distance between consecutive members being about twice as great as the diameter of the organs themselves. All three occupy a distance about twice as great as the corresponding body diameter. Each of these organs consists of an internal and an external portion, the internal portion being a flattish, hemispherical mass of tissue of very fine, or at least uniform texture. These have a diameter about twofifths as great as that of the body at the same part. The external portion of these organs consists of a ring slightly raised, from which projects a mammiform papilla which bears a minute median seta or nerve-ending. When this mammiform portion is traced inward it passes through the ring on the surface of the body and then bends forward and ends rather indefinitely in the anterior contour of the internal portion of the organ. These organs possess the peculiarity of staining strongly with carmine. In addition to the organs already described there are three others of smaller size, two very minute, one near the anus and the other opposite the proximal portions of the spicula. The third, which has a size intermediate between those just described and those first described, is located about half way between the anus and the posterior member of the larger grcup. These smaller organs are comparatively close counterparts of the larger, differing merely in size. In addition it may be noted that the cuticle on the ventral surface of the male throughout this region of the body, that is, as far forward as the most anterior of these organs, bears about twenty exceedingly minute structures on the ventral line, which appear to be innervated papillae. These also stain strongly with carmine, although they are so minute as to be difficult to observe. There are no papillae on the tail, but there are several short hairs, more particularly near the ventrally submedian lines. There is no bursa. The ejaculatory duct is coextensive with the supplementary organs. It is on the average nearly half 
as wide as the corresponding portion of the body. It is connected with the seminal vesicle by a narrow portion. The seminal vesicle is elongated, about half as wide as the body, and extends to a point about as far behind the neck as the base of the neck is from the anterior extremity. From this point the two nearly equal testicles extend in opposite directions, and end blind, one not far behind the base of the neck, and the other not far from the middle of the body. These possess the peculiarity of being slightly swollen at their proximal ends just where they join the vesicle. These swellings appear to constitute veritable cavities of small size. That region of the body corresponding to the ejaculatory duct is traversed by oblique striae, which, however, are arranged at right angles to the direction of such oblique striations as are found in other genera, and it is probable that they are connected with the duct itself, and are not muscles of the character which usually give rise to these oblique striations on the males of other genera. The two equal, arcuate slender spicula appear to be of rather uniform diameter when viewed laterally, and are one and one-half times as long as the anal body-diameter. Their proximal ends are cephalated by contraction. They are accompanied by accessory pieces two-fifths as long as the spicula themselves, and arranged parallel to the distal portions of the spicula. D. C.

Habitat: Mud, about the bases of aquatic plants, Potomac river,

\section{MESOMERMIS, Dady.}

Fig. 18, Plate VII. Fig. 24, Plate VIII.

\section{Mesomermis virginiana, n.sp. \begin{tabular}{ccccccc}
.8 & 6.3 & II. & $-\mathrm{M}-$ & 96. \\
\cline { 2 - 6 } & I.3 & 2. & I.8 & 2.3 & 2.2 & $1.8 \mathrm{~mm}$
\end{tabular} .}

The rather thick, transparent, colorless layers of the naked cuticle are destitute of transverse striations. There are minute longitudinal striations throughout the body. These are interrupted on the lateral lines, where there is a distinct wing. The conoid neck becomes convexconoid toward the lip region, which is not set off in any way. There are no cephalic setae, and there is no distinct pharynx. The mouth pore is very minute, and is a little toward the ventral side of the middle of the front of the head. The cuticle has about the same thickness on the head as elsewhere, being only a trifle thicker. The cuticle is penetrated on the head by a number of innervations. These end in minute depressions on the surface of the head. Near the mouth opening there is one of these depressions on the dorsal side, and apparently a similar one on the ventral side, while nearer the outer margin of the head there are two ventrally submedian and two dorsally submedian similar depressions. Pores occur also here and there on the body as well as on the neck. There are no eye-spots. The lateral organs present the 
following appearance when seen from the side: They appear to project from the surface of the body very slightly, beginning as a tube having a length about one-third as great as the corresponding diameter of the head. This tube has very thin walls, and a short distance in, apparently near the surface of the body, a second element appears in the form of a circle inside that representing the contour of the outer tube. This appears to constitute a sort of core in the midst of which are a number of refractive elements, resembling nerve fibers, which pass inward and backward toward the lumen of the oesophagus. Some of these elements are longer than others. The focus passing inward picks up one, then two, then several more, so that by the time a view is obtained that is wholly inside the body there are seen a half dozen or more of these elements. It is impossible in this view to pick up an internal connection of these refractive elements. The oesophagus begins just below the transparent cuticle as a very narrow tube, probably not more than one-eighth as wide as the corresponding portion of the neck. It continues to have this diameter until after it passes through the nervering. At a distance from the nerve-ring equal to about one body diameter, the oesophagus begins to change gradually into intestine. The intestine gradually widens out, so that at a point as far behind the nervering as the neck is wide it has a width one-third as great as that of the body. It goes on increasing in this way until at a distance from the nerve ring three times as great it is two-fifths as wide as the body. Near its middle the intestine is half as wide as the body. The cells of the intestine contain granules of variable size, the largest having a diameter fully twice as great as the thickness of the cuticle, and the smallest being very minute. The female has not been seen. The tail of the male is slightly arcuate, and is conoid to the blunt, rounded terminus, which has a diameter half as great as that of the base of the tail. There is no spinneret, and there are no caudal glands. The lateral fields are about one-third as wide as the body. The nervering surrounds the oesophagus somewhat obliquely. Nothing is known concerning the ventral glands or the excretory pore. The tail of the male bears several series of innervated papillae. These are sufficiently elevated at the surface of the cuticle to slightly break the contour when the tail is seen in exact profile. Where each nerve passes through the cuticle there is a very low, almost imperceptible elevation at the surface. These papillae are arranged on the ventral submedian lines as well as on the ventral line. The ventral papillae just in front of and just behind the anus are double. As to particulars of the submedian group, there are four on the tail, one member opposite the anus; one a little farther back, a third near the middle of the tail, and a fourth considerably farther back. In front of the anus on each side are eight submedian papillae. These occupy a distance more than twice as great as the length of the tail, and the distance between successive papillae in- 
creases with the distance from the anus, so that the space between the seventh and eighth is about two-thirds as great as the diameter of the body. Of the median papillae on the tail there are three; two near the anus, and one just in front of the middle of the tail, with possibly a fourth farther back. In front of the anus, of the median papillae there are two near the anus, and ten additional ones about coextensive with the submedian rows and distributed in the same manner, the distance between successive papillae increasing with the distance from the anus. The two equal, arcuate, uniform, blunt spicula are about one and one-third times as long as the anal body-diameter. Their proximal ends are not cephalated. The ejaculatory duct is about one-third as wide as the body. The vas deferens is about one-half as wide as the body. There are two outstretched testicles, the blind end of the anterior being about twice as far from the end of the neck as this latter is from the anterior extremity. The posterior testicle is a little shorter than the anterior.

Habitat: Cranberry bog, Arlington Farm.

ACHROMADORA, Cobb, 1914.

Fig. 11, Plate V.

28. Achromadora minima, Cobb.

For original description see Macleay Memorial Volume, Sydney, 1893; see also Journal Washington Academy of Sciences, Oct. 4, 1914. 


\section{EXPLANATION OF PLATES*}

Plate Ií.

IOTA, Cobb, 1913.

Fig I. Iota octangulare, n. sp. Lateral view of a female specimen. $a$, lip region; $b$, labial chitinous plate or expansion; $c$, protruding muscles of the spear; $d$, spear; $e$, trilobed base of the spear; $f$, lumen of the oesophagus; $g$, nerve-ring; $h$, beginning of the intestine; $i$, flexure in the single narrow ovary; $j$, excretory pore; $k$, ventral row of modified cuticular elements; $l$, submedian row of modified cuticular elements; $m$, ovum; $n$, muscular layer; $o$, anterior extremity of the uterus; $p$, uterus; $q$, cuticle; $r$, vulva; $s$, submedian row of modified cuticular elements; $t$, rectum; $u$, anus; $v$, terminus.

RHABDOLAIMUS, de Man, 1880.

Fig. 2. Rhabdolaimus minor, n. sp. I, lateral view of female; II, head of the same, showing amphid. The head in $\mathrm{I}$ is twisted, so that the amphid appears as if ventral, or nearly so.

$a$, amphid; $b$, long, narrow pharynx; $c$, anterior group of nerve cells; $d$, nerve-ring; $e$, cardiac bulb; $f$, wall of the intestine; $g$, flexure in anterior ovary; $h$, posterior group of nerve cells; $i$, body cavity; $j$, lumen of intestine; $k$, ovum; $l$, blind end of posterior ovary; $m$, egg; $n$, flexure in posterior ovary; $o$, cuticle; $p$, caudal glands; $q$, subcuticle; $r$, vulva; $s$, rectum; $t$, anus; $u$, nerve cells (?); $v$, duct of caudal glands; $w$, spinneret; $x$, lip region.

DIPLOGASTER, Max Schultz, 1857.

Fig. 3. Diplogaster fictor, Bastian. I, side view of female; II, head of the same seen in dorso-ventral view, lips nearly closed; III, head of the same, lateral view, lips nearly wide open; IV, head of the same, lateral view, lips partially closed; V, front view of mouth, partially closed; VI, lateral view, posterior portion of a male specimen; VII, somewhat diagrammatic perspective view showing markings of the cuticle.

$a$, one of the lips; $b$, one of the six cephalic setae; $c$, amphid; $d$, one of the two more or less evertible pharyngeal hook-shaped teeth; $e$, median oesophageal bulb; $f$, nerve-ring; $g$, anus ; $h$, rectum; $i$, intestine; $j$, terminus; $k$, posterior oesophageal bulb; $l$, nerve cells; $m$, renette cell (?); $n$, left spiculum; $o$, lumen of the intestine; $p^{\prime}$, preanal male seta; $p^{\prime \prime}, p^{\prime \prime \prime}$, $p^{\prime \prime \prime \prime}$, post-anal male setae and papillae; $q$, one of the cells of the intestine; $r$, accessory piece; $s$, flexure in anterior ovary; $t$, blind end of anterior ovary; $u$, longitudinal striae of the cuticle; $v$, vagina; $w$, synapsis in egg in the anterior uterus, the reduced number of chromosomes being seven; $x$, one of the spermatozoa in the ovary; $y$, uterus; $z$, vulva.

"The drawings are used by permission of the Secretary of Agriculture, and were made by Mr. W. E. Chambers, under the author's supervision. 


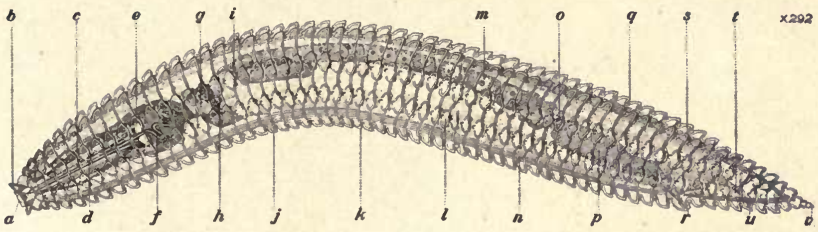

Fig. 1. Iota octangulare, n. sp.

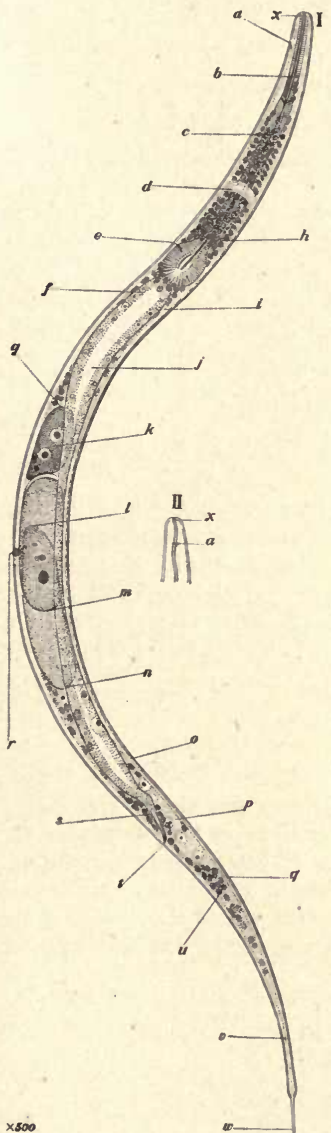

Fig. 2. Rhabdolaimus minor, n. sp

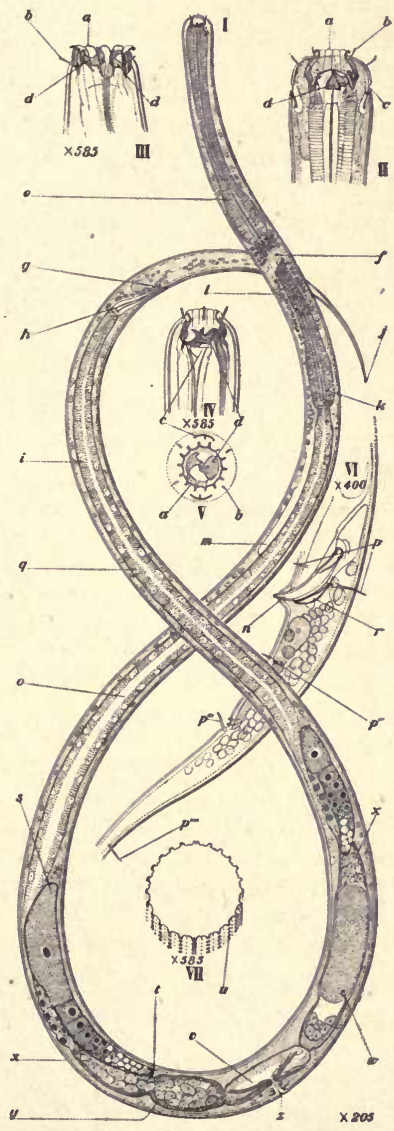

Fig. 3. Diplogaster fictor, Bastian.

Plate II. 


\section{Plate III.}

PRISMATOLAIMUS, de Man, I880.

Fig. 4. Prismatolaimus stenurus, n. sp. I, lateral view of a female; II, front view of head; III, side view of head.

$a$, one of the six-cephalic papillae; $b$, one of the ten cephalic setae; $c$, one of the six thin lips; $d$, pharynx; $e$, amphid; $f$, lumen of the oesophagus; $g$, nerve-ring; $h$, cuticle; $i$, nucleus of ovum; $j$, vulva; $k$, blind end of posterior ovary; $l$, egg; $m$, beginning of the intestine; $n$, one of the cells of the wall of the intestine; $o$, rectum; $p$, anus; $q$, one of the caudal glands; $r$, flexure in anterior ovary; $s$, spinneret.

\section{SPILOPHORA, Bastian, I865.}

Fig. 5. Spilophora canadensis, n. sp. Side view of male.

$a$, one of the lips; $b$, dorsal pharyngeal tooth partly exserted; $c$, pharynx; $d$, base of the pharynx; $e$, lumen of the oesophagus; $f$, nerve cells; $g$, nervering; $h$, excretory pore; $i$, chitinous valve of the cardiac bulb; $j$, one of the two lateral, longitudinal rows of cuticular markings; $k$, lumen of the intestine; $l$, renette cell; $m$, nucleus of renette cell; $n$, cell accessory to the renette cell; 0 , blind end of single testis; $p$, point in the cuticle where the striation is reversed; $q$, vas deferens; $r$, one of the spicula; $s$, anus; $t$, one of the caudal glands; $u$, spinneret.

\section{MICROLAIMUS, de Man, 1880.}

Fig. 6. Microlaimus fluviatilis, n. sp. I, lateral view of female; II, head of the same.

$a$, Mouth opening; $b$, one of the six cephalic papillae; $c$, one of the four cephalic setae; $d$, one of the small pharyngeal teeth; $e$, excretory pore; $f$, spiral amphid; $g$, oesophagus; $h$, nerve-ring; $i$, cardiac bulb; $j$, preliminary portion of the intestine; $k$, renette cell $; l$, body cavity; $m$, lumen of intestine; $n$, one of the cells of the intestine; 0 , anus; $p$, flexure in posterior ovary; $q$, uterus; $r$, blind end of posterior ovary; $s$, one of the three caudal glands; $t$, spinneret; $u$, eggs; $v$, vulva; $w$, cuticle.

\section{TRIPYLA, Bastian, 1865.}

Fig. 7. Tripyla lata, n. sp. Lateral view, male specimen.

$a$, cephalic seta; $b$, one of the three lips; $c$, amphid; $d$, spermatozoa; $e$. spermatocytes in anterior testis; $f$, posterior extremity of the oesophagus; $g$, nerve-ring; $h$, cuticle; $i$, oesophagus; $j$, lumen of oesophagus; $k$, intestine; $l$, spermatocytes in posterior testis; $m$, one of the numerous ventral male papillae; $n$, vas deferens; 0 , retractory muscle of spiculum; $p$, one of the spicula; $q$, lumen of the intestine; $r$, duct of one of the caudal glands; $s$, caudal gland; $t$, spinneret. 


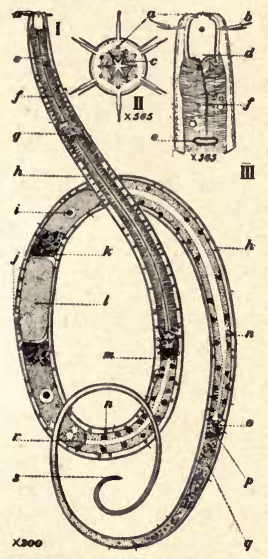

Fig. 4. Prismatolaimus stenurus, n. sp.

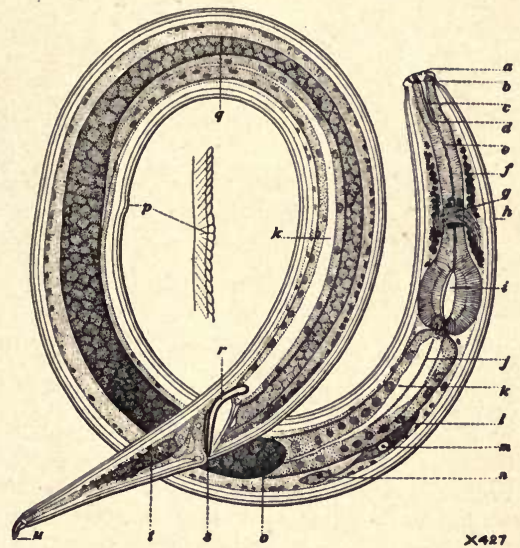

Fig. 5. Spilophora canadensis, n. sp.

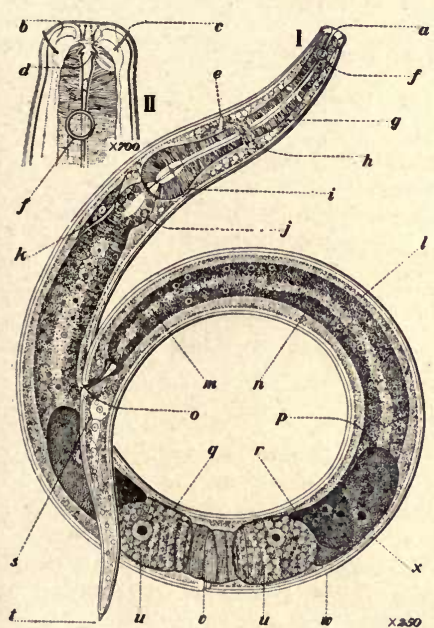

Fig. 6. Microlainus fluviatilis, n. sp.

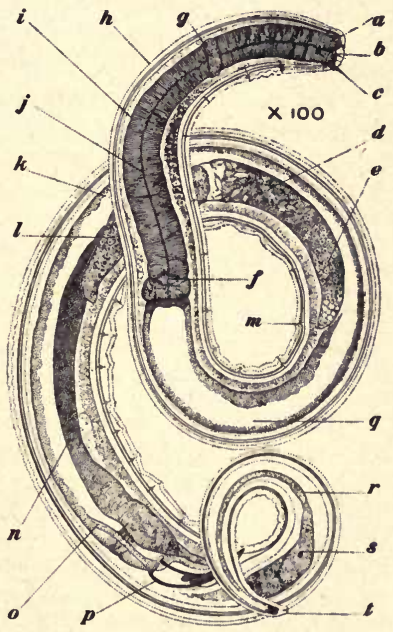

Fig. 7. Tripyla lata, n. sp.

Plate III. 


\section{Plate IV. •}

TERATOCEPHALUS, de Man, 1876.

Fig. 8. Teratocephalus cornutus, n. sp. I, lateral view of a female; II, lateral view of head, more highly magnified; III, front view of head; IV, dorso-ventral view showing lips wide open; V, cuticle showing lateral field.

$a$, one of the six movable, chitinous lips; $b$, one of the four submedian cephalic setae; $c$, amphid; $d$, nerve-ring; $e$, excretory pore; $f$, organ of unknown significance; $g$, cardiac bulb; $h$, intestine; $i$, anus; $j$, rectum; $k$, cuticle; $l$, one of the cells of the intestine; $m$, lumen of the intestine; $n n$, flexures in ovary; $o$, egg; $p$, vulva; $q$, blind end of posterior ovary; $u$, terminus.

ALAIMUS, de Man, I880.

Fig. 9. Alaimus simplex, n. sp. I, lateral view of a female; II, anterior extremity, lateral view; III, posterior extremity of a male, lateral view.

$a$, lip region; $b$, pharynx; $c$, amphid; $d$, amphid, enlarged; $e$, group of spermatozoa at the posterior portion of the ovary; $f$, blind end of ovary; $g$, male supplementary papillae; $h$, left spiculum; $i$, terminus; $j$, rudimentary submedian elevation or flap of the cuticle, indicating rudimentary bursa; $k$, egg; $l$, vulva; $m$, nerve-ring; $n$, posterior extremity of oesophagus; $p$, modified cells of anterior portion of the intestine; $q$, cuticle; $r$, wall of the intestine; $s$, lumen of the intestine; $t$, flexure in the single ovary.

The arrangement of the ova and eggs may need a few words of explanation. The rounded blind end of the reflexed part of the single ovary is shown near the group of spermatozoa, $e$. The blind end contains a large number of oocytes of small size. These oocytes have more than doubled in size by the time they have reached the position, $f$. Henceforth they become arrapged single file, always increasing in size until they pass the flexure, $t$. At $k$ a deceptive appearance is shown, inasmuch as the ripe ovum, $k$, has passed the flexure and in its passage to the uterus is pressing two of the unripe ova apart. This accounts for the apparent anomaly in relative size of the ova in the vicinity of the flexure.

\section{PLECTUS, Bastian, I865.}

Fig. Io. Plectus tubifer, ri. sp. Male specimen, lateral view.

$a$, lips; $b$, papilla-like cephalic seta; $c$, amphid; $d$, anterior chamber of pharynx; $e$, posterior chamber of pharynx; $f$, lumen of the oesophagus; $g$, nerve-ring; $h$, excretory pore; $i$, renette cell; $j$, cardiac bulb; $k$, vulva of cardiac bulb; $l$, cardia; $m$, lumen of intestine; $n$, blind end of anterior testis; $o$, spermatocyte; $p$, junction of testes; $q$, blind end of posterior testis; $r$, beginning of vas, deferens; $s$, spermatozoon; $t$, male glands; $u$, one of the caudal glands; $v$, anterior of the three tubular supplementary organs; $w$, spicula; $x$, anus; $y$, one of the nine male papillae; $z$, spinneret. 

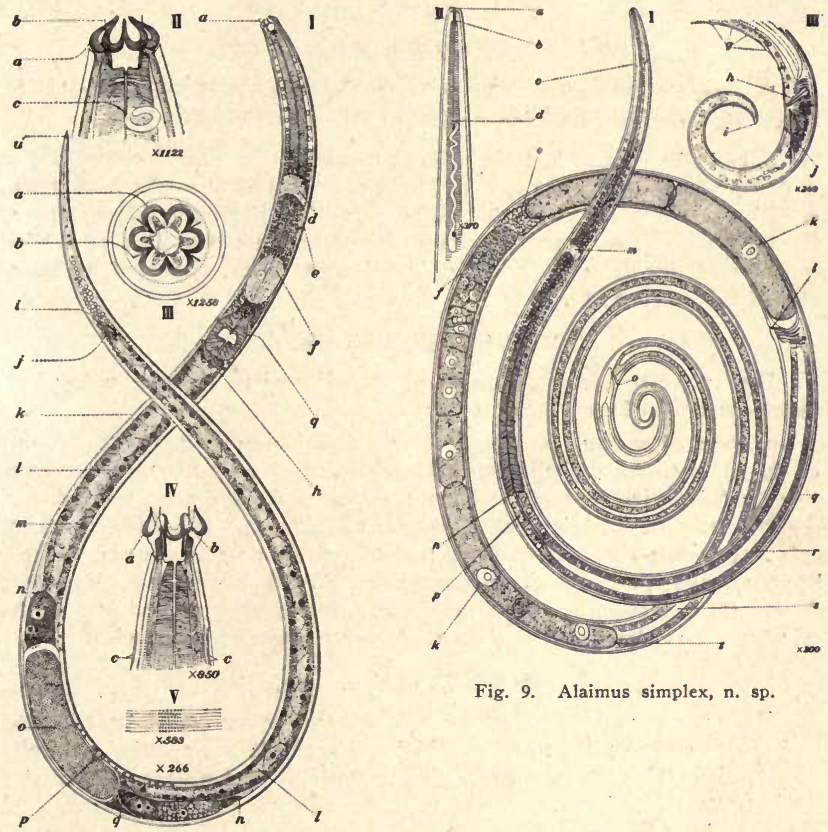

Fig. 9. Alaimus simplex, n. sp.

Fig. 8. Teratocephalus cornutus, n. sp.

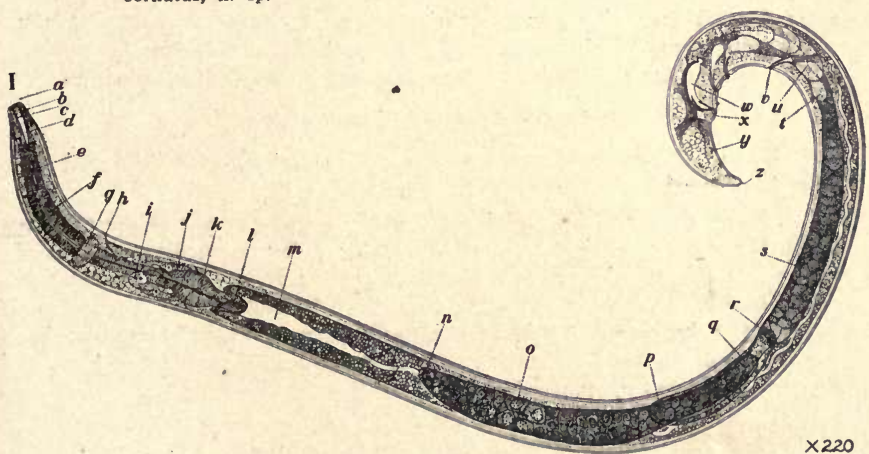

Fig. 10. Plectus tubifer, n. sp.

Plate IV. 


\section{Plate V.}

\section{ACHROMADORA.}

Fig. II. Achromadora minima, Cobb. I, lateral view of a female; II, lateral view showing cuticular markings; III, lateral view of head.

$a$, cephalic papilla; $b$, cephalic seta; $c$, one of the ribs of the pharynx; $d$, dorsal pharyngeal tooth; $e$, sub-ventral (?) pharyngeal tooth; $f$, pharynx; $g$, cuticular markings; $h$, amphid; $i$, nerve cell; $j$, nerve-ring; $k$, spinneret; $l$, excretory pore; $m$, flexure of ovary; $n$, one of the caudal glands; $o$, blind end of posterior ovary; $p$, anus; $q$, intestine; $r$, vulva; $s$, one of the granules of the intestine; $t$, egg.

DORYLAIMUS, Dujardin, 1845.

Fig. 12. Dorylaimus fecundus, n. sp. Side view of head and tail of female, and tail of male.

$a$, spear or onchus; $b$, one of the six anterior cephalic papillae; $c$, one of the six posterior cephalic papillae; $d$, guiding ring of the spear; $e$, oesophagus; $f$, intestine; $g$, rectum; $h$, anus; $i$, one of the anal muscles; $j$, one of the four lateral caudal, innervated papillae; $k$, cuticle; $l$, subcuticle; $m$, muscular layer; $n$, wall of the intestine; $o$, one of the numerous oblique ejaculatory muscles; $p$, one of the numerous ventral male papillae; $q$, ejaculatory duct; $r$, one of the two preanal papillae; $s$, retractor muscle of one of the spicula; $t$, muscular layer; $u$, right spiculum; $v$, right accessory piece.

\section{IRONUS, . Bastian, I865.}

Fig. 13. Ironus americanus, n. sp. I, anterior extremity, lateral view, oral organs everted; II, same, more highly magnified; III, anterior extremity of a specimen about to moult, showing two sets of oral organs, the anterior set in process of being replaced by the posterior; IV, posterior extremity of a female, lateral view.

$a$, one of the three chitinous oral teeth; $b$, cephalic papilla; $c$, cephalic seta; $d$, amphid; $e$, pharynx; $f$, anterior group of minute pharyngeal teeth; $g$, posterior group of pharyngeal teeth; $h$, oesophagus; $i$, lining of oesophagus; $j$, nerve-ring; $k$, intestine; $l$, anus; $m$, base of the tail; $n$, terminus.

\section{ONCHOLAIMUS, Dujardin, I865.}

Fig. 14. Oncholaimus punctatus, n. sp. Lateral view of head and tail of male specimen.

$a$, terminal flaps of lips; $b$, lip; $c$, cephalic papilla; $d$, one of the papillalike cephalic setae; $e$, right submedian onchus; $f$, left submedian onchus; $g$, wall of pharynx; $h$, intestine; $i$, ejaculatory duct; $j$, one of the spicula; $k$, dorsal onchus; $l$, amphid; $m$, duct of oesophageal gland leading into onchus; $n$, rectum; $o$, beginning of the oesophagus; $p$, anus; $q$, lining of the oesophagus; $r$, one of the caudal glands; $s$, one of the two male papillae; $t$, ducts of the caudal glands; $u$, spinneret. 


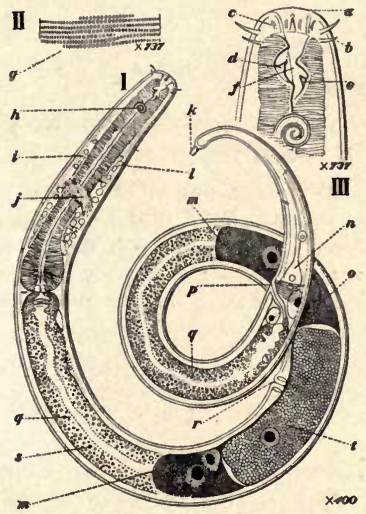

Fig. 11. Achromadora minima, Cobb

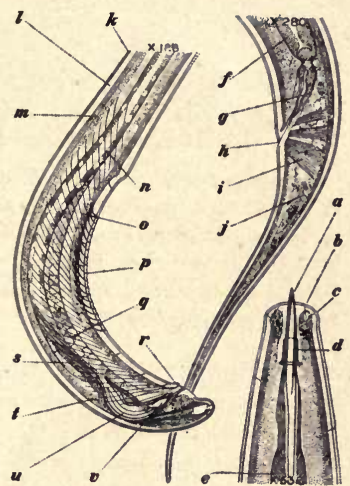

Fig. 12. Dorylaimus fecundus, n. sp.

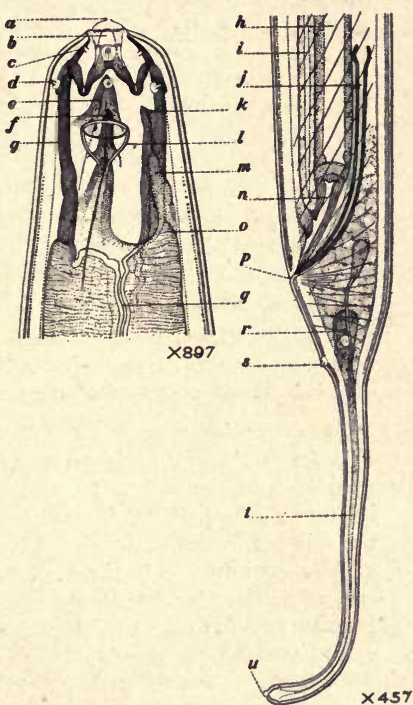

Fig. 14. Oncholaimus punctatus, n. sp.

Fig. 13. Ironus americanus, n. sp. Plate V. 


\section{Plate Vi.}

TRILOBUS, Bastian, 1865 .

Fig. 15. Trilobus longus, (Leidy), Bastian. I, male specimen, lateral view; II and III, head of the same, lateral view; IV, head, dorso-ventral view; V, VI, and VII, enlarged views of the ventral supplementary organs.

$a$, lateral cephalic seta; $b$, cephalic papilla; $c$, submedian cephalic setae; $d$, pharynx; $e$, amphid; $f$, anterior pharyngeal tooth; $g$, posterior pharyngeal tooth; $h$, oesophagus; $i$, nerve-ring; $j$, excretory pore; $k$, nerve cells $l l$ glandular (?)-organs; $m$, lumen of intestine; $n$, blind end of anterior testis; $o$, testis; $p$, junction of testes; $q$, blind end of posterior testis; $r$, vas deferens; $s$, anterior male supplenentary organ of the anterior series; $t$, anterior supplementary organ of the posterior series; $u$, spicula; $v$, accessory piece; $w$, caudal gland; $x$, anus; $y$, terminus; $z$, nerve ending of papilla of the supplementary organ.

\section{DOLICHODORUS, nov. gen.}

. Fig. r6. Dolichodorus heterocephalus, n. g., n. sp. I, nearly side view of a female; II, lateral view of surface of head, more highly enlarged; III, sagittal section of head; IV, dorso-ventral view of head; V, front view of head; VI, side view, posterior extremity of male; VII, ventral view of posterior extremity of female; VIII, ventral view of posterior extremity of male.

$a$, papilla ; $b$, cephalic organ of unknown significance; $c$, spear; $d$, base of spear; $e$, median bulb; $f$, nerve-ring; $g$, excretory pore; $h$, cardiac swelling; $i$, intestine ; $j$, anus ; $k$, lateral caudal pores ; $l$, terminus ; $m$, blind end of posterior ovary; $n$, ovary; $o$, left spiculum; $p$, accessory piece; $q$, distal end of accessory piece; $r$, left flap of bursa; $s$, terminus of male; $t$, ovum; $u$, spermatozoa ; $v$, vaginal muscles; $w$, uterus; $x$, vulva ; $y$, anus.

\section{CYATHOLAIMUS, Bastian, 1865.}

Fig. 17. Cyatholaimus truncatus, n. sp. I, side view of a female; II, side view of head; III, front view of the same head; IV, ventral view of anal region of male; V, lateral view of the same; VI, lateral view in the middle of the body showing cuticular markings and pores.

$a$, submedian cephalic seta; $b$, labial papillae; $c$, amphid; $d$, dorsal tooth; $e$, lateral cephalic seta; $f$, one of the twelve ribs of the vestibule; $g$, small submedian pharyngeal tooth; $h$, base of the pharynx; $i$, ejaculatory duct; $j$, intestine; $k$, one of the four male pre-anal supplementary organs; $l$, one of the spicula; $m$, anal muscles; $n$, one of the accessory pieces; 0 , nerve-ring; $p$, one of the cells of the intestine; $q$, lumen of the intestine; $r$, anus; $s$, blind end of reflexed ovary; $t$, egg; $u$, vulva; $v$, flexure in arterior ovary; $w$, junction of the ovary and uterus; $x$, pores in the cuticle; $y$, one of the three caudal glands; $z$, male gland (?). 


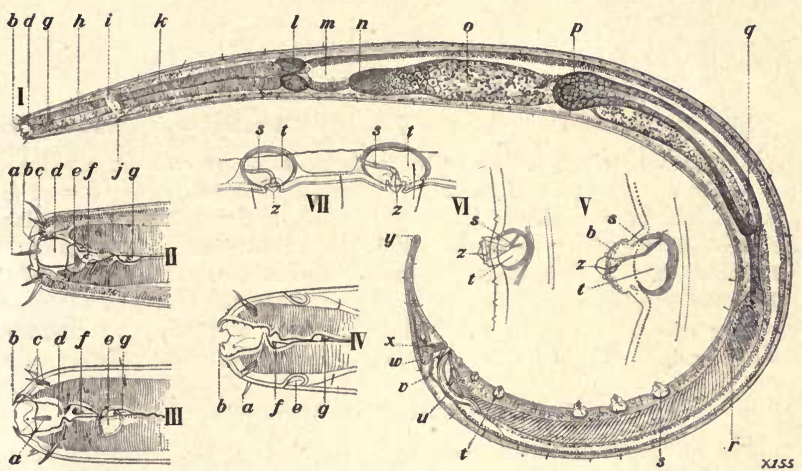

Fig. 15. Trilobus longus, (Leidy), Bastian
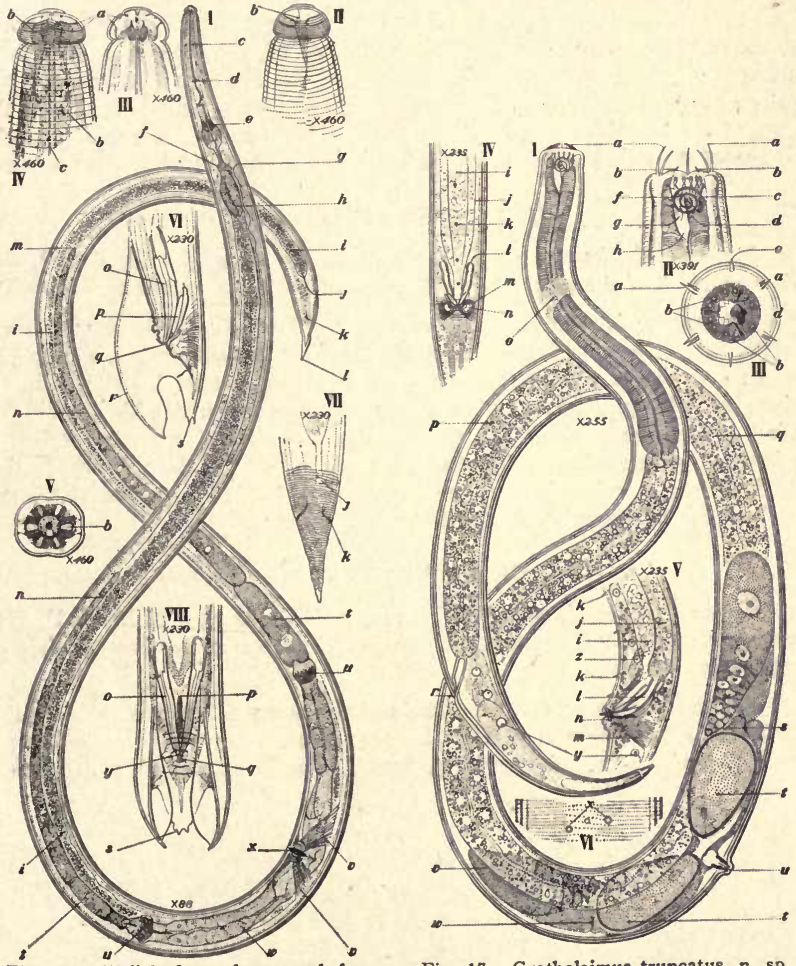

Fig. 16. Dolichodorus heterocephalus, Fig. 17. Cyatholaimus truncatus, n. sp. n. g., n. $8 p$.

Plate VI. 
Plate VII.

\section{MESOMERMIS.}

Fig. 18. Mesomermis virginiana, $\mathrm{n}$. sp. Lateral view of head and tail.

$a$. mouth opening; $b$, one of the anterior rows of cephalic papillae; $c$, one of the posterior row of cephalic papillae; $d$, pharynx; $e$, exterior of amphid; $f$, interior of amphid; $g$, oesophagus; $h$, innervated papilla; $i$, glandular cephalic organs; $j$, nerve-ring; $k$, lumen of oesophagus; $l$, intestine; $m$, ejaculatory duct; $n$, body cavity; $o$, oblique copulatory muscles; $p$, one of the submedian supplementary organs; $q$, one of the median supplementary organs; $r$, spicula; $s$, one of the median post-anal supplementary organs; $t$, final submedian supplementary organs; $u$, submedian supplementary organ.

ETHMOLAIMUS, de Man, I880.

Fig. 19. Ethmolaimus americanus, n. sp. Lateral view of a female.

$a$, lips; $b$, minute dorsal and ventral pharyngeal teeth; $c$, one of the four cephalic setae; $d$, amphid; $e$, pharynx; $f$, nerve-ring; $g$, excretory pore; $h$, nerve cells; $i$, cardiac bulb; $j$, beginning of the intestine; $k$, renette cell (?); $l$, beginning of main portion of the intestine; $m$, one of two pairs of unicellular organs of unknown significance; $n$, cuticle; 0 , one of the cells of the intestine; $p$, subcuticle; $q$ and $r$, body cavity; $s$, vulva; $t$, nuclei of one of the muscle cells; $u$, spinneret; $v$, one of the caudal glands; $w$, anus.

MONHYSTERA, Bastian, I865.

Fig. 20. Monhystera sentiens, n. sp. I, side view of a female; II, side view of head of the same; III, side view of posterior extremity of a male.

$a$, pharynx; $b$, submedian cephalic seta; $c$, lateral cephalic seta; $d$, spermatozoon; $e$, amphid; $f$, lining of oesophagus; $g$, oesophagus; $h$, subcephalic setae; $i$, lumen of intestine; $j$, nerve cells; $k$, nerve-ring; $l$, striated lip region; $m$, left spiculum; $n$, glandular structure associated with amphid; 0 , blind end of single ovary; $p p$, the three caudal glands; $q$, anal muscles; $r$, spinneret; $s$, beginning of intestine; $t$, anus; $u$, one of the cells composing the intestine; $v$, vulva; $w$, egg, the spermatozoa " $d$ " being outside of the egg " 2 "; $x$, egg in synapsis; $y$, vaginal glands; $z$, ovum.

\section{ONCHOLAIMELLUS, de Man, 1886.}

Fig. 21. Oncholaimellus heterurus, n. sp. I, side view of head; II, ventral view of head; III, side view of tail end of male; IV, ventral view of anal region of male.

$a, \epsilon x c r e t o r y$ pore; $b$, submedian cephalic seta; $c$, pharynx; $d$, left flap of bursa; $e$, oesophagus; $f$, left spiculum; $g$, accessory piece; $h$, amphid; $i$, male post-anal seta and papilla; $j$, lateral seta; $k$, spinneret; $l$, thin lips. 

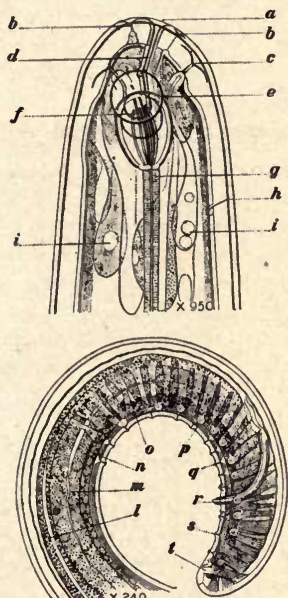

Fig. 18. Mesomermis virginiana, n. sp.

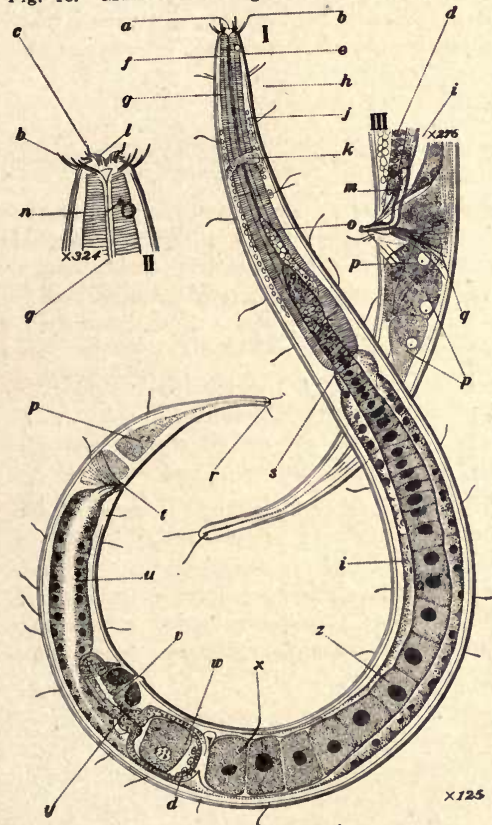

Fig. 20. Monhystera sentiens, n. sp. Plate VII

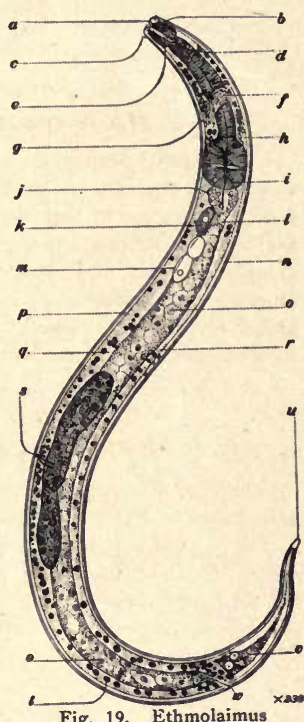

Fig. 19. Ethmolaimus americanus, n. sp.
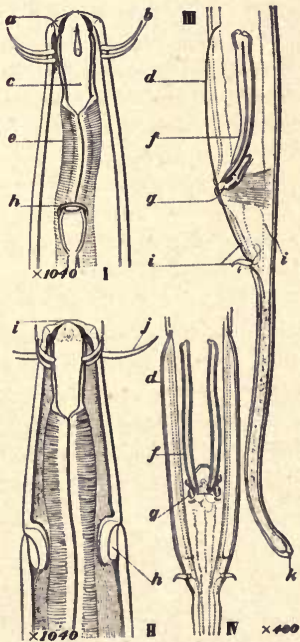

Fig. 21. Oncholaimellus heterurus, n. sp. 


\section{Plate Vili.}

CEPHALOBUS, Bastian, 1865.

Fig. 22. Cephalobus sub-elongatus, n. sp. Lateral view of a female.

$a$, lips; $b$, pharynx; $c$, anterior portion of oesophagus; $d$, posterior extremity of anterior portion of oesophagus; $e$, nerve-ring; $f$, cardiac bulb; $g$. beginning of intestine; $h$, one of the cells of the intestine; $i$, lumen of the intestine; $j$, excretory pore; $k$, cardiac valve; $l$, renette cell; $m$, flexure in single ovary; $n$, cuticle; 0 , ovary; $p$, spermatozoon in uterus; $q$, vulva; $r$, nucleus in ovum; $s$, body cavity; $t$, anus; $u$, ripe ovum; $v$, unripe ovum; $w$, oöcyte; $x$, blind end of ovary; $y$, rectum; $z$, terminus.

\section{BASTIANA, de Man, r876.}

Fig. 23. Bastiana exilis, n. sp. Lateral view of a male specimen.

$a$, one of the six cephalic papillae; $b$, one of the posterior set of four submedian cephalic setae; $c$, one of the anterior set of six cephalic setae; $d$. oesophagus; $e$, cervical seta; $f$, amphid; $g$, one of the cells of the intestine; $h$, one of the numerous male supplementary organs; $i$, blind end of the two testes; $j$, nerve-ring; $k$, posterior extremity of oesophagus, (pseudo-bulb); $l$, left spiculum; $m$, cuticle; $n$, spermatozoon; o, anal muscle; $p$, treminus; $q$, vas deferens; $r$, intestine.

\section{MESOMERMIS, Dady.}

Figure 24. Mesomermis virginia n. sp. Neck and head of the same species shown in figure 18 , showing how the deteriorated oesophagus changes rather gradually into the intestine.

$a$, mouth-opening; $b$, one of the anterior row of cephalic papillae; $c$, one of the posterior row of cephalic papillæ; $d$, pharynx; $e$, exterior of amphid; $f$, interior of amphid; $g$, oesophagus; $h$, innervated papilla; $i$, glandular cephalic organs; $j$, nervering; $k$, lumen of oesophagus; $l$, intestine.

\section{APHANOLAIMUS, de Man, I880.}

Fig. 25. Aphanolaimus spiriferus, n. sp. I, lateral view, anterior extremity of a female; II, lateral view, posterior extremity of a female; III, lateral view of head, more highly magnified; IV, one of the male supplementary organs; $\mathrm{V}$, lateral view of posterior extremity of male.

$a$, mouth opening; $b$, amphid ; $c$, lumen of oesophagus; $d$, pigmented eyespot (?) ; $e$, intestine; $f$, nerve-cell; $g$, rectum; $h$, nerve-ring; $i$, anus; $k$, oesophagus; $l$, caudal gland; $m$, duct of one of the caudal glands; $n$, glandular body at base of neck; $o$, spinneret; $p$, ejaculatory duct; $q$, intestine; $r$, anterior end of cloaca; $s$, right spiculum; $t$, backward pointing accessory piece; $u$, nerve-cells (?) ; $v$, one of the numerous male supplementary organs. 


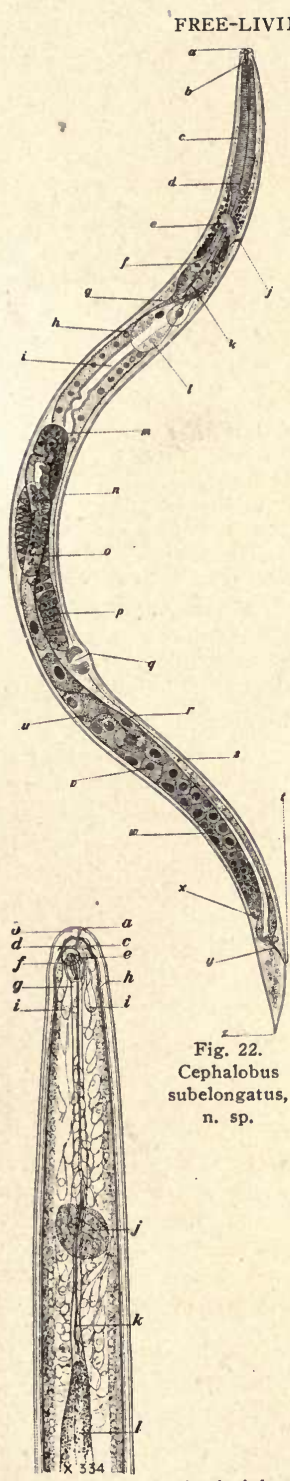

Fig. 24. Mesomermis virginiana, n. sp.

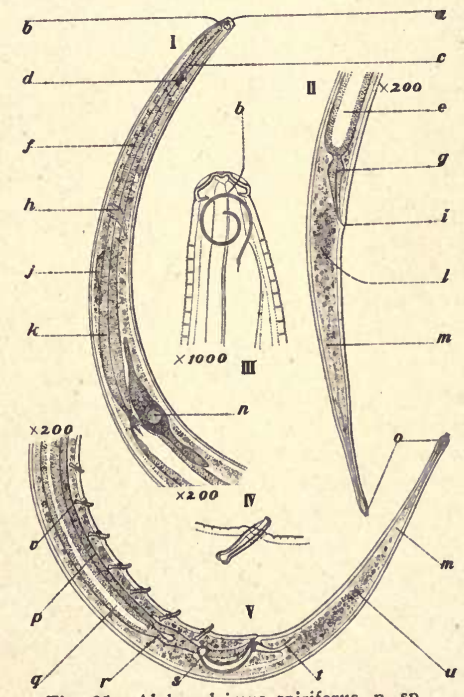

Fig. 25. Alphanolaimus spiriferus, n. sp. Plate Vili. 




\title{
THE
}

\section{ASYMMETRY OF THE NEMATODE \\ Bunonema inequale, n.sp.}

\author{
By N. A. Совв \\ Contributions to a Science of Nematology, III
}

The genus Bunonema was established in 1905 by Dr. L. A. Jägerskiöld for the reception of species from Kerguelen Island and from the Schwarzwald. ${ }^{1}$ Recently species belonging to this genus have been found in Switzerland and examined by Dr. G. Steiner. ${ }^{2}$

The present account deals with species found at Washington, D. C., U. S. A., in the rotting wood of red oak. The specimens have been examined with considerable care on account of their remarkable asymmetry. It seems probable that this asymmetry is characteristic of the genus. The following is an extended description of the generic characters of Bunonema.

\section{BUNONEMA Jägerskiöld 1905}

The rather thin layers of the transparent naked cuticle are traversed by four hundred to five hundred transverse striae, rather difficult of resolution: In at least a part of the circumference the annules are resolvable into dot-like elements, which apparently are the ends of minute, radial, rod-like elements in the cuticle. These elements are so arranged, at least on the right side of the body, as to give rise to a tessellation, in the midst of which, on the lateral field, there is usually a longitudinal series of tubercles of relatively large size, arranged in one or two rows, extending from near the head to near the terminus. When two rows of tubercles are present, one or more pairs at each end of the series are likely to be combined into a single tubercle. When viewed en face the surface of these tubercles presents a punctate appearance, and their circumferences not infre- quently show the presence of minute papilla-like elements, which do not break the contour, but which appear to have passing to them, from the body wall, linear elements, - suggesting that the tubercles are innervated. When seen in profile the tubercles appear to be made

1 "Bunonema richtersi, n. g., n. sp.," Zoologischer Anzeiger, 28 February, 1905.

2 "Freilebende Nematoden aus der Schweiz," Archiv für Hydrobiologie und Planktonkunde, 1913-14. 
of rods, or possibly laminae, corresponding in number with the annules of the adjacent cuticle. The dot-like elements of the cuticle lose their linear arrangement near the anterior extremity,--where the somatic cuticle joins the much thinner and more flexible cuticle of the head. This junction is of such a nature that the lip region can be partially withdrawn into the body cavity somewhat as into a shell. In other words, where the somatic cuticle ceases, there is a rather distinct shoulder and a rather rapid diminution in diameter to the base of the lip region. The expanded lip region is thus set off by a very distinct constriction. Apart from the transverse and punctate markings, the cuticle is traversed by five distinct longitudinal wings, one lateral, one ventral, one dorsal, and two left submedian. The existence of each of these wings is indicated by the presence of their two crenate refractive margins, close together. These lines extend from near the head to the anus, or beyond.

The posterior portion of the neck is more or less cylindroid, but it becomes convex-conoid toward the truncated head, which bears in the midst of the expanded lip region a somewhat depressed mouth opening, surrounded by obscure, more or less amalgamated lips. There are six inconspicuous, minute, innervated, more or less forward-pointing papillae, arranged in a rather asymmetrical fashion on the margin of the head. There are six spreading, more or less curved, tapering cephalic setae, arranged in an asymmetrical manner, the two largest being apparently dextral, but possibly median, the two next smaller left submedian, and the two smallest, which are in fact papilla-like, right submedian. The longest of these setae are usually nearly as long as the head is wide opposite the base of the pharynx. The setae often present the peculiarity of being saccate at the base, more especially the largest ones. The more or less triquetrous, regular, narrow pharynx is entered through a narrow vestibule, and has its walls rather distinctly chitinized, so that it is a very distinct feature of the head. There is no pharyngeal armature. No amphids have been seen, and there are no eye-spots. The rhabditoid oesophagus has an ellipsoidal median bulb, two-thirds as wide as the middle of the neck, and a prolate to pyriform cardiac bulb, two-thirds as wide as the base of the neck. The lining of the oesophagus is a distinct feature throughout its length, more particularly in its anterior half, where it is indicated by refractive elements occupying a considerable portion of the diameter. Both bulbs contain valves, that of the median bulb being simple and elongated, that of the cardiac bulb more complicated, 
and three-parted, but destitute of striations. The more or less thinwalled intestine, which is separated from the oesophagus by a very distinct constriction, becomes at once about two-fifths as wide as the body, and is made up of cells of such a size that about two are required to complete its circumference. The lumen is often a distinct feature, on account of the refractive nature of the lining of the intestine, and its zig-zag course indicates clearly the small number of cells composing the circumference of the intestine. The intestine often expands a short distance behind the cardiac collum, so as to be two-thirds as wide as the bulb. The cells of the intestine contain colorless, more or less scattered granules of variable size, the largest of which have a diameter nearly equal to that of the pharynx, the smallest of which are very much smaller. There is an indistinct flattish cardia. There is no pre-rectum. The rectum is narrow and rather long and slender, usually about one and one-half times as long as the anal body diameter. The anus of the female is usually rather conspicuous, on account of the development of the anterior lip, which in adults constitutes a flap extending backward over the anus and outward from the surface of the body at a slight angle. This anal flap is less pronounced in the young, and is almost altogether absent in very young larvae. The tail begins to taper from some distance in front of the anus, and is first convex-conoid and finally concaveconoid, to the exceedingly acute, simple, unarmed, symmetrical terminus. There is no spinneret, and there are no caudal glands, nor have any definite lateral papillae, such as those of the female of Rhabditis, been seen. The renette cell is probably located in the vicinity of the cardia. The excretory pore occurs opposite the anterior portion of the cardiac bulb, and usually empties on the right side of the ventral wing. The rather inconspicuous nerve ring surrounds the oesophagus somewhat obliquely. From the fairly well-developed vulva the vagina leads inward at right angles to the ventral surface about two-fifths the distance across the body, where it joins the two symmetrically placed uteri, each of which is fully as long as the body is wide. The reflexed ovaries extend back toward the vulva. The eggs are of such a size that each uterus probably will hold only one at a time. They appear usually to be about as long as the body is wide, and about two-thirds as wide as long. The tail of the male has the same general form as that of the female, but is characterized by the development of the cuticle into a very rudimentary bursa. The two equal, simple, frail, sub-arcuate, relatively long and slender, acute 
spicula are usually about two and one-half times as long as the anal body diameter, and are accompanied by an accessory piece about half as long, and arranged parallel to them. The spicula are more or less cephalated by expansion. The accessory piece is usually quite as conspicuous a feature as the spicula, since it is often slightly colored, while the spicula are colorless. The male papillae are reminiscent of those of Cephalobus. In the only males examined there appear to be eight somewhat asymmetrically placed pairs, one pair opposite the anus, two pre-anal, and five post-anal. These papillae are flattish, innervated, minute, and inconspicuous.

The young larvae of Bunonema do not bear tubercles. On the species that bear them the tubercles become permanent elevations during the last two moults. Just previous to the final moult they appear to be smaller and simpler than when fully developed. There is little if any evidence on the right side of very young larvae of the very remarkable transformation which is to take place in the course of growth; the cuticle differs from that of the left side so little that it is only by careful scrutiny that the differences can be detected. There are no reticulations and there is very little evidence of punctation. Previous to the two last moults the cuticle of the right side is differentiated from that of the left to a greater or less extent. Probably at the first moult, the dexter cuticle becomes slightly thicker and almost imperceptibly colored. The anal flap does not exist, or at least is in no wise prominent, on young larvae. The cephalic setae and other cephalic organs are neither so strongly developed, nor so complicated, on the larvae as on the adults.

When a specimen of Bunonema is mounted in water, with just sufficient space between the coverglass and the slide for the nematode to move about freely and without friction, it is seen to have the characteristic movement of nematodes, that is to say, the normal flexures of the body are in the dorso-ventral plane. This brings the tubercles either squarely toward the observer, or squarely on the far side of the body. At the same time the excretory pore, the anus and the vulva come into exact profile.

In manipulating specimens on the microscope slides it sometimes happens that a tubercle is torn loose, and in such cases the tubercle often comes off entire, as if there existed a definite junction along its periphery, or at least as if the peripheral attachment were weak. Such losses may in part account for the occasional irregular arrangement of the tubercles. Specimens are not infrequently seen in which 
one member of a pair of tubercles is missing, or where one member of a moniliform series appears to be missing, and the natural inference is that such tubercles never existed. This inference may, however, be incorrect, as there is very little evidence left to tell of the accidental removal of a tubercle; no very definite scar remains to tell the tale.

Bunonema can exert suction so as to attach itself by the aid of its lips even to smooth surfaces like glass. Specimens sometimes may be seen to attach themselves firmly by the head, and to wave back and forth through the water in which they are mounted.

It is clear that Bunonema is closely related to Rhabditis. This is shown by the structure of the pharynx, oesophagus, intestine, excretory system, and sexual organs. With reference to these latter, there is the difference that in Bunonema the bursa is rudimentary and the male papillae reduced in size so as to resemble those of Cephalobus. Bunonema appears therefore to be related to Cephalobus, especially that group of Cephalobi in which the labial structures are somewhat elaborate.

The most obvious source of food for the species of Bunonema here described is the mycelium of wood-infesting fungi, which is abundant in the cells of the rotting wood inhabited by the Bunonema. It may be that the peculiar modifications of the cuticle are triturating organs enabling the nematodes to break down the walls of fungus mycelium and so obtain the contents of the mycelial cells with greater ease. No organized food has been seen in the intestine of Bunonema, but occasionally granules have been seen that resembled those of the cell contents of the fungi.

\section{DESCRIPTIONS OF THE SPECIES}

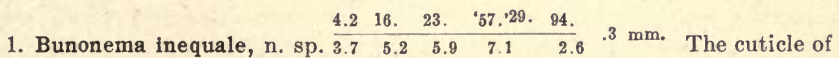
about three-fourths of the right side of the body presents a rudely hexagonal network or tessellation. The meshes of this network are of variable size, those nearest the dorsal and ventral lines being the smaller. The division lines of the tessellation of the cuticle are due to the peculiar arrangement of the minute strongly refractive cuticular elements into which portions of the transverse striae are resolvable. The tessellation becomes much finer toward the extremities of the body, where the dot-like refractive elements are no longer arranged regularly. Thence onward toward the extremities the cuticle is not striated and is more nearly colorless, and very transparent.

Opposite the right lateral line there is a very prominent single series of slightly yellowish, relatively large, projecting, cushion-shaped, striated, chitinous ap- 
pendages or tubercles, which through the middle of the body are about half as high as they are wide. The tubercles are of rounded or ellipsoidal form, and when seen en face sometimes show on their punctate surface the presence of three, four, five, or more exceedingly minute papilla-like elements encircling the axis of the appendage, this axis of course being radial and perpendicular to the surface of the body. In the specimen from which the description was derived, which appeared to be an average female about to undergo the final moult, there were sixteen of these appendages, forming a dextral row extending from the lips to near the anus. Those near the ends of the series were smaller than those near the middle, that on the lips being the smallest of all. Throughout the middle portion of the body the tubercles are of rather uniform size. Anteriorly theybegin to diminish in size near the base of the neck, so that the bluntly conoid tubercle opposite the base of the pharynx has a diameter only one-third as great as that of the one near the middle of the body. The posterior tubercle, near the anus, is of about the same size as that opposite the base of the pharynx. When seen in profile, that is, when the nematode is viewed dorso-ventrally, the tubercles exhibit somewhat the same degree of striation as the adjacent cuticle, but the striae, or elements thereof, appear as if arranged in a somewhat fan-shaped manner. When the body is arcuate and twisted, so that the row of appendages is on the outside of the curve, the distance between the appendages at the middle of the body nearly equals the diameter of one of the appendages. When the body is curved in the other direction, the tubercles may be nearly in contact. The exterior of the tubercular appendages is distinctly striated, and sometimes tessellated, the striae being of about the same size and nature as those of the rest of the cuticle.

The following is a detailed description of these interesting tubercles as they occurred on an adult male specimen fixed in Fleming's solution, and examined soon afterward in water. The specimen was arcuate and the tubercles were on the inside of the curve. The first or labial tubercle, standing halfway between the two relatively large and apparently dextral submedian cephalic setae, appeared somewhat thumb-shaped in contour, and when seen in optical section seemed to have a refractive chitinous core of a slightly greenish color. This tubercle was arcuate, and its apex was turned outward so as to be nearly at right angles to the body axis. The second tubercle, slightly behind the base of the pharynx, was bluntly conoid, the longitudinal optical section being approximately equilateral. The cap or outer half of the cone was a grayish green refractive chitinous element, whose structure showed comparatively little detail. The contour of this cap when seen in optical longitudinal section indicated the presence of a slight constriction half way between its apex and its base. Inside the cap the tubercle appeared as if radially striated, the number of striae being about ten. As these striae appeared to be more or less visible in every longitudinal optical section, it would seem as if they must be the optical expression of a columnar or laminated structure. When the tubercles were viewed in longitudinal optical section, the intermediate somatic cuticle, that is the cuticle between successive tubercles, presented refractive features related to the tesscllation so strikingly displayed when the animal is viewed from the right side. The plainer or less complicated portions of the cuticle, as thus seen in longitudinal section, represent the "lacunae" of the tessellation, while the refractive places represent the 
Fig. 1. Bunonema inequale, n. sp. I, ventral view of male; $I I$, ventral view of the head of larva; $I I I, I V, V$, ventral, front and lateral views of the head of adult specimens; the three figures are derived Irom three ditferent specimens; $V I$, lateral view of tail end of the male; $V I I$, cross-section taken near the middle of the hody and passing through one of the tubercles; VIII, ventral surface view of a segment of the body, showing details of the tessellation, rodlike structures, tubercle and subtubercles.

Nearly all the figures illustrate the remarkableasymmetry of Bunonema, but this feature is most noticeable in figures I, III, IV and VII.

$a$, forward projecting membrane half encircling the mouth-opening on the right side of the front of the head; $t$, dextral sub-median (?) cephalic seta; $c$, labial tubercle; $d$, one of the two left-hand sub-median cephalic setac; e, pharynx; $f$, anterior border of the dextral cuticular armament; $g$, memhers of the dextral series of tubercles; $h$, one of the subtubercles; $i$, repetitive elements connected with the large median (?) cephalic setae; $j$, saccate base of one of the largest setae; $k$, nerve ring; $l$, excretory pore; $m$, cardiac bulb; $n$, border of the dextral cuticular armament; $o$, median wing; $p$, reflexed blind end of the single testis; $q$, spicula; $r$, anterior pair of male papillae; $s$, right hand member of the second pair of male papillae; $t$, accessory piece; $u$, right hand member of the third pair of male papillae; $v$, fourth pair of male papillae; $u$, right hand member of the fifth pair of male papillae; $x$, sixth and seventh pairs of male papillae,-dorsally submedian; $y$, hindermost pair of male papillae-subventral; $z$, terminus, from which the posterior end of the cuticular armament is separated by a furcation.

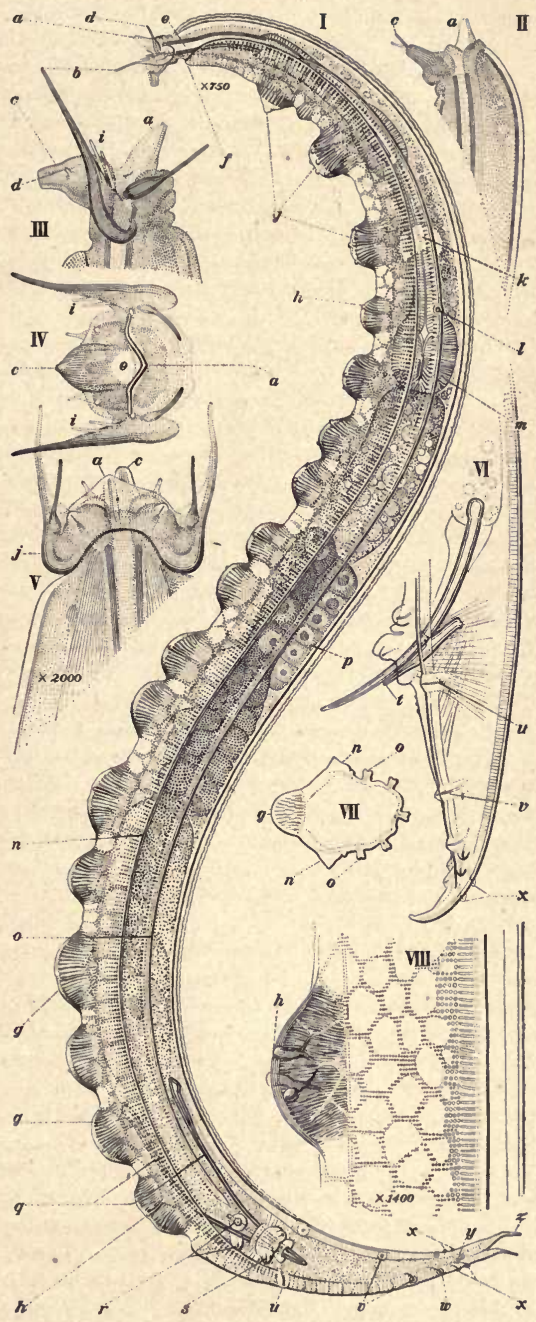


elements composing the net. There were about five of these refractive places between the second and third tubercles, four between the third and fourth, two between the fourth and fifth, and fifth and sixth, these numbers corresponding to the increasing coarseness of the tessellation on the posterior part of the neck.

The third tubercle had the form of a truncated cone. It was a little higher than the second, and the sides of the cone, were somewhat steeper. It had a chitinous cap like that of the second tubercle but truncated, and the outer surface of this cap bore on its margin exceedingly minute, round, flatish sub-tubercles - perhaps four only - so arranged that in optical section the middle of the face of the tubercle appeared almost imperceptibly depressed. The chitinous cap was also hollowed on its inner concave face, the depth of the excavation being about three-fifths the entire depth of the cap. This tubercle was also columnar in structure, the number of elements appearing in the optical section being slightly greater than in number two,-perhaps 12 . Tubercle number four was shaped very much like number thrce, but was a little more rounded on its exterior surface, so that the entire tubercle in optical section presented a rounded contour; its cap being about as thin as that of number three, but extending farther over the surface of the tubercle, extending in fact. nearly to the general body surface. The internal structure was like that in numbers two and three, except that the number of elements was slightly greater, and the outer elements were spread apart as they extended outward, so that the striation appeared somewhat fanshaped. This fourth tubercle stood half way between the median and cardiac bulbs, and its surface bore the same kind of minute subsidiary surface tubercles as were noted on the third. The number of these subsidiary tubercles was slightly greater on the fourth than on the third; there appeared to be at least five or six of them. The fifth tubercle was very much like the fourth, but slightly smaller, and also bore the minute subsidiary surface tubercles mentioned in connection with the third and fourth, but fewer of them, in fact there was perhaps only one. The sixth tubercle was somewhat asymmetrical in optical section, wider than any of the preceding, and composed of a greater number of elements. Its chitinous cap was slightly thinner than that of number five, just as that of number five was slightly thinner than that of number four. This tubercle was located opposite the anterior portion of the intestine. It remained uncertain whether this sixth tubercle bore any of the minute subsidiary surface tubercles. The columnar structure of the third, fourth and fifth tubercles, especially the two former, did not appear to be absolutely uniform, and it seemed likely that this lack of uniformity was due to the optical effect of special elements passing radially through the tubcrcle to the minute subtubercles, and suggested the possibility of the presence in the tubercles of nerye elements connected with the small subsidiary tubercles. From the sixth onward the tubercles were of varying size, sometimes a iittle smaller than number five, sometimes as large as number six, the six or seven along the middle portion of the body tending to be smaller, and two or three in the proximity of the spicula tending to be larger. The chitinous caps were less pronounced through the middle of the body and, on account of their retractive properties, appeared merely as an accentuated contour; that is to say, when seen in exact optical longitudinal section the chitinous covering gave rise to a sharp dark contour, which contrasted strongly with the contour produced by the corresponding portion of the somatic cuticle between the tubercles. 
Through the middle of the body the tubercles showed an obscure tendency to group themselves in twos.

Starting now at the other end of the body, the hindermost tubercle was a broad flattish and rather inconspicuous affair, whose contour did not differ very much from that of the remainder of the cuticle on the right side of the tail. This posterior tubercle lay slightly in front of the anus. The penultimate and antepenultimate tubercles were rounded and closely resembled the majority of the middle members of the series, though they differed in presenting more clearly the subordinate minute tubercles on their surfaces. Here too, in some cases, it was possible to demonstrate the presence of refractive elements-perhaps nerve elements-passing to the subsidiary tubercles. Behind the flattish posterior tubercle the general character of the cuticle of the right side was maintained to near the terminus, and the arrangement was such that the posterior extremity appeared as if somewhat unequally bifurcated, one fork being the terminus proper, that is, the end of the tail, the other being the slightly arcuate outturned loose terminal portion of the dexter cuticular armament.

The lip region is a rather prominently expanded markedly asymmetrical structure. The flexibility of the neck enables the lip region to be swung with comparative rapidity back and forth through an angle of nearly ninety degrees. There are six tapering, spreading, nearly straight cephalic setae, arranged in very unequal pairs, a left submedian slender pair, one dorsally submedian, the other ventrally submedian; a large pair, correspondingly arranged, but extending in the opposite direction, thus appearing to be right submedian; and a very small right submedian pair. The members of the large pair are two to three times as long as those of the left hand pair; these latter being about one-third as long as the pharynx, whereas the former are more than halt as long as the pharnyx. The right submedian setae are reduced and papilla-like. Each of these six setae has a swollen or even bladder-like base, which is very much more pronounced in the case of the largest setae. The dexter labial tubercle is located between the two small right-hand setae. Between these latter and the larger setae there may usually be seen two or three exceedingly delicate refractive elements somewhat suggestive of the repetitive elements in the nematode genus Wilsonema; these are beyond question connected with the largest setae. Between the left submedian setae, that is to say, on the left lateral line, careful focusing shows in the optical longitudinal section of the head what appears to be a minute pore, ending in a depression on the outer surface of a minute labial elevation. On each side of this "pore" are two minute papillae, corresponding to two similar ones on the right side of the head. Between the left-hand setae and papillae and the border of the mouth opening, there is a thin semi-circular, curved, projecting oral membrane, extending forward and outward. This membrane is widest, that is, extends farthest forward, in the lateral region, and at this point its altitude is somewhat less than the length of the left-hand setae. At their bases, which are relatively broad, the setae are inflated, more especially the largest ones, and are so set on the margin of the head that their proximal parts produce a very pronounced shoulder. The rather narrow triquetrous pharynx, which ends abruptly at the base, is of equal diameter throughout, and has a length about equal to the diameter of the head measured opposite its base; it is entered through a rather narrow mouth opening. The anterior end of the oesophagus receives the 
base of the pharynx. The oesophagus begins as a tube about half as wide as the corresponding portion of the neck. It continues to have this diameter, or a slightly greater, until near the middle of the neck it expands to form the median swelling, which is nearly two-thirds as wide as the corresponding portion of the neck, and has a distinct, simple, elongated, chitinous valvular apparatus, the length of which is nearly equal to the corresponding radius of the neck. Behind this bulb the oesophagus diminishes suddenly in diameter so that it is only onefourth as wide as the corresponding portion of the neck. It finally expands to form the pyriform cardiac bulb, which is two-thirds as wide as the base of the neck, and contains a distinct, three-fold, more or less complicated, non-striated valvular apparatus. The intestine becomes at once three-fourths as wide as the body. There is an obscure flattish cardia a little more than one-third as wide as the corresponding portion of the body. The cells of the intestine contain a few scattered granules of variable size, the largest of which, fatty granules, are relatively large and have a diameter nearly equaling that of the pharynx; the smallest, probably of another character, being very much smaller. They are less numerous and smaller toward the posterior part of the intestine. No doubly refractive granules have been seen. The lining of the intestine is somewhat refractive, so that, especially toward the middle of the body, the sinuous lumen is of ten quite a distinct feature. The excretory pore is somewhat behind the middle of the neck. The tail end begins to taper from some distance in front of the anus, and tapers at about the same rate until after it passes the anus, where it soon begins to taper more rapidly, so that the posterior somewhat concave conoid half of the tail is relatively narrow, and the terminus itself hairfine. The diameter of the tail at the middle is about one-sixth as great as at the anus. From the rather prominent, salient, two-lipped vulva the vagina leads inward at right angles to the ventral surface nearly halfway across the body. A mature ovum is about as long as the body is wide, and fully one-third as wide as long, and therefore of such a size as to indicate that each uterus can probably contain only one egg at a time.

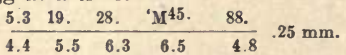

The tail of the male is like that of his mate in form and size, though it tapers more regularly. The two equal, uniform, long and very slender, slightly arcuate, colorless, inconspicuous spicula are about two and one-half times as long as the anal body diameter. Their proximal ends are slightly cephalated by expansion. They are accompanied by a more plainly visible, simple, somewhat wider, slightly colored accessory piece which is nearly straight, and about half as long as themselves. Springing from a little in front of the anus there is a very weakly developed or rudimentary almost invisible bursa, whose somewhat asymmetrically arranged ribs or papillae, difficult of observation, are located as follows:

Almost exactly on the ventral line, in front of the anus, at a distance from it equal to the corresponding body diameter, there is a circular marking ( $r$, Fig. 1 ), which is larger than any other marking of this character, and seems to represent one of the male papillae. To the right of it, and a trifle behind it, rather nearer the right submedian line, is another papilla of similar character, slightly smaller. Opposite the anus, on the left submedian line, there is a small papilla; the mate of this on the right submedian line $(u)$, seems to be in the border of the 
thickened dexter cuticle, and is a little larger. Slightly in front of the anus, and near the median line, both on the left and on the right, there is a papilla $(s)$, that on the right being a little the larger, and not quite so near the median line, but still outside the dexter cuticle. A little behind the middle of the tail there is a submedian pair $(v)$, of which the right hand one is in the edge of the thickened dexter cuticle, while the left hand one is very inconspicuous and somewhat farther forward. Considerably farther back there is a group of about eight papillae, $(w, x, y)$, arranged in four pairs, some of which are exceedingly obscure. The anterior pair, $(w)$, is ventrally submedian. This pair is followed by two dorsally submedian pairs, $(x)$, nearly opposite to which is a ventral pair, $(y)$, one nearly in front of the other. It will be noted that these supplementary organs are arranged much as in some species of Cephalobus.

The ejaculatory duct is about two-fitths as wide as the body. It has the same width as the single testis, whose reflexed blind end is located not far behind the base of the oesophagus, and extends backward a distance about equal to the length of the body diameter.

Habitat. Found in the rotting wood of red oak, Washington, D. C., by Dr. Howard Crawley.

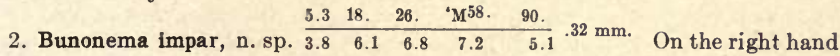
side there are two rows of yellowish tubercles, each row consisting of about eighteen to twenty members. The lip region bears a somewhat thumb-shaped lateral, more or less forward-pointing, protuberance which is probably homologous with the dextral tubercles. Opposite the base of the pharynx is a single tubercle, the first genuine pair of the lateral series being a little in front of the median bulb; thence backward all the tubercles are in somewhat irregular pairs, except one or two of those near the tail end. The members of each pair are placed side by side. These tubercles are rounded elevations having a height about equal to the width of eight of the annules of the cuticle. When the nematode is in a straightened-out attitude the distance between the pairs of tubercles is about equal to their width. The posterior tubercles tend to run together, and are slightly lower and flatter than the anterior. The anterior tubercles, especially those on the neck, are capped with slightly thickened, rather uniform chitin, which bears extremely minute markings similar to those noted on Bunonema inequale. The face of the tubercles appears punctate, though on focusing deeper they appear striated. The lip region is very similar to that of Bunonema inequale. The largest setae are of about the same size and proportions as in inequale, as are also the ventrally submedian setae, though the latter are hardly inclined to be inflated at the base. Between the papillae there seem to be structures very similar to those of inequale. When seen from the right side the adjacent lip region presents the following appearances: the two setae with saccate bases appear to be nearly at right angles to each other, and have their tips located at a distance from each other equal to the length of the pharynx. Each of these setae is about three-fourths as long as the chitinous portion of the pharynx. Between these two setae is the very pronounced, concave-conoid, blunt, rather forward-pointing, almost thumb-shaped protuberance already mentioned, which is probably homologous with the lateral tubercles, though it presents little or none of the brownish or yellowish coloration characteristic of the tubercles. On each side 
of this lateral protuberance there is a rounded innervated papilla, which breaks the contour of the curved line between the protuberance and the adjacent seta. On the lateral line opposite the base of the protuberance there seems to be a third papilla of the same character as the two last described. The head is separated from the neck by a very distinct constriction. Where the somatic cuticle ends, a little in tront of the middle of the phorynx, there is a rather distinct shoulder, and from this shoulder the diameter decreases rapidly to the base of the lips opposite the anterior end of the chitinous portion of the pharynx. Here the diameter is only about three-fifths as great as at the shoulder before mentioned. The saccate basal portions of the large apparently dextral setae extend backward somewhat from the collum or constriction separating the lip region from the neck. Viewed from the left side the left submedian setae are' seen to be not inflated at the base. Three labial papillae are also to be seen from this side, one lateral and two submedian. These, together with those seen on the right side, complete the complement of six minute and very inconspicuous labial papillae encircling the head. There is no forward-pointing thin semicircular border to the mouth opening, as in inequale. The pharynx is triquetrous, and about as long as the posterior bulb, or a little longer, in other words about one and one-third times as long as the diameter of the head measured opposite the base of the pharynx. As seen in optical longitudinal section the pharynx is about one-sixth to one-eighth as wide as long. Its cross-section is somewhat similar to that of the oesophagus; in other words, it is not prismoid, and yet is more or less triquetrous; each of the angles is composed of a thin, refractive, simple, chitinous element. The mouth opening is hardly wider than the pharynx. There are one or two innervated papillae on the dorsal side of the tail near the rectum. Nothing is known concerning the female sexual organs, but from the rudiments seen in male specimens which probably have yet to undergo a final moult, it seems clear that the testis is single and has its blind end reflexed a distance about equal to the diameter of the body.

Habitat. Found with Bunonema inequale in the rotting wood of red oak. Bunonema impar is very closely related to Bunonema inequale, and may indeed prove to be merely a variety of that species.

3. Bunonema dactylicum, n. sp. Species with about the same dimensions and form as the two preceding. As only the young forms have been scen it remains

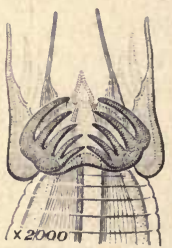

Fig. 2. He a d of Bunonema dactylicum, n. 8 p. somewhat uncertain whether the thickened dextral cuticle bears tubercles, but it is believed not. The cuticle is tessellated and striated as in the first species described. The two large saccate setae are arranged very much as in $B$. inequale, and there is a dextral labial "tubercle" as in that species. The left submedian appendages are palmate, as shown in Figure 2, and have about five finger-shaped processes. Inside the saccate setae is a forward-pointing, more slender pair of equal length.

Habitat. Found in the rotting wood of red oak, with the two preceding, Washington, D. C., U. S. A.

May, 1915 


\title{
SELACHINEMA \\ A NEW NEMATODE GENUS WITH REMARKABLE MANDIBLES.
}

\author{
By N. A. Совв \\ Contributions to a Science of Nematology, IV
}

SELACHINEMA, new genus.

Body slender; cuticle finely transversely striated; head armed with powerful non-retractile dentate chitinous mandibles; amphids spiral; tail armed with caudal glands and spinneret; oesophagus plain; renette present; minute pores on the lateral lines.

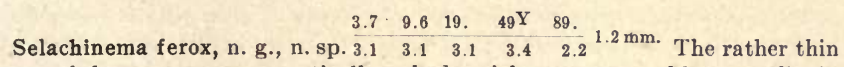
layers of the transparent, practically naked cuticle are traversed by exceedingly

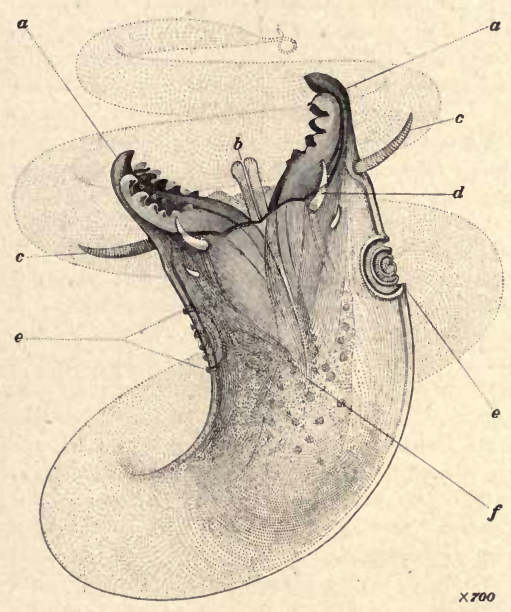

Fig. 1. Selachinema ferox, n.g., n.sp. $a$, the two powerful submedian chitinous jaws, each armed with tour rows of retrorse teeth; $b$, rudiment or remnant of ventral jaw; $c$, lateral setae (restoration); $d$, submedian setae (restoration)-behind may be seen two ot the four small subcephalic setae; $e$, spiral amphids; $f$, base of the pharynx. The head is a correct camera lucida drawing. The body and tail have been drawn in by the artist to assist the imagination, and the perspective though fairly correct is not from nature. fine transverse striae resolvable only with the aid of the highest powers under favorable conditions. Only a very few minute hairs, hardly more than twice as long as the cuticle is thick are to be found on the body. The cylindroid neck ends in a head which diminishes in size very slightly just beyond the amphids, and bears three (?) large, solid, slightly inwardly arcuate, projecting, powerful chitinous jaws armed on the inner surface with four longitudinal rows of somewhat backward pointing conical teeth, each row consisting of four or five teeth. Each of the two submedian jaws is composed of two parts of unequal size, that toward the dorsal side being the shorter, and armed with two rows of teeth of about equal size, while one of the two rows on the other half of the mandible is composed of exceedingly minute dentations. Between the two 
unequal halves of the mandible is a thin, arcuate, toothless element, which ends in an incurved point forming the distal end of the mandible. The chitinous projecting portion of these jaws is slightly inwardly arcuate. Each jaw is somewhat longer than the head is wide, although the projecting portion is only about half as long as the head is wide. These jaws are permanently extended, and cannot be withdrawn into the pharynx. They are used in a manner somewhat like that of the projecting mandibles of certain insects. It appears as if a dorsal jaw or mandible had been broken away from the only specimen so far seen-a young one. There is a circlet of cephalic setae opposite the base of the chitinous, extended portion of the jaws. There are six of these setae in one circlet, and behind each of the submedian setae of this circlet there is a smaller seta slightly farther back so that the total number of the cephalic setae may be said to be ten. The length of the six main setae is unknown, as they are all broken away from the only specimen hitherto seen. A restoration of these six main setae is attempted in the illustration; hence these setae are shown by means of dotted lines. The spiral amphids consist of about three and one-half winds, and are nearly half as wide as the head, and are placed opposite the base of the pharynx, their anterior borders being about half as far from the anterior extremity as are the bases of the before-mentioned naked chitinous portions of the jaws. There are no eye-spots. The phar$y n x$ is of the form consonant with the strongly developed jaws, that is to say, it is triangularly concave-pyramidal. Its base is rather plainly indicated by the proximal ends of the chitinous roots of the jaws, and lies opposite the posterior borders of the amphids. The oesophagus begins as a tube about two-fifths as wide as the base of the head, and continues to have this diameter throughout the greater portion of its length. It is separated from the oesophagus by a constriction. There is no bulb and there does not appear to be any distinct cardia. The intestine, which is made up of cells of such a size that probably four to six are required to build a circumference, is rather thick-walled. The small granules in the cells are of rather uniform size, and do not give rise to any distinct tessellation. The rectum appears to be shorter than the anal body diameter. The tail is convex-conoid in the anterior part in such fashion that at a distance from the anus at least as great as the anal body diameter, it has diminished so that it is only about one-eighth as wide as at the anus. Thence onward it is nearly cylindroid, although it still diminishes slightly in diameter. It ends in a slightly swollen terminus bearing a slightly elongated, plain, unarmed spinneret. It seems likely that the tail is prehensile. The caudal glands are located in the vicinity of the anus. The renette cell lies opposite the cardiac constriction, and is somewhat dumbbell shaped, and placed transversely; the slender duct leads forward from it, and connects with the prominent pyriform ampulla, which is one-fourth as wide as the corresponding portion of the neck, and this in turn leads to the excretory pore near by, opposite the nerve-ring. This latter surrounds the oesophagus somewhat squarely. In front of the exceedingly inconspicuous anus there are two lateral rows of seven or eight minute, equidistant pores. These occupy a space about equal to twice the length of the corresponding body diameter. The distance between these small circular organs is three to four times as great as the diameter of one of them. They appear merely to penetrate the cuticle. so that the extent of their chitinous outlines in a radial direction is about equal to theirwidth. There are three or four similar pores on 
each lateral line opposite the posterior end of the oesophagus, occupying a distance equal to the body radius. Nothing is known concerning the sexualorgans, as the description is derived from a single immature specimen.

Habitat. Found on calcareous algae, near low-tide mark, Colon, Panama; where it was feeding on other nematodes.

Selachinema seems without doubt to belong to the family Enoplidae, which may now be defined as follows: Free-living, marine, carnivorous nematodes with three mandibles; head armed with setae and papillae; body naked; çuticle transversely striated, sometimes also obliquely striated; oesophagus plain; intestine with a variety of well-developed specialized cells indicating definite division of digestive functions; renette and caudal glands present; male and female sexual organs double, spicula two, equal; amphids usually small, inconspicuous, oval or spiral.

The following genera may be included in the family: 1 . Demonema, Cobb 2. Enoploides, Ssaweljev 3. Enoplolaimus, de Man 4. Enoplus, Dujardin-type genus 5. Labyrinthostoma, Cobb 6. Selachinema, Cobb 7. Triodontolaimus, de Man.

The sub-family Enoplinae should contain Enoplus, Enoplolaimus, Enoploides and Labyrinthostoma; the sub-family Selachineminae, Selachinema. Demonema and Triodontolaimus probably represent distinct sub-families. There is probably confusion of characters in Enoplolaimus, Enoploides and Labyrinthostoma, and it may become necessary to rearrange the species now included under these generic names.

Enoplidae occur in all parts of the world, the following being the present known geographical distribution of the various genera.

Demonema...... Italy, California Labyrinthostoma........ Ceylon Enoploides...Arctic, Massachusetts Selachinema..... Panama (Colou) Enoplolaimus...... Cosmopolitan Triodontolaimus .English Channel Enoplus.......... Cosmopolitan

Demonema, Enoplus and Selachinema all contain species known to devour other nematodes. The granules in the intestinal cells of the Enoplidae give rise to the rather distinctly tessellated appearance which I have observed to be more or less characteristic of the free-living nematodes which feed upon animal organisms, or at least those which feed in whole, or in part, upon other nematodes. The following description, derived from the examination of an Enoplus whose intestine 
contained nematode remains, indicates characteristics of these carnivorous species.

The cells of the intestine, separated from each other by thin refractive walls, had the form of rather regular polyhedrons, and each contained a distinct subspherical nucleus having a diameter about onethird as great as that of the cell itself. This nucleus was supplied with a distinct nucleolus. Between its nucleus and the wall each cell contained a large number of brownish-yellow spherical granules of somewhat variable size, the largest having a diameter, one-fourth to one-fifth as great as that of the nucleus, and the smaller varying from this size downward. These granules had a tendency to gather next to the intestinal lumen. The nucleus, therefore, lay nearer the outer surface of the intestine than the inner. In the anterior part of the intestine all the cells except the cardiac cells had the same character. Through the middle of the body the intestinal cells varied from those in the anterior part of the body in that the granules were somewhat smaller, and less numerous, so that the clear spaces in the cells had relatively greater volume. Behind the middle of the body certain of the cells constituting the wall of the intestine were markedly different from those just described, although these latter continued to constitute the main portion of the intestinal wall. These markedly differentiated cells were twice as large as the others and contained larger granules of more uniform size. One of these larger cells may be most advantageously examined when it occurs opposite the lateral field, where the refractions due to the muscular cells of the body wall do not interfere with the view. It may then be seen to have an elliptical contour, at least when seen face view, and to contain a nucleus half as wide as itself. The nucleus in turn contains a nucleolus having a diameter one-third as great as its own. The total number of granules in one of these giant cells was between 50 and 100, and owing to the relatively large size of the nucleus they were located more particularly toward the ends of the cell. The granules were of rather uniform size and had a diameter about twice as great as that of the largest granules in the ordinary intestinal cells. There were 20 to 30 of these scattered giant cells in the posterior fourth of the intestine. 


\title{
NO'TES ON NEMAS*
}

(1) Segmentation of Their Organs, Illustrated by three new free-living marine genera. (2) Intravitam Color Reactions, (3) the Nema population of Beach

Sand, (4) Locational Terms for the Cytology of Descent, and

(5) Functions of the Amphids.

\author{
By N. A. Совв \\ Contributions to a Science of Nematology V
}

1

\section{SEGMENTATION IN NEMATODES}

\section{Observations Bearing on the Unsettled Question of the Relationship of Nematodes to Other Branches of the Animal Kingdom}

I have long been impressed by certain evidences of segmentation in nematodes. My first impressions arose from a study of the distribution of the setae on aquatic forms. This distribution was in those days, and is even yet, described as irregular; the setae are said to be "scattered" on the body. Charting all the setae on a given specimen led to the conclusion that they were not scattered ("zerstreut"); that, rather on the contrary, they constituted a series of more or less harmonious groups. The cephalic setae, it is well known, have an orderly arrangement. The study of a large number of cases leads me to the conclusion that those setae, some distance behind the cephalic setae, denominated subcephalic setae, are also orderly in arrangement, and might, in some instances at least, be regarded as repetitive of the cephalic setae.

Later I was able to show that the transverse striae of the cuticle are retrorse on the posterior half of the body, and the reverse on the front half. (See Fig. 1.) This reversal in the cuticle at the middle of the body, or thereabouts, occurs in a very wide range of gen-

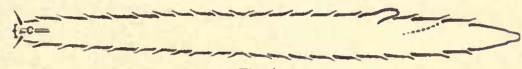

Fig 1

Fig. 1. Diagram of the reversal of the striae of the cuticle of a nematode. The reversal is shown just above the letter $F$. era, is independent of age and of sex, and seems a character of fundamental significance.

*Waverly Press, Baltimore, May 8, 1917. 
Recently I have discovered that the principal cephalic organs are made up of segments which, while simple in character, bear no small re-

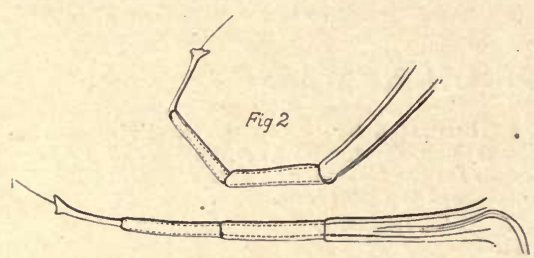

Fig. 2. Cephalic seta of a nematode, showing segmentation. Two different attitudes of the same seta. semblance to corresponding features in segmented organisms. The nature of these segmented appendages will be more easily understood by consulting the illustrations in Fig. 2.

The articulations in the cephalic organs of nemas are not easy to discover, owing to the small size of the organs and the transparency of the tissues. Some of these segmented organs are under muscular control, and can be extended and inflexed. This is true of some of the labial organs, which unfortunately are usually so small as to be difficult to observe. The cephalic setae, however, are larger, being particularly well developed on some marine forms, and in this case observation on living specimens affords evidence of the articulations when they might be overlooked if they were sought by other methods; for if a seta is obstructed it takes on the attitude natural to an organ composed of flexible joints and more or less inflexible segments, as shown in the upper illustration, Fig. 2. Here again, once having established the fact and learned how to make the observations, it proves

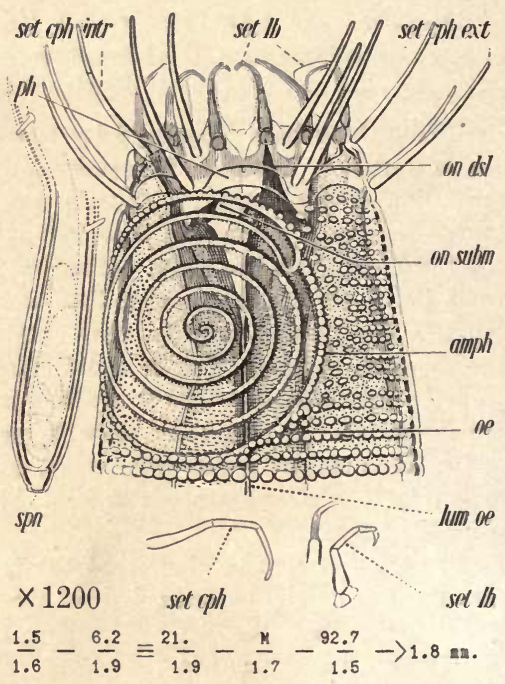

Fig. 3. Pomponema mirabile n.g., n.sp., a nems with jointed cephalic organs, and resembling Cyatholaimus, but with 3 onchi, more complicated amphids, long. jointed, labial palps, wings as in Spilophor,, spicula as in Cyatholaimus, with 20 chromadoroid, male supplementary organs. Characters of $P$. mirabile, type species, given in the illustrations and formula.

that the setae of a wide range of genera are jointed, though the number of segments is often reduced to only one or two. 
One recalls that a number of observers have noted the presence of longitudinal series of repetitive organs in the lateral fields of nem-

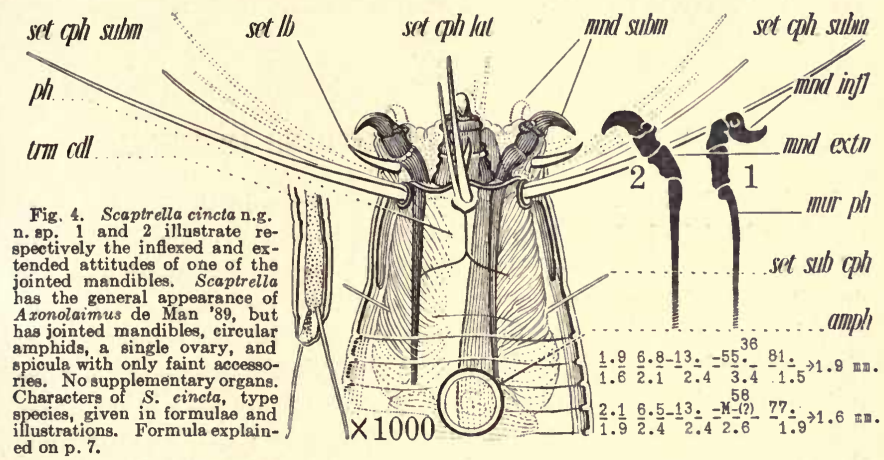

atodes, though attention has never been called to the fact that these organs on opposite sides of the body may be symmetrical to each other. Sometimes they are exactly so.

How is the prominent trilateral symmetry of the nematode head to be expressed in terms of bilateral symmetry? Selachinema and Cheironchus assist in answering this question. A second species of Selachinema, not yet described, has little or no trace of the vestigial dorsal jaw present in $S$. vorax (p. 113), so that the two projecting mandibles become practically lateral, and are bilaterally symmetrical. An even more complete transformation occurs in Cheironchus (Fig. 5), where by a complete disappearance of the dorsal sector of the pharynx the submedian ones have become truly lateral. In both these genera the resulting transformation to a two-jawed animal gives rise to symmetrical mandibles, acting from side to side.

It was, of course, conceivable that any such transformation might take the form of a union of the submedian

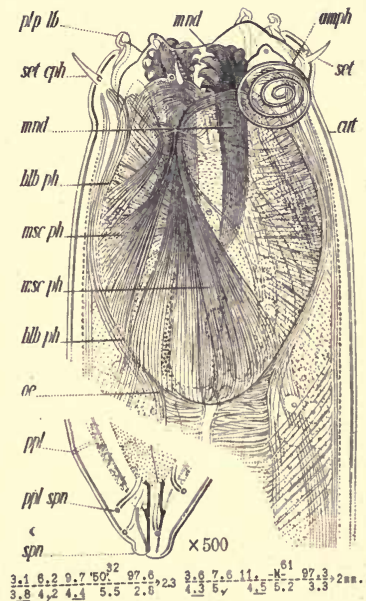

Fig. 5. Cheironchus vorax n.g., n.sp., a nems whose bilaterally symmetrical mandibles have arisen from the ventrally submedian sectors of the head at the same time that the dorsal elements of the pharynx have disappeared. Cheironchus has the general appearance of Selachinema but has no vestigial dorsal jaw, and presents both pharyngeal and cardiac bulbs. Characters of $\boldsymbol{C}$. vorax, type species, given in the illustrations and formulae. 
sectors, these united elements then acting in opposition to the dorsal sector, the two jaws thus developed becoming one dorsal and one ventral. There is no evidence of such a transformation.

\section{INTRA VITAM COLOR REACTIONS IN NEMAS}

We have slowly come to have great confidence in the specificity of certain physiological actions. The known cases of specificity are relatively few, and have been discovered largely by accident. We do not know the exact nature or cause of this specificity. We introduce into an organism certain substances, and definite results follow; about the only thing we know in the matter is that these definite results follow with certainty. What the reactions are that bring about the result we do not know. Our ignorance is so great that even our theories are very vague. In such cases, if only we could see what it is that happens while it is happening, it seems certain that important advances would be made in our knowledge of nutrition, growth, and decay,- of physiology, pathology and medicine.

If substances giving color reactions in living tissues could be applied to small, transparent, varied and highly complex living organisms, under circumstances that would permit microscopic examination while the reactions are in progress, we might hope for more light on this exceedingly important subject. Experiments I have made lead to the belief that many of the conditions requisite for success in this line of investigation can be much better realized than hitherto by feeding colored substances, notably coal-tar dyes, to free-living nematodes.

These minute, transparent animals are comparatively highly organized; not only this, but also extremely varied in their habitats and mode of life. Some are exclusively vegetarian, others exclusively carnivorous, and others omnivorous. They constitute a group composed probably of hundreds of thousands of species, embodying an almost inconceivable number of kinds of physiological action. Their organs are enclosed in a thin transparent cuticle, and are strung out so as to make them unusually suitable for intra vitam examination. Under slight pressure the nema flattens out more or less without losing its vitality sufficiently to preclude satisfactory intra vitam examination under the highest powers of the microscope. 
Observing certain precautions, I find that a great variety of coal-tar compounds and other colored compounds can be fed to nemas, apparently without interfering materially with their normal metabolism. Some nemas are resistant to chemicals that to most organisms are poisonous. Often I have had the best results by cumulative action, using small quantities of color dissolved in the medium in which the nema lived, and allowing the dye to act for days or weeks.

Not infrequently the dyes prove to be highly specific in their action. Only certain cells, or only definite parts of certain cells, exhibit visible reactions in the form of colorations. The results obtained by the use of any given dye may be quite varied. It is evident in many cases that the dye is digested and assimilated, thereby undergoing molecular changes by which it is converted into new compounds in a manner analogous to the processes exemplified in chemical laboratories devoted to the production of aniline dyes. Thus, a dye may give rise to several different colors, none of them like that of the dye itself, and all of them very likely due to new compounds. I have seen considerable evidence pointing to the conclusion that in some cases the dye fed is converted into colorless compounds during the process of digestion (a reduction phenomenon?), and these colorless compounds re-converted into colored substances after they arrive at certain destinations or conditions. The number of changes these "living laboratories" can ring on the molecular structure of a given dye must in some cases be very considerable. Two or more dyes fed simultaneously sometimes produce results more or less independent of each other. The spectacles are very brilliant.

Using these methods I have been able to demonstrate within the confines of a single cell the existence of an unsuspected number of kinds of "granules," manifestly playing different rôles. After the differences among these bodies have been shown in this way, it is sometimes possible to perceive corresponding morphological differences; but without the aid of the color reactions the differences would never have been suspected.

The main thing to bear in mind is that on the basis of our present more complete knowledge of the chemical and physical properties of coal-tar compounds these color reactions in living nemas may be made the index of physiological characters possessed by the cells and their components. In view of the great variety of the known coal-tar derivatives, and the wonderfully varied metabolism of the free-living nemas, it seems to me a very reasonable hope that researches directed 
along this line will lead to important results, and that the nemas may become classical objects in cell and general physiology, as they have already become in sex physiology.

A new and rather extensive nomenclature will become necessary. It will be needful to distinguish between the results of intra vitam, intra mortem and post mortem staining; for these three terms, the last two new in this connection, represent as many different phases in the reactions that take place during the course of the experiments. As the cells lose vitality, new color reactions occur, and the death of the cell is followed by further equally marked changes in the reactions.

The cell elements I have mentioned vary in size, but most of them

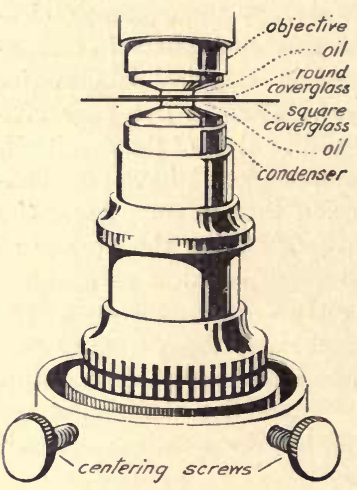

Fig. 6. Diagram of a pair cf objectives, the lower used as a condenser for the upper. are exceedingly small, many so small that they are on the limits of visibility using the very best instruments with the greatest skill and under the most favorable conditions. On the other hand, some of them are large enough so that they can be examined in considerable detail and their structures made out. Among them are the bodies currently referred to under the name mitochondria, and other more or less synonymous words.

As it will be some time before we can establish a rational nomenclature for these numerous intracellular structures it is desirable meanwhile to adopt terms that will permit intelligent discussion of our discoveries as they are made. While the principles underlying such a nomenclature are easily defined, it is by no means easy, in the present condition of things, to suggest suitable short and expressive loots to be used as a basis. In the long run there'll be less confusion if meanings of the terms first employed relate to form, size, and position rather than function.

Investigations of this character are not unlikely to stimulate further research in connection with aniline derivatives. Present efforts are directed toward the discovery of dyes of greater or less permanency. Permanency, however, is of little moment in these investigations; what is of moment is the chemical and physical nature of the dyes. No doubt dyes of a greater range of composition can be produced if 
permanency be disregarded. Furthermore, as already hinted, colorless compounds may be used if in the course of the metabolism they are converted into colored compounds. The results of recent studies of dyes as chemical indicators come into play, and give valuable evidence in determining acidity and alkalinity.

I am almost ready to express the opinion that a small army of investigators should be engaged on the problems opened up in this way. The equipment needed by the investigator is as follows: (1) He must be a good microscopist, versed in physiology, cytology, and histology. (2) He should be conversant with the chemistry of the coal-tar compounds, not so much from the viewpoint of the maker of dyes as from that of the broad-minded chemist, freed from the economic domination of the dye industry, for, as before remarked, fugitive dyes, and even colorless compounds, are possible factors in such investigations as are here under discussion. edge of nemas.

(3) He should have a working knowl-

\section{ILLUMINATION}

In order to distinguish with accuracy among intra vitam color reactions it is necessary to be very particular about illumination. The most perfectly corrected lenses must be used, both as condenser and objective, and the light used must be as nearly white as possible. The best source of light known to me for these researches is bright sunlight reflected from a plane, matte, white reflector. The reflector should be several feet across, and placed at a distance from the microscope several times its own diameter. It should be universally adjustable, so that it can be set to reflect a maximum of light to the mirror of the microscope,-all the better if it is heliostatic. A good surface for the screen is made by whitewashing a rather finely woven cotton cloth.

The best optical arrangement I have tried is the use of one apochromat objective as a condenser for another apochromat. I have been using with success a $2 \mathrm{~mm}$. apochromat as a condenser for a 2 $\mathrm{mm}$. or $1.5 \mathrm{~mm}$. apochromat ob-

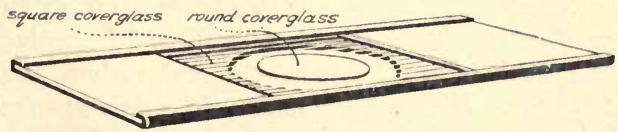

Fig. 7. Microscope slide used for intra vitam examinations as indicated in Fig. 6 .

jective. These precautions are necessary if fine color distinctions are to be made with the greatest possible accuracy. If these precautions 
are taken, it will be found that fine distinctions can be made with such precision as to dispel all doubt as to the existence, side by side, in the same cell, of bodies of quite different character that it otherwise would be either impossible or exceedingly difficult to distinguish from each other.

The use of an ordinary apochromatic objective as a condenser necessitates the use of a special object slide, consisting essentially of a carrier, and two cover glasses. The object is mounted between the cover glasses. Such a slide is shown in the accompanying illustration. The substage of the microscope should have a centering arrangement and a rack and pinion, or screw focusing adjustment. A little experience with an apparatus of this sort, in which all known precautions are taken to remove color from the optical system leads one to distrust the ordinary Abbé substage condenser when fine distinctions are to be made between colors in the microscopic object, especially if the colors are of similar character.

\section{NEMATODE POPULATION OF BEACH SAND}

Through the courtesy of the U. S. Bureau of Fisheries I was able to make quantitative observations during the summer of 1916 on the nemas of ordinary beach sand, between tide-marks, at Woods Hole, Buzzard's Bay, U. S. A. Nemas were plentiful; it was calculated that on one beach in the top 3 inches of sand there were at least 527 millions per acre. On another beach there were at least 1040 millions in the topmost inch of sand. The nemas varied from a fraction of a millimeter to 10 millimeters in length, averaging 2 to 3 millimeters. On muddy shores where organic matter is more abundant, the nematode population is much more dense,- thousands of millions per acre.

Many of these nemas were strictly vegetarian, and fed on microscopic plants present in the beach sand,--plants both green and colorless. Experiment showed that a considerable amount of light penetrates average beach sand to a depth of $\frac{3}{4}$ inch, thus rendering possible the growth of green protophytes and various algae at slight depths in the sand. Some of the nemas were strictly carnivorous, feeding on protozoa and other small animals.

On trial it proved that the top layers of sand, between high and low tides, under ordinary circumstances, afford the nemas a habitat of considerable stability, since the shifting of these layers during the 
rise and fall of the tides is so little as to interfere in no way with the life activities of the nemas. On open ocean beaches, where the force of the breakers is greatest the nemas take on forms and acquire habits that protect them from destruction,- - a tougher cuticle, and the habits of burrowing and of coiling themselves into a "ball."

Beach nemas in their turn are devoured by the larger animals dwelling in and on the sand, and thus form one of the links in a chain from the most minute forms of life to those of largest size.

Beach nemas lead a very active life, winding in and out among the grains of sand as do snakes in a pile of stones. The earth's hundreds of thousands of miles of beach sand, far from being barren, must be reckoned as a productive area of some little importance.

4

\section{LOCATIONAL TERMS FOR THE CYTOLOGY OF DESCENT}

There is no satisfactory locational terminology connected with parthenogenesis, hermaphroditism and bisexuality; in other words, with the space relationships of the reproductive cells and their essential elements, - a terminology enabling us to answer succinctly such questions as, "How are these cells and their elements located with reference to each other?" Such nomenclature as we have for this purpose has accumulated, bit by bit, through successive contributions of more or less independent investigators, and, naturally enough, has become a very heterogeneous mixture of terms and phrases.

Aside from standing open to the criticism of being inadequate and an incongruous mixture, such terms as are in current use, at least a considerable portion of them, date from a time when our knowledge of the chromosomes and their relationships to each other and to heredity was either non-existent or much less complete than at present. Most of these terms, therefore, are based on the assumption that the body or soma is the principal or predominant feature of the organism, and, philologically speaking, they take little or no account of the modern view of the importance of the gametes and of the rôle they play.

As we seem in need of a more adequate and homogeneous set of terms based on the relationship to each other in space of the gonads, the gametes, and the chromosomes and other intra-cellular elements, I call attention to the following series of terms, positional rather than physiological, I have been using to meet this need: Just as we have "cone" and "conic" evolved from the Greek kovos, I derive the 
words "gone" and gonic" from rovos. Primarily the word "gone" refers to the generative portion of a sexual organ. By metonomy "gone" designates an organism or species having gones; thus we have two kinds of organisms- "gones" and "agones."

From "gone" come the verb "to gone," and the substantives "syngone," "digone," "amphigone," "homogone," and "heterogone." From syngone come the words "syngonic," syngonically," and "syngony;" and corresponding words from digone, amphigone, homogone and heterogone.

Gone. To produce gones.

Gonic. Of or relating to a gone.

Syngonic. Having macro-("female") and micro-("male") gametes in the same gone; e.g. as in many nemas.

Digonic. Having macro-("female") and micro-("male") gametes in separate gones in the same individual; e.g. as in many hermaphrodites.

Amphigonic. Having macro-("female") and micro-("male") gametes in separate gones that are in separate individuals; e.g. as in all bisexual forms.

Homogonic. Having gones all of the same kind.

Heterogonic. Having gones of various kinds; e.g. as in a species presenting both syngony and amphigony.

Syngone. A gone bearing both macro-("female") and micro-("male") gametes. By synecdoche syngone also designates an organism or species containing, or characterized by, syngones; similarly with the following four terms.

Digone. A digonic individual or species.

Amphigone. An amphigonic species.

Heterogone. A species presenting both amphigony and syngony, or both digony and amphigony, etc. A heterogonic species.

Homogone. A species or individual presenting uniformity in the space relationships of its gonic cells. A homogonic species.

Kinetogone. A gone whose gametes are active, aggressive, or "male."

Statogone. A gone whose gametes are passive or "female."

Entering now a more or less theoretical domain whose permanency will depend on the results of future investigations, and carrying the analysis a step farther by taking into consideration the space relationships of the chromosomes and other intra-cellular elements that according to an increasingly prevalent modern belief themselves carry, or determine the factors of descent, and using the Greek word kvoros as a basis, I derive, as may be necessary, "syncyst," "amphicyst," 'heterocyst," etc., and their adjective, adverbial, and substantive derivatives ( e.g., syncystic, syncystically, syncysty) to aid in expressing positional relationships. Thus the word "syncyst" refers to a gonic cell, such as a parthenogenetic ovum, containing all the elements, 
factors, or determinants necessary to the production of an individual (except, of course, the environmental ones), of which the chromosomes are the familiar example, expressed in some physical form, often, though not necessarily, of definite conformation. It is assumed that these factors belong to two classes, having opposite, vital, chemical, or physical properties. By synecdoche the word syncyst may also designate a gone, individual or species containing, or characterized by syncysts; similarly throughout the series of terms. In dicysty the inheritance mechanism is such that the full complement of factors necessary to the production of an individual is distributed to functional cells of two kinds, the macro-("female") and micro-("male") gametes, neither normally capable of producing an individual, but which, pairing, form zygotes capable of producing an individual.

Syncystic. Of, or relating to, a gonic cell containing all the factors necessary to the production of an individual, - as in the parthenogenetic ovum. The word syncystic may also be applied to a gone, individual, or species bearing syncysts. Syncysty,-state of being syncystic; and so with the following five words.

Dicystic. Of, or relating to, a gone bearing in separate cells the different factors necessary for the production of an individual; as in syngones. The word dicystic may also be applied to individuals and species.

Amphicystic. Of, or relating to, an individual in which the different groups of factors necessary for the production of a new individual occur in separate cells that are in separate gones; as in many hermaphrodites.

Telecystic. Of, or relating to, amphicystic species in which the different groups of factors necessary for the production of an individual are borne in separate cells in separate gones that are in separate somas or individuals; as in all bisexual forms.

Homocystic. Having or producing gonic cells all of the same kind.

Heterocystic. Having or producing gonic cells of more than one kind.

Syncyst. A gonic cell, or by synecdoche, a gone, individual, or species, presenting syncysty.

Dicyst. A gone, or by synecdoche, an individual or species, presenting dicysty.

Amphicyst. An individual or species presenting amphicysty.

Telecyst. A species presenting telecysty.

Heterocyst. An individual or species presenting both amphicysty and syncysty. or both dicysty and amphicysty, etc.

Homocyst. A species presenting uniformity in the space relationships of its different kinds of gonic intracellular reproductive elements.

Kinetocyst. A gonic cell whose elements are active, aggressive, or "male." A "male" gamete; a spermatozoon.

Statocyst. A gonic cell whose elements are passive, or "female." A "female" gamete; an ovum. 
Digones, amphigones and heterogones, and many syngones, are dicystic. The word syncysty and its immediate relatives may be used to designate the conditions present in parthenogenetic organisms, in which single gonic cells (not zygotes, at least not in the ordinary sense of the word) contain all the factors necessary to the production of an individual. My (thus far theoretical) cryptogenetic organisms are syncysts.

\section{FUNCTIONS OF THE AMPHIDS}

My published observations emphasize the junction of each amphid, by means of a duct, with a chain of internal lateral organs. Bütschli and de Man each record an instance of definite outflow from the amphids. I now find this outflow in many different genera, when specimens are fixed with Flemming solution; from a definite part of each amphid there issues a coiled, or irregular "string" or "ribbon," the volume of -which precludes attributing it to an evagination. Occasionally an "axis" is seen in the "string," but nothing warrants the belief that this "axis" is other than a coagulation phenomenon, just as it is in the similar coagulation "strings" occasionally seen at the spinneret. I mention evagination because some investigators declare the amphids to be supplied with special nerves, and because it is conceivable that death spasms might so act on a nerve organ as to cause an evagination. However, my numerous observations do not at all support the idea that the appearances I have studied are evaginations. In many cases I have traced inward and backward from the amphids structures whose histology in no way suggests nerve organs, but does correspond in every respect with the histology of ducts, especially those of nemas.

These new observations of mine afford, I think, a better basis for speculation as to the function of the amphids, and lead away from the idea that they are simply organs of sensation. I observe in Mononchus, and other genera, that invariably there is an innervated papilla very close to the amphid. May not confusion have arisen here through different observers having studied similar-looking, but unrelated structures?

I have instances of amphids so obscure that it would have been impossible to discover them had it not been for the issuance from them during fixation of the fluid matter described. 


\title{
THE MONONCHS
}

\author{
(Mononchus Bastian 1866)
}

\section{A GENUS OF FREE-LIVING PREDATORY NEMATODES}

\author{
CONTRIBUTIONS TO A SCIENCE OF NEMATOLOGY VI
}

(With 75 illustrations in the text) ${ }^{2}$

By

\author{
N. A. Совв
}

\section{INTRODUCTION}

\section{Mononchs are Predaceous}

No free-living nematodes have gained more in interest during recent years than those constituting the genus Mononchus; this is because the latest discoveries have led to a complete change of view concerning their economic relationships. Careful examination, here recorded, of a large number of specimens belonging to many different species of Mononchus, has fully demonstrated the predaceous character of certain common and widely spread soil-inhabiting species-which are found to feed on other small animal organisms, such as protozoa and rotifers, and, most interest-

\footnotetext{
1 Reprinted from Soil Science, May, 1917, with renumbering of pages and errata, (p. 184).

2 For the most part the illustrations were prepared under the author's personal supervision by Mr. W. E. Chambers of the Bureau of Plant Industry. Many features set forth in them cannot be seen in the natural object except with the aid of the best immersion lenses skillfully used under favorable conditions. Frequently no further allusion is made to facts thus elucidated. So, too, information contained in the key may not be repeated elsewhere; the key should therefore be consulted in connection with each description. Most of the illustrations are original; when not so, their source is indicated. The species figures are all at the same magnification, so that the
} reader may judge of the relative sizes of the nemas. Explanatory abbreviations follow.

amp-ampulla
amph-amphid
an-anus
an gl-anal gland
ar dnt-denticular area or rasp
cav som-body cavity
cl int-intestinal cell
cl msc-muscle cell
cl nrv-nerve cell
cl nrv subm-submedian nerve
col crd-cardiac collum
crd-cardia
cst ph-pharyngeal rib
cut-cuticle
dct-duct
dct gl cdl-duct of one of the
dnt-denticules
flx ovr post-flexure of poster-
gl-gland
gl an-anal gland
gl cdl-caudal gland
gl oe-asophageal gland
grn-granule
gl sal-salivary gland
ing-ingested material
ing nematod - ingested nema-
int-intestine
inc-iunction
lam lh-lahial lamina

lb-lips

lum int-lumen of intestine

lum oe-lumen of cesophagus

lum som-body cavity

ms an-anal muscle

msc oe-cesophageal muscle

msc som-body muscle

mur ex-external wall

mur int-intestinal wall

mur ph-pharyngeal wall

mur ut-wall of uterus

ncl-nucleus

$\mathrm{ncl} \mathrm{cl}$ int - nucleus of intesti. nal cell

ncl lat-lateral nucleus

ncl ov-nucleus of ovum

nrv-nerve

nrv $r$-nerver ring

oe-csophagus

oes lum-asophageal lumen

on ds!-dorsal tooth

on $\mathrm{rtr}$ dsl-retrorse dorsal tooth

on $\mathrm{rtr}$ subm-retrorse submed. ian tooth

on subm dxt-right submedian tooth

on subm snst-left submedian tooth

org-organ

ov-ovum

ov det-oviduct

ov frt-fertilized egg

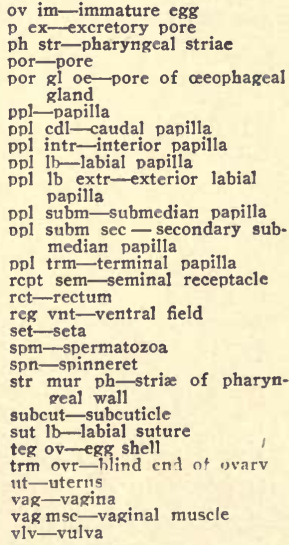

ov im-immature egg

$p$ ex-excretory pore

ph str-pharyngeal striae por-pore

por gl oe-pore of ceophageal gland ppl-papilla

ppl cdl-caudal papilla

$\mathrm{ppl}$ intr-interior papilla

Dpl lb-labial papilla

ppl lb extr-exterior labial papilla

ppl subm-submedian papilla

opl subm sec - secondary sub. median papilla

ppl trm-terminal papilla

rcpt sem-seminal receptacle

rct-rectum

reg vnt-ventral field

set-seta

spm-spermatozoa

spn-spinneret

str mur ph-stria of pharynreal wall

subcut-subcuticle

sut lb-labial suture

teg ov-egg shell

trm ovr-hlind cnt of ovarv

ut-uterns

vag - vagina

vag msc-vaginal muscle

vlv-vulva

(S. S. 431) 129 . 
ing of all, on other nemas-and has made it practically certain that all mononchs are predaceous.

Economic Importance. If, as is often the case, the nemas destroyed by the mononchs are nemas injurious to agriculture, then the mononchs are beneficial to man. The first definite instance of this kind was reported in the Journal of Agricultural Research in September, 1914: Mononchus papillatus was shown to feed upon Tylenchulus semipenetrans, the latter a nema infesting the roots of citrus trees. Since that time the writer has observed many similar instances, fourteen of which are recorded herein.

Formerly mononchs were considered harmful to vegetation. The basis of this opinion was twofold: first, they were known to congregate about the roots and between the leaf sheaths of plants, especially succulent plants, in sufficient number to justify the opinion that they would be harmful, provided they were vegetarians; second, vegetable matter was often found in their intestines.

More careful investigation, however, has disclosed other facts incompatible with this opinion that mononchs are harmful to vegetation. The food-habits of mononchs have now been more carefully investigated, as herein recorded, and each species so studied has proved to be carnivorous. The presence of vegetable matter in a mononch's intestine proves to be no very definite criterion of its food habits, appearances to the contrary not-

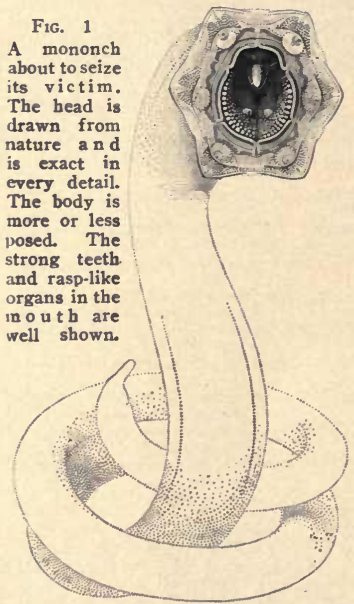
withstanding. A deception has arisen that is now easily explained. The occurrence of vegetable organisms in the intestine of a mononch is merely incidental; in many instances it would be impossible for the mononch to devour its prey without at the same time swallowing vegetable matter. For instance, suppose a mononch to bolt, as one of its victims, a monhystera - a common occurrence. Monhysteras are vegetarians, some of them feeding almost exclusively upon diatoms, others upon other kinds of unicellular algx. Both on account of their peculiar structure and their bright green color, these diatoms and other algæ are very conspicuous objects in the intestine of the monhystera. Imagine now the appearances presented by the body of an ingested monhystera, swallowed whole, or nearly so, and lying lengthwise in the intestine of a mononch. Under such circumstances, which are entirely normal, the diatoms and other algæ in the intestine of the monhystera may be observed through the colorless tissues 
of both the mononch and the monhystera, without the body wall of either nema being very plainly visible: it is somewhat as if one looked through the walls of two glass tubes, one within the other, to view comparatively conspicuous objects in the inner tube. Such an observation might easily lead to the conclusion that the mononch had been feeding upon unicellular algæ. The deception is heightened after the body of the monhystera is partially digested, for then all vestiges of the monhystera become practically invisible, and there are left as comparatively conspicuous remains the less readily digested vegetable matter, lying loose in the intestine of the mononch, - the frustules of diatoms for instance, which of course are wholly indigestible. Taking everything into consideration, it is not in the least surprising that hitherto observers have been deceived as to the food habits of mononchs.

As nematologists heretofore have not carefully studied the food habits of mononchs, particular attention was given them in the course of these investigations; but in spite of the care exercised much remains to be discovered. The mouth parts and digestive organs have come in for special study, since it is mainly here that we get clues, faint and puzzling though they be, that help interpret the little we can glimpse of the food habits of these organisms in their natural state. Soil mononchs live in darkness and, as might be expected, behave in a wholly unnatural way when placed in water on a microscope slide and brought in a drowning condition into the blaze of light necessary to a microscopic examination. The difficulties and delays attendant on such a line of research are manifest. Nevertheless, certain fundamental facts have been established; new organs have been discovered and light has been thrown on the true nature and function of organs not hitherto understood. It has seemed best to dwell on the various habits and functions while describing the structures with which they are associated.

As we gain familiarity with the food habits of nemas, it becomes possible on this new basis to make comparative anatomical studies, the results of which may be applied in determining the food habits of newly discovered genera and species.

\section{Structure, Functions, and Distribution of Mononchs}

The mononchs constitute a genus of free-living, predatory nematodes inhabiting soil and fresh water, as well as the above-ground parts of certain plants. The average form and size of a mononch is indicated by the following average formula:

$$
\begin{array}{ccccc}
2.2 & 7.4 & 25 . & 63 \text { '28 } & 92.7 \\
\hline 2.1 & 2.6 & 3 . & 3.2 & 2 .
\end{array} 2.4 \mathrm{~mm} \text {. }
$$

- This formula, which is now coming into more general use, is simply a decimal method of stating concisely the necessary measurements. The formula is fully explained in the appendix, p. 184. 
which corresponds very closely with that of the type species of the

II $\mathrm{PP}^{\prime}$

at st

ph. . .

aner $p$

$M x \times C$.

lint of

d nor suba

d aro ot

not of .

exp?

ex det?

d no soth.

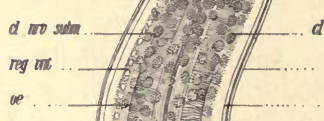

ax

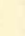

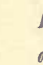
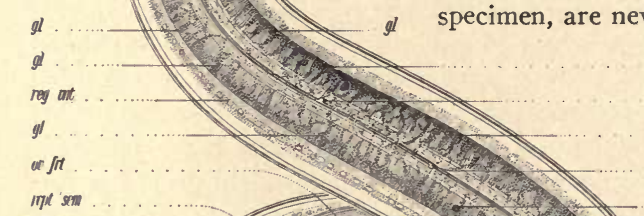

res

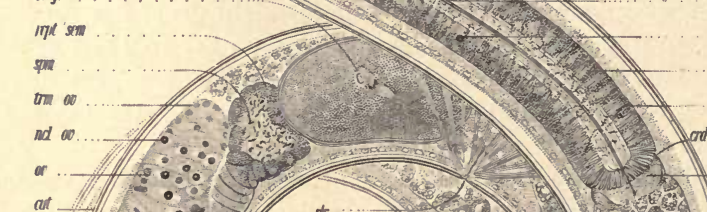


resolve the striæ into components; occasionally, however, they are resolvable into rows of excessively fine dot-like elements, as for instance in bathybius, reversus and incurvus. Lateral wings to the cuticle are almost unknown, and the striæ are not perceptibly altered on the lateral fields; papillatus and macrostoma furnish a slight exception to this rule.

Though the muscles of the body wall are relatively powerful, their attachments to the subcuticle are not a conspicuous feature; hence longitudinal striæ due to this cause, though of rather common occurrence, are not very readily observed. There are no longitudinal striations in the cuticle proper. This apparent complete absence of dermal markings often imparts a glassy appearance to living mononchs when examined casually under the microscope, an effect that is heightened by the fact that the colorless body wall is, as a rule, of more than average thickness. Despite their rather thick cuticle, mononchs are very flexible. They coil and uncoil themselves with facility,-a useful gift in a struggle with active prey.

Moulting. From researches on a considerable variety of genera, Maupas concluded that nematodes undergo four moults, and that, correspondingly, the life of each individual is divided into five periods. He examined no mononchs. The accompanying sketch of a portion of the pharynx of a moulting Monochus brachyuris, from Arlington, is therefore of interest, as indicating that this specimen had yet to moult three times; and since it was already half-grown $(0.8 \mathrm{~mm}$. long) it is fair to presume it had already moulted once, although of course there is no proof of this. The sketch shows four successive dorsal onchi, numbered in chronological order, each the representative of a separate cuticle. The fourth onchus is in a very rudimentary state. This record accords with the observa-

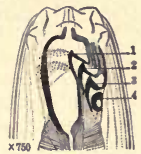

Fic. 3.- - Head of a moulting mon on c h, showing suc. cessive dorsal teeth, $1,2,3,4$. tions of Maupas, and indicates the probability that mononchs also moult four times.

Strength and Activity. Various features of the cuticle and bodymuscles seem to stamp the mononchs as powerful nemas, and when they are taken from their natural haunts and placed in a glass of water for examination, their active serpentine movements fully justify the inference. Though less agile than species belonging to some other genera, they are anything but sluggish. The movements of the anterior extremity are especially striking, a certain suppleness of neck enabling them to dart the head suddenly here or there in almost any direction,- - a faculty enabling them with ease to capture their prey, even though it be active.

\section{Nemas Sometimes Float}

Distribution by flotation. Ditlevsen notes a very interesting trait of his Mononchus spectabilis, namely, that of floating on the surface of water. A number of free-living nemas belonging to other genera are 
known to have this faculty, which is due to repulsion between the cuticle and water; de Man found it to exist in the case of Oncholaimus viscosus, and the writer has observed it in the case of the larvæ of Diplogaster aerivora, although a second larval form of this same species does not exhibit the property, nor do the adults. Ability to float is known also of a number of other species, and, as Ditlevsen's observations show, is not absent in the genus Mononchus. Whether flotation plays any part in the economy of such nemas is not known with certainty. Floating on the surface of moving water, nemas would doubtless sometimes be quickly and widely distributed, and it is therefore easily conceivable that flotation has some definite bearing on the life history of species exhibiting it.

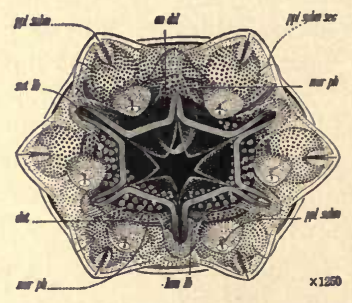

Fig. 4.-Front view of the head of M. tenuis. Compare with Fig. 1. Here the lips are nearly closed, but the dorsal tooth and rasps can be seen through the six labial flaps. The two circlets of papillæ are seen to good advantage. The large dorsal tooth shows faintly through the two upper lips.

The surface of a mononch seems almost entirely destitute of setæ and papillæ, if we except those on the lips. Nevertheless, future researches will probably reveal superficial nerve-endings hitherto overlooked. In rare instances a few fairly well-developed setæ occur on the tail, as in the case of $M$. sigmaturus; papillæ also occur sparingly on the tails of the males of various species, and near the vulva on the females of a number of species. No pores are known to occur in the cuticle, except, of course, the spinneret pore, and the pore observed near the nerve-ring and supposed to be the renette pore.

\section{General Form of Body}

Neck and Head. The various mononchs are considerably alike in external form. In front the body tapers but little; the neck is nearly always more or less cylindroid, and almost invariably ends in a head not set off from the neck in any way, though there is usually a slight expansion at the lip-region, due to the strong development of the labial papillæ.

Tail and Spinneret. The posterior portion of the body usually tapers from some distance in front of the anus, but in the pre-anal region the diminution is slight and very gradual. The tail may be either simply conoid, or first conoid and then cylindroid, and usually ends in a spinneret, though in about one-fourth of the species the caudal glands and spinneret are absent. When the tail is conoid the spinneret is usually a simple structure, whose existence is indicated mainly by the fact that the terminus is sub-truncate in form, and presents an inconspicuous axial, or sub-dorsal, or sub-ventral pore. This form of spinneret appears to be entirely unarmed, though inconspicuous innervations probably occur. 
When the posterior portion of the tail is cylindroid, the terminus is usually almost imperceptibly expanded, and the spinneret is then somewhat differently developed, and is usually armed with obscure, innervated, submedian papillæ or setæ.

\section{Head}

Lips. In the view usually obtained the six lips appear to be more or less confluent, and if it were not for their papillæ it would be difficult to count them; but when thrown apart, an attitude in which, however, they are seldom seen, they are more easily counted, since in this attitude the refractive "ceratinous" internal elements of the lips are separated

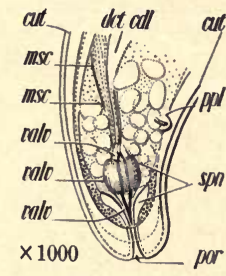

FIG. 5. - Spinneret of $M$. lacustris showing mus. cle, msc., whose contrac. tion opens the spinneret valve. from each other and more readily distinguishable, as shown in figure 1. When closed, these six, broad, flat lips meet together in such a way that the star-shaped mouth opening usually lies in a slightly depressed area on the middle of the front of the head. In some species the inner walls of the lips are strongly "ceratinized," and may be developed into six rather definite, retrorse, subacute points, which, in assaults on other animals, serve as grappling hooks, and act in opposition to the dorsal tooth of the pharynx.

Labial papilla. Each lip is supplied with at least two, usually more or less conical, innervated papillæ; one on the outer margin and somewhat outward pointing, the other situated about half way between the outer papilla and the centre of the head, and forward-pointing. The front of the head therefore presents two circlets of papillæ, one on its outer margin, and one more closely surrounding the mouth-opening, as shown in figures 1 and 4, and many others. These papillæ may be so flat as to play no conspicuous part in the external conformation of the head, or they may be conical and raised, so as to give to the front of the head a more or less angular contour. The papillæ are slightly mobile, and hence vary somewhat in appearance at different times on the same specimen, a matter depending on the attitude of the lips. Drowned specimens with relaxed lips present a slightly different appearance from those that have been fixed for examination by means of chemicals.

Some of the innervations that frequently occur on the head near the lips may have special functions. Structurally they are sometimes indistinguishable from the subordinate labial papillæ. The species figures show the location of some of these innervations. One pair of them is very near the amphids - the only nerves so far observed to be definitely associated with the amphids. These particular papillæ.are so uniform in their-occurrence that it seems likely they have a special function, connected with the use of the amphids. 
Mononchus: Number of Labial Papillæ in the Outer Set

\begin{tabular}{|c|c|c|c|c|c|}
\hline 14 & \multicolumn{2}{|c|}{10} & 6 & \multicolumn{2}{|c|}{ Doubtful: said to be 6} \\
\hline acutus & bathybius & $\begin{array}{c}\text { muscorum var. } \\
\text { macrolaimus }\end{array}$ & brachyuris & dadayi & obtusicaudatus \\
\hline $\begin{array}{l}\text { brachylaimus } \\
\text { dentatus } \\
\text { gracilicaudatus } \\
\text { major } \\
\text { monhystera } \\
\text { muscorum } \\
\text { papillatus } \\
\text { rapax } \\
\text { regius }\end{array}$ & $\begin{array}{l}\text { brevicaudatus } \\
\text { consimilis } \\
\text { denticulatus } \\
\text { gerlachei } \\
\text { incurvis } \\
\text { longicaudatus } \\
\text { longicollis } \\
\text { micrurus }\end{array}$ & $\begin{array}{l}\text { obliquus } \\
\text { radiatus } \\
\text { reversus } \\
\text { sigmaturus } \\
\text { subsimilis } \\
\text { angustus } \\
\text { trichurus } \\
\text { vorax }\end{array}$ & $\begin{array}{l}\text { lacustris } \\
\text { megalaimus } \\
\text { minor } \\
\text { palustris } \\
\text { sparsus } \\
\text { teres } \\
\text { tunbridgensis }\end{array}$ & $\begin{array}{l}\text { digiturus } \\
\text { dolichurus } \\
\text { exilis } \\
\text { fovearum } \\
\text { gymnolaimus } \\
\text { index } \\
\text { intermedius } \\
\text { japonicus } \\
\text { macrostoma }\end{array}$ & $\begin{array}{l}\text { parvus } \\
\text { punctatus } \\
\text { rex } \\
\text { spectabilis } \\
\text { studeri } \\
\text { tenuicaudatus } \\
\text { tridentatus } \\
\text { truncatus } \\
\text { zschokkei }\end{array}$ \\
\hline
\end{tabular}

Comparative morphology of the cephalic papilla. The outer set of cephalic papillæ is the morphological equivalent of the circlet of cephalic setæ often to be seen on other free-living nemas. In harmony with this fact, the submedian papillæ of this outer set are sometimes two or three in number to each lip, the numerical and morphological factors harmonizing with the law of arrangement of the tactile cephalic setæ of nemas, namely:-when six are present one is found on each of the two lateral lines and one on each of the four submedian lines; when more than six are present the increase occurs first on the submedian lines, the commonest number being ten,- - one on each lateral line and two on each of the four submedian lines; when the number is in excess of ten the increase is again more commonly found on the submedian lines. Illustrations of this law of arrangement are seen on the heads of many mononchs. The secondary submedian papillæ of mononchs are sometimes minute and more or less difficult to detect.

\section{Method of Hunting}

Senses of Smell and Taste. Obviously mononchs hunt by the aid of some other sense than that of sight, since both they and their prey usually live in subterranean darkness. It is, therefore, exceedingly likely that the nerve endings on the head, terminating in the labial papillæ of the inner row, serve as organs of taste and smell, and that it is by the aid of these senses that they trail their quarry.

Picture these ferocious little mononchs engaged in a ruthless chase in the midst of stygian darkness. We may imagine them taking up the scent of the various small animals upon which they feed, among which almost anything they can lay mouth to seems not to come amiss, and pursuing them with a relentless zeal that knows no limit but repletion. How many acres have their organic balance determined by their millions of prowling mononchs? 


\section{Pharynx}

The six powerful lips arch together over a large characteristic pharynx, consisting of a rather simple, ellipsoidal or elongated cavity, whose cross-section is more or less irregularly circular, and of which there are two distinct types.

Types of Maw. Teeth. In one type, characteristic of the larger species, there are longitudinal ribs, sometimes simple, sometimes duplex, situated mainly on the median and submedian lines (subgenus Iotonchus). ${ }^{3}$ When these longitudinal ribs are well-developed there is a tendency for the dorsal tooth or onchus to be farther back, and for the submedian teeth, smaller still, to be located toward the base of the pharynx, and in extreme cases all three teeth may be located actually at the base of the pharynx, or indeed be quite vestigial. The farther back the teeth, the smaller they are, and when located actually at the base of the pharynx they are often exceedingly small.

In the other type of pharynx the dorsal

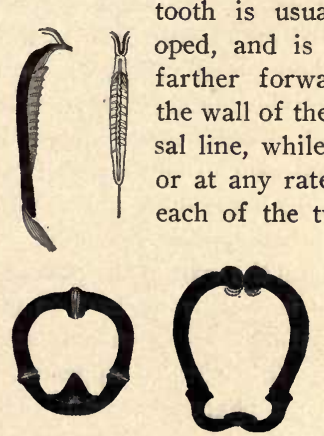

Fig. 7.-Ahove, profile and front view of the same series of denticles in the throat of $M$. muscorum. Below, two cross-sections of the same specimen, showing end view of the same denticles; at left the section is through the dorsal tooth (shown black); at right the section is above the tooth. The denticles are shown in the upper part of these two lower figures. The two joints shown in cross. section impart a slight degree of mohility to the pharyngeal walls. Compare with Fig. 6.

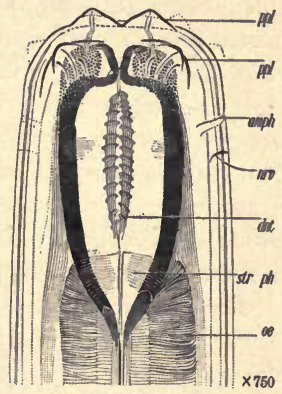

FiG. 6.-Dorsal view of the throat of $M$. muscorum, to show the arrangement of den. ticles (dnt.) characteristic of the subgenus Prionchulus, ; they constitute a rude "saw." Com. pare with Fig. 7. which in some species are two submedian

Iotonchus, insignificant tooth; "Prionchulus, a small saw-tooth; 5 Anatonchus, reversed tooth. 
areas, armed with minute denticles arranged in transverse rows like the teeth of a rasp (subgenus Mylonchulus ${ }^{6}$ ). While these denticles are usually distributed in two distinct areas, the two sometimes coalesce on the ventral line, so that the denticulated area, or rasp, is continuous and bilaterally symmetrical. As a rule, however, there is a distinct, narrow, ventral interruption, so that the right and left rasps are rather clearly separated from each other. These rasps are widest near the ventral line, and become gradually narrower as they recede, and usually end near the lateral line, or near the dorsally submedian line. On the margins of the rasps the denticles are arranged in distinct rows; elsewhere the arrangement is more or less irregular, though in certain species the arrangement is rather orderly throughout. Each rasp is composed of about six rows of denticles, except in the species denticulatus, where the number of rows is about twice (?) as great, and in the new species sparsus and reversus, where there are only one or two rows. Often at the base of each rasp there is an exceedingly minute submedian onchus.

Method of Attack. When used in conjunction with the strong dorsal tooth and the powerful lips, the rasps are remarkably efficient organs. The mononch glides up to its quarry and makes its onslaught by a quick snap of the head, throwing its jaws suddenly wide open, and grappling its prey by means of the inner armature of the lips. As the jaws close in, the victim's body is jammed against the point of the dorsal tooth, as well as against the rasps, and in this way is at once both punctured by the tooth and lacerated or milled by the rasps. In most cases the onchi seem to be solid bodies, containing no duct through which venom could empty. If any poison is injected into the victim it would seem to be derived from œsophageal glands emptying into the pharynx by some other road. The axial element sometimes seen in the onchus is probably a nerve ending.

Minutice of the Wall of the Throat. In general the wall of the pharynx is well developed and strongly refractive, and is usually thickest on the dorsal side where it gives support to the dorsal onchus; often it is very finely transversely striated. In many species this striated appearance seems to be due to a transverse lamination of the pharyngeal wall. The striation, or lamination, may be so pronounced as to give rise to transverse ridges on the interior surface of the pharynx like those of the teeth of a mill-saw file, and no doubt these ridges have somewhat the same function as the rasps just described. The onchi are sometimes seen to be longitudinally striated. The strix of the pharyngeal walls are most clearly visible during the moulting period. In the original species-figures to follow, what appears merely as shading on the pharyngeal wall is a carefully worked-out chart of the position, number and direction of these laminations. 
Contour of the Maw. The contour of the pharynx differs somewhat in the various species. If the dorsal onchus is strongly developed, the profile indicates a roughly goblet-shaped or ellipsoidal cavity, and the onchus extends well into the cavity so that its apex lies near the axis of the head, sometimes up front and close to the inner margins of the lips. If, on the other hand, the dorsal onchus is weak; and especially in species

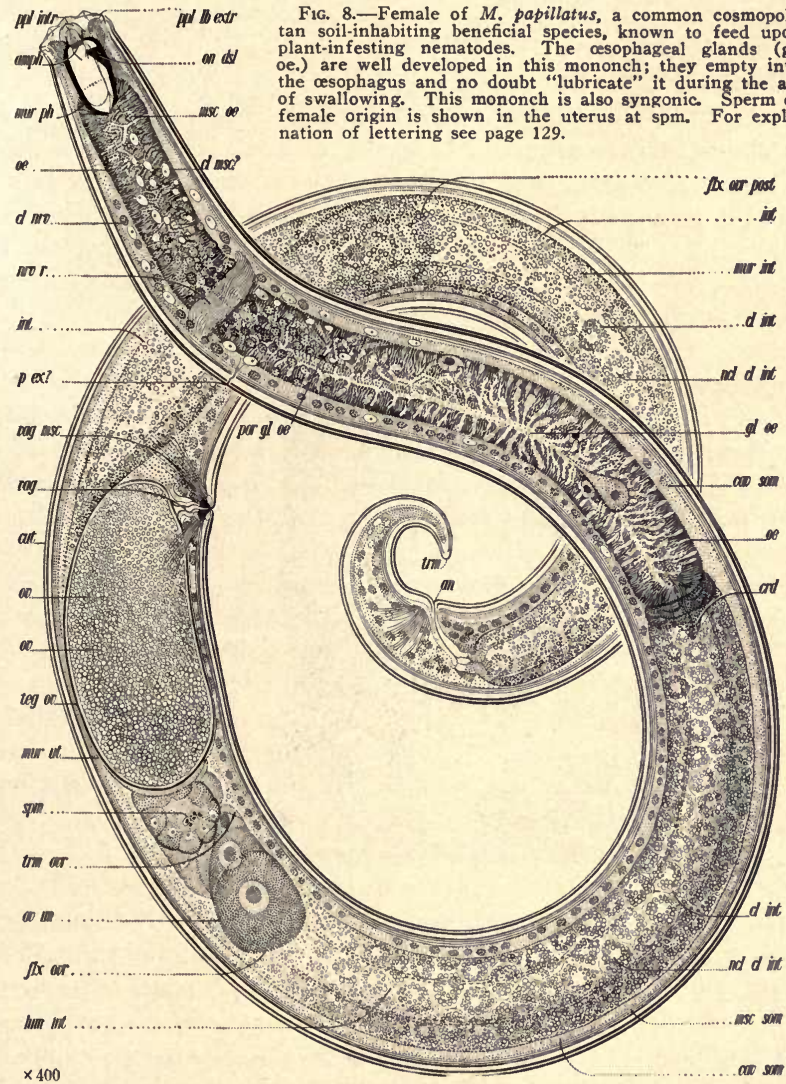

where the wall is not much reinforced by longitudinal ribs, the contour of the pharynx tends to be more or less oblong, so that its floor, lying in the anterior end of the œsophagus, though irregular, is likely to be, on the whole, more nearly flat. Opposite the dorsal onchus there is frequently to be seen a faint transverse seam, most prominent along the ventral side 
of the pharynx, indicating a division of the wall into anterior and posterior elements. Sometimes this pharyngeal suture is rather prominent.

Jaw Muscles. The pharyngeal muscles of various species of mononchs have been observed, figured and commented upon by one or two investigators, but only in a vague way. Much remains to be learned concerning them. The following incomplete observations have been made in the course of these investigations.

Under suitable conditions, and especially in some species, long, slender muscles may be seen passing from the lip region backward and outward, so that their proximal attachment is to the body-wall a short distance behind the pharynx. These muscles are the flexor muscles of

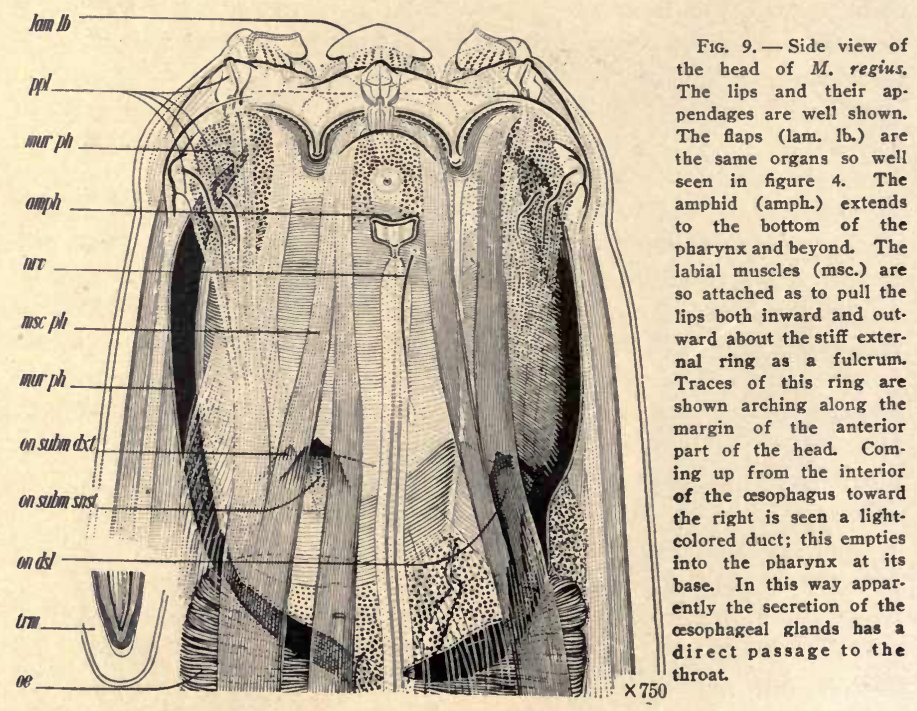

the lips. It is certain that each lip, probably each pair of adjacent lips, is connected with a muscle whose function is to pull the lip toward the body axis about a fulcrum near the surface of the head. These muscles, then, shut the mouth. It seems likely that the elasticity of the various parts composing the frame-work of the lips and pharynx may play some part in mastication and deglutition, and act in opposition to some of the muscular elements of the pharynx. The extent to which a mononch can throw its jaws open is shown in the sketch, figure 1, made from a preserved specimen. This act is accomplished by the aid of a second similar set of muscles acting on the outside of the same fulcra. It is unlikely 
that the sketch represents the maximum gape, for the effect of chemical fixatives seems to be to lock the jaws tightly rather than leave them agape. When examined alive mononchs are seldom seen to move their mouth parts.

\section{The Mononch and Its Victim}

Fate of Victim. So far as I am aware, the struggle of a mononch with its victim has never been witnessed by human eyes. A struggle it must be, for mononchs are sometimes discovered that have gulped down other nemas nearly half as long as themselves. Such a dénoument must be the result of a dramatic conflict. A similar gastronomic exploit on the part of a man would be the gulping down of a string of bologna sausage several feet in length. In one instance in the course of my experience, a mononch was caught with its quarry, another nematode, still gripped in its jaws. Seized by its middle, the victim had been bitten nearly in two. This is a common fate. Sometimes, however, the ingested nema is but little mangled. I imagine the mononch swallows its prey somewhat as the python does, though less deliberately. As the œsophagus seldom occupies over one-fourth of the length of the body, and as the mononch sometimes swallows other nemas nearly half as long as itself, manifestly in such cases one end of the victim's body must reach the mononch's stomach before the other end has disappeared down its throat.

Gluttonous Appetite. While many mononchs bolt their food, it is evident that the food of some among them receives a certain amount of mastication, for the body of the victim is bitten into fragments and swallowed piece-meal. In some cases the degree of mastication may be greater still. Figure 10, for instance, shows the head of a preserved specimen, in the mouth of which lies a portion of the "gizzard" of a rotifer, which has been almost completely denuded of the muscular tissue originally attached to it. It is the thick-lipped species like $M$. muscorum that masticate their food in this way.

\section{Esophagus or Gullet}

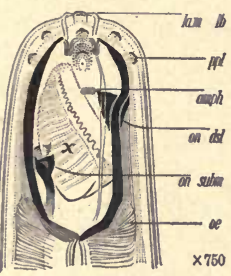

Fig. 10.-Pharynx of a female $M$. palustris with portion of the "gizzard"
of a rotifer ( $x)$ being masticated.

Lining of the Gullet. Salivary (?) Glands. The œsophagus is more or less cylinfroid, a form of œsophagus common in nemas that engorge relatively large objects. The anterior end of the œsophagus, where it receives the base of the pharynx, is usually about one-half to two-thirds as wide as the base of the head, and is occasionally somewhat swollen, so that one may speak with propriety of an obscure pharyngeal bulb. The œsophagus has nearly the same diameter throughout the greater portion of 
its length; it may diminish a trifle in the vicinity of the nerve-ring, while in the posterior half it usually expands a trifle, so that finally it is onehalf to two-thirds as wide as the corresponding portion of the body. The refractive lining of the œsophagus is strongly developed, and is of such a character that its longitudinal optical section often occupies as much as one-third of the diameter of the whole organ, sometimes one-half, exceptionally even more. The radial musculature of the œsophagus is strongly developed, and usually appears coarser posteriorly than anteriorly.

Esophageal Glands. In some species, very likely in all, among the interstices of the œsophageal muscles there exist glandular tissues, which empty their secretion into the lumen of the œsophagus through minute pores in the lining, situated from place to place throughout a considerable portion of the length of the organ. Some of these glands appear to empty into the pharynx, but the best developed ones occur in the dorsal section of the œsophagus near the middle.

De Man and Micoletzky mention structures in the wall of the pharynx of Mononchus, about the nature of which they seem doubtful, but suggest the possibility that they are pores. My observations lead me to the conclusion that some of the more minute so-called "teeth" or denticles of authors, at the bottom of the pharynx, and even some of those higher up, are in reality elevated pores connected with glandular structures in the anterior part of the œsophagus. These pores appear to me to be the homologues of the pores which I have demonstrated undoubtedly to exist farther back in the osophagus, and to be the outlets of glands located in the interstices of the radial muscles. See fig. 9.

It is easy to believe that the œsophageal glands are salivary glands, and that they serve the mononchs in gorging down food in somewhat the

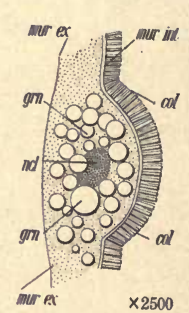
FIG. $11 .-$ Portion of
cross section of intestine of $M$. longicaudatus to show columnar structure of testine, col.

fourths as wide as the body, and is usually made up of cells of such a size that about 8 to 20 are required to build a circumference. These cells contain numerous granules of somewhat variable size, often so arranged 
about the large central nucleus as to give rise to a beautifully regular appearance simulating tessellation. As a rule, the columnar lining of the intestine (fig. 11) is not very refractive. Owing to the low visibility of this lining, and the difficulty of seeing through the mass of granules contained in the intestinal cells, the lumen is usually hard to decipher; toward the anus, however, it may become more distinctly visible. Occasionally, the granules are so few or so transparent that the contents of the intestine can be seen with ease, but this is rather exceptional.

Granules. The structure and arrangement of the granules in the intestinal cells of Mononchus lacustris may be taken as somewhat typical. The granules of this species are numerous and packed rather closely in the cells, and give rise to a more or less distinct tessellation. Specimens fixed with Flemming solution, and mounted in glycerine jelly, show many of the granules as refractive shells, enclosing a relatively large inner spherical mass, which appears dark or light according to the nature of the focus of the microscope. This structure is characteristic of some of the smallest, as well as some of the largest, granules. These appearances are not uniform throughout the intestine, the shell-like structures being more apparent in the posterior part than elsewhere, and less apparent through the middle portion of the body, where the granules are largest and most abundant.

These intestinal granules play an important role in the economy of the nema-some of them are in fact indispensable intracellular organs. Little as we know about their functions in detail, it is already certain that they have to do not only with the secretion of various digestive fluids, but also with the transformation and storage of the digested matter. The granules in any given cell may be of several kinds, doubtless serving entirely different functions. These facts the author has demonstrated by intra-vitam staining.

\section{Digestion}

Food Remnants. The digestive fluids of the mononch must be well fitted for dissolving ceratin, supposedly the main component of nematode cuticle, for the cuticle, as well as most other parts of the ingested victim,

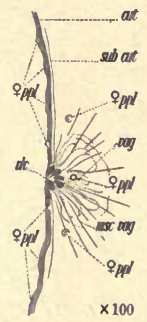

Fig. 12. - Vulva region of $M$. major showing vulvar papillæ, $P p p l$. disappears completely in the course of digestion. The parts that resist digestion longest are the spicula of male nemas and the oral spears of the spear-bearing sorts. These organs, the spicula and the spear, are composed of relatively thick layers of solid "ceratin," and furthermore are protected from the action of the digestive fluids because of their situation in the interior of the ingested nema, and hence are among the last to be dissolved. Such an undigested remnant, a spear or a spiculum, consti- 
tutes one of the most frequent, as w.ll as most reliable, bits of evidence concerning th 2 food-habits of mononchs.

Voracity. Mononchs are voracious. Often the remains of several other nemas are to be seen in the intestine of a single one of them. The writer once had under observation a specimen that had swallowed four large rotifers in quick succession, all tail-end first. The file of rotifers could be clearly seen in the intestine of the mononch. Aquatic mononchs are fond of rotifers. The characteristic "gizzards" of the rotifers, being rather indigestible, are often found in the posterior part of the intestine.

Rectum. From the slightly depressed anus, the lips of which are sometimes rather pronounced, the refractive rectum, usually about as long as the anal body diameter, extends inward and forward, and is separated from the intestine by a distinct constriction. There is no prerectum, though the contrary impression is sometimes created by a constriction caused by a mural commissure (?) some little distance in front of the rectum.

Feces. From the region of the anus relatively powerful transverse muscles pass obliquely backward to the body wall, and find their proximal attachment in the dorsally submedian regions. These anal muscles are more strongly developed in mononchs than in most nemas. A powerful defecating apparatus is needful to such gluttons, since their feces are sometimes bulky, owing, apparently, to the fact that the contents of the intestines of their victims often prove indigestible. Probably this indigestible material is to a considerable extent vegetable in its nature; at any rate, it frequently happens that the feces are bulky, and this fact seems to account for the strong development of the rectum and the anal muscles.

\section{Caudal Glands; Spinneret}

The Cement. The caudal glands, when present, are always three in number, and are arranged in a cluster or series opposite to or immediately behind the anus. If the tail is short, the foremost gland is usually

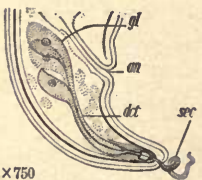

Fic, 13. - Mononch tail showing the three caudal glands, their ducts, the spinneret, and its secretion (sec.). dorsal while the two submedian glands are arranged side by side; if the tail is long all three may be arranged tandem. Apparently there are always three separate ducts, one from each gland, each having a separate ampulla; these latter are arranged side by side in a group at the spinneret. Often, however, the spinneret is simple in form and presents ducts that form only indistinct ampullæ. Some idea of the manner and rate of secretion of the caudal glands may be derived from an examination of the adjacent illustration, which shows a spiral mass of the cement substance secreted by them. This spiral mass was coagulated by the fixing fluid in which the specimen met its death. 
Action of Spinneret. The structure of the spinneret is very similar to that of Mononchulus, a related genus whose unusually large spinneret has proven especially favorable for detailed study, and may be described as follows. The three somewhat elongated ampullæ at the ends of the caudal ducts empty into a single sac located in the spinneret. From the front wall of this sac there projects backward into its cavity an elongated element whose free conical distal extremity fits into the outlet of the spinneret, where it acts as a plug or valve. Apparently, this valve must arise through an invagination of the sac during development. The plug is withdrawn by muscles attached to it and to the dorsal body-wall near the end of the tail. A contraction of these muscles elongates the sac containing the caudal secretion and draws the plug away from the aperture of the spinneret, thus allowing the secretion to flow out through the terminal pore. The plug appears to be returned to its position and held there either by the pressure of the secretions in the sac, or by the general internal body pressure exerted on it through the sac. Figure 5 shows the similar spinneret of a mononch.

Use of Spinneret. In any genus consisting of many species most of which have caudal glands, aberrant species usually occur in which these organs are absent. This is true of Mononchus. A spinneret would appear to be a particularly useful organ to a predaceous nema, permitting it at will instantly to cement itself to a relatively fixed object, and thus more easily master an active victim; nevertheless, about one-fourth of the mononchs have no trace of such an organ.

The lateral fields are always comparatively well-developed, and are usually one-third to one-fourth as wide as the body. The cells composing them often contain spherical granules not differing greatly in size from those of the intestinal cells. No cuticular pores have ever been seen connecting elements of the lateral fields with the exterior.

\section{Renette}

Nothing is known about the structure of the renette-in fact, it is not yet definitely known to exist in mononchs. In most species there is a ventral pore immediately behind the nerve-ring, which bears a close resemblance to the excretory pore of nemas possessing a well-developed renette; but no internal structures have as yet been found to connect with this pore.

\section{Centrai Nervous System}

The nerve-ring surrounds the œsophagus somewhat squarely near the front end of the middle third of the neck. It is usually well developed and easily seen - a distinct refractive collar with groups of nerve cells both in front of it and behind it. Otherwise than this very little is known about the central nervous system of Mononchus. 
Nerve Commissures. A constriction in the intestine is sometimes observed at a point not far in front of the rectum, say at a distance equaling 3 to 5 body diameters. This constriction is sometimes so pronounced as to cause the posterior portion of the intestine to simulate the pre-rectum of Dorylaimus. There is here, however, no true pre-rectum. The constriction seems to be caused by mural commissures surrounding the intestine. The writer can only suggest the possibility of the existence of special nerves at this point, which leave the ventral field, pass slightly backward, then almost immediately become squarely transverse, encircling the intestine until they are very near the dorsal field, where they turn suddenly backward.

\section{Amphids}

If is almost certain that small amphids occur on all mononchs; the writer found them present on three-fourths of the known species. As their general characters have never been adequately described, their form and position are specially noted here, as well as more explicitly in the various illustrations. subat The external indica- som tions of the amphid are always located

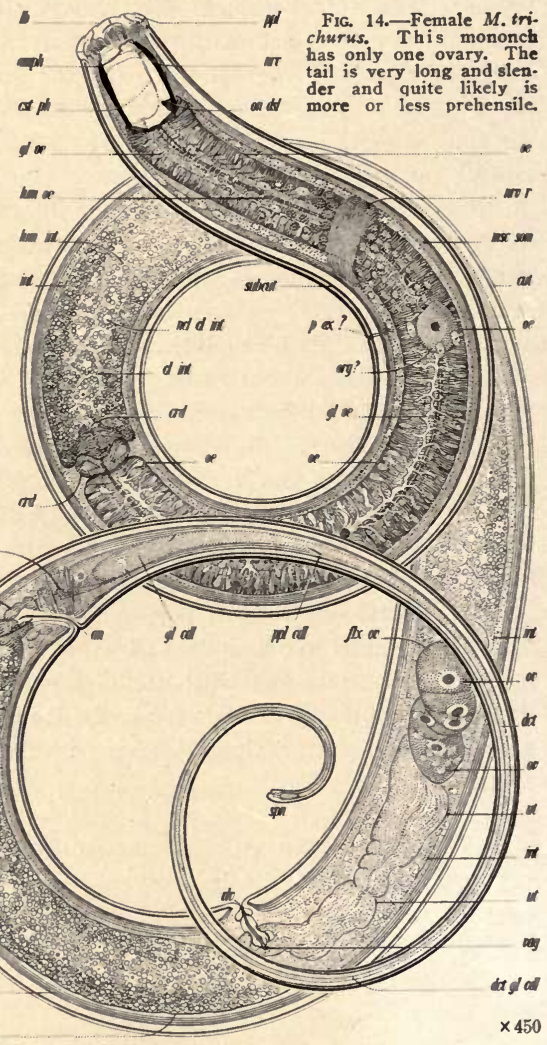
on the lateral lines somewhere between the base of the lips and a point opposite the middle of the pharynx. The periphery, which in some cases may be unclosed behind, is usually more or less elongated or elliptical in form, its long axis lying transversely on the head. These amphids are seldom more than one-sixth as long as the head is wide, and are usually 
very difficult of observation. They are most easily detected when viewed dorso-ventrally, and then appear as refractive oblique elements leading from the surface of the head inward and backward through the transparent cuticle, as shown for instance in the cases of subtenuis and regius (p. 167 and 140). They can seldom be followed more than a short distance inward, and their ultimate internal connections are unknown. A nerve-ending is nearly always to be seen in the cuticle immediately behind each amphid.

\section{No Eyes}

Mononchs have no definite organs of vision.

\section{Female Sexual Organs}

Organs Mostly Double. Eggs. Ovaries and Ova. From the slightly elevated and rather small vulva, the prominent refractive vagina leads inward at right angles to the ventral surface about one-third the distance across the body. Though comparatively small in size, the vulva is usually easily located on account of the refractive nature of the walls of the vagina. In nine-tenths of the species, the internal female organs are double, and symmetrically reflexed, though in some cases the posterior branch is somewhat smaller than the anterior. In the exceptional species the single ovary is also reflexed (fig. 14). The ovaries occupy relatively less space than is usual in nemas-less than one-fourth of the length of the body. Owing to the comparatively great length of the neck, the vulva is usually located somewhat behind the middle of the body even when the sexual organs are double and symmetrical, and may occur as far back as the junction of the third and final fourths. Each of the two uteri is about twice as long as the body is wide, that is to say, of a size to receive one or two eggs only. These latter are nearly always smooth, thin-shelled, ellipsoidal or elongated in contour, and appear about twice as long as the body is wide, though they are sometimes somewhat shorter, and more rarely longer. Only in a single species are the shells known to be sculptured. The shells are thick in obliquus. In all the species examined by the writer, the eggs are deposited before segmentation begins, and this is presumably true of the great majority, if not of all the species. The reflexed ovaries extend one-half to three-fourths the distance back to the vulva and contain a score or so of ova, which near the blind ends are packed in several rows - ova that by increase in size come, each one in turn, to occupy the whole of the width of the organ in its proximal half near the flexure. The ovaries are rather broad, and taper relatively little.

Syngonism. A considerable number of the mononchs are syngonic, and it is probable nearly all of them are so, since the males, if found at all, are nearly always rare. Only on a single occasion, so far as records go, have males been found to be as common as the females. Of most species 
the males never have been seen. At a time previous to the development of the vulva the gonads of the female produce minute spermatozoa, which are sent forward and stored in a special portion of the uterus next the proximal end of the ovary. In the only species so far carefully examined in this regard these minute spermatozoa are known to be functional, at least to the extent that they enter the ova, which then proceed to form polar bodies and begin to segment. However, these particular investigations, made by the author and further recorded in figure 2 , have been confined to the single species $M$. longicaudatus. Possibly the phenomena are different in other species.

\section{Male Sexual Organs}

Spicula. The tail end of the male is invariably like that of the female in general form and size, except that it is more strongly arcuate; it differs however, in some instances, probably in all, in the possession of

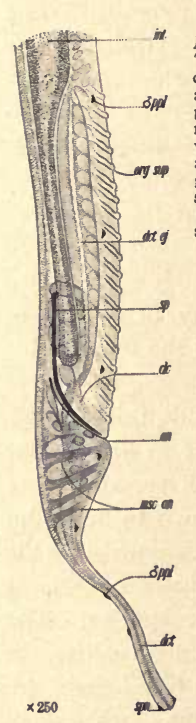

Fig. 15. - Tail end of M. obtusus after Bütschli. The spicula in this species are exceptionally long in the existence of a ventral row of and slender. The male 8 to 20 more or less equidistant sup-

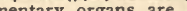
mentary organs are very plementary organs immediately in
well shown. The spicula pleme are protrudable and are front of the anus. The two well-
guided by slender acces- front sory pieces half as long developed arcuate spicula are of
as themselves-also shown.

equal size, and are always accompanied by two distinct though small accessory pieces, a right and a left. Only exceptionally are the spicula long and slender; usually they are somewhat uniformly arcuate, and appear to be subacute and of somewhat uniform width throughout, at least when viewed in profile, and about one and one-half times as long as the anal body diameter. Usually each is strengthened by a median longitudinal refractive piece. They are never prominently cephalated. The accessory pieces are located some little distance inside the anus, alongside the spicula, which they may appear to cross when viewed in profile. These accessory pieces are usually a little narrower than the spicula, and 2 to 4 times as long as wide. Distally they usually end in two subacute points, often so arranged as to form a small U-shaped figure. When at rest the accessory pieces usually appear as if at about right angles to the axis of the body. They are not cephalated.

Supplementary Organs. Sperm. The supplementary organs vary in number up to twenty. The hindmost is located immediately in front of the anus, and may be a little farther removed from its nearest neighbor 
than is usually the case in the remainder of the series; thence forward the organs are about equidistant. Usually they are more or less contiguous, though occasionally they are separated by short spaces, especially anteriorly. When most highly developed, each is a convex-conoid, innervated, very minutely papillated or echinulate, more or less protrudable organ, connected with the interior by an oblique, indistinct element along the axis of which a nerve passes inward and forward to join the ventral nerve of the body (v, fig. 16). It would appear that these organs are partly tactile and partly excitatory in function, and act as the complements of the vulvar papillæ of the female. There is no bursa. The series of oblique copulatory muscles is always coextensive with the series of supplementary or-

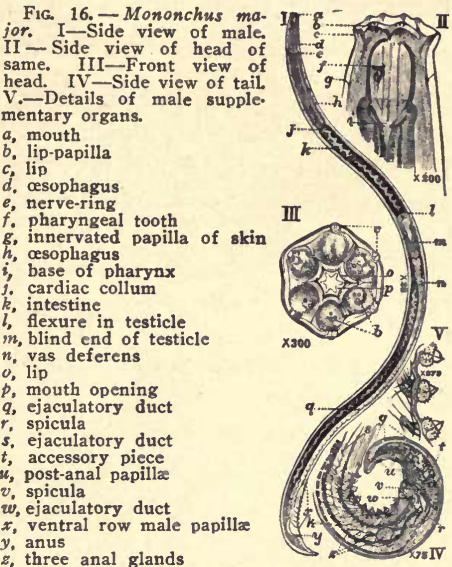
gans. The rather short testes seem to be invariably two in number and are outstretched in opposite directions. The spermatozoa of males are minute and present a more or less vermiform nucleus.

\section{Self-Fertilizing Females; Syngonism}

Males Rare. Conjugation of Syngonic Gametes. No trait of mononchs is more interesting than the capacity of the female to reproduce without the intervention of a male-by a peculiar sort of hermaproditism called syngonism. Of most of the species no males are known; in the remaining species, almost without exception the males are very scarce, and only in exceedingly rare cases are they present in anything more than small numbers. Usually hundreds, and even thousands, of specimens may be examined without the discovery of a single male. How then do the females reproduce? The answer is singularly interesting, for, as already explained, the females fertilize their own eggs by means of spermatozoa which they themselves produce in the same gonad. In the mononchs these spermatozoa produced by females are so exceedingly minute that they have hitherto escaped notice (fig. 2). Notwithstanding its small size, the syngonic sperm cell appears to be functional. It enters the egg, expands, and approaches the nucleus of the egg, which meanwhile throws off polar bodies and later proceeds with segmentation. Observations have not yet extended beyond this point, but it seems alto- 
gether likely that these minute spermatozoa produced by mononch syngones act in what would be called a normal manner, and as the spermatozoa are known to do in other syngonic nemas in which they are of much larger size, e. g. Rhabditis.

\section{Abundance; Distribution}

Frequency of Mononchs. Long ago Bütschli declared mononchs to be among the commonest of nemas, an assertion that has been corroborated by all investigators in this field, hardly any of whom have failed to note a number of species, occasionally new ones. Our knowledge of the genus has been augmented in this way by de Man, Micoletzky, Ditlevsen, Steiner, Hofmänner, Menzel, Daday, and the writer, until at the present time about sixty species are known. Doubtless this number will be very largely increased by future researches.

Geographic Distribution. Variety of Habitat. Mononchs are found in all the habitable regions of the world. They inhabit the soils and fresh waters of every clime, occurring even at great depths in lakes and at very high altitudes on mountains. While we have only just begun to learn the details of their geographic distribution, it is already manifest that some species are cosmopolitan. Several species occur both in Europe and in North America, and a few of these same species are known also from Australia. Considering the small number of observations hitherto made along this line, it seems safe to predict that many of the species will be found to be cosmopolitan. Some species adapt themselves to surprisingly varied conditions. Mononchus longicaudatus, for instance, is known from the tropics, from temperate regions, and from very cold regions, and inhabits both soil and fresh water. Another species, Mononchus brachyuris, is known both from warm springs and from cold Alpine lakes.

Abundance in Arable Soil. Mononchs occur in great numbers in arable soil. On one occasion the writer estimated that at least thirty millions of mononchs per acre were present in the top six inches of a field of maize in New Jersey, and the actual number present may have been much greater. They are regularly present in practically all arable land of a sandy or loamy nature.

The very numerous introductions of living plants into the United States, through the agency of the Office of Seed and Plant Introduction of the Department of Agriculture and other agencies, has brought about simultaneously the introduction of many species of Mononchus. Nemas existing in the soil about the roots of introduced plants are often placed under favorable conditions for propagation in this country. It is therefore certain that an unusual variety of mononchs exists in the soils of the United States. 
Interplay of Organisms. There are regions where certain nematode diseases of crops are very destructive, while other regions, the climate and soil conditions of which are apparently similar, suffer but little, or at least to a lesser degree, so far as we know. In such cases it is permissible to suppose that the nematodes in the area where the lesser damage is done are held in check by some as yet unknown agent. Is it not possible that the mononchs play some such rôle as this, and that just as certain insects hold other insects in check, so certain nemas hold other nemas in check?

We know relatively little about the life history of most of the mononchs, and as yet very little about the possibility of controlling their growth. One species, Mononchus longicandatus, occurs at certain times in almost inconceivable numbers in the sand of the slow filter beds of the water works of cities. Near the end of the period of use the top layers of the sand in these filter beds sometimes become in reality a huge culture of this species. The observations suggest at least the possibility of cultivating this species on a large scale, should it prove desirable to do so.

The discovery that the genus Mononchus is very large and to a considerable extent composed of common and cosmopolitan species that feed upon injurious plant-infesting organisms, suggests so many new lines of research in soil biology as to make it desirable that a clear and connected account of the members of the genus be available to investigators. This need is increased by the fact that the literature is a scattered and fragmentary one, difficult to assemble. To these facts it must be added that most of the new observations herein recorded have been made upon species previously unknown.

\section{II}

\section{Genus Mononchus Bastian, 1866}

This genus is composed of non-marine, free-living nemas, with naked cuticle and obscure amphids, and having a plain œsophagus preceded by a broad-that is, non-tubular - pharynx, armed with 1 to 3 more or less immobile teeth of which the dorsal is largest, and supplied with 6 powerful papillated lips. Ovaries reflexed, usually two. Testes two, outstretched; spicula two, equal, simple, as are their small accessories; there is a pre-anal ventral row of supplementary organs.

Genera Similar to Mononchus, With Distinguishing Characters

Oncholaimus Anonchus

Microlaimus Ironus

Nannonchus

Oionchus

Mononchulus
Marine; sometimes found in brackish soils. Has cephalic setæ. Has spiral amphids, and cephalic setr.

No thick muscular lips; amphids circular.

Pharynx tubular; teeth movable radially.

Has spiral amphids and cephalic setz.

Pharynx filled by single tooth, which is really spear-like.

Quite similar. Has much larger (ventral) spinneret; strongly developed lateral fields; smaller pharynx; female organ single. 


\section{Mononchus Bastian, 1866}

DORSAL TOOTH midway in pharynx or higher, usually massive Tooth not opposed by denticles; subg. MONONCHUS Cobb; type, $M$. truncatus Bast.; pharynx about 2 to 3 times as long as wide, goblet-shaped or ellipsoidal; wall smooth or transversely striated; spinneret usually present; males of about half the species known; female organs double (except monhystera)

Dorsal tooth faced by 2 ventrally submedian teeth

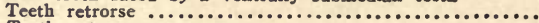

Teeth not retrorse

Tail conoid, then cylindroid; body $7 \mathrm{~mm}$. long.........

Tail simply conoid; body $3 \mathrm{~mm}$. long or less

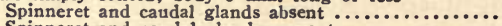

Spinneret and caudal glands present ...

Contour of the head angular; labial papilla 16

Contour of the head rounded; labial papilla 12

Pharynx longer than the head is wide...........

Pharynx only as long as the head is wide...........

Dorsal tooth not faced by submedian teeth

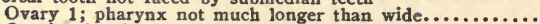
Ovaries 2; pharynx toward twice as long as wide

Tail simply conoid, 8 per cent or less

Spinneret none

Pharynx over half as wide as the head...........

Pharynx not over half as wide as the bead

Length about $1 \mathrm{~mm}$; tooth midway, small, digitate

Length 1.5 to $2 \mathrm{~mm}$; tooth beyond midway, massive Spinneret present

Body $2 \mathrm{~mm}$. long

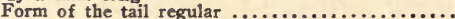

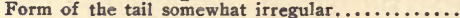

Body 3 to $4 \mathrm{~mm}$. long

Tooth midway, amphids a little behind the lips

Tooth and amphids at the base of the lips.......

Tail conoid, tuen cylindroid, about 10 per cent or more

Esophagus 15 per cent $\ldots \ldots \ldots \ldots \ldots \ldots \ldots \ldots \ldots$

Esophagus 22 per cent or more

Buccal cavity toward 2 times as long as head is wide Spinneret armed with 2 very small setæ..........

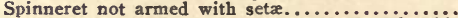

Buccal cavity only about half as long as head is wide Labial papille setose.$\ldots \ldots \ldots \ldots \ldots \ldots \ldots \ldots$. Labial papilla, at least the outer, not setose

Pharynx $1 / 3$ as wide as head; body $1.5 \mathrm{~mm}$. long Pharynx $1 / 2$ as wide as head or more; body 2 to $3 \mathrm{~mm}$.

Cavity about 2 times long as wide; tooth acute Cavity about 3 times as long as wide; tooth sub-acute

Inner labial papilla large; tail sub-conoid. Inner labial papilla normal; tail finaliy

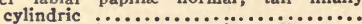

Tooth opposed by numerous denticles on opposite wall

Arrangement of the denticles irregular; characters otherwise as in Mylonchulus; subg. nov. SPORONCHULUS; type $S$. dentatus n. $\mathrm{sp}$.

The tooth behind midway; spinneret present; anus at 92 ; 'f

The tooth in front of midway; no spinneret; anus at 96 ;

Denticles about 20 ; pharynx half as wide as the head; ' $f$ '

Denticles about 50 ; pharynx one-third as wide as the head Arrangement of the denticles orderly

Denticles along a ventral, longitudinal pharyngeal rib; subg. PRIONCHULUS Cobb; type Pr. muscorum (Dui.) Bast.; pharynx ellipsoidal, about 2 times long as wide; males of one species known; female organs double; no spinneret

Eggs punctate or echinulate

Eggs not punctate or echinulate

Length 2.5 to $3.4 \mathrm{~mm}$; vulva at 65 per cent; anus at 94 per cent

Width 3.6 per cent; denticles forward pointing.....

Width 2.3 per cent; denticles inward pointing......

Length 4 mm.; vulva at 55 per cent; anus at 97 per cent

Denticles in transverse rows on 2 sometimes confluent, submedian, rasp-like areas, often with 2 very small submedian teeth at their bases; subg. MYLONCHULUS Cobb: type $M$ minor Cobb; pharynx goblet-shaped, tooth more or less arcuate; smaller species of which

\section{Subgenera \\ and \\ Species}

\section{MONONCHUS}

(tridentatus de Man 1876)

(rex Cobb 1904)

exilis n. sp.

radiatus $\mathrm{n}, \mathbf{s p}$

palustris n. $\mathrm{sp}$.

teres $\mathrm{n}$. $\mathrm{sp}$.

monhystera $\mathrm{n} . \mathrm{sp}$.

$\operatorname{vorax} \mathbf{n}$. SD.

parvus de Man 1879

papillatus Bastian 1866

intermedius Cobb 1893

tenuicaudatus Stefanski 1914

maior Cobb 1893

gerlachei de Man 1904

fovearum (Dujardin) Bastian '66 13

megalaimus n. sp.

macrostoma Bastian 1866

obtusus n. sp.

tunbridgensis Bastian 1866

truncatus Bastian 1866

dadayi Micoletzky 1904

longicaudatus Cobb 1893

\section{SPORONCHULUS}

recessus n. $\mathrm{sp}$.

dentatus n. sp.

decurrens $n$. sp.

\section{PRIONCHULUS}

punctatus n. sp.

muscorum (Duiardin) Bast. '66 23

longicollis n. sp.

spectabilis Ditlevsen 1911 
the males are unknown (except tenuis); female organs double (except index and reversus); spinneret present (except similis)

Ovary 1; tail rapidly diminished, then digitate

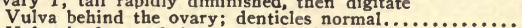

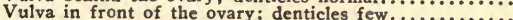

Ovaries 2; tail not digitate (Ex. perhaps sigmaturus)

Dorsal tooth somewhat digitate, relatively small

Refractive ring about pharynx in front of tooth....... Refractive ring about pharynx not prominent............. Dorsal tooth massive, not digitate

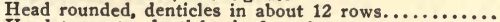

Head truncate, denticles in 2 to 6 rows

Rows of denticles $2 \ldots \ldots \ldots \ldots \ldots \ldots \ldots \ldots \ldots \ldots \ldots . \ldots \ldots$. Rows of denticles 4 to 6

Denticles about 4 rows; spinneret small........... Denticles about 6 rows; spinneret larger

Tail bent ventrally near the middle

Caudal setæ none; tail conoid .............. Caudal setæ ( 3 pairs) present; tail not conoid Tail arcuate or straightish

Width of adults 2.3 per cent .............. Width of adults 2.7 per cent or more

Caudal glands and spinneret absent........ Caudal glands and spinneret present

Eggs thick shelled ...................

Eggs thin shelled

Buccal stria faint if any; anus 94 to 96 per cent

Species aquatic

Species not aquatic ...............

Buccal stria more pronounced; anus 98 per cent

Spinneret finally dorsally recurved..

Spinneret not dorsally recurved.....

DORSAL TOOTH and others small, basal or nearly so (Exc. 44)

Teeth retrorse, small, basal, exceptionally midway; subg. ANATONCHUS Cobb; tvpe A. tridentatus de Man; large species with roomy elongated pharynx and smallish retrorse teeth; tail long and usually becoming cylindroid; female organs double; males of most of the species known

Body 2 to $4 \mathrm{~mm}$; ; teeth equal, midway; anus 90 per cent....

Body 5 to $6 \mathrm{~mm}$; teeth sub-basal; anus 80 to 85 per cent

Submedian teeth equaling the dorsal; body $6 \mathrm{~mm} . . . . .$. .

Submedian teeth smaller than the dorsal; bodv $5 \mathrm{~mm}$.....

Teeth not retrorse, small, or even minute; subg. IOTONCHUंS Cobb; type $I$. gvmnolaimus Cobb; large species with roomy elongated harynx having longitudinal ribs; tail rather long, and often slender; males of more than balf the species known: female organs double or single; most species with spinneret

Ovary single; 1 to 3 rudimentary basal teeth

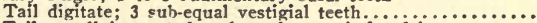

Tail not digitate 1 dorsal tooth, remainder faint or none

Posterior extremity setaceous .......................

Posterior extromity slender but not setaceous

Pharynx as long as wide: few submedian denticles...

Pharynx about 2 times long as wide; faint submedian teeth

Amphid simnle; buccal wall thin, transversely striated

Amphid duplex; buccal wall thick, not striated.....

Ovaries 2; 1 to 3 small, sub-basal teeth

Body 4 to ? mm.: tail finally cylindroid; 3 teeth

Spinnere: present; lips rather plain

Length $4 \mathrm{~mm}$; anus 90 per cent; the 3 teeth subequal

Lengtb 6 to $7 \mathrm{~mm}$; ; anus 80 per cent; submedian teeth

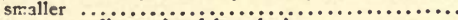

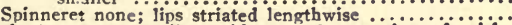

Body 1 to $4 \mathrm{~mm}$; tail conoid; 1 tooth, sometimes denticles Anus $2^{*}$ about 86 per cent; tail conoid, or at last sub. cylindroid

Lips not set off by constriction; onchi unequal or one Onchus with small companions; head rounded....... Onchus one only: head truncate; pharynx striated...

Lips set off by a deep constriction; onchi three, equal

Anus at about 94 per cent; tail simply conoid

Dorsal tooth small, others basal, minute, indefinite....

Dorsal tooth distinct

Buccal cavity balf as wide as long: tooth sub-basal. Buccal cavity as wide as long; main tooth basal

Spinneret present ..........................

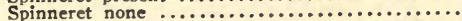

\section{MYLONCHULUS}

index Cobb 1907

reversus n. sp.

obtusicaudatus Daday 1901

brachyuris Bütschli 1873

denticulatus n. sp.

sparsus n. sp.

micrurus n. sp.

incurvus n. sp.

sigmaturus n. sp.

subtenuis n. $\mathrm{sp}$.

subsimilis n. sp.

obliquus n. sp.

lacustris Cobb 1915

polonicus Stefanski 1915

minor Cobb 1893

brevicaudatus n. sp.

japonicus n. sp.

\section{ANATONCHUS}

tridentatus de Man 1876

gracilicaudatus n. SD. dolichurus Ditlevsen 1911

\section{IOTONCHUS}

digiturus Cobb 1893

trichurus n. sp.

(bathybius Micoletzky 1913)

47

gymnolaimus Cobb 1893

consimilis n. sp.

rex Cobb 1904

bathybius Micoletzky 1913

similis Cobb 1893

fasciatus $\mathrm{n}$. $\mathrm{sp}$.

studeri Steiner 1914 
I. M. exilis, n. sp. The cylindroid neck ends in a rounded head with a distinctly expanded lip region. The large pyriform pharynx is armed with three subequal teeth. The wider anterior part of the pharynx is two-thirds as wide as the *

\begin{tabular}{ccccc}
1.8 & 6.2 & 27. & 678,15 & 98.2 \\
\hline 1.8 & 1.8 & 2. & 2.3 & 1.
\end{tabular} head. The apices of the teeth are midway, the dorsal one being a little the farthest forard. Amphids somewhat behind the lips, 1.6 6. 24. $-M_{-} 38$ 98. but farther forward than the teeth, consisting $\begin{array}{lllll}1.6,1.8 & 2 . & 2.2 & 1.2 & 2\end{array}$ of somewhat rectangular markings one-fifth as wide as the head, longest in the transverse direction, and with the lateral and front margins plainer than the posterior. Lining of the œsophagus not so prominent as in most mononchs. Intestine greenish, finely granular, obscurely tessellated. Cardia long and plainly to be seen through the flat, colorless pseudo-bulb at the beginning of the intestine. The rectum has a thick lining of highly refractive ceratin, and is a prominent crgan one and one-half times as long as the anal bodydiameter. Terminus about one-fourth as wide as the base of the tail. The very broad vulva is only slightly elevated. Posterior sexual branch only two-thirds as long as the anterior. On the male there is a row of about 14 equidistant, rather closely approximated ventral ridges in front of the anus, extending forward a distance equal to 3 tail-lengths. These do not bear any prominet papillæ. The body is somewhat thicker in the region of these ridges. Spicula linear, uniformly $5 \mu$ in diameter when seen in profile, the proximal end being in no way distinguished from the rest of the shaft. The accessory pieces appear to rest rather closely against the distal halves of the spicula.

Found about the roots of moss, Moss Vale, New South Wales, 1894. Examined in water after fixation with osmic acid.

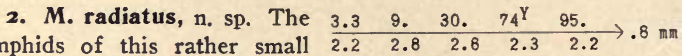
but elegant species are minute and inconspicuous,-about oneeighth as wide as the corresponding part of the head. The granules of the intestine are largest posteriorly, where they are about one-half as wide as the spinneret. The intestine is not tessellated. The tail is markedly arcuate, its spinneret about one-fifth as wide as its base. Anus very slightly raised. Caudal

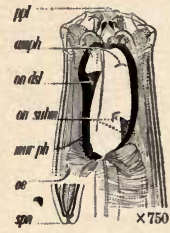
glands rather small, elongated. Sexual organs apparently double and symmetrically reflexed.

Description derived from a single young specimen from a cranberry bog in New Jersey, U. S. A. Flemming solution to glycerine. Fig. 17.**

3. M. palustris, n. sp. Intestine 12 to 15 cells in girth, more or less distinctly

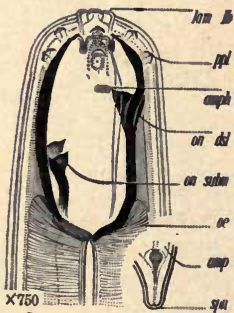
2.7 8. 30. 62,1597 . tessellated. Tail arc\begin{tabular}{ccccc}
2.7 & 8. & 30. & 62.15 & 97. \\
\hline 2. & 2.3 & 2.8 & 2.7 & 1.9
\end{tabular} $1.4 \mathrm{~mm}$ uate, its rather conoid spinneret about one-fifth as wide as its base. Caudal glands broad and saccate, their ampullæ occupying most of the posterior half of the tail. The elongated eggs are about twice as long as the body is wide, and occur one at a time in each uterus. The rather small, tapering ovaries contain 8 to 10 ova arranged more or less irregularly.

From a white cedar swamp, Jefferson County, Wisconsin, U. S. A. Feeds upon rotifers and probably upon other nematodes, which it appears to masticate (page 443, fig. 10). Sublimate to balsam. Fig. 18.

* This decimal formula for nemas is explained in the appendix.

**The species figures have the same magnification throughout, so that the reader may guage the relative sizes. 
4. M. teres, n. sp. This comparatively small, simple form has 2 submedian teeth of equal size. Walls of the intestine with numerous granules of rather uniform size, giving rise to a faint tessellation; the granules such that 2 to 3 would be

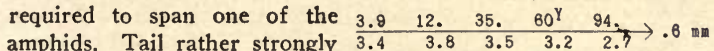
arcuate, its fairly well developed spinneret about one-fifth as wide as its base. Caudal glands somewhat elongated, their small but rather conspicuous ampullæ nearly filling the posterior fifth of the tail.

Description prepared from a single, more or less shrunken specimen, found in the sphagnum of a pot in which blueberry plants

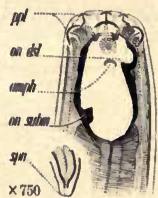
were being cultivated. Bears a general resemblance to papillatus, but differs in the form of the pharyngeal teeth and of the terminus. Flemming solution to glycerine. Fig. 19.

5. M. monhystera, n. sp. Amphids not seen. Lining of the rectum and of the intestine rather distinct and refractive. Intestine only faintly tessellated, if at all.

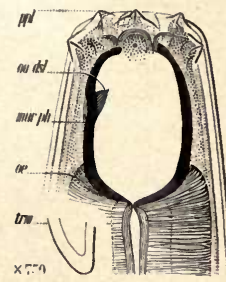

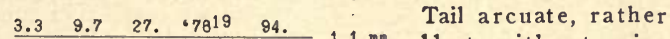

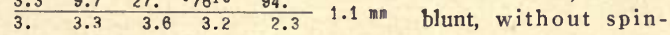
neret. From the rather conspicuous, more or less elevated vulva, the vagina, which is two-thirds as long as the body is wide and tubular, extends obliquely inward and forward. Nothing definite is known with regard to the eggs or the form of the ovary.

Found about the roots of orange trees, Bahia, Brazil. Bears a general resemblance to $M$. papillatus, but has only one ovary. This species is nemativorous. Flemming solution to glycerine. Fig. 20.

6. M. vorax, n. sp. This voracious species has a large mouth cavity and the œsophagus is therefore at first slightly swollen. Intestine 12 to 20 cells in girth,

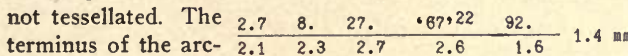
uate tail is about one-seventh as wide as its base. The elongated eggs are about twice as long as the body is wide. Each ovary contains about 30 developing ova, arranged irregularly. Small sperm cells were seen in abundance at the flexure, in a condition indicating that the species is syngonic.

Found in soil from a white cedar swamp in Wisconsin, and from a cranberry bog in New Jersey, U. S. A. This is apparently a voracious feeder on other nema-

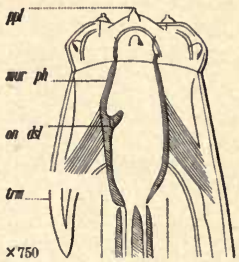
todes. Remains of Dorylaimus and Ironus were seen in the intestine. Resembles $M$. mac-

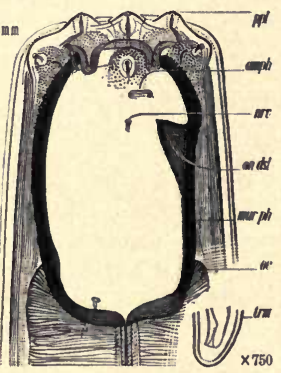
rostoma, but the tail is conoid and has no spinneret. Sublimate to balsam. Fig. 21.

7. M. parvus de Man. De Man mentions the presence of minute teeth at the base of the pharynx, near the be3.1 - 9.5 29. '63, 93. ginning of the œsoph-

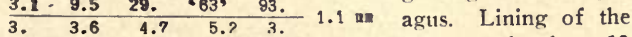
œsophagus well developed. Intestine apparently about 10 to 12 cells in girth, tessellated. Rectum somewhat shorter than the anal body-diameter. Tail arcuate; its terminus only about one-eighth as 
wide as its base. An egg seen in the uterus measured $100 \mu$ in length. It is not certain that caudal glands are present.

Knowledge of this species rests very largely on the observations of Dr. J. G. de Man, who says that it is an active species, common in sandy soils of the dune districts of Holland. Occurs also in Germany, according to Brakenhoff, whose specimens, however, have the dorsal tooth close to the lips instead of midway as in the type form. Fig 22, previous page (after de Man).

8. M. papillatus Bastian. Glands are present in the segments of the cesophagus, and are most strongly developed in the posterior half. The secretion of the glands

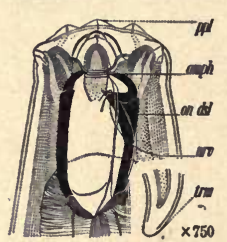

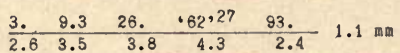

in the dorsal segment pours into the lumen of the œesophagus through a minute pore near the middle of the neck, a short distance behind the nerve-ring. Under favorable conditions lenses of the highest power show transverse striæ to be interrupted on the lateral lines, where there are 2 refractive longitudinal striations very close together. What appears to be an ordinary ventral renette pore is found a short distance behind the nerve-ring. The writer's investigations prove this species to be syngonic. The longitudinal ribs of the pharynx, probably 3 in number, are a little more prominently developed than usual. Occasionally a subventral or submedian rib of the pharynx shows traces of most exceedingly fine denticles. Only the most careful examination of favorable specimens shows these denticles. The writer has never observed a denticulated ventral rib like that of muscorum, as mentioned by Menzel.

A common, voracious, cosmopolitan, nemativorous species. Found in many parts of Europe and of the United States, and also in Hawaii, Australia, South America and Asia. Fig. 23. See also fig. 8.

9. M. intermedius Cobb. Truncate head having 6 lips, each with 2 papillæ. The elongated-oval amphids are half as wide as the base of the lip. The rather long, goblet-shaped pharynx is half as long $2.5 \quad 7.4 \quad 24 .{ }_{61}^{61}, 35 \quad 94$. as the head is wide, and presents a mod- $\begin{array}{llllll}2.4 & 2.4 & 2.8 & 3.3 & 2.4 & 1.9 \\ \mathrm{~mm}\end{array}$ erate-sized dorsal tooth two-thirds the way from the base to the lips. The intestine, whose commencement is marked by the presence of a pseudo-bulb, shows a rather indistinct tessellation. What appears to be a ventral renette pore occurs just behind the nerve-ring. The lateral fields are one-fourth as wide as the body. Caudal glands are present in the conical, arcuate tail. Spinneret almost pointed. Anus depressed, consequently conspicuous. Vulva conspicuous. The reflexed portions of the ovaries extend one-half way back to the vulva.

Found about the roots of sugar cane, Harwood, Clarence River, New South Wales, Australia. Examined in water after fixation with osmic acid vapor.

ro. M. tenuicaudatus Stefanski. The lips are said to bear 6 large, spheroidal papillæ. A transverse element is said to traverse the wall of the pharynx opposite ? ? 21. '50+, 94. $\rightarrow 2.2 \mathrm{~mm}$ the apex of the ? 4 . ? ? to bear 2 slightly curved projections. The csophagus is muscular. At first the tail diminishes regularly in diameter, but presents 2 swellings in the posterior part.

Found among algæ, in the Rhone River, Switzerland. This species is said to resemble $M$. macrostoma Bastian in the form of the buccal cavity, but to differ from that species in the details of the structure of the mouth, as well as in the form and length of the tail. Fig. 24, after Stefanski.

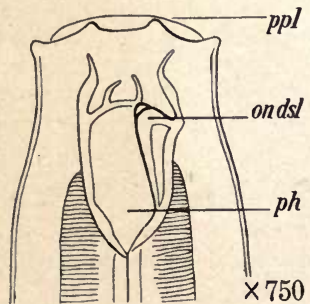


11. M. major Cobb. Labial papillæ of the inner row each with 3 (?) nerveendings. Amphids slightly farther forward than the apex of the dorsal tooth, small. Lateral fields one-fifth as wide as the body. Intestine composed of rather

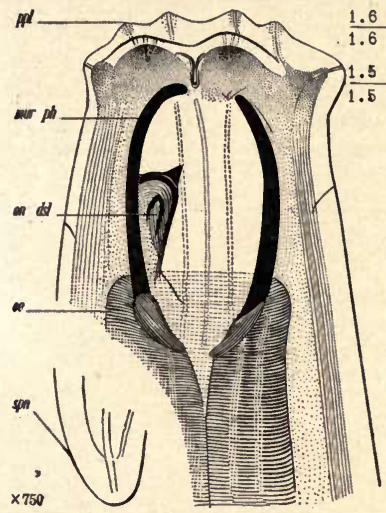
small cells, showing an indistinct tessellation. The are probably less than twice as long as wide. Each of the 12 rather conspicuous, echinulate, mammiform accessory organs is situated on the posterior side of a transverse ceratinous ridge extending one-fourth the distance around the body. The anterior two or three and the posterior one of these organs are smaller than the others. There are 2 pairs of ventrally submedian papillæ, also innervated, on the anterior third of the tail, the posterior pair being near the end of the anterior third, and the other pair half way between that point and the anus. There are other papillæ faintly visible on the dorsal side of the tail and elsewhere (fig. 16). The females of this species also present papillæ near the vulva: 3 small, ventrally submedian papilla on each side of the body, one opposite the vulva, one a short distance in front of it and another a short distance behind it, and in addition a tandem series of 3 papillæ, both in front of and behind the vulva, on the ventral line. The location and conformation of these papillæ is shown in figure 12. Doubtless the females of other species possess similar papillæ. Fixed with osmic acid; examined in water.

Found about the roots of plants, in damp soil, Moss Vale, New South Wales, Australia. Also about turnips in Tasmania. This species resembles the following. Fig. 25.

12. M. gerlachei de Man. Lateral fields about one-third as wide as the body. In transverse section the buccal cavity, though nearly round, is obscurely threesided, and shows the existence of 3 small, longitudinal grooves in the wall of the

\begin{tabular}{|c|c|c|c|c|c|}
\hline • & 5.2 & 18. & $\cdot 50^{, 33}$ & 92.5 & \\
\hline .8 & ? & $?$ & 3.7 & 2. & \\
\hline & $\begin{array}{l}5.6 \\
? ?\end{array}$ & 20. & $\frac{-x_{-}}{3.7}$ & $\frac{95.5}{2.3}$ & \\
\hline
\end{tabular}

pharynx, presumably so functioning as to increase the elasticity and mobility of the pharyngeal walls. What appears to be a renette pore occurs immediately behind the nerve-ring. Anal muscles are well developed in the female. Spicula arcuate, tapering both ways, about one and one-third times as long as the anal body-diameter, and in their widest part about one-sixth as wide as the corresponding portion of the body. The accessory pieces are about one-third as long as the spicula, relatively small and poorly developed, appearing to be parallel to them when viewed in profile. The 
pre-anal ventral row of supplementary organs is essentially the same as in major (fig. 16). According to Steiner's observations the number of supplementary organs in this species may at times be as great as that noted in major. Steiner calls attention to the existence of a submedian row of pre-anal papillæ, lying on either side of the ventral row. The tail bears 3 pairs of papillæ, one submedian or subventral pair near the anus; a second subventral pair slightly behind the middlè; and a third dorsally submedian pair at the beginning of the final fourth of the tail. In the male the slightly conoid spinneret is one-sixth to one-seventh as wide as the base of the arcuate tail. The proportions are about the same as in the female, although the tail of the female is slightly longer and more slender, and not so strongly arcuate.

Found among fresh water algæ, Cape Van Beneden, Danco Land. It is rather difficult to point out satisfactory differences between this species and major. The pharynx of gerlachei is relatively smaller and is thicker walled, and its dorsal tooth is slightly farther forward. The number of labial papillæ-sixteen-appears to be the same in each. The amphids are perhaps slightly farther back in major than in gerlachei. The number of papillæ noted on the tail of the male of major is greater than that noted on the tail of gerlachei, but so far as the distribution of the dorsal papillæ on the tail of gerlachei is concerned, they appear to accord very closely with those of major. The two species appear to be closely related. Fig. 26 on the previous page (after de Man).

13. M. fovearum (Dujardin) Bastian. This is an insufficiently known species and may have to be declared nomen nudum. Head a trifle angular. Pharynx angular, "armed with 2 to 3 straight pieces, each (?) with a pronounced tooth in front of the

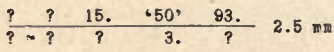
middle." Tail tapering at first but retaining the same diameter through its posterior half, and ending in a sort of spinneret (?). Eggs in single series.

Found at Rennes, France, in a fresh water ditch, along with Branchipus, entomostrica, Euglena etc. De Man suggests that this species is related to tridentatus.

14. M. megalaimus, n. sp. Labial papillæ obscure. Amphids obscure, half way between the apex of the dorsal tooth and the anterior extremity. Cardia large and

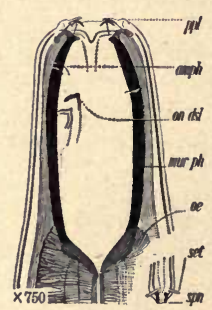

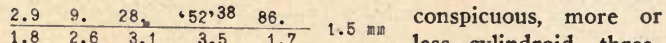
less cylindroid, threefourths as wide as the body. Intestine about 12 cells in girth, more or less distinctly tessellated. At the end of the anterior third the tail is one-fourth as wide as at the anus. Spinneret slightly swollen, with 2 very small digitate setæ.

About the roots of plants, in a tamarack swamp, Wisconsin, U. S. A. This mononch is peculiar in the relatively meager development of the labial papillæ and the pharyngeal teeth, and the weakness of the pharyngeal muscles. Flemming solution to glycerine. Fig. 27.

15. M. macrostoma Bastian. Inner set of labial papillæ inconspicuous; outer set fairly well developed. The neck tapers but little. The head, however, is rather markedly convex-conoid. De Man describes and figures 2 exceedingly minute submedian teeth at the base of the pharynx. Csophageal lining strongly developed. Intestine 12 to 15 cells in girth, tessellated. Posterior half or two-thirds of the tail of the female of practically uniform diameter, tapering but very slightly. Spicula arcuate, very slender, about twice as long as the anal body-diameter. Their proximal ends are not cephalated. They are accompanied by 2 accessory pieces, hardly one-third as long, arranged parallel to their distal parts. Supplementary organs, about 20 , in the form of innervated papillæ, spread over a distance nearly 
equal to the length of the tail, the posterior member of the series being a short distance in front of the anus. There are 3 pairs of somewhat equidistant subventral post-anal papillæ on the anterior part of the tail. There are also lateral papillæ on the tail.

This beautiful, active, rather common species is found in the soils of meadows and marshes in many parts of Europe; it is also aquatic. The writer has followed Dr. de Man's rehabilitation of Bastian's macrostoma. Corresponding females which the writer has found at the Arlington Farm, Virginia, U. S. A., indicate the presence of exceedingly fine transverse striæ, and of inconspicuous wings. Amphids obscure, just behind the lips, difficult to see except in dorso-ventral view. The œsophagus receives the basal fourth of the pharynx, and in this region is very slightly swollen. The writer

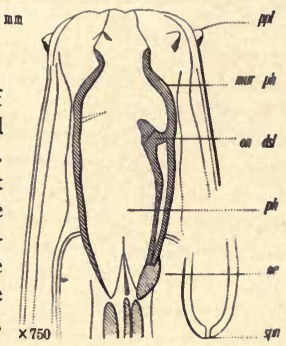
failed to see the minute basal onchi mentioned by de Man. The thin-shelled eggs, which occur in the uteri one at a time, are about one and one-fourth times as long as the body is wide and about half as wide as long, and are apparently deposited before segmentation begins. The writer has no doubt this species is syngonic. A female which had yet to undergo the final moult contained an egg which had its shell completely formed, and was evidently in a state preparatory to segmentation. Flemming solution to glycerine. Fig. 28 (after de Man).

According to Bastian the following differences exist between macrostoma and truncatus; macrostoma is 50 per cent longer, and is relatively narrower in the ratio of 3.5 to 5.3. Macrostoma tapers less in the neck, and has papilla, while truncatus has none. While macrostoma is the larger, its pharynx is no longer; the onchus is a little farther forward. The intestine in macrostoma is less distinctly tessellated. The posterior part of the œsophagus in macrostoma is figured as having an internal expansion. These characters seem to the writer sufficient to separate the two species. It is probable that papillæ on the head of truncatus were overlooked by Bastian. This would have been easy, for instance, if the papillæ on truncatus are similar to those on megalaimus. See figure 27.

16. M. obtusus, n. sp. Papillæ setose. The adjacent figure (29) is after

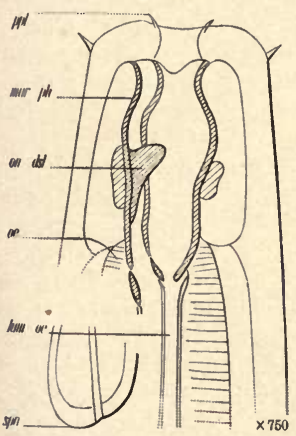
\begin{tabular}{cccccc}
2.4 & $?$ & 25. & $650+$ & 87. & \\
\hline 2.5 & $?$ & 4.2 & $?$ & 3.3 & $1.7 \mathrm{~mm}$ Bütschli, whose
\end{tabular} represent a different species from that figured by de Man under the name macrostoma, notwithstanding the general resemblances. The proportions of the tail and of the spicula are different in the two forms; the distribution of the papillæ on the tail is also different, and, while the number and position of the pre-anal ventral supplementary organs are about the same, their form as shown by Bütschli is decidedly different from that shown by de Man. In addition, Bütschli's figure shows 3 pairs of pre-anal, ventrally submedian papillæ coextensive with the ventral row. Biitschli's data may therefore, the writer thinks, be taken as establishing this new species. Synonym, M. truncatus Bastian of Bütschli.

Found in the River Main, Germany; common in mud and in aquaria and among aquatic plants, in moving water not foul. See also fig. 15. 
17. M. tunbridgensis Bastian. The Tunbridge mononch is interesting historically as well as intrinsically, for it was an examination of numerous specimens of this aquatic nema that marked the beginning of the classical researches of the

\begin{tabular}{|c|c|c|c|c|}
\hline .4 & 6.7 & 24. & $\cdot 52,20$ & 89. \\
\hline 2. & 2.8 & 3.6 & 3.7 & $2.3 \rightarrow 1.8$ \\
\hline & 7. & 23. & $\cdot 53,24$ & 90. \\
\hline & 2.5 & 2.6 & 2.7 & 1.7 \\
\hline
\end{tabular}
well-known English nematologist, $\mathrm{H}$. Charlton Bastian. The upper formula is the average of glycerine specimens from the Arlington Farm, Virginia, U. S. A., while the lower formula is the average of the writer's balsam specimens from Tunbridge Wells, Eng-

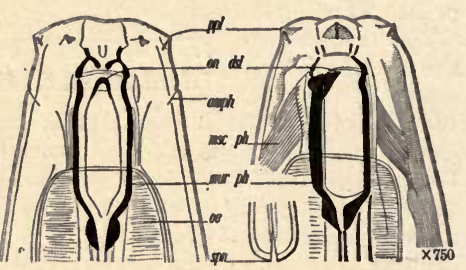
land. As a rule the head is rather suddenly contracted opposite the pharynx, and this is perhaps the best distinguishing mark of the species. Bastian, who had an abundance of specimens, emphasizes this character. The adjacent figures are those of Dr. de Man, and show the pharynx relatively a little narrower than is usual. The tails of the writer's Tunbridge Wells specimens accord rather with Bastian's figure than with his description. As is often the case in other mononchs, the labial papillæ stain more strongly with carmine than do adjacent tissues, so that the lip region as a whole appears strongly colored. Opposite the dorsal tooth there is an exceedingly minute, low, subventral projection. The form of the amphids is not fully determined; they are located nearly opposite the base of the pharyngeal tooth, and have a width one-half to one-third as great as that of the pharyngeal cavity. Near the cardia the œsophageal lining occupies about one-fourth of the optical longitudinal section of the cesophagus. The intestine, which may present a certain amount of tessellation, is about 10 to 12 cells in girth. The anterior two-fifths to one-half of the tail is conoid in such a fashion that at the middle the diameter is about one-sixth as great as at the anus. Thence onward the tail is nearly cylindrical, and ends in a very slightly expanded terminus armed with one or two very inconspicuous papillæ. That a sticky substance often exists on the surface of the terminus of the tail is evident from the accumulation there of minute particles of foreign matter. Caudal glands appear to exist immediately behind the anus, but their nuclei have not been definitely made out as yet. The lateral fields appear to be one-third as wide as the body. Each ovary contains a score or more of developing ova, arranged in several tiers in the distal half of the organ, but single file elsewhere. The somewhat elongated eggs are about one and one-third times as long as the body is wide, and about half as wide as long, and occur in the uteri one at a time.

This seems to be primarily an aquatic species, though the writer has found it also in soil in the vicinity of rivers and streams. Fig. 30 (after de Man).

18. M. truncatus Bastian. Esophagus conoid, its lining well developed. Intes-

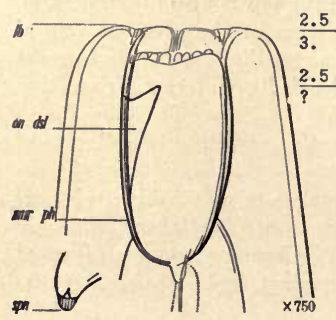
\begin{tabular}{lccccc}
2.5 & $?$ & 25. & $450+9$ & 86. \\
\hline 3. & $?$ & 5. & 5.3 & 3.4 \\
1.8 & $=1$ \\
2.5 & $?$ & 25. & $M$ & 92. \\
$? ?$ & $?$ & $?$ & $?$ & ?
\end{tabular} tine about 12 cells in girth, more or less distinctly tessellated. Lateral fields broad and distinct. It is a question whether to retain this species on the basis of Bastian's original description, or to accept Bütschli's description as a rehabilitation of Bastian's species. It is hardly likely that any species of Mononchus is destitute of labial papillæ, and in this respect Bastian's description seems defective. The extreme reduction of the 
labial papillæ so far recorded is shown in megalaimus. Bütschli and later authors describe and figure the papillæ of truncatus as setose. It seems hardly likely that Bastian would have overlooked setose papillæ such as those figured by Bütschli. In that case Bastian's truncatus should be retained as a species, probably having very inconspicuous labial papillæ similar to those of megalaimus, while the data given by Bütschli may be taken as establishing a new species, for which the name obtusus is proposed. (See No. 16.)

Found in a small pool, among decaying moss and liverwort, England. Fig. 31 (after Bastian).

19. M. dadayi Micoletzky. A striking character of this species, "M. macrostoma Bastian var. armatus Daday," as described by its author, is the presence of 6 small,

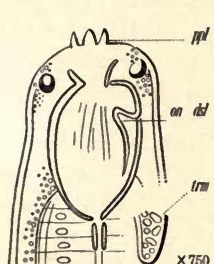
$\begin{array}{ccccccc}? & ? & 22 . & ? & 86 . & & \text { tooth-like cuticular spines close } \\ ? & ? & ? & 4.2 & 2.6 & 1.6 \mathrm{~mm} & \text { around the mouth. No such }\end{array}$ structures have been seen in other species, and accordingly Micoletzky has proposed for the form the name dadayi.

This is a littoral species. The description and drawings rest upon the examination of a single specimen. The original description is very brief and the accompanying illustrations, one of which is here reproduced, are meager sketches of the head and tail ends. A more complete examination of further x750 specimens is desirable. Fig. 32 (after Daday).

20. M. Iongicaudatus Cobb. Opposite the apex of the dorsal tooth there are refractive transverse thickenings of the wall of the pharynx in the ventrally submedian region, and in front of these thickenings there are a few transverse strix. The lateral fields are two-fifths as wide as the body, and are distinctly to be seen. since they have definite lateral contours, doubtless owing to the thickness of the muscular layer. The lateral fields contain scattered, nearly colorless granules somewhat smaller than those contained in the intestinal cells. In young specimens the vagina is distinctly separated from the uterus by a deep constriction, and the uterus itself is bulbous near this constriction and narrower farther away. Longi$\begin{array}{lrcccc}\text { caudatus is a syn- } & 3 . & 6.7 & 26 . & \cdot 55,23 & 88 . \\ \text { gonic species. In } & 2 . & 2.4 & 3 . & 3 . & 2.4\end{array} 2 . \mathrm{mm}$ the adult and egg-producing specimens the eggs are seldom or never seen more than two at a time, one in each uterus.

A predacious species, feeding upon other nematodes, upon rotifers and protozoa. It is cosmopolitan, and is found in rivers, ditches and pools, as well as in the soils of swamps and meadows. It occurs, sometimes in vast numbers, in the sand of the slow filter beds of the water-works of cities and towns. Flemming solution to glycerine. Fig. 33; also fig. 2, p. 132.

Longicaudatus suffers from an internal disease caused by a fungus having a branched mycelium. The width

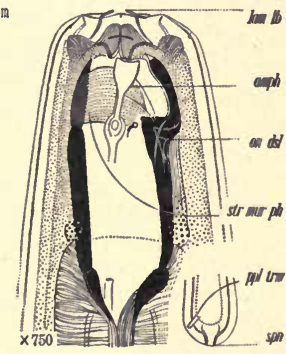
of the mycelium is atout half as great as the thickness of the body wall of the nema, and the septa of the cells are separated from each other by a distance equal to the diameter of the body. There are two other diseases affecting this nema, one of microbe origin, the other of fungous origin; they appear, however, to be confined to the cuticle. One consists of short-styled, elongated-ellipsoidal elements on the surface of the body extending outward at right angles. These have been seen on the tail end. The microbe growth appears as a coating, sometimes of considerable thickness, and occurs on various parts of the body. It has been seen 
at both extremities. The adjacent figure (34) depicts the tail end of a male found in Hawaii about the roots of sugar-cane. The general resemblance to the male as-

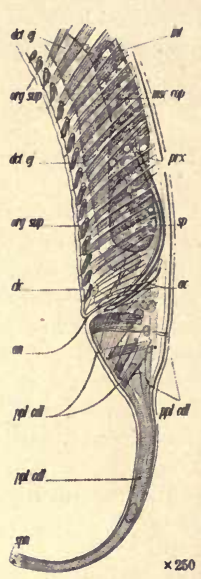
signed by Bütschli to $M$. truncatus Bastian is very striking, yet the differences in detail are also pronounced. The number of supplementary organs is 16 instead of about 20 , and the organs themselves relatively shorter and more plump. The spicula and their accessory pieces have the same general proportions and size. The tail also has the same form and proportions, but the terminal portion is more slender in the Hawaiian specimen, and the distribution of the papillæ thereon is decidedly different, as will be seen by comparing figures 15 and 34 . Male mononchs are so rare that the amount of variation that may exist in a given species is a nearly unknown quantity, so that it is not exactly easy to make intelligent comparisons between the males in these two cases. The Hawaiian male is supposed to be the male of $M$. longicaudatus, the only one that has ever been seen. It is interesting to note that when the spicula become long and slender the accessory pieces also become long and slender, but maintain their general form and are bifurcated at the distal end. Fig. 33 (on the previous page) and fig. 34 . It is a curious fact that this, the only male of $M$. longicaudatus ever seen among the many thousands examined, should have come from soil, since longicaudatus is much less common in soil than in water.

21a. M. dentatus, $n$. sp. This representative of a new subgenus is especially interesting as a guide to speculation concerning the relationship of Mononchus to

\begin{tabular}{llcccc}
3. & 9. & 27. & 62,14 & 95. & \\
\hline 2.6 & 2.8 & 3.3 & 3.3 & 1.8 & $1.1 \mathrm{~mm}$
\end{tabular}

other genera. The scattered denticles are paralleled in certain marine nemas. The arcuate tail is conoid from the raised anus. The rather blunt terminus is about onefourth as wide as the base of the tail. There is a pair of ventrally submedian, innervated papillæ a little in front of the middle of

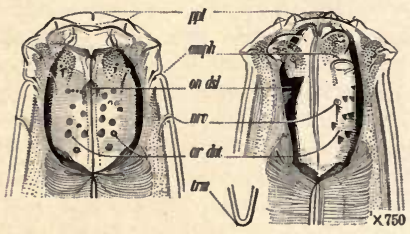
the tail. The amphid is sometimes more elongated than shown in the illustrations.

Description of a young female, from roots of orange trees, Bahia, Brazil. Fig. 35. For 21b, $M$. recessus, and 21c, M. decurrens, see Appendix, p. 184.

22. M. punctatus, n. sp. This species is proposed for the reception of the specimen described by Brakenhoff under the name $M$. papillatus Bastian, which appears to differ from any mononch hitherto

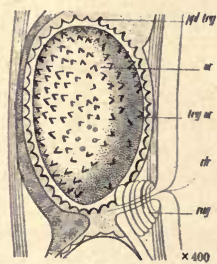

$$
\begin{array}{ccccc}
2.6 & \text { ? } & 25 . & 65,26 & 93 . \\
2.2 & \text { ? } & \text { ? } & 3.5 & \text { ? }
\end{array}
$$

described in that the shells of the eggs are echinulate. It is placed in the subgenus Prionchulus, though with some doubt, principally because both the description and the figure of Brakenhoff show the presence of denticles on the ventral rib of the pharynx. The author says: "Hier findet sich

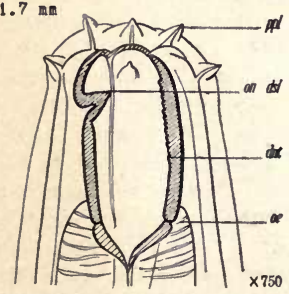
nämlich eine, in der Mitte (und an den 2 Seiten) fein gezähnelte Leiste. Diese 
Zähnelung erstreckt sich über eine etwa $0.016 \mathrm{~mm}$. lange Strecke und beginnt distalwärts etwa in der Hōhe der Spitze des dorsalen Zahns." The ellipsoidal eggs occur in the uteri one at a time, are about as long as the body is wide, and two-thirds as wide as long.

Found among the roots of Alopecurus denticulatus, as well as aquatic habitats, in the bottom of ditches and lakes in Germany. Fig. 36 and 36a (after Brakenhoff).

23. M. muscorum (Dujardin) Bastian. The oldest, and one of the best known species. Intestine tessellated. Tail conoid and arcuate. The reflexed ovaries, each containing about a dozen ova arranged more or less in single file, extend half way
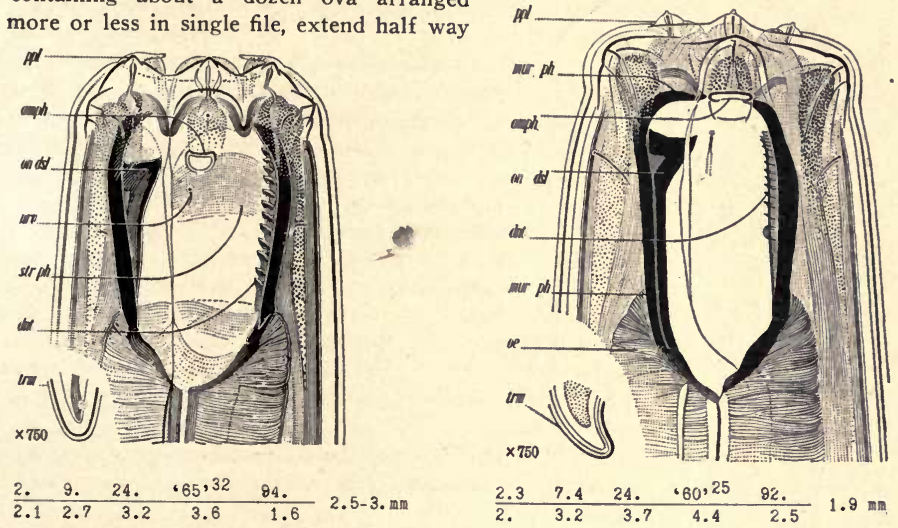

back to the projecting vulva. The eggs are one and one-half times as long as the body is wide and three-fourths as wide as long. The anterior sexual organ is somewhat the larger. Fig. 37, left.

This is a beautiful and rather common cosmopolitan species, occurring in swamps, marshes, meadows and moorlands. It has been found in various parts of Europe and is not uncommon in the United States. It was first found by Dujardin in the Jardin des Plantes, Paris, where it still thrives; the writer recently found it about the roots of some heather imported thence. It feeds on smaller animal organisms, among them other nematodes. The following is a variety:

M. muscorum (Dujardin) Bastian macrolaimus, n. var. Besides differing slightly in proportions from the type form of the species, the variety has a somewhat larger pharynx, with smaller denticles on the ventral rib. The submedian papillæ of the outer sets are apparently double instead of triple, though at some distance behind the outer submedian papillæ there is a special submedian innervation. Most of these minor differences are set forth in figures 36 and 37.

Found in Cladonia rangiferina, tamarack swamp, Wisconsin U. S. A. Fig. 38 , right.

24. M. longicollis, $\mathrm{n}$. sp. A species resembling $M$. muscorum, but which differs in the proportions of the various parts $\begin{array}{lllllll}\text { and in the conformation of } & 3.1 & 10 . & 27 . & \text { Y } & 93 . & \\ \text { the pharynx, the denticles } & \frac{2.2}{2.2} & 2.4 & 2.3 & 2.1 & 1.8 & 1.2\end{array}$ of which are irregular and inward pointing. Submedian papillæ of the outer row double, instead of triple as in muscorum.

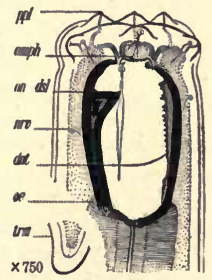

Amphids relatively 
larger than in muscorum. Striæ very difficult of resolution. Found about the roots of pitcher-plants and tamarack, in a swamp, Wisconsin, U. S. A. The difference between this and muscorum may not be very important. Both are nemativorous. Flemming mixture to glycerine. Fig 39 (at bottom previous page).

25. M. spectabilis Ditlevsen. The muscular œsophagus encompasses the proximal third of the pharynx, and has a conspicuous lining. The uteri may contain

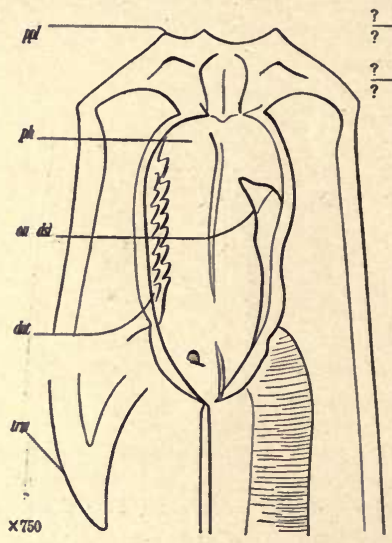
from 2 to 4 eggs, usually 2. The acute conical tail is strongly arcuate. The male is a little more slender than the female, and increases in size from a point where the supplementary organs commence, in such a way that the body assumes a maximum circumference near the anus. The copulatory muscles are strongly developed, and the numerous, prominent, supplementary organs appear to have a subventral position, forming two longitudinal rows, between which is a groove. Fourteen of these supplementary organs are figured by Ditlevsen-manifestly not the whole series. The arcuate spicula, which have a strengthening piece in their distal halves, are about one and one-half times as long as the anal body-diameter. Their proximal ends are not cephalated. At the widest part, toward the proximal end, they are about one-fifth to one-sixth as wide as the body; thence toward the distal end they taper gently. The obscurely bifurcated accessory pieces, which in profile view appear to cross the spicula, are of the usual form, and are about one-third as long as the spicula, and about one-third to one-fourth as wide as long. Their distal extremities lie somewhat in front of the tips of the spicula and toward the ventral side of the body. The elongated eggs are nearly one and onehalf times as long as the body is wide, and less than half as wide as long.

Numerous specimens, the males as numerous as the females, found at Hellerup, near Oresund, Denmark. This species exhibits the phenomenon of flotation. Ditlevsen says: "If some material (meadow soil) is spread in a flat glass cup and water is poured over it, the mononchs will mount rapidly and be lying on the surface dry and shining." Fig. 40 (after Ditlevsen).

26. $M$. index Cobb. No striæ seen. The neck diminishes opposite the base of the pharynx to form a rather cylindrical, truncated head which is slightly expanded at the lip region. Amphids, one-fifth as wide $\begin{array}{cccccc}2.1 & 8.8 & 30 . & 69^{15} & 95.8 \\ \text { as the head, are indicated by transverse mark- } & 2.4 & 3.2 & 3.5 & 3.8 & 2 \text {. }\end{array} .9 \mathrm{~mm}$ ings, bent backward at each end, and located opposite the middle of the dorsal tooth. Denticles in about five rows, the outer rows more distinct. Pharynx half as wide as the head, and about three times as deep as wide. Esophagus more or less conoid, with a massive lining occupying about one fourth of the optical section. Cardia pointed. Intestine few cells in girth; rather obscurely tessellated. Rectum half as long as the anal body-diameter. Longitudinal fields are visible throughout most of the length, and are about two-fifths as wide as the body. The diameter of the body increases somewhat just in front of the anus, and then diminishes suddenly at the anus, so that the beginning of the tail is very considerably less in diameter than the portion of the body immediately in front of the anus. 
The diameter of the cylindrical portion of the tail is about one-fourth that of the body at the anus. Caudal glands 3, egg-shaped, opposite the anus. Spinneret slightly apiculate and apparently unarmed. The eggs are evidently of large size. A single egg, not yet passed on to the uterus, was four-fifths to five-sixths as wide as the body, and about three times as long as wide.

Common about the roots of sugar cane on various plantations on the island of Hawaii. Flemming solution to glycerine.

27. M. reversus, n. sp. This very interesting abnormal form possesses striæ that appear resolvable into rows of dots. Intestine about 6 cells in girth, faintly

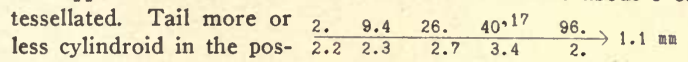
terior half, its terminus one-fourth to one-fifth as wide as its base. Lateral fields two-fifths as wide as the body. The eggs are of relatively large size, since an ovum not yet passed on to the uterus is 5 to 6 times as long as the body is wide. It is therefore likely that the eggs occur in the uterus only one at a time. The relatively broad ovary tapers but

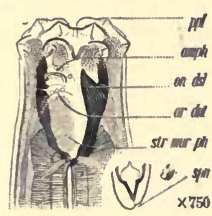
little; it contains about a dozen developing ova.

Rio Janiero, Brazil, about the roots of Platonia insignis Mart. In general this species resembles incurvus and minor, but is readily distinguishable by the form of the sexual organ, and by the sparseness of the pharyngeal denticles, only a few of which are to be seen. When the female sexual organs reduce to one, this remaining one usually extends forward from the vulva. Here the reverse is the case; hence the specific name. Flemming solution to glycerine. Fig. 41.

28. M. obtusicaudatus Daday. Tail conoid, slightly arcuate; toward the end

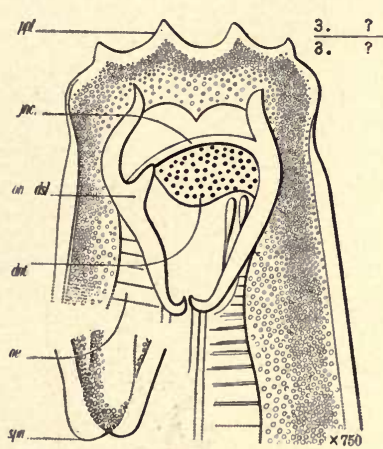
rather suddenly diminished in size. The spinneret well marked.

Found at Berlinhaven, Island of Salao, New Guinea, in fresh water. It seems probable from Daday's description that there are 3 teeth in the pharynx, 1 dorsal and 2 smaller submedian ones just at the base of the rasps. Probably the junction between the posterior and anterior elements of the pharyngeal walls is unusually prominent; this would account for the "bogige leiste" mentioned in the original description and shown in the original figure along the anterior margin of the rasp. Fig. 42 (after Daday). Assuming Daday's drawing to be more or less conventionalized, this species might be regarded as identical with minor.

29. M. brachyuris Bütschli. Amphids appear as transverse slits opposite the onchus. Lining of the œsophagus strongly developed. Cardia plainly to be seen. Intestine tessellated.

Rectum half as long \begin{tabular}{cccccc}
2.2 & 8. & 28. & 662,18 & 96. & \\
\hline 2.4 & 2.9 & 3.6 & 3.9 & 2.2 & $1.2 .2 . \mathrm{mm}$
\end{tabular} as the anal body-diameter. Caudal glands more or less saccate, opposite the somewhat raised anus. Tail somewhat arcuate. Spinneret pore a little to the dorsal side of the middle of the terminus. The vulva is a conspicuous feature, owing to the thickness of the walls of the vagina, which is one-half as long as the body-diameter. Series of

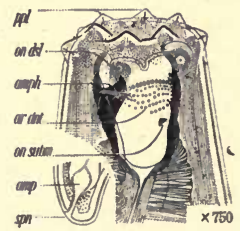


male supplementary organs, according to de Man, about 12, mammiform and apparently protrudable except the two anterior and the posterior, which are more or less rudimentary. Series about twice as long as the tail.

A cosmopolitan species. In Florida, the writer found this species feeding on the larvæ of Heterodera radicicola, an extremely serious root pest. Fig. 43, bottom p. 467.

30. M. denticulatus, n. sp. Onchus opposed by two small subventral onchi

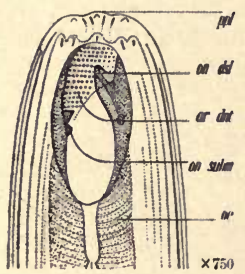
farther back, the left a little farther forward than the right.

Found in the Zambezi River, South Africa, among fresh water algæ. The peculiar form of the head and pharynx, and the unusually large number of denticles are the prominent characters of this species. Only a single mutilated specimen has been seen by Dr. Micoletzky. As it is unlikely that the large number of denticles shown in the original figures can be due to moulting phenomena, it would seem that this form differs distinctly from all others. Fig. 44 (after Micoletzky). As neither Micoletzky's text nor figure suggests moulting, the numerous rows of denticles are assumed to be normal.

31. M. sparsus, n. sp. Each rasp consisting of but 2 rows of denticles. The \begin{tabular}{lcccccc} 
tail diminishes suddenly in size & 2.9 & 12. & 32. & $54 \mathrm{Y}$ & 94. & \\
behind the elevated anus, and & 3.4 & 4.3 & 4.2 & 3.8 & 2.7 & .5 \\
\hline
\end{tabular} tapers somewhat in the posterior three-fourths. The terminus is about one-fourth as wide as the base of the tail. The caudal glands (?) lie opposite the rectum.

Found in sphagnum from greenhouses, Department of Agriculture, Washington, D. C., U. S. A. Flemming solution to glycerine. Fig. 45.

32. M. micrurus, n. sp. This odd form appears to have no submedian onchi. Rasps of only about four rows of denticles. Amphids not seen. Lining of the œsophagus well-developed, occupying three-fifths of the optical longitudinal section.

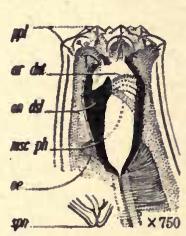

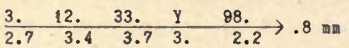

Intestine about 5 cells in girth, not tessellated, or only faintly

so. Tail very short and strongly arcuate or bent. Spinneret about one-fifth as wide as the base of the tail. Rectum somewhat shorter than the anal body-diameter; longer than the tail. Caudal glands not clearly seen, probably opposite the rectum. Ampullæ of the caudal glands not very strongly developed.

Described from young specimens found about Litchi roots, Fukien, China. Resembles brevicaudatus. Characterized by the extremely small and very strongly arcuate tail. The spinneret is sometimes turned nearly at right angles to the axis of the tail. Flemming solution to glycerine. Fig. 46.

33. M. incurvus, n. sp. Striæ resolvable with difficulty and into rows of dots. Dorsal onchus opposed by 2 inconspic-

wous, ventrally \begin{tabular}{ccccc}
2.1 & 2.4 & 28. & 663,21 & 98.2 \\
\cline { 2 - 3 } & 2.9 & 3.2 & 2.1
\end{tabular} $1.4 \mathrm{~mm}$ submedian onchi of smaller size opposite its base. The lining of the œsophagus is a prominent feature, occupying about two-thirds of the apparent width of the œsophagus. Intestine 12 to 15 cells in girth, not tessellated, its granules numerous and fine. Caudal glands 3, broadly saccate, opposite the anus, their ampullæ long and filling the posterior part of the tail.

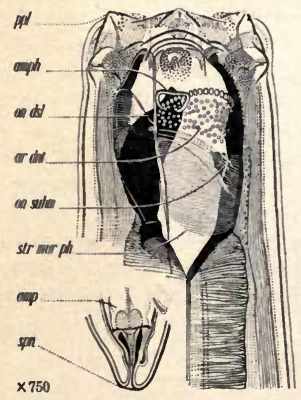


There is a flattish cardia. Anus raised. Longitudinal fields two-fifths to one-third as wide as the body. From the more or less elevated vulva the vagina leads inward at right angles to the ventral surface one-third the distance across the body. The eggs are one and one-half times as long as the body is wide, and occur one at a time in each uterus. The ovaries contain about a dozen developing ova.

Sandy soil in a cranberry bog, Arlington Farm, Virginia, U. S. A. Also on the margin of the spring that gives rise to Salt River, Jamaica. Flemming solution to glycerine. Fig. 47 (at bottom of previous page).

34. M. sigmaturus, n. sp. Two very inconspicuous submedian onchi are found opposite the base of the dorsal onchus, just at the base of the rasps. Intestine 10 cells in girth, comparatively distinctly tessellated. The rectum is about as long as the anal body-diam-

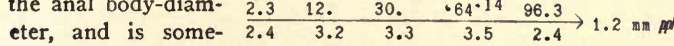
what sigmoid. The anus is very distinctly visible on account of its contour, and on account of the refractive nature of the lining of the rectum. What appears to be a renette pore exists immediately behind the nerve-ring. The distinct lateral fields are about one-fourth as wide as the body, and are characterized by the presence in them of scattered granules much smaller in size than those of the intestine. The posterior part of the tail is

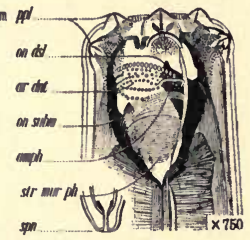
somewhat digitate, and the whole is slightly sigmoid. From the anus the tail tapers rapidly to near the middle, so that if the posterior half were lacking the tail would be rounded. The diameter of the tail at the middle is about one-third as great as at the base. From the middle onward the tail for a short distance is of uniform diameter, and then tapers rather rapidly in the posterior fourth to a rounded or subtruncated spinneret. Small, somewhat finger-shaped setæ are found on the tail; one dorsally sublateral pair, a trifle in front of the anus; another dorsally sublateral pair near the middle of the tail but located on the more bulky part; finally, a third ventrally sublateral pair a little in front of the digitoid part of the tail. The caudal glands are opposite the rectum. The eggs are about two and onehalf times as long as the body is wide. The tapering ovaries contain 10 to 12 developing ova, arranged irregularly.

Found in various parts of the United States and Mexico. Resembles minor and brachyuris, from which it may be distinguished by the form and structure of the tail. Flemming solution to glycerine. Fig. 48.

35. $M$. subtenuis, n. sp. Wall and dorsal tooth of the pharynx rather strongly developed. When the lips are closed the pharynx is hardly half as wide as long. Dorsal tooth slightly arcuate, its point located close to the base of the lips. The

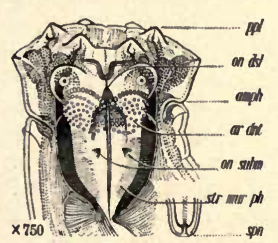

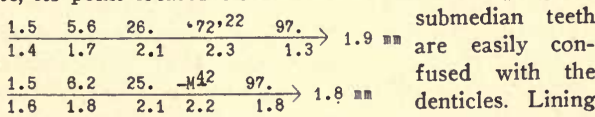
of the œsophagus well developed, occupying one-fourth of the longitudinal optical section. There is a small conoid cardia. Intestine probably about 6 cells in girth, not definitely tessellated. Anus raised and conspicuous, the anterior lip somewhat massive and overhanging. The arcuate, conoid tail ends in a spinneret one-fifth to one-sixth as wide as its base. The ovaries, of which the posterior is somewhat the smaller, appear to contain about a dozen ova. Inconspicuous papillæ appear on the ventral side of the female near the vulva. Two were noted behind the vulva and one or more in 
front of it. The tail of the male is somewhat like that of the female but dimin ishes very suddenly behind the anus. Five pairs of innervated papillæ have been seen on the tail of the male, two of them, however, exceedingly inconspicuous and easily overlooked: Of the three more conspicuous, one ventrally submedian pair is located a short distance behind the anus; a second dorsally sublateral pair occurs a little behind the middle of the tail, and a third subventral pair occurs a short distance in front of the spinneret. Of the two more inconspicuous pairs, one is nearer the spinneret than that just mentioned, and the other, lateral, and slightly behind the middle of the tail. The arcuate spicula are about one and one-fourth times as long as the anal body-diameter. At their widest part, near the middle, they are about one-fifth to one-sixth as wide as the corresponding part of the body and thence taper in both directions; they are not cephalated. The distal ends are obscurely two-pronged. The obscurely bifurcated accessory pieces are of typical form and size, about one-third as long as the spicula and about one-fourth as wide as long. Fourteen rather closely approximated, equidistant supplementary organs occur in front of the anus, occupying a distance about three times as great as the length of the tail. Internally the organs seem to be short, broad tubes of slightly varying diameter; these are probably slightly protrudable. The protrudable portion is not hispid as is the case in $M$. major; - on the contrary, it appears to be smooth. The anterior one and the posterior three of these organs are not so well developed as the others, that near the anus being reduced to a mere innervation; the distance between this latter and its nearest neighbor is about twice as great as between any other adjacent members of the series. These organs give to the ventral contour a crenate or serrate appearance. The anal muscles are prominently developed. The ejaculatory duct is often filled with elongated spermatozoa, somewhat resembling those of Dorylaimus, and similar in form to those figured by Dr. de Man for $M$. gerlachei. Each one may be one-fourth as long as the body is wide, or thereabouts. There are two outstretched testes. The blind end of the anterior is about as far behind the base of the neck as the latter is behind the anterior extremity. The blind end of the posterior seems to lie about twice as far in front of the foremost supplementary organ as this latter is in front of the anus. The spicula have a median stiffening piece, and their proximal ends lie toward the dorsal side of the body.

Found about the roots of plants on the Arlington Farm, Virginia, U. S. A. Resembles $M$. minor, but in the proportions of the pharynx there are notable differences. The walls are here thicker; the amphids are larger and farther back; the lips and onchus are strongly developed, so that when the pharynx is closed the cavity appears smaller than in minor. Opposite the anterior supplementary organ there is a fibrous ring, probably nervous. A similar structure has been noted in other species. There probably exist at this point in the body special nerve commissures. Flemming solution to glycerine. Fig. 49 (near bottom of previous page).

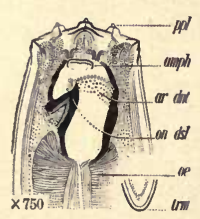

36. M. subsimilis, n. sp. Striæ of the cuticle more or less easy of resolution. Lining of the œsophagus strongly developed. 3. 10. 34. Y 98. $\quad$ occupying three-fifths of the

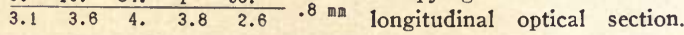
Anus slightly elevated, especially the anterior lip. The scattered granules in the cells of the intestine are small, but variable in size, and do not give rise to tessellation. The more or less arcuate tail is conoid to the blunt terminus, which is about one-fourth as wide as the base of the tail. A ventrally sublateral innervated papilla occurs on each side near the middle of the tail. There are no caudal glands. Description derived from a single young female. 
About the roots of banana plants imported from Paris, France. Resembles brachyuris and minor, but differs in having no spinneret. The dorsal onchus is set farther back in the pharynx than in either of those species, and there are no traces of submedian teeth. The rasps also are less strongly developed. Flemming solution to glycerine. Fig. 50 (at bottom of previous page).

37. M. obliquus, n. sp. Two small, ventrally submedian onchi present, opposite the base of the dorsal onchus. Esophagus at first a little swollen; its lining well developed, and occupy- 2.6 9. 31. $.63,2498$.

ing one-fourth of the $\begin{array}{llllll}2.6 & 3.4 & 3.4 & 4.1 & 2.4 & 1.4 \\ \mathrm{~mm}\end{array}$ optical longitudinal section. Intestine about 8 cells in girth, as a rule not distinctly tessellated. The conoid tail is slightly arcuate, and obliquely truncate at the spinneret, which is about one-third as wide as the base of the tail. The three caudal glands are located opposite the rectum, which ends externally in a slightly elevated anus. The ampullæ of the caudal glands are well developed, and occupy the posterior two-thirds of the tail. Though the vuiva is more or less continuous with the ventral surface, it is conspicuous on account of the refractive nature of the walls of the vagina. The

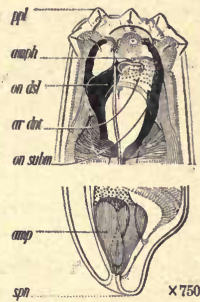
elongated thick-shelled eggs are two and one-half times as long as the body is wide, and appear to be deposited before segmentation begins. The ovaries contain about a dozen developing ova, arranged partly single file, partly irregularly. A pair of ventrally submedian innervated papillæ were noted near the middle of the tail of the female.

From soil from Germany, along with specimens of Heterodera schachtii. Resembles brachyuris, from which it seems easily distinguishable by the large thickshelled eggs. Flemming solution to glycerine. The habitat led to the suspicion that it was feeding on $H$. schachtii, but the writer was unable to establish the fact from the few specimens available for examination. Fig. 51.

38. M. lacustris Cobb. The amphids have the form of "slits," 3 to 4 times as long as wide, and are placed at the base of the lips nearly opposite the apex of

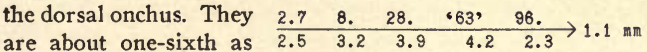
wide as the corresponding portion of the head. Intestine from 15 to 20 cells in girth, the cells closely packed with granules of variable size in such a manner as to give rise to a close and obscure tessellation. The well developed lateral fields are one-third as wide as the body. The anus is - slightly raised. The lining of the rectum is distinctly refractive. The tail is ventrally arcuate, and ends in a blunt spinneret one-fourth as wide as its base, containing a well developed, internally ceratinized spinneret. The three caudal glands are arranged tandem in the anterior

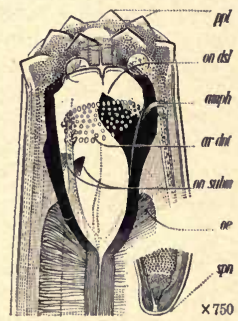
half of the tail, the foremost being opposite the rectum. The spinneret appears to have a needle-shaped valve. A pair of ventrally submedian innervated papillæ has been noted a little in front of the spinneret on the female. The eggs, which appear to occur one at a time in the uteri, are about one and one-third times as long as the body is wide and four-fifths to five-sixths as wide as the body.

Found in fresh water lakes, Michigan, U. S. A.; about the roots of ferns, Panama Canal Zone; and in the Silver Springs, Florida. Resembles M. brachyuris Bütschli, from which it differs in the form of the tail and spinneret. $M$. polonicus Stefanski seems to closely resemble this species. Sublimate to balsam. Fig. 52. 
39. M. polonicus Stefanski. Two minute teeth are found at the base of the buccal cavity. The conoid tail is somewhat arcuate from the raised anus, and ends in a truncated spinneret one-fourth as wide as its base. The three caudal glands are located in a tandem series in the anterior third of the tail.

Description derived from young specimens found in vegetable detritus in the Czarna River, Poland. Said by its author to resemble the next species, No. 40.

40. M. minor Cobb. There are 2 minute, submedian onchi, easily overlooked. Amphids, only one-sixth as wide as the corresponding portion of the head, are

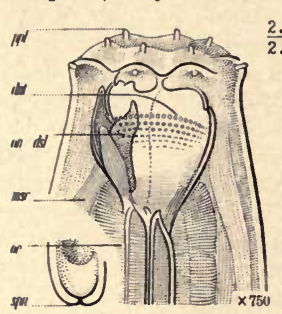
present opposite the

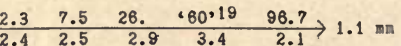
apex of the dorsal tooth, and consist of arcuate, refractive markings having their convex side toward the lips. They appear to be about one-fourth as wide as the corresponding portion of the head. There are excessively minute striæ or dentations of the inner surface of the margins of the lips; these structures are so fine that they might easily escape observation-much finer than the rasp-like teeth of the pharynx. The lining of the œsophagus occupies nearly one-third of the optical longitudinal section. The cells of the intestine contain small, somewhat uniform. rather evenly distributed granules. The anus is slightly raised, the anterior lip being a little more pronounced than the posterior. The conoid tail is rather strongly arcuate, or even more or less bent near the middle. The comparatively well developed spinneret is one-third as wide as the base of the tail, and possesses a valve similar to that found in Mononchulus. The caudal glands are located opposite to, or a little behind the rectum. The lateral fields are twofifths as wide as the body.

This is a cosmopolitan species, found in tropical and temperate regions. It closely resembles $M$. brachyuris of Bütschli, but is smaller and differs somewhat both in the structure of the tail and in that of the pharynx. Osmic acid to water. Fig. 53.

4I. M. brevicaudatus, n. sp. Walls of the pharynx unusually thick. Two exceedingly small, ventrally submedian onchi, opposite the middle of the dorsal onchus.

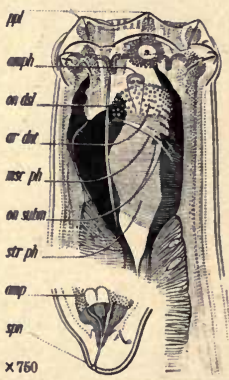

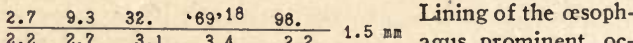
cupying one-third of the optical section. Intestine 6 to 8 cells in girth, faintly tessellated. Contour of the tail like that of the head of a duck whose beak is very short; from the anus onward having roughly an equilateral contaur. Spinneret more elaborate than usual. The ceratinized walls of the vagina are plainly visible. The elongated eggs are two to two and one-half times as long as the body is wide. The ovaries taper but little. About a dozen females have been examined. No males have been seen. Sperm has been seen at the flexure in the ovaries. The species is probably syngonic.

Found about the roots of plants in a cranberry bog, New Jersey, U. S. A. Resembles micrurus and brachyuris in its general form, but differs in the details of the pharynx and in those of the tail. The species is nemativorous, and also feeds upon rotifers. Sublimate to balsam. Fig. 54, in which, as in many of the original illustrations used in this chapter, what at first sight appear to be merely lines used as shading, are in reality carefully charted striæ or laminations of the wall of the pharynx. 
42. M. japonicus, n. sp. The head is not set off in any marked fashion. Amphids present in the form of elliptical markings nearly opposite the apex of the

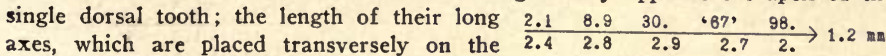
head, is probably about one-sixth as great as that of the diameter of the head. The capacious pharynx is somewhat deeper than the head is wide; the anterior portion has a diameter more than half as great as that of the corresponding portion of the head, while the posterior portion has a diameter about two-fifths as great as that of the base of the head. The single, highly refractive dorsal tooth has its apex somewhat in front of the middle of the pharynx. The rasps consist of 5 to 6 rows of teeth, forming a group whose width is about one-fifth as great as the depth of the pharynx. The wall of the pharynx is strongly developed, and is very finely transversely striated in the posterior part. The lining of the esophagus is an exceedingly distinct feature throughout its length, and appears to occupy about one-fourth of the optical longitudinal section. There is a small cardia. Intestine, about 8 cells in girth, very obscurely tessellated. The short, blunt, arcuate conoid tail is truncated at the terminus, which has a diameter about one-fourth as great as that of the base. The lateral fields are about one-third as wide as the body, and are composed of 2 rows of cells containing relatively large nuclei.

This species rather closely resembles a number of others, and it is by no means certain that it is not identical with some one of them, perhaps consituting a variety. Curiously enough, it was found in Mississippi Bay, Yokohama, Japan. Sublimate to balsam.

43. M. tridentatus de Man. At the base of the pharynx are 2 minute denticles. Amphids unknown. Pharynx more or less triquetrous, with three well developed,

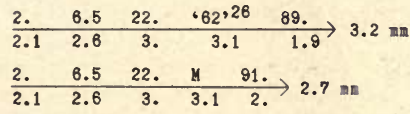

double, longitudinal ribs reaching from end to end. Esophagus at first slightly swollen. Intestine distinctly tessellated. The arcuate tail is conoid to the terminus, which is about one-sixth as wide as the base of the tail. Each of the slender, arcuate spicula is cephalated by contraction and is supplied throughout its length with a median stiffening piece, and is crossed near its distal extremity on the outside by one of the rather broad, two-pointed accessory pieces, which are one-half as long as the spicula. Supplementary organs 15 to 17 , apparently conical, 2 to 3 times as far apart anteriorly as posteriorly, where they are nearly contiguous. The posterior member of the series, the whole of which is about one and one-half times as long as the tail, is somewhat smaller than the other members, and is located a short distance in front of the anus. On the tail itself are a number of papillæ, dorsal as well as ventral; 2 pairs on the anterior half, ventral, and 2 pairs on the posterior half, dorsal. It remains uncertain whether there is a spinneret and caudal glands.

This striking species seems to be widespread in Europe in moist soil, and is rather common. Fig. 55 (after de Man). 
44. M. gracilicaudatus n. sp. Intestine about 12 cells in girth. The anterior fifth of the tail is arcuate conoid in such fashion that at the beginning of the second

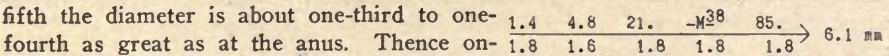
ward the tail tapers very gradually through the middle third, the posterior portion being cylindroid and somewhat narrower than the spicula. These latter are uniformly arcuate, and about one and one-fourth times as long as the anal bodydiameter. At their widest part, toward the proximal end, they are about onefifth as wide as the corresponding part of the body. They taper gently in each direction, and are not cephalated. The small accessory pieces are of typical form, only about one-fourth as long as the spicula. The series of 19 contiguous supplementary organs is twice as long as the spicula, or equals 3 body diameters. Anteriorly the organs are somewhat larger and also somewhat farther apart. The conical exterior portion of each organ seems to be more or less protrudable. These organs

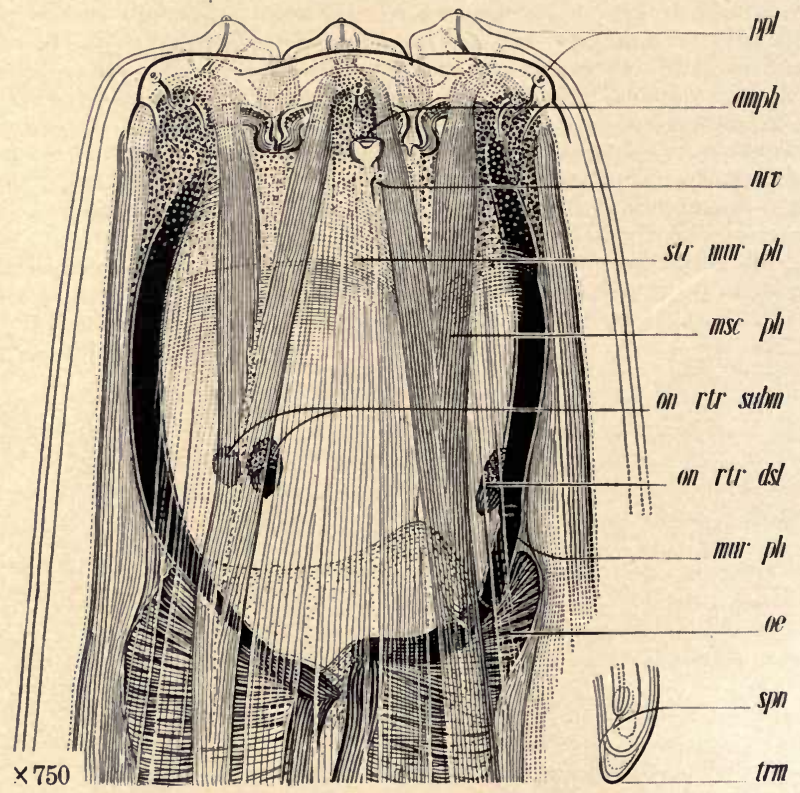

are similar in form to those of major, though they are not echinulate. The anal and post-anal muscles are strongly developed. The anal muscles are found throughout the bulkier portion of the tail. The protruding muscles of the spicula extend backward in the tail to near the point where the tail diminishes rapidly in diameter, and there join the ventral part of the caudal wall. The post-anal papillæ are confined largely to the more massive anterior fifth of the tail. On each side there is a ventrally submedian row about as long as the spicula, consisting of 4 to 5 members. Coextensive with them is a ventral row of 3 to 4 papillæ. A little distance behind these, where the tail begins to be smaller, there are 2 dorsally submedian 
innervated papillæ of smaller size. The supplementary organs are plainly innervated and the nerves can be traced through the cuticle and through the body musculature, and seem to be connected with internal more or less ellipsoidal cells whose nature remains unknown. The spinneret is very inconspicuous. The nature of the caudal glands remains uncertain.

Found in marshy ground, Arlington Farm, Virginia, U. S. A., about the roots of Impatiens, in black, clayey soil, with mud. Resembles $M$. dolichurus to a certain extent. It is regrettable that only a few specimens have been available for examination, as it is a species well adapted to throw light on various features of mononch anatomy. Fig. 56 (on the previous page).

Examination of the pharyngeal muscles of this species leads to a partial understanding of the mechanics of the lips. The muscles which move the lips are long and slender, and pass from the lips backward along the surface of the pharyngeal capsule. They join the body wall some distance behind the pharynx. There are 2 separate groups of muscles, extensors and flexors. Of the 6 extensors 2 are shown in the illustration; these are furcated opposite the middle of the pharynx, one half of each going to the lateral lip; the other half to a submedian lip. The joined fulcra of the 6 lips form a framework encircling the head. The extensors pass outside this framework. The flexor muscles are less clearly shown, but are manifestly better developed. A group of 3 is shown lying between the 2 extensors. They appear as faint bands outside the pharynx passing forward, and beyond doubt are attached to the lips inside the ring of fulcra. The musculature of the lips is shown in the illustration and is further explained on page 140 in connection with the general description of the head.

45. M. dolichurus Ditlevsen. Neck tapering but little. Pharynx probably prismatic, and in transverse section somewhat triangular. About 7 denticles about the base, or near the base, of the pharynx. The arcuate tail tapers to the terminus, which is about one-eighth as wide as the base. The author mentions

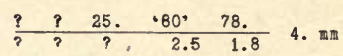

the presence of 3 or 4 inconspicuously developed lobes at the base of the csophagus, and it would appear from his figures that a spinneret and caudal glands are present, the latter forming a tandem series near the anus. Menzel finds in the pharynx of Swiss specimens of this species 3 teeth of equal size; that is to say, the ventrally submedian teeth are equal in size to the dorsal tooth. His specimens are $4 \frac{1}{2}$ to $5 \frac{1}{2} \mathrm{~mm}$. long, and this probably represents the adult size.

Found in moist soil and meadow land, Jutland; Switzerland. Fig. 57 (after Ditlevsen). Menzel shows the papillæ as much less conspicuous, and without the spherical tips. The original material consisted of

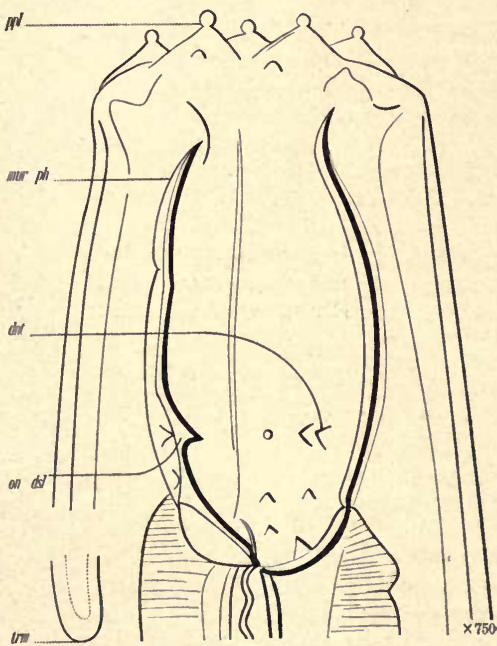
but a single immature female. 
46. M. digiturus Cobb. Amphids occur opposite the anterior part of the pharynx. What appears to be a renette pore occurs just behind the nerve-ring. The anal region is somewhat

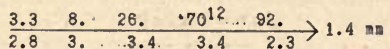
raised. Behind the anus the tail diminishes rapidly in diameter, so that at the end of the anterior fourth it is about three-fifths as wide as at the anus. Thence, for some distance it is cylindrical, but becomes somewhat abruptly convex-conoid in the posterior fifth and ends in a rather narrow, inconspicuous spinneret. The lining of the œsophagus is a conspicuous feature. Intestine not tessellated. The longitudinal ribs of the inner wall of the pharynx are rather conspicuous features, and extend from end to end of the pharynx.

Found about the roots of banana plants, Fij1. Fig. 58.

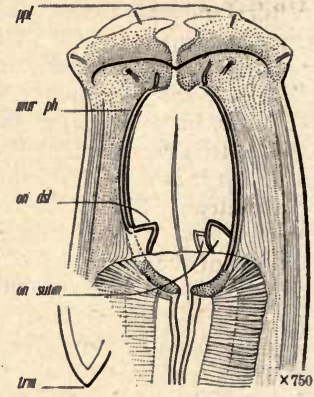

47. M. trichurus, n. sp. This outstanding form has an œsophagus that is slightly swollen where it receives the pharynx. The lining of the cesophagus is

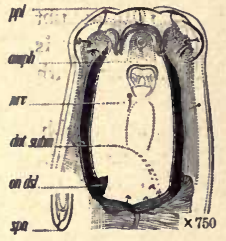

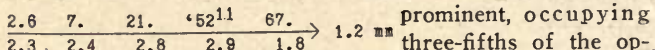
tical section. Longitudinal fields one-fourth as wide as the body. Characters well set forth in fig. 14, p. 146.

Found about the roots of. orange trees, Bahia, Brazil. Bcars considerable resemblance to $M$. gymnolaimus. It appears possible from these investigations that the tropical mononchs, when more fully known, will prove especially interesting. Nearly all the more striking species here recorded are from the tropics. The warm soils of the tropical regions teem with nematodes, many of them no doubt, especialy adapted to the peculiar conditions found there. The predatory mononchs in such soils will naturally enough have responded in structure not only to the climatic conditions, but also to the form and habits of their quarry. Fig. 59 (just above).

48. M. gymnolaimus Cobb. The more or less triquetrous pharynx is strongly three-ribbed. Cardia of such

$$
\begin{array}{rrrrr}
2.6 & 6.8 & 24 . & 67^{15} & 86 . \\
\hline 2.2 & 2.3 & 2.7 & 2.5 & 1.6
\end{array} 2.9 \mathrm{~mm}
$$

a nature as to give rise to a double constriction in the cardiac region. Intestine about 12 cells in girth. What appears to be a renette pore occurs immediately behind the nerve-ring. The lateral fields are about one-fifth as wide as the bodv. The tail tapers regularly to near the terminus; it is,

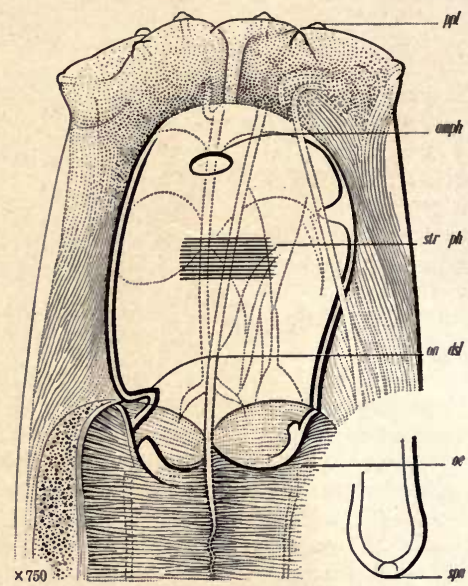
however, cylindroid for a short distance in front of the spinneret. Vulva not prominent. The uterus is as long as the reflexed portion of the ovary, which 
reaches two-fifths the way back to the vulva. The ova are for the most part arranged single file.

This nemativorous species will probably prove cosmopolitan. About roots of banana, Fiji; of Platonia insignis, Rio Janeiro, Brazil; roots of various plants, Arlington Farm, Virginia, U. S. A. Fig. 60 (on the previous page).

49. M. consimilis, n. sp. Amphids, unlike those of gymnolaimus, apparently duplex, located a little behind the base of the lips; one-fourth as wide as the corresponding portion of the head. Wall of the pharynx strongly developed.

Rather closely resem- $3.2 \quad 9.1 \quad 26 . \quad 68^{1} \quad 86$. bles $M$. gymnolaimus, $\begin{array}{lllllll}2.4 & 2.7 & 2.7 & 2.4 & 2.1 & 1.1 & \mathrm{~mm}\end{array}$ but has the walls of the pharynx much more strongly ceratinized, and is of much smaller size. The pharynx is relatively 50 per cent longer. The spinneret is not swollen as in gymnolaimus. Description and figures derived from a single, immature female specimen from about the roots of Platonia insignis Mart., Brazil. The figure of gymnolaimus shows, on the ventral side, near the front of the pharynx, an inward projection, probably representing the optical section of the junction of elements in the pharyngeal wall. No such appearance was observed in consimilis. Fig. 61.

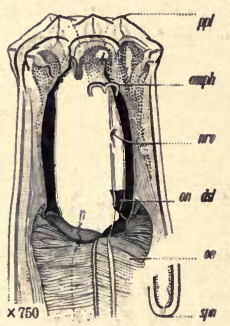

5o. M. rapax, n. sp. Intestine about 12 to 20 cells in girth, faintly tessellated. The female organs are probably double and symmetrical. The conoid tail tapers

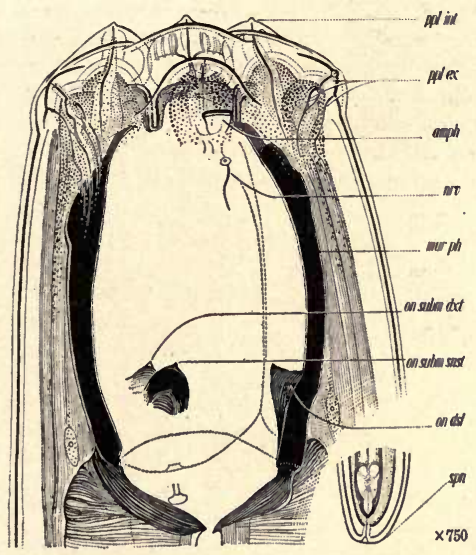
somewhat in front of the anus to a plain, symmetrical, unarmed spin-

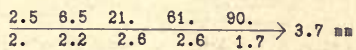

neret about one-sixth as wide as its base. One and one-half tail-lengths in front of the anus there is a constriction in the intestine, which appears to be due to the presence in that region of commissures, as if, possibly, nerves encircled the intestine at that part.

Found about the roots of plants, Arlington Farm, Virginia, U. S. A. Nemativorous. Only young females have been seen. As its name, rapax, indicates, this species is a rapacious one, swallowing other nemas whole, even when half as long as itself. Fig. 62.

51. M. rex Cobb. This "king" of the mononchs has low, broad, inconspicuous labial papillæ that do not interfere materially with the rounded contour of the front of the head. The lips are bulky and powerful. No amphids have been seen. The pharynx is armed with very powerful muscles. The intestine is tessellated. The lateral fields are one-fifth as wide as the body. The conoid tail tapers more rapidly

\begin{tabular}{|c|c|c|c|c|c|}
\hline 2. & 6. & 20. & $\cdot 58,37$ & 81. & \\
\hline 1.8 & 1.7 & 2. & 2.2 & 1.4 & \\
\hline & 5.5 & 20. & $M^{50}$ & 88. & 5 \\
\hline
\end{tabular}
at first, being nearly cylindroid in the posterior two-thirds, where it is about oneeighth as wide as at the anus. The spinneret, which is slightly expanded, bears two ventrally submedian papillæ, after the manner of longicaudatus, but slightly larger 
in proportion. Three caudal glands are present. Each uterus is capable of carrying one and possibly two eggs at a time. These are about one and one-fourth times as long as the body is wide, and half as wide as long. The tail of the male tapers more rapidly at first than that of his mate. Supplementary organs 17 , prominent, closely approximated, equidistant, occupying a space equal to one and one-half times the length of the tail. The spicula are about twice as long as the anal bodydiameter.

Found at depths of from about 200 to 1200 feet in Lakes Manapouri and Wakatipu, New Zealand.

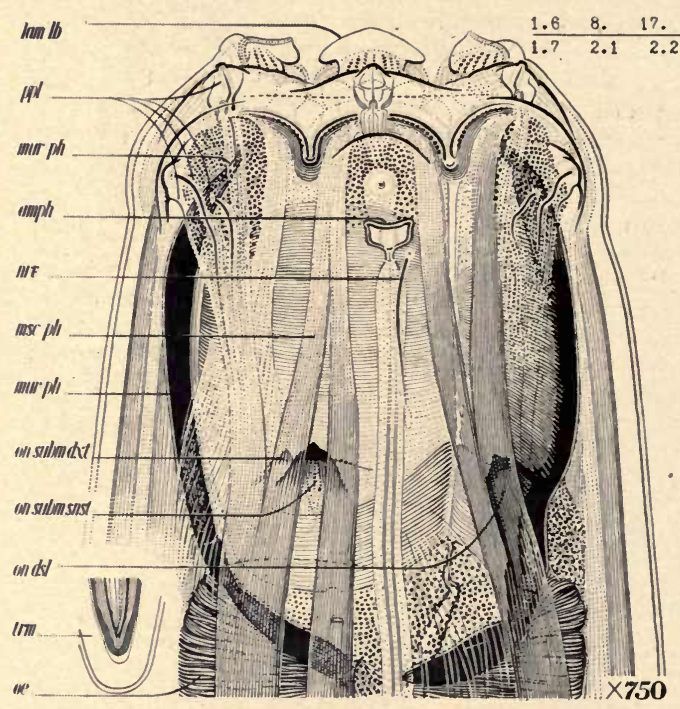

$\frac{63,26 \quad 90}{2.3} \quad 6.2=$

52. M. regius, $n$.

sp. Amphids relatively small, connected with a narrow, internal, apparently tubular element passing backward past the base of the pharynx. Pharyngeal muscles strongly developed, extending backward from the base of the lips to the body wall behind the pharynx. Each of the six lips with a flat, flap-like outer accessory part, which is longitudinally striated at the base. Onchi subequal, the dorsal slightly the stronger. Found about the roots of plants, Arlington Farm, Virginia, U. S. A. Fig. 63.

53. $M$. bathybius Micoletzky. In the figure the onchus labeled "submedian" is really a subdorsal onchus. In addition to this subdorsal onchus there are still

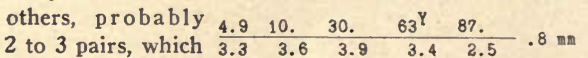
in profile view have a double contour. It remains uncertain whether the other markings seen on the wall of the pharynx are projections or pores.

The author considers this species to be related to $M$. gerlachei de Man, the lip region and vestibule of the two species being similar. However, the armature of the pharynx in the two species is quite different.

Found in mud at a depth of about 300 feet in the Atter Lake, Austria. The description and figures rest

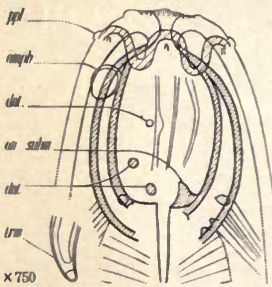
upon the examination of a single immature specimen, and it is probable that the examination of further specimens will improve our knowledge of this interesting form. Fig. 64 (after Micoletzky). For 53b, $M$. fasciatus, n. sp., and 53c, $M$. similis Cobb, see Appendix, p. 184. 
54. M. studeri Steiner. Wall of the pharynx presenting 5 longitudinal ribs, four of them in 2 pairs, the fifth single. At the base of the pharynx numerous small denticles, arranged in rows of from three to four. Esophagus powerful.
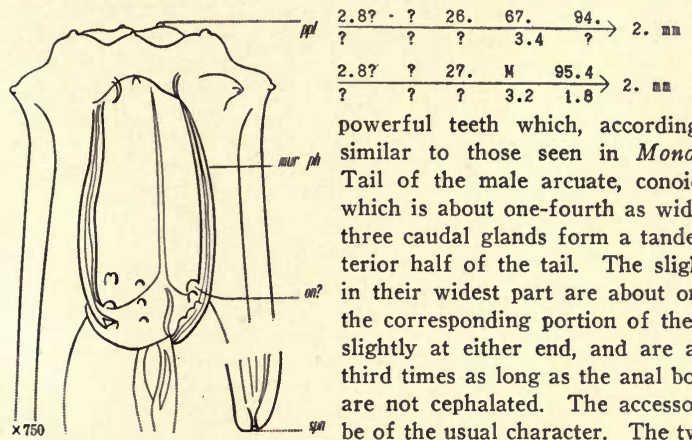
A little in front of the posterior end of the œsophagus there are 3 powerful teeth which, according to Steiner, "are similar to those seen in Mononchus dolichurus." Tail of the male arcuate, conoid to the spinneret, which is about one-fourth as wide as the base. The three caudal glands form a tandem series in the anterior half of the tail. The slightly arcuate spicula in their widest part are about one-sixth as wide as the corresponding portion of the body. They taper slightly at either end, and are about one and onethird times as long as the anal body-diameter. They are not cephalated. The accessory pieces appear to be of the usual character. The twelve short, tubular, equidistant supplementary organs, occupying a space equal to twice the length of the tail, do not interfere materially with the ventral contour. The internal tubular parts are about as long as the spicula are wide. Distance from the anus to the posterior supplementary organ is nearly twice as great as the distance between the adjacent organs. Two pairs of post-anal papillæ were noted.

Found on the island of Ufenau in the Zürich Lake, under moss. Fig. 65 (after Steiner).

55. M. zschokkei Menzel. This species, one male to each three to four females, is found in various parts of the Austrian and Swiss Alps. Neck and œsophagus \begin{tabular}{cccccc}
$3 . ?$ & $?$ & 25. & 666, & 85. & \\
\hline$?$ & $?$ & $?$ & 3.4 & 2.1 & $2.3 .2 \mathrm{~mm}$
\end{tabular} \begin{tabular}{lccccc}
$?$ & $?$ & 25. & $\mathrm{M}$ & 96. & \\
\hline$?$ & $?$ & $?$ & 3.6 & 2.4 & $2.4-3.5 \mathrm{~mm}$
\end{tabular} tapering but little. Small denticles often occur at the base of the pharyngeal cavity. Uterus capable of containing one or two eggs. The blunted terminus of the conoid arcuate tail is about one-eighth as wide as the base. The slightly arcuate spicula are rather slender, each, however, having a stiffening piece in its distal half. Accessory pieces as figured by de Man for his $M$. brachyuris Bütschli. Beginning near the anus the 21 projecting, obliquely conical, equidistant, contiguous supplementary organs, each as high as wide, occupy a space 3 times as long as the tail.

Found in the high Swiss Alps. Fig. 67, after Menzel, who considers that

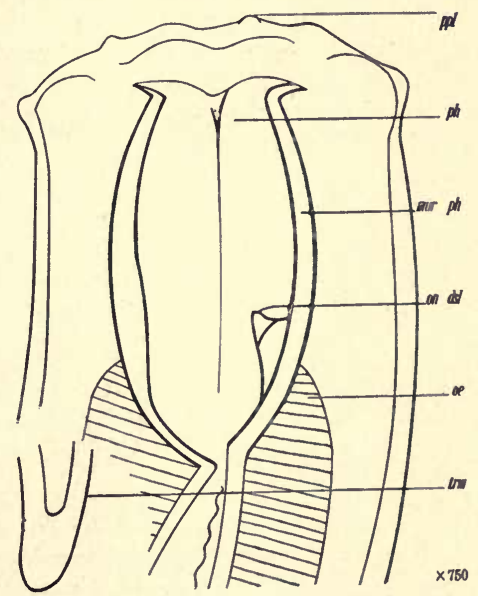
of all the European mononchs at present known, zschokkei has close affinity only with $M$. parous de Man, from which it differs in the position of the onchus and the relative abundance of males. 
56. M. brachylaimus, n. sp. There are two exceedingly minute, almost invisible, apparently rudimentary, ventrally submedian, conical, forward-pointing toothlets at the base of the pharynx. These are not shown in the illustration. Three slightly

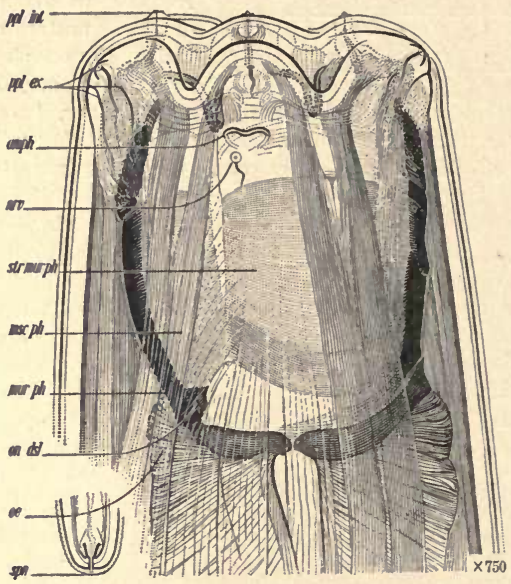

\begin{tabular}{|c|c|c|c|c|c|}
\hline 2.4 & $?$. & 21. & $65^{14}$ & 94. & \\
\hline 2.4 & 2.4 & 2.8 & 3. & 1.9 & 1 \\
\hline 2.3 & 7.4 & 19. & $-m-42$ & 94. & \\
\hline 2.2 & 2.3 & 2.5 & 2.5 & 2. & \\
\hline
\end{tabular}

arcuate, duplex, longitudinal ribs strengthen the walls of the pharynx and reach from end to end, one being dorsal, the other two ventrally submedian. Amphids difficult to see. Esophagus at first slightly expanded. The œsophageal lining is not so prominent as in many other species; its optical section finds expression in 3 or 4 parallel lines, occupying about one-fourth of the longitudinal optical section. There is an inconspicuous, flattish cardia. Intestine about 8 to 12 cells in girth, faintly tessellated. The tail is conoid to the plain, unarmed conoid terminus. The base of the spinneret is about one-fifth as wide as the base of the tail. The clavate caudal glands form a close tandem in the anterior third of the tail. The anus has rather conspicuous, with well developed, though not protruding lips. There is a pair of innervated lateral papillæ near the middle of the tail of the female. Both in front of and behind the vulva, especially behind, there are inconspicuous papillæ, occupying a distance equal to twice to thrice the length of the body-diameter. The two equal, arcuate, rather slender spicula are one and one-half times as long as the anal body-diameter, and are of nearly uniform size throughout the greater part of their length. However, beginning near the slightly truncated tips they taper slightly in the distal sixth. The proximal ends are also narrowed slightly and lie well toward the dorsal side of the body, at any rate when the body is incurved. The two accessory pieces, each about two-thirds as wide as the spicula, are located opposite the distal portions of these latter. When seen in profile they appear to lie nearly at right angles to the body axis, and seem to cross the distal parts of the spicula. They are about two to three times as long as wide, and their distal extremities are diminished and bifurcated, the two prongs of the fork forming a U-shaped figure. When at rest these accessory pieces, as usual, are well inside the anus, in fact appear as if lying immediately under the lateral fields. In front of the anus there is a uniform series of about 16 equidistant, closely approximated, innervated supplementary organs, occupying a distance about equal to the length of the tail. When the tail end of the body is incurved, each organ appears on the ventral contour as a flattish cone, at the apex of which is a nerve ending, which from this point extends inward and forward.

Brown, sandy soil, Arlington Farm, Virginia, U. S. A. This nemątivorous 'mononch is distinguished from all others by the relative broadness of the pharynx, and by the position and size of the basal onchi. Especially well adapted to show the structure of the lip muscles and other organs of the head. Fig. 67. 
57. M. acutus, n. sp. Esophagus bulbous at first, the swelling being prolate Intestine about 10 cells in girth. The arcuate conoid tail is practically acute. There is a pair of sublateral, $\begin{array}{llcccc}2.7 & 3.2 & 3.3 & 3.4 & .2 .6 & 2.1 \mathrm{~mm}\end{array}$ innervated papillæ near the beginning of the posterior third of the tail. Anus not raised. Fig. 68.

Found about the roots of rhubarb in loose, brown, sandy soil, Arlington Farm, Virginia, U. S. A. Nemativorous. One specimen was observed which had swallowed another mononch. This is one of the most instructive forms. It is desirable that the more minute structural details of nemas be very carefully investigated with a view to increasing our knowledge of their comparative anatomy. The different parts of the digestive organs of nemas, small as they are, are as profoundly modified in harmony with the nature of the

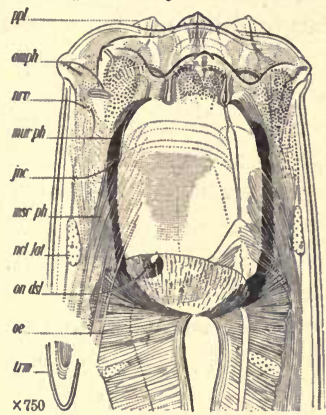
food as those of higher animals. The digestion of starch requires a different organic mechanism from that for digesting meat. The nemas have specialized to a high degree in the matter of food, and their digestive organs are correspondingly diversified.

\section{Nemas and Soll Fertility}

No one with a grain of imagination can engage in such studies as the foregoing without sooner or later asking himself questions of a general nature concerning the biology of the soil, for he gradually comes to sec how almost infinitely numerous and varied are the organisms inhabiting it; a population in which the nemas are but an element. The answers to these questions will lead to a new view of soil fertility.

The revolution wrought by Liebig's ideas concerning the chemistry of the soil spent itself only to show us that, grand as it was, it was little more than an overture. Subtler forces and more intricate relationships than any indicated in these earlier conceptions must be considered. We now see, or are beginning to see, that the value of manure and of the rotation of crops is to be explained not simply on the basis of the exhaustion of certain elements in the soil, but also, and probably in some instances mainly, on the basis of an opposite process, an accumulation of certain organisms and substances in the soil. Who knows but that the existence of annuals may be due in some measure to this latter fact? May not these elaborate provisions for the distribution of the seeds of annuals be in some measure a means of escaping these accumulated hostile forces in the soil?

After all the word Agriculture is more or less of a misnomer. We cultivate, not so much the field, as plants. What we are really after is sunshine, for we are lost unless we can convert our infinitesimal part of the energy of this nearest star into food, clothing and shelter. This we do by utilizing the life forces of certain plants and animals, and these are not so few as we dreamed in our older philosophy, for none of our "domesticated organisms" can any longer be considered by itself. Every 
such organism is reared in the midst of a host of other organisms, visible and invisible, and often it is these others that determine agricultural success or failure.

The soil is the habitation of a vast community of beings with all the attributes of other huge agglomerations of living things having varying needs, instincts and aspirations; and it is just as inappropriate to look upon it as inorganic as it would be to look upon a great city as merely an agglomeration of hills, streets and houses. Here in the soil are beings in enormous variety; multiplying, growing, dying ; competing, fighting, cooperating one with another, with an activity almost if not quite defying the imagination, and we need what may be called soil biologists or geobiologists, who shall understand, as far as possible, this interplay of life forces that gives us food, fiber and fuel. To a considerable degree our progress in agricultural knowledge in the not distant future will be in proportion to the firmness with which we lay hold of and act on this idea.

\section{SUMMARY}

1. The genus Mononchus is composed of scores, possibly hundreds, of species, divisible into distinct subgenera. The number of known species is hereby more than doubled.

2. The genus is of world-wide distribution, and many of the species are cosmopolitan. Mononchs occur in all kinds of arable soil, sometimes in hundreds of millions per acre.

3. Most mononchs, probably all, are strictly carnivorous. They feed on a variety of living microzoa, prominent among which are other nemas.

4. Injurious nemas are devoured by mononchs, and it is desirable that this trait of mononchs be carefully investigated with a view to utilizing it, if possible, in diminishing the enormous crop losses due to plant infesting nemas-losses amounting to many millions of dollars annually.

5. The lips are moved by long muscles, connecting proximally with the body wall behind the pharynx. These muscles lie along the outer surface of the pharyngeal capsule and act in such a way as to pull the lips inward and outward radially about a series of fulcra existing in a framework encircling the head along the margin of the pharynx. The lips are the mechanical complements of the dorsal tooth and denticles. While most mononchs bolt their food, some give it a certain degree of mastication. The appetite is sometimes voracious.

6. Many mononchs, probably most, are hermaphroditic, even to the degree of syngonism. In the typical case investigated the minute sperm cells of female origin are functional.

7. Well developed glands, salivary in character, occur in the mononch œsophagus, and empty dis ectly into its lumen, and both indirectly and directly into the mouth cavity. 
8. What appears to be an excretory pore of the usual type seems universal near the nerve ring.

9. The outer labial papillæ are the homologues of the ordinary cephalic setæ of other nemas, and are therefore most probably tactile in function. This leaves it probable that the inner papillæ are devoted to the senses of taste and smell.

10. Amphids are always present in the form of small lateral more or less elliptical structures near the lips, and are connected with internal elements extending inward and backward.

11. A functional spinneret is present in a majority of the species.

12. The cuticle is always finely transversely striated.

13. Mononchs probably moult four times.

\section{BIBLIOGRAPHY}

(1) Annett, Dutton, and Elifott.

1901. Report of the Malaria Expedition to Nigeria, Part 2. Liverpool School of Trop. Med., p. 1-92.

(2) Bastian, H. C.

1866. Monograph on the Anguillulidae. In Trans. Linn. Soc. London, v. 25, p. $73-184,6$ pl.

(3) BRAKENhoFf, $\mathrm{H}$.

1914. Nematodenfauna des Nordwest-deut. Flachlandes. In Abhandl. Naturw. Ver. Bremen, Bd. 22, p. 267-311, 3 pl.

(4) Bütschli, O.

1873. Beiträge zur Kenntnis der freilebenden Nematoden. In Nova Acta Acad. Leopold.-Carol., Bd. 36, p. 1-124, 11 pl.

(5) Bürschli, O.

1876. Freilebenden Nematoden und die Gattung Chaetonotus. In Ztschr. Wiss. Zool., Bd. 26, p. 363-413, 4 pl.

(6) Совв, M. V.

1915. Nematodes of the Michigan Lakes. In Trans. Amer. Micros. Soc., v. 34, p. $20-47$.

(7) Совв, N. А.

1888. Beiträge zur Anatomie und Ontogonie der Nematoden. In Jenaischen Ztschr. Naturw., Bd. 23, p. 41-76, pl. 3.

(8) Совв, N. A.

1890. A nematode formula. In Agr. Gaz. N. S. Wales, v. 1, p. 131-136.

(9) Совв, N. А.

1893 Plant diseases and their remedies; sugar cane. In Agr. Gaz. N. S. Wales, v. 4 , p. $808-833$, 16 fig.

(10) Совв, N. А.

1893. Plant diseases and their remedies; sugar cane. Separate Ed. for Queensland Govt., p. 31-56, 16 fig.

(11) Совв, N. A.

1893. Nematodes, mostly Australian and Fijian. Macleay Memorial Vol.,

Dept. Agr. N. S. Wales, Misc. Pub. No. 13, p. 3-59, 7 pl., 10 fig.

(12) Совв, N. A.

1904. Free-living fresh-water New Zealand nematodes. In Proc. Cambridge Phil. Soc., London, p. 363-374, 4 fig. 
(13) Совв, N. A. 1907. Fungus maladies of the sugar cane. Hawaiian Sugar Planters' Exp. Sta., Div. Path. and Physiol. Bul. 5, p. 163-196, 4 fig.

(14) Совв, N. A.

1913. Notes on Mononchus and Tylenchulus. In Jour. Wash. Acad. Sci., v. 3 , p. $287-88,2$ fig.

(15) Совв, N. A.

1914. Nematodes and their relationships. In U. S. Dept. Agr. Yearbook, p. $457-490,19$ fig.

(16) DADAY, J.

1897. Die freilebenden Süsswasser. Nematoden Ungarns. In Zool. Jahrb., Abt. System. Geogr. u. Biol. Thiere, Bd. 10, p. 91-134, 4 pl.

(17) DANAY, J.

1901. Mikroskopische Süsswasserthiere aus Deutsch-Neu-Guinea. In Természet. Füzetek, Bd. 2-25, p. 1-56, 3 pl.

(18) DADAY, J.

1908. Adatok német-kelet Afrika Mikrofauna. In Math és Természet. Ertesitö, Bd. 26, p. 1-57, 27 fig.

(19) DADAY, J.

1910. Fresh-Water Nematodes of German East Africa. In Zoologica, v. 23.

(20) DE MAN, J. G.

1876. Onderzoekingen over vrij levende Nematoden. In Tijdschr. Nederland. Dierk. Ver., Deel 2, p. 78-196, 11 pl.

(21) DE MAN, J. G.

1880. Die einheimischen Nematoden. In Tijdschr. Nederland. Dierk. Ver., Deel 5, p. 1-104.

(22) DE MAN, J. G.

1884. Die Nematoden der niederländischen Fauna. 206 p. 34 pl. Leiden.

(23) DE MAN, J. G.

1885. Helminthologische Beiträge. In Tijdschr. Nederland. Dierk. Ver., Ser. 2, Deel 1, p. 1-10, 3 pl.

(24) DE MAN, J. G.

1904. Nématodes Libres. In Résultats du Voyage du S. Y. Belgica, Expédition Antarctique Belge, 1897-99, Zoologie. 51., 11 pl. Anvers.

(25) DE MAN, J. G.

1906. Nématodes de l'Ile de Walcheren. In Ann. Soc. Roy. Zool. Malacol. Belg., p. 156-174, 17 fig.

(26) dE MAN, J. G.

1907. Nématodes libres de la Seine. In Ann. Biol. Lacustre, t. 2, p. 9-29, $3 \mathrm{pl}$.

(27) DE MaN, J. G.

1912. Helminthologische Beiträge. In Zool. Jahrb., Sup. 15, Bd. 1, p. 439-464, 2 pl.

(28) Ditlevsen, $\mathrm{H}$.

1911. Danish free-living nematodes. In Vidensk. Meddel. Naturhist. Forening, Bd. 63, Heft 1, p. 213-256, 5 pl.

(29) Dujardin, F.

1845. Histoire Naturelle des Helminthes. 654 p., 12 pl. Paris.

(30) HOFMÄNNER, B.

1913. Freilebenden Nematoden. In Zool. Anz., Bd. 42, p. 413-418, 4 fig.

(31) HOFMÄNNER, B.

1913. Nématodes libres du Lac Leman. In Rev. Suisse Zool., t. 21, no. 16 , p. $589-658,2$ pl. 
(32) Hofmänner, B., and Menzel, R.

1915. Freilenbenden Nematoden aus der Schweiz. In Rev. Suisse Zool., t. 23 , p. $109-243,3$ pl.

(33) JÄGERSKIÖLD, L. A.

1909. Freilenbende Süsswassernematoden. In Süsswasserfauna Deutschlands, (Brauer), Jena, Heft 15, p. 1-46, 65 fig.

(34) Klausener, C.

1908. Fauna eines hochgelegen Alpensees. In Internat. Rev. Hydrobiol., Bd. 1, p. 142-152, 1 pl.

(35) Marcinowski, Kati.

1909. Parasitisch Nematoden. In Arb. K. Biol Anst. Land. u. Forstw. Bd. 7, Heft 1, p. 1-192, 1 pl., 76 fig.

(36) Menzel, R.

1912. Freilebande Nematoden, Umgebung von Triest. In Rev. Suisse Zool., t. 20, p. 536-542, 2 fig.

(37) Menzel, $R$.

1913. Mononchus zschokkei n. sp. In Zool. Anz., Bd. 42, p. 408-413, 4 fig.

(38) MFnzel, R.

1914. Mikroskopische Landfauna der Schweiz Hochalpen. In Arch. Naturgesch., Abt. A, Heft 3, p. 1-98, 1 pl., 16 fig.

(39) Micoletzky, H.

1912. Fauna einiger Seen Salzburgs. In Zool. Jahrb., Abt. System., Geogr. u. Biol. Thiere, Bd. 33, p. 421-444.

(40) Micoletzky, H.

1913. Nematoden der Ostalpen. In Sitzber. K. Akad. Wiss. (Vienna), Math. Naturw. K1., Bd. 122, Heft 4, p. 543-548.

(41) Micoletzky, H.

1914 Nematoden der Ostalpen. In Zool. Jahrb., Abt. System., Geogr. u. Biol. Thiere, Bd. 36, p. 331-546, 10 pl., 1 fig.

(42) Micoletzky, H.

1915. Fresh-water nematodes of South Africa. In Ergeb. Bot. Forsch. Reise Deut. Ostafrika.

(43) Oerley, L.

1880. Monographie der Anguilluliden. In Természet. Füzetek, Bd. 4, Heft 1-2, p. 1-165, 7 pl.

(44) Plotnikoff, B.

1901. Nematoda, Oligochaeta, und Hirudinea. In Ber. Biol. SüsswasserStat. K. Naturf. Gesell., St. Petersburg, Bd, 1, p. 244-251, 2 fig.

(45) Stefanski, W.

1914. Nématodes du Bassin du Léman. Thése de l'Université de Genève.

(46) Stefanski, W.

1915. Nématodes de Pologne. In Zool. Anz., Bd. 45, p. 346-349, 6 fig.

(47) Steiner, G.

1913. Freilebenden Nematoden aus der Schweiz. In Arch. Hydrobiol. Planktonk., Bd. 9, p. 259-276, $29 \cdot$ fig.

(48) Steiner, G.

1916. Freilebenden Nematoden. In Zool. Anz., Bd. 46, p. 336-368, 11 fig.

(49) Steiner, G.

1916. Freilebenden Nematoden von Nowaja-Semlja. In Zool. Anz., Bd. 47.

(50) ZsснокKE, F.

1900. Die Tierwelt der Hochgebirgsseen. In Nouvean Mém. Soc. Helvetique Sci, Nat., t. 37, p. $1-400,8$ pl. 


\section{APPENDIX}

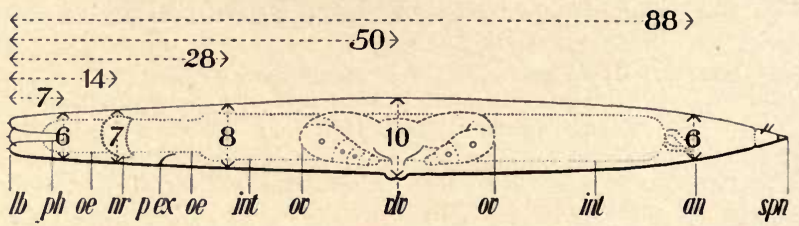

The above diagram illustrates the decimal formula used herein. $6,7,8,10,6$ are the transverse measurements, while $7,14,28,50,88$ are the corresponding longitudi-

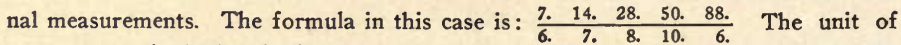
measurement is the hundredth part of the length of the body, whatever that may be. The measurements become, therefore, percentages of the length. The absolute length is given in millimeters as a final non-paired term. The measurements are taken with the nema viewed in profile; the first are taken at the base of the pharynx, the second at the nerve-ring, the third at the cardiac collum or end of the neck, the fourth at the vulva in females and at the middle (M) in males, the fifth at the anus.

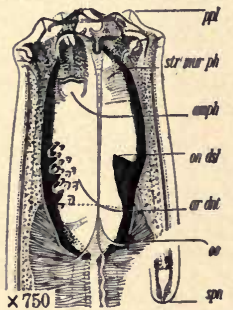

Fig. 68. M. recessus, n. sp. No. 21-b. Striæ excessively fine. Lips with 14 papillæ. Intestine about 5 cells in girth, $2.8 \quad 8.8 \quad 30 . \quad 63^{12} \quad 91.5 \rightarrow 1.4$ faintly tessellated. Cau-

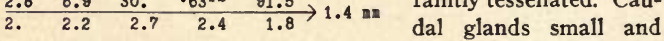
inconspicuous. Tail tapering rapidly on the ventral side behind the anus; thence onward arcuate and conoid, with spinneret. Habitat: soil of a tomato field, Naranjo, Fla. This species, together with decurrens and dentatus, constitute the new sub-genus Sporonchulus; it is interesting to note that so far as known all of them are tropical and below the average size.

Fig. 69. M. decurrens, n. sp. No. 21-c. Striæ excessively fine. Intestine 7 to 8 cells in girth, the cells packed with granules, which, however do not give rise to a tessel- $\begin{array}{ccccccc}2.9 & 4 . & 4.2 & 4.8 & 2 . & 1.2\end{array}$ lated appearance. Tail arcuate conoid, tapering from in front of the anus. No spinneret, or doubtful; no caudal glands. Excretory pore (?) just behind the nerve ring. Anus more or less elevated. Habitat: Plant Introduction Gardens, Miami, Fla.

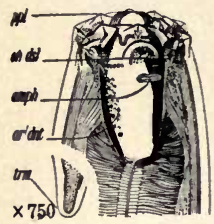

53-b. M. fasciatus, n. sp. Like M. bathybius, No. 53. Characterized by large, powerful lips, set off by a deep constriction, in $\begin{array}{llllll}2.4 & 5.6 & 16 . & 644^{\prime 27} & 90 .\end{array}$ the depths of which is a double refractive line. $\begin{array}{lllllll}2.3 & 2.3 & 2.4 & 2.4 & 1.8 & 2.2 \mathrm{~mm}\end{array}$ Teeth 3, small, equal, sub-basal; tail conoid, no spinneret. Papillæ 14. Habitat: About roots of camphor, Florida. Nemativorous.

\section{Erratum.}

After Soil Science went to press it was found that similis and tenuis are invalid as designations of new mononchs, owing to Mononchus similis Cobb 1893, and Mononchus tenuis Daday 1908, the latter not a mononch. The present $M$. similis is therefore hereby changed to $M$. subsimilis, and $M$. tenuis to $M$. subtenuis. The reference to $M$. megalaimus in "Nematodes mostly Australian and Fijian," Cobb, is a misprint for $M$. gumnolaimus. The overlooked $M$. similis is as follows:

53c. M. similis Cobb. 3.3 8. 24. 11' $58^{\prime \prime 1} \quad 86 . \quad$ Pharynx three-fifth as

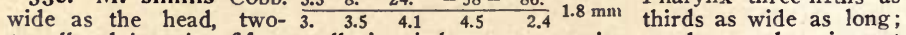
tessellated intestine fifteen cells in girth; anus conspicuous, depressed; spinneret one-eighth as wide as the base of the tail. Habitat: About roots of cane, Harwood, Clarence River, Australia. 

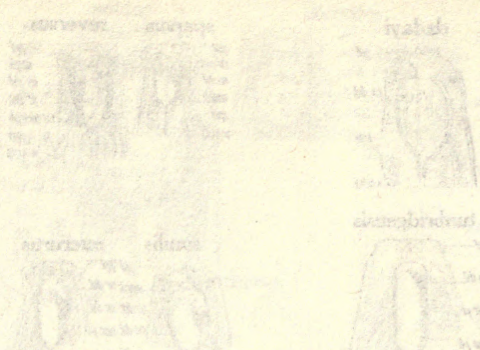

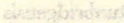

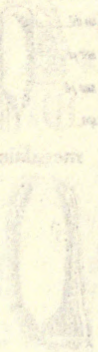

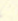
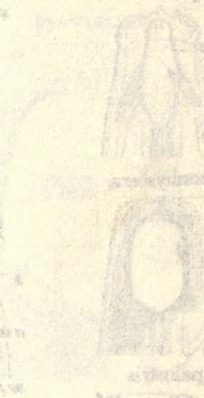

$$
\begin{aligned}
& \text { ausafictio }
\end{aligned}
$$

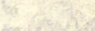

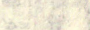$$
x=2-5
$$

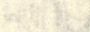$$
\text { bilangate }
$$
zorivistit

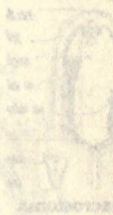

(25)
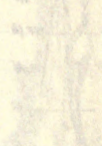

$$
\text { sit. }
$$
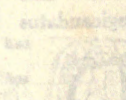

$\sqrt{2}+5$
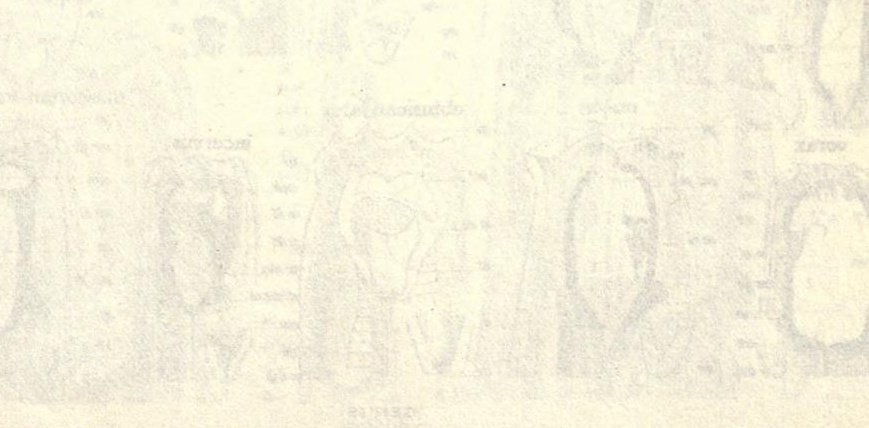
SUBGENERA

MONONCHUS

MYLONCHULUS
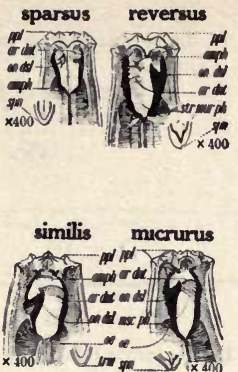

-

woo 1 .

monhystera

\% Rave

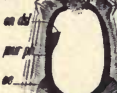

iny

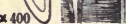

palustris

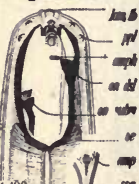

macrostoma

त5 ग >

$\int \sqrt{-m}$ 1) 100

${ }^{1} f^{*}$ $\times 400$ InIII longicaudatus (1) vorax

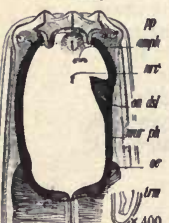

tunbridgensis

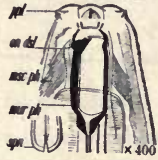

dadayi
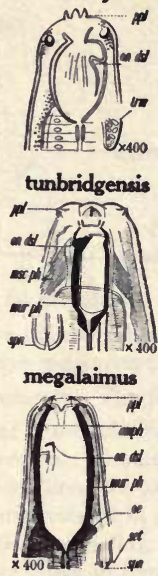

truncatus

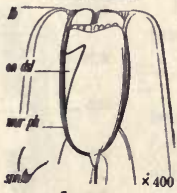

obtusus

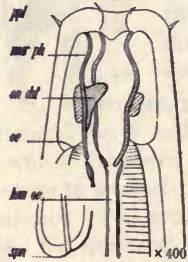

gerlachei
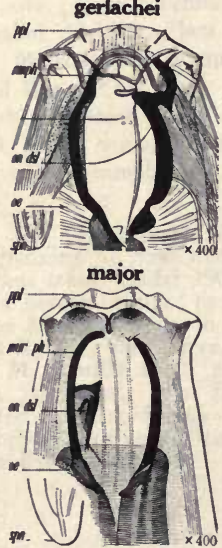
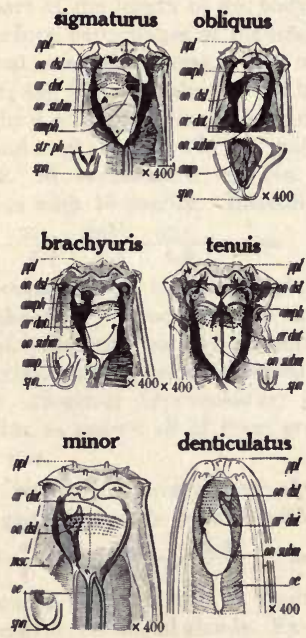

brevicaudatus lacustris

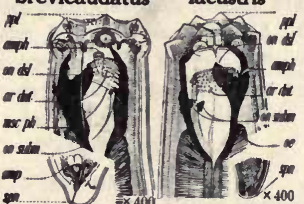

obtusicaudatus

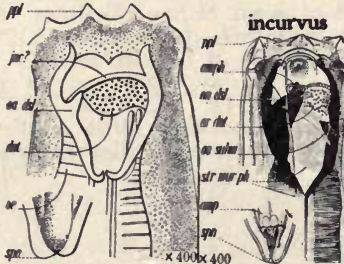

PRIONCHULUS
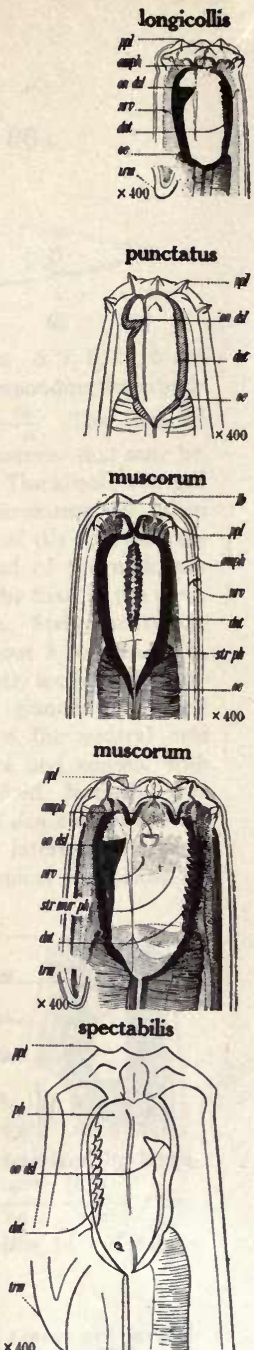

muscorum var microlaimus

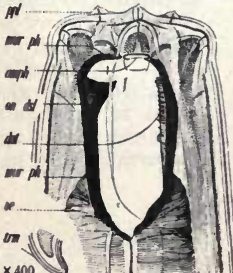

GENUS

MONONCHUS 


\section{Cesmantary}

knongt wat

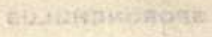

Mirkinobis?

cototions

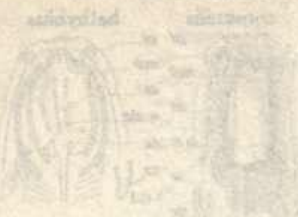

tratit

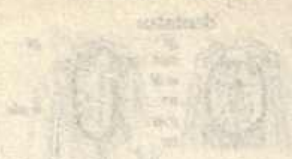

armed $x^{2}=$

rabuits

mentanets

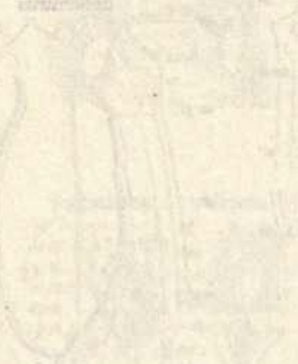


Filter-bed Nemas:

\section{NEMATODES OF THE SLOW SAND FILTER-BEDS OF AMERICAN CITIES}

(Including new genera and species)

\section{WITH NOTES ON HERMAPHRODITISM AND PARTHENOGENESIS*}

Contributions to a Science of Nematology, ViI

By N. A. Совв

United States Department of Agriculture

The nemas here described were collected from the filter-beds of several American cities, incident to a rather extensive investigation of nemas of economic interest. A study of them has afforded me such an interesting and suggestive glimpse of the biological conditions in slow sand filter-beds that I venture to think an account of it may be of some slight use to sanitarians, and to engineers connected with city and town water works. The hiological observations, more particularly those on the vanishing series of spermatozoa in syngones, may prove of interest to zoologists and geneticists.

\section{NATURE AND NUMBER OF THE ORGANISMS FOUND}

Few Green Organisms. Comparatively few green organisms occur in covered slow sand filter-beds, especially if the water passes through a subsidence reservoir before entering the beds. Practically all the living forms found are colorless or nearly so, and most of the larger ones are animal. To me the most striking organisms in every sample of sand examined were the nemas.

Period of Use. Renewal. From time to time a few inches of the topmost sand of slow filter-beds is renewed; the old sand is removed and fresh sand spread in its place. The period of use, the time between any two successive renewals, varies from a few weeks to a few months, according to the practice of the engineer in charge. Toward the end of a period of use the number of nemas in the topmost three inches of a bed often mounts to hundreds of millions per acre, and sometimes exceeds a thousand millions per acre. At this latter figure each glass of drinking water must percolate through sand containing at least about a thousand nemas.

Dozens of Species Found. Often the nema population is of a mixed character, but sometimes it is comparatively homogeneous. On

*Waverly Press, Baltimore, Jan. 11, 1918. 
one occasion, at the end of a period of use, I found ninety-six per cent of the nemas to consist of but a single species. About thirty species were found inhabiting the various beds examined, twenty-five species being found to inhabit the beds of one city. My observations make no pretence of being exhaustive, and I presume further research may easily double these numbers. Most of the species are of only occasional occurrence; those figuring prominently in the activities of the beds are only about half a dozen in number, and of these not all are equally important.

THE MORE COMMON SPECIES

Commonest Species Carnivorous. Of the four more important species, (1) Mononchus longicaudatus, (2) Ironus ignavus, (3) Tripyla monohystera and in a lesser degree (4) Ironus longicaudatus, I have shown that all are carnivorous, that they feed upon a variety of living organisms, and that no one of them confines itself to a single kind of food. Mononchus longicaudatus, for instance, feeds upon several species of nemas, upon rotifers, and upon a variety of protozoa. The same is true of Tripyla monhystera. In the struggle among these filter-bed organisms it appears that sometimes one species may almost annihilate others. This accounts for such cases as that in which Mononchus longicaudatus constituted ninety-six per cent of the nema population of a bed.

\section{ROTATION OF THE FLORA AND FAUNA}

Seasonal Fluctuations. I know little about the seasonal fluctuations; merely that they exist and that sometimes they are very marked. For instance, on January 6 samples of sand were gathered from various locations on a Washington filter-bed. This bed had been in operation about six months, a period longer than in the case of any previous examination (summer collections). About the same range of species was found in this sand as had been found in all previous experience taken together, but the smaller nemas, including Monhystera, were much more abundant in this than in previous collections, and seemed to be thriving. A large Dorylaimus that had been extremely rare in previous collections was fairly common in this January collection. Achromadora minima was also more common. Finally there were one or two small species not hitherto found, Cylindrolaimus obtusus and a Rhabdolaimus. Whether these faunal differences were due to the winter season or to the long time the bed had been in use remains in certain instances undetermined,--very likely some of them were due to both causes.

Economic Bearings. Apart from seasonal fluctuations, there is a rotation in the fauna and flora incidental to the management of the 
beds. Beginning with a new period of use, it appears that minute species, e.g., bacteria and protozoa, having a short life cycle and suited to the new conditions, first make their appearance; these multiply and become the food of succeeding species, which in their turn give place to others. The problems presented are of great biological interest, and may not be without some bearing on public health. Beyond doubt they have a more or less important relation to the economical and effective management of the filter-beds.

\section{DETERMINING FACTORS OF FILTER-BED POPULATION}

Organisms other than Nemas. Many other kinds of organisms are found in filter-beds, some of them in far greater numbers than the nemas. Bacteria, fungi and protozoa occur, of course, in abundance, and are, I believe, the organic basis on which is built up the later animal population consisting of organisms of larger size, such as the nemas. Rotifers are not uncommon. Small oligochaetes,--earthworms,--occur, especially after long use, but never in such myriads as in sewage. Small crustaceans, such as daphnia and cyclops, sometimes occur, but I have never seen them in large numbers. Occasionally aquatic insects are found. The filter-beds of each city present biological peculiarities dependent upon the source of the water supply, for the biological characteristics of filter-beds depend to a considerable extent on the climatic and geological conditions prevalent on the water-shed from which the supply is drawn.

\section{PHYSIOLOGICAL SIGNIFICANCE OF FILTER-BED NEMAS}

Illness from Change of Water. It is a well-accepted idea among physicians, as well as laymen, that a change of drinking-water may cause intestinal disorders; why they are thus caused is not always clear. If the waters in question are widely different in composition, for instance one soft and the other hard, it is easy to understand how intestinal derangements might follow a change from one to the other; but are the derangements due to change of water always associated with such marked chemical differences? Do not intestinal disturbances follow changes of water in which the usual tests would show but very slight differences?

Soluble Excreta in Drinking-water. Is it possible that slight quantities of organic substances found in drinking water and of a character as yet unknown, might, under some circumstances, exert a powerful physiological influence? If this question be answered affirmatively, a wide field of investigation is opened up in connection with potable waters, and it is in this connection that the present researches are 
definitely suggestive. The filter-beds, after they have been cleaned and again put to use, soon become the habitat of a succession of various organisms, animal as well as vegetable, so that at the end of a periorl of use it is no exaggeration to say that the sand through which the water percolates swarms with them. To state a definite case quantitatively, it has been shown during these examinations that such filter-bed sand may contain hundreds of millions of nemas per acre in the top three inches. Each of these nemas is excreting material of which the soluble portions must pass into the city's water supply, and if in the course of its passage through the filters, flumes, and delivery pipes this soluble matter is not precipitated or otherwise altered, it is present in every glass of drinking-water.

FLAVOR OF DRINKING WATERS

Drinking-water Connoisseurs? The excreta of any given filter-bed organism must be different from that of any other, and though the differences may be slight between similar organisms, there are good reasons for thinking that the differences among the organisms of the filter-beds of different cities are great enough to cause material differences in the nature of their excreta. Such soluble parts of the excreta as pass into the drinking-water must play a rôle in imparting to the water its flavor and other qualities. This is enough to make one wish that we had connoisseurs to assist us in the selection and control of drinking-water, as we have connoisseurs in wine and tea,--connoisseurs or experts capable of distinguishing minute differences in the flavor of drinking-waters. At first thought this may seem too fine-spun, and yet when we think of the care exercised in selecting wines, teas, and other beverages, and compare their actual importance with that of drinkingwater, it may not be going too far to suggest that consideration be given to the possibility of determining the qualities of drinking-water by flavor and other tests in addition to those now in use. I think experienced persons with a delicate sense of taste will bear out the statement that the drinking-water of each city has its characteristic flavor. If half a dozen glasses of fresh drinking-water could be assembled from the water supplies of as many cities, I have little doubt that a person with a delicate sense of taste would be able to tell one from another blindfolded, at any rate where the differences were most pronounced.

In this discussion it matters little that the amount of the dissolved substances thus suggested as a possible cause of differences in the physiological action of drinking-water is minute, for it is a well-established fact that very minute quantities of various substances may have a pro- 
found effect upon the human organism. Such reflections lead to the suggestion that the study of filter-bed organisms is one that should be prosecuted more vigorously. We have developed a few excellent chemical tests, and, so far as it goes, an excellent system for determining the bacterial content of drinking-water. Why not go a step farther and make at least an attempt to determine the nature of the minute quantities of soluble organic substances of physiological significance which may be present, and the origin and nature of these substances.

\section{POSSIBLE CONTROL OF FILTER-BED ORGANISMS}

If it should be found that the presence of a particular micro-organism in filter-beds is deleterious, is it possible so to manage the beds as to exclude the micro-organism, or counteract its effect? Already I feel sufficiently conversant with some of the facts to predict that such control will prove feasible, at least in some instances. In the case of Mononchus longicaudatus for instance, as soon as we know the natural distribution of the Mononchus; its relationship to the seasons of the year, if it has any significant relationship of that kind; the period of its life cycle; its rate and method of reproduction; its food; its enemies; then, almost beyond doubt we shall be able to suggest means for its control. So with other organisms.

To secure a reliable filter-bed census it is necessary to examine the sand as soon as collected. After a short period under laboratory conditions the population begins to change: e.g., sand which at the time it was removed from the bed contained many specimens of Mononchus and a few of Ironus ignavus, after ten days yielded no Mononchus, though it continued to yield Ironus, and in addition a few adult specimens of Tripyla and Monkystera,-differences no doubt due in part to the fact that in stagnant collections Mononchus longicaudatus is subject to the attacks of a variety of fatal diseases caused by fungi and microbes.

\section{NEMAS A CLUE TO THE FLOW}

Nemas Unequally Distributed in Beds. The distribution of organisms in filter-beds is not uniform. For example, if a series of samples be collected, one each from near the main drain-pipe, near a lateral, between the laterals, and at the margin of the bed, the numbers and kinds of nemas will be found to differ in the various samples. This unequal distribution is doubtless a function of the flow of the water, for where the flow is rapid the biological environment differs from that where it is slower; there is a greater supply of oxygen, a greater supply of watersoluble food, and a greater supply of such free micro-organisms as may pass between the grains of sand. It follows that to some extent the 
fauna and flora of filter-bed sand may be made a criterion of the rate and nature of the filtration.

The general appearance of the sand in any particular part of the bed is a clue to the rate and nature of the filtration there, but this rough method is capable of refinement through the aid of a biological census. The difficulty is we do not yet know what significance to attach to the presence or absence of particular species. Should an attempt be made to devise and apply such a method, it might be found that the presence or absence of a few species would constitute a sufficient test.

\title{
PARTIAL LIST OF THE NEMAS FROM AMERICAN SLOW SAND FILTER-BEDS*
}

\begin{abstract}
Achromadora minima Cobb.....Washington, D. C. Actinolaimus radiatus Cobb.... Washington, D. C. $\Delta$ phanolaimus ?...................Philadelphia A phelenchus sp......................Pittsburg Atylenchus sp..................... New Bedford Cephalobus sp.....................Philadelphia Cylindrolaimus obtusus Cobb...Washington, D. C. Dorylaimus fecundus Cobb..... Washington, D. C. Dorylaimus sp................Washington, D. C. Dorylaimus sp .....................Philadelphia Dorylaimus sp......................Philadelphia Dorylaimus sp................Washington, D. C. Iota simile n. sp ............Washington, D. C. Ironus americanus Cobb.............Philadelphia Ironus ignavus Bastian......... Washington, D. C. Ironus longicaudatus de Man... Washington, D. C. Monhystera subfiliformis n. sp..Washington, D. C. Monhystera dispar Bastian..... Washington, D. C. Monhystera sp.....................Philadelphia Monhystrella plectoides n. subg., n. sp.
\end{abstract}
Mononchulus ventralis n. g., n. sp.
Washington, D. C. Mononchus longicaudatus Cobb
Washington, D. C. Mononchus sp...................Philadelphia Plectus cirratus Bastian........Washington, D. C. Prismatolaimus sp...................Philadelphia Rhabditis sp....................... Philadelphia Rhabdolaimus sp..............Washington, D. C. Spilophora sp.......................Harrisburg Teratocephalus sp.....................Pittsburg Trilobus longus Leidy ................Philadelphia. Tripyla monohystera de Man... Washington, D. C. Tylencholaimus sp............ South Bethlehem, Pittsburg
Tylenchus filviformis Bütschli (?)
Washington, D. C. Tylenchus sp.....................Philadelphia Xiphinema americanum Cobb........Philadelphis

- The larger number of species from the Washington beds is due to the examination of a larger number of samples than frcm beds elsewhere. The more important of these species are described below. The drawings are from nature and were made under the author's personal supervision by Mr. W. E. Chambers.

\section{MONONCHUS Bastian 1865}

1. Mononchus longicaudatus Cobb (See p. 161). The feeding habits of Mononchus are distinctly different from those of either of its frequent companions, Tripyla and Ironus. In capturing food Mononchus depends largely upon the grip of its powerful jaws. Tripyla depends upon its agility and its flexibility, and possibly coils itself about its victim, after the manner of a boa constrictor. Mononchus and Tripyla bolt their food, but Ironus feeds in an entirely different way; attaching its lips to its food, it rips a hole in the external layers by the outward stroke of its three, extremely sharp, radially acting onchi. As these move forward their points move outward, and two or three such movements serve partially to imbed the head of the Ironus. It would appear that then the more or less fluid parts only are imbibed, for the contents of the intestine of Ironus hardly ever present optically identifiable substances. It is manifest, however, that the contents of the intestine are animal in character, and so it seems beyond reasonable question that Ironus is carnivorous. I have never found the contents of the intestine to respond to the starch test. 
Intra vitam staining shows the existence of a dorso-ventral physiological differentiation in the intestine of Mononchus longicaudatus.

Renette. When this well-known mononch was stained intra vitam with trypan blue, the ampulla and a short portion of the duct of the renette was so distinctly seen as to leave almost no shadow of doubt that this portion of the renette structure is normal. I am inclined to think that the duct soon takes a lateral turn, and possibly becomes connected with the lateral field; thus far it has not been possible to follow it more than a distance
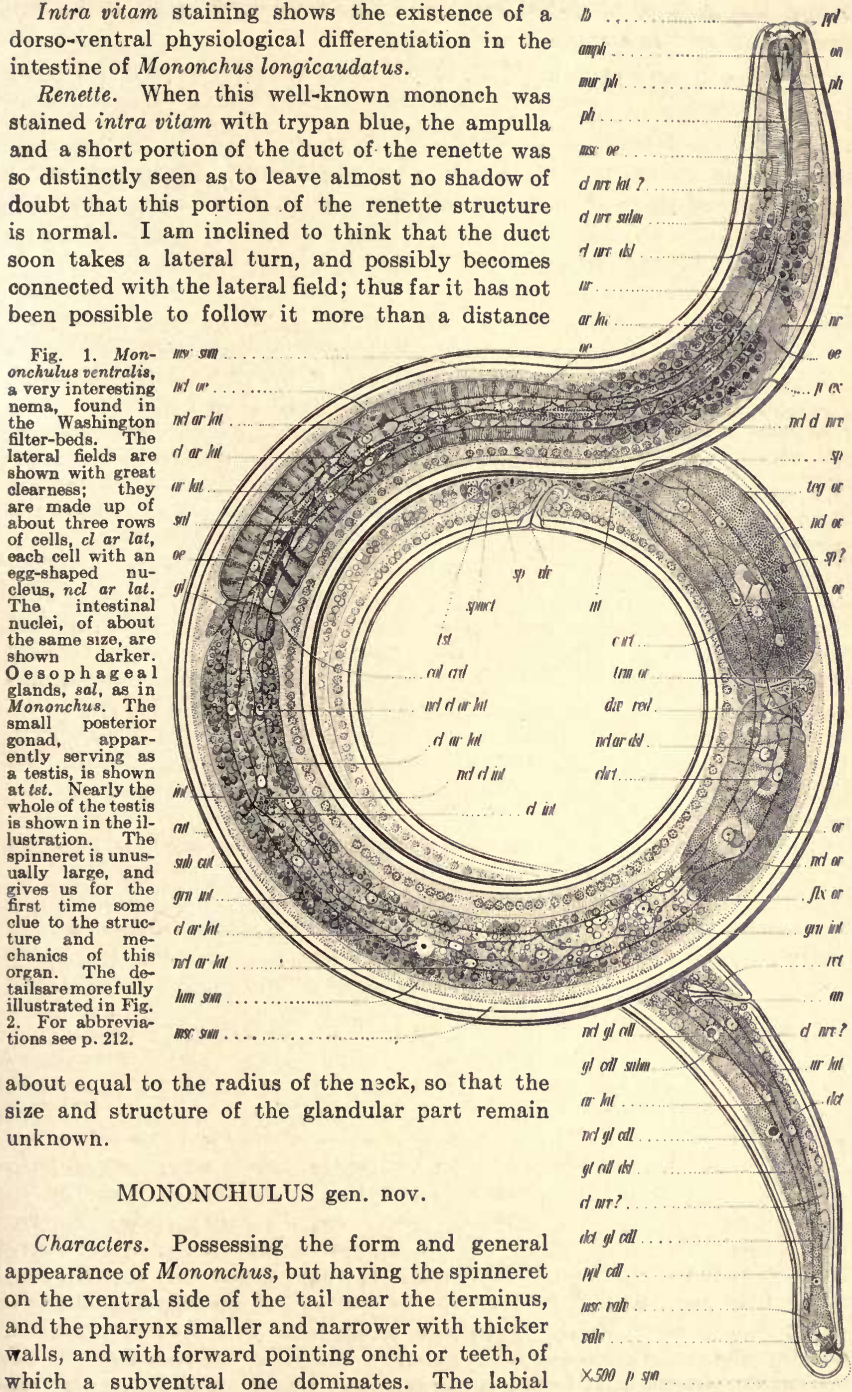
which a subventral one dominates. The labial papillae are smaller than is usual in Mononchus, in fact are barely visible. 
The entire pharynx is about twice as long as the cavity bearing the onchi, but the posterior part is narrower and somewhat obscure.

Anterior gonad reflexed. Posterior gonad outstretched, very small, producing spermatozoa. The development of the oöcytes follows about the same course as that of the spermatocytes, which they resemble to a certain extent, though they are larger. Occasionally the oöcytes so closely resemble the spermatocytes that it is rather difficult to make a clear distinction. The very youngest stages of the female gonad have not been seen, but in the youngest stages observed there were no indications of spermatozoa;-yet at the same time spermatozoa were developing in the minute posterior straight gonad.

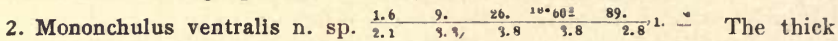
layers of the transparent, colorless, naked cuticle appear to be devoid of any but the very finest of transverse striae; but longitudinal striations are visible throughout the length of the body. Six rather thoroughly amalgamated lips of considerable thickness arch together over the pharynx and normally nearly close the mouth opening. There are six slightly spreading inconspicuous papillae scarcely interfering with the rounded contour of the front of the head; in addition, surrounding the mouth, there are six forward pointing papillae. There are no eye-spots. Very inconspicuous amphids occur opposite the middle of the largest pharyngeal tooth in the form of small semi-circumferences opening backward and having a breadth about one-fifth as great as that of the corresponding part of the bead. The anterior main thick-walled portion of the pharynx is about as long as the head is wide. When the pharyngeal organs are at rest this front cavity is comparatively well filled by the large, acute, forward pointing right submedian tooth. There are two other teeth, a small dorsal, forward pointing tooth having its apex near the middle of the pharynx, and an exceedingly minute inward pointing tooth, or spur, in the left submedian portion of the cavity near the base. Opposite the anterior portion of the main tooth the walls of the pharynx are armed with several dozens of minute, rasp-like teeth or denticles.

Close scrutiny of that part of the wall of the pharynx immediately behind the rasp-like area discloses that it is transversely striated to near the base. These pharyngeal striae can be seen only with the highest powers of the microscope under favorable circumstances. Behind this anterior portion of the pharynx is a narrower, unarmed portion, of equal length, making the total length of the pharynx about twice as great as the diameter of the head. The cells of the thick walled, narrow-lumened intestine contain scattered brownish granules. There are three unicellular caudal glands; two opposite each other immediately behind the anus, and a third, more or less dorsally located, behind and between the first two but emptying through a submedian ampulla and hence really submedian. The single reflexed ovary, except when pushed forward by the presence of an egg in the uterus, reaches nearly back to the vulva.

Habitat: This interesting digonic species has been found in peat soil, west of Fort Lauderdale, Fla., along a canal for drainage of land formerly covered with water, and at Miami, Fla.; in the sand of the filter beds at Washington, D. C. ; and also in the Potomac River.

A species that appears to belong to the genus Mononchulus is described by Daday under the name Prismatolaimus nodicaudatus, n. sp., in his "Mikro. skopische Süsswasserthiere aus Deutsch Neu Guinea." 


\section{STRUCTURE OF THE SPINNERET}

I am not aware that anyone has ever attempted to explain the mechanism of the spinneret of nemas. Manifestly the flow of the caudal secretion is controlled at will. Watching this operation as performed by a free-living nema, one is forcibly reminded of the facility with which spiders regulate the operation of their spinnerets, and, as in spiders, so in nemas, there must be a definite con. trollable mechanism for performing these operations. The structure of the spinneret in Mononchulus ventralis may at least suggest the mechanical principles exemplified.

The Needle-I'alve. As a rule the nema spinneret is so extremely minute that its details cannot be deciphered. In Mononchulus ventralis the spinneret is relatively large and its elements more or less resolvable, but since it is ventral in this species instead of terminal, as is usual, its form may not be entirely typical. In $M$. ventralis we find the duct of the spinneret to end externally in a conical depression near the end of the tail. This conical depression leads to a short oblique tube terminating internally at the valve of the spinneret, $v l v-v l v$, Fig. 2. The valve belongs to the class known as needle-valves, and the needle, if such it be called, is an acute, fusiform affair, duplex in cross section, and nearly half as long as the terminus of the tail is wide. It is placed at an angle of about 45 degrees with the axis of the tail, and while its acute free distal end lies loose in a cavity of obverse mold, its more or less cephalated proximal end is connected with the dorsal side of the tail by means of oblique muscular fibres. The proximal part of the valve is located in the midst of a vesicle (?) to the dorsal side of which its proximal extremity seems to be attached; the embryological development has not been investigated, but conceivably the needle is formed by an invagination of the wall of the "vesicle." Into the base of the more or less ellipsoidal "vesicle" the ducts of the caudal glands empty. The operation of the needlevalve is now easily understood. The internal body pressure will of itself keep the needlevalve closed. Contraction of the muscular fibres already described serves to pull the needle loose from the mouth of the valve,

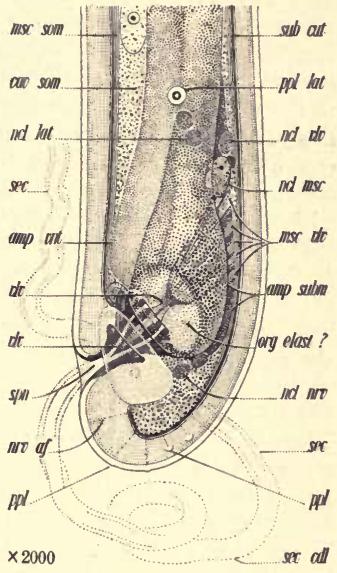

Fig. 2. Spinneret of Mononchulus ventralis, with coagulated secretion attached. The valve is partly open and the secretion is pouring out. The three ampullae empty into a common space round the proximal part of the spindle-shaped valve-plug, $v l v-v l v$, so that the body pressure, presumably assisted by the elasticity of the surrounding parts, (org. elast) holds the spinneret valve shut except when it is pulled open by the muscle, msc vlv. All the smaller nuclei shown are believed to be nerve nuclei. For abbreviations see p. 212 and so permit an outflow of the secretion. The "needle" is composed of two elongated, ceratinous lateral elements joined side by side, and the orifice of the valve is composed of three elements, disposed in the form of a hollow cone about the distal half of the needle.

Nervous Apparatus of Valve. Most of the foregoing features are shown in Fig. 2. Necessarily the apparatus is supplied with the appropriate sensory and moto: nerves. The details of these latter have not yet been made out with 
certainty; however, innervated papillae occur near the terminus,-doubtless tactile in function. The shortest, i.e., dorsal, unpaired caudal gland empties through a dorsally left submedian duct. Of the two longer and paired glands, one, the left submedian, empties through a ventral ampulla, while the other empties through a dorsally right submedian one; the details of the debouchment remain somewhat obscure and no attempt has been made to indicate all of them in the illustration. The three pores, however, are just in front of the "equator" of the valve.

Digonic. This species presents the unusual peculiarity for an hermaphroditic nema of developing its ova in one gone, and its sperm in another gone of much smaller size, -in a word is digonic. So great is the disparity between these two branches of the sexual apparatus that at first glance one almost inevitably concludes that the very small posterior branch is a mere functionless vestige. A careful examination seems to prove that only ova are produced in the anterior branch, while the very small outstretched posterior gonad functions as a testis; the evidence for this latter conclusion being the occurrence of spermatozoa and spermatocytes in small numbers arranged in the order to be expected if the organ were a small gonad devoted solely to the production of sperm. The cells in this minute gonad are so few that their precise order is not a striking feature, and yet an examination of a series selected from among individuals in which the ova in the anterior gonad are still quite young and small, enables one to demonstrate that the cells near the blind end of the small gonad correspond in structure with primary spermatocytes, and that the succeeding cells, sometimes as few as two to three in number, represent successive steps in the development of the spermatozoa. Occasionally one finds here a pair of gonic cells lying side by side, each containing about six chromosomes, practically as definite as those to be seen in the testes of male individuals of typical free-living amphigonic nemas. Often the perfected spermatozoa appear not to exceed eight to twelve in number. There is an obscure tendency for them to be located in groups of four, such as should exist if they were produced in situ in quartets in the manner characteristic of the spermatozoa of nemas. Once an egg was observed containing near its equatorial periphery a body corresponding in size and staining properties to one of the spermatozoa to be seen free in the uterus. The nucleus of this egg showed signs of being affected by the presence of the spermatozoön, though it appeared not yet to have produced polar bodies. All these appearances are in harmony with the supposition that the posterior branch of the sexual apparatus, small as it is, functions as a testis.

\section{TRIPYLA Bastian 1865}

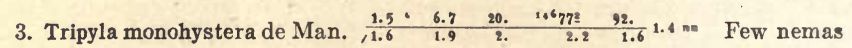
are more agile than this. Its movements are extremely rapid, especially those of the head end. It is unusually flexible, coiling and uncoiling all parts of its body with great rapidity. It is of a restless disposition, at least so appears when brought under the microscope for examination.

I have often seen this Tripyla attach itself to glass and then exhibit the evolutions characteristic of nemas possessing well developed spinnerets, but in spite of careful examination of living specimens, and specimens preserved both in glycerine and balsam, I have seen no definite traces of caudal glands. The cephalic setae are segmented and consist of two or more joints.

Harbors a Parasite. Tripyla monohystera of the Washington filter-beds often contains what appears to be a peculiar spherical parasite. The parasites (?) 
seem usually to gain entrance to the body at or near the caudal extremity, for in almost all cases where their number is few they are confined to the tail. See Fig. 3. Thence they seem to work their way forward, especially along the lateral fields, so that finally they may occur throughout the length of the body in hundreds of thousands. These objects are extremely minute and can be satisfactorily examined only with the aid of the highest powers of the microscope. At first sight they appear to be crescent shaped, a deception due to their peculiar refractive properties. Careful focusing shows that the greater portion of the sphere, an eccentric portion, is but slightly refractive. The remaining

Fig. 3. Tripyla monohystera. This active, voracious little nema is very common in filter-beds. Often the remains of several other nemas are to be found in its intes tine. The specimen figured had been feeding on a variety of microzoa. To be seen in the intestine are a nema, nematod ing: the "gizzard" of a rotifer, rot ing; and a number of protozos, ing. The egg shown has just received one of the syngonic sperm cells $s p$, and has thrown off the first polar body, corp plr I. The beginning of a sporozoön (?) infestation is shown in the tail, par. The renette of this nems (ren; ex $p$ ) has bitherto remained unknown An organ of considerable size, but of unknown significance, org?, is also now for the first time shown to exist in the neck. For abbreviations see p. 212.

portion is more easily seen, and, when it comes into view in optical section, presents the contour of a crescent;--is therefore in reality bowl-shaped. I have made no serious attempt to classify these objects and can only suggest that we have here a new sporozoön. If so it may be the cause of a serious disease of the nematode; often 10 to 20 per cent of the individuals appear to contain it. In some collections it occurs in practically every individual.

Nemativorous. Nematodes having a plain oesophagus, such as Tripyla, Mononchus, and Monhystera, of ten exhibit a marvelous capacity for swallowing relatively large objects. Some spe-

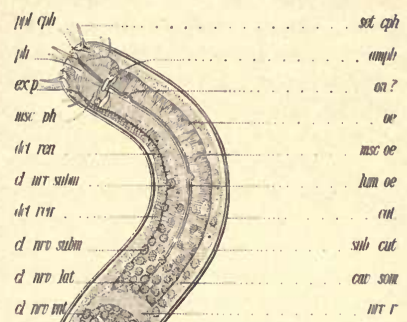

d IIr dd

max som

d $n \pi \mathrm{h} / \mathrm{t}$

d nv sum

det $n$th

ang?

cies of Monhystera are able to swallow diatoms one-half to two-thirds as wide as themselves, and one-fifth to one-sixth as long. Tripyla monhystera is rather 
remarkable in this respect. It has little difficulty in swallowing nemas half as wide as itself; and the partly digested remains of several such may sometimes be seen in its intestine. (See Fig. 3.)

\section{IRONUS Bastian 1865}

4. Ironus longicaudatus de Man.

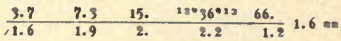

As regards the pronounced dorsoventral differences in the intestine of Ironus longicaudatus, when specimens of this species are stained in acid carmine it is noticeable that the large granules characteristic of the dorsal side take the stain. From an examination of living specimens one would think these granules probably fatty in their nature. The fact that they stain as they do seems to exclude this supposition.

No Sperm Seen. In spite of very careful examination I was unable to discover spermatozoa in the females of this species. Nevertheless I am strongly inclined to think that further investigation will reveal the presence of spermatozoa, and show that this species also is syngonic. I have seen no males.

I believe the foodhabits of Ironus longicaudatus to be much the same as those of Ironus ignavus, but having had less opportunity to investigate them I have fewer data from which to form an opinion.

Habitat: Washington filter-beds; sometimes abundant. Quite active.
Fig. 4. Ironus Fig. 4. Ironus The excretory pore is at the lips. The submedian salivary glands empty opposite the amphids, the dorsal one farther back. The dorsal cells of the intestine differ very much from the vantral.

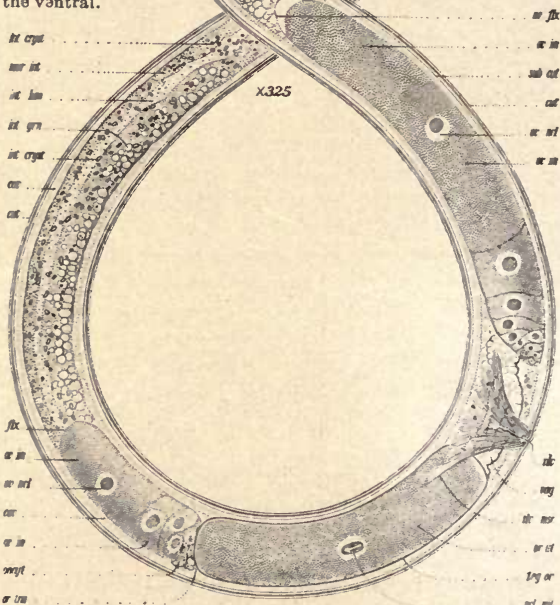




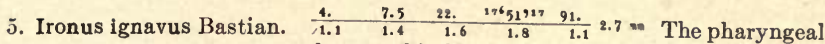
onchi are tissue tearers. The three onchi of Ironus ignavus are of unequal form and size. At first glance they appear equal, but even when the onchi are drawn in and the head viewed in profile it is sometimes possible to see that the dorsal onchus differs from the other two in being duplex. When the onchi are exserted the expansion necessary to this operation throws the two apices of the dorsal onchus wide apart, and they can then with ease be brought separately into focus. A front view of the head also emphasizes this double structure of the dorsal onchus. Either half of this onchus when seen in profile seems to be of almost exactly the same size and contour as one of the submedian onchi. The action of the onchi in Ironus is like that in some species of Diplogaster, of most species of Axonolaimus and of one or two other marine genera, and is the exact reverse of that of the onchi of Enoplus and its relatives. The outward action of the onchi in Ironus is adapted to tearing open the tissues upon which it feeds, the fluid and semi-fluid portions of which are then imbibed. In harmony with this is the liquid or finely divided character of the contents of the intestine in Ironus. Enoplidae, on the other hand, bolt their food. The movements of the onchi do not appear to be so quick as those of the onchi of Diplogaster. The outward throw and return occupied about one-quarter second in a specimen which though stained intra vitam with neutral red, yet appeared to be as active as the average living specimen.

Characteristic Intestinal Crystals. The doubly refractive crystals found in the intestinal cells of Ironus are different from those found in certain Rhabdites, to which I have given the name Rhabditin. The doubly refractive crystals of Ironus are not spherical,- on the contrary are distinctly angular in contour and have a definite polyhedral form. These doubly refractive bodies are absent from the anterior part of the intestine for a distance about equalling half the length of the neck, indicating a different physiological condition here. Some of the intestinal cells are very distinctly specialized. (See Fig. 5.)

Carnivorous. The following are strong reasons for regarding Ironus as carnivorous; (1) Recognizable plant remains are rarely if ever found in the intestine. (2) Ironus abounds in places where there is little plant food of any kind, but where animal food is plentiful. (3) The peculiar mouth parts can hardly be explained in any other way than by supposing them to be special organs for ripping open tissues of the food, and the only filter-bed plants that could furnish adequate food for Ironus are entirely too small to be operated upon by these mouth parts.

A very noticeable feature in the development of the eggs of Ironus ignavus is the appearance in the ripening ova of numerous protoplasmic structures which stain rather strongly in acid carmine. Toward a dozen of these structures may be seen in the full grown ovum when about to turn and pass into the uterus. The younger ovum immediately following it also shows these same structures, more closely packed together, but of about the same size. After the egg has passed into the uterus these bodies sometimes completely disappear.

Newly Discovered Organs. Among the numerous new facts here brought to light in connection with Ironus none appear more interesting than the pronounced dorso-ventral differentiation of the intestine. In both ignavus and longicaudatus this differentiation is pronounced, especially in the latter, where from one end of the intestine to the other the difference in structure between the dorsal and ventral sides is very striking. In ignavus the same quality of dif- 
ferentiation occurs, but the cells having the coarser structure are more scattered. They nevertheless are here also commonly dorsal or sub-dorsal.

What seems to be a very long and narrow tubular organ (org ?) exists in ignavus. I have been unable to determine the function of this interesting organ. The mycelium of various parasitic fungi is not altogether uncommon in the bodies of fresh water nemas, and sometimes presents highly deceptive appearances, but it did not seem possible to me that this tubular structure could be other than an integral part of the Ironus.

It is interesting to note that the most common species of Ironus in American filterbeds are identical with those of Europe. Ironus ignavus is widely spread in the United States, as I have collected it from spring, lake and river waters of many of the northern states, from the Atlantic as far west as Colorado and from widely varying altitudes.
Fig. 5. Ironus ignavus. Excretory pore and salivary glands gs in

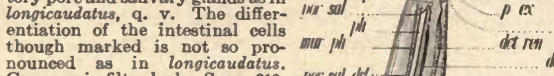

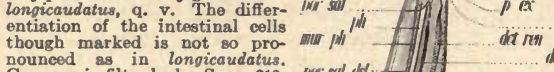

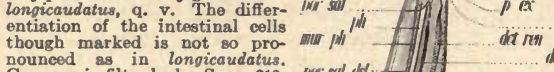

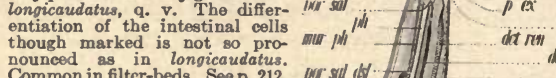
dat $s$ I 1 t

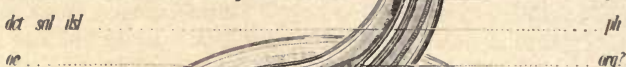

ac

$n \pi r$ $d n \pi$ and of ac

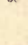

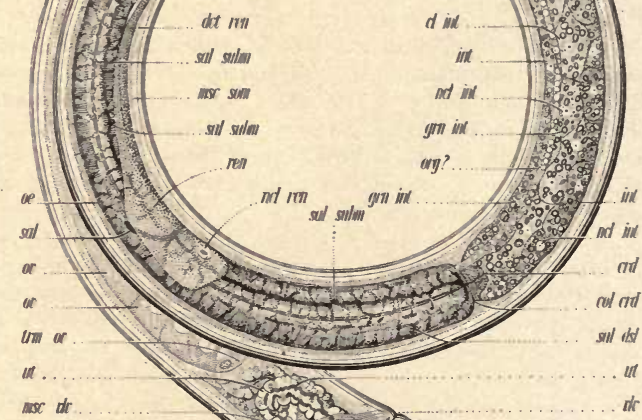
ang? $\ldots \ldots \ldots \ldots$. ary int

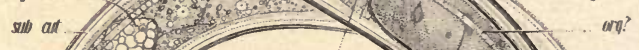

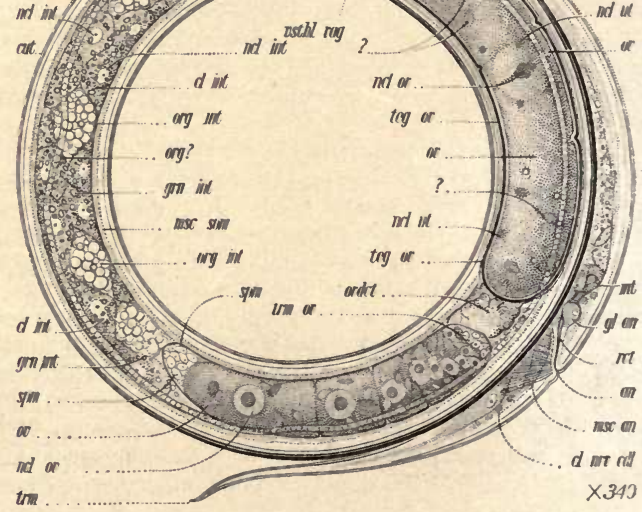


Fig. 6. Monhystrella plectoides n. subg. n. sp. For some distance in front of the nerve ring the oesophagus is distinctly though slightly altered.

A critical review of the Monhysteras so far discovered and described will undoubtedly result in the establishment of a number of fairly well defined groups, some of subgeneric rank, some of generic. The divisions may be made on the basis of the anatomy of the male organs as well as on that of the mouth parts. These anatomical differences are the outward expression of differences in food habits, and of special activities due to differences in habitat. The Monhysteras constitute a huge group, of considerable biological significance. Some species are specially adapted to studies of problems in genetics. The mode of development of the spermatozoa of certain species is worthy of study.

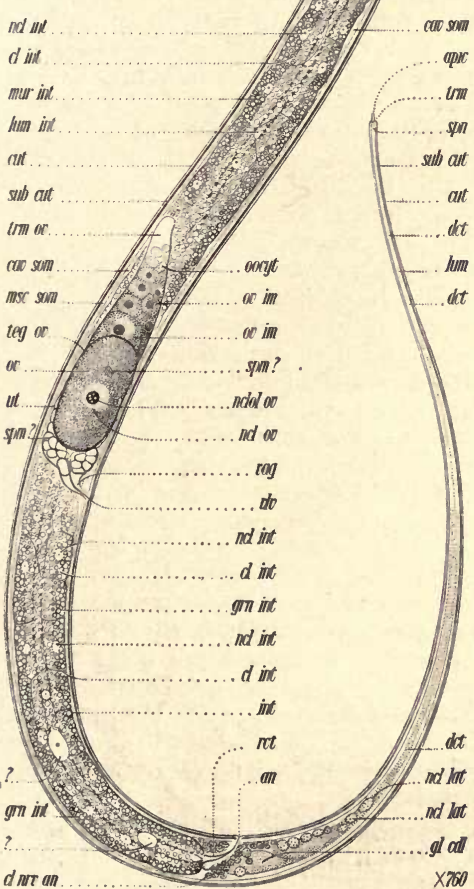

MONHYSTRELLA subg. nov.

Subgeneric characters. Has the general form and appearance of Monhystera, but differs in the following respects:-The pharynx is more elongated, somewhat resembling that of Cylindrolaimus, but tapers slightly. The oesophagus is not quite of uniform diameter, in this respect somewhat resembling that of Plectus in the anterior part, but with all the variations less pronounced, so that, at first glance, the oesophagus seems to be rather uniform in structure from the pharynx backward to the cardiac swelling. There is a rather distinct pyriform cardiac swelling, with clear indications of the presence of glands among its muscular tissues. One or two problematical unicellular organs exist in the lateral fields on each side of the body some distance in front of the anus. Otherwise very much as in Monhystera. Possibly Monhystera bulbifera de Man belongs to this sub-genus.

6. Monhystrella plectoides $\mathrm{n}$. $\mathrm{sp}$. \begin{tabular}{ccccc}
1.2 & 10. & 17. & $2-44$. & 65. \\
\hline 1.5 & 2.4 & 2.6 & 3.1 & 2.
\end{tabular} $.43 \mathrm{~mm}$

The rather thin, transparent, colorless cuticle is traversed by exceedingly fine transverse striae, resolvable only with high powers under most favorable conditions. The thin-shelled eggs appear to be deposited before segmentation begins.

Habitat: Sand, Washington filter beds; uncommon. Nemas of the character represented by Monhystrella and Monhystera are largely, if not entirely, vegetarian. As a rule they are not abundant in covered slow sand filter beds. On one occasion, however, after a long period of winter use, I found such nemas to be fairly abundant. 
They appeared to have been feeding upon bacteria and other similar microphytes. Monhysteras are more common in open filter beds and in reservoirs, where they appear to feed principally on green unicellular algae, which naturally do not flourish in covered filter beds, since sunlight, direct or diffused, is necessary to their growth.

\section{IOTA Cobb 1913}

7. Iota simile, n. sp. $\frac{12.5}{8.9}-\frac{20 .}{9.9}-\frac{24 .}{19.8} \frac{6599 .}{7.2}-\frac{95 .}{6.6 .6} \mathrm{~m}$ The colorless layers of the thick cuticle are traversed by ninety to one hundred plain, transverse striae of such a nature as to give a relatively coarse serrate-crenate appearance to the contour of the body. The annules of the cuticle are complete rings. Only at rare intervals is there a trace of anastomosis; occasionally it will happen that two semi-annules on one side of the body are joined to one on the other. The convex-conoid neck ends in a somewhat rounded head surmounted by a flat lip region composed probably of six very flat lips placed in the slightly depressed front surface of the first annule. The first two or three annules are packed closely together, and the prevalent crenate-serrate appearance of the contour begins probably with the fourth annule, possibly with the third. No labial papillae have been seen. Nothing is known concerning the amphids.

Spear. The spear is rather more slender than in most Iotas, and tapers throughout the main portion of the shaft, which constitutes fully two-thirds of the length. Where it reaches its maximum development this shaft has a diameter about half as great as the width of one of the neighboring annules of the cuticle. Behind the shaft the hilt of the spear has a diameter only a trifle larger than that of the main portion of the shaft. The hilt increases in diameter steadily posteriorly, and finally expands suddenly into a large three-fold bulb nearly one-fourth as wide as the corresponding portion of the neck. The central canal of the spear is plainly visible, and is continuous with that of the oesophagus.

Oesophagus and Intestine. The oesophagus is about as wide as the base of the spear, and appears to present no very definite median bulb, though there is a slight change near the middle of the neck which probably indicates the position of some such structure. The details of the intestine are somewhat obscure, but it appears to begin as a tube about half as wide as the body, and to continue at this width for some distance. The anus seems to be located between the fourth and fifth annules, counting from the posterior extremity. The excretory pore is located near the twenty-sixth annule counting from the head end where the annules first become distinctly developed; this means probably at about the thirtieth annule counting from the mouth itself. The tail is conoid to the blunt terminus, which is destitute of a spinneret.

Sexual Organs. The depressed vulva is located at about the seventh annule from the caudal extremity. The vagina leads inward and forward a distance fully equal to the corresponding body width. In front of this is the uterus, which appears to be once and one-half to twice as long as the corresponding body-diameter. The ovary extends directly forward and its blind end lies some little distance behind the base of the neck. The eggs occur one at a time in the uterus. They are thin-shelled, a little longer than the corresponding body diameter and about half as wide as long.

Habitat: Aberrant in filter beds, Washington, D. C.; roots of grape-vine, Herman, Mo. Synonymous with Iota are Ogma Southern and Criconema Hofmänner and Menzel. Iota consists of many species. I have specimens and full MS. descriptions of a number of new species collected during the last twenty years in widely different parts of the world. All are $-f$ and have the vulva hidden among the annules near the anus; tiny, wide nemas with retrorse annules. 


\section{MONHYSTERA Bastian 1865}

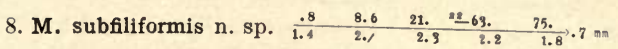

This form, which appears to be new, is closely related to Monhystera filiformis Bastian, but differs from the descriptions given by various authors in the following respects:-There are 10 cephalic setre on subfiliformis instead of six. The amphids are somewhat larger than given by most authors for filiformis. In the cardiac region I have noticed in the single specimen of the present species so far examined, a very definite pseudobulb. There is a definite renette, as shown in the figure, with an excretory pore opposite the nerve ring. The eggs are relatively larger than described for filiformis. The anatomical details are clearly shown in Fig. 7. This species also shows a certain amount of resemblance to Monhystera vulgaris, de Man, but differs in having setae on the body, and in having 10 cephalic setae, and in the amphids being slightly different in position, form and size. The tail of vulgaris also seems to be more slender.

Habitat: Sand, Washington filter beds; $\begin{gathered}\text { Fig. } 7 . \\ \text { on } h y g^{-}\end{gathered}$ usually uncommon. Of the numerous tera subspecies of Monhystera I have had occa- filiformis. sion to examine with respect to their mon in food habits, all appear to be largely if beds. not wholly vegetarian. The various species specialize to a considerable degree in the matter of food. For instance, many marine species feed almost exclusively on diatoms. If the filterber Monhysteras are not an exception to the rule, they would seem to be dependent on fungi and bacteria as a source of food, except in open beds, where of course they would find an abundance of green microphytes.

\section{CYLINDROLAIMUS de Man 1884}

Sexual Organs. Cylindrolaimus typically has but a single outstretched ovary; from the observations of various authors it would appear that this may extend either forward or backward, usually forward. As there is some doubt about the shape of the organ in the type species communis, it is desirable that specimens of that species be reëxamined. Inasmuch as the male of only one of the six true species of Cylindrolaimus has been seen, it would seem that the genus is typically
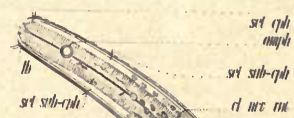

of $\mathrm{HI}$ IN

IIr $r$.

of twr htt

of urr sulue

(N)

that or

WOS w

ind $7 m /$

$n i l$.

IIt

hati wnt $^{\prime}$

twh int.

int ins

otr sell

shl (III
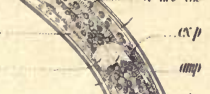

\section{.}
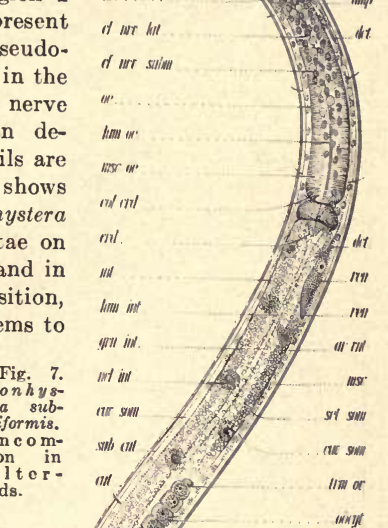

InKyt

dis I17??

or in

Int or iil

or in

.

.


syngonic or digonic. Such observations as I have been able to make upon glycerine specimens of obtusus lead me to conclude that this species at least is probably digonic; if it is syngonic we are confronted with the phenomenon of the ovary first acting as a testis and sending over into the rudimentary posterior part spermatocytes, probably primary spermatocytes, there to continue their development.

9. Cylindrolaimus obtusus Cobb.

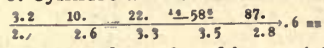

The moderately thin cuticle is traversed by five to six hundred transverse striae, which do not appear to be further resolvable. In glycerine specimens there are very faint indications of longitudinal striations, which are more pronounced toward the anterior extremity; these may be due merely to the attachments of the somatic muscles. There appear to be no very distinct lips, though probably minute lips are present, - so small and so closely amalgamated as easily to escape observation. Occasionally six excessively minute papillae have been seen immediately round the mouth opening; though no doubt always present, these papillae usually escape observation. When the lips are closed the small mouth opening appears as a simple pore in the middle of the front of the head, where there is an exceedingly minute depression. Four submedian, somewhat papilla-like, widelyspreading cephalic setae occur on the margin of the head about half way between the anterior extremity and the front of the amphids; each of these setae is about one-fourth as long as the corresponding diameter of the head. The well-developed lateral fields are about one-third as wide as the body. There do not appear to be any distinct wings.

Intestine. Ovary. The somewhat cylindroid cardia is of relatively large size, and is separated from the oesophagus by a constriction; it is about two. fifths as wide as the base of the neck, and is surrounded by about seven unicellular organs, probably glandular in nature. There is a small posterior branch of the sexual apparatus, extending backward a distance about equal to the length of the body diameter, serving either as a testis or spermatheca, apparently the former. The ellipsoidal, thin-shelled, smooth eggs are five-sixths as wide as 
the body, and about two to two and one-half times as long as wide. They appear in the uterus one at a time and seem to be deposited before segmentation begins.

Habitat: Sand, Washington filter beds, early in January 1916, at the end of about five months' use. Not common. No males seen.

\section{PLECTUS Bastian 1865}

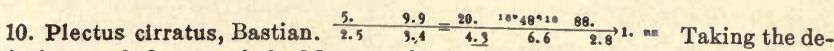
scriptions and figures of de Man as the basis of his identification, Maupas made numerous and very careful observations upon this species; he never found males, and concluded from this and his other observations that the species is purely parthenogenetic. As my own observations do not agree with those of Maupas, it is well to consider carefully whether the species observed by us are the same. My specimens came from the Potomac River near Washington, D. C., U.S.A., and in size and proportions agree in all respects with the figures and descriptions of de Man. The anatomy of my specimens also appears to agree in all essential respects with that set forth by de Man. There is a little uncertainty about the number of eephalic setae as reported by de Man;-in one instance he says four, in another six. Bastian's original description, made from specimens $\frac{2}{16}$ in. long, says four. In the description in which de Man placed the number at six his corresponding illustrations are possibly open to the interpretation that four only were present. The Potomac specimens always present four cephalic setae. The caudal setae of the Potomac specimens are not so prominent as indicated in de Man's figures. My specimens present the amphids at precisely the point indicated by de Man, and of very nearly the same size. De Man does not give a very clear indication of the shape of the amphids, but I see no reason to consider the two forms specifically or varietally different on the basis of the shape and position of the amphids. There exists, therefore, only the uncertainty with regard to the cephalic setae, and this may not in fact be a discrepancy. Maupas shows five eggs in each uterus. It is uncommon for the Potomac specimens to present as many eggs as this in the uterus; the common number is one or two, but it may rise to four. De Man describes the egg-shells as smooth; Maupas, however, says they are covered with minute points. The Potomac specimens agree with Maupas' description. I am therefore most strongly inclined to believe that the forms examined by Maupas and myself are the same.

Maupas, in his description of the development of the egg, notes the following points: In one case only among a large number of developing ova examined did he see more than a single nuclear figure. He considers this an important point in his demonstration of the parthenogenetic character of the development. He observed that when the egg entered the uterus it lost its definite nucleus for an hour or more, and concluded that during this period the polar bodies were formed, although, as he says, he never saw any polar bodies, but observed amoeboid movements in the ovum during the absence of the nucleus. Maupas says that it was with much difficulty that he convinced himself that no spermatozoa were present in the sexual organs of this Plectus, but that in spite of careful examination he never succeeded in seeing any. Nor did he see the slightest trace of spermatozoa on removing the sexual organs and treating them with acetic acid. He therefore took the species to be parthenogenetic. Nevertheless, he noted occasionally in the distal end of the uterus refractive bodies of exceedingly small size. As will appear later, it is not at all improbable that these minute bodies were really spermatozoa which he failed to recognize as such. 
Chromosomes. Zygosis. Using the best modern instruments, under favorable conditions, it is possible to see the spermatozoa of Plectus cirratus in living

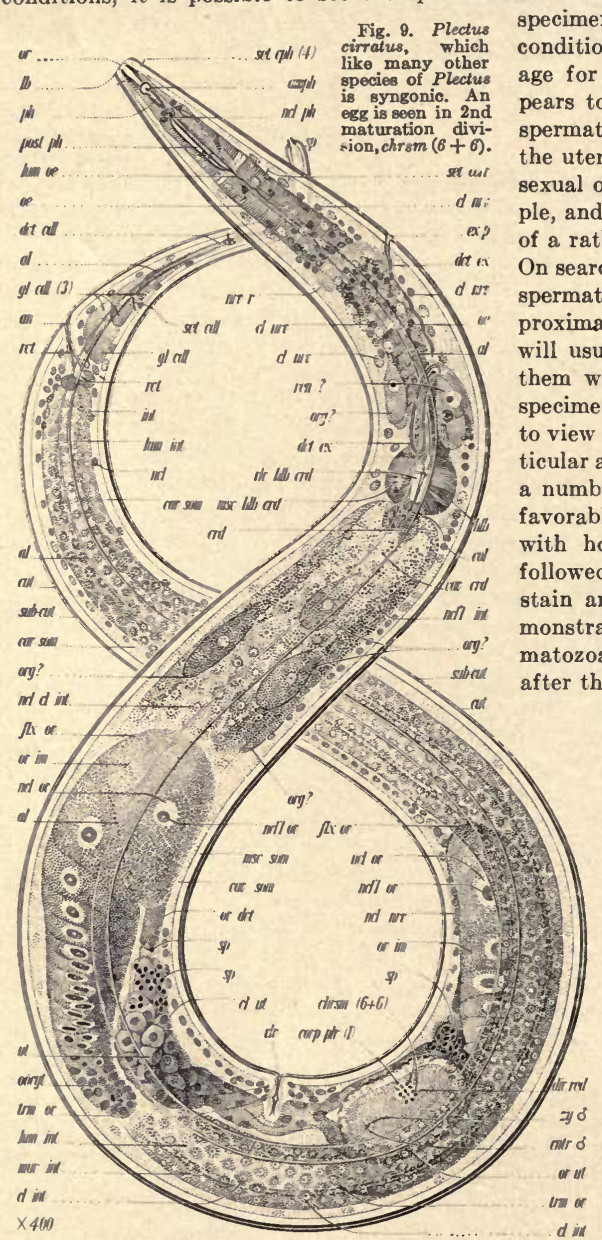

Fig. 9. Plectus like many other species of Plectus 8yngonic. An sion, $\operatorname{chrsm}(6+6)$

dt $a$ specimens of the right age and pears to be that at which the he uterus, - the stage when the al organs appear quite simand the uterus has the form a rather narrow empty tube. n searching for spermatozoa or ocytes near the large or end of the ovary it lly be possible to find hout examining many It may be necessary the specimens at a pargle, and it may require of trials to secure a specimen. Fixation t corrosive sublimate, by an acid carmine d long destaining, dees the presence of speralmost any stage formed, up to the time when all have been utilized. Using such material, I have been able to trace the spermatozoon into the egg, and show that the spermatozoon displays about six chromosomes at a time when its two centrosomes appear on the side next the eggnucleus, which under its influence divides, and forms two distinct groups of chromosomes consisting of six each. Indefinite bodies are formed in the vicinity of the egg nucleus which appear to have the sig-

nificance of polar bodies; see Fig. 9 , where one is shown faintly at corp plr (I).

Syngonic. My observations leave me without the slightest doubt that this Plectus is syngonic, and that the spermatozoa are functional. Rather casual observations I have made on other species of Plectus prove that syngonism is 
common in this genus, and that similar spermatozoa are present in a number of other species, but thus far I have no proof that in these other species the spermatozoa are functional. These results lend additional emphasis to my suggestion, made in previous papers, that parthenogenetie species in general be reëxamined with a view to ascertaining whether some of them do not present spermatozoa of minute size. As in the present case, species hitherto regarded as parthenogenetic may prove to be syngonic. Probably thousands of nemas are syngonic.

Hermaphroditism in Nemas. Dr. E. Maupas in 1900 summarized to that date a list of 34 hermaphroditic species belonging to 12 different genera, as follows:Rhabditis, Diplogaster, Cephalobus, Plectus, Allantonema, Bradynema, Microlaimus, Angiostomum, Strongyloides, Dorylaimus, Aphelenchus, and Alaimus:four of them parasitic, the remainder free-living. These 34 species represent varying degrees of hermaphroditism,-from species with two sexes, both funetional, but presenting also females capable of developing their own spermatozoa, to those in which only female forms are known, but in the gonads of which first spermatozoa then ova are produced, the spermatozoa serving to fertilize ova from the same gonad. Of these 34 species, over half belong to Rhabditis.

Syngonic Forms.* I have recently taken occasion to look somewhat carefully into the embryology of a number of species, all of which prove to be syngones or digones. These are the filter-bed species Mononchus longicaudatus Cobb, Ironus ignavus Bastian, Ironus longicaudatus de Man, Plectus cirratus Bastian and Tripyla monohystera de Man, all species that have been repeatedly investigated by different observers in various parts of the world. To three of them males are unknown; in the case of the fourth, Ironus ignavus, the males have been seen but rarely. In addition I have examined an interesting new genus, Mononchulus, also hermaphroditic. In the course of my investigations extensive series of individuals have been examined, in nearly every case several hundred, all of which proved to be syngonic females (digonic in Mononchulus). As in most of the other hermaphroditic nemas, the spermatozoa are produced in the young gonad and are early sent forward,- - often to a special receptacle,- -where they await the arrival of the ova.

Potency of Syngonic Sperm. In the light of reeent researches on the fertilization of the ovum several interesting questions again arise in connection with the origin, development and function of these spermatozoa produced by gones which simultaneously or subsequently produce ova. Are these spermatozon functional? That is to say, do they fertilize the ova in the "regular" manner? Do the syngonic spermatozoa enter the egg and behave in every respect like those produced in a separate male organism, or do they behave in some other way? To answer these questions, among other things the history of the ehromosomes throughout the ripening of both sperm and ova should be accurately known, and then compared with the corresponding facts in typical amphigonic species.

Vanishing Series of Spermatozoa. Mainly, previous researches have given us the records of species in which the spermatozoa produced by syngonic females were of the same size and form as those produced by the few males that a so occurred, that is to say relatively of very considerable size. In some species the males of which are unknown, the recorded spermatozoa, found in the female, are relatively small and difficult to observe. My own researches have led me to cases more and more difficult to decipher, owing to the smaller and smaller size of the spermatozoa discovered, and ended in eases in which I was left completely in doubt as to the existence of spermatozoa; I could find none, but the nature of my experience did not permit me to conclude that therefore none

*For the terminology used in the following discussion, see pp. 126, 127. 
existed. Sometimes the spermatozoa are so small, and so difficult to observe, even under the best of conditions and with the best instruments used by experienced observers, as to lead me to begin to question the adequacy of the evidence upon which we base our belief in some cases of alleged parthenogenesis. May it not be that sperm cells, small and difficult to observe, have escaped notice?

Potency. As to the efficacy of these small spermatozoa, in all the cases I have observed there seems to be a fair volume of evidence that the eggs are fertilized by the entrance of a body so closely resembling one of these small spermatozoa as to leave either no or little doubt that, so far, the phenomena are identical with those of bisexual fertilization. Spindles and polar bodies are formed, though sometimes the evidence is not complete. Unfortunately, in most of the cases I have observed, the chromosomes are so small and crowded as thus far to preclude exact counting; I am therefore unable to say, on the basis of fully satisfactory observations, that fertilization always takes place in the same manner as in the casc of females fertilized by copulation with males. I can only say that my cvidence, as far as it goes, points that way in a considerable number of species.

In view of the present developments this would seem to be a matter in which it is well to keep clearly in mind that no amount of not seeing a thing proves that it does not exist. It is no longer the case, as it formerly was, that non-existence of males may be regarded as proof of parthenogenesis. We must prove that there are no functional spermatozoa produced by the females themselves. Has this always been done? I think the answer must be "No," or "Not satisfactorily."

Road to Parthenogenesis? With Maupas one may suspect syngonic nemas to be on the road to parthenogenesis. This suspicion may be justified on the ground that we find species producing two groups of sexual cells, one male, the other female, variously arranged with respect to each other all the way from amphigony to the most intimate syngony, a series at once suggesting the hypothesis that bisexual nemas may be evolving along this road to parthenogenesis. What would be the ulterior (post-parthenogenetic) steps in such an evolution?

Consider for a moment the origin of the gonic cells in a syngonic nema: At some time in the growth of its gone all the future ova and spermatozoa exist in the form of a single or primary gonic cell, destined to give rise both to spermatocytes and oöcytes. The spermatocy tes usually take the lead in development and give rise to spermatozoa that are lodged in the uterus. In some cases almost simultaneously, in others only a few hours later, the oöcytes begin to develop, and give rise to ova, which in turn pass onward to be fertilized by the spermatozoa already produced by the same gone. Admitting the crudeness of the questions,-Is not this conceivably a wasteful method? Under the circumstances might it not be more "economical" to produce only one kind of cell, each such cell to contain both male and female elements? Why separate thesc complements only to bring them together again so soon? These queries are of course merely suggestive, and are not meant to outline the whole subject.

The original gonic cell of a syngone gives rise to both spermatozoa and ova; hence there exist in it, among other things, both male and female potentialities: these must have some physical embodiment, - of course not necessarily discernable. Should these potentialities, male and female, ${ }^{*}$ be separately embodied in the original gonic cell in numbers suitable each to cach, why might they not there and then, in ways harmonious with those familiar in amphigony, segregate their parts and regroup them, and afterward devclop in the form of some fractional number of syncysts? Is this anything more than following to what would appear to be a logical conclusion, the tendencies apparently existing in

" "Potentialities, male and female", being, of course, merely members of a series of potentialities subject to heredity. 
syngonic nemas? Proof of the existence of this form of genesis, for which I have suggested the name cryptogenesis, ${ }^{*}$ will place parthenogenesis in a new light. Evidence for or against cryptogenesis should be sought in the structure and behavior of the "oöcytes" and "ova" of syncystic forms. From some forms of syngony it would appear to be hardly more than a few steps along this road to parthenogenesis, itself perhaps, as it were, a waystation en route to cryptogenesis.

There have been three main theories of natural parthenogenesis:

1. Owen's, that not all the germinal matter is necessary for the production of the new organism, and that after the new organism has matured, a left over portion of the germinal matter within it proceeds to develop new organisms.

2. Huxley's, that the parthenogenetic "egg" is not in reality an ovum, and that its development is comparable to the growth of an organism from a bud.

3. Hertwig's, that parthenogenesis is a degenerate fertilization.

Parthenogenesis as commonly understood may be said to be of three kinds: $a$, The resultant generation is all female (homocystic). $b$, The resultant generation is all male (homocystic). c, The resultant generation is heterocystic. Furthermore it may be divided into three cases:

1. The parthenogenetic generation alternates strictly with a bisexual onc.

2. Several parthenogenetic generations occur between the bisexual ones.

3. Pure syncysty; i.e., no reproduction other than parthenogenesis is known.

A common supposition is that the parthenogenetic gamete is an ovum or macrogamete pure and simple. It seems more difficult to explain the three kinds of parthenogenesis on the supposition that the gamete of the parthenogenetic organism is essentially or only an ovum or macrogamete, than on the supposition that this gamete is syncystic; for this latter supposition makes it easy to imagine the different results of natural parthenogenesis to arise by processes similar to those already familiar in heterocysty.

Cryptogenetically considered even parthenogenesis may be conceived of as a concealed, (often perhaps unseeable or at least hitherto unseen) but more or less ."normal" genesis.

A strict construction of the phase of cryptogenesis discussed rests on the supposition that what we have been calling parthenogenesis is one phenomenon, and not a collection of more or less related phenomena. My own present view is that this latter clause probably comes nearer the truth, and that parthenogenesis as we have broadly understood it may possibly cover cases in accord with most of the theories that have been proposed.**

Cryptogenesis may not exhibit all the phases investigation has disclosed in heterocysty; the suggestion is rather not only that all the results accomplished or supposed to be accomplished in natural parthenogenesis may be explicable along the lines of ordinary fertilization, but that parthenogenesis, and cryptogenesis if it exist, is not so much a distinct method of generation, or even a degenerate fertilization, as an evolved amphigony.

There is a certain amount of evidence often interpreted as showing that fertilization cannot be superimposed on parthenogenesis,- - e.g., parthenogenetic eggs may "resist" sperm of the same species. In normal fertilization once an egg entered by a spermatozoon it thereafter "resists" the entrance of other spermatozoa. If what we have been calling parthenogenesis is, in any given case, in reality cryptogenesis, then the parthenogenetic eggs may be regarded as

* This conception differs from earlier ones in its space-and time-limits, (time, antecedent; space the confines of ancestral gonic cells), and in that its methods and mechanisin are extonded to possibly include all the phases known for, or postulated of, the forms of genesis from wnich it is supposed to bo evolvod.

** Here we seem unconsciously dominated by our terminology, some of which is outgrown and, as applied, even misleading. The facts and ideas need critical analysis, as well as the benefit of an adequate terminology, as Sir E. Ray Lankester has just indicated in the August number of "Nature" (1917). 
already having the substances or factors imparted by the spermatozoon,or their equivalent, - and we might therefore expect such eggs to be, as they are, "resistant" to sperm. Whether or not this suggestion has a basis of fact, the theory of cryptogenesis harmonizes with what is known about the incompatibility of parthenogenesis and fertilization. Of course ova may be too young to be fertilized, or too old to be fertilized, and their parts may be so acted upon by a variety of forces as to bring them into a more responsive or less responsive condition; needless to say, these various facts must also be taken carefully into account in any such speculation as the foregoing.

\section{Abbreviations $U_{\text {sed }}$ in the Illustrations}

$\mathrm{A}^{m p, \text { ampulla }}$ mp sal dct, ampulla of salivary duct

amp subm, submedian ampulla $a m p$ vnt, ventral ampulla

amph, amphid

an, anus

$a n g l$, anal gland

apic, apiculum

ar dnt, rasp

ar lat, lateral field

ar vnt, ventral field

\section{Bas ph, base of pharynx}

blb crd, cardiac bulb

Cav som, body cavity

chrsm, chromosome

cl ar lat, cell of the lateral field

$c l$ crd, cell of cardia

cl int, intestinal cell

cl lat, lateral cell

cl msc, muscle cell

cl nrv. nerve cell

cl nov an, snal nerve-cell

cl nro cdl, caudal nerve-cell

cl nro crd, cardiac nerve-cell

cl nro dsl, dorsal nerve-cell

cl nro lat, lateral nerve-cell

d nro subm., submedian nervecoll

cl nro vnt, ventral nerve-cell cntr, centrosome

corp pol $I$, Ist polar body

c $p h$ h $p l$, cophalic papilla

cph set, cephalic seta

crd, cardia

$c$ at $p h$, pharyngeal rib

cut, cuticle

Dct, duct gland

dct ren, renette duct

dct sal dsl, dorsal salivary duct div red, reduction division $d n t$, denticles

$\mathrm{F}^{x} p$, excretory pore

$\mathrm{F}^{l x}$ ov, flexure of ovary

flx oor post, flexure of pos-

Gl, gland

$U^{\prime}$ gl an, anal gland

gl cdl, caudal gland

ol cdl subm, submedian caudal gland

$o l$ oe, oesophageal gland

ol sal, salivary gland

gng, ganglion

grn, granule

grn int, intestinal granule

grn int maj, larger intestinal granule

grn int $\min$, smaller intestinal granule
$\mathrm{I}^{n g}$, ingested material

ing nematod, ingested nenatode

int, intestine

int cryst, intestinal crystal int lum, intestinal lumen

Jnc. junction

L $a m l b$, labial lamina

$l b$, lips

ub $p p l$, labial papilla

lum, lumen

lum int, intestinal lumen

lum oe, oesophageal lumen

lum som, body cavity

Mit, mitosis figure

MI $m s c$ an, anal musclo $m s c$ oe, oesophagus muscle msc $p h$, pharyngeal muscle msc som, body muscle msc valv, valve muscle $m a c$ olv, vulva muscle mur int, intestinal wall mur $p h$, pharyngeal wall mur ut, wall of uterus

Nol, nucleus ncl ar lat, nucleus of lateral field

ncl cl int, nucleus of intestins] cell

ncl $c l$ nro. nucleus of nerve cell

ncl $o l$ cdl, nucleus of a caudal

gland

ncl lat, lateral nucleus

ncl msc, nucleus of muscle

ncl nro, nerve nucleus

ncl $o e$, oesophageal nucleus

ncl ov, nucleus of egg

ncl oo im, nucleus of ovum

ncl ren, renette nucleus

ncl $u t$, nucleus of an uterine cell $n c l v l v$, nerve nucleus of valve ncl vnt, nucleus of the ventral field

$n r$, nerve

nrv, nerve

nro af, afferent nerve

nro $r$, nerve ring

nro ent, ventral nerve

C, oesophagus

oes lum, oesphageal lumen on onchus

on' dsl, dorsal tooth

on $r t r$ dsl, retrorse dorsal onchus

on $\mathrm{rtr} s u b m$, retrorse submedian

$$
\text { tooth }
$$

on submd $x t$, right submedian tootb

on subm snst, left submedian tooth

oöcyt, oöcyte

org?, organ of unknown significance

org elast, elastic organ

org int, intestinal organ

ov, oviun oo dct, oviduct

ov $\mathrm{frt}$, fertilized egg

ov im, immature egg

oor rud, rudimentary ovary

ov ut, uterine egg

P spn, mouth of spinneret par, parasite

$p h$, pharynx

$p h$ str, pharyngeal striae

por or $p$; pore

por $g l$ oe, pore of cesophageal gland

por sal, mouth of the salivary gland

por sal dsl, mouth of dorsal sal-

ivary glane

$p p l$, papilla

$p p l$ cdl, caudal papilla

ppl $c p h$, cephalic papilla

$p p l$ intr, interior panilla

$p p l$ lat, lateral papilla

$p p l l b$, labial papilla

$p p l l b$ extr, exterior labial papilla

ppl subm. submedian papilla

$p p l$ subm sec, secondary submedian papilla

$p p l$ trm, terminal papilla

$\mathrm{R}^{c p t \text { sem, seminal receptacle }}$

I ret, rectum

reg ont, ventral field

ren, renette

rot, rotifer

rot ing, ingested rotifer

Sal, salivary gland

$S$ sal dct, alivary gland duct sal dsl, dorsal salivary gland

sal $g l d s l$, dorsal salivary gland

sal subm, submedian salivary gland

sec, secretion

sec cdl, caudal secretion

set, seta

set $c p h$, cephalic seta

set sub-cph, subcephalic sets

$s p$, spiculum

spm, spermatozoa

$\boldsymbol{s p n}$, spinneret

spndl, spindle

spthc, spermatheca

str $m u r p h$, striae of plaryngeal wall

subcut, subcuticle

sut $l b$, labial suture

Trm, terminus

1 trm oo, terminus of ovary trm oor, blind end of ovary

U $\iota$, uterus

Vag, vagina

vag msc, vaginal muscle valv, valve

vlv, vulva, valve

vatbl vag, vestibule of vagins 


\title{
THE ORDERS AND CLASSES OF NEMAS*
}

\author{
Contributions to a Science of Nematology, ViII
}

\section{By N. A. Совв}

Our first adequate conception of the nema phylum will come from a study of the free-living forms, for in parasitic nemas it frequently happens that the structure of important organs, especially those of the mouth and alimentary canal, is greatly simplified through degeneration. This degeneration proves to be relatively as marked among the parasitic nemas as it is among parasitic species in other phyla containing both free-living and parasitic forms, so that from the standpoint of eomparative morphology, they are often very highly perplexing. On the other hand, it is already possible, through comparative study of the known free-living genera, to begin formulating some of the principal features of the orders and classes of nemas and to do it with sufficient truth and elarity to make the results useful to investigators, teachers and students.

The following table outlines a classification of the phylum, based on a study of several hundred genera. It is a comprehensive classification I have long employed, that has proved useful in many ways. It sets forth relationships based on and correlated with the mouthparts. In most animal phyla where there is a distinct mouth, oesophagus and stomach, experience has amply proved the utility of these portions of the anatomy as a guide to phylogenetic relationships. While some parts of the following scheme appear to outline clearly and permanently certain fundamental truths, other parts undoubtedly will have to be expanded or altered, $\dagger$ since our knowledge of details is still inadequate to a clear view of all the larger relationships.

In the table, the order columns are staggered, in an attempt to indicate relative importance,- the farther to the left the words, the more comprehensive or significant is the meaning to be attached to the corresponding order name.

\footnotetext{
* Waverly Press, July 19, 1919.

$\uparrow$ Perhaps it may not be going too far to suggest that, in any such expansion and alteration, the aim be, among other things, to make the terminology of the phylum both euphonious and characteristic. Too few appear to realize how important it is that such a terminology be (1) As descriptive and as nearly self-explanatory as possible. (2) Few, short and consistent in its roots. (3) Simple and brief in jts terms. (4) Characteristic and euphonious. (5) Such as lends itself readily to modern inflections and derivations.
} 
Phylum NEMATES

(Nematoidea sensu restricto)

Subphylum ALAIMIA*

Class Alaimia

Subclass Manitinia
1. Order.........Litinia
Litinian

Angliciscd

Subclass Kinetinia

2. Order.........Bolbinia

Bolbinian forms; the Bolbinia

Subphylum LAIMIA

Class Anonchia

Subclass Anodontia

3. Order......... Cytolaimia

4. Order........ Isolaimia

Cytolaimian forms; the Cytolaims

Isolaimian forms; the Isolaims

5. Order..........Polylaimia

Subclass Odontia

6. Order........ A podontia

Polylaimian forms; the Polylaims

7. Order......... Synodontia

Class Onchia

Subclass Homonchia

8. Order....... Synonchia Synonchian forms; the Synonchs

9. Order..........Mesonchia Mesonchian forms; the Mesonchs

10. Order........Aponchia Aponchian forms; the Aponchs

11. Order....... Triplonchia Triplonchian forms; the Triplonchs

Subclass Heteronchia

12. Order.......Axonchia Axonchian forms; the Axonchs

13. Order........ Anaxonchia Anaxonchian forms; the Anaxonchs

*The names are the plurals of latinised Greek diminutives of the words, ' $l$, $\lambda \alpha \iota \mu \dot{\sigma} \sigma,{ }^{\prime} \gamma \chi \sigma \sigma$, with descriptive prefixes.

\section{ALAIMIA}

\section{Nemas without distinct pharynx}

Nemas devoid of pharynx may be conceiyed to be so either because they have never developed a pharynx or have evolved ("deteriorated") from forms having a pharynx. In the latter case, the group ALAIMIA may contain obscured equivalents of the subdivisions of the LAIMIA, the nemas with pharynx, It may therefore be possible to subdivide the ALAIMIA more fully after these forms, many of which are parasitic, have been further considered in their broad relationships. In this article only two subdivisions are suggested, of more or less superordinal rank, the Litinia, having a simple oesophagus without bulb or swelling, and the Bolbinia, having an oesophagus with a posterior or median swelling, or both. 


\section{LAIMIA}

\section{Nemas having a more or less distinct pharynx}

A distinction is here made among the mouth-parts of nemas, based on their origin, location and method of use. Sometimes these organs are labial, and sometimes they arise from elements deeper in the pharynx and more closely associated with the oesophagus proper. When arising by modification of the labial region they are here termed odontia,singular odontium. The odontia are usually of smaller size than the second class of mouth-parts that arise from elements farther back and more closely associated with the oesophagus proper, and called onchs or onchia,- -singular onchium. The odontia are seldom less than three in number, and may constitute a labial circlet of a dozen or more elements. The onchia are almost never more than three in number, and even then often only one of them is well developed.

According as they are characterized by the absence or presence of onchia, LAIMIA may be divided into two classes, the Anonchia and the Onchia. The Anonchia may in turn be divided into the subclasses Anodontia and Odontia, according as they lack or possess odontia. There are three anodontian orders, Cytolaimia, Isolaimia and Polylaimia; and two odontian orders, A podontia and Synodontia. Of these five orders, the first three are characterized by the pharynx being wholly unarmed, the pharynx of the cytolaims being a plain conoid, subspheroidal, or somewhat irregular cavity, the pharynx of the isolaims being mainly cylindroid or prismoid, i.e., parallel-sided, and the pharynx of the polylaims being more complex and composed of two or three successive chambers more or less distinctly separated from each other; while the latter two orders have a pharynx armed with odontia, the odontia of the Apodontia having an outward stroke, while those of the Synodontia have an inward stroke.

The second class of the LAIMIA, the Onchia, is characterized by a pharynx armed with onchia or with a spear composed of amalgamated onchia, and is divided into two subclasses, the Homonchia and the Heteronchia, differentiated from each other by the fact that in the homonchs the onchia are similar to each other and symmetrically arranged and nearly always three in number, while the heteronchs are characterized by asymmetrical onchia, the number of which may be reduced to one. There are four orders of homonchs and two of heteronchs. The four homonchian orders are the Synonchia, in which the onchia, nearly always three in number and of equal size, have an inward stroke; the Mesonchia, composed of forms intermediate between 
the aponchs and the synonchs, and having three usually equal onchia, movable in a direction parallel to the body axis; the Aponchia, in which the onchia, separate and nearly always three or six in number, have an outward stroke; and the Triplonchia, having a spear composed of three more or less equal, slender onchial elements which have become amalgamated. The heteronchian orders are, Axonchia, having a single axial onchium or spear, and the Anaxonchia, in which the main onchium is not axial and may or may not be accompanied by one or two others, usually of smaller size.

Onchia and odontia seem more or less mutually exclusive, but the presence of one does not necessarily exclude the presence of the other. When both are present, experience appears to indicate clearly that the onchia are the more advantageously used as a guide to broad relationships.

In a later publication the proposed orders will be further defined by describing new type genera. In the meantime, the following table gives a tentative assignment of a few known genera, for explanatory purposes only. Reference to families composing the orders is omitted, because it appears to me after studying a very large number of undescribed species in addition to those described, that a number of the families that have from time to time been proposed may have to be recast.

Order

LITINIA ....... Bastiana

BOLBINIA ...... Laxus

CYTOLAIMIA... Monhystera

ISOLAIMIA..... Rhabdolaimus

POLYLAIMIA... Bathylaimus, Plectus

APODONTIA.... Axonolaimus

SYNODONTIA.. Teratocephalus

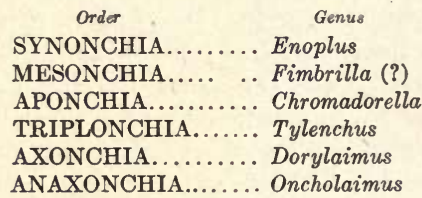

SYNONCHIA ........ Enoplus

MESONCHIA..... .. Fimbrilla (?)

APONCHIA ........... Chromadorella

TRIPLONCHIA....... Tylenchus

ANAXONCHIA........ Oncholaimus

The above linear tabular arrangements of the orders do not bring out fully the natural relationships of the different groups. These relationships will be discussed more fully later.

While the nomenclature here proposed is dominated by a few linguistic roots, it of course by no means follows that in assigning genera to the orders designated, due attention should not be given to other portions of the anatomy than those indicated by the nomenclature. This is a commonplace of taxonomy. The relationships of an organism are fully disclosed only on consideration of all its parts, and undue weight given to a particular feature will here, as elsewhere, result in confusion. 


\title{
ONE HUNDRED NEW NEMAS
}

\author{
(Type Species of 100 New Genera)
}

Contributions to a Science of Nematology, IX

\section{By N. A. Совв}

The arrangement of the genera in the following pages will serve, to a considerable extent, to define and illustrate the orders proposed on page 214. This is especially true of all except the Litinia, Bolbinia, Mesonchs and Aponchs. In each order, a genus has been selected and given a name philologically connected with that of the order, in fact, the singular of the order name, and, in most cases, these genera may be considered as genera typical of the orders,-for instance, Axonchium may be taken as typical of the order Axonchia.

So far as it is found advisable to accept the classification proposed, it might be well to keep in mind in the establishment of the many new genera which the future will undoubtedly disclose, the application of similar names to those genera which most nearly represent the average structure of the order. In carrying out this idea, such names as Cytolaimella, Isolaimella, and other derivatives at once suggest themselves.

It is already becoming evident that some of these groups may probably early be advantageously subdivided; e.g., Cytolaimia, Anaxonchia. In case of subdivision, the principles alluded to in the footnote to page 213 might lead to some such action as the following: Amending the definition of the existing order and segregating the new order, and utilizing for the new order-name the roots already suggested (see p. 214, lines 27-28) together with appropriate prefixes. This would result in building up a comparatively simple, rather homogenous and characteristic nomenclature for the nema phylum.

In each order the genera are arranged somewhat in accordance with their relationships. Genera of doubtful relationship are usually placed near the beginning or near the end of the order series, and not infrequently appear, in the light of our present knowledge, to be intermediate, or indeterminate, forms. Thus, Rhadinema flexile at the beginning of the Isolaimia, p. 256, is doubtfully placed, and may be a cytolaim; so Nannolaimus, p. 255, may perhaps be a litinian form. Most of the order series present these special cases. 
Oesophagus with median or posterior bulb or swelling, or both

A mphids none, so far as known; or difficult to see and therefore easily overlooked

Lateral wings to cuticle present; naked; striae fine; spinneret none; pharynx obscure

Mouth depr.; faint apophyses in pharyngeal bulb;-f-; excretory pore behind neck. Hyalaimus

Mouth not depr.; pharynx obscure, not apophysate; oesophagus faintly cephaloboid

Head with papillae minute lat. markings (amphids?); excrt. pore front of nerve ring. Litonema

Head without papillae or lateral markings; excretory pore behind the nerve ring.... Choroneme

Lateral wings to the cuticle none

Spinneret abs.; naked; oesoph. faintly cephsloboid; striae very fine; faint wings.. (Choronema) Spinneret present; cephalic setae present

Amphids invisible, known only as outlets of secretion; striae coarse; setae long. (Leptonemella)

Amphids tiny, tubular, labial, forward-pointing, easily overlooked; striae plain

Striae fine; male postanal tubular organs submedian; pharyngeal swelling present. (Catanema)

Striae coarse; pharyngeal swelling none; cephalic setae 4 or more; head nonstriate

Mouth cavity present, very small; males without supp. organs; nema $10 \mathrm{~mm}$...(Laxonema)

Mouth cavity none; vent. row acornshaped organs toward head; setae numerous. (Stilbonema)

Amphids present

Form of the amphids more or less irregular, symmetrical to at least one line

The amphids linear; cephalic setae $12+6$ small; striae coarse; spinneret present. (Leptonemella)

The amphids not linear; no spinneret; naked; labial region with papillae only

Winged; 'f; striae rather coarse; a mphids semi-ellipses; oesoph. faintly cephaloboid.Iotalaimus

Wings none; striae very fine; amphids with large internal connections............Bolbinium

Form of the amphids spiral, circular, or elliptical; spinneret present

Shape of the amphids distinctly spiral; striae fine, resolvable

Pharynx devoid of teeth; head expanded; cephalic setae 4 ; body setose..........Bolbonema

Pharynx with 3 minute teeth; setae in longitudinal rows.................... (Alaimonema)

Shape of the amphids circular; sometimes small, well forward on the head, and obscure

Lateral wings present; cephalic setae 4; amphids large

Striae coarse, altered on the lateral fields; lateral wing distinct.............. Antomicron

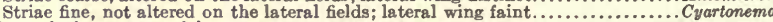

Iateral wings none or faint

External amphid circular, at least apparently, well developed; head set off

Body setose; amphids really spiral, on the head; striae rather fine, resolvable.... (Bolbonema)

Body naked; amphid circular

Position of amphid, on head; striae fine; contour not crenate; wings faint.... ( $C$ yartonema)

Position of amphid, behind head; striae coarse; contour crenate............. Cinctonema

External amphid minute, on the front of the head, tubular, easily overlooked; neck 2-5\%

Striae fine; male postanal tubular organs submedian; pharyn. swelling present.. (Catanema)

Striae coarse, plain; cephalic setae 4 or more; head without striae

Mouth cavity very small; nema $10 \mathrm{~mm}$; males without supplementary organs. . (Laxonema)

Mouth cavity none; cephalic setae in 3 or more circlets; males without caudal supplement

Supplementary organs of acorn shape, found in a ventral row near the head.. (Stilbonema)

Supplementary organs none, but with special pre-and postanal submed.setae. (Stilbonema)

Oesophagus plain, i.e. without median or posterior bulb or swelling of palpable size

Amphids none so far as known, or difficult to see and easily overlooked

Wings $10-12$; spinneret present; $\mathrm{f}=$; striae fine, plain; ceph. setae 6 , subceph. 4....Porocoma

Wings 2 , if any, lateral only, in any case faint

Spinneret abs.; oesoph. faintly cephaloboid; striae very fine; no setae; faint wings. . (Choronema)

Spinneret present; amphids minute if any, unclosed

Female organ single, f; 6 cephalic papillae only; spinneret doubtful; nema $0.28 \mathrm{~mm}$.. Litotes

Female organs double, 'f'; striae fine, plain; cephalic setae 4; 2 huge somatic glands.. (Ionema)

Amphids present

Form of the amphids more or less irregular, often symmetrical to at least one line

Contour of the amphids linear, i.e. long and narrow

Lateral wings none; cephalic setae 6 , subcephalic $4 ;$ ' $\mathrm{f}$ '..................Tycnodoro

Lateral wings present; head naked or its setae irregular; spinneret present

Wings prominent; striae coarse; ' $\mathrm{f}$ '; inconspicuous pharynx (?)............. (Actinonema)

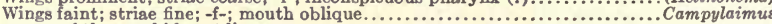

Contour of the amphids not linear

Striae interrupted on the lateral fields by wings, plain

Spinneret absent; oeosphagus faintly cephaloboid; ' $f$ ' ...................(Iotalaimus)

Spinneret present; oesophagus narrow, one to two-fifths as wide as neck

Anterior extremity mitriform, very distinctly set off; wings to the cuticle $6-8 . .$. Xennello

Ant. extremity not mitriform; coarse cuticular elements in rows like tiles; ' $f$ '. . (Ceramonema)

Striae uninterrupted, fine, plain; spinneret present; slender nemas, up to $1.2 \%$

Head without setae; amphids relatively large and deep; not ocellate........... Schistoder

Head with 4 cephalic setae; amphids small with obvious internal elements; ocellate. Nemella

Form of the amphids spiral, circular, or elliptical (doubtful in Litotes)

Shape of the amphids spiral; striae resolvable, not altered on the lateral fields

Spinneret absent; striae coarse; probably f' (?); contour crenate............... (Alaimella)

Spinneret present; striae rather coarse; a mphids large

Head expanded; lip-region elevated, not papillate; ceph. setae 6 plus 10; ‘f’... (Nannolaimus)

Head not expanded; lips 6 , papillate; cephalic setae $4 ; f^{\prime}(?) \ldots \ldots \ldots \ldots \ldots \ldots . .$. Alaimella

Shape of the amphids circular or elliptical; spinneret present except perhaps in Litotes

Lateral wings perhaps pres.; f'; striae plain; lipless; pharynx very minute, conoid. . Nemanema

Lateral wings none (or very faint); head rounded; striae none, or very fine

Cephalic setae ten or more, in two circlets

Contour of amphids unclosed behind; setae 12, 4 subcephalic; moutb a mere pore. Litinium

Contour of amphids closed; setae 10, no subcephalic; pharynx minute, conoid. (Linhomoella)

Cephalic setae none, or small ( 4 or 6 )

Setre 4, small; ocellate; amphids small, labial, unclosed; body contains huge glands. Ionema

Setae none; nemas of very simple structure; wings none or doubtful

Papillae representing 6 setae; amphid (?) minute; $-\mathrm{f}$; nema $0.28 \mathrm{~mm}$; width $5 \%$. Litotes

Papillae none; amphids fairly developed, closed; f'; nema $2.5 \mathrm{~mm}$; width $1.4 \%$. (Nemanema)

* For abbreviations used in the Key, see foot-note p. 223 . 
Wall of the pharynx unarmed (for alternative see page 221, midway)

Cavity of the pharynz conoid, or irregular

Oeosphagus with median or posterior swelling or both

Amphids none, or obscure; striae plain, not altered on the lateral fields

Spinneret none; head subtruncate, naked; contour almost imperceptibly crenate. Cephalobellus Spinneret present

Striae coarse; pharynx minute (none?) without swelling; head non-striated.... (Leptonemella)

Striae fine; pharynx narrow, enclosed in a swelling; amphids minute labial tubes. (Catanema) Amphids present

The amphids linear; pharynx (?); striae coarse, plain; no wings; setae, 3 circlets. . Leptonemella

The amphids spiral, circular, or elliptical; when tubular, surface contour eircular

Form of the amphids a distinct spiral; spinneret present

Striae coarse; oesophagus \pm oxyuroid; wings 8 ; cephalic setae 6 , subcephalic $4 \ldots$ Dasynema

Striae fine, plain

Lateral wings only on tail of male; ' $f$ '; pharynx somewhat zigzag; setae minute.. (Zalonema)

Lateral wings absent

Head with numerous seattered setae; pharynx small, narrow; onchi vestigial. (Alaimonema)

Head not with numerous setae; pharynx fairly well developed; teeth unobvious; ' $f$ '

Cheeks thickly cutinized; cuticle thick . . ................................ Zalonema

Cheeks, if thick, not heavily cutinized; lips 2, large, lateral.................Pseudonchus

Form of the amphids a circle or ellipse; striae unaltered laterally

Spinneret absent; striae fine

Intestinal cells with crystals; 6 lips, appendiculate; -f; cephalic setae $10 \ldots$. (Crystallonema)

Intestinal cells not with crystals; lips none; -f-; cephalic setae papilloid....... Anticyathus

Spinneret present

Head swollen, unstriated; no pharyngeal swelling; striae coarse, plain

Cephalic setae 4; lips none; no lahial papillae; amphids a single circle...... (Cinctonema)

Cephalic setae none; lips 6 , unipapillate; a mphids two concentric circles..... Micromicron

Head not swollen or set off; pharyngeal swelling anon present; striae fine, plain

Pnarynx narrow; amphids minute; male postanal organs tubular; setae $4+6$. (Catanema)

Pharynx capacious; amphids well developed, opposite the pharynx or behind it

Lips 2, lateral; pharyngeal ridges simulate teeth; ' $\mathrm{f}$ '; cephalic setae 4...... (Pseudonch us)

Lips 6, each with a digitate appendage; cephalic setae $10 \ldots \ldots . . . \ldots \ldots \ldots$ (Crystallonema)

Oesophagus plain, i.e. without median or posterior bulb or swelling

Amphids irregular

Spinneret none; striae unaltered laterally; -f-; spiral amphid placed on a deltoid part. Didelta Spinneret present

Striaealtered laterally; 'f'; head naked, set off; cuticle thick; pharynx armed (?).(Actinonema)

Striae not altered on the lateral fields

Cephalic setae $12+6$, in two circlets; $-\mathrm{f}$; amphid inconspicuous........... Leptogastrella

Cephalic setae 6 , in one circlet; amphid $\frac{1}{2}$ the width of the head; spinneret (?). Rhabdocoma

Amphids spiral, circular, or elliptical

The amphids in the form of a spiral; striae not altered on the lateral fields

Spinneret none (?); amphid $\frac{1}{3}$ the width of the head; naked; wings two.......... Neurella

Spinneret present

Buccal cavity with cutinous frame, and 3 apophyses with small apical teeth..(Trogolaimus)

Buccal cavity without distinct framework and without teeth of any kind; striae fine

Ovaries 'f'; striae resolvable; amphid of 4 winds; cephalic setae $6+10 \ldots$... Nannolaimus

Ovaries -f-; striae plain; amphids simulating a circle

Mouth cavity large and obvious; cephalic setae unknown, in any case few....Margonema

Mouth cavity small; head rounded; cephalic setae large, 4 (special) plus 6 ..... Linhomoella

The amphids in the form of circles or ellipses

Striae altered on the lateral fields by wings: spinneret present

Head set off by a groove, its appendages varied; striae coarse; wings numerous. . Xenolaimus

Head not strongly set off, though usually well developed

Ovaries reflexed, f'; naked; striae fine, plain; pharynx small, obsolescent....(Nemanema)

Ovaries outstretched; pharynx well developed, but not large; cephalic setae present

Striae fine, plain; cephalic setae 6 , segmented; $-f-\ldots \ldots \ldots \ldots \ldots \ldots \ldots \ldots$ (Cytolaimium)

Striae coarse; $-\mathrm{f}$

Cuticle rough from subdivided wings; setae many; lips 3 , mandibulate (?)...(X (Xala)

Cuticle normal; lips 6 , papillate, appearing somewhat fimbriate; striae plain. Daptonema

Striae not altered on the lateral fields

Spinneret absent; striae fine, plain; female organs outstretched, except Rhabdocoma

Ovaries two, $-\mathrm{f}-$

Cephalic setae 6, plainly segmented; lips three, papillate............... Cytolaimium

Cephalic setae 4, not segmented; lip-region punctate, amalgamated............ Didelta

Ovary one, subcephalic setae 4

Cephalic setae $6 ; f^{\prime} ;$ lips 3 , flattish, not digitate....................Rhabdocoma

Cephalic setae 10 ; -f; lips 6, small, digitate

Cells of intestine with crystals; amphids not raised; excretory pore labial. Crystallonemo

Cells of intestine without crystals; amphids raised; tail with spicate setae...... Zanema

Spinneret present

Pharynx obscure

Lips in two sets, outer large, low; inner soft extensions of the oesophagus. .Zygonemella

Lips not in 2 sets, amalgamated; ceph. setae 4,3-jointed; phar. prismoid cup. (Rhadinema)

Pharynx more or less obvious; striae plain

Female organs reflexed, $f^{\prime} ;$ striae fine; head naked; pharynx small..........(Nemanema)

Female organs outstretched

Ovaries two; striae fine

Mouth cavity small, inconspicuous; cephalic setae 4 (special) plus 6.... (Linhomoella)

Mouth cavity small, inconspicuous; cephalic setae 4 (special) plus 6 .... (Linhomoella)

Ovary one -f; striae coarse; setae 12; lips 6 , striate

Lips conoid, striated, large, tipped with setae, and with a seta near base. . Dactylaimus

Lips flat, papillate, appearing fimbriate, three-ribbed................. (Daptonema) 
Oesophagus with median or posterior bulb or swelling, or both

Amphids none so far as known, or diflicult to see and easily overlooked

Lateral wings present; spinneret absent

Striae none; wings 2; naked; pharynx like oesophagus lumen................. (Litonema)

Striae fine; wing 1 ; seta-like papillae 6 ; pharynx contains glottoid organ....... Cephalobium

Lateral wings absent; striae plain

Spinneret present

Striae very coarse; phar. cupshaped; nema $10 \mathrm{~mm}$; male, no supplement. organs. (Laxonema)

Striae fine; phar. small, enclosed in bulb; male postanal tub. organs submedian.. (Catanema)

Spinneret absent; setae none

Phar. very long, nar., ending in cylindroid bulb; ' $\mathrm{f}$ '; nema $1 \mathrm{~mm}$; lips conoid ....Myctolaimus

Pharynx very short; cardiac bulb oblate or spherical; $f^{\prime \prime}$

Nema $3.3 \mathrm{~mm}$; width $9 \%$; pharyngeal apophyses present; oesophagus clavate. Blattophila

Nema $6 \mathrm{~mm}$; width $3 \%$; pharyngeal apophyses absent; oesophagus cylindrical. Protrellus Amphids present.

The amphids are somewhat irregular, symmetrical to one line; striae fine, plain; wingless

Form of amphids not lin.; no spinneret; naked; lips 6; phar. tub.; amphids large.Colpurella

Form of amphids linear

Ovary one, 'f; spinneret absent; cephalic setae 6 plus 4; pharynx tubular....(Myolaimus)

Ovaries two, -f-; spinneret present; ceph. setae 4; ventral (?) onchus near mouth.Pseudolella

The amphids are spiral, circular, or elliptical

Shape of the amphids a distinct spiral

Striae coarse; oesoph. \pm oxy uroid; wings 8 ; ceph. setae 6 , subceph. 4 ; head unstr.. (Dasynema)

Striae fine

Lateral wings present; striae plain; spinneret present; head thick-walled....Pycnolaimus

Lateral wings absent

Spinneret none; -f-; cephalic setae 10, subcephalic 4 ; pharynx obscure.......Laimella

Spinneret present

Phar. armed in front with 3 obscure equal teeth; setae numerous on head. . (Alaimonema)

Pharynx without teeth; head rounded

Lips 2, latera]; ' $\mathrm{f}$ '; striae plain; pharyngeal ridges simulating teeth..........(Pseudonchus)

Lips none, or very indistinct

Cephalic setae 10, subcephalic 4; pharynx obscure, has minute onchus...... Laimella

Ceph. setae four? (or none); lips set off; phar. 2-chamb., 2d tapering .... (Polylaimium)

Shape of the amphid circular or elliptical (anon tubular); striae unaltered laterally

Spinneret absent; striae plain, fine

Ovary one, -f; ceph. setae 10, subceph. 6 ; a mphids circular; pharynx broad. (Crystallonema)

Ovaries two

Cuticle naked; spear (?) minute; ceph. setae 0,6 pap.; amphids ellip.; 'f'. . (Triplonchium)

Cuticle not naked; without spear; ceph. setae present; amphids circular; $-\mathrm{f}-$. A nticyathu.

Spinneret present

Buccal cavity very small and easily overlooked; cephalic setae present; striae plain

Striae coarse; phar. minute, cupshaped; males without supplementary organs.Laxonema

Striae fine; phar. narrow, bulbed; males with postanal rows of tubular organs. Catanema

Buccal cavity very long; 'f'; striae fine; cephalic setae small or none

Cephalic setae four (?) or none; amphids large, deep; pharynx 2-chambered... Polylaimium

Cephalic setae 10, short; amphids small; ocellate; pharynx very long......(Catalaimus)

Oesophagus plain, i.e, without median or posterior bulb or swelling

Amphids none so far as known, or difficult to see and easily overlooked

Wings present; spinneret present; striae fine; ovaries two, $\mathrm{f}=$; phar. vestigial. (Porocoma)

Wings absent; striae fine

Ceph. setae 10, long, segmented; lips 6 , thin, each with seta; amphid minute slit. . (Trilepta)

Cephalic setae none, or not long or obviously segmented; lips without setae, or lipless

Buccal cavity tubular; spinneret absent; ' $f$ '; lipless; papillae depressed.....(Isolaimium)

Buccal cavity not long and tubular; spinneret present

Ceph. setae 10; lips 6, obvious; dorsal phar. element bent in at lips; ' $\mathrm{f}$ ' (?)...(Asymmetrella)

Cephalic setae 6 , papilloid; lipless; $-\mathrm{f} \ldots \ldots \ldots \ldots \ldots \ldots \ldots \ldots \ldots \ldots \ldots \ldots \ldots \ldots$ (Litotes $)$

Amphids present

Structure of the amphids more or less irregular, usually symmetrical to at least one line

Contour of the amphids linear; striae not altered on the lateral fields

Ceph. setae long, segmented; lips 6 , thin, each with seta; amphid minute slit. . (Trilepta)

Cephalic setae none, or not long and not obviously segmented

Spinneret absent; 'f'; striae fine; phar. tub.; lips confluent; papillae depressed..Isolaimium

Spinneret present; female sexual organs double; striae fine

Buc. cavity tub.; amphids long; striae resolve; onchus ventral (?), obscure.. (Pseudolella)

Buccal cavity not tubular; amphids more or less stirrup form; striae not resolvable

Ocellate; cephalic setae none; lips faint; amphids distinct, though small...... (Illium

Ocelli 0; setae 10; cutinized dorsal pharyn. element bent inward at lips.. ( $A$ symmetrella)

Contour of the amphids not linear

Striae altered on the lateral fields; ovaries two, reflexed; spinneret present

Lateral wings present; striae coarse, like rows of tiles; pharynx narrow ...... Ceramonema

Lateral wings none; striae fine, plain; onchia vestigial; six cervical glands. (Anoncholaimus)

Striae not altered on the lateral fields, fine

Spinneret none

Cephalic setae 4, forward-pointing; amphids more or less deltoid............(Didelta)

Cephalic setae 0 ; amphids more or less reniform ...................... Colpurella Spinneret present

Cheeks thickly cutinized; head conoid; small forward-pointing onchia (?).(Isonemella)

Cheeks not thickly cutin; head not prom. conoid; no small onchia exc. in Anoncholaimus

Ovaries outstretched, -f-; ocellate; phar, tubular; nearly lipless; ceph. setae 4. (Coinonema)

Ovaries reflexed, 'f'; striae fine, plain; pharynx not tubular; head truncate

Cephalic setae none; ocellate; lips faint; amphids distinct, though small........ Illium Cephalic setae 10; ocelli none; amphids indistinct

Dorsal cutinized element of the pharynx bent inward at the lips......(Asymmetrella)

Dorsal wall of the pharynx not differentiated; onchia minute......... (Anoncholaimus)

Structure of the amphids spiral, circular, or elliptical

12

54 
Form of the amphids a distinct spiral; spinneret present

Winged; ' $\mathrm{f}$ '; striae coarse, plain; spinneret heavily cutinized; cephalic setae $4 \ldots .$. . Cynura Wings none; female sexual organs double; striae fine, plain

Ovaries reflexed; no lips; pharynx none or nearly obsolete; osellate.............(Ionema)

Ovaries outstretched, -f-; cephalic setae 4 ; pharynx tubular, narrow

Spiral amphid of one wind, somewhat hook-form; ocellate; pharynx obscure. . Coinonema

Spiral amphid of several winds; not ocellate; pharynx well developed...........Xinema

Form of the amphids circular or elliptical

Striae interrupted on the lateral fields; lateral wings present; spinneret present

Female organs single, $-\mathrm{f}$; a mphids 2 concentric circles; setae in groups of 3 ...... (Omicronema)

Female organs double, 'f'; amphids not 2 concentric circles; setae not in groups

Spinneret heavily cutinized; cephalic setae 4; a mphids really spiral..............(Cynura)

Spinneret simple, normal; cephalic setae 10 ; amphids small, elliptical.... (Anoncholaimus)

Striae not interrupted on the lateral fields

Spinneret none; striae fine, plain

Ovarios 2,-f-; setae 4; amphids more or less deltoid; phar. irregularly napiform.. (Didelta)

Ovary 1, outstretched; a mphids not deltoid

Amphids very small; ceph. setae in 3 circlets of $6 ; \mathrm{f}-;$ cuticle reticulated.... Halanonchus

Amphids large; setae 10 , with 6 subcephalic; $-f$; lips with digitate processes

Intestinal cells with crystals; caudal setae none; amphids not raised.......(Crystallonema)

Intest. cells without crystals; amphids raised; about 10 thorn-like caudal setae. (Zanema) Spinneret present

Ceph. setae in $3 \mathrm{~s}$; coarse, transverse striae resolvable into elongated elements. .Omicronema

Cephalic setae not in groups; striae, if resolvable, not into elongated elements

Buccal cavity more or less obscure, easily overlooked; wings none

Ceph. setae 4, 3-jointed; lips confluent; amphid $\frac{1}{3}$ wide as trunc. head, closed. Rhadinema Cephalic setae 6 or more, minute or papilloid

Lips none; amphid (?) unclosed behind, minute; head rounded; -f......... (Litotes)

Lips soft and rather obscure; amphid fairly well developed, closed.......(Zygonemella)

Buccal cavity obvious; setae only in Halinema long, then twice as long as head is wide

Ocelli present; ceph. cuticle thick; head distinctly conoid; minute onchia (?).Isonemella

Ocelli none; cephalic cuticle not thickened; head not distinctly conoid; no onchia

Ovaries two, 'f'; striae fine, plain; onchia vestigial; 6 cervical glands..... Anoncholaimus

Ovary one, -f; striae usually rather coarse; pharynx quite unarmed

Head end diminished to form a sort of beak; pharynx tubular.......... Rhynchonema

Head end not diminished to form a beak; pharynx not tubular

Setae and amphids large; phar. shorter than head is wide; caudal setae pres.. Halineme

Setae and amphids medium size; lips conoid, tips with minute "claws". (Dactylaimus)

\section{Wall of the pharynx armed with teeth or onchia}

Armature spear-like; caudal glands and spinneret typically (usually) absent

Spear with a bulbous base; striae fine, plain

Oesophagus plain, faintly cephaloboid; -f; cuticle with wings.

Oeosphagus with a posterior bulb or swelling; cuticle without wings

Amphids ellip., well devel. internally; 'f'; spear faint; lips 6 , faint, unipapillate. Triplonchium

Amphids linear, transverse; oesophagus dorylaimoid; f'; no labial papilae...... Doryllium

Spear plain, i.e., without bulbous base; striae fine, nearly always plain

Oesophagus plain, i.e. without median or postcrior swelling; spinneret present

Amphids 0; -f; cephalic setae 0; bead truncate; lip-region set off; spear small "prod" .Iotonchium

Amphids present; lip-region not set of

Amphids symmetrical to one line only; setae 10; pharynx long; spear toothlike.(Catalaimus)

Amphids spiral, sometimes faint; 'f'; striae unaltered laterally; cephalie setae 4

Ocellate; amphids labial; "spear" slender in nar. phar.; lips and papillae none. Onchulella

Ocelli none; lips 3, papillate; "spear" toothlike, in an open cavity.......... (Digitonchus)

Oesophagus with posterior swelling which sometimes occupies $\frac{1}{2}$ the neck

Spinneret present; amphids spiral (?); eyes present; ceph. setae 4 ; lipless............ Onchium

Spinneret none; striae fine

Amphids 0; -f; no ceph. setae; head truncate; lip-region set off; spear a small prod.Iotonchium

Amphids present

The amphids elliptical; spear-tip bent; ‘f; lips 6 , bipapillate............... Campydora

The amphids symmetrical to one line only; lips set off by constriction

Female organs double, $f^{\prime}$; spear long and very slender; pre-rectum very long... Leptonchus

Female organ single, $f^{\prime}$; spear short, not slender; pre-rectum short

Posterior oesophageal swelling short, not set off by constriction in front....(Doryllium)

Posterior oesophageal swelling long, set off by constriction from previous part. Axonchium

Armature of one or more teeth

Tooth one (for alternative see top Page 223: "Teeth two-"

Oesophagus with a median or posterior bulb or swelling, or both (for alternative see Page 222)

Amphids none or unknown, or so inconspicuous or faint as to be difficult to see

Striae fine, plain; spinneret none; 'f; naked; lips 6 , bipapillate; spear bent at apex. Campydora

Striae altered on the lateral fields

Wingless; spinneret present; onchulus with large dorsal muscle; setae small....(Iotadorus)

Wings present

Spinneret present; striae coarse, resolvable; 'f'; lips retractile; setae 4.....(Ptycholaimellus) 116

Spinneret absent; striae fine, plain; ‘f; lips not retractile, naked; spear bent..(Campydora) 88

Amphids present

Structure of amphids more or less irreg., of ten symmetrical to one line (for alternative see Page 222)

Contour of the amphids not linear; spinneret present

Striae altered laterally; onchus small, well forward; setse obscure; lips $12 \ldots \ldots$ Iotadorus 115

Striae unaltered laterally; ' $\mathrm{f}$ '

Setae foscieule

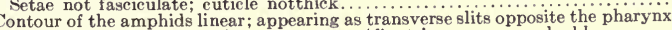

Striae altered laterally; spinneret present; ' $\mathrm{f}$ '; striae coarse, resolvable . 
New Genera

Lip-region retractile; cephslic setae 4, rather obviously segmented........Ptycholaimellus 116

Lip-region non-retractile; cephalic setae 10, not obviously segmented.............Rhips 118 Strise not altered laters!ly, or very little; cephalic setae 10

Lips thin and flap-like; cephalic setae long snd obviously segmented..

Lips not thin or flap-like; cephalic setae shorter, not obviously segmented; $\because \ldots$. . Trilepto

Striae resolvable; pharynx not subdivided; onchus not spear-like; no cervicsl setae.Rhips

Strise plain: pharyn in distinct chambers;

Structure of the amphids spiral, circulsr, or ellipticsl

Form of the smphids more or less distinctly spiral; spinneret present, doubtful in Laimella

Strise altered on the lateral fields; cephalic setae 4

Wings present; striae fine, resolvable; lips 6, confluent, bipapillate; -f-......Pepsonema

Wingless; tooth with apophysis faintly y-shaped; striae cosrse; amphids labial.. Ypsilon

Strise not sltered on the lateral fields

Onchus one, with its apophysis somewhat y-shsped; striae cosrse; setae 4......Ypsilon

Onchus not $y$-shsped, of ten with smsll compsnions

Amphids distinctly spiral

Setse 0; body stristed (exc. hesd); phsryn. bulb faint, cardisc nspiform. . Amphispira 103

Setse, cephalic 10, subcephalic 4; non-striated; $-f-$; onchus spesr-like....... (Laimella) 44

Amphids sppearing circular

Lips 12-fold; onchus somewhst spear-like; cephalic setae small, 6 plus 10-12..Mesodorus 105

Lips faint, not over 6; onchus not spesr-like

Ovsries -f-; phar. bulbous; smphids circular (?); setae 6 + 6; striae fine. (Bolbolaimus) 100

Ovaries reflexed, ' $\mathrm{f}$ '

Strise fine, plain; onchuli minute; lips fsint, 6; pspillse conicsl; setse 4.. (Polysigma) 106

Striae cosrse; head not striated; cuticle thick; cephalic setae fasciculate...Xanthodora 98

Form of the amphids circular or elliptical

Striae altered on the lateral fields; spinneret present

Wingless; labial amphids elliptic; strise resolvable; pharyngeal bulb one-sided.Iotadorus 11

Wings present; striae coarse

Female sexual organs single, -f; cephalic setae 4, labial setae 6; wings numerous.. Nudora 113

Female sexual organs double, ' $f$ '

Hesd expanded, nsked; striae not retrorse; tooth smsll; lips minute, obscure. Xenonema 102

Hesd not expanded, its setae 4; lips obvious; striae appar. retrorse throughout. Rhinema 114

Striae not sltered on the lateral fields

Cephalic setse none

Head hemispher., expanded; lips 6, conoid; spinneret pres.; onchus obscure. (Micromicron) 104

Head not expanded; lips set off, confluent; no spinneret; "f; "spesr" bent.. (Campydora) 88

Cephalic setae and spinneret present

Onchus spear-like; amphids resemble concentric circles; setae in 2-3 rows.... (Mesodorus) 105

Onchus not spear-like; smphids not concentric circles; setae, if numerous, fascicled

Female sexual organs outstretched

Ovsries two; cephalic setae $6+6$; submedian onchia present.............(Bolbolaimus) 100

Ovsry single; ceph. setae 4; submed. onchia none or vestigial..............Synonema 110

Femsle sexusl organs reflexed, ' $f$ '; amphids really spiral

Submedian onchi present; labial papillae conical; setae 4; striae fine......(Polysigma) 106

Submedian onchi abs.; setse in fascicles; head non-striated; cuticle thick. (X Xnthodora) 98 Oesophagus plain, i.e. without median or posterior bulb or swelling; smphids present

Form of the smphids more or less irregular, usually symmetricsl to at least one line

The amphids linear; spinneret present; 'f'; striae cosrse, sltered laterslly

Striae plain; head naked, set off by constriction; wing 1, prom.; cuticle thick. . Actinonema 117

Striae resolvable into rods; ceph. setae 10; wingless; smphid 5-6 times as wide as long. Rhips 118

The smphids not linear; strise not sltered on the latersl fields

Head conoid; cheeks thick; ocellate; onchus minute; phar. narrow; setae 10.....(Isonemella) 109

Head not conoid; cuticle of the cheeks not thick; ' $f$ '; striae fine, plsin

Spinneret absent; lips 6, unipapillate; cephslic setse 6; amphid behind pharynx.Onchulus 94

Spinneret present

Buccal cavity extending beyond the nerve-ring; onchus spesr-like; ocellate.Catalaimus 89

Buc. cavity not unususlly long, its dors. element bent at lips; not ocellate. Asymmetrella 70

Form of the amphids spiral, circular, or elliptical

The shape of the amphids a more or less distinct spiral; spinneret present; ' $f$ ' or $-f$

Striae sltered laterally, fine, resolvable; wings present; cephslic setae 10; 'f'. . Acanthonchus 101

Striae not altered on the lateral fields, coarse, plain

Spinneret unknown, abs. (?); -f; setae 10; onchi submedian; contour crenste..(Gonionchus) 97

Spinneret present; ' $\mathrm{f}$ '

Lips three; setae 6 ; smphids labial; onchus digitate................. Digitonchus 95

Lips more thsn 3 ; setse num., scattered; amphids not lab.; onchi more than 1 .(Croconema) 112

The shspe of the amphids circulsr or ellipticsl

Striae sltered laterslly; wings numerous with retrorse bristles; cephalic setae 4..Rhinema 114

Striae not sltered on the latersl fields

Head conoid; cephslic setae 10; spinneret present

Ocelli none; ' $\mathrm{f}$ '; rear portion of oesoph. succession of bulbs; strise fine, plain.(Bolbella)

Ocelli present; no succession of bulbs in the posterior part of the oesophsgus

Cheeks thick; onchus appressed, minute; pharynx rather long snd narrrow. (Isonemella) 108

Cheeks not thick; onchus digitate, well developed ;pharynx not narrow, short. Cophonchus 108

Head not conoid; not ocellate; pharynx not long and narrow

Spinneret unknown; a mphids spiral; -f; setaé 10; onchi submed.; contour cren.(Gonionchus) 97

Spinneret present

Onchi 3, clustered; phsr. unobv.; setse scsttered; cuticle thick; contour cren.. (Croconema) 112

Onchus single, dorsal; - f; cuticle thin

Setae 4; phsrynx small, closed; diameter of amphid 1 the width of the head. . Synonema 110

Setae in $2 \mathrm{~s}$ and $3 \mathrm{~s}$; phsr. obv., open; diam. of amphid about $\frac{1}{2}$ width of head. Anticyclus 111 
Teeth two or more

New Genero

The teeth more or less equal in size, and usually mobile

Oesophagus with median or posterior bulb or swelling, or both

Onchi or teeth have an outward throw; amphids circular; spinneret present; -f.. A ponchium

Onchi or teeth have an inward throw

Amphids none, or so obscure as easily to escape notice; f-; teeth (?) $12 \ldots . .$. (Synodontium)

Amphids present

The amphids horse-shoe shaped, faint; striae fine, unaltered laterally; f-.... Synodontium

The amphids spiral, circular, or elliptical; striae fine, plain

Shape of amphids spiral; winged; spinneret pres.; naked; head expanded. .(Pycnolaimus)

Shape of amphids round or elliptical

Terminus hamate; amphid minute, $\frac{x}{10}$ the width of the head; winged....Chambersiello

Terminus not hamate; amphid $t$ the width of the head; wingless; $-\mathrm{f} . . . . .$. (A ponchium)

Oesophagus plain, i.e. without median or posterior bulb or swelling

Onchi or teeth have an outward throw; amphids present; spinneret present

Form of amphids symmetrical to one line only; striae fine, plain; pharynx bulbous

Ovaries two, 'f'; onchi 3, duplex; cephalic setae 6 plus 6 ; head not set off........Ironella

Ovary single, f'; lip-region set off; lips 6 , bipapillate; spinneret ventral...... Trissonchulus

Form of the amphids spiral, circular, or elliptical

The amphids spiral; winged; 'f' (or -f-); lips bipapillate; cephalie setae 4...... Mesonchium

The amphids elliptical; striae fine, resolvable, unaltered laterally; setae $4 \ldots \ldots$ A podontium

Onchi or teeth have an inward throw

Amphids 0 or unknown; onchi small, apophysate, lips 12, appendiculate...... Gammanema

Amphids present; spinneret present (questionable in Crystallonema)

Contour of the amphids circular

Wings many; 3 mandibulate lips; setae in 3 circlets; cuticle rough............Xyalo

Wing one or none; striae not altered on the lateral fields

Cephalic setae none; armature of three biting plates or hooks; wingless......Tripylium

Cephalic setae ten

Armature, 18 duplex radiating refractive ribs; winged; $-\mathrm{f} \ldots \ldots \ldots \ldots \ldots \ldots$ Monhystrium

Armature, 6 talon-like or digitate labial elements; $-\mathrm{f} . \ldots \ldots \ldots \ldots \ldots \ldots \ldots \ldots$ (Crystallonema)

Contour of the amphids spiral; striae resolvable

Winged; ovaries reflexed or outstretched; cephalic setae 4; pharynx cylindroid. Mesonchium

Wings absent; the 3 onchia enclosed in a bulb

Onchi small, with heavy apophyses; , encl. in swelling; a mphid of $2 \frac{1}{2}-3 \frac{1}{2}$ winds.Trogolaimus

Onchi large, without apophyses; phar. bulb spheroidal, amphid of $1 \frac{1}{2}$ winds. . Synonchium The teeth usually unequal in size, not mobile

Oesophagus plain, i.e. without median or posterior bulh or swelling

Amphids none, or obscure and escaping notice; pharynx with many denticles; 'f'. (Thoönchus)

Amphids present

The amphids irregular, usually symmetrical to one line; spinneret present; ' $\mathrm{f}$

Form of the amphids not linear; striae fine, plain; pharynx denticulate.......Thoönchus 91

Form of the amphids linear; striae coarse, resolvable; cephalic setae $10 \ldots . . . . . . . .(R h i p s)$

The amphids spiral, circular, or elliptical

Form of the amphids spiral; striae coarse, plain, unaltered; spinneret present

Pharynx and onchi minute, obsc.; lips not thin; setae scattered; amphids small. Croconema 112

Pharynx obv.; onchi plate-like, submedian; lips thin; setae 10; amphids large.Gonionchus 97

Form of amphids circular or elliptical; spinneret present (unknown in Gonionchus)

Winged; amphids large, faint; lips appendiculate; striae coarse, resolvable.. Anoxonchium 99

Wings none; cephalic setae 10; striae plain; lips thin

Ovaries 'f'; striae fine; phar. with many denticles; amphids ohscure, ellip...(Thoönchus) 91

Ovary -f; striae coarse, contour cren.; onchi plate-like; amphids spiral......(Gonionchus) 97

Oesophagus with median or posterior bulb or swelling, or both

Amphids none or unknown; pharynx bulbous; striae fine, resolvable........(Bolbolaimus) 100 Amphids present

Form of the amphids linear; wingless; ovaries reflexed

Spinneret present; 'f'; striae coarse, resolvable; cephalic setae $10 \ldots \ldots \ldots \ldots \ldots \ldots$. Rhips 118

Spinneret absent; ‘f; striae fine, plain; cephalic setae 6 plus $4 \ldots \ldots \ldots \ldots \ldots \ldots$ Myolaimus 60

Form of the amphids spiral, circular, or elliptical

Shape of the amphids circular; striae fine, unaltered laterally; spinneret present

Ovaries reflexed, 'f; lips bipapil.; setae 4; submed. onchi minute; striae plain. (Polysigma) 106

Ovaries outstretched

Pharynx with distinct spherical bulb; striae resolvable; setae $6+6+4$. Bolbolaimus 100

Pharyngeal bulb a faint swelling; striae plain; cephalic setae $4 \ldots . . . . . . .($ A ponchium) 79

Shape of the amphids a distinct spiral; cuticle wingless; spinneret present

Onchus spear-like; lips minute, 12 ; cephalic setae $6+12+6$; striae fine, plain.Mesodorus 105

Onchus very small, not spear-like; lips none or obscure

Lips bipapillate, papillae conical; 'f'; amphids labial; cephalic setae 4.......Polysigma 106

Lips non-papillate

Setae in 6 longitudinal rows; amphids opp. pharynx, $\frac{1}{2}$ the width of the head. Alaimonema 107

Setae none; amphids not opposite the pharynx, $\frac{1}{8}$ the width of the head.... Amphispira 103

abs, absent

apophys, apophysate

appar, apparently

arc, arcuate

buc, buccal

chamb, chambered

clav, clavate

col, colored

cren, crenate
Abbreviations used in Key, not found on p. 341

cutin, cutinized

depr, depressed

devel, developed

digit, digitate

dist, distally

ea, each

encl, enclosed

lab, labial lin, linear

nar, narrow

numer, numerous

obsc, obscure

obv, obvious

pap, papillae

pres, present

prom, prominent sim simple

swol, swolle

spin, spinneret

term, terminu

thr, through

tub, tubular

uni, uniform

unstr, unstriated
65

91

$\frac{1}{8}$

(1)

2

9

7

0

18

60


BURSA PRESENT: spicula two, equal; tail more or less arcuste-conoid; no spinneret

Spiculum one, straight, uncephalated, 'm; spinneret none; tail acute

Accessories pres.; 1;()1;1, simple (postanals, single); spicula $2^{\text {ce }}$ anal body width.. Cephalobellus Accessories none; supplements present, papilloid; found in insects

Supplements 2();2; spiculum one-fourth as long as anal body diameter; tail conoid. Blattophila Supplements simple; spiculum twice as long as anal body diameter

Anus $90 ; 10,1 ; 1$; small access. (?); tail hemispheroid, then conoid; terminus conical. Protrellus

Anus 80 ; accessories (?); tail conical, its terminus pointed.................. Cepholobellus

Spicula two, equal, more or less arcuate (exc. Cophonchus, Synonchium, Iotalaimus, Ironella, Bolbinium, Myctolaimus?)

The spicula jointed, long, not distinctly cephalated, of uniform width; spinneret present

Supplements none; -m-; terminus clavate; spinneret armed; accessories apophysate....Xinema

Supplements 25 , vent., preanal, simple, rather faint; $-m$; tsil conoid; accs. not apophys..Rhip

The spicula not jointed; tail conoid or subconoid (cylindroid posteriorly in Ancicyathus, Anticyclus, Cophonchus, Cyartonema, Gonionchus, Daptonema, Halanonchus, Ironella, Anaxonchium, Monhystrium, Schistodera, Pepsonema, Myctolaimus, Rhabdocoma, Bolbella, Thoönchus, Trilepta, Mesonchium, Cytolaimium, Tripylium, Zygonemella, Synodontium and (?) Xennella) Accessories to the spicula none, or very faint

Supplements present; spinneret absent, except in Synonchium

Supps. dorylaimoid; spic. cephalated by expans., width unif.; -m-; term. rounded. Doryllium

Supps. all ventral; spicula about $1 \frac{1}{2}-2^{\mathrm{ce}}$ as long as anal body diameter, uncephalsted

Position of supps. not preanal only; 4 preanal, simple; 1 postanal, not simple; $=m$ ?. Colpurella Position of supplements preanal only; supplements simple

No, of supps. 4; spicula not of uniform widtb; ?=m; tail rather irreg. conoid. Colpurelle

No. of supps. 2 ; spicula straight, width rather unif.; tail conoid-hemispherical. Synonchium Supp? ements none or exceedingly faint

Spicula slightly cephalated by expansion, unif,; tail conoid, then cylindroid; $-\mathrm{m}-Z_{\text {ygonemella }}$ Spicula not cephalated

Width of the spicula not uniform; spinneret absent (?)

Spicula straight; about $\frac{1}{2}$ as long as anal body diam.; tail short, bluntly conoid. Bolbinium

Spicula arcuate; $-\mathrm{m}$; tail conoid, then cylindroid, its term. rounded, $\frac{1}{3}$ as wide as base. Trilepto

Width of the spicula more or less uniform; spinneret present, except in Triplonchium

Spicula straight; tail conoid-hemispherical; term. broad; spinneret a pore.. (Synonchium)

Spicula arcuate

Spinneret absent

Spicula very strongly arcuate; bursa (?); terminus broad, rounded; $-\mathbf{m}$.... (Triplonchium)

Spicula not very strongly arcuate; no bursa; terminus not broad............ (Rhabdocoma)

Spinneret present; spicula not strongly arcuate; no bursa

Anus 85 ; tail conoid, then cylindroid; width over $5 \%$; spic. only slightly arcu..Zygonemella

Anus 97; term. broad, rounded; width about $1 \% ;-m ;$ single questionable supp.. Nemaneme Accessories to the spicula present

Supplementary organs present near the anus (for alternative see middle opposite page)

The supplements not all ventral; spicula $1 \frac{1}{4}-1 \frac{1}{2}$ as long as anal body diameter

Position, preanal; not simp.; 2 rows 38 ea.; non-unif. spic. uncephalated; $-\mathrm{m}$....... Polysigma

Position, not preanal only; no spinneret (except in Catanema and (?) Cytolaimium)

Form of supplements simple; -m-; spicula uncephalated, width rather uniform

Supplements papilloid, 5 preanal, 4 postanal in pairs . . . . . . . . . . . . . . . . .

Supplements discoid, 11 pre-, 5 postanal pairs; spic. $1 \frac{1}{6}$ long as anal body diam. .Cytolaimium

Form of the supplements not simple

Spiculs not cephalated; 16 pairs of supplements; terminus barely swollen;-m-. Cytolaimium Spicula cephalated

Cephalated by constriction, straight?, width not uniform; 9 pairs papillae; ' $m$. Myctolaimus Cephalated by expansion: width of spicula rather uniform

Arrangement of papilloid supps. $1,1,1,1,1,1,1(1), 2,1,1 ; 3,{ }^{4} \mathrm{~m}$; term. hooked. Chambersielln

Arrangement of tub. supps. postanal, submed., about 7 prs.; -m; term. straight Catanem

The supplements all ventral; spinneret present (exc. Anticyathus, (?) Anticyclus, Neurella)

Position, preanal 17, postanal few, simple; spicula uncephalated, tapering (?); -m-. Anticyclus

Position of supplements preanal only

Structure of supplements not simple (for alternative see page 225)

Proximal ends of the spicula not cephalated

Width not uniform, length $1 \frac{1}{2}$ anal body diam.; 37 "campanulate" supps.; -m. Alaimonema Width of the spicula more or less uniform

No. of supplements 1 ; spicula $2 \frac{2}{2}$ times as long as anal body diam., straight; $=\mathrm{m}$. (Ironella) No. of supplements 4 or more; terminus more or less blunt

Apophysis present; spicula $3^{\text {ce }}$ s s long as anal body width; supps. $12 ;{ }^{\dagger} \mathrm{m}$. . A ponchium

A pophysis none: spicula long as anal body diam; acc. prom; supps, 4 ; -m. Acanthonchu

Proximal ends of the spicula cephalated

Spicula cephalated by constriction, twice as long as anal body diameter

Supplements 2, not simple; spicula rather uniform, their cephalation faint; $-\mathrm{m}-$. Bolbello Supplements 6 , mammiform; width of spicula not uniform; -m(?) ........ Xanthodora

Spicula cephalated by expansion or contraction; width of spicula rather uniform

Cephalated by contraction, strongly arcuate, as long as anal body diam.; -m. (Stilbonema) Cephalated by expansion

Supplement one, tubular; spicula nearly straight; tail conoid, then eylindroid

Length of spicula $5-6$ anal body diameters; -m-; spinneret bluntly conoid... Cophonchus

Length of spicula $2 \frac{1}{2}$ anal body diameters; $=\mathrm{m}$; supplement bent toward anus.. Ironella 
Supplements numerous, 8-21

New Genera

Length of spic. $2^{\mathrm{ce}}$ anal body width; proximae hooked; supps. 21 , flat; -m-. Trogolaimus

Length of spicula 1-1 times as great as the anal body diameter

No. of protrusile(?) tub. supps. 8 ; acc. $\frac{?}{3}$ as long as spic., bends away; -m'. Antomicran

No. of faint, non-tubular supps. 16 ; acc. parallel, ta as long as spicula;-m-.Gammanema Structure of supplements simple

Proximal ends of the spicula not cephalated

Width of spicula not uniform

No. of supplements one, flat, faint; accessories, simple, faint; $=m(?) \ldots . . .$. Neurella

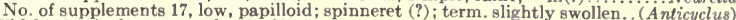

Width of spicula more or less uniform

No. of supplements two, obscure; accessories stout; term. convex-conoid, acute. Nudor

No. of supplements 15-20; terminus not acute

Length of the spicula twice as great as the anal body diameter; ' $m$...... Anaxonchium

Length of the spicula as great as the anal body diameter; $-\mathrm{m}-\ldots . . .$. . Halanonchus

Proximal ends of the spicula cephalated

Cephalated by constriction, about $1 \frac{1}{4}$ as long as anal body diameter; supplements 6-40

Width of spicula rather uniform; accessories 2 , apophysate; $-\mathrm{m}$; term. blunt. Margcnema

Width of spicula not uniform

Accessory with apophysis:-m-; tail fine, cylindroid; term. blunt; no spin. (Anticyathus)

Accessories without apophysis, two; terminus convex-conoid, acute . Bolbolaimus

Cephalated by expansion or contraction

The cephalation is by expansion

Width not unif.; supps. $5-6$; -m-; accessory with apophysis; spinneret armed. Halinema

Width of spicula uniform or apparently so

No. of supplements 3 ; spicula 1 times as long as anal body diameter; $-\mathrm{m}$ Rhynchonema

No. of supplements $25-65$; spicula 1 or $1 \frac{1}{2}$ times as long as anal body diameter

Apophysis to the accessory present; -m-; spinneret armed, 3-lobed.... Synodentium

A pophysis to the accessory none; $-\mathrm{m}$; spinneret unarmed; anus raised.... Mesodorus

The cephalation is by contraction; width of the spicula not uniform

No. of the supplements 1-3

Spicula $1 \frac{1}{2}$ times as long as the anal body diam; supps. mere innervations... Ypsilon

Spicula twice as long as the anal body diam; single elevated supp; $-\mathrm{m}-\ldots .$. Thaonchus

No. of the supplements 4 or more; spicula tapering both ways

Spicula long as anal body diam; accessory apophysate;-m-; no spinneret.. Anticyathus

Spicula twice as long as anal body diameter; accessory not apophysate

Anus 98 ; widtb $1.6 \% ;-\mathrm{m}-;$ single elevated supp; 2 dozen innervations. ... Thaönchus

Anus 92 ; width $3.8 \% ;-\mathrm{m}$; series of ventral supps.; nar. spinneret-tube. Micromicron

Supp. organs 0; spinneret pres. (exc. Crystallonema, Triplonchium, Iocalaimus, Cephalobium) Inner ends of the spicula not cephalated, or not obviously so

Width of spicula not uniform; spicula 1-13 times as long as anal body diameter

Form of spic. subarcuate; spin. midway on tail, ventral; ${ }^{4} \mathrm{~m}-;$ term. rounded ...Trissanchulus

Form of spicula arcuate

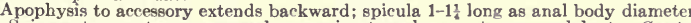

Spinneret none; term. unarmed; $-\mathrm{m}$; spic. strongly arcuate; no caudal setae. Crystallanemo

Spinneret present; term. faintly armed; $-\mathrm{m}-;$ minute, inconspic. caudal setce. Linhomoello

Apophysis to accessory none

Wings to the cuticle present, also in anal region; spicula strongly arcuate...... Xennella

Wings to the cuticle none

Accessory inconspicuous; spinneret (?); terminus $\frac{1}{3}$ as wide as base of tail; $-\mathrm{m}$. Trilepto

Accessories parallel, then bent away; spin. armed; term. $\frac{1}{2}$ wide as base; $-\mathrm{m}$.... (Stilbonema) Width of the spicula more or less uniform

Iength of spicula 2-3 times as great as anal body diameter; acc. with backward apophysis

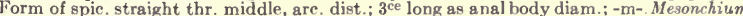

Form of spicula arcuate throughout, about $t$ wice as long as anal body dia.m.; $-m$. Cyartonema

Length of the spicula $1 \frac{1}{4}-1 \frac{1}{2}$ times as great as the anal body diameter

Accessories massive; supps. 2, obscure; term. convex-conoid; spin. large, acute....( $\mathrm{N} u d o r a)$

Accessories obscure; no suppelments; terminus blunt

Form of spic. very strongly arcuate; rudiment. burea pres,; no spinneret; -m Triplonchium

Form of spic not strongly arcuste, rather stout, blunt; no bursa; spinneret? Rhabdocom

Inner ends of spicula cephalated; spinneret present, except in Iatalaimus and Cephalobium

Proximal ends of the spicula cephalated by constriction, width not uniform

Length of the spicula twice or nearly twice or great as anal body diameter

A pophysis to acc. tapering; tail not striated; spinneret swan's-head form, unarmed. Nemella

A pophysis to acc. none; tail striated; spin. elongated-conoid, armed; $-\mathrm{m}$...Ptychalaimellus

Length of the spicula $1 \frac{1}{4}$ times as great as anal body diameter

Form of spicula straightish; ' $\mathrm{m}$; term. blunt, nearly $\frac{1}{2}$ as wide as base; no spin.. Iotalaimus

Form of spicula arcuate

Spinneret none; $-\mathrm{m}$; terminus acute, unarmed; width of the body $3 \% \ldots$.... Cephalobium

Spinneret present

Spinneret tub.; anus 90 ; width of body more than $3 \%$; term. unarmed; -m. . Monhystrium

Spinneret simple; width of body less than $1 \%$

Terminus swollen; accessories 2 ; anus $95 \%$; caudal setae faint, anal only; -m-?. Schistodera

Terminus not swol.; acc. 1 , more massive dist.; anus 97.5 ; setae not anal only . Laxonema

Proximal ends of the spicula cephalated by contraction or expansion

Ends of the spicula cephalated by contraction (for alternative see top next page)

Width of the spicula uniform; spicula about as long as anal body diameter

Anus 98:-m; cephalum short, faint; anal annules strongly modified ventrally... Stilbonema

A nus $94 ;-\mathrm{m}-;$ cephalum elongated, contraction distinct...................

Width of the spicula not uniform

Length of the spiculs twice the snal body diameter; -m-; terminus rounded. . (Thoönchus)

Length of the spicula equaling anal body diameter

Cuticle $=\frac{1}{2}$ radius; annules $t$ anal body width, elements not tile-shaped. Actinomema

Cuticle less tban $\frac{1}{2}$ radius; annules $\frac{1}{2}$ anal body width, elements tile-shaped. Ceramonema 


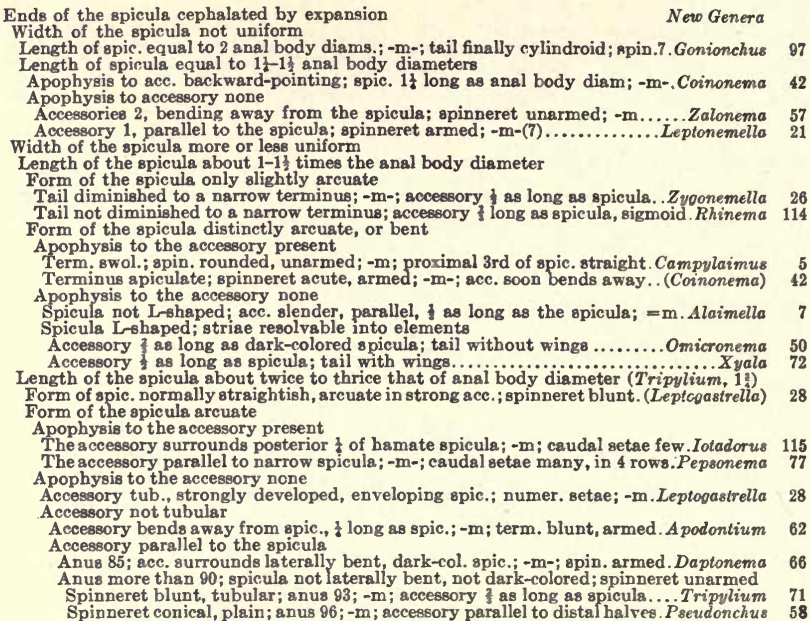
TIHE NEMA FORMULA

Nema dimensions sometimes vary perceptibly with varying technique. As a rule, information conveyed in the keys, formulae and illustrations is not repeated elsewhere. Following other authors, the measurements for the ovaries are given separately instead of in the form of a single measurement as formerly. Furthermore, the oblique stroke indicating the presence and position of the excretory pore is here, in No. IX, placed below the formula line, the assumption being that the formula shows the nema ventral side down. The general form of the spicula and accessories is shown in the formula for the male, as is also the presence, number and position of the supplements,--indicated by small suffixes before and after the anal measurement figure. The spicula diagrams are of three different degrees of curvature, indicating nearly straight, arcuate and strongly arcuate spicula. The nature of the proximal ends of the spicula is indicated, i. e. whether cephalated, and if cephalated, whether by contraction, constriction or expansion. Also, if it exists, the presence of an apophysis to the gubernaculum is indicated. The short, horizontal marks indicating the wings and the presence and relative diameter of the oesophageal swellings are only dotted when these features are not of a pronounced nature. The mark indicating the presence of a spinneret is either plain or so conventionalized as to indicate that the spinneret is supplied with setae. The absence of any particular mark in the formula is practically always to be taken as indicating that the particular feature in question is non-existent. Doubt is expressed by a question mark. The reason 
for calling attention to these special marks is that until one becomes accustomed to them they may appear rather insignificant. Once recognized, they may save the reader considerable time and trouble.

The adjacent table shows the various formula lines by which the number of striae per millimeter is indicated. See also pages 6 and 7 , where the various signs used in the formula are explained in full.

Terainology Relating to Striation of Catiele Nuwber of Striee
to the nillimeter
text term
texpond

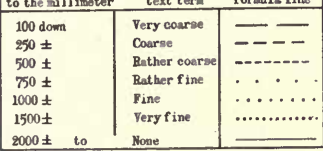
In this number, the presence of a bursa is indicated by a curved stroke under the transverse anal measurement figure, and the number of bursal ribs in front of and behind the anus is indicated by suffixes in front of and behind the anal measurement figure. Furthermore, as already noted, the form of the spicula and gubernacula is indicated by conventionalized sketches appropriately placed. See, for instance, page 279 .

\section{BURSAL FORMULA FOR NEMAS}

In the following rather arbitrary designations, which are expressed in a written formula, only the papillae and ribs on one side of the bursa are considered. They are designated according to their proximity to each other and not according to their anatomical and physiological characters. They are regarded as either anal, pre-anal, or post-anal, according as they are opposite to, in front of, or behind the anus. In the graphical bursal formula, the anus is represented by a pair of parenthesis marks; all papillae opposite the anus are indicated in the parenthesis, the pre-anal papillae are indicated in front of the parenthesis, and the post-anal papillae after the parenthesis. The papillae and ribs are considered as a single longitudinal series, and each group is indicated by a digit representing the number

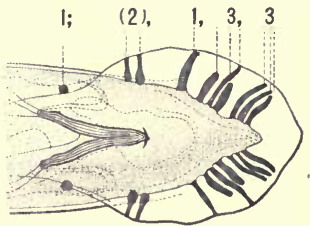

1; (2), 1,3,3 of ribs or papillae in the group. The longitudinal spaces separating the groups of papillae and ribs are indicated by commas and scmicolons, the comma representing a short space, the semicolon a longer space. In some cases before and after the parenthesis, the punctuation mark may be omitted, thus indicating that the ribs or papillae are even nearer to the anus than in those cases where the separation is indicated by a comma or semicolon. A blank space in the type after the comma, or after the semicolon, indicates a longer space than is indicated by the comma or semicolon alone. By such simple means it is possible to indicate with considerable accuracy the grouping and latitude of these various organs and groups of organs. A glance at the above illustration, Fig. 0, and formula immediately underneath will make the matter clear. 
General-Unless otherwise indicated,-

1. The species described under a generic name is to be regarded as the type species of the genus; where more than one species is described, the type species is specifically designated.

2. The text terms and formulae apply to the nemas as viewed in profile. References to stain are restricted to acid carmine.

3 . The cuticle is colorless.

4. The cuticle is striated,- - sometimes, however, so finely striated as to be resolvable only with the highest powers of the microseope used skillfully under favorable conditions. The division line of the formula is used to indicate approximately the number of striae per millimeter. (See page 227.)

5. The striae are approximately uniform in width throughout the length of the body except toward the extremities.

6. The contour is plain.

7. The striae are not resolvable into secondary elements. The presence of secondary elements is indicated in the formula. (See page 7.)

8. The longitudinal striations are due to the attachments of the musculature.

9. There are no obvious series of pores in the cuticle.

10. There are no eye-spots.

11. There are no definite valves in the oesophageal bulbs.

12. The lining of the oesophagus is a distinct, but not conspicuous, feature, practically throughout its length.

13. The intestine is set off from the oesophagus by a distinct constriction.

14. The arrangement of the granules in the cells of the intestine is not such as to give rise to a tessellated effect.

15. There is no pre-rectum.

16. The rectum extends inward and forward at an angle of about thirty to forty degrees.

17. The tail, at least that of the female, is straight, or nearly so.

18. The somatic, as well as the posterior cervical, setae, are perpendicular to the cuticle, or nearly so.

19. The renette cell lies behind the base of the neck.

20. The nerve-ring surrounds the oesophagus rather squarely.

21. Absence of the formula for either sex indicates that that sex is unknown at the present time.

Female-Unless otherwise indicated,-

22. The vagina is understood to lead inward at right angles to the ventral surface.

23. The uterus is more or less straight.

24. The eggs are thin-shelled and smooth, and are deposited before segmentation begins.

MALE-Unless otherwise indicated,-

25. There is no bursa.

26. The tail of the male is similar to that of the female in form.

27. The spicula are two in number, equal in size, and appear moderately arcuate when viewed in profile, and are simple, i.e., without obvious extra component longitudinal stiffening elements.

28. The accessory piece (or pieces), gubernaculum, lies more or less parallel to the spicula, and has no inward or backward-pointing apophysis. 


\section{New Data}

Apart from indicating that the nema phylum can be divided into valid, natural orders on the basis of the structure of the mouth parts and related organs, this article records numerous additions to our knowledge of the morphology of nemas. Facts announced in a sentence or two, or through the medium of illustrations, might perhaps have been advantageously made the subject of separate papers. Among these additions to our knowledge, the following may be mentioned:

1. In a miscellaneous lot of over one hundred new genera of nemas, thirty per cent prove to have jointed setae. Probably a much larger percentage have jointed setae. Among the nemas having setae, jointed setae are probably the rule rather than the exception.

2. The distal ends of the cephalic setae sometimes exhibit considerable complexity, indicating that they are specially developed sense organs; e.g., in Linhomoella and Crystallonema.

3. Additional evidence that bilateral mouth-parts arise by elimination of dorsal elements exists in Pseudonchus.

4. There is a large group of nemas with six well-developed onchia having an outward stroke, adapted for digging; e.g., A podontium and relatives.

5. More than ever, it is clear that amphids are practically universal among the free-living nemas. They may be small and difficult to see; as in Stilbonema and Laxonema. In the past, they have been sometimes overlooked. Some Triplonchs possess amphids; e.g., Onchium, Triplonchium.

6. All amphids have backward connections, - often, perhaps always, tubular and containing coagulable substance. Of unusual interest are the huge glands of Ionema.

7. Lateral series of organs occur in so many of the genera described, as to lend additional weight to the opinion that such series are a normal element of the nema structure.

8. There is much additional evidence of differentiation of form and structure among the various cells of the intestine.

9. The presence of oesophageal glands is established for Axonchium and its relatives, (Dorylaimus, etc.) They occur in the posterior enlargement of the oesophagus, one emptying into the lumen dorsally in its anterior part, and often two others emptying into the lumen farther back.

10. Non-terminal spinnerets and mouth openings seem practically always ventral; e.g., Trissonchulus, Campylaimus.

11. A large unicellular gland is connected with each supplement of the males of Bolbella and Eurystoma.

12. The presence of pairs of cloacal glands in the male, first observed by de Man in Euchromadora, is established for many other genera.

13. Cytolaimian transition-forms exist between the parasitic and free-living nemas; e.g., Monhystrium.

14. The existence of double-jointed spicula is established for Rhips and Xinema.

15. Nemas are disclosed in which the sperm cells are of extraordinary size; such species may prove useful in studies in genetics.

16. Rhynchonemas are distributed in various oceans. They are free-living nemas with beak-like heads, but otherwise normal in form. Presumably, this beak serves to extract food from receptacles the entrances to which are narrow.

17. There is a marked absence of syngonism in marine forms.

18. The existence is demonstrated of special spermatheca, in the form of special separate branches of the female sexual organs. 


\section{Order Litinia}

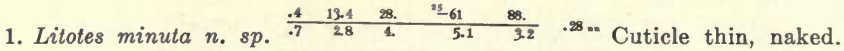
The mouth opening is almost imperceptibly depressed. The conoid neck carries a conoid oesophagus. The presence of amphids is indicated by slight, narrow external markings of a very faint character. In the region of the nerve-ring the oesophagus is three-fifths as wide as the corresponding portion of the neck, while finally it is five-sixths as wide as the base of the neck; its lining is indistinct, its musculature fine and colorless. No oesophageal glands. The intestine becomes at once three-fourths as wide as the body, is thin-walled, and but few of its cells are required to complete its girth. The intestinal lumen is distinct. or a $q p h p l(6)$ The distinct cardiac collum is two-thirds as wide as the body. amph 14 tr Near its beginning the intestine is pressed well to one side by of $\times 750$ the renette cell, which is more or less ellipsoidal in form and and about half as wide as long, and contains a very indistinct nucleus of medium size. There are few granules in the cells composing the intestine. The tail is conoid. Nothing is known concerning the longitudinal fields. The nervering is medium-sized and accompanied by more or less distinct groups of nerve cells. The small indistinct vulva is more or less continuous with the ventral surface of the body. The rather weak, tubular, non-refractive vagina extends obliquely forward apparently about half way across the body. The ellipsoidal granular eggs are about three times as long as the body is wide, and nearly onethird as wide as long. The broad, tapering ovary contains six to eight ova arranged single file.

Habitat: Algae, near the lighthouse, Bahia, Brazil. Bears some resemblance to Monhystera and Oxystoma. Differs from Monhystera in having no pharynx and no distinct amphids; from Oxystoma in the form of the amphids and the form of the female sexual organs. Sublimate to balsam. Fig. 1.*

2. Nemanema simplex $n$. $s p$. Head with faint traces of papillae on the outer part, indicated by refractions in the cuticle. Possibly the exceedingly minute

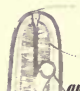

or pharynx contains an acute, forward-pointing projection or tooth, but of all details are so exceedingly minute that even under the very highest powers of the microscope they are resolvable with difficulty. The amph oesophagus expands but little until after it passes through the nerve$\times 750$ ring; thereafter it expands until near its posterior extremity, where 20 it is two-thirds as wide as the base of the neck;

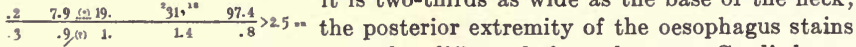
somewhat differently from the rest. Cardia large, $.29 .2 \ldots 21 . \quad{ }_{-12}^{51} x \quad 97.1>2.2 \ldots$ conoid, half as wide as the base of the neck. From the distinct cardiac collum the intestine becomes at once two-thirds as wide as the body; its cross-section comprises about six cells. The lateral fields are about half as wide as the body. When viewed dorsoventrally the amphids are seen to be connected with refractive, cutinized ducts which lead inward and backward for a short distance and then become indefinite. Tail conoid, its terminus three-fifths as wide as its base. The large, elongated, narrow caudal glands lie tandem on the ventral side in front of the anus, the foremost being removed from the anus a distance three to six times as great as the length of the tail. Observations on the ventral gland and excretory pore

* Each figure is designated by the number of the genus it illustrates. If two species of the genus are illustrated, the figures are designated $a$ and $b$, respectively. 
have not been satisfactory, but it appears as if there is a small, narrow, elongated ventral gland a short distance behind the cardiac constriction, where the intestine lies a little to one side. Each lateral field contains large ellipsoidal cells, too large to be placed side by side within its limits; they are placed alternately to one side and then the other. Their size is such that, in the neck at least, if they were arranged single file they would make a continuous series a little more than half as wide as the field and about one-third as wide as the body. From the raised vulva, the vagina leads half way across the body, where it joins the single uterus, which extends backward. The ovary reaches about half way back to the vulva and contains a score or more of developing ova, which are arranged for the most part single file. Anterior branch rudimentary. The elongated eggs are about four times as long as the body is wide and about one-sixth as wide as long. The tail of the male is slightly more arcuate than that of his mate. Opposite the proximal ends of the spicula there is a very low, rounded, ventral elevation. Near the ventral line in front of the anus for a distance two to three times as great as the length of the tail there are a few minute setae, about one-eighth as long as the body is wide, and immediately behind the anus there are one or two similar setae; otherwise there are no indications of special papillae or setae. Spicula about one and one-half times as long as the anal body diameter. Their proximal ends lie near the dorsal side of the body.

Habitat: Algae and marine sand at their bases, in surf, Island off Port Royal, Jamaica. Sublimate to balsam. Fig. 2, p. 230.

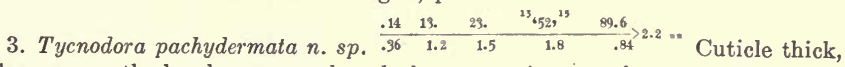
glassy; near the head more nearly colorless, sometimes smoky, at any rate after treatment with Flemming's solution; near the middle of the body, occupying one-sixth the radius; near the spinneret much thinner than elsewhere; rather suddenly diminishing in thickness near the nerve-ring. Lining of oesophagus subdistinct; the musculature fine and colorless. There is no cardia. The thin-walled intestine is separated from the oesophagus by a collum one-fourth as wide as the body. The lumen of the intestine is faint. From the depressed anus the inconspicuous rectum extends inward a distance twice as great as the anal body diameter. Very few granules are to be seen in the intestinal cells. The contents of the intestine are finely granular. The more or less arcuate tail is first conoid, then cylindroid in the posterior fourth, where it is about one-fourth as wide as at the anus. The lateral fields are onehalf as wide as the body. From the inconspicuous vulva, the cutinized vagina extends one-third the way across the body. The somewhat cylindroid ovaries extend two-fifths the way back to the vulva and carry twelve to twenty ova arranged more or less single file.

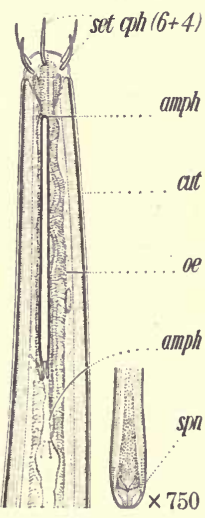

Habitat: "Seagrass," shoal, two miles off Key West, Fla., U. S. A. Flemming to glycerine jelly. Resembles Halalaimus, but the female has two reflexed ovaries. Fig. 3.

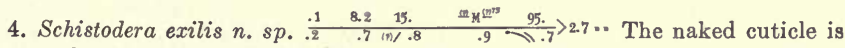
extremely thin. From the mouth about one-third the way to the anterior margin of the lateral organs, the lining of the oesophageal tube is a little more massive than farther back. The amphids are interesting on account of the presence 
in the posterior portion of the cavity of each of a strongly staining body, which may be assumed to be protoplasmic. The bottoms of the amphids are strongly cutinized. Oesophagus conoid, finally about half as wide as the base of the neck; just where it joins the intestine it appears to expand suddenly, although ffl (4) or this expansion could hardly be called a bulb. It is possible that this expansion indicates the presence of glands about the base hIm $\infty$ e $\quad \ldots$ of the oesophagus. The intestine becomes at once three-fourths subar In sam as wide as the body and in cross-section appears to present only amph amph two cells. Little that is definite is known concerning the lateral fields, but it is presumed that they are well-developed. The (1) $\begin{aligned} & \text { median fields are rather distinct. They contain a series of cells, } \\ & \text { at any rate in the neck; these cells are about half as long as the }\end{aligned}$ 11. neck is wide and are separated from each other by distances about twice as great as their length. Tail of the male at first conoid, then cylindroid in the posterior half, where it is about chrmt dirmt one-fourth as wide as at the base. The spinneret has a very $\times 750$ amph straight refractive piece appears to subtend the arc of the spicula. These latter are barely cephalated by an almost imperceptible expansion and a previous constriction. Immediately behind the anus there are two or three minute submedian setae; similarly, close to the anus, and in front, there is a pair of subventral setae,-all seen with considerable difficulty. The balance of evidence is in favor of the existence of two testes.

Habitat: Algae and sand at their bases, in surf, Island off Port Royal, Jamaica. Fig. 4.

5. Campylaimus inequalis $n$. sp. Striae more easily seen along the margin of the amphids. Wings are indicated by the presence in the lateral fields of two

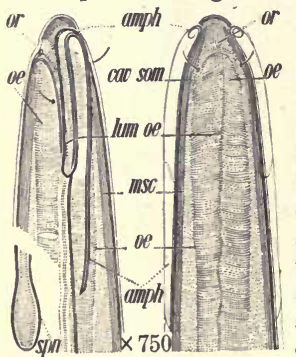

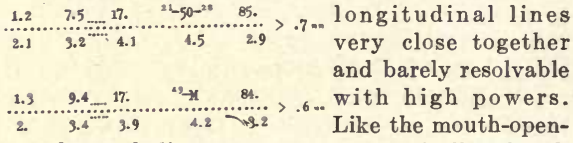
ing the cephalic setae are asymmetrically placed. The mouth is a simple, unarmed, conoid depression on the ventral side of the head a little behind the anterior extremity. There are no distinct lips, but the anterior extremity is modified so as to resemble a lip-region; it is set off by a minute constriction and presents a minute depression that takes acidcarmine stain more strongly than do the adjacent parts. No doubt this terminal "cap" serves some distinct function,--as tactile or gustatory. The conoid oesophagus finally becomes almost three-fourths as wide as the base of the neck. There does not appear to be any distinct cardia. Around the base of the oesophagus there is a circlet of small cells which stain in the same manner as do the cells of the intestine. This latter becomes at once about three-fourths as wide as the body. The lateral fields have not been distinctly seen. Nothing definite is known about the renette and excretory pore. Tail conoid to the simple terminus, which is set off by a broad, shallow constriction. The caudal glands appear to be located in front of the anus, probably a long distance in front of it. In the male the anus is slightly raised. Spicula 
about one and one-half times as long as the anal body diameter, their proximal ends slightly cephalated by expansion. They are rather markedly arcuate in their distal two-thirds, but nearly straight in the proximal third. There seems to be but a single testis.

Habitat: Marine sand and mud, San Pedro, California. Fig. 5.

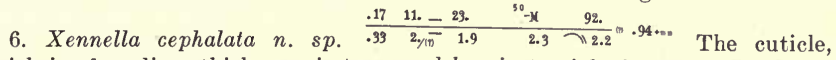
which is of medium thickness, is traversed by six to eight longitudinal ribs or wings on each side. There are at least four cephalic setae, possibly six; otherwise the cuticle seems to be naked. The mitriform head is set off by a distinct constriction. The neck is cylindroid. The somewhat conoid oesophagus is at first one-ninth, near the nerve-ring one-fifth, and at last two-fifths as wide as the corresponding portion of the neck. A final obscure swelling contains one or more nuclei, which differ from all other nuclei in the organ. The lining of the oesophagus is indistinct. The intestine is separated from the oesophagus by a collum onefourth as wide as the base of the neck, and becomes

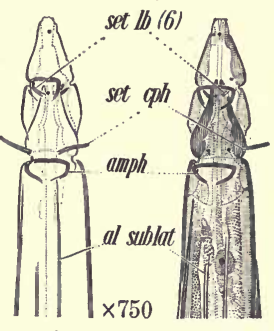
at once two-thirds as wide as the body, and in cross-section presents but few cells. The anterior part of the intestine for a distance about equal to the length

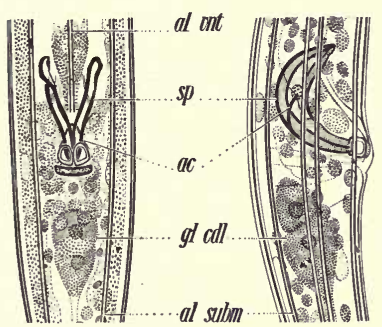
of the corresponding body-diameter has a structure different from the portion that follows. The cells of the intestine contain numerous very minute granules. The elongated granular renette cell is about as long as the corresponding body diameter, and lies a little in front of the cardia. It is not reflexed. Apparently the excretory pore is located near the nerve-ring. This latter is oblique, distinct, and broad. The spicula when viewed dorso-ventrally appear to make an angle of about thirty degrees with each other. The specimens were molting.

Habitat: "Sea-grass," shoal, two miles off Key West, Florida. Figs. $6 a$ and $b$. It is believed only the spinneret was missing from the single specimen measured.

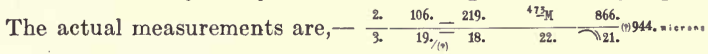

7a. Alaimella cincta $n$. sp. Cuticle relatively thick. The secondary elements of the striae are on the linits of visibility and reach nearly across the annule. There are probably six, flat, amalgamated lips. The conoid neck contains an oesophagus which is more or less clavate at the rear end. Near the mouth it is one-half, at the nerve-ring two-fifths, and finally three-fifths, as wide as the corresponding portion of the neck; its lining is indistinct. There may be a faint, conoid cardia. Intestine at once about three-fourths as wide as the body, more or less thin-walled, and presenting but few cells in cross-section. The intesti-

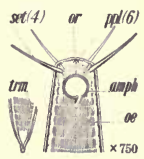

$$
\begin{aligned}
& \frac{.0}{1 .}-\frac{9.6}{1.1} \div \frac{19 .}{1.3}-\frac{92,(t)}{1.5}-\frac{92.5}{1 .}>1.3 . \\
& \frac{.0}{.5}-\frac{9.7}{1.1} \div-\frac{18}{1.5}-0-\frac{x}{1.4}=\frac{90.3}{1.1}>1.3=
\end{aligned}
$$

nal cells contain numerous, rather uniform

granules, regularly placed. From the continuous anus, the cutinized rectum 
is about twice as long as the anal body diameter. Tail conoid. The eggs are two to three times as long as the body is wide. The ova are arranged more or less in single file, and the ovary extends two-thirds the way back to the vulva. The proximal ends of the slender, somewhat tapering, rather frail, subacute spicula lie opposite the body axis. There are two rather frail, simple accessory pieces.

Habitat: Sand-bar, Biscayne Bay, Florida, U. S. A. Flemming to glycer ne jelly. Fig. 7a, p. 233.

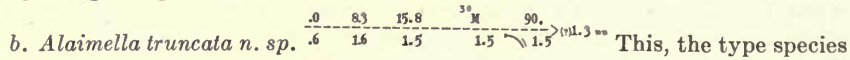
of Alaimella, rather closely resembles Alaimella cincta. Secondary markings of the cuticle faint, if any. Oesophagus as in cincta, except relatively a trifle narrower. Intestine as in cincta, but narrow and displaying only two cells in crosssection, the cells containing minute inconspicuous granules.

Proximally the slender, uniform, simple, frail and subacute spicula expand much and suddenly, to form flattish cephala, an som (Q) $a m p h$ located about opposite the body axis. There seem to be two mssom ann simple, frail, sub-slender, more or less arcuate accessory pieces, which are probably joined together at the anus. There $\mathrm{sm}$ number of nuclei ventrad from the narrow intestine, which keeps well to the dorsal side. Both before and behind the anus there are some very slender ventrally submedian setae half as long as the body is wide. Ejaculatory duct about one-third as wide as the body. Forward-pointing testes two-fifths as wide as the body. In the single male examined, which appears to be immature, one testis ended four tail-lengths from the anus, the other five tail-lengths from the anus. At one tail-length in front of the anus there occurred one or two glands (?) with very brilliantly staining nuclei, whose connections remain unknown.

Habitat: Algae, near lighthouse, Bahia, Brazil. Sublimate to balsam. Fig. 7b.

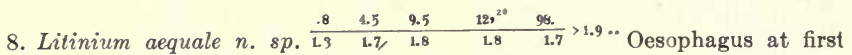
three-sevenths, near the nerve-ring one-third, and finally three-sevenths, as wide as the corresponding portion of the neck. Its lining is indistinct. There seems

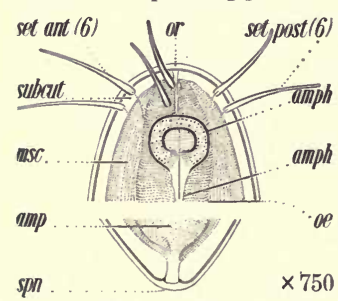

to be no distinct cardia. The intestine, which is separated from the oesophagus by a collum three-sevenths as wide as the neck, becomes at once half as wide as the body. Its walls are thick and its lumen faint. The granules contained in the cells of the intestine are numerous and more or less uniform. The anus is more or less continuous, the rectum inconspicuous. The hemispherical-conoid tail tapers from the anus to the terminus. There are no caudal setae. She caudal glands are located in front of the anus. The lateral fields are about one-fourth as wide as the body. The nerve ring is of medium size and on either side of it are obscure nerve cells. From the large, depressed, but rather conspicuous vulva, the vagina leads obliquely backward a distance two-thirds as great as the body diameter. Judging from the size of the ovum just before it leaves the ovary, the eggs are about one and one-half times as long as the body is wide. In the broad, cylindroid ovary are twenty to forty ova arranged single file except in the distal half, where they are arranged irregularly.

Habitat: Sand, Ocean Beach, Miami, Florida; U. S. A. Flemming to glycerine jelly. Fig. 8 . 


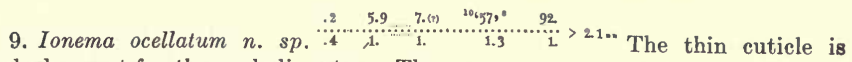
naked except for the cephalic setae. There are no traces of lips. The amphids are very inconspicuous. The neck is cylindroid posteriorly, and convex-conoid anteriorly, especially near the head. The eye-spots are light brown in color, and each has a refractive element in front. In the pigmented portion of each eye there is a more or less central nucleus with a subsidiary, small companion object. The cylindroid oesophagus is at first one-third, near the nerve-ring onefourth, and finally one-fifth, as wide as the corresponding portion of the body. Its lining is indistinct. There is no cardia. Owing to the presence of large somatic glands, observation of the junction of the oesophagus with the intestine is difficult. The collum appears to be one-fifth as wide as the corresponding portion of the body. The intestine is two-thirds to three-fourths as wide as the corresponding portion of the body, and is thick-walled, and has a very faint lumen. The cells of the intestine, which appear somewhat as if overlapping, contain more or less uniform, yellowish, scattered granules; they have large, spherical, granular nuclei with conspicuous nucleoli. The arcuate, conoid tail tapers from the anus to the unarmed convex-conoid terminus. The conoid spinneret is simple in structure. The ellipsoidal caudal glands occur in a loose tandem series in the anterior half of the tail. There are no caudal setae. The lateral fields are two-fifths as wide as the body. The granular, elongated, pyriform renette is one and one-half times as long as the body is wide, and one-half as wide as long. It is not reflexed and lies near the middle of the body, a little in front of the flexure of the front ovary. The nerve-ring is of medium size and is accompanied by rather obscure nerve cells. From the small, elevated, more or less inconspicuous vulva the medium-sized vagina leads inward half way across the body; it is more or less strongly cutinized. The size, form and covering of the eggs is unknown, but the ripe ova set(4) are about as long as the body is wide. The broad, cylindroid ovaries extend five-sixths the way back to the vulva, and contain about twenty ova, arranged single file in the proximal half, but irregularly in the distal half. The duct of the renette is necessarily very long and is slender; there is a distinct, elon- $\infty$ gated ampulla, with a very long and very slender duct leading from it to the pore. This latter duct is about as long as the body is wide. This genus, of which there are several species, spo is made very remarkable by the possession of a pair of relatively huge glands filling most of the body cavity behind the base of $\times 750$

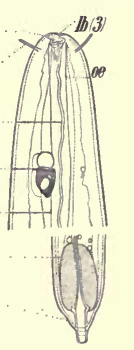
the neck and ending two and one-half times as far behind the neck as this latter is behind the head. The points of exit of these glands appear to be at the head end. Each gland has a nucleus near its blind end. In the vicinity of the nervering the glands diminish in size, and half way from this point to the head appear to come to a narrow, rounded end, strictly lateral in position, and from thence there appear to be narrow ducts leading toward the lip-region to pores, where foreign particles are seen to cling, and which are designated in the drawing as amphids. Near the middle these glands are pressed to one side by two subdorsal cells, apparently nerve cells.

Habitat: Marine algae, Panama. Hot sublimate to balsam. This genus is of wide occurrence in tropical oceans; specimens from both the East and West Indies are known to the writer. While specifically different, these forms do not vary much one from another. Fig. 9. 


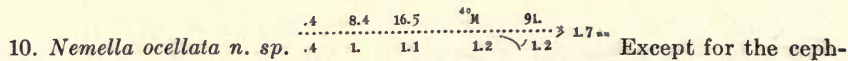
alic setae, the thin cuticle is naked. At a distance from the anterior extremity about equal to the width of the head, the striation of the cuticle ceases, so that the head is set off by an absence of striations, as well as by a slight thickening of the cuticle. The neck is cylindroid. From the faint amphids, an inner element can be traced backward to near the base of the head. The two eyespots are brownish in color, and each has a colorless lens. The oesophagus, which has an indistinct lining, is at first one-half, near the nerve-ring also one-half, and finally three-fifths, as wide as the corresponding portion of the neck. There is no cardia. The intestine, which is set off from the oesophagus by a collum one-half as wide as the neck, becomes at once three-fourths as wide as the body. Its wall is thick, its lumen faint, and it is made up of cells of such a size that probably only two are required to complete a circumference. These cells are packed with colorless granules of variable size, the largest of which are about onetwentieth as wide as the body. The tail is conoid to the convex-conoid spin-

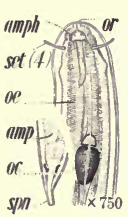
neret, which at its base is one-third as wide as the base of the tail, and has somewhat the profile of a swan's head. No setae were seen on the tail. At their widest part the simple, frail, tapering, subacute spicula are about one-fifth as wide as the corresponding part of the body. Their proximal ends, which are minutely cephalated by contraction and constriction, appear to lie a little ventrad from the body axis. They are supported by a simple, frail, slender, faintly S-shaped accessory, with a tapering apophysis (?) extending backward from the spicula at an angle of about ninety degrees, and about one-third as long as the anal body-diameter, its proximal end lying opposite the caudal axis. Whether there is one testis or two remains to be determined.

Habitat: Eel-grass, Biscayne Bay, Miami, Florida, U. S. A. Flemming to glycerine jelly. Fig. 10.

11. Porocoma striata n. sp. cle appears to be destitute of setae except at the extremities. The body is traversed nearly from end to end by about ten to twelve wings. Between the wings the transverse striae seem to be resolvable into excessively minute elements. In addition to the cephalic setae, there is a ventral seta-like organ, opposite the sub-cephalic setae and just behind the excretory pore. This is a little stouter, longer, and more blunt than the cephalic setae, and seems to have a special relation to the excretory pore. The mouth is surrounded by relatively thick, minute lips. It is possible that a more or less vestigial pharynx extends back to near the cephalic setae, its presence being indicated by a slight difference in the lining of the canal, and by a break in the musculature. Posteriorly the neck is cylindroid, anteriorly convex-conoid. No distinct traces of amphids have been seen, but possibly obscure ones exist opposite the bases of the lateral setae. The oesophagus is cylindroid, then conoid in its posterior eighth. Near the head it is one-half, near the nerve-ring two-sevenths, and finally threefourths, as wide as the corresponding portion of the neck. Its lining is indistinct. There is a flattish cardia, one-half as wide as the base of the neck. The thick-walled intestine is separated from the oesophagus by a collum one-half as wide as the base of the neck and becomes at once two-thirds as wide as the body, and would present two to three cells in cross-section. Its cells contain scattered granules of variable size, the largest of which have a diameter about equal 
to the width of two of the adjacent annules; tessellated effect faint. The tail. which tapers from in front of the anus, is conoid, and then cylindroid in the posterior half, where it is about one-half as wide as at the base Apparently the ellipsoidal caudal glands are packed in a close tandem in the anterior fourth of the tail. The lateral fields are one-half as wide as the body. The elongated, outstretched, granular renette cell lies one to two body-widths behind the base of the neck. It is two to three times as long as the base of the neck is wide and about one-fourth as wide as long. The broad oblique nerve-ring is accompanied by obscure nerve cells. While both ovaries are essentially behind the vulva, one of them extends forward a distance about twice as great as the corresponding body diameter, and is then reflexed, and extends backward, so

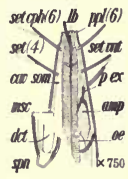
as to lie parallel to the othcr. The medium-sized, but rather conspicuous vulva is continuous with the ventral surface. The rather strongly cutinized vagina extends inward two-fifths the way across the body. It is possible that the narrow, cylindroid ovaries are reflexed for a short distance near their blind ends.

Habitat: Biscayne Bay, from sponges and associated material. Flemming to glycerine jelly. Fig. 11.

\section{Order Bolbinia}

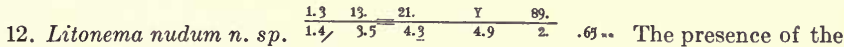
two wings, which begin on the neck and end near the anus, is indicated by two refractive, longitudinal markings, which occupy a space equal to one-fourth to one-third the width of the body. The cuticle is entirely naked. There appears to be a vestigial pharynx nearly as long as the base of the head is wide,- - simple, narrow, tubular, obscure. The neck is conoid. Almost on the front of the head, but near its margin, there are two minute, lateral pores, which may possibly be amphids. Connected with these pores are inner tubular elements, which may be followed backward for a considerable distance. The more or less cephaloboid oesophagus has a very faint, elongated, posterior swelling, one-fourth as wide as the base of the neck. Near the pharynx the oesophagus is one- reglb third, near the nerve-ring one-eighth, and in front of the cardiac swelling one-seventh, as wide as the corresponding portion of the neck. The optical expression of the lining of the oesophagus consists of two distinct, refractive lines, having a distance apart about equal to one-tenth the width of msc the oesophagus. There is no cardia. The thick-walled intestine presents a faint lumen, and becomes at once five-sixths as wide as the body. Anus continuous; rectum inconspicu- $p e x-$ ous. The cells of the intestine are packed with granules of rather uniform size, the largest of which are one-seventh $\times 750$. as wide as the body. The conoid tail tapers from in front of the anus. There are no caudal glands. The lateral fields are probably one-third as wide as the body. The excretory pore and the duct leading to it are so refractive as to be easily visible.

Habitat: About the roots of the lady's slipper, Cypripedium acaule Linn., sphagnum swamp, Wisconsin, U. S. A. Flemming to balsam. Fig. 12. 


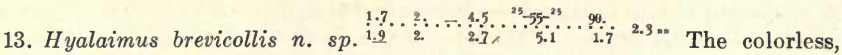
naked skin displays irregular markings and a finely crenate contour. The colorless, transparent, conoid neck ends in a truncate head without setae and having very flat lips, if any. Six rather conspicuous, sub-marginal, wart-like papillae occur in a circlet on the front of the head. No amphids are to be seen. The mouth is a mere depression one-sixth as deep as the head is wide; from it faint apophyses, of which the ventral is the longest and most conspicuous, extend backward and seem to indicate that the real depth of the pharynx is equal to the length of the distinct pharyngeal bulb, and accordingly the dimensions are so given in the above formula. The somewhat phalangiform oesophagus begins with the bulb just mentioned as filling the head, and which is two-fifths as long as the neck. Behind this bulb there is a broad, shallow constriction, the remainder of the oesophagus being fusiform and in its widest part two-thirds as wide as the neck. For a short distance the irregular intestine, three-fourths as wide as the body and separated from the oesophagus by a not very deep constriction, appears transparent and almost bulbous. The cardiac cavity is small and the cardia very flat. The large cells composing the intestine are filled with small granules, displaying no very definite arrangement. The narrow, colorless, transparent rectum is thrice as long as the anal body-diameter, and has a distinct lining. The ventral excretory pore is situated as far behind the cardia as the head is in front of it, the duct in the immediate vicinity being very transparent and distinct, and having a distinct lining. The granular lateral fields are one-fourth as wide as the body, and from head to tail a finely crenulate, cuticular wing extends along each lateral line. The tail of the female is conical to the pointed terminus. The unusually large, flat, elevated vulva is twothirds as wide as the body, and from it the vagina extends backward a distance greater than the body-diameter. The two straight uteri in the only specimen seen contained six to eight eggs, each a little longer than the body width, and measuring 56-60 x 132-140 microns. The ovaries extend two-thirds the distance to the cardia and anus respectively and contain ova arranged single file.

Habitat: Intestine of an earth-worm, Moss Vale, New South Wales, Australia, April, 1894. It is not certain that this nema may not be con-generic with one or more of those mentioned by earlier authors under the generic names Anguilula, Nematodum, etc., nemas also found in earth-worms, but insufficiently described; hence the proposal of a new genus for its reception.

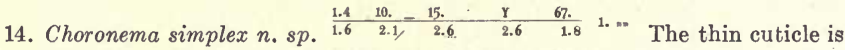
entirely naked. The wing, which begins near the head and ends on the tail, is possibly double in structure. There is no cardia. The rather thick-walled intestine is separated from the oesophagus by an indistinct collum one-half as wide as the base of the neck, and becomes at once about three-fifths as wide as the body. It has a faint lumen and presents few cells in cross-section. Anus subcontinuous; rectum inconspicuous. The cells of the intestine contain scattered colorless granules of variable size, the largest of which are one-fifteenth as wide as the body. In the specimen examined the tail was destined at the next molt to become very much shorter, so that the anus would lie at about $90 \%$, and the tail would therefore be about four times as long as the anal bodydiameter. Two sublateral papillae existed on the tail nearly opposite each other.

Habitat: Soil about the roots of plants, Arlington Farm, Virginia, opposite 
the city of Washington, U. S. A. Flemming to glycerine jelly. This species, of which but a single young specimen has been seen, has the general appearance of being a deteriorated Cephalobus or Diplogaster. The specimen figured was

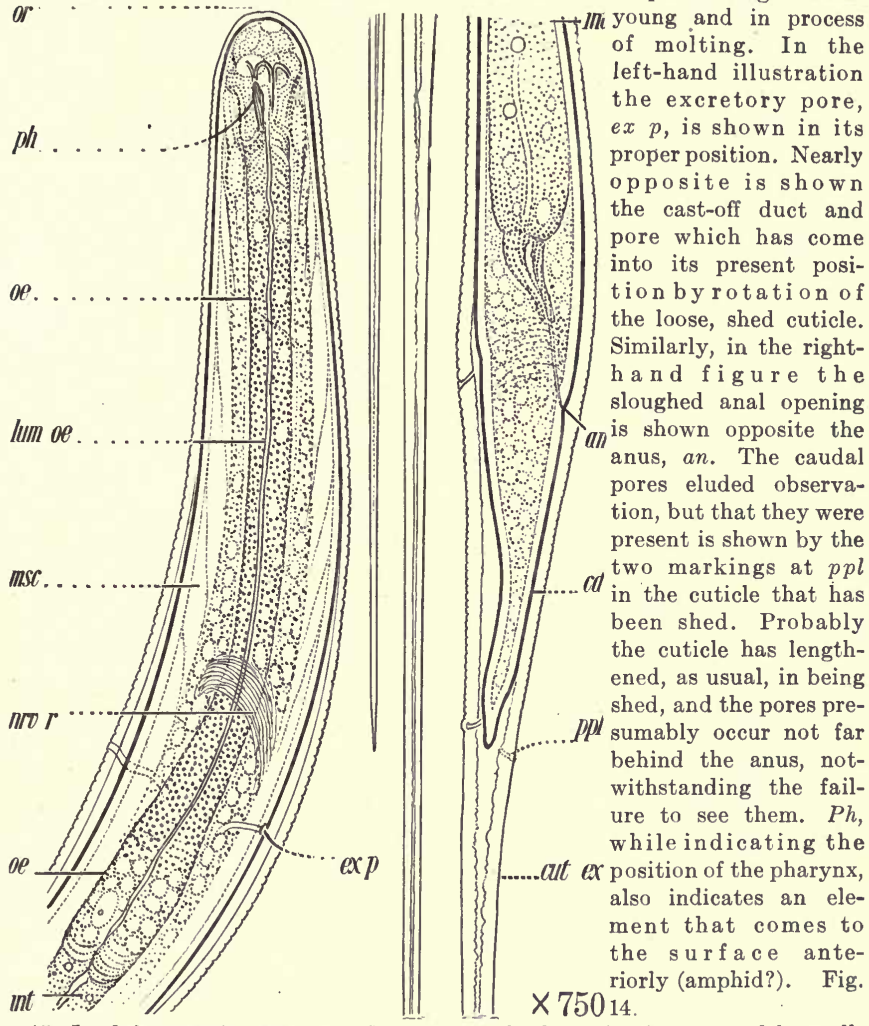

15. Iotalaimus striatus $n$. $s p$. Cuticle naked; the striae interrupted by welldeveloped crenate wings one-fifth as wide as the body. Between the wings is a straightish refractive line, due to a continuous, cuticular structure. On some specimens the outer contour of the wings consists of a doubly refractive line. There appear to be four faint, submedian cephalic papillae. The amphids are faint. It is possible that there is a vestigial pharynx. The oesophagus is very faintly cephaloboid in form. The anterior two-fifths is cylindroid and averages only about half as wide as the corresponding portion of the neck; thence backward the oesophagus diminishes gradually so that opposite the nervering it is only about one-fourth as wide as the middle of the neck. It continues to have this diameter for some distance, but finally begins slowly to expand, so 
that at last it is about half as wide as the base of the neck. The lining is faint, and there are no refractive breaks in its structure to indicate the presence of vestigial bulbs. There is no distinct cardia. The intestine becomes at once two- to three-fifths as wide as the body. It is composed of cells containing scattered groups of granules. The distinct "lumen," instead of appearing as

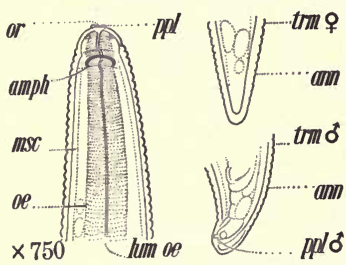

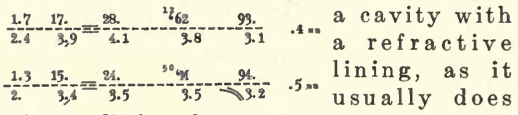
when a distinct feature, seems to have a different structure of about the same general appearance. From the slightly depressed anus the conspicuous rectum extends a distance about $p p l \delta$ one and one-half times as great as the anal body diameter. The lateral fields are one-third as wide as the body. It appears as if there is a renette cell opposite the posterior portion of the oesophagus. The tail is conoid to the blunt terminus, which has a diameter about one-third as great as that of the base of the tail. There are no caudal glands. It is possible there is a pair of subventral papillae immediately behind the anus. A trifle behind the middle of the tail, there are two faint, lateral innervations close together on each side, one in front of the other, doubtless connected with surface papillae. Ventrad from these, it is possible there is a third papilla belonging to the same group. There are probably one or two pairs of subventral papillae near the terminus. The proximal ends of the tapering, very slightly arcuate spicula are bent in a ventral direction so as $\quad m /(6)$ to appear to lie on the ventral side of the body-axis, and
so appear cephalated. The accessory piece is more
strongly refractive than the spicula themselves, the frame-
work of which is not particularly conspicuous.

Habitat: About the roots of Bamboo, Yuma, Arizona,

ne

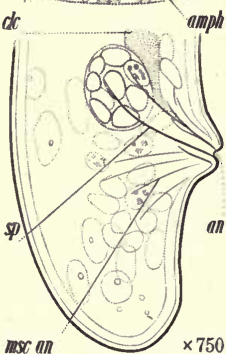

amph U. S. A. Fig. 15, above.

16. Bolbinium brevicolle $n$. sp. Cuticle of medium thickness, naked. Posterior half of the neck cylindroid, anterior half convex-conoid. Amphids unusually large, with large internal connections that can be followed backward for some distance. The narrow oesophagus continues to have the same diameter until near the posterior, pyriform, cardiac swelling, which is one-half as wide as the base of the neck. Many of the nuclei connected with the cells of the neck are large and well-developed. In the dorsal and ventral fields there are strands that an appear to be composed of closely-packed cells. The cardiac bulb has no distinct valve.

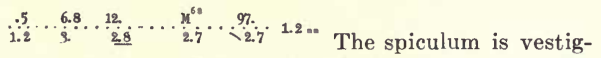
ial. In a variety of ways this nema seems related to the Mermithidae,- - for instance, in the structure of the mouth, the cephalic papillae, the amphids, the internal structure of the tissue of the neck and of the body, the absence of spinneret, and the short, rounded, broad tail.

Habitat: Soil, about the roots of citrus plants, Florida, U. S. A. Fig. 16. 


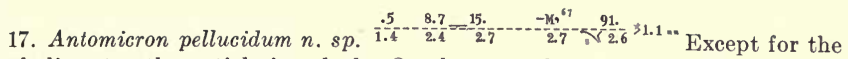
cephalic setae the cuticle is naked. On the ventral side of the distinctly projecting wing there is a subordinate line to be seen throughout the greater portion of the length of the body, which is not as conspicuous as the main part of the wing. Neck conoid. The very minute pharynx (?) is apparently conoid;whether armed or not is unknown. The lip-region has not been examined with success on account of deficient material. That portion of the amphid inside the two ellipses stains more strongly than the tissues elsewhere. The borders of the two amphids approach each other so closely on the dorsal side that they sometimes almost touch, in fact appear to be connected by a special cuticular element. Oesophagus cylindroid for some distance back, and then expanding rather suddenly to form an almost imperceptible swelling near the beginning of the middle third of the neck; théreafter it diminishes almost imperceptibly to near the nervering, then begins to expand gradually until near the end, where it rapidly expands to form an obscure, small, elongated, pyriform cardiac bulb, almost half as wide as the base of the neck. There is a rounded cardia one-fourth as wide as the base of the neck. Considering the size of the oesophagus its lining is prominent.

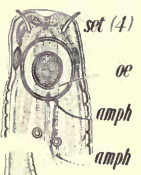
set $t / m$ (iv) $\times 750$ The intestine is separated from the oesophagus by a broad, shallow constriction, and soon becomes half as wide as the body, and apparently would present only two or three cells in cross-section. The lateral fields appear to be nearly onethird as wide as the body. It seems probable that a renette cell occurs at some distance behind the base of the neck, but no excretory pore has been seen. The tail of the male is conoid to near the terminus. The caudal glands are located in an open tandem series in front of and behind the anus. The eight supplementary organs occupy a distance nearly four times as great as the length of the tail; each is a nearly straight, cutinous tube, half as long as the body is wide, arranged at an angle of forty-five degrees with the body axis, and having its distal extremity protruding backward slightly through the cuticle. The organs taper slightly in the vicinity of the distal end, which is suddenly somewhat ventrally arcuate at the terminus. They are probably protrusile, though they have not been seen in a protruded position. These tubes have a diameter approximately equal to the width of one of the adjacent annules of the cuticle, and their proximal ends show indications of an attachment extending forward. There are a number of specially-developed setae in the vicinity of the anus; especially prominent are two submedian setae, one on each side of the anus, one-third as long as the anal body-diameter. A little in front of these is another pair, one on each side, of slightly smaller size, and on the tail there are a few similar ventrally submedian setae of smaller size. Spicula a little longer than the anal bodydiameter, and tapering to a point in their distal thirds. The accessory piece surrounds the spicula in their distal fourths, and then extends forward to the dorsal side of the body from the middle of the spicula at a small angle. The appearance of the proximal portion of the accessory piece somewhat resembles that of the proximal ends of the spicula, though it is straighter and more solid. From its cephalated proximal end a muscular strand joins the body wall on the dorsal side of the anterior portion of the tail. The posterior testis is the smaller.

Habitat: Marine; Punta Arenas, Pacific Coast of Costa Rica. Unfortunately the single specimen examined is of such a character that the details of the pharynx must be left undetermined. Fig. 17. 
18. Cyartonema flexile $n$. $s p$. Except for the cephalic setae the cuticle is naked. There appears to be a circlet of six, inconspicuous, minute papillae

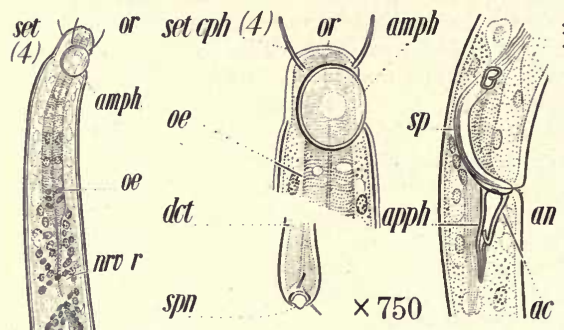

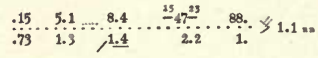

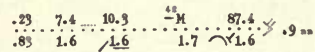
on the margin of the head. The form and structure of the gland at the base of the In narrow oesophagus is reminiscent of the salivary glands in Tylenchus. The intestine is made up of cells so arranged that only one

ae to two are required to build a circumference. The parts of these cells are so differentiated that the groups of granules characteristic of the cells occur at intervals equal to one to two bodydiameters, and are more or less alternated with finely granular intestinal elements, or what are suspected to be such (see Figure 18, int.?). The tail, tapering from in front of the anus, is conoid then cylindroid in the posterior fifth. Spinneret with minute setae. A single caudal gland was seen near the middle of the tail. A few very inconspicuous setae were observed on the tail. Concerning the lateral fields, I was at times uncertain whether the cells marked int? were not in some way connected with these fields. From the somewhat elevated vulva, the relatively large vagina leads inward two-fifths the way across the body. The eggs are three to four times as long as the body is wide, and about one-fourth as wide as long. The broad, more or less cylindroid ovaries suddenly taper near the blind end. They contain apparently only four to five ova, arranged single file. The clavate testis is about two-thirds as wide as the body.

Habitat: Clean, white sand, in about five feet of water, in a cove near the entrance to Buzzard's Bay, Mass., U. S. A. Sublimate to balsam. Fig. 18.

19. Stilbonema brevicollen.sp. Annules plain, retrorse in the posterior half of the body, the reverse in the anterior. Exceedingly short, submedian, slender setae are found throughout the length of the body, six to nine annules apart, those at the extremities being somewhat the longer. Neck cylindroid. Immediately around the mouth there is probably a row of exceedingly minute papilIae. In most of the neck the diameter of the oesophagus hardly exceeds the spn

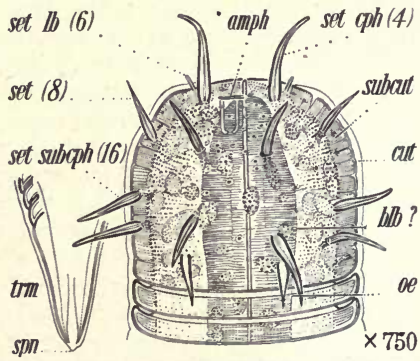
thickness of the cuticle; it finally expands to form a pyriform bulb about threefifths as wide as the base of the neck. There is no distinct cardia. The very 
narrow intestine, joined to a depression in the posterior surface of the cardiac bulb, becomes at once about one-sixth as wide as the body, and then enlarges

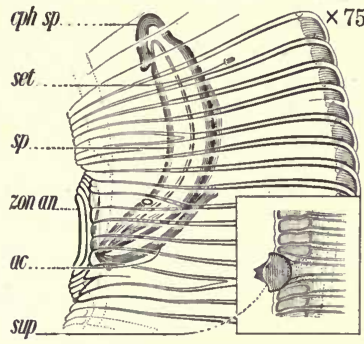
gradually until it is one-fourth as wide. Its cross-section would show two cells. The lateral fields are about one-fourth as wide as the body, and contain numerous nuclei. Nerve-ring oblique. Tail conoid, arcuate. 1. $\frac{3}{2}-\cdots \frac{3 .}{1.4}-\frac{4.5}{1.4}-\frac{M}{1.5}=\frac{98 .}{4.6}>3$. . Spicula slender. "Acorn-shaped" accessory organs (see figure) far forward, so that the anterior one is a little behind the neck. The cup-shaped part of these organs has slightly different refractive properties from the "acorn" itself, which is plainly innervated.

Habitat: Shoal in Kingston Harbor, Jamaica, in one foot of water. Sublimate to balsam. Fig. 19a, p. 242; Fig. $19 b$.

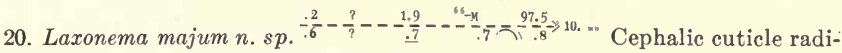
ally striated; the basal part of the cephalic setae penetrate and interrupt the cuticle. Inside the cephalic setae, as shown in the sketch, other interruptions occur in the cuticle; possibly in some cases these are the "stumps" of lost setae. There are two circlets of papillae inside the cephalic setae, one at a distance from the mouth pore somewhat greater than the thickness of the body cuticle, and the other twice as far away. Oesophagus nearly cylindroid, finally expanding to form a pyriform cardiac bulb two-thirds as wide as the base of the neck. There is no cardia. The intestine soon becomes about one-fifth as wide as the body; at a distance back about twice as great as the body-diameter it expands and becomes thicker-walled and one-third to one-half as wide as the body. Its cross-section is composed of about four cells, each with ten to twenty brownish granules, the largest of ppl?. which have a diameter about one-fourth as great as the thickness of the cuticle, murph and the smaller one-third to one-fourth this size. Renette unknown. The lateral fields are about onefourth as wide as the body, and contain a double row of nucleated cells, generally somewhat rectangular in subat. form and separated into two series. The nerve-ring is probably a little behind the middle of the oesophagus. The tail of the male is $\times 750$

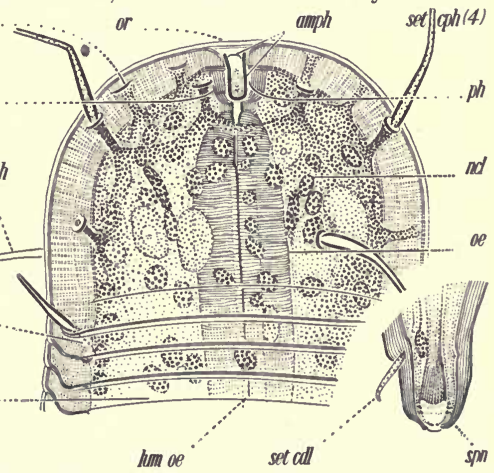
arcuate-conoid and ends in a spinneret destitute of striations and having a length about equal to the sum of the widths of the last eight striae. The tip of the spinneret is somewhat differentiated, and its core presents minute longitudinal "striations" which end just anterior to the terminal pore. The caudal 
glands are probably located in front of the anus. No supplementary organs have been seen. There are no caudal papillae, but both in front of and behind the anus there are a number of ventrally submedian setae; five or six on each side of the anterior half of the tail; while an equal number in front of the anus gradually merge into the scattered setae found all over the body. The proximal ends of the rather stout acute spicula are somewhat diminished and set off by a broad and deep constriction. The framework composing the spicula is relatively massive. The rather straight accessory piece is half as long as the spicula. The ejaculatory duct is about one-fourth as wide as the body. There appears to be a single outstretched testis, though there remains a little doubt on this point.

Habitat: Shoal in Kingston Harbor, Jamaica, in about one foot of water. Fig. 20 , p. 243 . Sublimate to balsam.

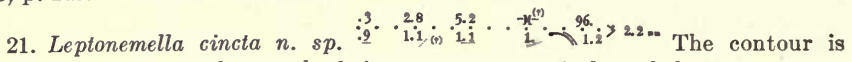
more or less serrate, the annules being retrorse posteriorly and the reverse anteriorly. In addition to the cephalic setae there are scattered cervical setae arranged at right angles to the surface and one-third as long as the neck is wide. No somatic setae have been seen. Apparently at every eight to ten annules along the lateral lines there are pores, each with its longest diameter arranged set sulm Ib transversely. Seemingly there are six, very minute, more
or less amalgamated lips. Posteriorly the neck is cylin-
droid, anteriorly conoid. Amphids are present in the
form of straight transverse slits, one-sixth as long as the
corresponding diameter of the head, and located between
the bases of the submedian cephalic setae. Their presence
and their form is proved by the ribbon-shaped outflow
plainly seen issuing from each amphid. The cylindroid
oesophageal tube ends behind in a broad, pyriform cardiac
bulb, two-thirds as wide as the base of the neck. There
is no cardia. The thick-walled intestine is separated
from the oesophagus by a collum one-tenth as wide as
the neck, and becomes at once one-fourth as wide as the
body. Its lumen is indistinct. Its cells contain fine,
rather numerous granules of more or less uniform size.
Several submedian, slender, tapering, cuticular "thorns" are seen on the tail, each about one-fourth as long as the anal body-diameter. The lateral fields are one-fourth as wide as the body. The nerve-ring is accompanied by obscure nerve cells. The tail is more or less conoid from the anus, but tapers more rapidly in the posterior half. The strong, tapering, acute, colorless spicula are more or less compound in structure and their extremities appear to lie somewhat ventrad from the body axis. The single, slender, rather strong, simple parallel accessory is three-fifths as long as the spicula.

Habitat: Sand, Ocean Beach, Miami, Florida, U. S. A. Flemming to glycerine jelly. Fig. 21.

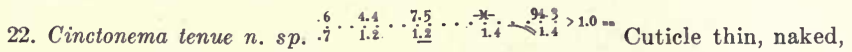
its striae not further resolvable. Neck, cylindroid posteriorly, conoid anteriorly. In the specimen examined the lip-region was not favorable for observation. Oesophagus about half as wide as the head and continuing to have this diameter until after it passes through the nerve-ring, behind which it begins to increase 
a little, and finally enlarges to form an elongated, pyriform cardiac bulb, threefourths as wide as the base of the neck. The intestine is very narrow where it joins the center of the posterior face of the cardiac bulb,wider than one of the annules. Very soon, however, it becomes two-fifths as wide as the body. The lateral fields are about onefifth as wide as the body and contain nuclei, two of which placed and side by side would span the field. Tail of the male conoid; on it there are a few setae in front of the anus. The rather slender,

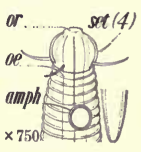
acute spicula are about one and one-fourth times as long as the anal bodydiameter.

Habitat: Sand and algae near East Drive, east shore of Kingston Harbor, Jamaica. Sublimate to balsam. Fig. 22.

\section{Order Cytolaimia.}

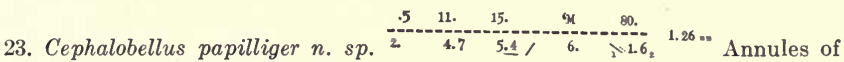
the cuticle 2.2 microns wide. Head continuous with the conoid neck. There are probably six lips, but no labial papillae have been seen. The pharynx resembles that found in the genus Monhystera, being a conoid or more or less pyramidal depression one-third as wide as the head. Oesophagus cylindroid, one-fourth as wide as the middle of the neck, joining a pyriform cardiac bulb two-thirds as wide as the base of the neck and containing a distinct valve. Lining of the oesophagus rather faint. The rather thick-walled intestine is three-fifths as wide as the body, and is separated from the neck by a shallow, cardiac constriction. The ventral renette cell is situated just in front of the flexure in the testis. From the somewhat elevated anus the tail is conical to the acute terminus. A pair of tall, conical, sub-ventral papillae are located opposite the middle of the spiculum. Taken altogether, the papillae are situated as follows: 1 ; () $1 ; 1$. Spiculum slender, somewhat irregular, twice as long as the anal body diameter, placed at angle of forty-five degrees with the bodyaxis. The ejaculatory duct was of equal length with the testis, and was connected with it by a tube also of the same length.

Habitat: Intestine of the larva of a lamellicorn beetle, from soil under cowdung, Moss Vale, New South Wales, Australia. 1893.

24. Anticyathus tenuicaudatus n. sp. Body wall thick. In addition to cephalic setae there are scattered papilloid cervical setae. Conoid oesophagus at first

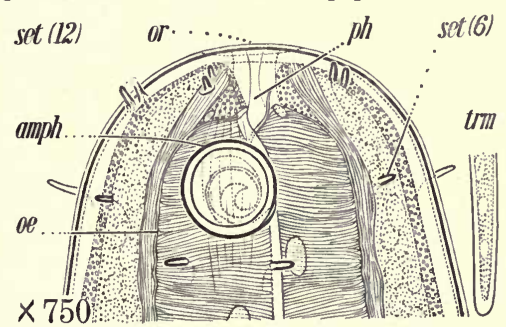

\begin{tabular}{rrrrrr}
.1 & 2.2 & 5. & ${ }^{3}-50-^{29}$ & 85. & $6.5=$ \\
\hline .8 & 1.6 & 1.6 & 2.7 & 1.3 & \\
.2 & 3.7 & 7.5 & $-M-{ }^{45}$ & 86. & \\
\hline 9 & 1.7 & 1.8 & 2.1 & $25 \times 1.7$ & $5.2=$
\end{tabular}

IIMI two-thirds, near the nerve-ring four-sevenths, finally four-fifths, as wide as the corresponding portion of the neck. Oesophageal lining subdistinct. There is a somewhat obconoid more or less "structureless"-looking anterior element of the intestine threefourths as long as the body is wide; this may be regarded as a cardia one-half as wide as the neck. The thick-walled intestine, which has a faint lumen, is set off 
by a collum one-fifth as wide as the base of the neck, and becomes more or less gradually three-fourths to five-sixths as wide as the body. Its cross-section is composed of twenty to fifty cells. These cells contain numerous granules of variable size. The tail tapers from in front of the anus; it is first conoid, then cylindroid in the posterior fourth, where it becomes one-fifth as wide as at the anus. There are no caudal glands. Fifteen to twenty almost invisible setae, as long as the cuticle is thick, occur on each ventrally submedian line on the tail. The longitudinal fields are one-third as wide as the body. Near the excretory pore is an ampulla one-sixth as wide as the corresponding portion of the neck. The nerve-ring is accompanied by obscure nerve-cells. Vulva medium-sized, more or less elevated; vagina non-cutinized. Near the proximal end, each ovary presents a double flexure, occupying a distance about equal to one body-width; thence onward the ovaries are outstretched in opposite directions, - at last not over one-tenth as wide as the body. The eggs occur in the uteri about nine at a time. They are about one-half to two-thirds as long as the body is wide, though they are often so crowded together in the uterus that they appear wider than long. The narrow tapering ovaries contain seventy to eighty ova, arranged single file. The strong, rather simple, stoutish, tapering, rather blunt spicula are as long as the anal body-diameter, and are so placed that their proximal ends, which are cephalated by expansion, appear to lie somewhat dorsad from the bodyaxis. At their widest part the spicula are about one-eighth as wide as the corresponding portion of the body; the apophysis is more or less uniform, and onefourth as long as the anal body-diameter, so that its proximal extremity appears to lie opposite to or dorsad from the axis of the tail. The 25 supplementary organs, hardly more than innervations, are papilloid and of slight elevation, rather farther apart anteriorly, and occupy a distance five to six times as great as the corresponding body-diameter. There is a single papilla-like ventral seta close to the anus.

Habitat: Sand, Coco-plum Beach, Miami, Fla., U. S. A. Flemming to glycerine jelly. Fig. 24 , p. 245.

25. Neurella simplex $n . s p$. Striae resolvable with great difficulty into secondary elements. The narrow, sharply-defined wings begin near the head and end on the tail. The cuticle appears to be naked, except that at the base of the amphids there are elements of problematical significance and number. The neck

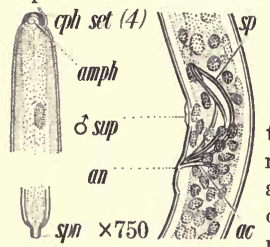

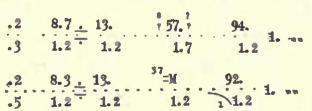

is cylindroid posteriorly, conoid anteriorly, -convex-conoid toward

the head. There seem to be three lips surrounding the somewhat irregular but more or less distinct, relatively small pharynx. Oesophagus cylindroid; at first one-half, near the nerve-ring one-third, as wide as the corresponding portion of the neck; its lining is indistinct. The separation between the oesophagus and the intestine was not very clear-cut. The thick-walled intestine gradually becomes one-half as wide as the body; its section is made up of two cells containing scattered granules of variable size, the largest about one-eighth as wide as the body. Not until near the middle of the body does the intestine acquire very definite characteristics. Anus more or less elevated. Tail of the male, as far as seen, conoid; apparently only its tip was missing. Located well toward the middle of the body there is a long, granular cell empty- 
ing forward;- probably the renette cell. Excretory pore unknown. Nerve-ring oblique, accompanied by distinct cells arranged in groups.

Habitat: "Sea-grass," shoal, two miles off' Key West, Florida, U. S. A. Sublimate to balsam. The view of the tail of the female was slightly foreshortened; the formula must be interpreted accordingly. Fig. 25, p. 246.

26. Zygonemella striata n. $s p . \frac{1.4}{3.1}-\frac{7.3}{4.1}-\frac{13.6}{4.6}-\frac{-K^{60}}{5.3}-\frac{84.8}{3.6}>1.2 \ldots$ In addition to the cephalic setae, scattered on the anterior part of the neck, there are a considerable number of other similar setae, some of which are longer than those near the margin of the head. Lips three, massive but low, faintly bi-lobed; within these there is a rather broad inner mouth consisting of three soft, low, flat lobes, which appear to be extensions of the tissue of the oesophagus. These latter present faint refractive elements, the expression of minute foldings of the lip tissues, that make possible the great expansion necessary for the deglutition of the relatively large diatoms constituting the food. In appearance the pharynx closely resembles the lumen of the oesophagus. It would, in fact, be indefinable, were it not for the pharyngeal swelling which is half as wide as the head, that is to say, a very little wider than the remainder of the oesophagus. Immediately behind the pharyngeal swelling the oesophagus diminishes gradually, so that where it passes through the nerve-ring it is about set ll (6?) two-fifths as wide as the neck; thence onward it is cylindroid. The lining of the oesophagus is a conspicuous feature set cph (4) throughout its length. The tubular cardia is prominent, about one-fourth as wide as the base of the neck, and about one and one-half times as wide as long. The intes- $\times 750$ a tine is almost at once fully half as wide as the body and is separated from the oesophagus by a broad and deep constriction. Its cross-section probably comprises only two cells. The cells contain scattered granules of small size and rather uniform diameter. The lateral fields, about one-fourth as wide as the body, contain cells packed with fine uniform granules. Renette unknown. The tail of the male is conoid in such fashion that at the beginning of the final third it has a diameter about equal to the width of two of the corresponding annules; thence onward it is very nearly cylindrical. The anus is slightly raised,-its posterior lip elevated. The caudal glands are packed in a tandem series opposite the anus and occupy a space somewhat longer than the anal body-diameter; their ducts are narrow. Spicula acute, not quite as long as the anal body-diameter; their width about equal to the width of one of the adjacent annules. They taper in the distal fourths to slender, acute points. The posterior testis is only about half as long as the anterior. Ten unicellular glands are a prominent feature of the anatomy of the male. (1) A pair of clavate glands immediately behind the blind end of the reflexed posterior testis. Each of these glands has a length nearly equal to that of the adjacent body-diameter, and empties through a duct extending backward. The ducts have a width somewhat greater than that of one of the adjacent annules. (2) Just behind the pair of glands already mentioned is a second clavate pair of larger size and very similar; these are about half as wide as the body and somewhat longer than the body is wide, and the ducts extending backward from them have a width greater than that of two of the adjacent annules. (3) Behind the second pair of glands there are six small, pyriform glands, i.e., a set of three on each side of the body, arranged longitudinally close together, but having separate ducts, also extending backward. It 
has been impossible to determine with exactitude the entire course of the ducts of these various glands, but most of them have been seen to be connected with the rectum, and all are believed to be so connected, though it is possible that some of them may deliver into a common duct before reaching the rectum. As to the structure of these glands, the description of one of them will answer fairly well for all the others. In the fixed specimens, the anterior extremity of each contains a spherical nucleus with a strongly staining nucleolus: the nucleus lies in the midst of chromatin matter which stains rather strongly with acid carmine. The nucleus and chromatin occupy the anterior fourth, or third, of the glandular cell. The remainder of the contents is of a uniformly fine, granular nature. This granulation is also characteristic of all the ducts.

Habitat: Punta Arenas, Pacific Coast of Costa Rica. Diatomivorous. Sublimate to balsam. Fig. 26, p. 247.

27. Margonema ringens $n$. $s p$. Striae unaltered on the lateral fields. Cuticle apparently naked, but it is possible that through rough handling cephalic setae Ib ... pllb may have been broken off, as was the case with some other ah amph specimens in the same collection. Lips three and double. Neck cylindroid. Amphids faint. Oesophagus at first twoary? 1 subat thirds, near the nerve-ring one-half, and finally two-thirds, 11 of as wide as the corresponding portion of the neck; its lining indistinct. There is a faint, rather flat cardia about half as lumoe I la $99 m$ wide as the base of the neck. The cross-section of the thickdet 1 .... set sp walled intestine is made up of two cells. These transparent, elongated cells have very distinct nuclei, but few and small ${ }^{\times} \mathrm{x} n \mathrm{750}$ inconspicuous granules. The intestine becomes at once two-

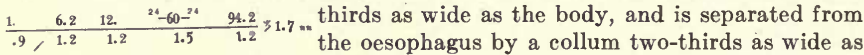
the base of the neck, and has a faint zig-zag lumen.

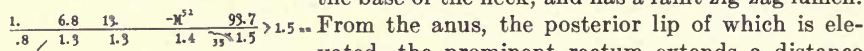
vated, the prominent rectum extends a distance about as long as the anal body-diameter. The conoid tail tapers from the anus. The three ellipsoidal caudal glands lie in a loose tandem in the anterior twofifths of the tail. There are no caudal setae. The lateral fields are about one-third as wide as the body, and contain both small and large nuclei. At a distance behind the neck equal to twice the width of the body lies the ellipsoidal renette cell, which is about as long as the body is wide and about half as wide as long, It empties through an ampulla about as long as the neck is wide, and about one-fourth as wide as long. This latter is connected with the excretory pore by a very short duct. The nerve-ring surrounds the oesophagus squarely, and is accompanied by distinct cells apparently not very definitely arranged. From the somewhat depressed, more or less continuous vulva, the tubular vagina extends nearly half way across the body. The vagina is bifurcated, each branch being about half as long as the body is wide. The uteri are narrow. The eggs are three-fifths as wide as the body, and six times as long as the body is wide. The narrow ovaries taper but little, and contain about a dozen ova approximately in single file. At their widest part, the slender, acute spicula are about one-eighth as wide as the corresponding portion of the body. Their arcuate, cephalic portions, viewed in profile, appear to lie somewhat ventrad from the body-axis. The subarcuate, rather slender, frail, simple accessory pieces are joined together at the anus. The separate apophysis 
is uniform and one-fourth as long as the corresponding body-diameter. There are thirty to forty low, about equidistant supplementary organs, of such a character that the ventral contour becomes crenate when the tail end is incurved; the crenations are then nearly contiguous. There are a few, scattered ventrally submedian setae on the tail. The acute ends of the spicula are minutely and sharply curved through an angle of 180 degrees at the very tip. One male specimen was seen in which there were two renette cells, each with a separate duct for some distance.

Habitat: Salavery, Peru. Marine. Sublimate to balsam. Fig. 27, p. 248.

28. Leptogastrella pellucida n. sp. Except for the setae near the head and on the tail of the male, the cuticle appears to be naked. The neck is cylindroid posteriorly, becoming faintly convex-conoid toward the rounded head, which may be set off by an almost imperceptible, broad constriction opposite the base of the pharynx. The membranous lips, are either six in number, or three and each two-parted. The oesophagus is cylindroid. There is an elongated cardia, one-half as long as the body is wide, and about two-thirds as wide as long. The intestine, separated from the oesophagus by a shallow constriction, becomes at once two-fifths as wide as the body, and then diminishes slightly so that it has about one-third the width of the body. Its cross-section would appear to be made up of not more than two cells. The intestine is so narrow as to give the nema quite an unusual appearance. It is thick-walled and has an exceedingly narrow lumen. Its cells are packed with exceedingly minute yellowish granules. Correspondingly, the body-wall is unusually thick and muscular, and slightly oblique longitudinal striations due to the refractions of the muscular fibres can

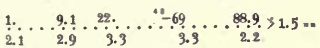

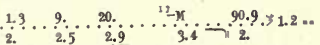

be seen throughout the length of the body. The lateral fields appear to be about one-third as wide as the body. The renette has not been seen. Vulva slightly elevated. The outstretched ovary contains forty or more ova arranged single file. The blind gl adl end of the ovary lies not far behind the nerve-ring. The eggs occurring in the uterus appear to be $a c$ about as long as the body is wide and one-third to one-fourth as wide as long. The tail of the $g l c d l$ male is conoid to near the terminus, where it has a diameter about one-fifth as great as at the anus.
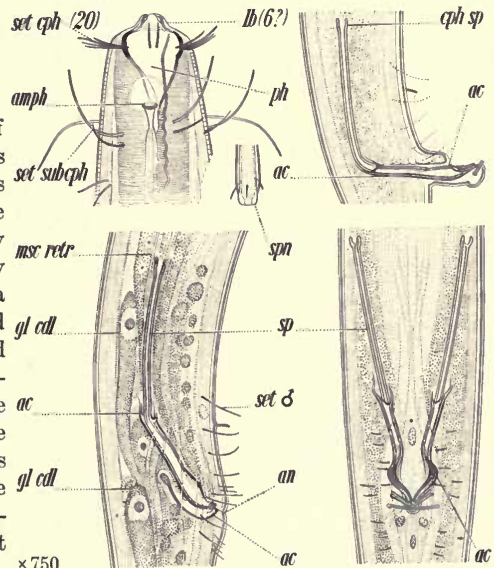
Caudal glands are located in the base of the tail. There are no supplementary organs, nor have any special papillae or setae been seen either in front of the anus or behind it. The spicula slide in tubular accessory pieces.

Habitat: Marine mud, San Pedro, California, U. S. A. At first glance this 
nema appears to be a typical Monhystera, but careful examination fails to reveal any such well-developed amphids as are characteristic of Monhystera. The spicula are quite different in form from the typical spicula of Monhystera. The intestine also is highly peculiar. Occurs also at Woods Hole, Mass. Fig. 28, p. 249.

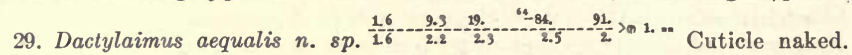
Three of the six lips are somewhat more substantial than those alternating with them. Distal thirds of the lips free, the remaining portions webbed. From the head, the oesophagus continues to have the same diameter to near the oblique nerve-ring, but then begins to swell gradually so that ph finally it is two-thirds as wide as the base of the neck. There is a small cylindroid cardia. The intestine, separated from the oesophagus by a deep constriction, becomes at once about two-thirds as wide as the body. Its circuit appears to comprise about four cells. The

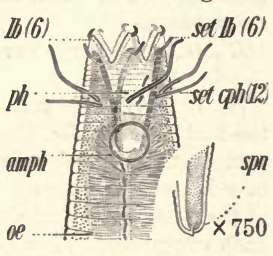
lumen of the intestine presents a refractive and distinct contour. From the inconspicuous, slightly depressed anus, the rectum is considerably shorter than the anal body-diameter. The distinct lateral fields are about one-third as wide as the body and contain numerous cells whose nuclei are arranged in two indistinct rows along the margins of the field, which they fill fairly well. Renette unknown. The description is derived from a single young female, with the vulva in process of development. The tail is conoid in such fashion that at a distance from the anus about five times as great as the length of the anal bodydiameter, where the annules vanish, it has a width about one-sixth as great as at the anus. An indefinite, but probably not considerable portion of the tail of the specimen examined was possibly missing. Hence, the above formula may be only approximately correct. The following formula is in terms of absolute

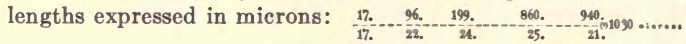

Habitat: Fine marine mud, San Francisco Bay, California, U. S. A. Sublimate to balsam. Fig. 29 .

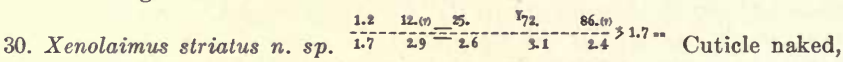
and complicated, the annules retrorse posteriorly and the reverse anteriorly. The twelve longitudinal wings extend to near the spinneret, becoming fewer on the tail. The thick, somewhat digitate lips are united by a membrane. The set lat set lb set subm portion of the head containing the pharynx is protrusile and appears as if surrounded by a balustrade composed of the anterior annules of the cervical cuticle. The large, simple, regular, conoid pharynx is fully three-fourths as long as the head is wide. Each of the six lips bears a two-jointed seta, and appears to be armed internally with a slender, flexible element considerably longer than the seta. In the illustration, what appear to represent two minute setae on the foremost annule of the neck are probably the optical expression of an exceedingly tenuous membrane surrounding the head. Although the oesophagus was not plainly seen, it is evident that it is cylindroid as in Trachynema. The intestine becomes at once three-fourths as wide as the body. The anus appears continuous. The cells of the intestine con- 
tain scattered granules of variable size, the largest of which are one-half as wide as one of the annules. The conoid tail tapers from in front of the anus and ends in a spinneret.

Habitat: Marine mud from near the government-dredged cut, Biscayne Bay, Florida, U. S. A. Sublimate to balsam. Fig. 30, p. 250.

31. Cytolaimium exile $n$. $s p$. Cuticle thin, without setae except those on the head. Lips with thin distal flaps as in Monhystera. The cylindroid oesophagus is at first three-fifths, near the nerve-ring one-half, and finally three-fifths, as wide as the corresponding portion of the neck. There is a conoid cardia onethird as wide as the base of the neck. The thick-walled intestine, which has a faint lumen, becomes at once three-fourths as wide as the body. Its cells contain scattered granules of variable size, the largest of which, near the neck, are onefifteenth as wide as the corresponding portion of the body, but near the middle of the nema are one-tenth as wide as the body. The conoid tail tapers rather regularly from somewhat in front of the anus, but faster near the anus. No clear evidence of the presence of caudal glands. The lateral fields are about one-half as wide as the body. The narrow nerve-ring is accompanied by obscure nerve cells. From the small, continuous, inconspicuous vulva, a small, more or less weak, non-cutinized vagina extends inward one-third the distance across the body. In the narrow, tapering ovaries, the ova are arranged single file. Only one egg at a time occurs in each uterus. The eggs are three times as long as the body is wide and appear about three-fourths as wide as the body. The tail of the male is first conoid, then cylindroid in the posterior two-fifths, where it is one-third as wide as at the base. The somewhat stout, rather blunt spicula are strong, non-cephalated, and so situated that their proximal ends appear to lie

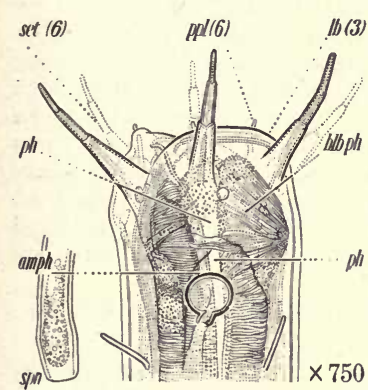

opposite the

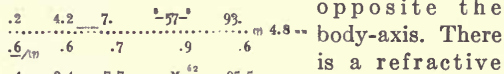
$\begin{array}{lllll}4 & 3.4 & 7.7 & & \text { is a refractive }\end{array}$

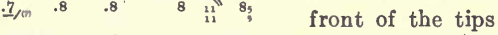
of the spicula. Accessory piece slender, apparently strong, one-third as long as the spicula. The 16 pairs of submedian, equidistant, discoid, slightly-elevated supplements are not cupch shaped, and are relatively less cutinized than in well-developed Chromadora supplements. The posterior pre-anal pair lies opposite the distal parts of the spicula. Thence, forward, $\sin$ $\times 750$ placed at intervals about equal to three-fourths the body-diameter, the organs occupy a space eight to nine times as long as the anal body-diameter. The post-anal five pairs occupy the anterior three-fifths of the tail. The discs are about one-fourth as wide as the body, and one-fourth as wide as high, the distance between them being about one and one-half times their diameter. There appears to be an innervated element that projects from near the center of each disc. The ejaculatory duct, which is co-extensive with the pre-anal supplementary organs, is one-half as wide as the corresponding portion of the body. The vas deferens is one-half to onethird as wide as the body. Each testis is at first about one-third as wide as the body and cylindroid, but afterwards tapers. The anterior testis is much the longer.

Habitat: Sand-bar opposite Miami River, Biscayne Bay, Fla., U. S. A. Sublimate to balsam. Fig. 31 . 
32a. Rhabdocoma americanum n.sp. Type species. Cuticle thin, naked except for the cephalic setae. Lips three. Wall to the pharyngeal cavity in optical section showing three somewhat thickened elements, that are possibly minutely transversely ribbed; these elements, however, are very small land difficult of

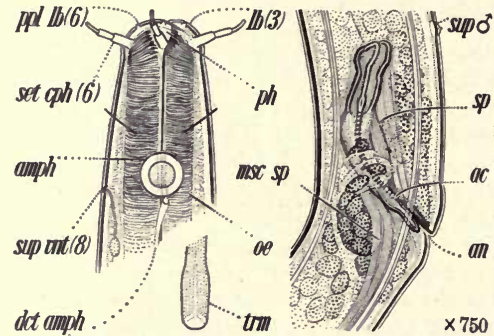

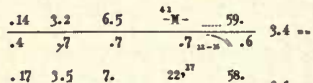

\begin{tabular}{rrrrr}
.17 & 3.5 & 7. & 22. & 58. \\
\hline .4 & .6 & .7 & .9 & .6
\end{tabular} $.1=$

resolution. Neck somewhat conoid anteriorly. Oesophagus at the nerve-ring two-fifths, at the cardia two-thirds, as wide as the corresponding portion of the neck, and containing glands, as is indicated by fine granular matter in branched $\times 750$ cavities. Cardia more or less spheroidal, one-half as wide as the base of the neck. The rather thick-walled intestine presents a faint lumen and becomes at once about one-half as wide as the body; its cross-section presents but few cells. Anus continuous; rectum about as long as the anal body-diameter. The intestinal cells contain granules of variable diameter, the largest one-tenth as wide as the body. Doubly. refractive granules occur in all parts of the intestine, but are not numerous; these tend to have quadrate contours and do not present St. Andrew's crosses. The tail tapers very gradually throughout, commencing well in front of the anus. Renette unknown. There is an anterior rudimentary branch to the female sexual organ, about as long as the body is wide. From the rather large, more or less depressed vulva the well-developed cutinized vagina extends inward three-fifths the distance across the body. The elongated eggs are twice as long as the body is wide. The broad, cylindroid ovary reaches three-fourths the distance back to the vulva and contains very many ova, those of the two-thirds next the uterus being arranged single file. The frail spicula are about one and two-thirds times as long as the anal body diameter and are rather wide apart. Accessory pieces faint. Supplements papilloid, twelve to sixteen, occupying a distance in front of the anus ten to twelve times as long as the body-diameter, while a second series of about eight, occurs on the neck. The members of the anal series are somewhat farther apart anteriorly; of the cervical series, posteriorly.

Habitat: Sand among mussels, Devil's Island, Woods Hole, Mass. Fig. 32.

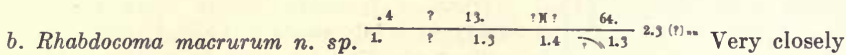
resembles the preceding, but differs in the following respects: straight setae, nearly as long as the head is wide, digitate, with a minute setose mucro; amphids a trifle farther forward, somewhat elongated; apparently four, submedian, minute, short setae opposite the posterior margin of the amphids; accessory pieces one-half as long as the spicula and parallel to them.

Habitat: Sulphurous sand, Bay of Naples, toward Vesuvius, 1888.

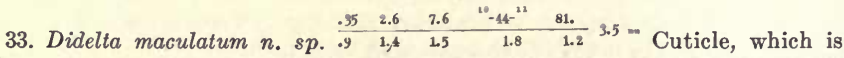
above medium thickness and naked except for the setae on the head, is characterized by the presence of peculiar transversely-elongated refractive subcuticular 
markings, reminiscent of the bubbles in defective window glass. The simple, subregular, somewhat asymmetrical, napiform pharynx is about one-third as wide as the head. On the dorsal side of the base of the pharynx there is a more or less glottoid, low, flat elevation, so that the more or less refractive dorsal wall of the pharynx appears only about half as long as the ventral wall. Posteriorly the neck is cylindroid, anteriorly, more or less conoid. The elliptical amphids are in reality spirals of about one wind. They occur on large, somewhat equilaterally triangular or deltoid areas on the sides of the head; hence, the name Didelta. The oesophagus is at first about three-fourths, near the nervering one-half, and finally two-thirds, as wide as the corresponding part of the neck. The lining is distinctly indicated by a more or less zig-zag, refractive line; the musculature is coarse and

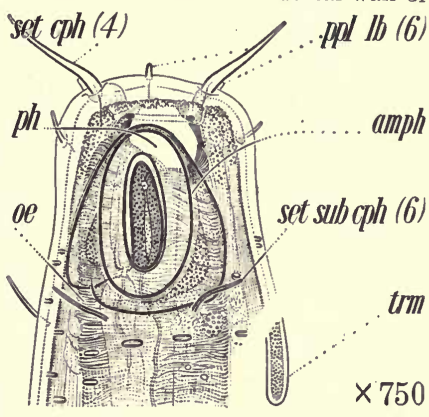
colorless. There are no valves, and there is no cardia. The thick-walled intestine, which has a distinct refractive lining, becomes at once three-fourths as wide as the body; its cross-section being composed of about six cells. It is separated from the oesophagus by a collum one-third as wide as the base of the neck. The cells of the intestine are packed with more or less uniform granules, having a diameter about one-ninetieth that of the body. Arranged in elliptical clusters, they give rise to a distinct tessellation. The tail, which tapers from the anus, is first conoid, and then more or less cylindroid in the very narrow posterior half. It really tapers throughout, but is nearly cylindroid in the setaceous part. There is no spinneret. The lateral fields are about one-fourth as wide as the body. The nerve-ring is of medium size, and is accompanied by obscure nerve cells. From the rather inconspicuous, but somewhat elevated vulva, the cutinized vagina leads inward two-fifths the distance across the body. The tapering ovaries were not favorable to detailed observation.

Habitat: "Sea-grass," shoal, two miles off Key West, Florida, U. S. A. Flemming to glycerine jelly. Fig. 33 .

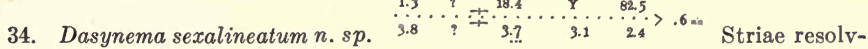
able into secondary, elongated elements. Annules retrorse posteriorly, and the reverse anteriorly. Cuticle naked except for the setae on or near the head. set $q h(6)$
set sub cph
acute, possibly conoid, mobile; the appearance is
that of three segments of the head, each armed at
the summit with a short, inward-pointing, dark,
eutinized apex, having an inward stroke. There
does not appear to be a distinct cardia, but a num-
ber of small cells forming the beginning of the
intestine are manifestly different in structure from those directly behind. The rather thin-walled intestine, which has a more or less distinct, refractive lumen, soon becomes two-thirds as wide as the body, and its cross-section is composed of two to four cells in which there are few or 
no granules. From the anus, the posterior lip of which is elevated, the prominent rectum leads inward a distance one and one-half times as great as the ph...... $\operatorname{set} q h(6)$ anal body-diameter. The tail is sub-conoid,-at first conoid, then more or less cylindroid. The elongated caudal glands appear to lie in the anterior half of the tail.

Habitat: Eel-grass, Woods Hole, Mass., U. S. A. Sublimate to balsam. Described from a young specimen. Fig. 34a, p. 253; Fig. $34 b$.

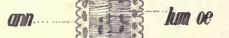

35. Linhomoella exilis n. $s p$. The shorter cephalic setae are differentiated at the extremity, suggesting that they bear special sensory organs. Cuticle naked except for the setae on or near the head. Lips apparently confluent. Possibly there are papillae immediately around the mouth opening. It sometimes appears as if there is a minute
pharynx like that of Monhystera, but this may be a decepnor $r$ tion due to the structure of the front part of the oesophagus; possibly there is no true buccal cavity. Neck cylinLib.

and oal droid. The more or less cylindroid oesophagus is at first three-fifths, near the nerve-ring two-fifths, and finally twothirds, as wide as the corresponding portion of the neck.

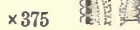

Itt It is colorless and has a fine musculature. The cylindroid cardia is three-fourths as wide as the neck. The intestine, which is separated from the oesophagus by a collum three-fifths as wide as the base of the neck, becomes at once three-fourths as wide as the body. Near its anterior end, however, there is a broad, shallow constriction, in which region its lumen is faint. Anteriorly the walls are thick, while posteriorly they are thin. A cross-section of the intestine is probably made up of about three cells. These cells contain scattered granules of variable size, the largest of which are about one-twelfth as wide as the body and appear to have the structure of spherical shells. The arcuate tail tapers from the anus to the terminus, where it is one-fourth as wide as at the anus. Caudal

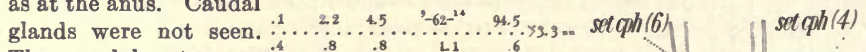

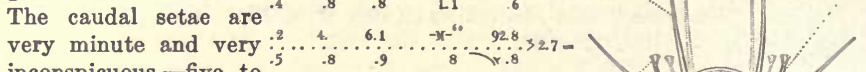
inconspicuous, - five to ${ }^{.5}$.8 six on each ventrally submedian line. From the con- ary? spicuous, fairly large, elevated vulva the cutinized vagina extends one-third the distance across the body. The slender eggs are ten times as long as the body is wide, and appear to be about three-fourths as wide as the body. The narrow tapering ovaries contain twenty or more ova arranged single file. The proxi- amph.. mal extremities of the stoutish, tapering, acute, rather strong spicula appear to lie ventrad from the $\times 750$ body axis, and are not materially cephalated. The part of the accessory piece near the spicula is refractive; the apophysis, however, is faint, and a little more than half as long as the anal body-diameter. As to supplementary organs, there are almost invisible ventral innervations, equidistant and separated from each other by spaces about two-thirds as great as the body-diameter. The ejaculatory duct and vas deferens are about one-third as wide as the body. The tapering testes are very narrow. 
Habitat: Sand-bar, Biscayne Bay, off the mouth of the Miami River, Florida, U. S. A. Flemming to glycerine jelly. Fig. 35, p. 254. The almost imperceptible constriction occurring in the cephalic region, as shown in the illustration, is somewhat more marked when seen dorso-ventrally; the amphids are located in the midst of a broad and shallow depression.

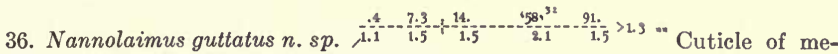
dium thickness, naked except for the cephalic setae, of which there is a circlet of ten, with a circlet of six immediately preceding it. There are, however, a few, scattered setae on the neck, one-third as long as the neck is wide, arranged at right angles to the surface. Lips confluent, very small, thin and minute, probably six in number. No labial papillae have been seen. Pharynx almost nonexistent, simple, conoid. Neck cylindroid. The oesophagus is at first one-half, near the nerve-ring two-sevenths, and finally two-thirds, as wide as the corresponding portion of the neck; its lining is subdistinct, its musculature fine and colorless. There is a hemispherical cardia, one-fourth as wide as the base of the parlat setghlol neck. The thick-walled intestine, which is set off by a amp...1(6) amph collum one-fourth as wide as the base of the neck, becomes at once two-thirds as wide as the body. Anteriorly its lumen is faint, but posteriorly it is distinct. set The cross-section of the intestine is composed of about three cells. The granules contained in these cells are scattered to numerous, and are of variable size, the spn. $=5750$ largest having a diameter equal to the width of two of the adjacent annules. They have the appearance of hollow shells, more or less thick-walled. The conoid tail tapers from the anus to its terminus, which is one-third as wide as its base. Though the caudal glands were not clearly seen, they appear to be broadly saccate and to be arranged in a close tandem behind the anus in the anterior half of the tail. There appear to be about three slender, somewhat cylindroid, subacute setae on each submedian line on the tail. The lateral fields are two-fifths as wide as the body. The huge renette cell begins at the cardia and extends backward a distance equal to the length of the neck. It is two-fifths as wide as the body, and, of course, is not reflexed. It is granular and possesses a large nucleus. The elongated ampulla is one-third as wide as the head and empties through a short duct near the base of the lips. Vulva more or less continuous, small; cutinized vagina of medium size. The thin-shelled, elongated eggs are probably five to six times as long as the body is wide. According to the condition of the uterus, the broad tapering ovaries reach from three-fifths to the whole of the distance back to the vulva. They contain about a dozen ova arranged more or less single file, except near the blind end.

Habitat: Sand, Cape Florida, Biscayne Bay, Florida, U. S. A. Flemming to glycerine jelly. This genus resembles Cyatholaimus more or less but has no onchia and the excretory pore is farther forward. The labial features are smaller and less definite than in Cyatholaimus. It is notable also that no pores are to be seen in the cuticle. The oesophagus ends behind in an almost imperceptible elongated swelling. The color of the ocelli, through oversight, was not noted. Until the male is known, the affinities will remain doubtful. Fig. 36. 


\section{Order Isolaimia}

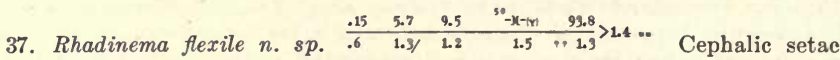
3-jointed; cuticle otherwise naked. The thick, small lips confluent. Pharynx exceedingly minute, two-thirds as deep as the head is wide; wall characterized by six longitudinal elements of minute size, exactly parallel to the axis of the head, so that the pharynx appears to be a more or less shallow, cylindroid or

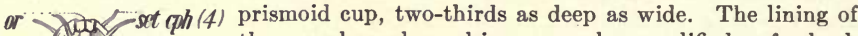
of the oesophageal canal is more or less modified as far back ph 1 elmt ph $(3)$ as the base of the amphids. The circumference of the amph 1 . 15 side. The somewhat cylindroid oesophagus is almost $\times 750^{\circ}$. $.5 p n$ imperceptibly swollen in the posterior fifth. At first it is three-fifths, near the nerve-ring one-half, and finally two-thirds, as wide as the corresponding portion of the neck, The lining is subdistinct. There is a more or less hemispherical cardia three-fifths as wide as the base of the neck. The thick-walled intestine, which has a distinct, refractive, more or less zig-zag lumen, becomes at once three-fourths as wide as the body. Its cells are packed with uniform colorless granules of variable size, the largest of which are onesixteenth as wide as the body. They do not give rise to more than a faint tessellation. The tail is conoid. The more or less narrow elongated caudal glands are arranged in a loose tandem behind the anus in the anterior third of the tail. The elongated renette cell lies at a distance behind the base of the neck equal to twice the body-diameter; it is one body-width long, about one-third as wide as long, and contains a rather large nucleus. The rather broad oblique nerve-ring is accompanied by somewhat obscure nerve cells.

Habitat: Clear, "white" sand, 5 feet of water, cove at the entrance to Buzzard's Bay, Mass., U. S. A. Sublimate to balsam. Fig. 37.

38. Protrellus aureus n. sp. $\frac{-4}{.7}-\frac{3.2}{2.4}-\frac{7.3}{3} \cdot--\frac{5.3}{3} \cdot-\frac{94.7}{1.7}-6 . .$. Annules as much as 20 microns wide. Striae are visible throughout the length of young specimens, which also differ from the adults in not having the cuticle inflated on the neck. No setae or amphids. The conoid neck ends in a truncate head, bearing a lip-region set off by constriction, and apparently composed of six lips. There is at least one papilla on each lip. The prismoid pharynx is half as wide as deep. The major part of the oesophagus is simple and cylindrical, having the same width as the lip-region, and is separated from the cardiac bulb by a broad constriction. The triquetrous lining of the oesophageal tube is easily seen, and ends in a distinct manner near the base of the pharynx. The intestine is modified, i.e., enlarged, to form a sort of "stomach," for a distance equal to twice the width of the body; thereafter its width does not exceed one-fourth that of the body. The length of the rectum is one and one-half times that of the anal body-diameter, and it has the peculiarity of lying close to the ventral side of the body. Region of the ventral excretory pore conspicuously marked by a large colorless ampulla. The lateral fields are one-third as wide as the body. The nerve-ring encircles the oesophagus squarely. The anterior fourth of the tail is somewhat hemispherical, and at the end of this part one-third as wide as at the anus; thence onward it is conical to the acute terminus. The large, elevated, and very prominent vulva is situated far forward, somewhat in front of 
the cardiac bulb, in fact, and from it the vagina and long single uterus are directed backward, the vagina being highly muscular, and in its contracted condition thrice as long as the body is wide. The uterus ends and the ovaries begin near the commencement of the posterior third of the body. There are numerous fusiform, slightly curved eggs, measuring $50 \times 104$ microns, the shells of which acquire a bright yellow color on entering the uterus. In many cases these goldenshelled eggs give the nemas, when seen with the unaided eye, a bright yellow appearance. The ovaries extend forward, then backward again; the ova are arranged single file. The eggs in the proximal part of the ovaries and in the uterus have the less-pointed end saddled with a peculiar organ. The saddled end of the egg is directed away from the vulva.

$\frac{.8}{2.5}-\frac{10}{4.5},-\frac{21 .}{6}-6$. transverse striae measure 3 microns apart on the neck, and 2 microns on the body. There is no distinct boundary between the rounded head and the conoid neck. The lips are very small and connate. Small, low, refractive papillae occur near the margin of the head. The somewhat irregular pharynx is half as deep as the head is wide, and about two-thirds as wide as deep. The anterior three-fifths of the oesophagus is a tube two-fifths as wide as the middle of the neck, the remaining two-fifths being flask-shaped, the neck of the flask being somewhat narrower than the anterior tube, and the ovoid bulb being two-thirds as wide as the neck. Cardiac collum, though shallow, distinct. The thinwalled intestine is at first at least two-thirds as wide as the body. The nervering encircles the oesophagus squarely. The tail diminishes rapidly behind the anus, until only one-third as wide as at the anus. There are three pairs of papillae, all sub-ventral:- one pair of conical papillae, the largest of all, just in front of the anus; a second smaller pair somewhat behind the anus; and a third pair near the middle of the tail. The single linear, acute spiculum makes an angle of $45^{\circ}$ with the axis of the body; its length equal to that of the narrow part of the tail. Testis-flexure such that the cardia lies half way between it and the nerve-ring.

Habitat: Rectum of the cock-roach, Polyzostaria melanaria Erich., Moss Vale, New South Wales, Australia, August 17, 1894. Examined in water.

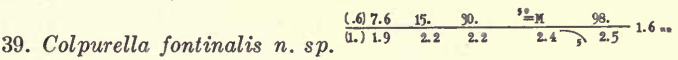
naked. No labial papillae. Pharynx very long and tubular. Neck cylindroid posteriorly. The oesophagus is at first threefifths, near the nerve-ring two-fifths, and finally two-thirds, as wide as the base of the pharynx. It is a little narrower through the middle, so that one may detect a faint posterior swelling. No cardia. The thick-walled intestine, which has a faint lumen, is separated from the oesophagus by a collum one-fourth as wide as the neck, and becomes at once one-half as wide as the body. Numerous, minute, uniform granules occur in the cells $\times 750$ of the intestine. Renette unknown. Female unknown. Five somewhat equidistant, papillate supplementary organs, onethird as high as the cuticle is thick, occupy a distance equal to two and one-half times the length of the body-diameter. The posterior supplement is near the proximal part of the spicula. The organs are wider apart anteriorly. Oblique copulatory tm.

Cuticle thick.
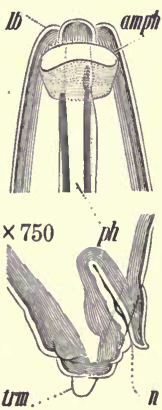
muscles coextensive with the supplements. Exact form of the testes unknown.

Habitat: Spring, at Country Club, Washington, D. C., U. S. A. Sublimate to balsam. Fig. 39. n, designates a caudal pocket, and not the anus, which is farther forward. 


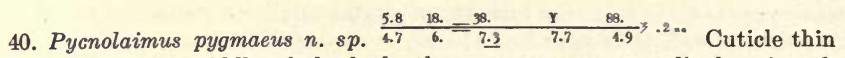
naked. Near the middle of the body the very narrow, exceedingly minutely crenate wings occupy a space about equal to the width of two annules of the cuticle. Neck conoid. From the amphids an internal element extends inward and backward a distance twice as great as the width of the corresponding portion of the neck. Lip-region difficult to decipher. Cephalic cuticle very thick, especially near the base of the lips. When the head is viewed in profile and in optical section there are seen connected with this thickened portion of the labial cuticle elements that extend forward over the vestibule. At first sight these extensions appear to be thin, cutinized flaps, that are very minutely transversely striated. A careful examination, however, appears to indicate that they may have somewhat the structure of odontia, inasmuch as a short distance behind the apices, which are decidedly sharp, the inner contours curve in toward the axis of the head, and the longitudinal section of the elements, including the bases, above described, appear claw-shaped. The dorsal and ventral elements, thus viewed, are very much alike. Concentrating attention on either lateral surface of the head one finds, opposite the axis, two elements which extend forward parallel to each other and then bend toward the axis, arching inward to meet the dorsal and ventral elements already described, and appear to be the median elements of lateral labial organs. These elements are not so finely striated as the labial elements first mentioned, and appear to be slightly darker. The napiform vestibule leads to the pharynx, the entrance to which seems to be nearly closed webl. mnd by a very slight curvature on the anterior part of one of the cutinan? Is ous elements marking its contour. The oesophagus diminishes 11 slightly in diameter as it passes backward, so that its middle part th $f \int^{\text {ph }}$ is hardly two-fifths as wide as the corresponding portion of the and I I neck. It then diminishes more rapidly, so that just in front of the

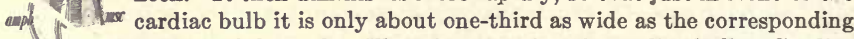
spn $\times 750$ portion of the neck. There is no vestige of a median bulb. Cardiac bulb somewhat pear-shaped, or sub-spherical, three-fifths as wide as the base of the neck, and containing a rather complex central valve half as wide as the bulb itself. Two small, refractive elements occur one in front of the other in the midst of the valve. There is a sub-spherical cardia one-third as wide as the base of the neck. The intestine becomes at once three-fourths as wide as the body; its cross-section presents apparently only two cells. These cells contain granules of various sizes and kinds; the largest and clearest are spherical, and have a width two to three times that of one of the annules of the cuticle. The smaller and darker granules are not distinctly spherical and vary in size down to those of very minute size. From the inconspicuous but very slightly raised anus, the refractive rectum extends inward a distance a little greater than the anal bodydiameter. The tail is conoid from the anus, but is convex-conoid at the terminus. The description is derived from a specimen much too young to give any indication as to the position and form of the sexual organs. The measurements must be correspondingly interpreted.

Habitat: From green-house soil, Ann Arbor, Mich., U. S. A. Fixed in Flemming; examined in water. Fig. 40.

41. Isolaimium papillatum n. $s p$. Cuticle rather thin, naked. Neck convexconoid anteriorly. Cuticle with surface innervations along the edges of the lateral field. Nerve-ring oblique. Pharynx tubular, one-sixth as wide as the head measured opposite its base. Tail of the male conoid, slightly arcuate, a 
little longer than the anal body-diameter, blunt, its ventral contour nearly straight, its dorsal contour arcuate. The rather simple, blunt, uniform, more or

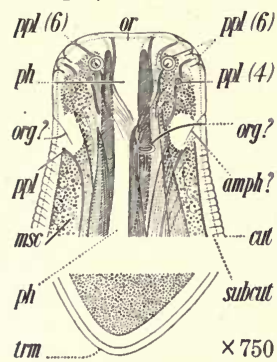

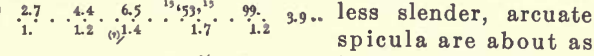
2.9
1. .5 .2
1.2
(i) 1.6 2.9
1. .5 .2
1.2
(i) 1.6 cephalated proximal ends lie ventrad from the bodyaxis. The frail, slender, arcuate accessory piece is mph? parallel to the spicula and one-third as long; at its proximal end it has a very small, backward-pointing apophat ysis from which muscular strands pass forward to the dorsally submedian lines and backward to the ventral

subrut field near the middle of the tail. The six papilloid or mammiform, sub-equidistant supplements occupy a $\times 750$ space about three times as great as the anal body-diameter. Each comprises about six annules of the cuticle and has the same slightly crenate contour as the ventral surface. The tail bears four pairs of flattish, conoid papillae, a little behind its middle; one dorsally submedian, one lateral, one ventrally submedian and one subventral.

Habitat: Soil, Plummer's Island, Potomac River, Virginia, U. S. A. Flemming to glycerine jelly. Fig. 41.

42. Coinonema punctatum n. sp. Lips thick. Neck conoid. Eye-spots two, far apart, brown to yellow, solid, with backward connections. The cylindroid oesophagus is at first one-half, at the nerve-ring one-third, and finally also onethird, as wide as the corresponding portion of the neck; lining subdistinct. There is a cylindroid cardia one-fourth as wide as the base of the neck. The intestine, set off by a collum one-fifth as wide as the base of the neck, becomes at once three-fourths as wide as the body. Anteriorly it is thick-walled, posteriorly thin-walled. Its cross-section is composed of four to five cells. These cells contain numerous granules packed together in such a way as to give rise to a faint tessellation. The granules are of variable size, the largest set stbm $1 / b / J^{\text {set }} 7 \mathrm{hh}$ being one-tenth to one-sixtcenth as wide as the body. The anus is continuous, the rectum three-fourths as long as the anal body-diameter. The conoid tail tapers from the anus to the 19 , mmph rather minute spinneret. The three, broadly-saccate caudal pex f t Imph glands lie in a close tandem in the anterior third of the tail. The ellipsoidal renette cell stretches along behind the base of the neck for a distance equal to

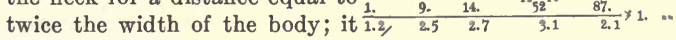
is two-thirds as wide as. long.

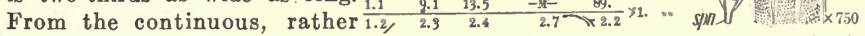
inconspicuous vulva, the rather weak, non-cutinized, medium-sized vagina leads inward one-third the way across the body. The eggs occur one at a time in each uterus, and are one and one-half times as long as the body is wide. They appear elongated in form and are deposited after segmentation begins. In the mediumsized, tapering ovaries are ten to twelve ova arranged single file. The acute spicula are rather frail and slender. Their proximal ends appear to lie ventrad from the body-axis. A refractive "chord" appears to subtend the arc of the spicula. The rather frail, somewhat slender, arcuate accessory pieces have an 
applied part one-third as long as the spicula, and projecting at right angles to this is a uniform, blunt apophysis, one-half as long as the anal body-diameter having its proximal end opposite the axis of the tail. The testes are wide and more or less tapering.

Habitat: Key West and Biscayne Bay, U. S. A., on algae. Flemming to glycerine jelly. Fig. 42 , p. 259.

43. Rhynchonema cinctum $n$. sp. Annules of the cuticle retrorse posteriorly, the reverse anteriorly. Neck cylindroid, very rapidly narrowing just behind the

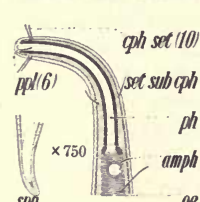
head, and thence nearly cylindroid, so that the entire head spm forms a kind of flexible beak. There are no eye-spots. Conoid oesophagus at the nerve-ring two-fifths, and finally onehalf, as wide as the corresponding part of the neck; it has a

ph fine colorless musculature with no indication of glands. The ph lining is indistinct. There is no cardia. The intestine, set off by a collum one-fifth as wide as the neck, becomes at once -

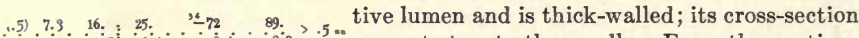

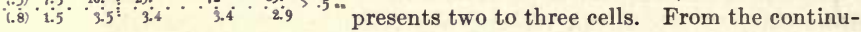
(.5) 7.4 16. 26. $\quad-2 \quad 87,>5 \ldots$ ous anus, the rather inconspicuous though (.9) 1.5 . $3.3^{3}+3.3$ cutinized rectum is three-fourths as long as the anal body-diameter. Only the first few cells of the intestine show granulation. The conoid, sub-arcuate tail tapers from the anus to the non-striated spinneret, whose width is about equal to that of three of the caudal annules. At the base of the spinneret there are always about three minute nuclei that stain strongly. The ellipsoidal caudal glands form a close tandem in the anterior two-fifths of the tail. The lateral fields are about one-third as wide as the body, but are more or less indistinct. Renette unknown. In the vicinity of the vulva about eight of the annules present simply a crenate, instead of a serrate contour. The broadly elevated vulva is rather conspicuous, particularly in front. Vagina small. A mass of small sperm cells has been noted near the vulva. The eggs are about eight times as long as the body is wide and onetwelfth as wide as long. They are granular and have a prominent central nucleus. The narrow cylindroid ovaries contain ova arranged single file. The spicula of the male, about as wide as one of the adjacent annules, are slender, rather frail, and sub-acute, and when seen in profile appear to have their proximal ends about opposite, or a little dorsad from, the body-axis. There is a slender, frail accessory piece. The three minute papilloid supplementary organs occupy a space three times as great as the body-diameter, and are about one body-width apart; the posterior member is about two body-widths in front of the anus. They hardly more than accentuate the annules on which they occur, but are rendered visible by carmine stains. The ejaculatory duct is one-fourth as wide as the corresponding portion of the body, the cylindroid testis one-half as wide.

Habitat: Salaverry, Peru, near low tide mark; marine. Rhynchonema is composed of a considerable number of species occurring in at least the Atlantic and Pacific Oceans. With low powers it is difficult to distinguish the head end from the tail end. The flexible, narrow head suggests the functions of a beak. One may imagine it to probe the depths of some receptacle containing food, for instance the neck of an algal oögonium. However, nothing is known with certainty concerning the food habits. Fig. 43. 


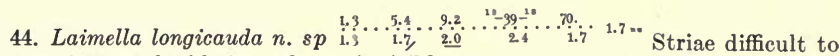
detect, but resolvable into almost invisible dots. Cuticle naked. Labial papillae obscure. Pharynx closed, inconspicuous, but undoubtedly present. There is a slight thickening or extra-refractiveness at one portion of the pharyngeal wall that suggests the presence of an extremely minute onchium. The cylindroid oesophagus near the nerve-ring is two-fifths as wide as the neck, and ends in an ellipsoidal valveless bulb three-fourths as wide as the base of the neck. The oesophagus has a fine colorless musculature. Hemispherical eardia, two-sevenths as wide as the base of the neck. The thick-walled intestine, set off by a cardiac collum one-fifth as wide as the neck, becomes gradually five-sixths as wide as the body, its cross-section presenting about six cells. From the somewhat depressed anus, the rather prominent cutinized rectum extends inward a distance equal to the anal body-diameter. The colorless, scattered to numerous intestinal granules are of variable size, the largest of them at first only twice as wide as one of the annules, but near the middle of the body six to eight times as wide.

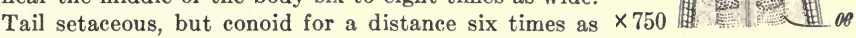
great as the anal body-diameter, at which distance it is one-fifth as wide as at the base; thence it tapers very gradually to the terminus. It is very doubtful if there is a spinneret. There are elements in the base of the tail, simulating caudal glands, but no definite ducts have been seen, and the terminus seems too fine to afford space for a spinneret. About six very slender caudal setae, each about two-thirds as long as the anal body-diameter, occur on each of the four submedian lines. Similar setae occur throughout the body. The lateral fields are two-fifths as wide as the body. The large, non-granular, elongated renette cell lies just behind the base of the neck, and empties through an ellipsoidal ampulla, one-third as wide as the neck. The broad, oblique nerve-ring is accompanied by rather obscure nerve cells. From the small, more or less continuous vulva, the moderate-sized, conoid, cutinized vagina extends threefifths the distance across the body. The elongated eggs are one to one and onehalf times as long as the body is wide. The ellipsoidal sperm cells in the uterus are granular and about haif as long as the body is wide. The cylindroid ovaries contain at least ten ova, arranged single file.

Habitat: Algae, Key West; also mud-flat, Biscayne Bay, Florida, U. S. A. Flemming to glycerine jelly. Fig. 44. Terminus, trm, shown foreshortened.

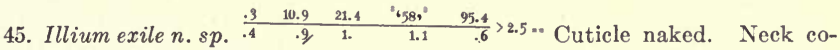
noid. Oesophagus conoid, finally about three-fourths as wide as the base of the neck. Cardia elongated, half as wide as the base of the neck. The intestine IJ (6) is about as long as the anal body-diameter. The lateral fields are a little more than one-third as wide as the body. The ventral gland is a much elongated cell, lying at a distance behind the base of the neck equal to about three to four body-diameters, and having a width about one-fourth as great as that of the corresponding portion of the body. There is a distinct ellipsoidal ampulla 
one-third as wide as the middle of the neck, connected with the excretory pore by a short, rather sharply curved, minute duct. The caudal glands are located in a tandem series a considerable distance in front of the anus, that which is farthest forward being somewhat farther from the anus than is the spinneret. The tail is conoid in the anterior three-fifths in such a fashion that at the beginning of the fourth fifth it has a diameter about one-fourth as great as at the anus; thence onward the tail is cylindroid to the terminus which bears a slightly oblique spinneret. From the inconspicuous vulva the rather strongly cutinized vagina leads inward more than half way across the body. The ovaries reach about twothirds the distance back to the vulva, at any rate in the only specimen so far examined, which was a rather immature female. Nothing is known concerning the number, size and structure of the eggs.

Habitat: Green algae, Carlisle Bay, Jamaica. Fig. 45, p. 261.

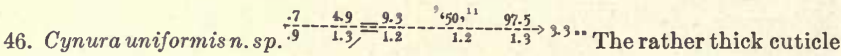
is naked. The two wings, occupying a space one-third as wide as the body, begin near the head and end on the tail. Pharynx very strongly reminiscent Ill...... pal (6) set (4) of that of Plectus; - is continued by a longer posterior part, whose presence is indicated mainly by alterations in the lining. This part, however, is rather easily disamph ... droid posteriorly, convex-conoid anteriorly. The cylindroid oesophagus near the nerve-ring is three-sevenths, finally three-fifths, as wide as the corresponding portion of the neck. The radial oesophageal tissue continues to the middle of the anterior chamber of the pharynx, but is narrower there. There is the faintest possible break in the lining of the oesophagus near the oe nerve-ring,-possibly the vestige of a bulb. There is a

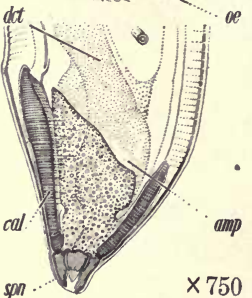
large cylindroid cardia, three-fifths as wide as the neck. The thick-walled intestine becomes at once two-thirds as wide as the body; while its lumen is faint anteriorly, it is very pronounced posteriorly. From the elevated anus, the strongly-built, prominent rectum,- the lining of which is somewhat cutinized,-extends a distance three-fourths as great as the anal body-diameter. The cells of the intestine are packed with granules of variable size, the largest of which have a width equal to that of spon

$\times 750$ one of the annules. The tail is cylindroid, then conoid in the posterior three-sevenths. The blunt, conoid terminus is strengthened by a large, obliquely-truncate cap, the several component plates of which are minutely punctate. The caudal glands probably lie in front of the anus; they end in the terminus in three simple, prominent ampullae. There are no caudal setae. The lateral fields appear to be about one-third as wide as the body. The granular ellipsoidal renette cell, two-thirds as long as the body is wide, and half as wide as long, lies about two body-widths behind the base of the neck. The mediumsized nerve-ring is accompanied by obscure nerve cells. From the mediumsized, more or less elevated, refractive and therefore conspicuous vulva, the vagina leads inward half way across the body. The inner wall of the vagina is also cutinized. The short, broad, cylindroid ovaries contain about fifteen ova arranged somewhat irregularly. Ellipsoidal granular spermatozoa, seen in the uteri, are one-eighth to one-tenth as wide as the body of the female. 
Habitat: Seaweed, washed up at Ocean Beach, Miami, Fla., U. S. A., after a storm. Flemming to glycerine jelly. Only a single specimen seen. Fig. 46, p. 262.

47. Xinema perfectum $n$. $s p$. Neck conoid. Somatic setae papilloid, or nearly so; cuticle becoming much thinner in the region of the lateral organs, thickening again in front of them and becoming again as thick as ever on the front of the head. The oesophagus continues to have the same diameter until after it passes through the nerve-ring, when it begins to expand gradually, until finally it is nearly three-fourths as wide as the base of the neck. The two ventrally submedian sectors of the oesophagus appear to contain glands, - at least in each there extends from near the posterior end of the oesophagus forward a minute duct, which stains distinctly with carmine. This duct can be traced at least as far

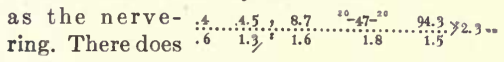

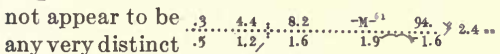
cardia. The intestine gradually becomes threefourths as wide as the body. In cross-section it is composed of six to eight cells, containing relatively large nuclei and numerous small, uniform granules. The renette cell, nearly as long as the body is wide and about one-third as wide som

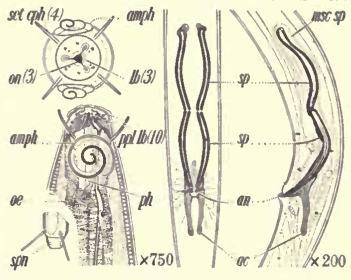
as long, is located just behind the base of the neck. Its ampulla, about onefourth as wide as the corresponding portion of the neck, is connected with the excretory pore by means of a very short duct. The lateral fields are about onethird as wide as the body. The nerve-ring surrounds the oesophagus squarely. The tail tapers from considerably in front of the anus, being convex-conoid in such a fashion that at the beginning of the final fifth it has a diameter about one-fifth as great as at the anus, or even less; thence onward, the tail expands a little. The caudal glands are located near the anus. From the depressed vulva, the vagina leads inward more than half way across the body, where it joins the two symmetrically-placed uteri; its internal walls are distinctly cutinized. The ovaries contain twelve to fifteen ova arranged single file. Occasionally the tips of the ovaries are reflexed. Apparently the females possess a pair of spermatheca, which when filled reach to near the bases of the ovaries. The spermatozoa appear to be elongated. The elongated eggs are about twice as long as the body is wide and a little less than half as wide as long; they have been seen in the uteri one at a time. Tail of the male a little more bulky than that of the female and a little narrower in the posterior part, which instead of constituting one-fourth of the tail, constitutes about one-third. No supplements or special setae. Spicula consisting of two, equal segments, one in front of the other. The muscular tunic enclosing the spicula is continuous at the elbow. While the spicula and their sheaths tend to stain in acid carmine, the accessory pieces do not do so, but retain a slight yellowish color of their own. The caudal glands are located alongside the accessory pieces, both in front of the anus and behind it. The ejaculatory duct is about one-fourth as wide as the body, the vas deferens about one-third.

Habitat: Marine mud, San Pedro, California, U. S. A. Specimens of this species appear to have a tendency, when killed with hot sublimate, to twist and present a dorsoventral view of the head instead of a lateral. Fig. 47. 


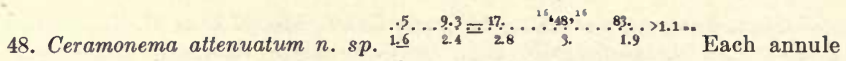
of the thick, transparent, naked cuticle is made up of eight elements so fitted together as to be reminiscent of the arrangement of corrugated roof-tiles; hence, the name "Ceramonema" (see an, Fig. 48). Neck cylindroid, with minute, somewhat forward-pointing setae one-fourth as long as the annules are wide. The setae occur in longitudinal rows, one on each annule, but whether on each of the longitudinal lines is problematical. Lips amalgamated. It is probable that there are minute papillae a little in front of the anterior row of cephalic setae. The very narrow pharynx joins the oesophageal lumen with little alteration. The oesophagus gradually narrows to near its posterior part, becoming at its narrowest point about one-fourth as wide as the neck; thence onward, however, it expands so that where it joins the intestine it is about half as wide as the base of the neck. There is no distinct cardia. The thin-walled intestine becomes at once two-thirds as wide as the body. Possibly its cross-section is composed of as few as three or four cells. Anus
inconspicuous; rectum fully twice as long as anal
body-diameter. Salivary glands unknown. The
renette appears to be a unicellular gland located
ammediately behind the cardiac constriction; it is
about half as long as the body is wide, and, as usual,
tapers anteriorly to join the narrow excretory tube.
The excretory pore is perhaps just to the rear of the
nerve-ring. It is very difficult to observe the longi-
tudinal fields, owing to the highly refractive nature
of the elements composing the cuticle. These latter
join each other in such fashion as to give rise to eight
longitudinal lines, two of which are, of course, sub-
lateral and span a distance equal to about one-third conoid; terminus having a diameter about half as great as that of the base of the tail. The final caudal segment of the cuticle is more than twice as long as the penultimate, though it seems also to be compound in its structure. The caudal glands appear to be located in front of the anus, near the place where the intestine joins the rectum. From the depressed and inconspicuous vulva the vagina leads inward nearly half way across the body. The reflexed portions of the ovaries reach well back toward the vulva. The eggs are long and narrow, though no specimens have been seen containing well-matured eggs.

Habitat: Mud among marine algae, shores of Kingston Harbor, Jamaica. Sublimate to balsam. The male of another undescribed species has stoutish, tapering, subacute, rather frail spicula, accompanied by a more or less arcuate, rather slender, frail, simple accessory piece half as long. There are no supplementary organs. This undescribed species is so similar to $C$. attenuatum that it is believed the male of attenuatum will be found to present similar features. Fig. 48.

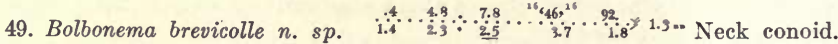
The walls of the pharynx are a little more strongly cutinized than those of the oesophagus, especially toward the minute mouth opening. The cylindroid oesophagus is about three-fifths as wide as the base of the head, but expands finally to form the pyriform cardiac bulb, which is about four-fifths as wide as the base of the neck. This bulb has no distinct valve, though the cutinous 
lining is more strongly developed in the bulb than it is elsewhere. There appears to be an elongated eardia,-perhaps one-third as long as the neck is wide. The intestine begins as a narrow tube only about one-fourth as wide as the base of the neck, and enlarges gradually so that near the middle of the nema it is about onehalf as wide as the corresponding portion of the body. For a distance behind the bulb as great as the body-diameter, the cells of the set (4) intestine contain nuclei which stain more strongly with carmine than do their neighbors. Thence onward the $1 / b$ cells contain granules of variable size, the largest being larger than any of the nuclei in any of the adjacent cells. The intestine shows about four cells in crosssection; their contents are so disposed that there is a distinct tessellated effect. Anus slightly raised; the spn- $\times 750$ rectum as long as the anal body-diameter. There appears to be no doubt about the existence of a unicellular renette cell just behind the neck, but the position of the excretory pore is unknown. Lateral fields about one-third as wide as the body. Tail conoid. From the slightly raised vulva, the vagina leads inward fully one-third the distance across the body. The eggs appear to occur one at a time in each uterus. They are fully twice as long as the body is wide, and considerably less than half as wide as long. It seems probable that segmentation sets in before the eggs are deposited.

Habitat: Mud, shallows of Kingston harbor, Jamaica. Sublimate to balsam. Fig. 49.

50. Omicronema litorium n. sp. $\frac{1.7}{1.5}-\frac{8.8}{2 .} \div \frac{19 .}{1.9}--\frac{48.85}{2.1}--\frac{92 .}{1.6}>1.23$.. Cuticle rather thin, naked, its striae more easily resolvable into rows of longitudinal markings near the extremities. Wings faint. No labial papillae. Amphids yellowish. Oesophagus at the nerve-ring one-half as wide as the middle of the neck; thereafter it expands somewhat, so that finally it is about two-thirds as wide as the base of the neck. The oesophagus has a somewhat wavy lining. There is a narrow, elongated cardia, nearly half as long as the body is wide. The intestine, set off by a deep and broad constriction, becomes at once about three-fourths as wide as the body. The lateral fields appear to be about one-fourth as wide as the body. The female has a single outstretched ovary extending forward. From the more or less conspicuous, depressed vulva, the rather weak, more or less cutinized vagina connects with the straight uterus, which contains elongated eggs, twice as long as the body is wide and about one-eighth as wide as long. The narrow ovary is first cylindroid, then tapering. The larger ova are arranged single file; toward the blind end, however, the ova are arranged irregularly. The conoid, arcuate tail tapers from the anus, or from somewhat in front of it. The three ellipsoidal caudal glands lie in a loose tandem in the anterior half of the tail.

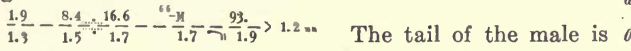
arcuate and conoid to the spinneret, which is one-sixth as wide as the base of the tail. No supplements, though

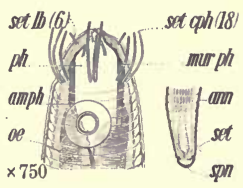
there are inconspicuous setae on the submedian lines, both behind and in front of the anus. The dark rather slender spicula attain their greatest width at their bent middle parts. Accessory piece nearly as long as the anal bodydiameter. Near the bends of the spicula the accessory piece takes on a sigmoid contour, and this portion is connected with the body wall both anteriorly and posteriorly by strands of muscle. The ejaculatory duct is about one-third as wide as the body. 
Habitat: Ocean beach-sand, vicinity of Los Angeles, Calif., U. S. A., near low tide mark. Fig. 50, p. 265.

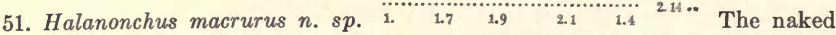
cuticle is very finely and somewhat irregularly reticulated upon the head. Outer row of cephalic setae stout, two-jointed. Lips probably distinct and six in number, thin, flap-like. Pharynx somewhat deeper than the base of the head is wide. The pharynx has a faint triquetrous framework, the three, slender, longitudinal elements of which are split behind (and also less conspicuously in front), the set (6) - of $\sec (6)$ branches thus made bowing round together; this imparts to the wall of the pharynx, opposite the beginning of the posterior fourth, a junction-like effect. As a whole the pharyn $\mathrm{x}$ is much like an elongated and naked and weakened Oncholaimus pharynx destitute of onchia. The cylindroid

ph to conoid oesophagus near the nerve-ring is three-fifths, and finally two-thirds, as wide as the neck. The distinct

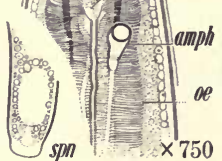
refractive lining appears to be composed of two to three faint elements, occupying a space three-fifths as wide as the oesophagus; the musculature is coarse. Probably glands are present in the oesophageal tissues. There is a more or less hemispherical cardia, one-third as wide as the neck. The thick-walled intestine is set off by a constriction one-third as wide as the base of the neck, and becomes at once two-thirds as wide as the body. In cross-section it is composed of about twelve cells. The rather inconspicuous rectum is as long as the anal body-diameter. The cells of the intestine contain scattered, colorless granules, of variable size, the largest of which are one-twentieth as wide as the body. The larger granules darken in Flemming's solution. The tail is first conoid, and then cylindroid in the posterior two-thirds, where it is one-third as wide as at the base. It tapers from in front of the anus to the terminus, which is apparently devoid of spinneret. The lateral fields are threefifths as wide as the body; their margins are rather distinctly indicated by the abrupt beginning of the longitudinal musculature. Renette unknown. Nervering accompanied by obscure nerve cells. From the large, conspicuous, somewhat elevated vulva the large, tubular to conoid, non-cutinized vagina extends obliquely backward a distance equal to the body-diameter. The uterus is twice as long as the body is wide, and contains four to seven eggs, which are probably deposited after segmentation begins. The narrow ovary tapers but little, and contains about thirty ova arranged single file. The sub-arcuate, rather simple, frail, blunt spicula are one-eighth as wide as the body; viewed in profile their non-cephalated proximal ends appear to lie dorsad from the body-axis. The single, more or less straight, very slender, frail, simple, parallel accessory piece is one-third as long as the spicula. The fifteen to eighteen very inconspicuous, sub-equidistant, papilloid, ventral supplementary organs occupy a space in front of the anus seven times as long as the body-diameter; the posterior one lies opposite the middle of the spicula. The distance between these organs is about one-fourth of one body-diameter. For each supplement there is a "pore" in the cuticle, and to this comes a "nerve-ending" that appears to project more or less. The sperm cells are long and cylindroid.

Habitat: Sand inside government cut, Biscayne Bay, Florida, U. S. A. Flemming to glycerine jelly. Fig. 51 . 
52. Halinema spinosum $n$. sp. There are a few, very inconspicuous cervical setae, one-third as long as the neck iswide. Cephalic setae three-jointed (Fig. 1,2,3). Lips three, possibly double. Neck more or less cylindroid. Oesophagus cylindroid, with an almost imperceptible cardiac swelling; - at the nerve-ring four-sevenths, and finally three-fourths as wide as the corresponding portion of the neck. The sub-distinct lining of the oesophagus is optically expressed by refractive lines occupying a space two-sevenths as wide as the organ itself. The colorless musculature is rather coarse. No cardia has been noted, but the intestine is at first more refractive in its lining, and otherwise altered. The thick walled intestine is set off by a collum one-fourth as wide as the neck, and has a faint, but somewhat refractive lining. It soon becomes two-thirds as wide as the body, and in cross-section is composed of few cells. From the anus, the posterior lip of which is elevated, the rather inconspicuous rectum leads inward a distance three-fourths as long as the anal body diameter. The intestinal cells contain scattered granules of variable size, the largest of which are about one-tenth as wide as the body; they darken on treatment with Flemming's solution, and are strongly refractive in glycerine jelly. The conoid tail tapers from the anus. The broadly saccate caudal glands, three in number and relatively small, form a close tandem in the anterior eighth of the tail. The posterior half of the tail bears eight pairs of ventrally submedian stiff setae. The rather fusiform, nongranular renette cell lies on the ventral side of the body a little behind the base of the neck; it empties through a rather conspicuous ampulla one-third to onefourth as wide as the corresponding portion of the neck. The nerve-ring is of medium size and

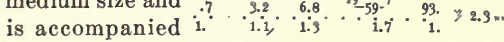

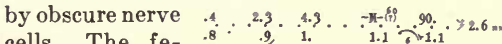

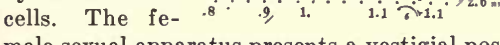
male sexual apparatus presents a vestigial posterior branch. Vulva rather large, somewhat elevated; vagina more or less cutinized. The eggs are five times as long as the body is wide, and evidently occur in the uterus one at a time. The narrow tapering ovaries contain few ova arranged single file. The spicula are rather strong, somewhat slender, tapering and acute. Their expanded proximal ends appear to lie ventrad from the body-axis. The applied parts of the two rather frail accessory pieces are onefourth as long as the spicula. They have taper- set $\lambda$

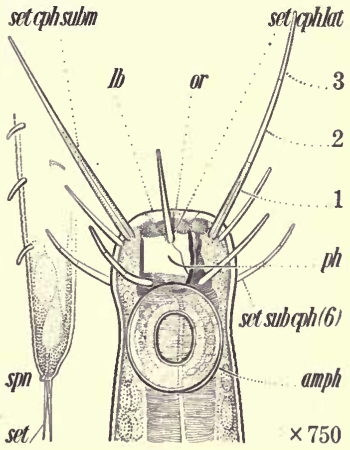
ing apophyses, one-third as long as the anal body diameter, whose proximal ends lie ventrad from the body-axis. Papilloid sub-equidistant supplementary organs on the ventral line occupy a space five to six times as long as the body diameter, the distance between them being about equal to three-fourths the corresponding body diameter. Of these almost imperceptible innervations, the posterior one lies opposite the distal portions of the spicula. Ejaculatory duct two-fifths as wide as the body. There seem to be two narrow, tapering testes, but there is a little uncertainty about the posterior one.

Habitat: Sand bar, opposite the mouth of the Miami River, Biscayne Bay, Florida, U. S. A. Flemming to glycerine jelly. Fig. 52. 


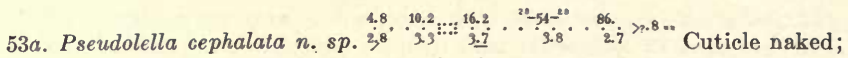
wings optically expressed in the form of closely approximated, indistinct longitudinal lines. The unusually long amphids are of such a character as to be mistaken at first for wings. Behind the neck the amphids gradually narrow, so that somewhat in front of the middle of the body, where they end, their width is only about one-third as great as opposite the base of the pharynx. Neck conoid. Cuticula much thinner on the head. Lips thick; six, or three and twoset qph(4)...... Internally the lips are armed with six very minute odontia (?), apparently having an outward stroke. From
the expanded base of the pharynx muscular fibres are seen
passing to the front, clearly indicating that the entire struc-
ture can be moved forward; such movement would be
entirely in accord with the structure of the labial region.
The oesophagus maintains the same diameter until after it
passes through the nerve-ring; it then expands rather rap-
idly so as to form the pyriform cardiac bulb nearly two-thirds
as wide as the base of the neck. There is a flattish-conoid
cardia, one-third as wide as the base of the neck. The front
end of the intestine is pressed to one side by the strongly
developed renette cell, but behind this cell it becomes about
two-thirds to three-fourths as wide as the body; its cross-
section probably is composed of six or more cells. These cells contain scattered yellowish to brownish granules of small but variable size. The anus is rather inconspicuous. The rectum is about as long as the anal body diameter. The tail is at first conoid, so that at a distance from the anus equal to five times the anal body diameter, its diameter is not over one-sixth as great as at the base. The remainder of the tail, probably a short part only, is missing. Caudal glands probably located in the base of the tail. The renette cell, half as wide as the body and about twice as long as it is wide, is located behind the base of the neck a distance equal to two body diameters. From the slightly elevated vulva the rather massive vagina leads inward fully half way across the body. About eight ova are arranged single file in each ovary. The eggs are two to three times as long as the body is wide, and a little less than one-third as wide as long. The spermatozoa seen in the uterus are about one-sixth as wide as the body, and are finely granular and have their chromatin in an ellipsoidal form surrounded by a clear area.

Habitat: Punta Arenas, Pacific Coast of Costa Rica. The following are the

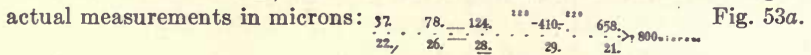

b. Pseudolella granulifera n. $s p$. Type species. Cuticle as in P. cephalata, but without wings. Neck convex-conoid anteriorly; especially at the head, which is somewhat bluntly pointed. Cephalic setae four, arcuate, spreading, and onethird as long as the corresponding diameter of the head, arranged about onefourth of the distance back to the beginning of the tubular portion of the pharynx. Lips confluent, obscure, probably three in number. No labial papillae seen. Amphids long, slender; beginning some distance behind the pharynx and extending forward in the form of a band or groove about half as wide as the pharynx, and having refractive cutinized edges. This band extends to very near the lips, then suddenly narrows and turns back on itself and ends indefinitely in front of 
the middle of the pharynx. At its widest, i.e., toward the front, the amphid is a little narrower than the pharynx. Lips relatively thick, closing to form an exceedingly narrow, cutinized vestibule. Pharynx and all parts of the alimentary canal like those of $P$. cephalata. Pharynx sub-uniform, about one-sixth as wide as the base of the head. A little behind the lips the lining of the pharynx is discontinuous and the cavity bends slightly toward the ventral side, and there appears to be a pair of very small, ventrally submedian onchia of equilateral profile. It is difficult to make out the details sufficiently well to state positively that these refractive, cutinous elements are homologous with ordinary onchia. Opposite the onchia on the dorsal side the cutinized, pharyngeal elements are discontinuous, two or three in number, but not very variable in character. The onchia and the elements opposite them and in front' of them are fully as robust as the walls of the pharynx, of which the ventral side supporting the "onchia" is considerably thicker than the dorsal. The cross-section of the intestine appears to be made up of few cells, perhaps only two or three. The granules in the intestinal cells, the largest of which are half as wide as the nucleus of the renette cell, are a very conspicuous feature and give rise to a very indistinct tessellated effect. In its anterior half the tail is conoid from the anus, and at the middle is one-fifth as wide as at the base. Thence onward, it tapers but little and ends in a somewhat rounded, unarmed spinneret. There are no caudal setae. Caudal glands occur in the anterior part of the tail. Lateral fields indistinct, apparently one-third as wide as the body. Both before and behind the renette cell there are bodies of unknown significance that stain with carmine. Nucleus of the renette cell distinct, slung in a conspicuous protoplasmic network. Behind the renette cell is a spindle-shaped, distinctly nucleated cell fully one-third as wide as the body and about twice as long as wide. Still further back, after a

$$
\begin{aligned}
& \text { 4.3. } \frac{8.5}{3.3} \cdot \frac{12.9}{3.6} \cdot \frac{23.3-33}{4.4} \cdot \frac{86 .}{2.7}>1 .=
\end{aligned}
$$

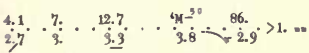

considerable interval, there is another similar cell of larger size; these two latter cells seem connected by a narrow process, and the anterior smaller cell presents a narrow process extending forward. Spicula uniform, arcuate, one and one-half times as long as the anal body

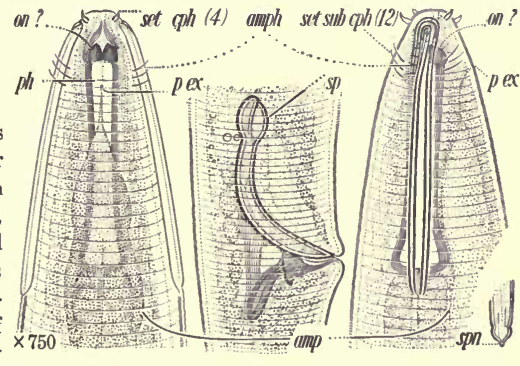
diameter; their proximal ends rather prominently and obliquely cephalated by expansion. Proximal ends a little dorsad from the body-axis. The spicula taper to a fairly acute point in the distal fourths and their cutinized framework is duplex; at their widest part, the middle part, the spicula are about one-sixth as wide as the corresponding portion of the body. Two accessory pieces adjoining the distal fourths of the spicula; extending backward at right angles to the spicula are the blunt apophyses three-fifths as long as the anal body diameter. The number and the structure of the testes is uncertain, but apparently there are two, of which the anterior is reflexed and the posterior outstretched. The sexual cells are of unusually large size and their walls appear to divide up the testes in an irregular geometric fashion.

Habitat: Marine mud and sand, Noımea, New Caledonia. Fig $53 b$. 


\section{Order Polylaimia}

This order cannot at present be so satisfactorily defined as most of the others herein represented. There probably can be removed from it sooner or later a number of groups of ordinal rank, but the time seems hardly yet ripe for such a removal. For the present, therefore, the genera and families ranged under this name are of a more heterogeneous character than under most of the other twelve orders here presented. Some slight effort has been made, here as elsewhere, to place these new genera in the order of their family relationships. In general, the genera that are first mentioned in an ordinal group, and the genera placed at the end of the group, as here presented, exhibit more or less manifest

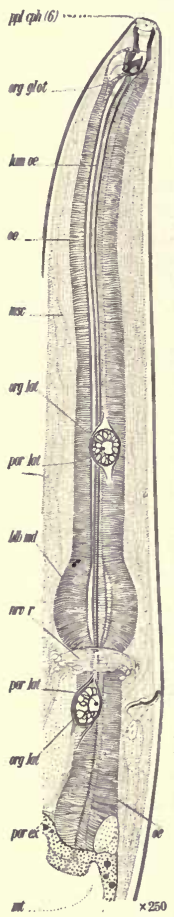
relationships to genera included in other orders. (See p. 217.)

54. Cephalobium microbivorum $n$. $s p$. The wing begins near the head and ends near the terminus. Its optical expression is either a pair of lines, or a single line, in the middle of a field onetwelfth as wide as the body. The contour of the body may become crenate in the anal region. There are about thirteen lateral organs in each lateral field connected with pores in cuticle (see org. lat., Fig. 54). Base of the pharynx containing a large, complicated and peculiar dorsal glottoid organ. No amphids. The rather thin-walled intestine is set off by a collum one-eighth as wide as the neck, and has a rather distinct lumen. It becomes at once five-sixths as wide as the body, and in crosssection presents two to four cells. From the somewhat depressed anus, the narrow, cutinized rectum extends inward a distance one and one-fourth times as great as the anal body diameter. Scattered yellowish granules of variable size occur in the cells of the intestine, the largest being one-twenty-fifth as wide as the body. In addition, there are numerous very small granules. The subarcuate, conoid tail tapers from in front of the anus to the acute fine terminus. There is no spinneret. From the elevated vulva, the rather small, somewhat weak vagina extends inward one-fourth the way across the body. Along the middle half of the body the two equal uteri contain ellipsoidal eggs two-thirds as long as the body is wide, which are deposited after segmentation begins. No embryos were seen in these eggs,only blastulas. For the most part the ova are arranged irregularly in the somewhat tapering ovaries. The rather strong, slender, tapering, subacute spicula, when seen in profile, have their proximal ends nearly opposite the body-axis. Toward

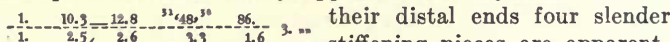
stiffening pieces are apparent. $-\frac{1.2}{1.2}-\frac{10}{2}=-\frac{19}{2.8}-74-x-90.0$ There is a strong, and rather bending back from the straight accessory piece, mal end lies opposite the body-axis. Near the beginning of the second quarter of the tail there is a pair of lateral pores (?) similar to those on the female. On the female these pores have been shown by intra vitam staining to be homologous with those mentioned above, and shown in the illustration at por lat. Pairs of papillae on the tail of the male flattish-conoid, plainly innervated, rather 
inconspicuous, occupying the anterior two-thirds of the tail, thus: 1() $1 ; 111 ; 1$. The members of the posterior four pairs are not located exactly opposite each other, the right hand member of ath each pair being slightly behind the left hand member. Spicula con- phe spicuous, rather close together; at the widest part about one-eighth as wide as the corresponding portion of the body, ending in minute "but- angla tons." The lateral pores on the tail are the final members of the series of lateral organs.

Habitat: Intestine of field cricket, Gryllus neglectus Scudd. Fig. 54a and $b$.

55. Blattophila sphaerolaima $n . s p$. 1.- $4.3-3.3$. marked with plain transverse striae, 3 microns apart on the head and 24 microns apart on the body, where they are much less plainly to be seen, though they cause a slight crenation of contour. No amphids or setae of any sort. The head is somewhat hemispherical, bearing very small lips almost in the form of an annular elevation about the mouth. The pharynx and its apophyses are contained in a small spherical pharyngeal bulb,-hence the specific name. When closed, the pharynx is a prismoid cavity one-fourth as deep as the head is wide and about half as wide as deep, and backward from its base spring apophyses, a dorsal one dominating. The mouth can probably be turned partially inside out. The anterior three-fourths of the oesophagus is clavate, averaging one-third as wide as the corresponding part of the neck; this anterior part is connected by a narrow and very short tube with an oblate cardiac bulb one-half as wide as the base of the neck. The intestine, which appears to be only one-third as wide as the body, is set off by a broad and very deep constriction. The rectum is one and one-half times as long as the anal body-diameter. The renette plexus is quadruple. The nerve-ring encircles the oesophagus obliquely. The acute, conoid tail tapers rapidly near the anus, where there is a somewhat indistinct constriction. The anterior lip of the anus projects. The vulva is not very prominent. The numerous elongated ellipsoidal eggs measure 40-48 $\times 88-104$ microns, and may segment before deposition.

$\frac{4}{1 .} \quad 3 . \quad \frac{7.7}{4.5}-\frac{15 .}{5.4}-3.1 \quad 2.52 \mathrm{~m}$

bre-anal, being large submedian ones adjoining the anus and having a truncate-conoid form with a mucro; there are also two post-anal papillae similar to the foregoing but smaller, and removed from the anus a distance equal to one-third of the body diameter. The spicula appear to be represented by a mere rudimentary point, -no longer than the larger papillae,-conical in form and projecting with the anus when the nema is killed with osmic acid. The single testis is reflexed at a point somewhat farther behind the cardia than the latter is behind the head.

Habitat: Intestine of Panesthia brevicollis Sauss., the common spiny-legged wild cockroach; Moss Vale, New South Wales, Australia. 1889. Osmic to water?

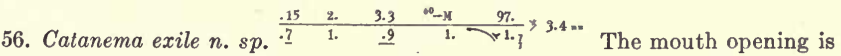
closed by three or six very minute, slightly refractive structures. About opposite the bases of the cephalic setae there are exceedingly minute onchia (?), possibly six,-difficult to see in profile; impossible to count because of their 
small size and their overlapping. Amphids forward-pointing, minute, difficult to see. The oesophagus becomes slightly narrower where it passes through the nerve-ring; posterior bulb napiform, three-fourths as wide as the base of the neck. There is no distinct cardia. The thick-walled intestine becomes at once about two-thirds as wide as the body, and its cross-section is made up of about

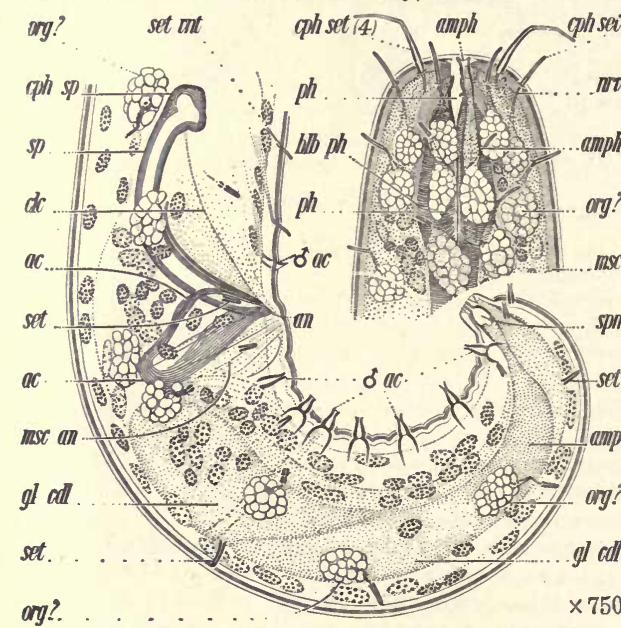
packed with granules of uniform size. The lateral fields are about one-third as wide as the body, and contain a series of cells of large org? size. The nerve-ring surrounds the oesophagus obliquely. There is a single, small, elonspn gated, very inconspicuous ventral suppleset mentary organ of the ordinary kind opposite the posterior part of the spicula, and in , front of it a number of minute ventral setae. Seven pairs of subventral tubular supplements occur on the tail. The ejaculatory duct is about two-fifths as wide as the body. The caudal glands are evidently located in front of the tail, but their exact position has not been made out.

Habitat: Marine sand about the bases of algae, in surf, Island off Port Royal, Jamaica. This genus resembles Laxonema in many respects, and also resembles Laxus but differs in the form of the lateral organs and in other ways. Sublimate to balsam. Fig. 56.

57. Zalonema nudum n. sp. Cuticle naked. The face view of the striae gives the impression of a series of narrow, contiguous hoops. The cuticle becomes thicker on the neck, and much thicker on the head. Lips, thick, small, plain. On the male there are very distinct and prominent wings, beginning about three times as far in front of the anus as the terminus is behind it. Each wing extends backward and ends opposite the proximal ends of the spicula. The width of this wing is about equal to the corresponding thickness of the dorsal or ventral cuticle as seen in optical section. None of the irregularities in the wall of the pharynx are suggestive of the ordinary pharyngeal organs, and yet they are very distinct features. Two of them are rather prominent, one on the dorsal side, opposite the anterior portion of the cephalic thickening, and the other on the ventral side opposite the middle or posterior portion of the cephalic thickening. The oesophagus continues with the same diameter until it expands to form the very broadly pyriform or napiform cardiac bulb, which is three-fourths as wide as the base of the neck. The lining of the oesophagus occupies nearly one-third of the optical section. The oesophageal lumen continues through the bulb. 
The intestine joins the middle of the posterior surface of the cardiac bulb, and at this point the collum is nearly one-third as wide as the base of the neck. The rather thin-walled intestine becomes at once about two-thirds as wide as the body. From the nearly continuous anus, the tail is arcuate and conoid. There

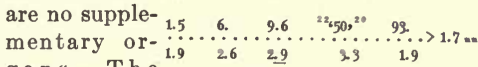
gans. The $\begin{array}{lllll}1.9 & 2.6 & 29 & 3.3 & 1.9\end{array}$

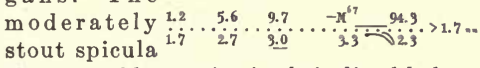

taper to a blunt point in their distal halves. At their widest part they are about one-fifth to one-sixth as wide as the corresponding part of the body; they are accompanied by $\times 750$

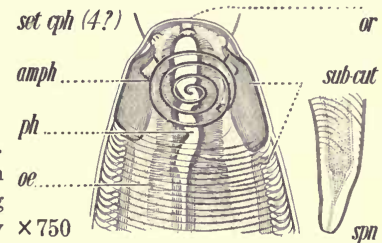
accessory pieces half as long. The distal ends of the accessory pieces are applied closely to the spicula, but the proximal ends curve away slightly and then recurve. The ejaculatory duct is about one-third as wide as the body. Apparently there is but a single testis.

Habitat. Marine mud and sand, Noumea, New Caledonia. Sublimate to balsam. Fig. 57.

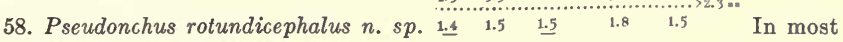
portions of the body longitudinal striations have been observed, apparently connected with the cuticle rather than with the musculature. Neck cylindroid. Lips two, each lateral. See Fig. 58. The mouth is very difficult to see in the lateral view. In the dorso-ventral view it is easily seen, and is found to be a dorso-ventral opening running across the head. Each lip has about six very minute, refractive, double, cutinous markings, each presenting two blunt-edged "teeth" directed toward the base of the pharynx. It appears that these represent foldings in the closed lips. In any case they represent, when viewed laterally, the roof of the pharynx, located opposite the bases of the anterior cephalic setae. The elements in the pharynx opposite the anterior borders of the amphids, which, at first sight, appear to be onchia, prove on careful examination to be ridges, (see pseudon, in the illustrations). The pharynx is unusual in structure and rather difficult to understand. The amphids of the female occur in about the same position as those of the male, but instead of appearing spiral appear

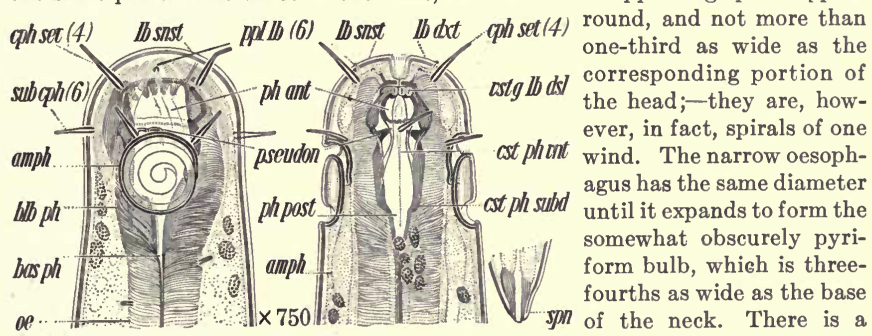

flattish cardia, about one-third as wide as the base of the neck. The intestine is set off by a constriction about one-half as wide as the body, and in cross-section presents about half a dozen cells. There appear to be faint indications of the presence of wings. Renette unknown. Lateral fields are about one-fourth as wide as the body. Nerve-ring oblique. The tail is convex-conoid to the termi- 
nus, where the conoid spinneret has a diameter about one-fifth as great as that of the base of the tail. From the slightly elevated vulva the vagina leads inward half way across the body, where it joins the two, symmetrically placed uteri. The ovaries reach about half way back to the vulva, at least in specimens in which the uteri are empty. There are eight to ten ova in each ovary, arranged single file.

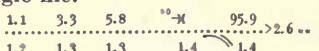

$\begin{array}{lllll}1.2 & 1.3 & 1.3 & 1.4 & 1.4\end{array}$ The spinneret of the male is about one-third as wide as the base of the tail. No supplements or special setae or papillae have been observed either in front of the anus or behind it. The spicula are rather uniform and taper to an acute point in their distal thirds; in profile their proximal ends are seen to lie near the ventral surface of the body. The ejaculatory duct is about half as wide as the body.

Habitat: Mud, tide pool, Portsmouth, N. H., U. S. A. Sublimate to balsam. Fig. 58, p. 273.

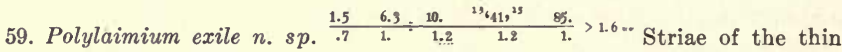
cuticle minute. It seems very likely that four cephalic setae have been lost from the specimen from which the description and illustration are drawn. Lips three, and double, (or possibly six), with six minute labial papillae. Cuticle naked. Neck convex-conoid, especially toward the head. The oesophagus at the nerve-ring is one-half, just in front of the cardiac swelling one-half, and finally two-thirds, as wide as the corresponding portion of the neck. The elon-

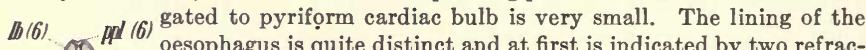
(10) oesophagus is quite distinct and at first is indicated by two refracph f ph tive lines, occupying a space one-sixth as wide as that of the amph (spl oesophagus. There appear to be no glands in its fine colorless 0 ( musculature. There is an indistinct cardia. The thick-walled 10 intestine is set off by a collum one-third as wide as the base of the neck; it has a faint lumen and becomes at once two-thirds as $\times 750$ th wide as the body. In cross-section it is made up of two to three cells. Anus more or less continuous; rectum somewhat cutinized, equal in length to the anal body-diameter. The granules contained in the intestinal cells are numerous, uniform and colorless. The tail is conoid from the anus, and then cylindroid in the posterior half, where it is two-thirds as wide as at the base. It is probable that the caudal glands are small and located near the anus. There are no caudal setae. Renette present; excretory pore probably located near the nerve-ring. Full-grown ova are twice as long as the body is wide, and nearly one-half as wide as long. The reflexed parts of the ovaries reach about three to four body-widths back toward the vulva.

Habitat: Sand below low-tide mark, Belmar, New Jersey, U. S. A. Sublimate to balsam. Apparently the species is syngonic. Up to the present time extremely few free-living marine nemas have been observed to be syngonic. A similar species with four setae occurs at Woods Hole, Mass, U. S. A. Fig. 59.

60. Myolaimus heterurus $n$. sp. Cuticle thin, naked; striae fine. Lips thin, low, flat, confluent; six, or three and two-parted. Amphids faint, in the form of transverse slits. That portion of the pharynx in front of the amphids is about half as wide as the lip region; this chamber is continued by a narrower tubular portion, nearly one-third as long as the neck and surrounded by a peculiar radial musculature, differing from that of the oesophagus proper. Nearly as far behind the amphids as these latter are behind the anterior extremity, there is a prominent infolding in the wall of the pharynx. The amphids are difficult 
to detect with the nema in profile,-more easily when viewed dorso-ventrally. Behind the anterior chamber of the pharynx, which in many respects resembles that of Diplogaster, there is a muscular portion closely resembling the oesophagus in size and structure, but which seems undoubtedly pharyngeal. This is indicated in two ways; first, the lining is different from that of the portion of the oesophagus immediately following it, and second, it behaves differently with staining material, indicating that its muscular wall also is considerably different in nature from that of the oesophagus. The dimensions given in the formulae relate to the anterior or labial chamber. At the base of the anterior pharyngeal chamber there appear to be minute cutinous pro- 1.2 .14 .4
cesses which perhaps may

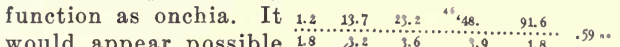

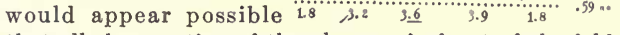
that all that portion of the pharynx in front of the folds in its lining are capable of being opened out or everted. From the pharynx the oesophagus is cylindrical to near the nerve-ring, where it diminishes rather suddenly in diameter near the very faint vestiges (?) of a median structure. The vestiges consist in internal modifications of the oesophagus only. From the nerve-ring onward the oesophageal tube is about two-fifths as wide as the corre-

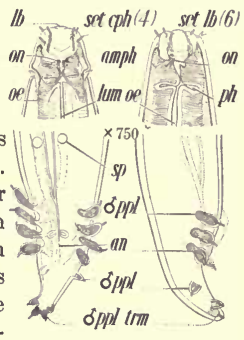
sponding portion of the neck. It finally expands to form the elongated-ellipsoidal cardiac bulb, which bears a more or less distinct cardia, and is separated from the intestine by a shallow constriction. The cardiac valvular apparatus appears to be comparatively simple. The intestine becomes at once four-fifths as wide as the body. The intestinal walls are rather thick, and the cells are packed with minute granules. The rectum is about twice as long as the anal body diameter. The lateral fields appear to be half as wide as the body. The tail of the female is conical to the acute terminus and presents the peculiarity of diminishing in diameter very suddenly immediately behind the anus, the diminution taking place on the ventral side. The anal region is, therefore, conspicuous on this account, as well as on account of the fact that it is slightly depressed, and again on account of the refractive nature of the lining of the comparatively long and large rectum. The salient lips of the vulva are conspicuous, a property that is increased by the refractive nature of the lining of the vagina, which extends inward two-fifths the distance across the body. Tail of the male very different from that of the female. There is a bursal membrane connected with the digitate organs shown in Fig. 60. The specimens seen were molting, so that the exact nature of the membrane, which is believed to connect these organs one with another, has not been deciphered. It seems also certain, however, that the anterior four are joined together by means of a ventrally submedian membrane somewhat as in the case of the bursa in Rhabditis. The spicula are very weak and poorly developed in the specimens thus far seen. They appear to be as long as the tail, and are probably cephalated at their proximal ends by constriction; but they are very difficult to discern, and it is possible that the appearances are deceptive and that they are altogether absent. The testis extends forward to near the posterior end of the neck and appears to be reflexed for a short distance, but this is somewhat uncertain.

Habitat: Loamy soil, El Paso, Texas, U. S. A. Flemming to glycerine jelly. Fig. 60 . 


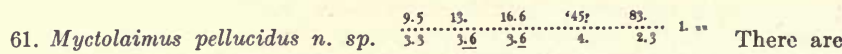
traces of a very fine transverse striation. No amphids or setae of any sort to be seen. Head continuous. Six relatively large, obtusely conical lips, each supplied with an obscure papilla, surround the mouth. The cuticle at the base of the lips, and at the beginning of the pharyngeal tube, is somewhat thickened, and this gives rise to obscure rings at the base of the lip-region. The unusually long pharynx is divided into two very distinct parts of about equal length, together occupying nearly two-thirds the length of the neck. The anterior part is a simple, narrow, straight strongly cutinized tube, having a lumen one-third as wide as the lip-region. The succeeding second part of the pharynx is a cylindroid bulb, rounded at both ends, and two-thirds as wide as the corresponding part of the neck. Its lumen is triangular and of about the same width as that of the anterior part of the pharynx, from which it is separated by a distinct break in the continuity of the cutinous lining. From this elongated bulb a tube one-sixth as wide as the corresponding part of the neck passes backward and gradually expands into a valveless bulb half as wide as the base of the neck, thus completing the oesophagus. The intestine, which at first is colorless and transparent and only one-fourth as wide as the body, gradually expands and becomes half as wide as the body and distinctly granular. The refractive lining of the intestine is a prominent feature. The cardia is unusually long and narrow, and opens into a small cardiac cavity. The cells composing the intestine are relatively large. The length of the well-cutinized rectum is about one and one-half times that of the anal body diameter. Renettte unknown. The width of the lateral fields is one-third that of the body. The nerve-ring is small and narrow. The acute tail is almost exactly conical. The vulva is very slightly elevated. The ovaries extend back as far as the vulva, and are often there again reflexed. Each uterus contains one to two eggs, measuring $32 \times 56$ microns. Segmentation takes place in the uterus, and proceeds to at least the gastrula stage, probably beyond it.

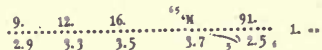

convex-conoid in the anterior half tail of the male instead of being conical is the middle of the tail is one-fourth as wide as the base; thence onward the tail is cylindroid to the acute terminus. While the anterior part of the tail is ventrally arcuate, the remainder is dorsally arcuate. No ventral supplements or bursa. Nine pairs of papillae, mostly finger-shaped, are arranged as follows: $1 ; 1(1) 1,1$; 1, 3. The linear, tapering, rather blunt spicula somewhat exceed the anal body diameter in length. The spicula are supported by sigmoid accessory pieces half as long as they themselves. The reflexed part of the testis is nearly equal to the neck in length; it can be seen to contain small glassy, globular spermatocytes, which reach their full growth near the middle of the body, becoming as long as the body is wide and two-thirds as wide as long. At this stage they are granular, with a large central nucleus containing a prominent nucleolus. By division these large cells give rise to the granular spermatozoa, an elongated parcel of a dozen or more of which is usually to be seen in each uterus of the female.

Habitat: Sheep-dung, Moss Vale, New South Wales, September 2, 1894. It will be seen that this genus is similar to Cephalobus. It is equally clear that it is generically distinct. It is unfortunate that no specimens of this species were preserved, and hence no drawings can be presented. This is all the more to be regretted because the genus is evidently closely related not only to Cephalobus but to one or two others of similar character. 


\section{Order Apodontia}

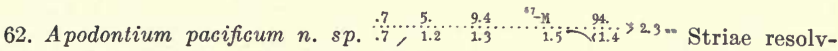
able into dot-like elements. Neck cylindroid posteriorly, convex-conoid anteriorly. The cylindroid oesophagus ends in an inconspicuous, flattish-conoid cardia one-third as wide as the base of the neck. The thin-walled intestine is set off by a collum two-thirds as wide as the base of the neck, has a somewhat distinct lumen, becomes at once two-thirds as wide as the body, and in crossset(10)? mnd(6) section presents three to four cells containing granules of setsubph body diameter. Tail conoid from the anus. The conspicu-
ous, more or less elongated caudal glands are packed in a
close tandem behind the anus in the anterior half of the
setcll cell, two-thirds as long as the body is wide, and two-thirds as wide as long, lies a short distance behind the base of the neck, and empties through an ellipsoidal, thin-walled ampulla. The nerve-ring is accompanied by obscure nerve cells. The yellowish spicula are slender and subacute. Their proximal ends appear to lie somewhat dorsad from the body-axis. There are two separate, strong, rather stout, simple accessory pieces. They are one-half to two-thirds as long as the anal body diameter, and their proximal ends lie near the dorsal body wall. There are no supplementary organs, special setae, or papillae on the tail of the male. The ejaculatory duct is one-third, the vas deferens one-third, and the testis one-half, as wide as the body. The testis tapers and is at last narrow.

Habitat: Marine; Mollendo and Salaverry, coast of Peru, S. A. Sublimate to balsam. Fig. 62 .

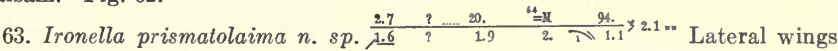
very faint. Body naked. Lips six, very tenuous, revolute, forming a short tube one-fourth as wide as the lip-region. There are six obscure, flat, conical, labial papillae, not shown in the illustration. Labial setae minutely "furcated" at the extremity. Each of the three odontia is duplex, the two parts of the submedian ones being of unequal size. The odontia are contained in a somewhat napiform cavity. Odontia acute, with an outward stroke; when at rest somewhat outwardpointing, and blocking the entrance to the pharynx. Posterior part of the napiform ph.. cavity apparently lined with exceedingly minute denticles. Neck cylindroid. $0 e$. Amphids faint, apparently consisting of an almost imperceptible transverse groove partially encircling the head. The cylin-

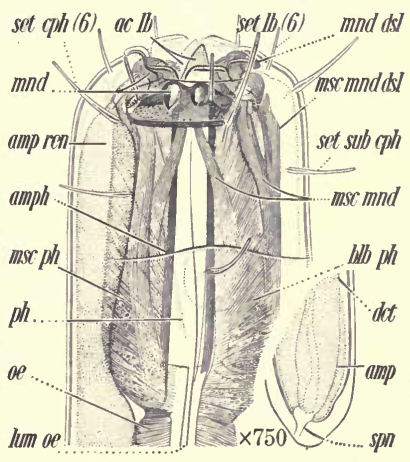
droid oesophagus is at the nerve-ring four-sevenths, and finally three-fifths, as wide as the corresponding portion of the neck; its lining is prominent, occupying onethird the diameter of the organ. In the coarse, colorless musculature there are 
three glands which empty at the mouth. There is a cylindroid cardia twosevenths as wide as the base of the neck. The thick-walled intestine is set off by a collum one-third as wide as the base of the neck, and becomes at once one-half to three-fifths as wide as the body. Its cross-section is composed of four to six cells. It has a faint zig-zag lumen. Very fine, colorless, more or less polyhedral granules of variable size are found scattered in the intestinal cells. The largest of these are one-twenty-fifth as wide as the body. They give rise to no more than a faint tessellation. The more or less arcuate tail of the male tapers from in front of the anus, and is first conoid then cylindroid and two-fifths as wide as at the anus. The caudal glands are probably preanal. There are no caudal setae. The lateral fields are one-fourth as wide as the body, and faintly granular. The very frail, straight, slender, subacute spicula become arcuate near the tips, and are faintly cephalated by expansion. Their proximal extremities appear to lie dorsad from the body-axis. The frail, simple, arcuate, slender accessory piece lies parallel to, and is two-fifths as long as, the spicula. There is a single supplementary organ opposite the posterior part of the spicula. The internal part is refractive and more or less irregularly cylindrical, and bent back parallel to the ventral surface. It is one-half as long as the body is wide, and one-fourth to one-fifth as wide as long, and is probably protrusile through the small, short, cylindrical element at the ventral surface, where there is also a single, strongly curved seta, of considerable size. The ejaculatory duct is one-third as wide as the body. The testes are characterized by the presence of numerous, elongated elements, which constitute one phase in the development of the sperm. These elements are apparently two-thirds as long as the body is wide and one-fifth to one-eighth as wide as long, and are rounded at the end. From the living nema.

Habitat: Sand, Nobsca Beach, Woods Hole, below low tide. Fig. 63.

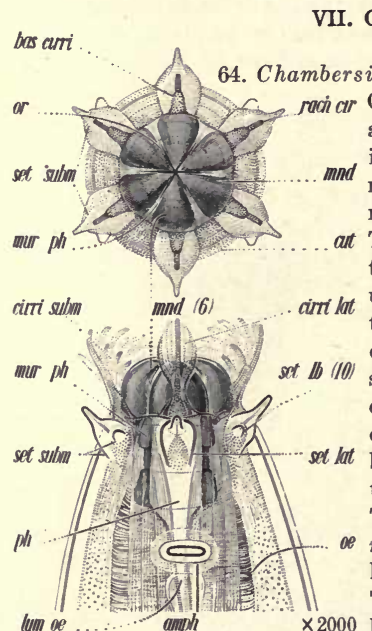

Frg. 64a. The cirri are omitted from the upper illustration in order to show more clearly the mandibles and the setae.

\section{Order Synodontia} forward. The eggs are elongated, apparently more or less long and slender. The gently tapering ovary contains 50-100 ova; reflexed to near anus. 


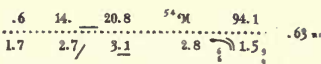

The males are less common than the females.

Habitat: Bark of a great variety of trees set ph lat set ph sulm md .... cir lb sulbm in many parts of the eastern United States; on "Spanish Moss," Miami, Florida. Flem- $m x \in$ ming's solution to glycerine jelly. This genus comprises a considerable number of species, all with a hooked caudal extremity. The species vary considerably in the labial accessories. The present species has the opl.... strongest mandibles so far known in the genus. These nemas are highly resistant ${ }^{0}$ to drought and cold, and may be kept on dried bark for several years and then be lum of fully revived by soaking the bark in water. They may be repeatedly frozen in and an thawed out without injury. Recently revived specimens are mostly immature $d$ nro and seem to present an empty intestinal canal, suggesting that on the approach of $\mathrm{gl} \mathrm{an}$ untoward conditions, they evacuate the canal. Not infrequently, the lip-region of cop mas revived specimens appears to exhibit a

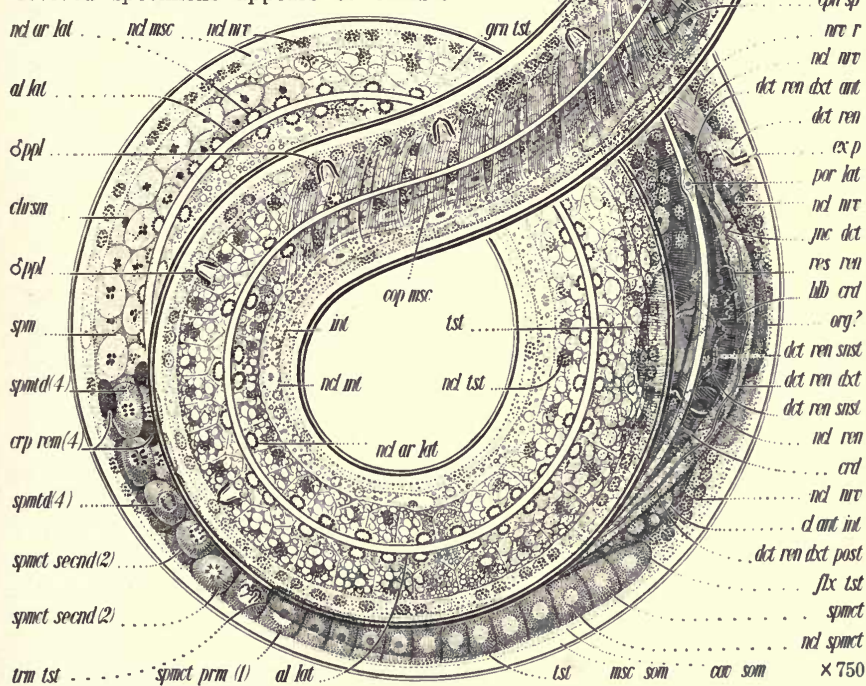

pluglike accession, seen also in the mouth opening of similarly revived Cephalobi. The opening is protected during "suspended animation" by means of this dried-up evacuation from the oesophagus. Fig. 64a, p. 278; Fig. $64 b$.

The name of this remarkable genus, the abundant species of which are widely distributed on trees, commemorates the classic work in this field of Mr. W. E. Chambers, through whose untimely death science has lost one of its greatest graphic interpreters. 
65. Synodontium fecundum n. sp. About forty setae occur on the neck; those on the anterior part are about one-half, while those on the posterior part are onefourth, as long as the corresponding portion of the neck is wide. There are six subdistinct lips, each with a somewhat forward-pointing, innervated, conoid papilla. The pharynx is fairly well cutinized, and bears probably twelve closely approximated odontia, forming a distinct circlet opposite the bases of the cephalic setae; only their points are easily visible. The conoid neck becomes more decidedly conoid near the head. At first sight the oesophagus appears as if composed mainly of glands, which extend backward to the cardia. These glands are, however, outside the oesophagus and empty near the bases of the odontia by means of three or four ducts. At the nerve-ring the oesophagus is three-sevenths, and finally one-half, as wide as the corresponding part of the neck, and ends posteriorly in a faint, elongated, pyriform, valveless bulb. The lining of the oesophagus is narrow; its musculature fine. The thin-walled intestine has a faint lumen and is set off by a collum two-fifths as wide as the base of the neck and becomes at once one-half as wide as the body. In cross-section it presents four to five

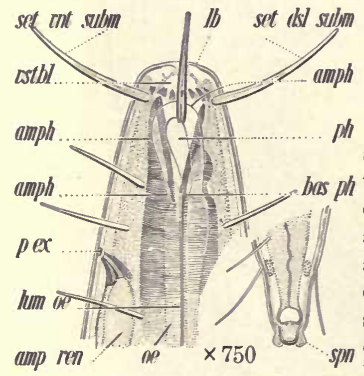

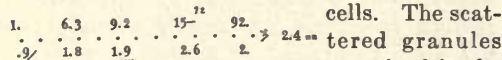
contained in the 1. 6.9 10. . ${ }^{-k} .93 .323$ intestinal cells $1.1_{1.6} \quad 2.3 \times 1.7$ are of variable size, the largest being one-fifteenth as wide as the body. The nuclei of the intestinal cells are one-seventh to one-eighth as wide as the body at the base of the neck. Tail conoid from the anus, but tapering faster near the anus. The end of the spinneret is in the form of a hemispherical button, the three sectors of which are sm eversible, so that the organ may appear threelobed. The ducts of the caudal glands are separate, practically to the spinneret pore. There are about fifteen ventrally submedian, and about ten dorsally submedian, slender, flexible, tapering setae on each side of the tail of the male. There is a much smaller number of setae on the tail of the female. The lateral fields are one-third as wide as the body. The granular, ellipsoidal renette cell, two-thirds as long as the body is wide and twothirds as wide as long, is located at a distance behind the neck equal to the body diameter. The obscure, rather broad nerve-ring is accompanied by distinct and numerous nerve cells of large size, extending back to the base of the neck. The large, elevated, conspicuous vulva leads to a conoid, rather muscular, cutinized vagina accompanied by small, ellpisoidal glands. There is a small anterior branch to the female sexual organs, about as long as the body is wide. The uterus extends back to near the middle of the body. The eggs are one and one-half to two times as long as the body is wide and one-fourth to one-third as wide as long. Numerous, granular, spherical sperm cells, one-fifteenth as wide as the body, are abundant near the middle of the body, disarranging the chain of eggs in such a way as to suggest the presence of a spermatheca. The broad ovary tapers gradually, and contains twenty to thirty ova arranged single file. The egg, just before deposition, lies opposite the vulva, one-half of it being in the small anterior branch of the uterus. The spicula are strong, somewhat slender, sub-uniform, and rather blunt. Their proximal ends appear to lie ventrad from the body-axis. The two strong, well-separated accessory pieces are one-fourth 
as long as the spicula. Their uniform, blunt apophyses extend backward at an angle of ninety degrees with the spicula, and are one-half as long as the anal body diameter. Fifty to seventy equidistant supplementary organs, in the form of a series of slight undulations, extend forward in front of the anus a distance three to four times as great as the length of the tail. The undulations are separated from each other by a space about equal to one-fourth the body diameter, though they become gradually a little farther apart anteriorly. Their elevation is slight, and every other undulation is opposite a granular, saccate gland, about one-third as wide as the body. These glands form a contiguous series and seem to empty ventrally. There is no bursa. The ejaculatory duct is onethird, and the testes are one-half, as wide as the body, these latter gradually tapering so that they are narrow at the extremities.

Habitat: Sand, below Bathing Beach, Woods Hole, Mass., U. S. A. Also beach-sand, Squibnocket, Martha's Vineyard, Mass., U. S. A. Flemming to glycerine jelly. Fig. 65, p. 280.

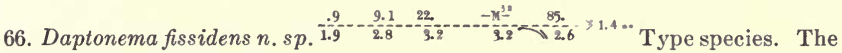
contour of the moderately thick cuticle is crenate, the annules being separated by refractive lines. Toward the head end the set cph (12).1 annules diminish in width until they elude detection. Neck conoid; head rounded. Here and there on the neck occur long, slender hairs, as long as those on the head, but colorless and very much more slender. Each of the six lips is strengthened by three, dark-brown, or blackish, strongly arcuate, incurved, slender, acute, cutinized ribs or teeth. Lips capable of being turned over into the pharynx so as to form a flattish, centrally indented dome. From the head backward the oesophagus narrows a little, becoming two-thirds as wide as the corresponding portion of the neck. There is no very distinct cardia. The rather thick-walled intestine becomes at once about half as wide as the body, and in cross-section presents about four cells. The cells contain scattered brownish granules, of variable size, the largest of which are nearly as wide as one of the annules of the cuticle. The lumen of the intestine is quite narrow, and its lining somewhat refractive. Renette unknown. The tail of the male is conoid from the anus to the somewhat blunt terminus, which has a plain, rather blunt spinneret, armed with two setae considerably longer than the diameter of the terminus. The caudal glands appear to be located in front of the anus. The brownish spicula have been seen only in dorso-ventral view. The accessory pieces are curved distally msc an

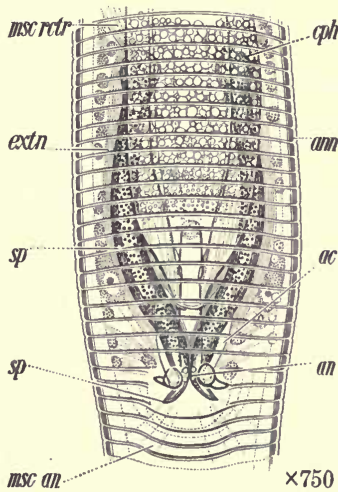
in the same way as the spicula, and end in minute points. The ejaculatory duct is about one-third, the vas deferens about one-half, as wide as the body.

Habitat: Larat, East Indies; marine. In the general form of the neck and pharynx these nemas closely resemble Monhystera, but the food habits are 
probably different, and the structure of the male differs materially from the structure of the male of Monhystera,-dark-colored, punctate, out-bending spicula; and two testes. A very similar marine

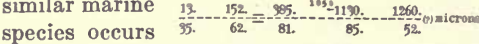
in the harbor at Portsmouth, N. H., and at Woods Hole, Mass., U. S. A., namely Daptonema fimbriatum, $n$. $s p$. of which the formulae and the figure of the head end are given on this page. The head of Daptonema fissidens differs but slightly from that of $D$. fimbriatum.

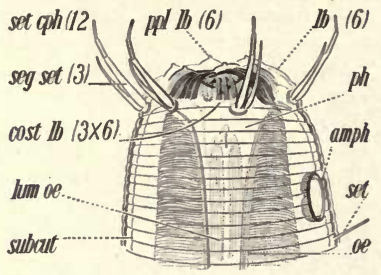
Daptonema is manifestly an offshoot of Monhystera. Probably the eighteen refractive labial elements are ribs attached to a thin membrane. They sometimes appear, however, as if free at the extremities, and if so, would function as combs in securing the food, which is probably combed or scraped from the surface of algae and other submarine objects. Fig. 66a, p. 281 (fissidens); Fig. $66 b$ (fimbriatum).

67. Crystallonema fuscacephalum $n . s p$.

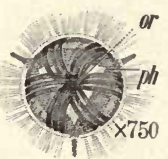

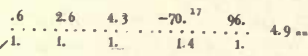
longer, setae are one-tenth as long as the neck is wide. Opposite the pharynx the head contains dark brownish gray pigment. The consistency of the labial palps (?) remains undetermined, that is to say, it is uncertain whether they are apodontia with an inward stroke and capable of executing a firm grip, or whether they are more or less flabby palps. Neck somewhat cylindroid, expanding a little toward the head. Amphid with a pore near the posterior margin, from which there set $(7 /(4) \ldots . . . . \quad$ plp $1 / 0$ set $10(6)$ leads inward and backward a narrow duct with granular contents. There is a faint,
elongated, more or less pyriform cardiac
swelling. Near the nerve-ring the oesoph-
agus is one-half, just in front of the cardiac
swelling three-fifths, and finally two-thirds, ules in the intestinal cells are scattered in groups and-are of variable size, the largest granules being about as wide as one of the annules on the neck. The nema is strongly characterized by the presence of more or less angular, doubly refractive crystalline masses, having about the same diameter in every direction, and being about one-sixth as wide as the body. The crystalline bodies are onehalf to one body-width apart and seem to lie in the longitudinal fields. The conoid tail tapers from the anus, but at last for a short distance is more or less 
cylindroid and one-fifth as wide as at the anus. There are no caudal setae. The ellipsoidal to prolate renette cell lies close behind the neck, and is one-half as long as the body is wide and five-sixths as wide as long. The large, continuous vulva is inconspicuous. The medium-sized vagina extends two-fifths the way across the body. There is a posterior rudimentary branch to the sexual organs, three times as long as the corresponding body diameter. The uterus is four times as long as the body is wide, and one-sixth to one-fifth as wide as long. The elongated eggs are three times as long as the body is wide. The relatively very small ovary contains twelve ova, which diminish rapidly in size toward the blind end, and are arranged single file. The finely-granular, ripe ova are three times as long as the body is wide, and three-fourths as wide as long.

Habitat: Sand, below Bathing Beach, Woods Hole, Mass., U. S. A. Flemming to water. The males of this species, C. fuscacephalum, are unknown, but the males of two other species are known to the writer. As these two are structurally closely related to $C$. fuscacephalum, it may be assumed that the spicula of C. fuscacephalum are

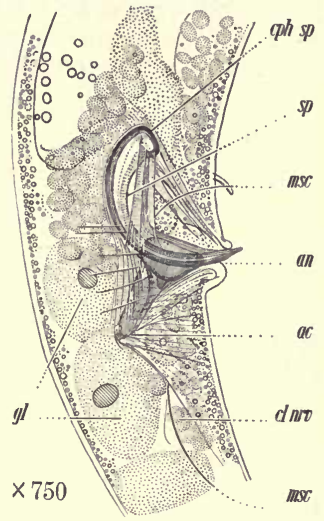

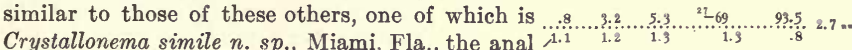
region of the male of which is shown in the adjacent illustration, together with the dimensions of both sexes. Besides being smaller, $C$. simile differs in lacking pigment at the head end; in having relatively slightly longer and more nearly equal cephalic setae; in having amphids relatively larger,- three-sevenths as wide as the head; in having the posterior oesophageal swelling almost indistinguishable; in having the preliminary segment of the intestine as long as the body is wide, and the intestine itself relatively a little wider,-three-fourths as wide as the body. Fig. $67 a$, p. 282, C. fuscacephalum; Fig. $67 b, C$. simile, n. sp., type species.

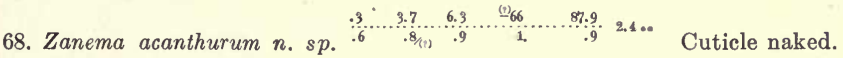
Neck cylindroid. It is possible that very small or broken
members of the submedian group of cephalic setae have
escaped notice, and that there are really three submedian about as long as the anal body diameter. The tail tapers from a little in front of the anus and soon is reduced to a diameter about half as great as at the base. Thence onward it tapers gently, so that near the terminus it has a diameter about 
one-fourth as great as at the anus. Posterior two-fifths of the tail armed with short, backward-pointing, arcuate, acute thorn-like setae to the number of ten on each ventrally submedian line. No caudal glands. The lateral fields appear to be fully one-third as wide as the body. The excretory pore appears to be connected with a large, ellipsoidal ampulla, nearly half as wide as the neck. The position of the renette cell has not been determined. From the depressed vulva the vagina leads slightly forward. Beyond this fact little is known concerning the internal organs. As no traces of ova have been seen behind the vulva it is assumed that the organs are asymmetrical.

Habitat: Mud, tide pool, low tide, Portsmouth, N. H., U. S. A. The single specimen examined contained numerous yellowish, spherical, crystalline bodies, arranged in an obscurely double series, beginning at the base of the neck and ending at the anus. Whether these are natural or have been induced by the method of preservation remains to be determined. The peculiarities of the species justifying the establishment of a new genus are as follows: (1) the formation of the pharynx and of the lips with their distinct forward-pointing appendages; (2) the unusual size and form of the amphids; (3) the absence of caudal glands; and (4) the peculiar thorn-like setae on the tail. These, combined with the very slender form, make it difficult to refer this specimen to any known genus. Fig. 68, p. 283.

69a. Monhystrium transitans $n$. $s p$. Type species. Cuticle naked, except for the setae on the head. Lips three, more or less confluent, thick and mobile. Pharynx double; the closed lips forming a narrow vestibule reaching one-third the way to the base of the pharynx. Anterior chamber of the pharynx pyramidal, the posterior more of less napiform. The lips come to a sharp edge, and are stiffened by several refractive "plates," whose direction is radial but indefinite, as if due to the folding of a cutinized membrane. Oesophagus cylindroid, ending posteriorly in a pyriform cardiac bulb five-sixths as wide as the base of the neck. Its lining finds expression in three longitudinal lines, occupying a space equal to two-sevenths the width of the whole organ. Its musculature is rather coarse. There is no cardia. The thick-walled intestine, which has a distinct, refractive lumen, is set off from the oesophagus by a constriction one-fourth as wide as the base of the neck, and becomes at once three-fourths as wide as the body. Its cross-section shows two cells. In contour, it is more or less crenate, at least in alcoholic specimens, because of the pronounced nature of the intestinal cells. These latter contain numerous granules of rather uniform size. The walls of the intestinal cells are refractive. The tail is at first conoid, then cylindroid in the posterior two-fifths. It tapers from in front of the anus to the almost imperceptibly swollen, more or less apiculate, unarmed, symmetrical terminus, which presents a profile similar to that of a swan's head. It bears a simple, unarmed, blunt spinneret. Only two caudal glands are to be seen; these are broadly saccate cells, forming a close tandem in the anterior third of the tail. The more or less finely granular longitudinal fields are one-third as wide as the body. The post-cervical renette cell empties through the excretory pore a little in front of the nerve-ring by means of a more or less spherical ampulla, and final slender duct one-half as long as the body is wide, leading therefrom to the excretory pore. The vulva is large and more or less elevated. The ellipsoidal eggs are three-fourths as long as the body is wide and are deposited after segmentation; it is probable that the species is viviparous, or ovoviviparous. The small posterior branch of the sexual organ is one-half as long as the body is wide. 
The tapering ovary is at first broad, and contains numerous ova arranged for the most part single file. At its blind end, it is one-eighth as wide as the base of the neck. At their widest part, that is, near the middle, the spicula are one-sixth as wide as the corresponding portion of the body. They taper to a rather blunt point, and present a faint, stiffening element. These colorless, rather frail spicula appear to have their proximal ends somewhat dorsad from the body-axis. There is a faint, frail, slender, arcuate accessory piece, lying parallel to the spicula, probably consisting merely of a differentiation of the lining of the cloaca. Obscure papillae occur on the middle part of the tail, near the place where it suddenly diminishes a little in diameter. These are probably as follows: one ventrally submedian pair opposite the middle of the spicula; three ventrally submedian pairs, one of them nearly anal, the other two at the beginning of the middle third, and occurring one in front of the other; one dorsally submedian pair. The large spermatocytes in the testes are three-fourths to twothirds as wide as the body. That portion of the testis, containing them is correspondingly wide, but the testis tapers so as finally to be only about one-fourth as wide as the body.

Habitat: Gill-chambers of the Land Crab, G. ruricola, Jamaica. Coll., Dr. C. B. Wilson, Westfield, Mass. Described from more or less shrunken alcoholic specimens, mounted in balsam and in glycerine jelly. May be looked upon as a transitional form between a freeliving and parasitic state. Hence, the specific name transitans. While much reduced and flabby, the cephalic setae still exist. Very
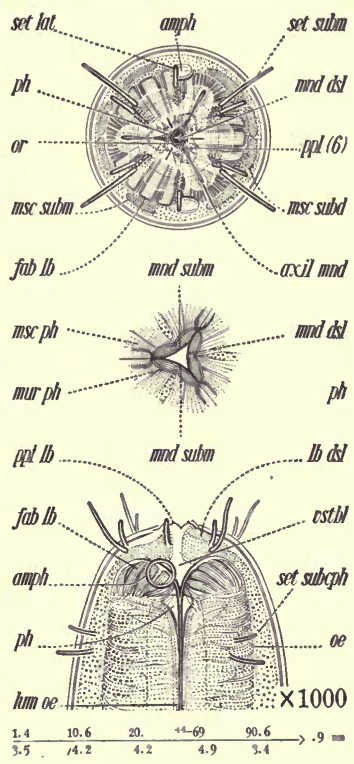
\begin{tabular}{rrrrr}
1.1 & 10.3 & 18.5 & \multicolumn{1}{c}{$66-M$} & 88.4 \\
\hline 2.8 & 13.5 & 3.6 & 4.2 & 3.1
\end{tabular} $.83=$ few parasitic nemas possess cephalic setae. Fig. $69 a$.

b. Monhystrium wilsoni (Baylis). Very narrow wings extend from the base of the neck to near the anus. Each wing is expressed as a double line. Eight to twelve longitudinal striations occur on each submedian field. The longitudinal striae do not anastomose, and seem to be in the outer cuticle. Lips confluent, elastic, closing to a small central pore. Pharynx of two chambers; the anterior, pyramidal, the posterior appearing roughly napiform in profile, but really three-sided as shown in the illustration. The distinct, refractive lining becomes rapidly thicker in the posterior half of the sccond chamber. About eighteen duplex radial refractive ribs of small size occur in the anterior chamber, somewhat as in Cyatholaimus; i.e., there are about six refractive radial markings on each of the three lips. These have a rather definite duplex outer end, or contour, but are not so definite as the "rods" of Cyatholaimus, being more like folds. Neck conoid; head convex-conoid. The cylindroid oesophagus near the nerve-ring is two-fifths, just in front of the pyriform cardiac swelling two-thirds, 
finally two-thirds, as wide as the corresponding part of the neck. There are no oesophageal bulbs, but there is a pyriform anterior segment of the intestine, set off on both sides by a distinct constriction, in some ways resembling a cardiac bulb. This pseudo-bulb is three-fourths as wide as the corresponding portion of the body, as measured in alcoholic specimens. The lining of the oesophagus is a prominent feature, and its optical expression consists in three refractive lines, occupying a space one-fourth as wide as the entire organ. The oesophageal musculature is coarse. At the cardiac constriction there are three elements, questionably glands, each one-third as wide as the corresponding portion of the body. There is no cardia. The thick-walled intestine has a very distinct refractive lumen and soon becomes five-sixths as wide as the body. The cells composing the intestine are about twice as long as he body is wide, and are so arranged that each cross-section presents practically only two of them. These cells are packed with exceedingly fine granules of more or less uniform size. The tail is first conoid, then cylindroid in the posterior half. It tapers from in front of the anus; the terminus has a contour more or less resembling that of a swan's head. The simple, unarmed, symmetrical, blunt spinneret is half as wide as the terminus. The three, broadly saccate caudal glands form a close tandem in the anterior fourth of the tail. Their ducts and ampullae are distinct. There are no caudal setae. The tail, like the body, is naked. The elongated renette cell lies two to four body-widths behind the neck, and empties through a wide duct separated from the ampulla by a constriction; the ampulla is one-fourth as wide as the corresponding portion of the neck, and empties through a pore located half-way back to the nerve-ring. The nerve-ring is accompanied by distinct nerve-cells arranged in groups. From the somewhat large, more or less conspicuous vulva, the large, rather muscular, cutinized vagina leads half-way across the body. The straight uterus is about fourteen times as long as the body is wide, and contains ellipsoidal eggs, each about as long as the body is wide, which are deposited after segmentation begins, -in fact, the species may be viviparous. Fully-formed embryos occur in the eggs near the vulva. Spermatozoa, half as wide as the body of the female, and with strong refractive nuclei, occur in the uterus. There is a rather narrow, ventral posterior rudimentary part to the female sexual organ, about one and one-half times as long as the corresponding portion of the body is wide. The ovary is nearly cylindroid, but tapers slightly, and contains numerous ova arranged single file. Toward its blind end, it suddenly narrows, and is reflexed, or thrown into an " $\mathrm{S}$ " form, in a space one to two times as long as the body is wide; here it is only one-sixth as wide as the body. The rather frail, somewhat simple, rather slender, subacute spicula are one and one-half times as long as the anal body diameter. At their widest part near the middle, they are about one-sixth as wide as the corresponding portion of the body. Their proximal ends appear to lie dorsad from the body-axis. The single, frail, very slender, more or less arcuate, accessory piece is somewhat removed from the spicula, at least from the refractive part. Its proximal extremity lies dorsad from the body-axis. There are no supplementary organs. About ten pairs of papillae oocur on the tail of the male. These are very inconspicuous and are arranged as follows: one ventral, single, and two submedian pairs just in.front of the anus; third, fourth, fifth and sixth pairs behind the anus, opposite the two anterior caudal glands; seventh and eighth, lateral, opposite the posterior caudal gland; ninth and tenth farther back, not so close together, averaging about twice as far from the anus, as the seventh and eighth. There is a rudimentary bursa; i.e., there are submedian longitudinal elevations of considerable magnitude 
beginning some distance in front of the anus and extending on to the tail to a point opposite the posterior caudal gland. Most of the papillae described occur on the ventral surface of these welt-like elevations. A cross-section taken near the anus clearly discloses these well-developed organs. The ejaculatory duct is one-fourth, the vas deferens one-fourth, the testis two-thirds, as wide as the corresponding portion of the body. Toward its blind end, however, the testis tapers so as to be only one-eighth as wide as the corresponding portion of the body. Spermatocytes, occupying a considerable portion of the length of the testis, are one-half to two-thirds as wide as the body, so that the testis bears considerable resemblance to an ovary. The nro set labllbsulm $\mathrm{ppl} / \mathrm{lb}$ set lat set sulfm deceptive appearance created by these large spermatocytes might easily lead to the conclusion that the males are hermaphrodites.

Habitat: Found with the preceding.

One is inclined to suggest that this Monhystrium also represents a transitional form $m$ from a free-living to a parasitic condition. A careful study of such forms is destined to throw much light on the relationship between the Laimia and Alaimia.

Although bearing considerable resemblance to Monhystera, this species and its close rela- $10 \mathrm{sthm}$ tive just described seem to make a new genus desirable for their reception. The distinguishing differences may be listed as follows: (1) In Monhystrium, the pharynx is a double chamber; moreover, the anterior chamber has a distinctly radiated structure, more easily seen if the specimen is viewed slightly obliquely instead of laterally, as shown in the illustration. These radial elements are stiffening factors enabling the lips to bite more efficiently. The margins of the lips are sharp-

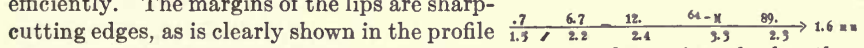
view in the illustration. Further study is necessary to determine whether these organs are odontia or onchia. (2) The pseudo-bulb, which is not common in Monhystera. (3) Monhystrium is ovoviviparous or viviparous. (4) There is a pronounced renette. (5) There are no cephalic setae or the setae are very weakly developed. (6) The species is presumably carnivorous; the more typical Monhysteras, perhaps all, are vegetarian.

Larvae removed fron the uterus of alcoholic specimens present differences from the adults as follows: (1) The amphids are farther back. (2) The pharynx is tubular, and not in two parts. (3) The head is more tapering. (4) The spinneret is not so well defined. (5) No wings were seen. (6) The pseudo-bulb is less conspicuous. Fig. 696 , on this page. Syn. Monhystera wilsoni Baylis.

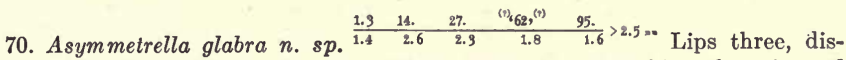
tinct, unequal, flat, thin. Pharynx with a peculiar dorsal onchium, bent inward at right angles at the apex. Oesophagus surrounding the posterior two-fifths of the pharynx in the form of three, somewhat finger-shaped processes. Wall of the pharynx fairly well cutinized, especially near the lips on the dorsal side, 
where it bends inward and forms an elbowed lip, giving to the front of the head an asymmetrical appearance; hence, the generic name. Neck cylindroid posteriorly, convex-conoid anteriorly. Amphids faint. At the base of the pharynx there are three spherical collections of black, loosely-placed granules (ocelli?), one collection dorsal and two submedian, the dorsal collection being the least pronounced. The cylindroid oesophagus at the nerve-ring is one-third, and finally is one-half, as wide as the corresponding portion of the neck; it has an indistinct lining, and a fine, colorless musculature. There is a more or less hemispherical cardia, three-fifths as wide as the base of the neck. The thick-walled intestine, which is set off by a constriction one-half as wide as the base of the neck, becomes at once three-fourths as wide as the body. It has a faint lumen, and its cross-section exhibits about four cells. In all parts of the intestine the cell walls are rather distinct and optically give rise to a network
effect. From the more or less continuous anus the prominent,
anmph cutinized rectum extends inward a distance three-fourths as great
as the anal body diameter. The rather numerous, more or less
yellowish granules contained in the cells of the intestine are of
variable size, the largest being about one-thirty-fifth as wide as
the body. They are so arranged as to give rise to a more or
less distinct tessellation. The lateral fields are one-fourth as wide as the body. The tail tapers from the anus, and is at first conoid, and then convex-conoid in the posterior eighth. It is arcuate to the unarmed, more or less symmetrical terminus. There is a conoid, symmetrical spinneret. In it a compound ampulla is apparent. Renette unknown. The female sexual organs are probably double and symmetrically placed.

Habitat: Marine tap water, laboratory, Woods Hole, Mass., U. S. A. Flemming to glycerine jelly. Fig. 70 .

\section{Order Synonchia}

71. Tripylium carcinicolum (Baylis). Cuticle naked. Cephalic setae papilloid. Wall of the pharynx fairly thick and refractive, and curved inward on the inner surface of each pair of lips so as to form three $120^{\circ}$ biting organs, which close together as shown in the illustration. Pharynx cylindroid. Neck cylindroid posteriorly, conoid anteriorly. Oesophagus cylindroid, its lining a trifle stronger behind the pharynx for a distance equal to the length of the pharynx. The anterior portion of the intestine is altered so as to produce the effect of a pyriform bulb three-fourths as wide as the base of the neck, set off on both sides by a constriction. At the nerve-ring, the oesophagus is two-thirds, and finally three-fifths, as wide as the corresponding part of the neck. The lining of the oesophagus finds optical expression in three refractive lines, occupying two-fifths of the width of the organ. There is no cardia. What appear to be glandular cells occur opposite the cardiac constriction,-two, one on each side, each ellipsoidal and half as wide as the neck, finely granular and with a prominent nucleus. The thick-walled intestine has a distinct, refractive, more or less zig-zag lumen, and becomes at once three-fourths as wide as the body; in crosssection it presents two cells. The cardiac collum is one-fourth as wide as the neck. From the more or less continuous anus, the cutinized rectum extends a distance equal to the length of the anal body diameter. Fine uniform granules pack the cells of the intestine; there is a faint tessellated effect due to the refractive nature of the cell walls. From in front of the anus, the straight tail is first 
conoid, then cylindroid in the posterior two-fifths, where it is one-eighth as wide as at the base. Terminus apiculate, bearing a simple, symmetrical spinneret. The three broadly-saccate caudal glands are packed into the anterior two-fifths of the tail, and empty by means of separate ducts. Three pairs of conoid, subacute, ventrally submedian papilloid setae occur on the tail of the female, one pair near the anus, one near the middle of the tail and one at the beginning of the posterior fourth. Midway on the tail there is a dorsally submedian pair of setae. The finely-granular lateral fields are half as wide as the body. There appears to be an elongated renette cell one body-diameter behind the neck; it is one-fifth as wide as the body. From the large, conspicuous, elevated vulva, the vagina, also large, extends inward half way across the body. Its wall is cutinized. There is no posterior vestigial portion to the sexual organ. The straight uterus contains twelve or more already liberated embryos. Ova are arranged single file. The simple, rather frail, slender, uniform, acute spicula are arcuate distally. They are three-fourths as long as the tail, and at their widest, about one-tenth as wide as the corresponding portion of the body. Their proximal ends seem to lie more or less dorsad from the body-axis. The simple, frail, very slender, arcuate accessory piece is more or less obscure in alcoholic specimens. It lies parallel to the spicula and its proximal end appears to be dorsad from the body-axis. There are three pairs of very flat, conoid, innervated, inconspicuous, nearly ventral papillae on the tail, one at the beginning of the fourth fifth, and two at equal distances between it and the anus. The ejaculatory duct is one-third, the testis two-thirds, as wide as $0 e \ldots+\cdots, 1000$ the body. Only the narrow blind end of the

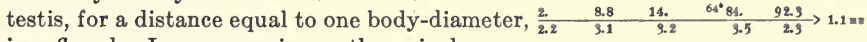
is reflexed. In one specimen, the spicula were more or less compound.

Habitat: Found in the gills of the Land Crabs,
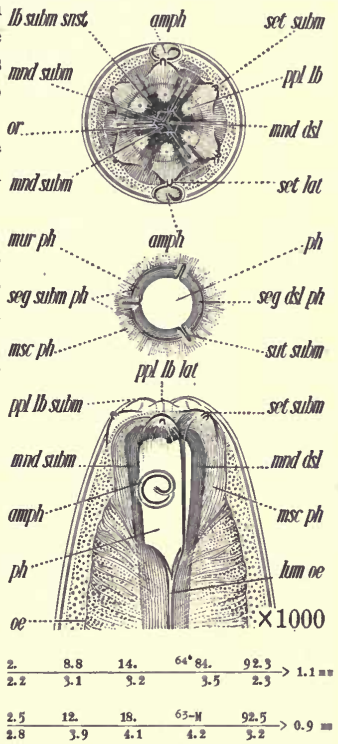
Gecarcinus ruricola and Cardisoma guanhumi, Jamaica. Collected by Dr. Chas. B. Wilson, Westfield, Mass. This genus differs from Monhystera as follows: (1) Male has caudal papillae; (2) Has a cardiac bulb or pseudo-bulb; (3) Is viviparous; (4) Is parasitic in crabs; (5) Has three biting edges to the segments of the pharynx. Fig. 71. Syn. Monhystera carcinicola Baylis.

72. Xyala striata $n$. sp. Cuticle with numerous wings, especially anteriorly, where there are sixteen, increasing to about thirty-two on the head; behind the vulva the number is twelve, at the terminus four. Contour dentate. There are a few cervical setae, about as long as the body is wide, occurring in groups of four; no somatic setae. Lips thick, armed with three, mandible-like odontia, or onchia, somewhat flap-shaped, and apparently very mobile. Pharynx simple, regular, more or less conoid, large and long, three-fourths as wide as the head, having the form of a deep cup. There is a distinct, transverse, cutinized raised 
circle on the inner wall of the pharynx, dividing it into two more or less distinct parts; nevertheless, the inner contour of the pharynx is rather regular on the whole. Neck cylindroid. Oesophagus plain, except that there is a pyriform bulb surrounding the pharynx, tapering gradually into the oesophagus, which is at first two-thirds, at the nerve-ring one-half, and finally two-fifths, as wide as the corresponding portion of the neck. There is a more or less cylindroid cardia one-third as wide as the base of the neck. The thick-walled intestine has a distinct lumen, and becomes at once two-thirds as wide as the body. Cardiac tcollum one-half as wide as the body. The scattered, rather uniform yellow

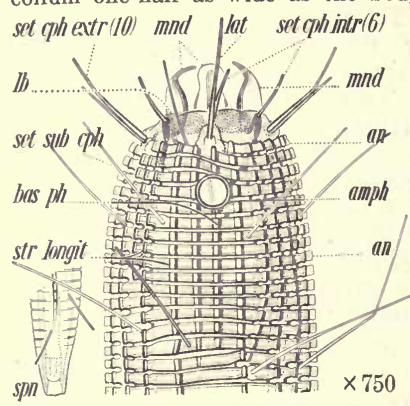

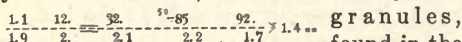
$1.1 \quad 7.6 \quad 25 . \quad-M^{65} \quad 91.70$ intestinal

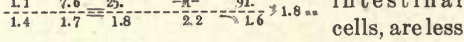
than half as wide as one of the annules. From the continuous anus the more or less cutinized rectum extends inward a distance hardly as great as the anal body diameter. The conoid tail tapers from the anus, near which, in the anterior third of the tail, the three ellipsoidal, caudal glands lie in a close tandem. There are very few, inconspicuous, slender, tapering, acute, dorsally submedian caudal setae. Renette unknown. The nervering surrounds the oesophagus obliquely and is accompanied by obscure nerve cells. From the large, conspicuous vulva, the large, tubular, muscular, cutinized vagina extends forward a distance equal to the width of the body, where it joins the straight uterus, which is six times as long as the body is wide and contains eggs two and one-half times as long as the body is wide, and one-third as wide as long,-if one may judge the size of the eggs from that of a full-grown ovum. There is a spermatheca extending forward a distance five to six times greater than the body diameter, the extent of which is plainly indicated by the definite contour of its blind end. The rather narrow, tapering ovary contains about twenty ova arranged single file. The spicula are slender, uniform, and acute. Their spherical proximal ends appear to lie dorsad from the body-axis. The two, separate, slender, acute, strong accessory pieces have proximal parts that appear to envelop the spicula. There are no pre-anal ventral supplementary organs, or special papillae. The ejaculatory duct is one-third as wide as the body. The narrow, cylindroid testes finally taper to a width two-fifths as great as that of the body.

Habitat: Eel-grass, Woods Hole, Mass.; also Belmar, New Jersey, U. S. A. Sublimate to balsam. Fig. 72 .

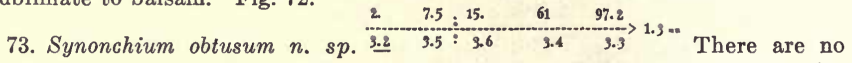
wings. On the lateral fields, which are about one-fourth as wide as the body, the secondary elements of the annules become scattered and coarser, and oval or elongated in contour. There are two laterally submedian, irregular rows of pores, one to two times as wide as the annules, lying along the margins of the lateral fields, and separated from each other transversely by a distance about equal to one-fourth the width of the body; measured in a longitudinal direction, the distances between the pores are about the same, but somewhat irregular. These pores have a special arrangement on the tail. (See illustration.) Neck cylin- 
droid; oesophagus somewhat cylindroid, but presenting a more or less spheroidal bulb about the mandibles. At the base of the neck, the oesophagus is two-thirds as wide as the corresponding portion of the body. Its lining is more or less distinct; its musculature colorless. The rather thick-walled intestine has a faint lumen, and becomes at once five-sixths as wide as the body. Throughout the

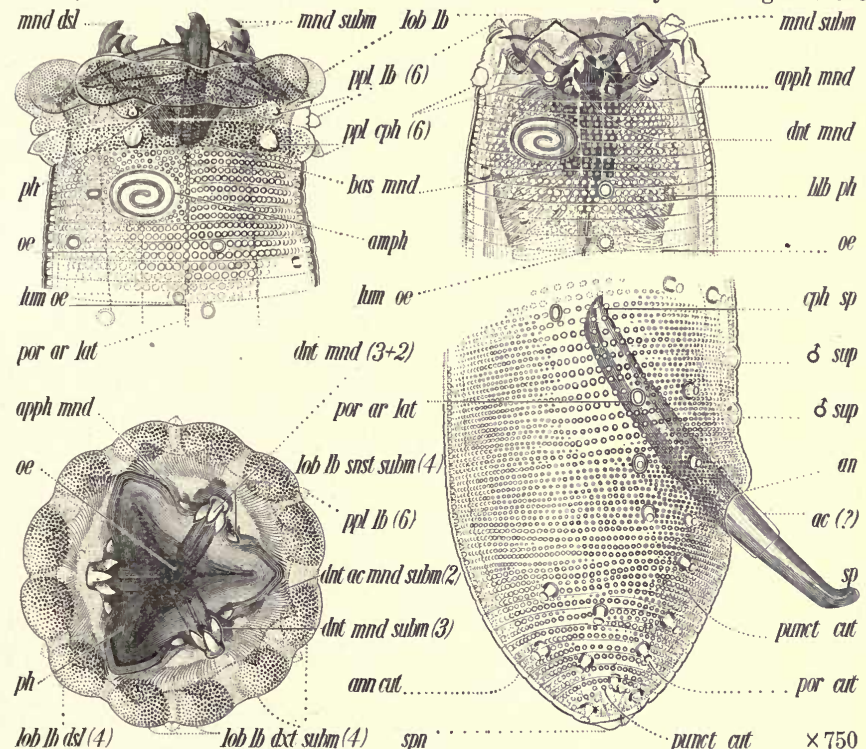

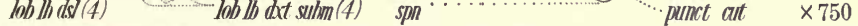
body under the cuticle, there are coarsely granular, spherical organs one-fourth to one-third as wide as the body, located in the lateral fields, and separated from each other longitudinally by a distance about equal to the body diameter. It is probable that the female organs are double, and symmetrically reflexed.

\begin{tabular}{|c|c|c|c|}
\hline .8 & 7. 13 & $44(t)$ & 98. \\
\hline
\end{tabular}

Apparently there are only two small supplementary organs in front of the anus. (See illustration, sup.) Resembles Enoplus, from which it is distinguishable; however, by the form of the spicula, and other organs of the male, as well as by the form of the amphids.

Habitat: Seaweed, drifted ashore, Ocean Beach, Miami, Florida, U. S. A. Fig. 73.

74. Gammanema ferox n. $s p$. Neck cylindroid. Cuticle naked, except for the setae on the head. Lips joined by a membrane,

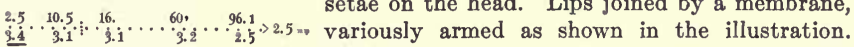
Probably the twelve "spatulate" appendages are

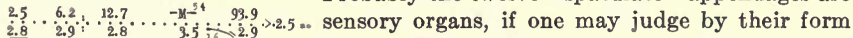
and position. The pharyngeal bulb contains three "apophyses," each carrying near its summit an inward-pointing, acute onchium,- - or perhaps it would be better to say, each of the three strong ribs of 
the posterior portion of the pharynx bears one of these onchia. The oesophagus gradually diminishes so that at no great distance behind the head it is but little more than half as wide as the neck, and continues thus throughout. There is a somewhat small, hemispherical cardia about one-third as wide as the base of the neck. The intestine becomes at once about three-fourths as wide as the body. In crosssection it presents four to six cells, manifestly of two different kinds, one of which, a minority, is much more distinctly granular than the other and is more numerous in the anterior portion of the intestine than in the posterior. The limits of each cell, of whichever kind, are rather clearly marked on account of the refractive nature of the cell-walls. The cells are packed with a multitude of fine, nearly colorless granules. The anus is prominently raised; the rectum is as long as the anal body diameter. The tail is conoid to the convex-conoid terminus, which forms a convex-conoid, somewhat truncate spinneret of large size, in which is the group of large ampullae of the caudal glands, which nearly fills the terminus, its diameter being about half as great as that of the base of the tail. The large caudal glands are located in a close tandem just behind the anus, in the anterior two-thirds of the tail. The lateral fields are fully one-third as wide as the body, and contain cells of unusual size,- of such a width as to reach nearly across the fields themselves. These cells are not contiguous, and there are found with them in the lateral field cells of small size. Renette unknown. From the elevated vulva the vagina leads inward half way across the body, where it joins the single uterus, which extends backward. The ovary reaches about half way back

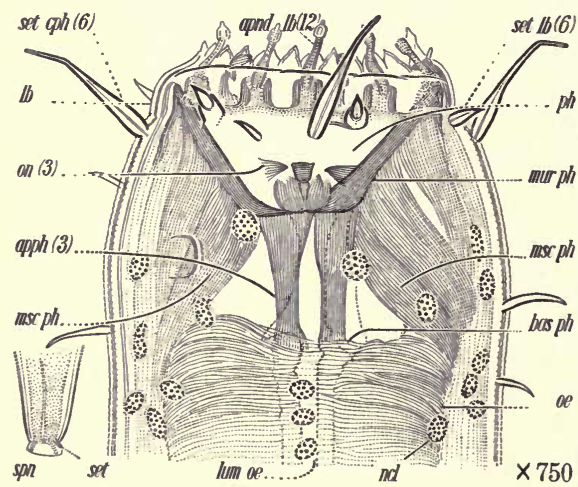
to the vulva and contains six to twelve developing ova, of which the larger are arranged single file. The eggs appear to be elongated, somewhat longer than the body is wide and about one-third as wide as long. The anus of the male is prominently raised. In front of it there is a ventral series of supplementary organs occupying a space equal to six oe to eight body-diameters. The hindermost of these organs is located opposite the posterior part of the spicula, and the penultimate opposite the middle of the spicula, and the third from the last nearly opposite the proximal ends of the spicula; the series continues along the ventral line with a somewhat equidistant arrangement, the total number of supplements being at least sixteen. Each supplement is a somewhat narrow cutinized structure of small size, extending outward and backward, and its distal extremity is capable of being exserted. These organs are not very conspicuous. A few submedian setae are seen near the anus, one pair opposite the distal parts of the spicula, and one or two on the bulky portion of the tail. The slender, uniform spicula have their proximal ends located opposite the bodyaxis. The width of the spicula is not much greater than that of the surrounding nuclei,-in other words, the spicula are quite slender. They are accompanied 
by obscure accessory pieces. The ejaculatory duct is about one-third as wide as the body, the vas deferens about half. The testes are unusually short and broad, only about four to six body-diameters long, and are, at their broadest, twothirds as wide as the body; they taper rapidly to the blind ends, which are only about one-fourth as wide as the body.

Habitat: Marine; coral sand, New Hebrides. Sublimate to balsam. Fig. 74 p. 292.

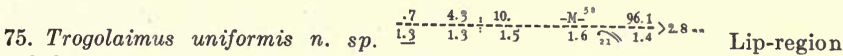
divided into twelve parts, readily distinguishable, though not cutinized. The broadly cup-shaped anterior portion of the pharynx is that part $10(6)$ referred to in the formula. From its rather flattish base three $s c p h$ apophyses extend backward close together, near the axis of the amph head, a distance about equal to the depth of the open portion of msc the pharynx, thus adding a second chamber. Each of these apophy- cut ses has a small, inward-pointing

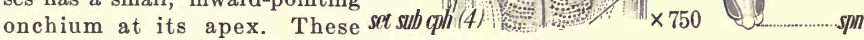
onchia meet together and cross at the middle of the floor of the anterior portion of the pharynx. As usual, the apophyses serve for the attachment of muscles, and all that portion of the pharyngeal bulb in front of the bases of the apophyses is different in character from the wall of the oesophagus, being more completely fibrous and destitute of the large nuclei that occur in the oesophageal musculature. The oesophagus continues with the same diameter until after it passes through the nerve ring, when it begins to expand gradually, so that finally it is more than half as wide as the base of the neck. In optical section the lining of the oesophagus appears wavy. There is no distinct cardia. The intest ne, which is separated from the oesophagus by a deep constriction, becomes at once about three-fourths as wide as the body. Its cross-section probably presents two to four cells. These contain a multitude of minute, evenly distributed granules. The walls of the cells are refractive. The lateral fields are about twofifths as wide as the body, and appear to be composed of two rows of cells. Renette unknown. The tail of the male is conoid from the prominently raised anus. It diminishes in diameter rather slowly until near the end. The diameter of the base of the spinneret is about one-sixth to one-eighth as great as that of the base of the tail. The caudal glands appear to be located in front of the anus. In Cyatholaimus, with which Trogolaimus may be compared, it is usually easy to discover the ventral gland and excretory pore. So far these have not been seen in the present species. The following is a description of the tail of a young female: The posterior lip of the anus is distinctly raised and rather broad. The rectum is somewhat longer than the anal body-diameter. The tail is cylindrical to the rounded or conoid-hemispherical terminus, which ends in a somewhat cylindrical spinneret, having a diameter about one-sixth as great as that of the base of the tail. The length of the tail is about two and one-half times as great as that of the anal body-diameter. This description is derived from a specimen in which the sexual organs are represented by an oval body consisting mainly of two cells. Supplements twenty-one, slightly elevated, flat, similar to those frequently seen on the males of Chromadora. These organs occupy a 
distance about three times as great as the length of the tail; the posterior ones are a little nearer together than the anterior ones. The two hindermost are opposite the anterior halves of the spicula, while the antepenultimate is a little in front of the proximal ends of the spicula. The average distance between the organs is one and one-half to two times as great as their diameter. No special setae or papillae have been seen on the tail either in front of the anus or behind it. The strongly developed spicula taper at both ends. The proximal ends are slightly hooked toward the ventral side of the body, and also slightly enlarged, so that they are distinctly cephalated. The main portion of the framework of each spiculum consists of four refractive elements arranged in two pairs,-one dorsal, the other ventral. The slightly curved accessory piece leaves the surface of the spicula somewhat and then recurves so as to touch them again. The ejaculatory duct is half as wide as the body.

Habitat: Mud, tide pool, low tide, Portsmouth, N. H., U. S. A. At first sight this species appears to be a Cyatholaimus but examination shows a number of important differences. The pharynx is not striated in the same way as that of Cyatholaimus, nor is there a dorsal onchium. The examination has not revealed the pores frequently to be seen along the borders of the lateral fields in Cyatholaimus. Most Cyatholaimi have a single testis. This genus also bears a considerable resemblance to Halichoanolaimus, but differs in that the numerous, minute pharyngeal denticles of Halichoanolaimus are lacking. Alternating with each pair of the twelve amalgamated lips there are papillae which extend an exceedingly short distance beyond the margin of the lips. Fig. 75, p. 293.

\section{Order Mesonchia.}

76. Mesonchium poriferum n. $s p$. The thickish, colorless cuticle has on the females three rows of round cuticular elements on the lateral fields, on the males, two; similar markings arranged irregularly occur on the ventral surface. Setae, half as long as the corresponding part of the body is wide, are found scattered on the neck. None are seen on the body. Rows of pores occur on the lateral fields, one row on each side just outside the longitudinal rows of lateral markings. The diameter of these pores is about equal to the width of two annules; the distances

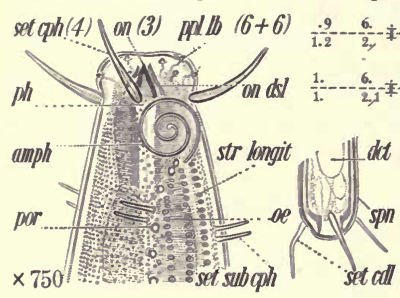
separating them longi-

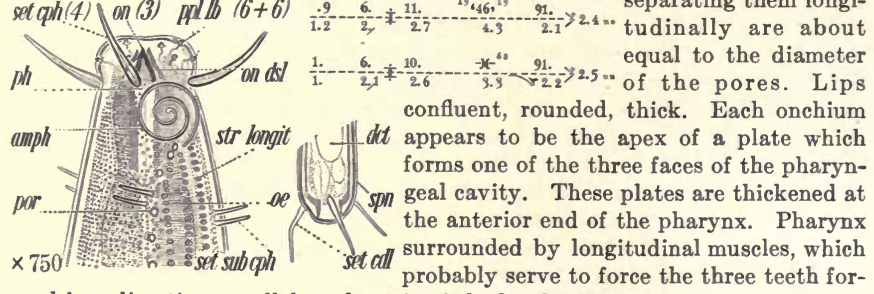
ward in a direction parallel to the axis of the head. Neck conoid. Oesophagus conoid, with a faint cardiac swelling, hardly to be called a bulb. Near the nerve-ring, the oesophagus is one-half, and finally three-fifths, as wide as the corresponding portion of the neck. The lining of the oesophagus finds optical expression in three parallel, refractive lines. Its musculature is rather coarse. There is a more or less hemispherical cardia, one-fourth as wide as the base of the neck. The intestine becomes at once three-fourths as wide as the body, its 
cells containing numerous granules of rather uniform size, and of about the same diameter as the dot-like lateral, cuticular elements. The conoid tail tapers from in front of the anus and becomes cylindroid in the posterior third, where it is one-seventh as wide as at the base. The elongated caudal glands, which empty by separate ducts, are packed in the anterior half of the tail. Slender, rather tapering setae, to the number of twelve occur on each ventrally submedian line in front of the anus; about seven setae occur on each of the submedian lines on the tail. The pre-anal setae are scattered through a distance about equal to the length of the tail, and are mostly very inconspicuous, especially anteriorly. The lateral fields are two-sevenths as wide as the body. The non-granular, ellipsoidal renette cell occurs near the cardia, and is one-third as wide as the base of the neck. The large, rather conspicuous, elevated vulva leads into a large, conoid, muscular, more or less cutinized vagina, which extends inward half way across the body. It is probable that the prolate eggs are about as long as the body is wide, but there is some uncertainty about this. An unusual feature is that the ovaries are reflexed only near their blind ends. Each contains about twenty ova, arranged more or less single file. The tail of the male is cylindroid in the posterior twofifths, where, at its widest, it is only one-tenth as wide as at the anus. The rather long, slender, uniform, more or less acute, colorless spicula are straight to near the middle, but are arcuate distally. Their proximal ends appear to lie more or less ventrad from the body-axis. The rather strong, slender accessory piece has an applied part one-fourth to one-third as long as the spicula; its uniform, more or less blunt, rather slender apophysis bends and extends backward parallel to the body-axis. The proximal extremity of the apophysis lies dorsad from the body-axis. To be seen along the posterior half of the region occupied by the oblique copulatory muscles, is a pre-anal series of almost invisible ventral innervations, the distances between which are about equal to one-third the body-diameter. The space occupied by the oblique copulatory muscles is about two and one-half times as long as the tail.

Habitat: Marine algae, Key West, Fla., U. S. A. Flemming to glycerine jelly. Fig. 76, p. 294.

77. Pepsonema pellucidum n. $s p$. Cuticle of medium thickness. Anteriorly the number of the longitudinal rows of "beads" appears fewer than near the tail, where there are sometimes six or possibly eight rows. Formation of the cuticula like that found in the genus Spilophora. Somatic setae very minute. Lips six, or three and twoparted. Mouth-opening about one-fourth as wide as the front of the head, and leading into a minute vestibule, which is $\times 750$

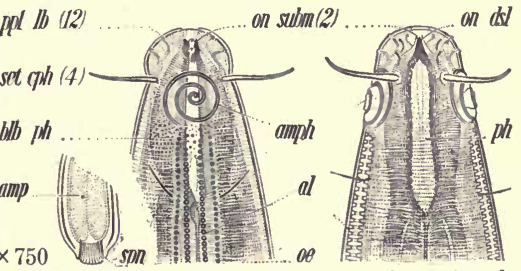
nearly twice as wide as the mouth opening and very shallow. The pointed, cutinized apices of three inner lips come together in this vestibule; they are of nearly equal size, the dorsal being only slightly larger than the two submedian ones. It may possibly be questionable whether this genus should not be placed in the Anaxonchia. At the base of the pharynx, there are one or two minute, onchium-like processes, which are so small as to be easily overlooked. The oesophagus continues to have the same width, or to decrease slightly, until it 
passes through the nerve-ring. Thence onward it increases rather regularly, though finally there is a tendency to form a much elongated bulb two-thirds to three-fourths as wide as the base of the neck. There is a distinct though small cardia. The intestine begins as a tube one-half to one-third as wide as the body, but soon expands so as to become two-thirds as wide as the body. It presents 1.2. . $5.4 \div 12 . \ldots 7^{20}+47^{20} .92 . \times 2.3 \ldots$ the very interesting peculiarity of containing in 1.3. 3.5 its anterior part about a score of cells whose

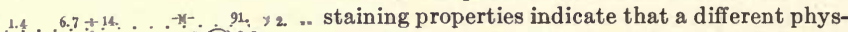

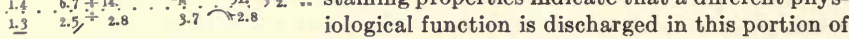
the intestine, possibly some gastric function. This is in line with other anatomical observations, indicating that the anterior portion of the nema intestine possesses functions differing from those of the middle and posterior portions. Opposite the cardiac constriction, there are two or three bodies closely resembling those usually seen in this position in the genus Trilobus. The nature of these organs still remains problematical. From the slightly depressed anus, the rectum, which is somewhat shorter than the anal body-diameter, extends inward almost at right angles to the ventral surface. Owing to the strong development of the somatic muscles, the intestine is only about one-half as wide as the body. The tissues of the oesophagus evidently contain glands, of which the dorsal is much the more highly developed; from its structure, it would be easy to suppose that the gland in the dorsal sector empties at the pharynx, but no conclusive proof of this has been obtained. In a similar way, it may be surmised that the glands in the submedian sectors empty into the lumen of the oesophagus in the vicinity of the nerve-ring. The tail is at first convex-conoid in such fashion that at the middle it is only about one-sixth as wide as at the anus; from the middle onward it is cylindroid, expanding slightly to form the rather rounded spinneret. The ovaries may be reflexed for a short distance near their blind ends. The tail of the male is rather strongly ventrally arcuate. For a distance in front of the anus equal to about five-body-diameters, there is found a ventral row of subequidistant innervations probably amounting, in fact, to a series of supplements. These begin near the anus, where they are only one-third as far apart as at the front end of the series. These very inconspicuous organs are nearly co-extensive with the series of oblique copulatory muscles. There are two lateral and two ventrally submedian rows of setae on the tail, the lateral rows lying close to the outside lateral rows of beads. Of these lateral pairs there are eight or nine. Each of the two sub-ventral rows has about twelve to fourteen setae. There are a few other setae scattered irregularly on the ventral side of the tail, as well as in the dorsally submedian positions. Spicula much elongated, narrow, acute; guided by accessory pieces about as long as the anal body-diameter, which have, projecting backward from their middle parts, processes nearly as long as they themselves are. The distal ends of the spicula are obscurely retrorsely barbed. The caudal glands seem to be located in the tail. It appears that sometimes the distal extremity of the anterior testis may be reflexed for a short distance. The posterior testis is considerably smaller than the anterior.

Habitat: Mud in the shallow portions of the harbor of Kingston, Jamaica. The name Pepsonema is suggested by the interesting cells found in the anterior portion of the intestinal wall. Such specially differentiated cells are now known for all parts of the nema intestine. In general the specialized cells of the posterior part of the intestine are histologically different from the specialized cells of the anterior portion. Fig. 77, p. 295. 


\section{Order Aponchia.}

78. Trissonchulus oceanus $n$. $s p$. Cuticle naked, practically without marks of any kind. Onchia refractive, in contour roughly equilateral, slightly curved, apparently with an outward stroke. When the mouth is slightly open, the apices of the teeth are on a level with the surface of the head. Neck slightly conoid, -convex-conoid at the head. Amphids very faint. Inner surface of each of the six lips armed with scores of exceedingly minute, closely set denticles. Oesophagus cylindroid, then conoid in the posterior fourth, where there is a faint cardiac swelling, which can hardly be called a bulb. At the nerve-ring the oesophagus is one-half, and finally three-fifths, as wide as the corresponding part of the neck. The indistinct lining is expressed by three parallel lines occupying a space two-sevenths as wide as the oesophagus. There is a conoid cardia one-half as wide as the base of the neck. The thin-walled intestine becomes at once three-fourths as wide as the body, at least when gorged. Its cross-section is probably made up of eight to ten cells. These cells contain scattered colorless granules of variable size, the largest of which are one-fortieth as wide as the body. Tail sub-cylindroid in the anterior three-fourths. The spinneret presents the peculiarity of being on the ventral surface of the tail some distance from the tip. (See illustration.) The elongated-ellipsoidal caudal glands, as long as the body is wide and one-third as wide as long, are located in front of the copulatory muscles. They form a loose tandem, and empty through distinct ducts and ampullae, the latter being large and filling the tail. There are no caudal setae. The lateral fields are one-third as wide as the body and contain large, ellipsoidal cells nearly as wide as the fields themselves, occurring at intervals of one to three body-widths; these are apparently glandular, and connect with the surface by means of pores on the lateral line. The excretory pore is at the lips and is directed forward. The renette cell, one neck-length behind the cardia, and as long as the neck, is granular and contains a large nucleus; its duct is about as wide as the cuticle is thick, and being coarsely granular,
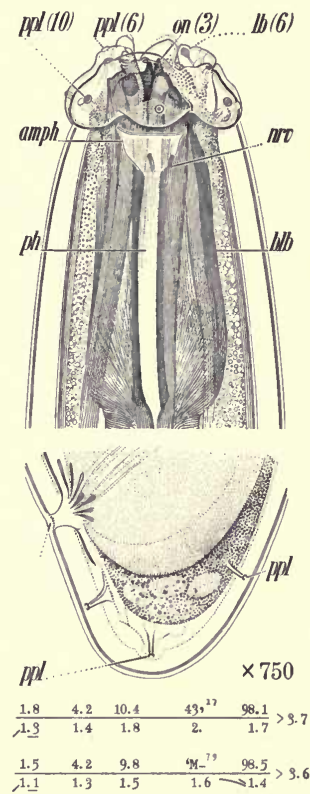
is rather easily followed. There is an anterior rudimentary part to the female sexual organs two to three body-widths long. From the continuous vulva, the medium-sized, more or less tubular vagina leads inward two-fifths the distance across the body. The broad, cylindroid ovary contains about thirty ova, arranged single file proximally, but irregularly distally. Tail of the male smaller and more arcuate than that of the female, and more nearly conoid. The proximal ends of the stoutish, tapering, rather blunt spicula appear to lie opposite the body-axis. There is a rather frail, slender accessory piece. There is a low and more or less obscure ventral pre-anal elevation at the anterior portion of the region occupied by the copulatory muscles. This may perhaps partake of the nature of a supplementary organ. On each side, in front of the anus, there are 
three, and behind the anus four, ventrally submedian papillae, the former separated by spaces about one and one-half times as great as the width of the body. Of those on the tail two are in front of the spinneret pore and two behind. Of the two testes the anterior one is much the longer; both are narrow. The ejaculatory duct is one-half as wide as the body, the vas deferens one-fifth to one-third. The copulatory muscles occupy a space five times as long as the tail. The spermatozoa are more or less ellipsoidal, and one-fifteenth to one-tenth as wide as the body of the female. In the male, at a distance in front of the anus four to eight times the length of the tail there are seven clavate, long glands connecting backward with the ejaculatory duct. These glands darken in Flemming's solution. In front of these glands, for a distance equal to four to five times the body-diameter, there are about seventeen spherical glands not darkening in Flemming. These also seem to connect with the ejaculatory duct.

Habitat: Seaweed that drifted ashore after a storm at Ocean Beach, Miami, Florida, U. S. A. Also found in beach sand at Ocean Beach. Flemming to glycerine jelly. Fig. 78, p. 297.

79. A ponchium cylindricolle $n . s p$. When the lips are closed the three onchia almost completely fill the pharyngeal cavity. Extending backward and slightly

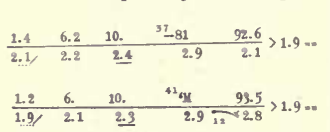
outward from the bases of the teeth are refractive markings in the pharynx, indicating folds,evidence that the cavity containing the teeth can be everted. The pharyngeal cavity seems tc merge gradually into the lumen of the oesophagus; it probably extends backward a distance equal to the diameter of the head. Onchia of very slightly unequal size; - the dorsal perhaps smallest, the submedian ones equal. The oesophagus continues to have the same diameter until after it passes through the nerve-ring, when it expands to form the elongated, pyriform, posterior bulb or swelling, three-fourths as wide as the base of the neck. There is a distinct, somewhat cylindroid cardia, about one-third as long as the neck is wide. The thick-walled intestine, which is separated from the oesophagus by a broad and deep constriction, soon becomes three-fourths as wide as the body. Its cross-section would display six to eight cells. The cells of the anterior portion of the intestine for a distance twice as great as the corresponding bodydiameter appear to be of a slightly different character from those farther back, as they almost invariably stain somewhat differently. The preliminary cells of the intestine, namely, a transverse series of about six cells, are undoubtedly of a larger size than those immediately following, and stain in a different manner; they fail to take acid carmine stain, when the cells immediately following stain well. These cells are packed with granules of rather uniform size. The succeeding intestinal cells contain numerous yellowish granules of variable size, which are rather irregularly disposed. A notable feature of the intestine is the lining, which is unusually thick, and consists of two elements; an interior thin, refractive membrane surrounded by a non-staining layer nearly as thick as that portion of the intestine containing the granules, already mentioned. In many parts of the intestine this lining, composed of the two above-mentioned elements, occupies fully half the optical longitudinal section. Anus raised, rectum somewhat shorter than the anal body-diameter. The tail begins to taper slowly from some little distance in front of the anus. Behind the anus it tapers somewhat more rapidly, and is conoid to the terminus. The three saccate caudal glands form a close tandem series in the anterior half of the tail. The nuclei of these cells 
are slung in a rather fine net-work of protoplasm. The lateral fields are fully one-third as wide as the body. The renette cell is not yet fully understood. There is a series of rather prominent cells a short distance behind the base of the neck on the ventral side of the intestine which undoubtedly constitutes the

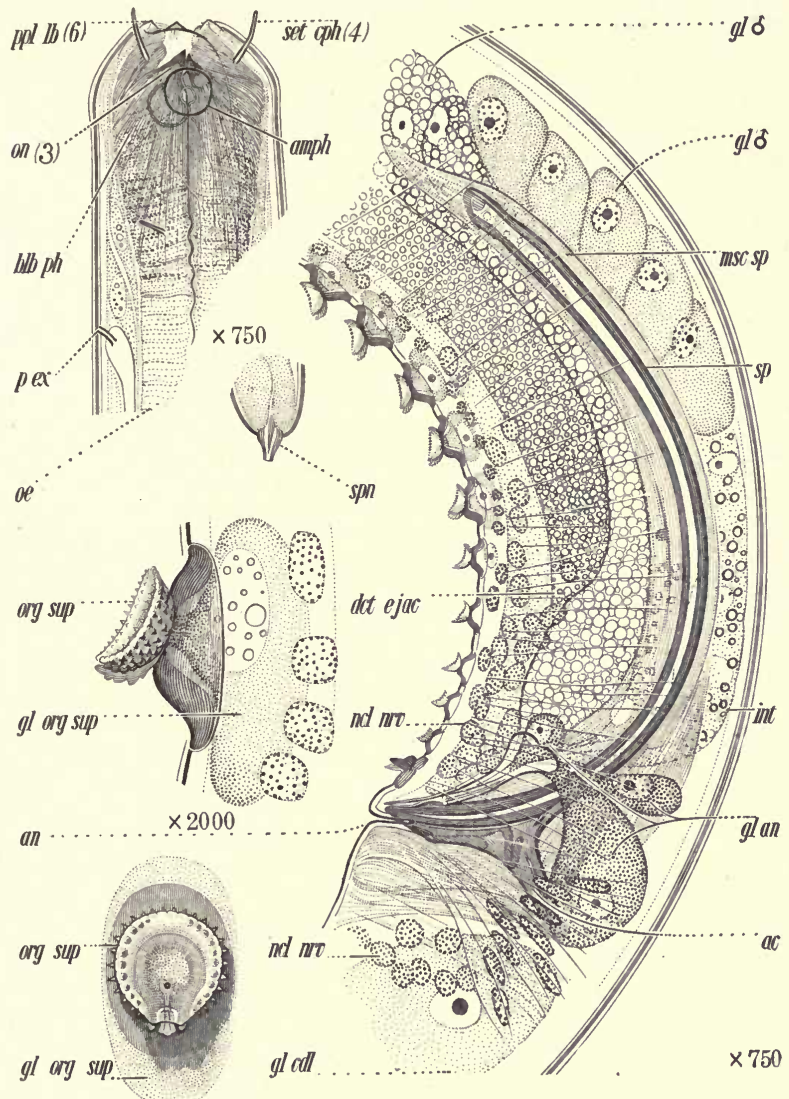

renette. In one typical specimen these cells were arranged as follows: First, an elongated-ellipsoidal cell, which failed to take the carmine stain, but contained a distinctly stained nucleolus in its nucleus. This. was followed by a cell which stained rather strongly, which in turn was followed by a pair of cells which did not stain, except in their nuclei; these two cells were arranged side by side, and were the largest of the series, being nearly half as wide as the body, and nearly twice as long as wide. Behind this pair of cells there was a single cell 
resembling the first member of the series, and behind this one or two others arranged tandem. This entire series occupies a distance equal to the length of the neck. From the raised vulva the vagina extends inward and forward. The outstretched ovary contains fifty or more developing ova arranged in double file, except near the blind end, which is located near the middle of the body. The eggs are of large size, elongated, about one and one-half times as long as the body is wide and about two-fifths as wide as long. In all those thus far seen the spherical nucleus is of unusually large size. There is no posterior rudimentary branch to the sexual organs. Well-developed accessory pieces surround the distal ends of the spicula. With them a median piece extends along the spicula and appears to end opposite the body-axis, near the base of the apophysis. The karyokinesis of the spermatocytes shows the arrangement of the chromosomes in spireme threads, something very uncommon in nemas.

Habitat: Larat, East Indies. Marine Fig. 79, p. 299.

\section{Order Triplonchia.}

80. Triplonchium cylindricum n. $s p$. Cuticle naked as in the case of nearly

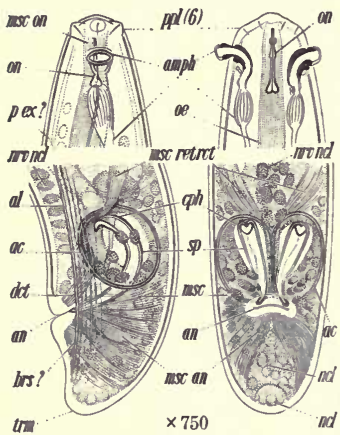

\begin{tabular}{ccccc}
1.8 & 6.9 & 13. & ${ }^{22}\left(54,{ }^{23}\right.$ & 98. \\
\hline $2.3 / 5)$ & 3.1 & 3.2 & 3.3 & 2.8 \\
1.2 & $=$
\end{tabular}
all Triplonchs. ending in a con\begin{tabular}{ccccccc}
2.5 & 7.8 & 15. & ${ }^{62}-x$ & 97. & ending in a con- \\
\hline $2.3 / 4.4$ & 3.4 & 4.2 & 9.7 & 1.
\end{tabular} Amphids more or less protrusile, projecting considerably in all specimens killed with Flemming's solution. (See illustration.). In some respects the amphids are reminiscent of those of certain Mermithidae. Oesophagus mainly cylindroid, but finally expanding to form a pyriform bulb nearly two-thirds as wide as the base of the neck. and having a rather indistinct valve one-sixth as wide as itself. The lining of the oesophagus is not a very distinct feature. There is no very distinct cardia. The intestine becomes at once

nd about two-thirds as wide as the body. The cells

contain numerous refractive spherical granules, the largest of which are about one-sixth as wide as the body, the smallest of which are very much smaller. These granules are packed so closely together as to obscure the details of the histological structure of the intestine. Anus continuous; rectum slightly refractive. The lateral fields have not been clearly seen, but appear to be about onefourth as wide as the body. Renette unknown. The nerve-ring surrounds the oesophagus squarely, and there are numerous nerve cells close together, both in front of the nerve-ring and behind it, filling the greater part of the cavity of the neck. From the slightly raised vulva the distinctly cutinized vagina leads backward a distance nearly equal to half the corresponding body diameter; it thell joins the single uterus, which extends first backward, then forward. This latter, when empty, appears to be about two-fifths as wide as the body. The end of the posterior ovary lies about one to two body-widths behind the vulva. Nothing is known concerning the size and form of the eggs. There is a cell located about as far behind the base of the neck as this latter is behind the anterior extremity, which attracts attention on account of its somewhat peculiar appearance. It presses the intestine to one side, and has a distinct nucleus, with a 
refractive nucleolus. Possibly this cell is the renette cell. The tail of the male is a little more strongly developed than that of his mate. No pre-anal supplements, though there appear to be one or two obscure papillae just in front of the anus. There are very obscure, straight accessory pieces; these may be little more than thickenings of the wall of the cloaca. The ejaculatory duct is twofifths, the vas deferens about two-thirds, as wide as the body. In glycerine specimens the spermatocytes are distinctly refractive, ellipsoidal bodies, about onesixth as long as the body is wide. The dorso-ventral view of the amphids is very instructive. As seen in glycerine preparations fixed with Flemming's solution, the external amphids appear as tubular protrusile organs, arcuate in form, and three to four times as long as wide. They may be protruded for at least half their length. Their outer surface is of a refractive character, and there appears to be a slightly refractive core. At the extremity they are almost imperceptibly expanded, somewhat hemispherical in form, with the terminal surface much thinner and less refractive than the lateral. They appear to slide in an inner tube located in the head, which is also cutinized. This tube extends inward and backward, and ends opposite the base of the pharynx, and is therefore arcuate like the amphid itself. Tubes leading back from the external amphid may be traced at least half way to the nerve-ring. The inner elements of the tube are refractive, and are seen to lie more or less parallel to the body-axis, but as yet have not been connected up with any internal cellular structure. There are comparatively few ova in the ovary, perhaps about a dozen in all, arranged somewhat irregularly.

Habitat: Humus, Plummer's Island, Potomac River, near the District of Columbia, U. S. A. Fig. 80 , p. 300 .

81. A phelenchulus mollis $n . s p$. Cuticle thin, naked; the wings, extending from the neck to near the terminus and bearing about twelve, very fine, longitudinal striae, are hardly raised at all. Contour, very minutely crenate. The head, which is more or less rounded in the adult, is sub-truncate in the young, and blb on the region is sometimes set off by an almost imperceptible broad, shallow constriction. The spear is nearly vestigial, can $\mathrm{smm}$. $\mathrm{lum}$ oe probably nearly functionless, and may act merely as a tube through which the food passes. It is not clear what the motive force in deglutition is, as the usual oesophageal bulbous pump is absent. Oesophagus cylindroid or faintly cephaloboid. In the larvae opposite the excretory pore are to be seen two successive breaks in the lining of the oesophagus, which are probably vestiges of a bulb. At first, the oesophagus is two-fifths, near the nerve-ring one-half, and finally one-fourth, as wide as the corresponding portion of the neck; the lining is subdistinct, the musculature fine and colorless. There is no cardia. The oesophagus changes gradually into the intestine, as in Aphelenchus. In the larvae, the

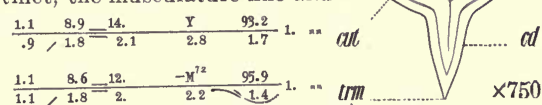

Upper Fig.-Adult male. Lower Figs.-Adult female. thick-walled intestine gradually becomes three-fifths as wide as the body. Its lining is refractive and in cross-section, it presents one to two cells only, which alternate with each other as in some Rhabdites. The anus is con- 
tinuous, the rectum inconspicuous and about as long as the anal body diameter. The nuclei of the intestinal cells are clear, and have twice the diameter of the largest intestinal granules, and present a nucleolus. The cells of the intestine are usually packed with granules of variable size, the largest of which are oneninth as wide as the body and are so arranged as to give rise to a more or less distinct tessellated effect. Tail of the adult, as shown in the illustration. The tail of a larva is more or less cylindroid, but slightly conoid and at the end bluntly rounded and almost imperceptibly apiculate. There are no caudal glands.

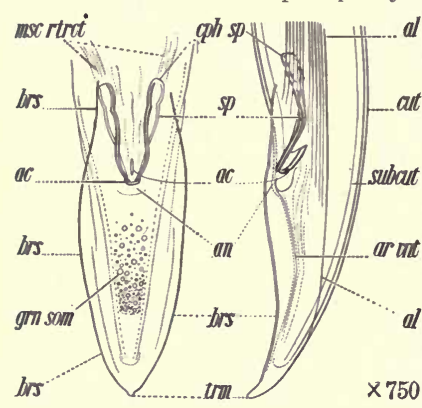

al Opposite the base of the oesophagus, there are two to three nuclei similar to those often found in the genera Tylenat chus and Aphelenchus, and probably having the same function, namely, that of salivary glands. The long renette, which appears to be sometimes on the right, sometimes on the left, extends back along the lateral field a long distance; it has been followed for three-fourths the length of the body. The nerve-ring is accom-

al panied by rather obscure nerve cells. Only the blind end of the tapering ovary is reflexed. The vulva is large and conspicuous, and is somewhat elevated, especially in front. The straight uterus contains ellipsoidal to elongated eggs, which are about as long as the body is wide, measuring forty by twenty-five microns. They begin segmenting before deposition.

Habitat: Found at Falls Church, Va., U. S. A., parasitic in a wood-boring beetle, determined by Mr. F. C. Craighead as Cyllene picta Drury. Sixteen thousand larvae and a few adult females were found in one beetle. Both male and female beetle are infested, usually with only a few female parasites; - at least such is the condition in the month of May. Of fourteen female insects, three were infested; of twelve males, seven were infested. As a rule, only a few adult females of Aphelenchulus mollis occur in one host, about one to eight. Two living adult males of Aphelenchulus mollis were found in the castings of beetles taken from infested logs. These are the only adult males so far seen. This finding may indicate that the females are fertilized before entering the host. The parasites are found in the thorax of the beetle, as well as the abdomen. Fig. $81 a$, p. 301 ; Fig. $81 b$.

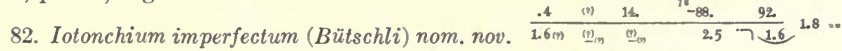
Striae fine; contour entire. Head abruptly truncate, set off by expansion. Spear exceedingly minute, eight microns long, about one-fourth to one-fifth as long as the head is wide, simple, plain, apparently not bulbed; in the male, the spear is difficult to see, being reduced to a mere point. Neck conoid; amphids?. Intestine very transparent; anus indistinct. Tail of the female conical from the vulva, acute. Excretory duct single, much coiled. Ovary outstretched forward; eggs somewhat longer than body is wide, half as wide as long, deposited after segmentation. Spicula L-form, tapering, cephalated by constriction, considerably longer than the anal body-diameter; accessory pieces none; bursa well-developed, springing from considerably in front of the spicula and extending somewhat beyond the terminus.

Habitat: In rotting fungi, Germany. Syn. Tylenchus imperfectus Btsli. 


\section{Order Axonchia}

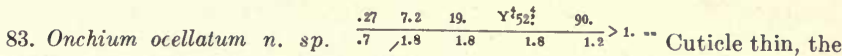
transverse striae almost impossible of resolution. Except for the cephalic setae, the cuticle is naked. Spear hollow, brownish, very thin-walled. Though the ycllowish, solid, refractive eye-spots are sublateral in position, they are rather near together. The specimens thus far examined do not enable one to make a clear distinction between the oesophagus and the intestine. Connected with the posterior part of the oesoph- set qph (4) agus are large glandular cells, probably three in number, judging by the number of nuclei. These structures seem to be homologous with those found in a similar position in Tylenchus and Aphelenchus, but their actual connection with the lumen of the oesophagus $\times 750$ is problematical, as no connecting duct has been seen. The best interpretation seems to be to consider these structures as a part of the oesophagus, but it is entirely possible that the oesophagus ends just in front of these glands. The thickwalled intestine, which has a very distinct, refractive zigzag lumen, becomes almost at once three-fourths as wide as the body. From the elevated anus, the prominent, cutinized rectum extends inward a distance equal to the anal bodydiameter. The cells of the intestine contain numerous somewhat variable yellowish granules, the largest of which are one-sixteenth as wide as the body. The conoid tail is arcuate. Caudal glands have not been clearly deciphered, but they probably lie behind the anus in the anterior half of the tail in a loose tandem. They are narrow and elongated. The elongated, granular renette cell, twice as long as the body is wide, and about one-third as wide as long, is located six to eight body-widths behind the neck. The single, reflexed sexual organ extends forward; the accompanying formula, correct for the larvae, does not pretend to indicate the true form of the developed organ. Males unknown.

Habitat: Clean "white" marine sand, in five feet of water, $\times 500$ from a cove near the entrance to Buzzard's Bay, Mass., U. S. A., Also from "sea-grass," Key West, Florida. Fig. 83.

84. Doryllium uniforme $n$. $s p$. Cuticle naked, very minutely transversely striated. Neck conoid. Spear minute, dorylaimoid, but with a distinct, somewhat refractive posterior swollen part. The oesophagus continues with slight variations until it joins the pineapple-shaped cardiac bulb, which is two-thirds as wide as the base of the neck and is separated from the preceding portion of the oesophageal tube by a constriction. This bulb contains, among other things, a spherical nucleus with a distinct nucleolus, presumably indicating the existence here of glands like those of Dorylaimus. The narrow intestine joins the middle of the posterior surface of the bulb, and becomes at once about two-fifths 
as wide as the body; its cross-section would present only about two cells. These cells contain scattered yellowish granules of uniform size. From the incouspicuous anus the rectum extends a distance longer than the anal body-diameter. The pre-rectum is about four times as long as the corresponding body-diameter,

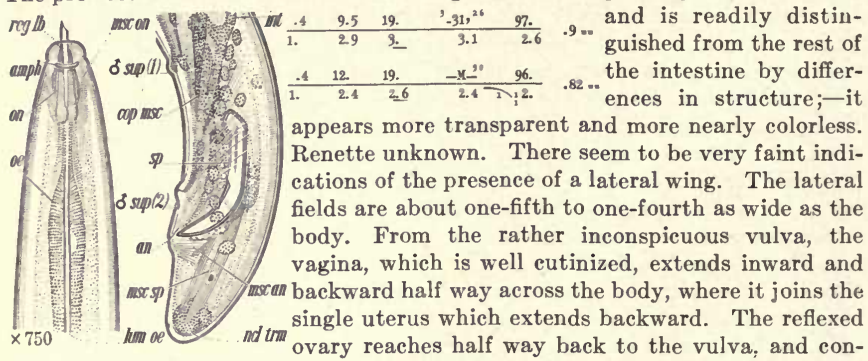
tains ten to twenty ova arranged for the most part single file. There is an anterior rudimentary branch to the uterus which is a little longer than the corresponding body-diameter. Eggs unknown; judging from the full-grown ova they are about as long as the body is wide and about half as wide as long. Single male supplement two body-diameters in front of the anus, four times as far away as the anal pair.

Habitat: Brackish soil on the banks of a marine estuary, Los Patos, California, U.S. A. Differs from Dorylaimus in the form of the spear, and the oesophagus. Most species of Dorylaimus have two ovaries. Differs also in the reduced number of supplementary organs; - outside the anal pair, there is only one. Differs in material respects also from Tylencholaimus, to which it may be compared. Fig. 84 .

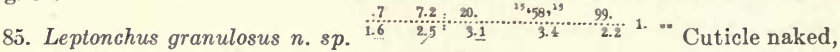
its transverse striae resolvable with difficulty into exceedingly minute dots. One of the inner striae seems to correspond to about four of the outer. Cuticle also possessed of inconspicuous longitudinal striae. Neck conoid. Amphids broad, faint, somewhat stirrup-shaped, their anterior contours opposite the labial constriction. The oesophagus begins as a tube about one-fourth as wide as the base of the head, and continues to have this diameter until it finally expands to form the clavate or elongated pyriform cardiac swelling, which is about one-half as wide as the base of the neck. There is an inconspicuous conoid cardia about one-third as wide as the body. The oesophagus is therefore essentially tubular throughout most of its length. It is, however, a trifle wider near the middle, in the neighborhood of the nerve-ring, usually behind it, and at this point there

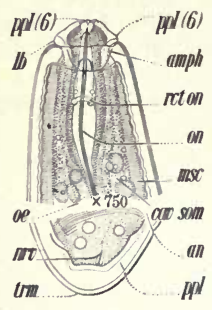
appears to be a slight alteration in the lining, suggesting in the faintest possible manner vestiges of a median bulb; at its widest part, however, the slender part of the oesophageal tube is not more than one-fourth to one-fifth as wide as the corresponding portion of the neck. At its narrowest part, just in front of the cardiac swelling, it is about half as wide as it is at its widest part. The lining of the oesophagus is not a conspicuous feature. The intestine, which becomes at once fully three-fourths as wide as the body, is made up of cells of such a size 
that its cross-section presents but two of them. These cells are packed with granules of variable size, the largest having a diameter one-eighth as great as that of the body, while the smallest are very minute. The tail begins to taper from some distance in front of the anus. There are a few inconspicuous papillae near the posterior extremity of the tail of the female. The pre-rectum is of most unusual length, extending to a little beyond the flexure of the anterior ovary, and hence occupying about half the length of the body. It is set off from the front portion of the intestinal tube by a distinct constriction, and differs also in structure. The lateral fields are about one-third as wide as the body. The ventral fields appear to be wider than the lateral,-nearly half as wide as the body, at least appearances half way between the vulva and the anus give rise to this measurement. The traces of the excretory pore are difficult to observe, but they are uniform in the different specimens, and as no other similar "break" in the cuticle is to be deciphered it seems impossible that the single "break" opposite the nerve-ring can be anything but an excretory pore. No indications are seen of the existence of an internal structure connecting with this pore, but the neck, especially the posterior portion of it in front of the cardiac swelling, is occupied by elongated structures, which may be of a glandular nature and connected with the aforesaid "pore." The slightly elevated vulva is a transverse slit about one-fourth as long as the body is wide. Radiating from its ends are four muscles passing to the submedian fields. The vagina leads about halfway across the body. The reflexed ovaries reach about three-fourths the distance back to the vulva, and contain a dozen or more developing ova, arranged somewhat irregularly. The elongated eggs are three to four times as long as the body is wide, and about one-fourth to one-fifth as wide as long.

Habitat: Soil about willow trees, Arlington Farm, Va., opposite the city of Washington, D. C., U. S. A. Fig. 85, p. 304.

86. Axonchium amplicollen. $s p$.

$$
\begin{array}{rrrrr}
.2 & 6.7 & 34 . & 55,{ }^{20} & 98.8 \\
\hline .6 & (\text { (7) } 1.9 & 2.8 & 3.1 & 1.7
\end{array} 2.2
$$

Cuticle naked. The two portions of the $l b(6)$ oesophagus are separated by a constriction, as shown in the figure; both parts eylindrical. Lining of the oesophagus well-devel- amph oped, but more or less obscured by the well marked, rather coarse, colorless musculature in the posterior part. Salivary glands in the posterior part of the oesophagus as in Dorylaimus, one gland emptying into the lumen near the anterior portion of the larger cylindroid half of the oesophagus, the others farther back. Anus subcon- $p p l$ tinuous; the rectum prominent and cutinized, and one and one-third times as long as the anal body-diameter. The anal mus-

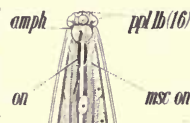

asc on

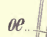

ato som

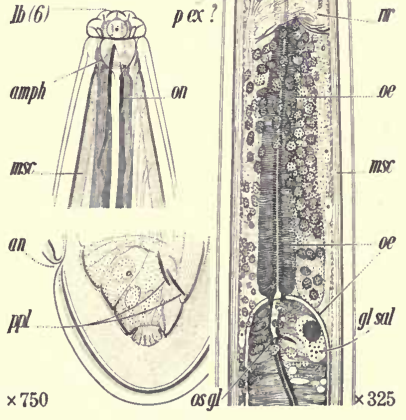
cles are clearly disclosed. The pre-rectum is five times as long as the anal bodydiameter, and set off by a definite but faint constriction. The cells of the intestine contain numerous, colorless granules of variable size, the largest of which are one-twenty-fifth as wide as the body. Tail of the adult female as shown in the illustration. In the youngest larvae, however, the tail is cylindroid in the 
posterior half, and in this part, about one-third as wide as at the anus, the terminus being rounded. The coarsely granular lateral fields are one-fourth to one-sixth as wide as the body. Renette problematical. From the small, inconspicuous, faintly elevated vulva, the rather weak, non-cutinized vagina leads inward two-fifths the distance across the body. The elongated eggs are three times as long as wide and twice as long as the body is wide. The tapering ovary reaches about half way back to the vulva, and contains about twenty ova, the largest of which are arranged single file, while the others are arranged irregularly.

Habitat: Soil about the roots of Luca da Persia, Brazil. The part of Brazil from which the plants came is unknown. The soil examined was removed from the roots after they were imported into the United States. It is probable that the species is syngonic. Another tropical species, originally described by the writer under the name of Dorylaimus longicollis, from about banana roots, Fiji, possibly also belongs to this genus. Fig. 86. p. 305 .

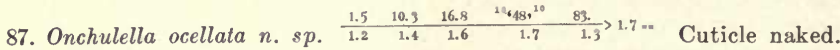
Striae almost impossible of resolution. Spear solid, refractive and apparently "structureless" throughout. The two yellowish ocelli are wide apart, their lenses colorless. The oesophagus is at first about one-half, near the nerve ring two-fifths, and finally five-sixths, as wide as the corresponding portion of the neck; the anterior part is cylindroid, the posterior part more or less obscure. The oesophageal region seems to be largely filled with a series of elongated, clavate glands, some granular and some not, and hence of two kinds. The hindermost of these is the largest, and with reference to the oesophagus lies in much

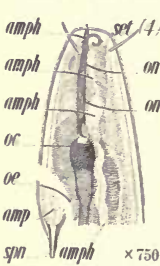
the same way as the larger salivary gland of Tylenchus. There is also a large spherical nucleus in this gland, as in Tylenchus. on This is the only one of the granular cells having a large conspicuan ous nucleus. The non-granular cells are smaller; two or three of them present nuclei. The measurements given in the formula for the base of the oesophagus really refer to the posterior limits of these glands. Just in front of the ocelli there are two lateral, external, elliptical, cuticular elements, two-thirds as long as the corresponding diameter of the neck, and one-third as wide as long. These connect backward by means of ducts, and it seems probable that they are connected with one of the two kinds of glandular cells contained in the neck. The lining of the oesophagus is indistinct, its musculature fine and colorless. No cardia has been seen. The thick-walled intestine, which has a distinct, refractive, ziz-zag lining, becomes at once five-sixths as wide as the body. The anterior lip of the anus is more or less elevated. The inconspicuous rectum is about three-fourths as long as the anal body-diameter; anal muscles arc dimly to be seen. The cells of the intestine, of which only two to three would be presented in cross-section, are packed with colorless granules of variable size, the largest of which are one-fifteenth as wide as the body. The arcuate tail tapers from the anus to the terminus, and presents a spinneret about one-sixth as wide as its base. Caudal glands tandem in front half of tail. No caudal setae. Renette unknown. From the somewhat depressed vulva, the more or less cutinized vagina extends inward three-fifths the distance across the body. An ovum, apparently full-grown and about to enter the uterus, is twice as long as the body is wide, and one-third as wide as long.

Habitat: Eel-grass, Biscayne Bay, Miami, Florida, U. S. A. Flemming to glycerine jelly. Ouly one specimen-that, a shrunken one-seen. Fig. 87. 


\section{Order Anaxonchia}

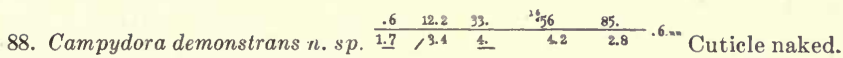
Eight longitudinal striations exist throughout the length of the body. In certain parts, especially along the neck, the longitudinal striae are more marked than elsewhere, attaining almost the magnitude of wings. There appear to be four such "wings," but an exact count has not yet been made. Neck conoid. There are six rounded, somewhat flat, partly confluent lips, bearing two circlets of labial papillae, as shown in the illustration. These are plainly innervated, and are of the sort commonly seen in Dorylaimus. The inner circlet has six members; the outer circlet twelve, i.e., six pairs. The six slender structures extending forward around the mouth, the inner circlet, may also end in organs having the nature of papillae, but if so, they are of a different character from the outer papillae. Base of the solid onchium axial, on or nearly so, the apex however, bent considerábly to the dorsal side. The onchium may be protruded by means of longitudinal muscles composing the pharyngeal bulb. Lining of the posterior portion of the pharynx considerably less refractive. The oesophagus has about the same relative diameter until it finally expands suddenly to form the somewhat elongated, msc cylindroid cardiac bulb, which is fully five-sixths as wide as the corresponding portion of the neck, and contains a rather faint, elongated, three-parted valve occupying about half $\times 750$

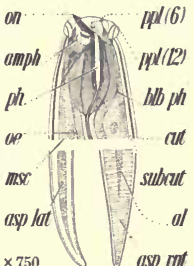
the optical longitudinal section. This cardiac bulb has a peculiar formation in that the valve extends very nearly from end to end of the bulb and is manifestly triquetrous, so that by the contraction of the radial muscles, the bulb can be thrown into the form of a chamber. Lining of the oesophagus unusually distinct. The central oesophageal tube, when seen in optical section presents each of its walls with a double contour, and the lumen has about the same width as the thickness of the walls. The entire width of this tube is considerably greater than the thickness of the cuticle, and is about equal to one-twelfth the diameter of the middle of the neck. The intestine becomes at once about five-sixths as wide as the body. There is a comparatively distinct, broad, low cardia. The walls of the intestine are comparatively thick. Its cross-section would probably present two to four cells. These cells contain doubly-refractive, spherical, scattered granules of variable size, the largest of which have a diameter one-tenth as great as that of the body, the smallest being very much smaller. When seen with crossed Nicols, these granules show a prominent St. Andrew's cross. All the granules appear to be of the same character; their walls, seen in optical section, present a double contour. Behind the base of the neck for a distance about equal to one body-diameter, the granules are few in number and small in size. Elsewhere, they are about equally distributed, or perhaps a little less abundant toward the posterior end. The tail is conoid from near the anus, and the cuticle becomes thicker on the posterior extremity and more strongly striated. In young specimens it expands laterally to form wings, which appear quite distinctly striated when viewed dorso-ventrally. From the conspicuous, depressed anus, the strongly refractive, cutinized rectum is considerably longer than the anal body-diameter. Excretory pore depressed and rather conspicuous. From it, the cutinized duct can be distinctly seen leading inward. From the depressed somewhat circular vulva, the cutinized 
vagina leads inward nearly half way across the body, where it joins the single uterus, which apparently extends forward. The ovary extends backward past the vulva. Nothing is known concerning the size and form of the eggs.

Habitat: About the roots of citrus trees, Corfu, Greece. Fig. 88, p. 304.

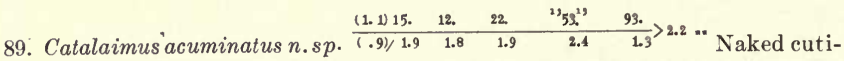
cle traversed by exceedingly fine transverse striae. Lips when closed exhibiting about a dozen folds which

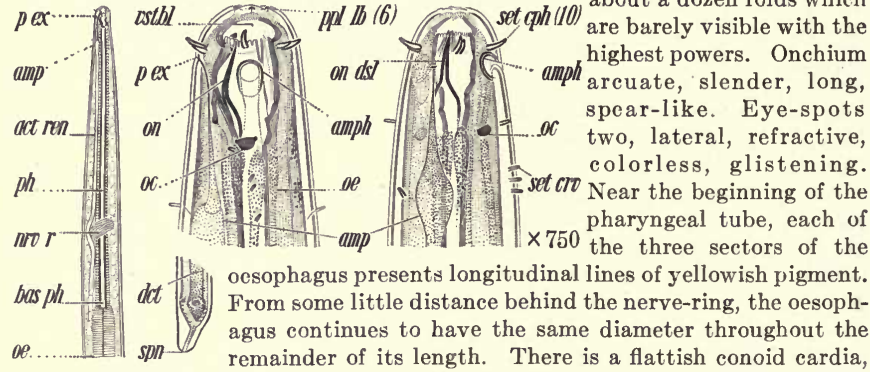
about one-third as wide as the base of the neck. Lining of the oesophagus apparently wavy, occupying one-third of the optical section. The intestine, which becomes at once three-fourths as wide as the body, is made up of cells of large size, at least some of the cells are of very large size,- - of such a size that probably two only would be required to build a circumference. Whether there are other smaller cells scattered among these larger cells remains to be determined. The large cells are packed with nearly colorless granules of rather uniform size, having a width about half as great as the thickness of the body wall. While the anus is depressed, its posterior lip is slightly raised. Rectum about as long as the anal body-diameter. The nema tapers gradually from a considerable distance in front of the anus. Near the anus, it begins to taper more rapidly, so that at the middle of the tail, the diameter is only about half as great as at the anus; thence onward, the tail is nearly cylindroid and ends in an elongated spinneret. No distinct traces of caudal glands are to be seen in the base of the tail; hence it is assumed that they are located in front of the anus. Obscure indications have been seen of their presence half as far in front of the anus as the terminus is behind it. The lateral fields have not been distinctly seen. The location of the renette cell remains undetermined. This description is derived from a young female, and all that can be said is that the ovaries are probably double and reflexed.

Habitat: Marine mud and sand, Noumea, New Caledonia, and Biscayne Bay, Fla., U. S. A. Other species of this genus occur in the Indian Ocean and in the Mediterranean Sea. Fig. 89.

90. Bolbella tenuidens $n$. $s p$. Transverse striae excessively fine, more readily seen in the subcuticle with high powers under fav-

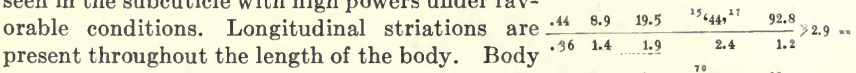

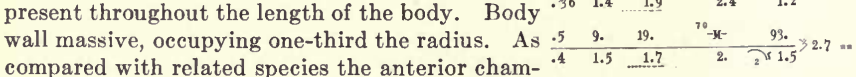
compared with related species the anterior cham- ${ }^{.4}$
ber of the pharynx is somewhat more foreshortened. There are two to three 
dozen very long and very slender cervical setae, those toward the head end being nearly twice as long as the cephalic setae,-more of them than are shown in the illustration. Glandular tissue exists in the oesophagus, the granular branches of which may be seen more particularly between the oesophageal bulbs: a duct appears to lead to the pharynx. A cross-section of the intestine would present four to six cells. In the female, from the more or less continuous anus. of which, however, the posterior lip is slightly elevated, the inconspicuous rectum extends inward a distance equal to the anal body-diameter. A narrow renette cell exists two to three body widths behind the cardia on the ventral side. The slightly elevated vulva is of medium size, and leads to a large tubular vagina, at first at right angles to the ventral surface and then leading obliquely forward. The wall of the vagina is well cutinized. Each branch of the two-horned uterus is about one and one-half times as long as the body is wide. Two eggs have been seen in a uterus at one time, each about twice as long as the body is wide. The spherical spermatozoa existing in the uterus are about one-sixteenth as wide as the body. The broad, more or less cylindroid ovaries extend two-fifths the way back to the vulva, and contain eight to ten countable ova arranged for the most part irregularly. The two supplementary organs of Bolbella, like the similar organs of Eurystoma, are connected with large glands, one to each supplement. One of these glands is very well shown in the illustration at $g l$ sup $\sigma^{7}$.

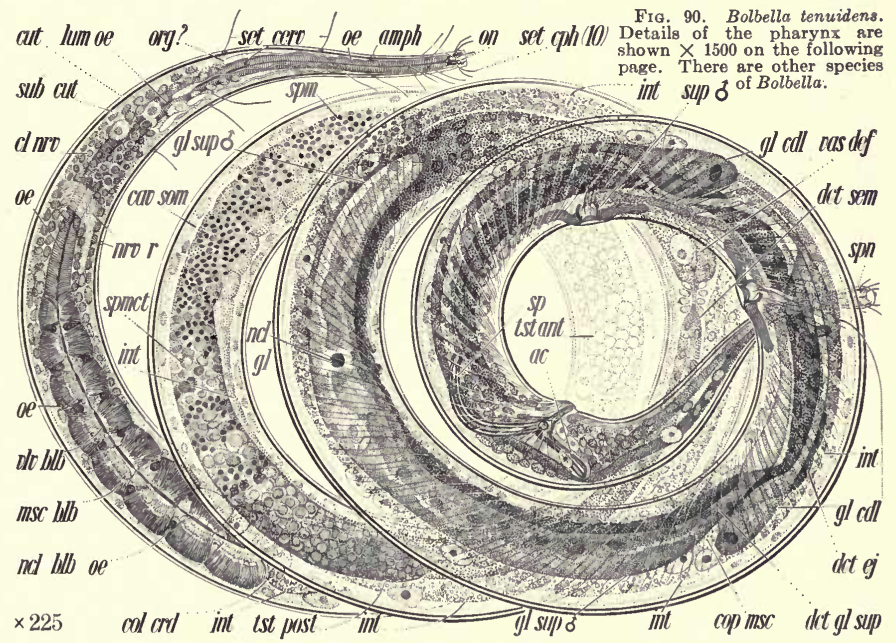

The gland is long and unicellular and its nucleus is shown at $n c l \mathrm{gl}$. A natural supposition is that these two supplements are cup-shaped organs attachable by suction. The supposition appears, at first thought, to find support in the existence of the "anchors," which are manipulated by special muscles. It is rather difficult to harmonize this theory with the existence of such large glands, so much so that one feels inclined to give up all idea that suction plays any part in the functioning of these organs. The so-called cups of the supplements are 
to a eonsiderable extent protrusile, just as in Eurystoma. The males of BolHabitat: Sand, Bathing Beach, Woods Hole,
Mass., U. S. A. Flemming to glycerine jelly.
the femablimate to balsam. Resembles Symploeos-
toma Bastian, but differs materially in the
structure of the posterior part of the oesoph-
agus, as well as in the absenee of eye-spots.
The males of Symplocostoma have no sup-
plements.
The strongly tapering neck of Bolbella results
in a head of small size. Notwithstanding its
small size, one is immediately struck by its
strong resemblance to the head of Eurystoma.
The following differences, however, are to be

91. Thoönchus ferox n. sp. Striae resolvable with difficulty. The thin, colorless lips are arehed over the pharynx as in Oncholaimus, but the minute flaps often seen on the tips of the lips of Oncholaimus appear to be absent in this speeies, or at any rate, mueh redueed. Labial papillae loeated in the midst of shallow depressions. Amphids faint. Pharynx dentieulate. The oesophagus has the \begin{tabular}{ccccc}
1.4 & 8.5 & 22. & ${ }^{17}{ }^{4} 63,{ }^{17}$ & 96.9 \\
\hline 1.2 & 1.4 & 1.6 & 1.8 & 1.1
\end{tabular} $2.2=$

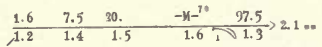
same diameter throughout its anterior half, after which it begins slowly to expand and finally is two-thirds as wide as the base of the neck. There is a distinct, bluntly eonvex-eonoid cardia, nearly half as wide as the base of the neck. The intestine, which is separated from the oesophagus by a deep constriction, beeomes at once three-fourths as wide as the body. It is rather thin walled and in cross section presents about six eells. These cells contain scattered, yellowish granules of variable sizes, so arranged as to give rise to an obscure tessellated effeet. The largest granules have a diameter nearly equal to the thickness of the body wall. Posterior lip of the anus strongly elevated. Reetum equal in length to the anal body-diameter. Renette elongated, located a little distance behind the eardia. The lateral fields appear to be rather narrow; they are characterized by the presenee in them of numerous, rather scattered, yellowish granules, which are more numerous toward the edges of the field, and which, therefore, define the fields more distinctly than is usually the case. At the base of the head, the fields are seen to widen out and terminate in a rounded contour; the margin of this contour marks the position of the amphids. The granules found along the lateral fields appear also in longitudinal rows outside these fields. Thus, on either side of the lateral fields, there is a single distinet row of these granules; on the submedian line, another very distinet assemblage of granules, eonsisting of several irregular rows. Then eomes another scattered faint row; then a ventral or sub-ventral assemblage consisting of several rows. There is thus produced on the neck as well as elsewhere quite a distinct longitudinal 
"striping" whose presence is indicated mainly by the arrangement of these Ininute yellowish granules. The stout tail diminishes rapidly from the posterior lip of the anus, so that at the middle it has a diameter about two-thirds as great as at the base; thence onward it is nearly cylindroid to the rounded terminus. The caudal glands are located in a tandem series considerably in front of the anus; the hindermost being about four times as far from the anus as the terminus. The two anterior glands are close together and are nearly as far in front of the hindermost as this latter is in front of the anus. From the elevated vulva, the vagina extends inward half way across the body. The ovaries reach about one-third the distance back to the vulva in specimens that contain three eggs in the uterus. Eggs elongated, about twice as long as the body is wide and about one-third as wide as long. Euch of the twc uteri may contain three eggs at one time. Tail of the male more strongly arcuate than that of his mate. Clavate caudal glands about as long as the body is wide and about one-third as wide as long, arranged some distance apart in a tandem series. When the body of the male is closely coiled, the ventral surface toward the posterior end becomes corrugated in a definite way for some distance in front of the anus, suggesting that this area may be systematically innervated and serve the purpose of a ventral series of about two dozen subequidistant supplementary organs. These corrugations are apparently innervated, but no distinct corresponding breaks or openings have been seen in the cuticle. On the ventral side at a distance in front of the anus three times as great as the length of the tail, there is an elevated supplementary organ in the form of a transverse ridge. This occurs in the midst of the corrugations just described,-at least, it appears that the corrugations extend beyond it, although they are not quite so close together or so marked in this region. This supplementary organ begins near the submedian lines and extends across the ventral region. Its internal structure has not been satisfactorily elucidated. It appears probable that the cuticle on the ventral line is interrupted, but even this is not clearly established. This swelling has two to three times the width of the corrugations and occurs at the same position on three different male specimens, so there can be

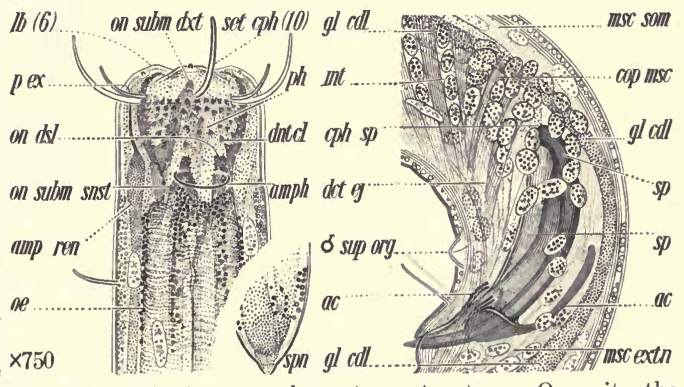
no doubt about its being a distinct supplementary structure. Opposite the middle of the spicula, also, there is a pair of subventral supplementary papillae, accompanied by slender subventral setae in the rear. There are a few short delicate setae on the tail, especially on the submedian lines, but also in front of the anus for some distance, where they are longer,-more nearly the size of those on the anterior extremity. About a dozen pairs of copulatory muscles occur in the ventrally submedian region, in a space four to five times as long as the tail. Testes relatively small.

Habitat: Open-ocean beach-sand, South California. Bay City, Huntington Beach U. S. A. Fig. 91. 


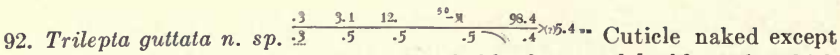
for the cephalic and cervical setae. Lips probably three, and double, rather thick and well-developed. When closed the pharynx appears as if longitudinally ribbed. This appearance, however, may be due to the folding of the rather massive lips, which close together and form a vestibule nearly one-half as long as the remainder of the pharynx. Neck cylindroid. The cylindroid oesophagus near the nerve-ring is one-half, and also finally one-half, as wide as the base of the neck; its musculature is fine and its lining indistinct. There is an indistinct cardia one-third as wide as the base of the neck. The thick-walled intestine, set off by a collum two-fifths as wide as the neck, and presenting a faint lumen, becomes at once two-thirds as wide as the body, and in cross-section is made up of but few cells containing scattered to numerous, small, more or less uniform, yellowish granules. Neither spinneret nor caudal glands have been distinctly seen. The lateral fields are one-fourth as wide as the body. Renette unknown. From the raised anus the tail diminishes rather suddenly, then tapers gently to the middle, where it is about one-half as wide as at the base; thence onward it is cylindroid to the terminus, which is one-third as wide as the base of the tail. The two simple, slender, rather frail and more or less tapering, acute to sub-acute spicula are arcuate or somewhat bent, and are one and one-half times as long

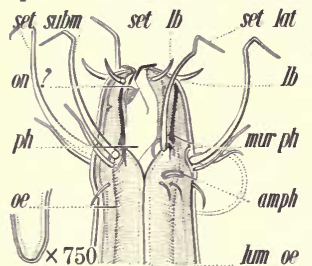

of lat as the anal body-diameter. Viewed in profile their proximal ends appear to lie dorsad from the bodyl) axis. The inconspicuous accessory piece lies parallel to the spicula. There are no supplementary organs. The ejaculatory duct is one-third, the vas deferens one-half, as wide as the body. The cylindroid testis is one-half as wide as the body, even at its rounded, blind end. Oblong, fusiform, chromatin masses are to be seen in the sperm cells.

Habitat: Sand, below low-tide mark Bathing Beach, Woods Hole, Mass., U. S. A. Sublimate to balsam. Fig. 92.

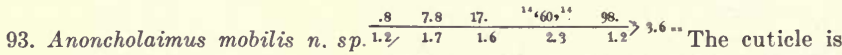
so very thin that it wrinkles on the inside of the curve when the body bends. Though there are no true wings, there is a faint, clear wing-space about one-eighth as wide as the body. The thin, flat lips resemble those of Oncholaimus. There is a vestigial dorsal onchium opposite the bases of the cephalic setae,-faintly shown in the illustration. The neck is cylindroid. In the oesophagus near the base of the pharynx there are scattered yellowish granules. Theoesophagus is a little wider at both ends than elsewhere. Near the pharynx it is two-thirds, at the nerve-ring two-fifths, and finally three-fifths, as wide as the corresponding portion of the neck. It has a distinct, refractive, apparently corrugated lining; among its radial muscles there is more or less of yellow pigmented matter, which occurs in straightish radial lines. There are at least two submedian glands in the tissues of the oesophagus, emptying at the pharynx. The thick-walled intestine is set off by a constriction half as wide as the neck, and has a faint ziz-zag lumen; it becomes at once two-thirds as wide as the body, and in cross-section presents about ten to twelve cells. There are numerous yellow granules of variable size in the cells of the intestine, the largest being one-thirtieth as wide as the body; owing to their arrangement there is a faint tessellated effect. From the anus, the posterior lip of which is elevated, the rather prominent rectum extends a distance one and one-fourth times as great as the anal body-diameter. 
The convex-conoid tail tapers from the anus to the spinneret, which is armed with submedian setae. The caudal glands lie in front of the anus. There are

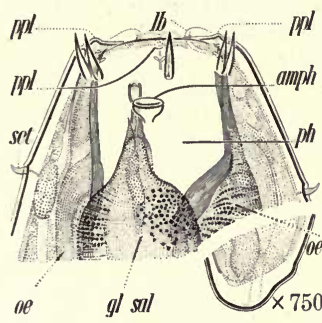

pll practically no caudal setae. The lateral fields are two-fifths as wide as the body and contain large cellular elements. Anoncholaimus has eight cervical glands, six emptying into the anterior part of the pharynx opposite the bases of the setae and two smaller, a little farther back, on the dorsally sublateral lines. The granular contents of the ducts of these glands, increased slightly in volume, form fo prominent feature of the head. These ampullae move back and forth during the various movements of the head. Hence the specific name "mobilis." The cervical glands lie behind the nerve-ring, since their ducts have been followed backward to behind the nerve-ring. There are also ducts of glands to be seen in the ventrally submedian sectors of the oesophagus. The pyriform renette cell, which lies a very short distance behind the base of the neck, empties through a rather distinct ampulla twice as far back as the base of the pharynx. The narrow nerve-ring is accompanied by large distinct nerve cells arranged in groups. From the rather large and conspicuous, more or less elevated vulva, the large muscular, non-cutinized vagina leads inward to the two straight uteri. The eggs are twice as long as the body is wide, and two and one-half times as long as wide. Judging by their size when empty, the uteri are adapted to receive one egg at a time, possibly two. The posterior ovary is a little the smaller; both are more or less cylindroid, finally tapering, of medium size, and reach three-fourths the distance back to the vulva. The ova in them are arranged single file. An ovum about to enter the oviduct is two to three times as long as the body is wide, and about one-fourth as wide as long.

Habitat: Sea-weed, Squibnocket, Martha's Vineyard, Mass., U. S. A.; Bathing Beach, Wood's Hole, Mass., U. S. A.; not common. Examined living, in water. Fig. 93.

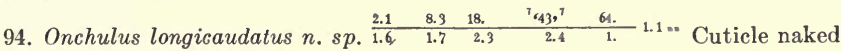
except for the setae on the head; its striae very difficult of resolution. Whether the onchium is hollow and perforated remains uncertain, but seems probable. Neck subcylindroid. The cylindroid oesophagus is at the nerve-ring three-fifths, and ostbl finally three-fourths, as wide as the corresponding portion of the neck. Throughout its length, the colorless, coarse musculature is interspersed with granular, glandular tissues, as in Oncholaimus, making it seem probable that the pharyngeal outlets are similar, but no duct has been definitely established in the dorsal onchium. There is a cylindroid cardia, about one-third as wide as the base of the neck. Scattered setae having a length equal to the breadth of two annules occur on the tail. There

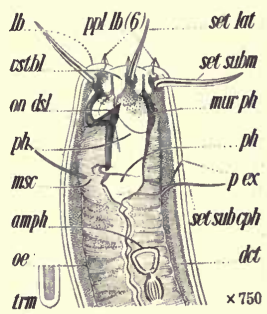
are no caudal glands. The description is derived from a single young specimen in which the vulva was not yet developed. The immature ovaries reached twothirds the way back to the location of the future vulva.

Habitat: Soil about the roots of living plants imported from Brazil into the United States. Flemming to glycerine jelly.' Fig. 94. 


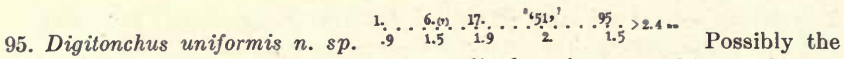
transverse striae are resolvable into exceedingly minute markings. Cuticle naked except for the cephalic setae. Lips three, possibly very faintly double. on $\quad \mathrm{d} /(6) \ldots . . \mathrm{lb}(3)$ Onchium spear-like, solid. Anteriorly, the neck is convexon ....... The oesophagus has not been well seen but ph A amph appears to be more or less cylindroid; its diameter at ad ( ) det neck. Its lining is subdistinct, its colorless musculature m 1 . the body. Cardiac collum one-third as wide as the base of the neck. From the more or less elevated anus, the prominent, cutinized rectum is about as long as the anal body-diameter. The conoid, arcuate tail tapers from the anus to the rather simple spinneret. The rather broadly saccate caudal glands are packed behind the anus in the anterior third of the tail; they empty by separate ducts. The lateral fields are one-third as wide as the body. Renette unknown. The nerve-ring is accompanied by obscure nerve cells. From the more or less continuous, inconspicuous vulva, the vagina extends inward three-fifths the distance across the body. It is muscular and more or less cutinized. The medium-sized, tapering ovaries reach half way back to the vulva.

Habitat: Beach sand, Squibnocket, Martha's Vineyard, Mass., U. S. A. Flemming to glycerine jelly. Fig; 95.

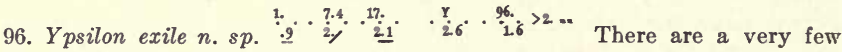
cervical setae about half as long as the neck is wide. Three flat, minute, confluent lips bear a circlet of six forward-pointing or somewhat spreading papillae around the mouth opening. Though there are six nerves, one to each papilla, they are not precisely equidistant, but seem to be arranged in three, somewhat distinct pairs. Pharynx relatively strongly cutinized. In optical contour, the anterior end of the onchium is more or less crescentshaped; this cup-shaped head is fixed obliquely on the strong, straight element that forms the main portion of the dorsal wall

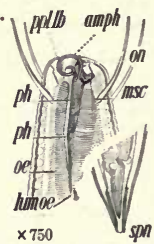
of the pharynx. The head of the onchium is yellowish in color. The neck is cylindroid posteriorly, convex-conoid anteriorly. The oesophagus, narrow and more or less cephaloboid in form, ends in a somewhat cylindroid cardiac swelling, two-sevenths as long as the neck, and three-fifths as wide as the base of the neck. At the nerve-ring, the oesophagus is one-third, just in front of the cardiac swelling also one-third, as wide as the corresponding portion of the neck. The lining of the oesophagus is rather prominent. The colorless musculature of the oesophagus is of fine texture; in it, traces of oesophageal glands have been seen. There is a distinct, refractive, cylindroid cardia, two-sevenths as wide as the base of the neck. The intestine, which is thick-walled and has a faint, zig-zag lumen, gradually becomes three-fourths as wide as the body. The cardiac collum is one-third as wide as the body. The anus is elevated, and the rectum is one and one-fourth times as long as the anal body-diameter. The intestinal cells are packed with pearly granules, such as are often seeu in Aphelenchus; the largest of these are one-eighth as wide as the body. These granules tend to be larger posteriorly than anteriorly, and sometimes appear to be polyhedral. The posterior part of the body tapers from in front of the anus, is at first conoid then convex-conoid, and ends in a spinneret one-sixth as wide as the base of 
the tail. The ellipsoidal to broadly saccate caudal glands are packed in a close tandem behind and opposite to the anus in the anterior three-fifths of the tail. Their large, distinct ampullae are two-sevenths as long as the tail. There are one or two slender setae at the middle of the tail, and near the terminus. The lateral fields are about one-fourth as wide as the body. The much elongated renette cell lies about one body-width behind the neck. The frail, strongly arcuate, rather slender, tapering, subacute spicula are cephalated by a constriction on the ventral side in such fashion that the proximal ends appear to be more or less barbed. The simple, frail, very slender, arcuate accessory piece is one-third as long as the spicula and presents a faint apophysis; its proximal end, like those of the spicula, lies dorsad from the body-axis. At first it is parallel to the spicula, then recedes and bends away at an angle of ninety degrees. There are three faint and minute supplementary organs in front of the anus, occupying a space one and one-half times as long as the body-diameter,-little more than innervations. The posterior of these is opposite the distal ends of the spicula. The distance between the first and second is equal to two-thirds, that between the second and third, to one-half, the body-diameter. At the posterior end of the middle fifth of the tail there is a somewhat raised small area supplied with two subventral nerve endings.

Habitat: Coarse sand, Beach, Devil's Island, Woods Hole, Mass, U. S. A. The sexual organs are difficult to decipher on account of the granules in the intestine. It does not seem advisable to refer this species to Acmaeolaimus Filipjev. The following differences may be noted: (1) The cuticle is coarsely striated. (2) The cephalic setae are in one circlet only. (3) The amphids are not "split." (4) The pharynx is not open. (5) The dorsal organ is "Y"-shaped. Possibly the Filipjevian specimen might have been referred to Camacolaimus de Man. Fig. 96, p. 314.

97. Gonionchus villosus $n$. sp. Annules separated by strongly refractive lines. Neck conoid in the anterior half. About twice as far from the anterior extremity as the amphids are four submedian groups of hairs, which are longer than the cephalic setae. These groups consist of three each, arranged one in front of the other, the members being separated by a distance equal to the width of one of the annules. Similar submedian long and slender hairs occur throughout the length of the body and are so numerous as to con- set qph (10) llo(6) (on/subm (2) stitute a very striking feature. The amphids at first appear "O"-shaped; in reality, however, they are spiral. The terminal flaps are hinged to the lips, and the anterior portion of each lip apparently is hinged to the main portion of the wall of amph the head, which is itself unusually thin. Onchia apparently more or less plate-like. The oesophagus continues to have the same diameter until after it passes through the nerve-ring; thereafter,

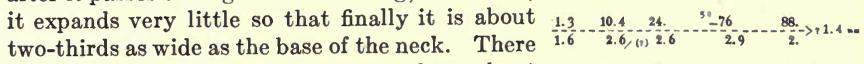
is a distinct conoid cardia, having a base about $1 .+9.5$
one-third as wide as the base of the neck. The $1.2 .22 .1,(1) 2.2$

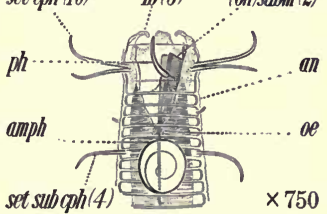
intestine becomes at once about two-thirds as wide as the body. It is rather thick-walled, and its cross-section would present about four to six cells. The lining of the intestine is refractive, so that the lumen is readily followed; in fact it is a rather striking feature. The cells contain scattered yel- 
lowish granules of variable size, arranged irregularly. The posterior lip of the anus is slightly raised. Rectum only about two-thirds as long as the anal bodydiameter. The tail is conoid in such fashion that, at a distance from the anus equal to eight times the anal body-diameter, it has a diameter about one-fourth as great as at the anus. The remainder of the tail has been lost from the specimen from which the description was drawn and is therefore of unknown form; hence the dimensions given in the formula must be interpreted accordingly. The tail is just as distinctly striated as the body. The lateral fields are about onethird as wide as the body. It is probable that the renette is located at a distance behind the base of the neck about equal to two body-diameters. At this point there is an elongated structure somewhat longer than the body is wide and about one-fifth as wide as it is long, which bears a general resemblance to a renette cell. From the slightly elevated vulva, the vagina extends inward and forward. The tapering ovary ends somewhat in front of the cardia where it is about onefourth as wide as the corresponding portion of the neck. The eggs are about three times as long as the body is wide, and about one-fourth as wide as long. There is a small posterior rudimentary branch to the sexual organs, extending back from the vulva a distance equal to two to three body-diameters. This has been seen to contain spermatozoa and may function as a spermatheca. The presence of caudal glands remains problematical. Unfortunately, also, in the case of the only male specimen available, the tail is broken, but that portion of it remaining is about two-thirds as long as the neck. It tapers a little more rapidly at first than it does farther back, - in fact, toward the terminus, it must be very nearly cylindroid. It seems probable that caudal glands are present, but there is some doubt about this. There are no special supplements, papillae or setae on the male. The long and slender hairs found on the body occur also on the tail, and are there equally long and slender so far as observed. The proximal ends of the acute, slender spicula present the peculiarity of being separated from the remainder of the spicula by straight shafts, having a length somewhat greater than that of the cephalic portions. The cephalic portion, together with this shaft constitutes about one-fourth of the spiculum; the remaining portion of the spiculum is uniformly arcuate, and tapers regularly to the subacute terminus. The proximal ends of the spicula lie toward the dorsal side of the body. There are inconspicuous accessory pieces arranged parallel to and close by the spicula, and which are about half as long as these latter. The ejaculatory duct is one-third, and the vas deferens about one-half, as wide as the body. Development of the spermatozoa in the two testes presents rather marked contrasts. From the blind end of the anterior testis, lying near the base of the neck, the spermatozoa develop regularly and form spermatocytes half as wide as the body, which develop into spermatozoa with nuclei that stain prominently. The blind end of the posterior testis contains spermatocytes which, for a short distance, resemble those already described, but they do not form large spermatocytes. They nevertheless develop into spermatozoa having the character just described. At a distance in front of the anus about equal to the length of the tail, there are three glands on each side of the body, arranged tandem. These glands are accessory to the male organs. Each is more or less homogeneous anteriorly, and granular posteriorly, the granules not retaining carmine stain, as do the nucleus and the anterior parts. The ducts of these glands lead backward to the cloaca.

Habitat: Mud, tide pool, near low tide mark, Portsmouth, New Hampshire, U. S. A. Fig. 97 , p. 315. 
98. Xanthodora nuda n. $s p$. The striae of the yellow, naked cuticle have the appearance of a series of hoops that are considerably wider on the neck, especially toward the head. The anterior half of the neck is convex-conoid, the convexity increasing steadily toward the head. From the point where the striae cease on the head, the cuticle diminishes

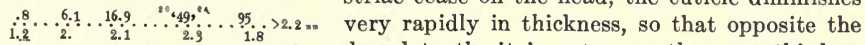
2.7. 20.6 dorsal tooth, it is not more than one-third as ${ }_{2.6}^{2.7} \ldots \frac{7.4}{3.5}+\frac{20.6}{3.8} \ldots{ }_{4.3}^{4.9}, \frac{92.3 .72}{2.9}=$ thick as a little farther back; thence onward, it is thin to a point half way between the circlet of setre and the axis of the mouth opening. At this point, the hard cuticle ceases and there is a very minute, faint groove encircling the lip-region. The lips come together so as to form a vestibule having a width about one-third as great as the diameter of the circlet of setae. The tissues of the lips apparently reach back a little farther than the apex of the dorsal tooth, and their limits are rather easily noted on account of the fact that they attract carmine stain more than the surrounding tissues do. The lip-region appears to be longitudinally striated internally, and probably to consist of about a dozen elements folded together. When the mouth is open and the onchium thrust forward, the labial region is seen to be practically continuous and entire on its anterior margin, from which it follows that any striations appearing when the mouth is closed are due to foldings of the lip-region. When the mouth is open and the lips expanded, the amphids become more nearly circular. When the lips are closed, the vestibule widens out but little in front of the dorsal tooth, whose apex lies in the axis of the vestibule. The pharynx is surrounded by muscles which appertain particularly to it, as is indicated by the fact that they are separated from the oesophagus by a broad but fairly distinct constriction. The oesophagus continues to have the same diameter until near the nerve-ring, where it diminishes somewhat in diameter. Very soon after passing through the nerve-ring, it enlarges, sometimes rather suddenly, to form the elongated posterior three-fifths of the organ. This enlarged part averages to be about three-fifths as wide as the corresponding portion of the neck, and contains a massive lining, which, when viewed in optical section, occupies one-fifth of the diameter. This enlarged portion of the oesophagus is divided into three regions by breaks in its radial musculature, of which one region comprises nearly the whole of the anterior half. The other two parts are of subequal size. Though the lining of the oesophagus is quite distinct in the narrow portion, it is considerably less so in the wider portion, and ceases a short distance in front of the posterior end of the oesophagus. Cardiac collum less than onethird as wide as the base of the neck. For a spn

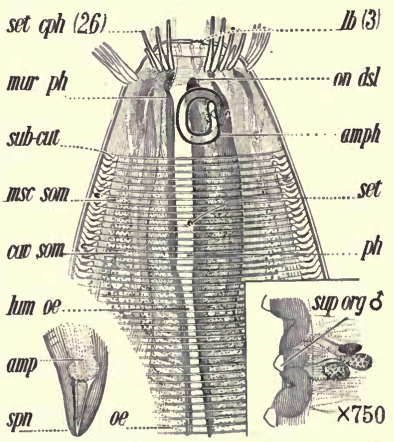
short distance, the intestine is composed of small cells differing from those which make up its main part. These cells stain more strongly with carmine and contain nuclei of relatively larger size. This region probably may be regarded as a sort of cardia. The intestine becomes almost at once two-fifths as wide as the body. Its cross-section would present about eight cells. The cells are packed 
with numerous yellowish granules. In the body cavity at the cardiac collum, there are three ellipsoidal groups of cells, two dorsally submedian and one ventral or nearly so. These groups contain eight to ten nuclei, and one of them is seen to be connected to a distinct, refractive strand, extending forward along the surface of the oesophagus. These groups of cells have their nuclei close together and seem to have a general membrane covering them. In addition to these groups of cells, there are two other granular bodies, ventrally submedian, each containing a single, strongly staining nucleus, and a second body which does not stain so strongly. The structure of these latter is suggestive of that of the ordinary renette cell, but their connections have not been made out. Rectum somewhat longer than the anal body diameter. The three caudal glands are arranged in a close tandem in front of, opposite to and behind the anus. Each is a large cell with a protoplasmic network, in the midst of which the single nucleus is slung. The lateral fields are about one fourth as wide as the body and are composed of two ranges of cells with a space between them, in which lie nuclei belonging to cells of a different group. Renette unknown. From the slightly raised anus, the arcuate tail is conoid to the terminus, which has a diameter about one third to one half as great as that of the base of the tail. From the very slightly elevated vulva, the well cutinized vagina extends fully half way across the body. The ovaries reach half way back to the vulva and contain ten to fifteen ova arranged, for the most part, single file. The moderately thin-shelled eggs are twice as long as the body is wide and one-third as wide as long. They occur in the uteri one at a time. The spermatozoa in the uterus are one-seventh as wide as the body. The conoid, arcuate tail of the male begins to taper from a short distance in front of the anus. The six supplementary organs occupy a distance equal to the length of the tail. The posterior supplement is nearly opposite the posterior thirds of the spicula; the second, third and fourth succeed each other without intervening spaces, in such a way that the third and fourth are pretty nearly opposite the proximal ends of the spicula. The fifth is separated by a greater space from the fourth, and the sixth is about as far from the fifth as the fifth is from the fourth. The fifth and sixth are of smaller size than the others, the inconspicuous sixth being the smallest of all. Their apices are more transparent than the other portions. An unusual feature is the presence of nerve endings between the organs. It is possible that nearer the anus still than any described, there is another inconspicuous organ of somewhat the same character. The strongly arcuate, somewhat acute, yellowish spicula are about twice as long as the anal body-diameter. They appear to be widest in the middle and to taper both ways. The cephalated portion of the spicula are cut off obliquely. The proximal ends lie a little to the dorsal side of the body-axis. The accessory pieces are for the main portion of their length not very conspicuous. Surrounding the distal ends of the spicula, however, there is a rather prominent portion which must be reckoned a portion of the accessory apparatus. Oblique copulatory muscles are present for a distance twice as great as the space occupied by the supplementary organs. The ejaculatory duct is about one-third, and the vas deferens a little more than one-third, as wide as the body. It remains uncertain whether there is one testis or two, but the evidence seems to favor the supposition that there is one only, and that its blind end lies about as far behind the base of the neck as the nerve-ring is in front of it.

Habitat: Larat, East Indies. Marine. Fig. 98, p. 317. 


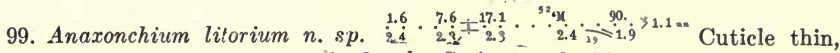
but considerably thicker near the head. Striae resolvable with difficulty into dot-like elements, which are close together on the body, more distant on the head. The striae are altered on the lateral fields so as to give rise to a ladder-like pattern, with the "rungs" very close together. Cephalic setae two-jointed. No pores are seen in the cuticle; nevertheless, they may have been present and escaped notice. The lips are thick and double. In the single male specimen examined, the conical branches of the lips end in minute setae, each about as long as the labial setae, and terminating in a "blob,"-this latter hardly an artefact. Dorsal onchium opposed on the ventral side by several irregularly arranged, small denticles, among which probably two submedian ones dominate. Apparently somewhat similar denticles occur behind and above the dorsal onchium. Amphids peculiar, large, faint. The plain oesophagus, near on the nerve-ring is one-half, and finally three-fourths, as wide as the corresponding portion of the neck. Its lining appears corrugated; its colorless musculature is more or less coarse, but there seem to be no indications of the presence of glands, and there is no distinct cardia. The thin-walled intestine, which has a rather faint, though distinctly visible lumen, becomes at once half as $\times 750$

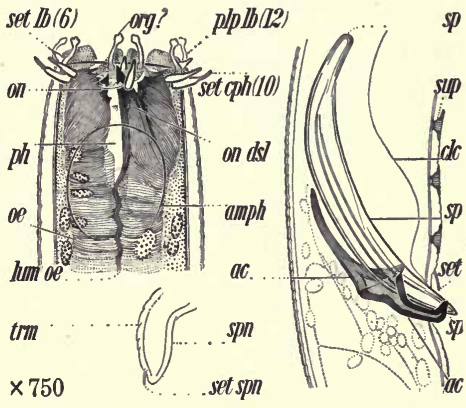
wide as the body, and in cross-section would present four to six cells. Cardiac collum one-half as wide as the neck. The cells of the intestine contain scattered yellow granules of variable size, having a diameter about equal to the width of two annules of the cuticle. The tail tapers from the anus, and is at first conoid then cylindroid in the posterior third, where it is about one-fourth as wide as at the anus. A few, rather small, tapering, subacute setae occur on the tail. The lateral fields are about one-third as wide as the body. The non-granular ellipsoidal renette cell lies only a short distance behind the neck, and is three-fourths as long as the body is wide, and one-third as wide as long. The rather pronounced clavate ampulla empties through the distinct pore lying close to its anterior end. The nerve-ring is accompanied by obscure nerve cells. The yellowish spicula are guided by two separate, strong, rather wide, more or less arcuate, somewhat shoe-shaped pieces near the anus, with a single median piece between and behind them. Ejaculatory duct one-fourth, testis two-thirds, as wide as the corresponding portion of the body.

Habilat: Marine; Belmar, N. J., U. S. A., below low tide mark in beach sand. Female unknown. Anaxonchium bears considerable resemblance to Cyatholaimus, but differs, among other ways, in the almost obsolete amphid (?), the reflexed testis, and in the large number of small supplementary organs. Fig. 99.

100a. Bolbolaimus pellucidus n. sp. Type species. Naked except for the setae on the head; possibly there are very inconspicuous wings. Lips subdistinct, the region elastic and finely subdivided. Margin of the lip-region serrated by the forward projection of about fifteen papillae (?) with minute bristles outside the serrations. The appearance is as if each papilla is armed with a pair of minute 
bristles very difficult to see. Onchium capable of being protruded past the lips. Opposite the onchium is a pseudo-onchium, somewhat smaller, and more distinctly visible in some attitudes of the pharynx than in others. Amphids exceedingly inconspicuous, nearly circular, a little the wider transversely, apparently circular but really consisting of spirals of one wind slightly open behind. At the nerve-ring, the oesophagus is three-fifths, just in front of the cardiac bulb one-half, and finally five-sixths, as wide as the base of the neck. This latter measurement is that of the elongated, ellipsoidal cardiac bulb. The rather prominent, apparently corrugated lining of the oesophagus is distinctly visible. set lb(6) $6.6 \quad 129 \quad-46.6=$ The musculature

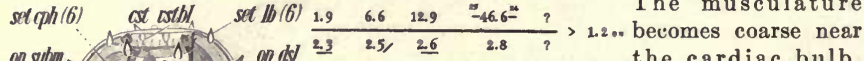

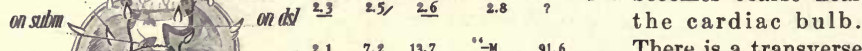

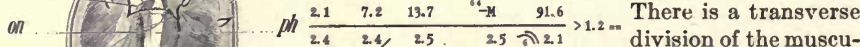

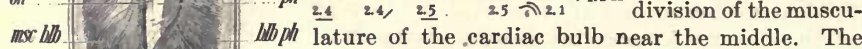
con sam - . of wall of the intestine is of variable thickness; the lat lum lumen is distinct. Gradually the intestine becomes sm ms oe ind $\times 750$ cross-section would present five to six cells. Often the lumen of the intestine presents the appearance of having certain cells bulging inward. Cardiac collum one-sixth as wide as the neck. From the more or less elevated anus, the cutinized rectum extends inward for a distance fivesixths as great as the body-diameter. From the anus, the tail is conoid to the rather blunt terminus. The long, simple spinneret is in its basal part onethird as wide as the base of the tail. The broadly saccate caudal glands form a loose tandem behind the anus in the anterior half of the tail; they empty through separate ducts and ampullae, the latter elongated. The lateral fields are one-third to two-fifths as wide as the body. The ellipsoidal, non-granular renette cell lies close behind the neck, and is two-thirds as long as the body is wide and two-thirds as wide as long. The ampulla is one-third as long as the neck is wide, and three-fourths as wide as long. The nerve-ring is accompanied by obscure nerve cells. From the small, inconspicuous, rather continuous vulva, the small tubular vagina extends inward one-third the distance across the body. Its inner wall is cutinized, so that its rectangular furcation is plainly to be seen. The ellipsoidal eggs are one and one-half times as long as the body is wide and appear to begin segmentation before being deposited. One or two at a time occur in the uteri, which are five times as long as the body is wide. The somewhat narrow, tapering ovaries contain about a dozen ova arranged single file for the most part, but irregularly near the blind end. The faint, acute, tapering, stoutish spicula are colorless and one and one-half times as long as the anal body-diameter. They are so located that their proximal ends appear to be opposite the body-axis. The simple, strong, separate accessory pieces are somewhat slender. They are yellow and quite as conspicuous as the spicula. Their applied parts are two-thirds as long as the spicula, from which they recede a little. Their proximal ends appear to lie dorsad from the body-axis. The six, very small and inconspicuous, equidistant supplementary organs occupy a distance somewhat less than the length of the tail. The last is opposite the proximal parts of the spicula. The spaces between them are about equal to half the body-diameter. They hardly disturb the ventral contour of the body. Possibly there is also one near the anus. They are papilloid and have but slight elevation. The ejaculatory duct is one-half, the vas deferens one-half, and 
the testes two-thirds, as wide as the corresponding portion of the body, but the testes taper so that at their blind ends they are only one-half as wide as the body. The various regions of the male sexual organs are separated from each other by narrower channels. After synapsis, the spermatozoa grow and become granular.

Habitat: Sand, below low tide mark, Belmar, N. J., U. S. A. Sublimate to balsam. Fig. $100 a$, p.. 320 .

b. Bolbolaimus punctatus n. sp. $\frac{1.8}{2.6}-\frac{5.5}{2.8}-\frac{13}{3}-\frac{34}{3}-\frac{46}{3.3}--\frac{93 .}{2.3}>1.6 \mathrm{~m}$ Striae resolvable into rows of dots, of which about every third row is a trifle coarser, thus indieating the real width of the annules. Labial papillae six, setose, about one-third as long as the cephalic setae. Around the mouth, there are fine, longitudinal striations due to foldings in the vestibule. The punctate walls of the pharynx are well-cutinized. There are two very small ventrally submedian projecting onchia opposite the dorsal onchium. The oesophagus near the nerve-ring is two-fifths, just in front of the cardiac bulb a little more than two-fifths, and finally three-fourths, as wide as the base of the neck. This latter measurement is the diameter of the somewhat elongated cardiac bulb, which is two-sevenths as long as the neck. There is an obscure, elongated, two-parted valve in the cardiac swelling, one-third as wide as the swelling set $\mathrm{Thh}$ set $1 \mathrm{~b} / \mathrm{ll}$ ) set subm itself. The cardiac valve is divided into three parts, corresponding to breaks in the muscula- on subm sut toh ture, the anterior part inconspicuous, the other two each occupying nearly one-half the length $p e x$ of the bulb. The thick-walled intestine becomes at once three-fifths as wide as the body. Its ph cross-section would present three to four cells, containing scattered granules of variable size, lum of the largest of which are about one-twenty-fifth as wide as the body. Cardiac collum one-fourth as wide as the base of the neck. From the con- spn

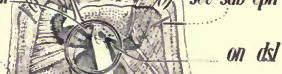
on dsl tinuous, inconspicuous anus, the rectum extends inward a distance two-thirds as great as the anal body-diameter. The conoid tail tapers from the anus to the blunt, conoid, symmetrical, unarmed spinneret. The broadly saccate caudal glands lie behind and opposite the anus in the anterior half of the tail. Measured near the neck, the lateral fields are one-fourth as wide as the body. The ellipsoidal, non-granular renette cell, one body-width behind the neck, is one-half as long as the body is wide and five-sixths as wide as long. From the small, inconspicuous, continuous vulva, the rather weak vagina extends inward two-fifths the distance across the body. The elongated eggs are as long as the body is wide and three-fourths as wide as long, and are packed three to five in each uterus at once. The tapering ovaries contain about twenty ova arranged single file.

Habitat: Marine sand, Nobsca Beach, Woods Hole, Mass., U. S. A. Flemming to glycerine jelly. Fig. $100 b$.

101. Acanthonchus viviparus $n$. $s p$. There are distinct wings, whose presence is indicated by about four to six longitudinal striations, resolvable, at least the outer ones, into rows of dots. The wings extend from near the head to near the tail. Neck conoid. Setae two- (or three-) jointed. The lip-region appears to be divided into twelve parts, or into six parts, each of which is double. The mouth cavity is cyathiform and shallow when the lips are closed and is entered 
through a narrow vestibule. The cavity itself is very small, and a good deal wider than it is high when the lips are closed. In fact, when the lips are closed they appear to form a depression on the front of the head, so that their interior margins actually rest on the dorsal tooth and on the base of the pharynx. The pharynx actually continues to twice the depth indicated in the formula, though when the mouth is closed this posterior portion appears to be very similar to the lumen of the oesophagus. The oesophagus swells a little in the anterior third, then diminishes almost imperceptibly, and finally swells toward the posterior extremity where it is two-thirds as wide as the base of the neck. The thick-walled intestine becomes at once two-thirds as wide as the body, and expands so as soon to be three-fourths to four-fifths as wide as the body. Its cross-section would probably present about eight cells. The elongated ventral

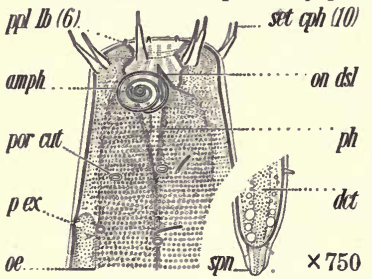

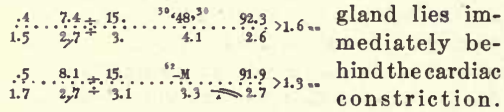
The lateral fields are about one-third as wide as the body, and are composed mainly of a double row of very prominent ellipsoidal cells, which in the females at least, may be most clearly seen in the region of the neck. From the slightly raised vulva, the vagina leads inward about half way across the body. The uteri are generally about one and one-half times as long as the neck, and contain numerous developing eggs or embryos. The ovaries are reflexed for a distance about twice as great as the body-diameter, and contain a dozen or more ova arranged in several series. This species is viviparous. Tail of the male is very much like that of the female, but more arcuate. Its terminus has a diameter one-fifth as great as the diameter of the base of the tail. The equidistant supplements are of unequal size, those more distant from the anus being much the larger. The first is opposite the proximal ends of the spicula and is very minute. It is visible mainly on account of the refractive nature of the material of which it is composed. The second is about twice as far from the anus as the first and of about the same size. The third, which is three times as far from the anus as the first, is very much larger, having a length half as great as the body-diameter; its internal walls are brown and strongly lined and refractive. It is somewhat curved and passes inward and forward; its proximal portion is slightly cephalated. The fourth of these organs is more than four times as far from the anus as the first, and is more than twice as long as the third, having a length approximately equal to the bodydiameter. Its internal walls are strongly cutinized, and it is a very prominent feature, almost as much so as the spicula. Where it passes through the cuticle, its point is curved backward; thence inward, it is somewhat sigmoid, enlarging all the time, so that its proximal portion is about one-fifth as wide as the corresponding portion of the body. The slightly arcuate, rather uniform spicula are somewhat longer than the anal body-diameter. Their proximal ends are not distinctly cephalated. Parallel to the spicula, and extending inward from the prominently raised anus for a distance three-fourths the length of the spicula, are the accessory pieces, whose distal extremities are two to three times as wide as the spicula, but which decrease in size internally. They are considerably more prominent than the spicula. The ejaculatory duct is about half as wide as the body. 
Habitat: Marine mud, San Pedro, California. Possibly another species exists at Woods Hole, Mass., U. S. A. Sublimate to balsam. Fig. 101, p. 322.

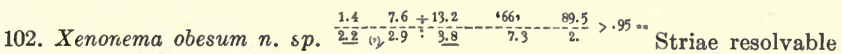
with high powers into rows of dots or elongated markings which are altered somewhat on the lateral field, where there is a wing extending from near the base of the neck to the base of the tail. There are setae on all parts of the body, but they do not appear to be very numerous. Pharynx apparently consisting of a cavity whose walls are folded and which is capable of being opened outward. The oesophagus continues to have the same diameter until near the posterior end, where it contracts slightly and then expands to form the pyriform cardiac bulb, four-fifths to five-sixths as wide as the base of the neck. There is no cardia. The intestine at first is only one-fourth as wide as the oesophageal bulb. Its cross-section presents about four or five cells; that those next the oesuphagus are physiologically different from those following is shown by the way in which they stain. The intestine soon enlarges so that its maximum diameter is twice as great as that presented near the bulb, but nowhere does the intestine become more than about half as wide as the body. Its cells contain a few yellowish or brownish granules of variable size, the largest of which have a diameter about equal to the distance between two striae of the cuticle, the smallest being not more than one-tenth as wide; otherwise, the cells of the intestine are very transparent so that their nuclei can readily be seen. These latter are of large size and each presents a distinct nucleolus; the average diameter of one of these nuclei is rather more than double the distance between two successive striations. The anus is elevated; the rectum is two and one-half to three times as long as the anal body-diameter. Immediately behind the anus, the tail diminishes abruptly in diameter, and in this region for a short distance near the anus on the ventral surface, the striations of the cuticle are much less conspicuous. The location of the excretory pore and the ventral gland remains uncertain, but it appears possible that the latter lies a short distance in front of the cardiac bulb. The lateral fields are about one-fourth as wide as the body, corresponding approxinately in width, at least in the anterior part of the body, with the alterations in the transverse striae which exist along the sides of the body. The tail is conical from a short distance behind the anus. Behind the anus, the tail diminishes so rapidly in diameter, especially on the ventral side, that it becomes almost at once only about two-thirds as wide as at the anus; thence ann onward, it is conical. The three caudal glands are located in a tandem series in front of the anus. From the enor-

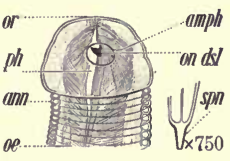
mously developed vulva, the vagina leads inward and forward at an angle of forty-five degrees with the body-axis a distance equal to the span of eight to ten striae. The walls of the vagina are brownish, thick, and highly refractive. There is a single uterus connected with two short ovaries, each containing three to four ova. This entire system of organs is clustered near the vulva and occupies a distance about five times as great as the average body-diameter. The size and nature of the eggs remains to be discovered. Overhanging the vulva is a flap-like expansion of the cuticle which adds much to the ventral prominence. Tissues of the body-wall are contained also in the cavity of this peculiar expansion of the cuticle. Owing to the peculiar development in this region, the diameter of the body here is more than twice the average body-diameter. In fact 
these structures are so very peculiar as to suggest abnormality, but as there is no evidence of any disease or malformation in the single, very transparent specimen examined, it seems best to regard it as the normal form of a very exceptional species.

Habitat: Salt River, Jamaica, in six inches of water. Fig. 102, p. 323.

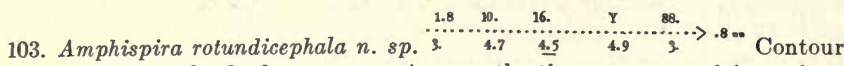
plain, or exceedingly finely crenate. Apparently there are very faint subcephalic setae near the base of the "cuirasse." At the mouth opening of the single specimen examined there are three punctate spherical elements, as shown in the illustration. The significance of these remains problematical; while they may possibly be fixation products, such a supposition is doubtful. Neck conoid. The oesophagus retains the same diameter until it expands to form the flattishpyriform cardiac bulb, which has a faint valve one-third as wide as itself. There appears to be an almost imperceptible break in the musculature near the middle of the oesophagus, and a corrresponding very slight swelling. The thick-walled intestine has a faint lumen and soon becomes one-half as wide as the correspond-

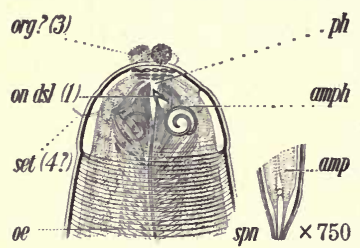

ph ing portion of the body. Its cross-section presents few cells. From the apparently continuous anus the prominent rectum extends a

amph distance equal to the anal body-diameter. The scattered to rather numerous, spherical granules found in the cells of the intestine are of variable size, the largest being one-thirtysixth as wide as the body. Tail arcuate, conoid, ending in a spinneret one-third as wide as its base. The broadly truncate caudal glands are located opposite to and behind the anus in the anterior third of the tail. The lateral fields are one-third as wide as the body. What appears to be the renette cell is located about three body-widths behind the neck; it is one-third as long as the body is wide and onehalf as wide as long. The location of the excretory pore remains unknown. Nerve-ring oblique. From the location of the rudimentary sexual organs in the single young specimen seen it seems probable that they will develop to be double and symmetrically reflexed.

Habitat: Soil, Arlington Farm, opposite the District of Columbia, U. S. A. Flemming to glycerine jelly. This species almost seems out of place in the habitat in which it was discovered, as it is most closely related to marine forms. Fig. 103.

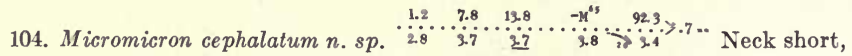
cylindroid. There seems little doubt that the mouth cavity is armed with a single, dorsal tooth, which can be placed so closely against the dorsal wall of the pharynx as to be difficult of detection, although the tooth itself is of considerable magnitude. It appears to have an acute apex, located opposite to or a little behind the labial constriction. Oesophagus cylindroid, ending behind in a prolate or pyriform cardiac bulb four-fifths as wide as the base of the neck. Cardia faint. The intestine, which joins the middle of the posterior surface of the cardiac bulb, is at first about one-third as wide as the neck. It widens out gradually so as to become about half as wide as the body, and its cross-section would appear to be composed of about six to eight cells containing yellowish granules of rather uniform size. The lateral fields are about one-fourth as wide as the 
body. Just behind the base of the neck, there are cells which stain a little more strongly than the adjacent structures,--possibly renette cells. The tail of the male is conoid to the terminus, where it has a width one-fourth as great as at its base. The caudal glands seem to be located in the base of the tail. The presence of a series of ventral supplementary organs is indicated by corrugations occurring in the cuticle on the ventral surface when the posterior extremity is incurved. This series extends from the anus forward a distance about equal to twice the length of the tail, and must be composed of about twenty elements. Each probably consists of an inconspicuous modification of the ordinary cuticle, in the midst of which there is a nerve ending. In front of the anus on the ventrally submedian lines there are series of setae, each seta being nearly onethird as long as the body is wide. There is one of these setae in front of the anus on each side, a second pair nearly opposite smo the proximal ends of the spicula, and so on. Similar setae occur on the anterior two-thirds of the tail on the submedian lines. When seen in profile, the spicula appear at the middle to be about one-third as wide as the body. They taper rapidly to the acute distal extremities. The proximal ends are slightly arcuate in a ventral direction. Near the proximal extremities there is a break in the framework on the ventral side. The framework of the spicula is rather frail, considering the size of the spicula themselves. Muscular fibers appear to pass from the inner free end of the accessory piece backward to the ventral surface of the anterior portion of the tail.

Habitat: Punta Arenas, Pacific Coast of Costa Rica. The intestine contained a considerable number of diatoms. Sublimate to balsam. Fig. 104.

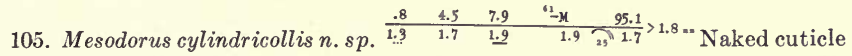
traversed by exceedingly fine, plain transverse striae. Neck cylindroid. In certain aspects, the amphids appear to consist of one circlet within another, but are in reality true spirals. No doubt the papillae surrounding the mouth opening correspond with folds in the lip-region; these, however, are so very minute as to be difficult of resolution. From the base of the onchium backward, the pharynx is narrow and tapering, and joins the lumen of the oesophagus in an indefinite way at a point distant from the anterior extremity nearly equal to the diameter of the front of the head. From behind the pharyngeal swelling, the oesophagus continues with about the same diameter until it expands to form the rather obscurely pyriform cardiac bulb, which is fully three-fourths as wide as the base of the neck, and contains a relatively large setcph(14) ppl(12) setll(6) though rather simple valvular apparatus, having a diameter half as great as that of the bulb itself. This valve is about twice as long as wide. The relatively narrow intestine joins the middle of the posterior surface of the cardiac bulb and soon enlarges so as to be two-fifths as wide as the body. There is no distinct cardia. In cross- setsuloph $\times 750$. $s \mathrm{gn}$ section, the intestine presents six to eight cells. The lateral fields are about one-fourth as wide as the body. Nothing is known concerning the renette or the excretory pore. The nerve-ring surrounds the oesophagus squarely. The tail of the male is strongly arcuate and conoid from the prominently raised anus. The terminus is blunt and bears a simple spinneret. The caudal glands are packed together in the anterior third of the tail, extending a little in front of the 
anus. The twenty-five supplements are rather simple and when the body is curved, take on the form of semi-cylindroid, transverse elevations, the distance between them increasing anteriorly. There are two opposite the spicula and a third a short distance in front of the proximal ends of the spicula; and then for a distance equal to four times the length of the tail, the organs are separated by spaces not greater than their own width. Thence forward, the organs are separated by wider spaces, and occasionally one of the series appears to be missing. The two anterior ones are separated by a distance about twice as great as the width of one of the organs. The entire series occupies a distance about eight times as long as the tail. No special papillae or setae have been seen on the tail end, either in front of the anus or behind it. The proximal ends of the strongly arcuate, slender-looking spicula appear to lie nearly opposite the body-axis. Apparently connecting the cephalic expansions with the anus are slightly curved, refractive lines, indicating that the spicula may be broader than would appear from an examination of only the more striking portions of the framework. Accessories parallel to, and two-fifths as long as the spicula. Ejaculatory duct onethird as wide as the body. The blind end of the testis is about half as wide as the body.

Habitat: Mud, tide pool, Portsmouth, N. H., U. S. A., near low tide mark. This genus resembles Onyx. Fig. 105, p. 325.

106. Polysigma uniforme n. $s p$. Cuticle, if striated at all, very finely so. Body naked except for the setae on the head. Lips minute, more or less confluent, relatively thick, six and double, or possibly twelve. Near the nerve-ring and just in front of the cardiac bulb, the oesophagus is one-half, and finally three-fourths, as wide as the corresponding portion of the neck, this latter figure being the measurement of the pyriform cardiac bulb. The refractive lining is one-twentieth as wide as the oesophagus. In the posterior half of the oesoph-
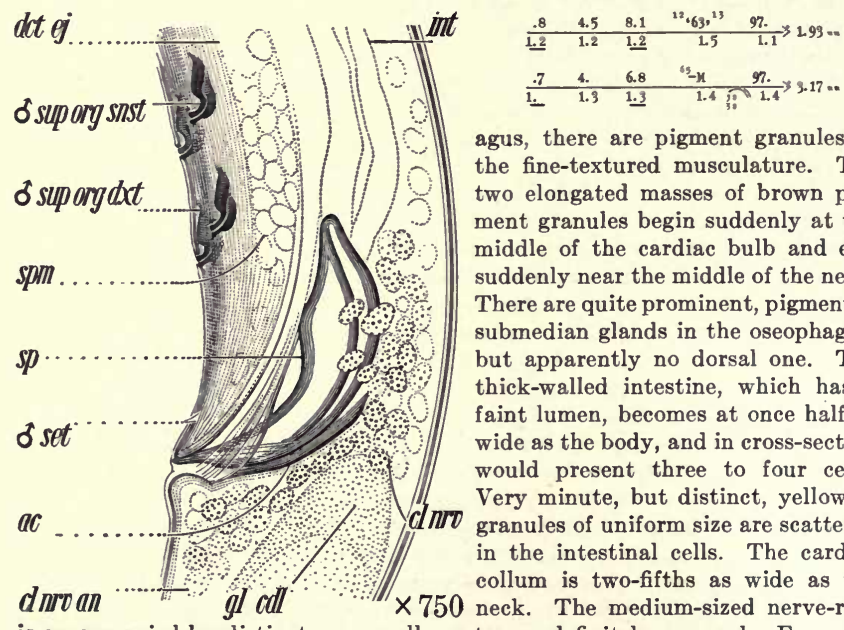

agus, there are pigment granules in the fine-textured musculature. The two elongated masses of brown pigment granules begin suddenly at the middle of the cardiac bulb and end suddenly near the middle of the neck. There are quite prominent, pigmented submedian glands in the oseophagus, but apparently no dorsal one. The thick-walled intestine, which has a faint lumen, becomes at once half as wide as the body, and in cross-section would present three to four cells. Very minute, but distinct, yellowish granules of uniform size are scattered in the intestinal cells. The cardiac collum is two-fifths as wide as the is accompanied by distinct nerve cells, not very definitely grouped. From the more or less elevated vulva, the fairly-well cutinized vagina extends inward half 
way across the body. The eggs are probably about three times as long as the body is wide. The broad, tapering ovaries, which contain about ten ova arranged mostly in single file,-but irregularly near the blind end,- reach about half way back to the vulva. The tail of the male is conoid to the spinneret, which is nearly one-third as wide as the base of the tail itself. The slightly yellowish, rather strong, subslender, rather acute

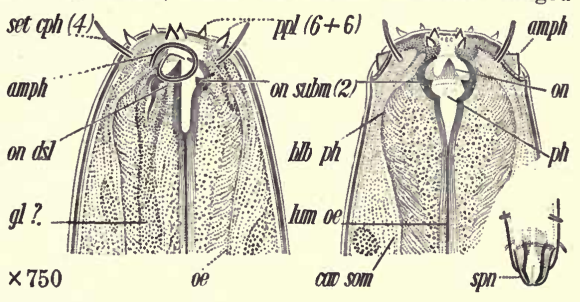
spicula appear to have their slightly expanded proximal extremities nearly opposite the body-axis. There are seventy-six yellowish, refractive supplementary organs in two subventral rows of thirty-eight each. They are subequidistant, but somewhat wider apart anteriorly. They appear to be more or less protrusile. Ejaculatory duct one-third to two-fifths, the testis one-half, as wide as the body. The testis is cylindroid, but tapers near its blind end.

Habitat: Marine mud, twenty-five fathoms deep, Woods Hole, Mass., U. S. A. Fig. 106a, p. 326; Fig. $106 b$.

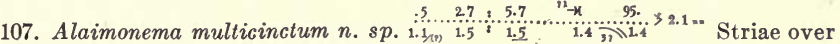
1500 to the millimeter, resolvable with difficulty into very exceedingly fine dotlike elements. Cephalic and subcephalic setae sixteen, in four sets of four. Cervical setae scattered, the first four to eight larger than the cephalic setae, the others smaller. Somatic setae scattered, inconspicuous, about one-sixth as long as the body is wide. In two series on the borders of the lateral fields, every

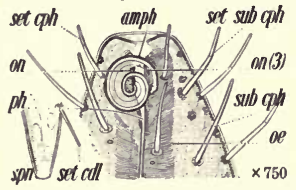
twenty to thirty annules throughout the nema, there are round, pore-like markings, twice as wide as the annules. The two rows of pores opposite the lateral fields are removed from each other by a distance about equal to three-fifths the bodydiameter. There are no lips. The simple, more or less triquetrous pharynx is so small as to be very easily overlooked. The three very small onchia are more or less equal in size. Their forward-pointing, acute apices are about opposite the anterior borders of the amphids. The oesophagus is at first three-fifths, near the nerve-ring and in front of the cardiac bulb two-sevenths, and finally three-fourths, as wide as the corresponding portion of the neck. The pyriform cardiac bulb contains a more or less spheroidal, simple valve, two-sevenths as wide as the bulb itself. The oesophagus has a subdistinct, narrow lining, and a fine musculature. The intestine, which has a more or less thick wall and a faint lumen, soon becomes half as wide as the body. In cross-section it presents but few cells. The cardiac collum is onefourth as wide as the base of the neck. The widest of the variable, colorless granules found scattered in the intestinal cells are twice as wide as one of the annules. The longitudinal fields are three-fifths as wide as the body. The tail is conoid from the rather prominently raised anus. The large, rather frail, tapering, somewhat blunt spicula, at their widest parts, near the proximal ends, are one-fourth as wide as the corresponding portion of the body. There is a rather frail, slender accessory piece. The elevated, "campanulate," subequidistant supplementary organs begin opposite the proximal parts of the spicula and are stationed at a 
distance from each other varying from one-sixth of one, to one, entire bodydiameter. The organs are nearly as high as they are wide, and are more or less asymmetrical. Each is connected with an internal, refractive piece, a little farther forward. They somewhat resemble the well-developed supplementary organs of Chromadora. The ejaculatory duct is one-half, the vas deferens threefifths, as wide as the body. A considerable portion of the male sexual organs is filled with spherical granular spermatocytes, one-third as wide as the body, and having a distinct ectosarc.

Habitat: Beach sand, Bathing Beach, Woods Hole, Mass., U. S. A. Flemming to water. Fig. 107, p. 327.

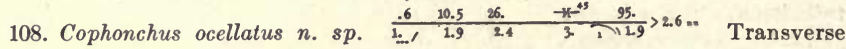
striae exceedingly minute; the cuticle is also longitudinally striated. The somepol lb Ib Ib on what cylindroid neck is almost imperceptibly convexset subm conoid in its anterior part. Cuticle relatively thicker pht on the head. A few short setae occur here and there on the neck. There appear to be three somewhat confluent 0 lips with papillae on their outer margins, probably to the number of six. There are two dark-brown dorsally submedian eye-spots and opposite them in the ventral portion of the oesophagus a linear collection of pig$o$ mented granules, from which there also extends backward a line of scattered granules. Similar granules occur $\operatorname{spn} \times 750 \quad$ Ium $0 e$ throughout the oesophagus in groups of a score or more. Spear-like onchium very slightly sigmoid. It is probable that the pharynx extends back to opposite the row of cephalic setae. The cuticle on the anterior surface of the head, as far back as the cephalic setae or a little farther, reminds one of the covering on the head in Thoracostoma, but the thickening is less pronounced, and the cuticle here is not materially different in color or texture from that elsewhere on the body. Where the cuticle reaches its maximum thickness, near the lips, it is three to four times as thick as elsewhere on the body. From this point backward to near the base of the head, it gradually grows thinner. The oesophagus continues to have the same diameter until after it passes through the nerve-ring; there it begins to enlarge, so that finally it is two-fifths as wide as the base of the neck. The intestine, set off by a deep constriction, becomes at once about two-thirds as wide as the body. Its cross-section would present four to six cells packed with minute granules. The conoid cardia is one-fourth as wide as the corresponding portion of the body. The renette cell is about half as wide as the body, and is located on the ventral side just in front of the cardia; its pyriform ampulla is one-fifth as wide as the corresponding portion of the neck. The lateral fields appear to be about one-third as wide as the body, and to contain numerous, rather small, scattered nuclei. The nerve-ring surrounds the oesophagus squarely. Tail of the male conoid in the anterior fourfifths; thence cylindroid for a short distance to the almost imperceptibly swollen terminus. The caudal glands are apparently located opposite to and a little behind the anus, and present the peculiarity that one of them, the one on the ventral side, has a separate duct leading to the separate smaller ampulla in the slightly enlarged spinneret, while the other two have their ducts parallel and close together and appear to join in a single, much larger ampulla opposite the smaller one. From these ampullae, minute ducts lead backward to the pore of the spinneret, which presents a minute, conoid depression on the terminus. Supplementary organ tubular, of about the same diameter as the spicula, extend- 
ing first inward, then directly forward. It is about one-third as long as the corresponding body-diameter. Its outlet is opposite the middle of the spicula. There appear to be some obscure papillae on the tail, evidenced by almost imperceptible ventral elevations. Mention may be made of one of these near the beginning of the middle third, and another near the beginning of the posterior third. Spicula slender, uniform, acute, one and one-half times as long as the tail, and five to six times as long as the anal body-diameter. Their proximal ends are cephalated by expansion. They are accompanied, both in front and behind, at their distal extremities, by accessory pieces two-thirds as long as the anal body-diameter. The ejaculatory duct is one-fourth as wide as the body, and extends forward to near the middle of the body, where it joins the testes.

Habitat: Shoal in Kingston Harbor, Jamaica, in about one foot of water. This genus bears a very considerable resemblance to Digitonchus. Fig. 108, p. 328

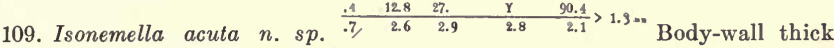
and muscular; in the middle of the single specimen seen it takes up about threefifths of the radius. There are a few exceedingly minute setae to be seen on the tail; otherwise than these, and those shown in the illustration, no others are known. There appear to be three lips, but they are so small and so thoroughly amalgamated that it is very difficult to decide on their structure, and it is possible that there is no division of the margin of the mouth into lips. The mouth opening is nearly as wide as the front end of the head. Between the thin lips there is a very short vestibule, whose limits are marked by the presence of an internal refractive structure on the inner wall of the pharynx very near the margin of the lips. It is impossible to say from the observations so far made whether this refractive marking is a circular affair or whether it is dis- set (10). continuous and represents special thickenings on the inner surface of the three components of the lip-region. The result of this thickening is a sudden, but very slight narrowing of the vestibule. The very short vestibule, there- $o c$ fore, begins nearly as wide as the front of the head, widens out posteriorly and then soon suddenly narrows to the beginning of the pharynx proper. At its base this latter tapers to join the lumen of the oesophagus. At an som first sight it appears that, with the exception of the abovementioned, excessively minute, cutinized processes at the base of the vestibule, the pharynx does not present any feature suggestive of a particular armature, but there is $\$ p n$

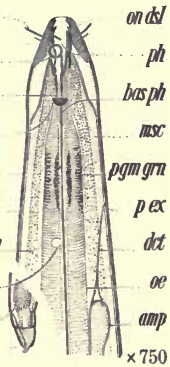
nevertheless a minute, forward-pointing ventral (?) onchium of small size. The walls of the pharynx; though distinctly outlined by the refractive nature of the cuticle, are very thin throughout. The lip-region stains more strongly than any of the succeeding tissues of the head, or of the anterior part of the neck. All that portion of the lip-region in front of the middle of the pharynx takes part in this staining, and the posterior contour of the stained portion extends outward and backward from the front part of the pharynx. Behind this stained portion, indicated in the illustration, there are two elongated "cavities," beginning opposite the middle of the pharynx and ending a little behind the eye-spots, one dorsal and one ventral, and the outer contour of the head opposite these "cavities" is very slightly elevated, in the single specimen so far examined. When viewed in profile these "cavities" have a size and contour 
somewhat similar to that of the pharynx. The amphids, difficult to observe, have a somewhat elongated, inward, refractive extension, as shown in the illustration. Eye-spots colorless or slightly yellow. There are rather inconspicuous collections of golden brown pigment granules in the anterior portion of the oesophagus. This latter continues with much the same diameter for some distance, but gradually enlarges. After it passes through the nerve-ring it is twofifths as wide as the corresponding portion of the neck, and it finally becomes three-fifths as wide as the base of the neck. Its radial musculature is very strongly marked in the posterior half, reminding one to some extent of the oesophagus in Bolbella. The nerve cells both before and behind the nerve-ring are an unusually prominent feature. A little more than half way from the head to the nerve-ring the foremost of these cells occur as two lateral ellipsoidal granular cells half as wide as long, and having a length one-third as great as the corresponding width of the neck. Almost on a level with these is a ventral cell, and immediately behind them are other lateral cells of somewhat smaller size, and thence onward the neck is packed with cells of the same character, which gradually beeome globular and continue behind the nerve-ring and cease about half way between the nerve-ring and the cardia. There is a collection of similar cells in front of and behind the anus. The cardia is convex-conoid and about onethird as wide as the corresponding portion of the body. The intestine becomes at once about two- to three-fifths as wide as the body. The anus is almost continuous, though the posterior lip is very slightly raised. The refractive, narrow rectum is about one and one-half times as long as the anal body-diameter. The tail begins to taper from a little in front of the anus, and tapers at much the same rate behind the anus, in such a fashion that at its middle the diameter is about one-third as great as at its base; thence onward, it tapers but little and ends in a terminus about one-fourth as wide as its base. Caudal glands appear to be located in the base of the tail. The renette cell is located just behind the base of the neck. The lateral fields appear to be about one-half as wide as the body, and are characterized by a succession of groups of cells, which are larger than usually seen in these regions. These groups contain dozens of nuclei packed rather closely together. The groups do not seem to be very regularly arranged, but succed each other with intervals between them that are shorter than the length of the groups. The groups are of rather uneven size, and are most conspicuous between the neck and the anus.

Habitat: Larat, East Indies. Marine. Fig. 109, p. 329.

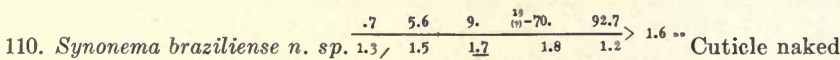
except for the setae at the anterior end. Mouth depressed. Lips subdistinct, flat, rather thin, fairly developed, flap-like; lip-region apparently twelve-ribbed, closing nearly together over the rather small pharynx, which may be reckoned to extend at least a short distance behind the base of the dorsal onchium. The oesophagus near the nerve-ring is one-half, just in front of the cardiac bulb onehalf, and finally three-fourths, as wide as the base of the neck. This latter measurement represents the diameter of the valveless, pyriform cardiac bulb. The musculature of the oesophagus is colorless, with no indication of the presence of glands. There is a cylindroid cardia one-third to two-fifths as wide as the base of the neck, and two-thirds as long as the body is wide. The thick-walled intestine, which has a distinct, refractive lining, becomes at once three-fourths as wide as the body, and in cross-section would present about four cells. Cardiac collum one-half as wide as the neck. From the more or less depressed anus, the 
prominent cutinized rectum extends inward a distance equal to the anal bodydiameter. Anal muscles are dimly to be seen. The intestinal cells have conspicuous nuclei, and as there is an almost entire absence of granules, the intestine is very transparent. Tail arcuate, conoid. The three saccate caudal glands form a close tandem in the anterior half of the tail; they empty through separate ducts and ampullae. No caudal setae are to be seen. The lateral fields have not been clearly seen; they are probably one-third as wide as the body and composed mainly of two rows of cells. The granular renette cell is three times as long as the body is wide and one-sixth as wide as long. It is placed from two to six body-widths behind the neck, and seems to be com-

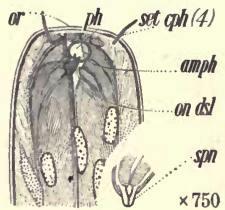
posed of about six ellipsoidal, granular cells, arranged more or less in pairs. The excretory pore is two-fifths the way back to the nerve-ring; there is no ampulla. The nerve-ring is accompanied by distinct nerve cells arranged in groups. The following description of the sexual organs is derived from an immature specimen: The tubular, muscular, more or less cutinized vagina extends obliquely forward two-thirds the distance across the body, where it joins the straight uterus, which is about twice as long as the body is wide. Ova prolate; the maturest six to eight being arranged single file, the others irregularly,-about thirty in all. Ovary medium-sized and tapering. Male unknown.

Habitat: Algae, near lighthouse, Bahia, Brazil. Sublimate to balsam. The multicellular renette is highly peculiar. Fig. 110.

111. Anticyclus exilis n. sp. Cephalic setae sixteen, subcephalic four; cuticle otherwise naked. The rather thin lips, apparently six in number, arch together over the pharynx, somewhat as in Oncholaimus. According to the state of the muscular contraction in the lips, the head is

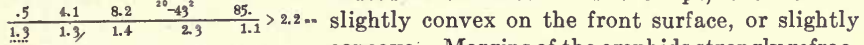
$3.7 \quad 7.7 \quad-\mathrm{M}^{-2} \quad 86 . \quad$ concave. Margins of the amphids strongly refrac-

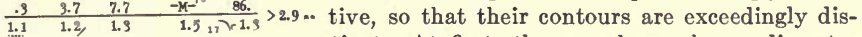
tinct. At first, the oesophagus has a diameter fully two-thirds as great as that of the corresponding portion of the neck, and it retains this diameter until after it passes through the nerve-ring; about half way between the nerve-ring and the intestine, it begins to expand, and continues to do so very gradually, so that finally it is about three-fourths as wide as the base of the neck. The anterior cells of the intestine are more transparent and otherwise different in appearance from those which succeed, and it not infrequently happens that they present a strong resemblance to the oesophagus, so that the real position of the cardiac constriction is likely to be mistaken. The intestine is at first about two-thirds as wide as the body. It soon, however, becomes three-fourths to four-fifths as wide as the body, and is composed of rather massive cells, so that the lumen is narrow. Its cross-section probably presents about six cells. These contain numerous granules, rather uniform in size and so arranged as to give rise to an indistinct tessellation. From the inconspicuous anus, the rectum, which has about the same length as the anal body-diameter, extends inward and forward. The lateral fields are onethird as wide as the body. Renette unknown; it is possible that the renette cell is unusually small and located near the cardiac constriction. The tail of the female is supposed to be like that of the male described below, but there is 
uncertainty on this point, as all the female specimens so far examined have lacked the posterior portion of the tail; - the portion present has the same form as the corresponding portion of the tail of the male. From the inconspicuous vulva, the vagina leads inward and probably slightly forward. The eggs are about three to four times as long as the body is wide and about one-fourth as wide as long. The tail of the male is conoid in the anterior fourth in such fashion that at the beginning of the second fourth, its diameter is about one-third as great as at the anus; thence onward, it tapers much less rapidly, and in the posterior half hardly tapers at all, the diameter for a considerable distance in front of the terminus being no greater than that of the thickness of the body-wall. There is present behind the anus a collection of cells which bear some resemblance to caudal glands, but whether they really are caudal glands and empty through a spinneret at the terminus remains uncertain. The seventeen equidistant supplementary organs are rounded, low, conoid papillae separated by distances about equal to their own diameters, and occupy a distance from two to three times as great as the corresponding body-diameter. These organs appear to be innervated, but they at times closely resemble mere transverse corrugations of the cuticle. Behind the anus, at least when the tail is incurved, there are folds in the cuticle that present a very similar appearance to those just described as supplementary organs, occurring in front of the anus; these are confined to the wide and muscular portion of the tail, and do not extend, as a rule, as far backward as the corresponding appearanoes in front of the anus extend forward. set sulm at sulm Spicula somewhat longer than the anal body-diameter,
more strongly arcuate toward their proximal extremities
than near the anus. They extend about two-thirds the
distance across the body and then curve around toward
the body-axis. There extend from the proximal ends
toward the anus nearly straight refractive elements that the body-axis. The ejaculatory duct is two-fifths as wide as the body. Posterior testis much the smaller.

Habitat: Bath Tub Springs, Jamaica, near Salt River. These are salt springs having about body temperature. Fig. 111.

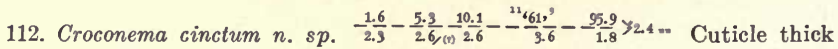
transparent, yellow, composed of about 500 annules imparting to the contour of the body throughout a distinctly crenate appearance, and bearing numerous short, stiff, slightly curved bristles, having a length somewhat greater than its own thickness. The cuticle of the head seems to consist of a single, somewhat hemispherical helmet-like piece. In all, there must be at least six irregular longitudinal rows of setae on the "helmet," but it seems impossible to pick out any single circlet of these setae that particularly merit the term cephalic setae. The spreading setae on the neck curve backward. Both here and toward the tail end, these setae are shorter than near the middle of the body where they are tapering, acute, nearly twice as long as the cuticle is thick and have a base one-fourth as wide as the distance between two successive annules of the cuticle. When the mouth is closed, the tips of the lips are bunched at the middle of the front of the head. There appear to be about twelve of these tips. The pharynx 
is about one-eighth as wide as the base of the head and is fairly well filled by the apices of the teeth. Two of these onchia (?) have been distinctly seen, a dorsal one which extends to the base of the lips and has a subacute, conical, refractive apex, and whose base is about one-eighth as wide as the corresponding diameter of the head, and a larger submedian one with a conical, refractive apex, and having a base somewhat wider than that of the dorsal one. This latter has a length somewhat greater than the width of the amphids, and its base lies near the center of the head and rather behind the middle of the pharyngeal bulb. The pharynx is strongly amph cutinized, and manifestly extends backward through the greater part of the region surrounded by the "helmet." The rather obscure change from pharynx to oesophagus takes place suddenly along two loci which extend obliquely outward and forward from the lumen of the oesophagus. The oesophagus retains the same diameter until after it passes through the nerve-ring; soon after that it begins to enlarge gradually in diameter, so that finally it is threefourths as wide as the base of the $\times 750$

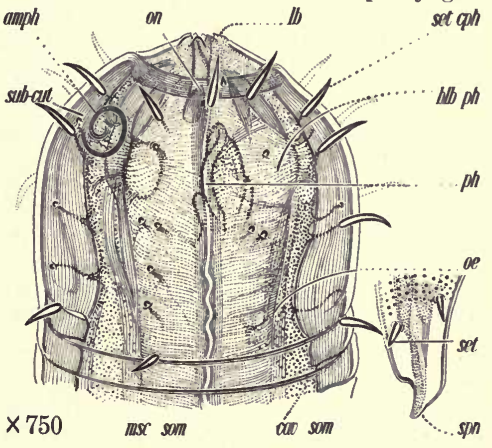
neck. The intestine is at first very narrow, about one-fifth as wide as the body, and for a short distance has a different structure from the portion that follows. Possibly this first part should be considered a cardia. In this cardiac portion, there are no cells containing granules. Immediately behind the "cardiac" portion, the intestine is half as wide as the body and would present eight to ten cells in cross-section. The cells contain brownish granules of uniform size, so arranged as to give rise to a distinct tessellation. The intestine is moderately thick-walled. From the raised anus, the strongly cutinized rectum, which is somewhat longer than the anal body-diameter, extends inward and then almost directly forward, so that it joins the intestine considerably ventrad from the body-axis. The tail is arcuate-conoid, and presents the peculiarity that the annules of the cuticle cease in front of the middle; thence onward, the cuticle is considerably thicker and is traversed by radial markings, which pass outward and a little backward and give to the surface of this portion of the tail a distinctly punctate appearance, which, however, is less marked than the similar appearance on the head. The caudal glands are arranged tandem immediately in front of the anus, extending from the end of the intestine proper to a little behind the anus. There is apparently a small renette cell immediately behind the oesophagus. The nerve-ring surrounds the oesophagus obliquely. From the slightly elevated, rather inconspicuous vulva the vagina extends nearly balf way across the body. The ovaries reach more than half way back to the vulva, and contain a dozen to twenty developing ova, arranged irregularly.

Habitat: Sand and marine algae, in surf, shore of small island off Port Royal, Jamaica. Fig. 112. 
113. Nudora lineata $n$. $s p$. Cuticle two to three times as thick at the head end as elsewhere; more or less like that of Desmodora, but with longitudinal striae somewhat like those of Monoposthia. The annules increase in width, and are more accentuated, toward the head. Each of the ten longitudinal wings appears to comprise a series of "V"-like markings on the anterior portion of the body, which are reversed on the posterior. Following the serrate contour of the head it is discovered that this reversal takes place not far behind the neck. There seem to be twelve, subdistinct, more or less conoid, minute, blunt labial elements. The labial papillae are more or less setose, one seta between each pair of labial elements. Limits of the pharynx rendered very definite on account of the elongated pharyngeal swelling. Anterior part of the pharynx cyathiform, posterior triquetrous-tubular. Posterior part of the neck cylin-

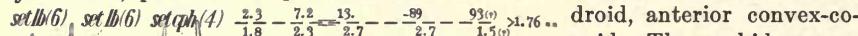

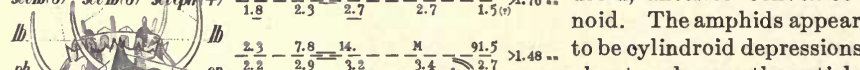
ph the on $2.2 \quad 2.9 \quad 3.2 \quad 3.4 \leqslant 2.7$ about as deep as the cuticle amph 8 II $1 \mathrm{mn}$ is thick. In front of the wide annule bearing the amphids, ae 1 . $\times 750$ which, however, does not thin out on its anterior edge, $\times 750$ strhngil sp as do the regular annules. Following this annule comes the lip-region, which in contra-distinction takes a carmine stain. The elongated, pharyngeal bulb, one-half as wide as the neck, is set off from the remainder of the oesophagus by a constriction. The oesophagus is at first one-third, near the nerve-ring one-third, preceding the cardiac bulb one-third, and finally fivesixths, as wide as the corresponding portion of the neck. The cardiac bulb is cylindroid and makes up one-third of the oesophagus. Its musculature is coarse and colorless. The oesophageal tube expands suddenly to form the cardiac bulb, which is divided into two main parts by a break in its musculature. There are smaller breaks, also, near each end. The lining of the oesophagus appears to occupy one-sixth of its width. The rather thin-walled, narrow intestine becomes at once one-fifth as wide as the body. At the cardiac constriction the intestine joins the center of the posterior surface of the oesophageal bulb. Minute granules are scattered in the intestinal cells. The conoid tail, on which there are obscure longitudinal striae, tapers from the anus to the non-striated, simple, symmetrical spinneret. The broadly saccate caudal glands form a close tandem in the anterior half of the tail. Slender caudal setae occur on the tail end, both preanal and postanal, three to four ventrally submedian, and three to four dorsally submedian, just in front of as well as behind the anus, about one and one-half body-widths apart, and shorter on the male than on the female. The rather broad ovary tapers near its blind end. The flattish ova are arranged for the most part single file. The spicula are frail, very slender, mostly uniform, but finally tapering and acute. Their proximal ends appear to lie about opposite the body-axis. The yellow accessory pieces are somewhat longer than the spicula. They are arcuate and stoutish, and their proximal ends appear to lie somewhat dorsad from the body-axis. There are two obscure supplementary organs, the anterior of which is located at a distance in front of the anus equal to one and one-half body-diameters, the posterior being opposite the middle of the spicula. These consist of slight elevations further accentuated by small alterations in about three consecutive annules.

Habitat: Sand, below low tide mark, Hull, Massachusetts, U. S. A. Fig. 113. 
114. Rhinema retrorsum n. $s p$. Cuticle thick, interrupted at twelve places so as to form exceedingly distinct longitudinal wings, which when brought into focus give a somewhat "fishbone"-like effect. The annules and their modifications give a retrorse appearance to the entire cuticle, but in reality the striae are retrorse in the posterior part of the body, and the reverse in the anterior part. Some of the cuticular markings cease in front of the anus on the male and are not continued on the tail, so that the tail has a somewhat unusual appearance. There are submedian longitudinal striations extending to the middle of the tail. Neck for the most part cylindroid. Opposite the dorsal tooth there is a distinct junction with the cuticle of the lip-region indicated by an almost imperceptible constriction encircling the head. In front of this constriction there are two others, close together, connected with the lip-region. Lips with

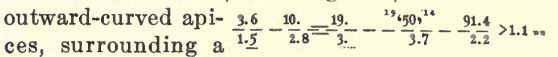
cyathiform cavity $\frac{2.9}{2}-\frac{8.9}{3.5}=\frac{-17.9}{3.8}--\frac{-\mathrm{M}^{52}}{3.6}=\frac{91 .}{2.8},>1.2$. having a diameter about three-fifths as great as that of the front of murph the head, and a depth not more than half that amount. Into the midst of this shallow cavity the dorsal onchium projects. Sometimes, however, this cavity is deeper, namely, when the dorsal tooth is withdrawn to a greater extent. Then the cavity is about half as wide as the head, and about as deep

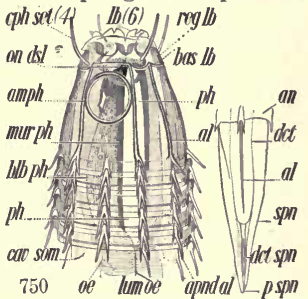
as wide, and the lip-region is manifestly more closely folded. Onchium faintly spear-like. Muscles of the pharynx of an entirely different character from those of the oesophagus, which begins as a tube about half as wide as the corresponding portion of the neck, and continues to have this diameter until after it passes through the nerve-ring, thereafter expanding gradually, but finally somewhat faster, so that at the end it becomes two-thirds as wide as the base of the neck. The posterior swelling is of such a character that one might speak of it as a bulb. There is no very distinct cardia. Renette unknown. Vagina reaching half way across the body. Mature ova at the flexure, ready to enter the uterus, are one and one-half times as long as the body is wide and about half as wide as long, with nuclei one-third as broad as themselves. The narrow ovaries are more or less cylindroid and contain comparatively few ova, apparently arranged more or less single file. Anus of the male distinctly raised. Tail arcuate and conoid to the naked terminus, which has a length about equal to that of five of the preceding annules. Position of the caudal glands doubtful, but it seems quite possible that they are located in the base of the tail. There are a few inconspicuous hairs on the smooth terminus that are not shown in the illustration, of which two have been seen in the ventrally submedian position, having a length about two-thirds as great as the corresponding diameter of the terminus. No supplements or special papillae or setae have been seen, though it is possible that very small setae may have escaped observation. The slender, slightly arcuate, rather uniform spicula have their proximal ends almost imperceptibly cephalated by expansion. The uniform proximal three-fourths of the spicula, when viewed in profile appear to have approximately the width of one of the adjacent annules. In the distal fourths the spicula taper to an acute point. There is a single, nearly straight but slightly " $\mathrm{S}$ "-shaped accessory piece, two-thirds as long as the spicula, which is acute at its distal extremity and capable of protrusion to a certain extent. It is fully as wide as the spicula. 
Its proximal half curves away from the spicula and then recurves, and is connected with the ventral part of the body-wall a short distance behind the anus by means of oblique muscles.

Habitat: Coral sand, New Hebrides. This genus differs from Chromadora, Spilophora and Euchromadora in the possession of distinct circular amphids, and also in the possession of twelve longitudinal cuticular costae. In this latter respect it resembles Monoposthia, but here the spicula are double, and there are no supplementary organs or swellings. The lip-region and pharynx also present contrasts with any of the above genera. The striking differences are: (1) The continuation of the pharynx in its narrow part as a definite well lined posterior chamber, ending definitely opposite the point where the pharyngeal bulb is separated from the oesophagus by a distinct constriction. (2) By the form of the lips, which, instead of being soft and internally folded so as to give rise to about twelve refractive ribs, or folds, are more strongly built, cutinized and outwardly recurved, and are only six in number. (3) -M-Fig. 114, p. 335.

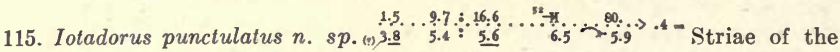
naked cuticle resolvable into rows of dots, which are modified a little on the lateral fields, where there are distinguishable two longitudinal rows of markings close together on each side of the lateral line, at least toward the anterior extremity and on the neck. The neck is cylindroid. There are probably four or six short and inconspicuous cephalic setae in the midst of the labial constriction. There are twelve lips of the character usually found in the genus Cyatholaimus, but smaller, and in such a position in the specimen examined that it is impossible to make out the details, except that when the mouth is open about twelve papilla-like lips of small size are to be seen toward the margin of the head. Behind the pharyngeal bulb the oesophagus is about one-half as wide as the base of the head. It continues to have this diameter until after it passes through U63 on If the nerve-ring, when it expands to form the pyriform cardiac spn bulb, which contains a distinct but simple valvular apparatus. The intestine joins the middle of the posterior surface of the ph cardiac bulb, and is at that point only about one-fourth as wide as the base of the neck; it gradually widens out so as to become half as wide as the body. It would present few cells in crosssection, perhaps only two. The renette cell is about one-third as wide as the body, and fully twice as long as wide, and lies a short distance behind the base of the neck. The tail of the male is conoid in the anterior threefourths; thence onward it tapers more rapidly to the minute spinneret. The posterior fourth of the tail is more finely striated than the remainder. Caudal setae few, minute, and scattered. The proximal ends of the uniform spicula are bent over toward the ventral side of the body. The spicula are of very peculiar form, the proximal halves being much more strongly arcuate than the distal halves. At the distal extremity each spiculum is bifurcated, one fork, the anterior, being rudimentary, while the other, the posterior, is somewhat S-shaped and acute distally. The finger-shaped apophysis of the accessory extends backward nearly parallel to the body-axis. The apophysis is nearly as strongly cutinized as the spicula themselves, and has a length nearly equal to that of the anal body-diameter. The apophysis is nearly twice as wide as the spicula, which have a width about equal to that of three of the adjacent annules of the cuticle. Ejaculatory duct about one-third as wide as the body; vas deferens about half.

Habitat: Punta Arenas, Pacific coast of Costa Rica. Marine. Fig. 115. 
116. Ptycholaimellus carinatus n. sp. Cuticle rather thin and naked, resolvable near the anterior extremity into elements which are interrupted on the lateral fields. Near the middle of the body, the division line between the annules is resolvable into dotlike elements; these are interrupted by two prominent lateral wings. These wings begin near the head and end behind the middle of the tail, and near the middle of the body occupy a space about equal to the width of one

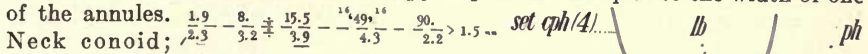

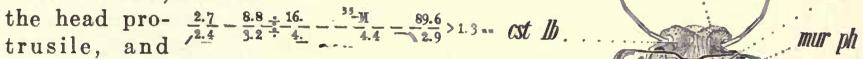
connected with the anterior margin of the cervical cuticle by a thin, flexible membrane. When the mouth is closed, it is surrounded by twelve folds in the lip-region, which come together and form a striated, somewhat tubular vestibule, having a length about equal to the width of two of the adjacent annules of the cuticle. The lip-region stains strongly with carmine, and is a very striking feature in stained specimens. The lips arch together $S p n$

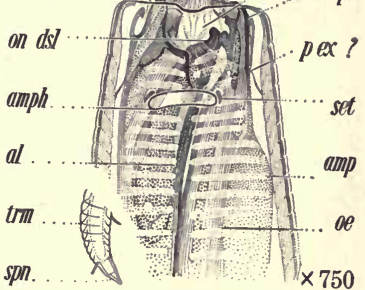
over the somewhat spheroidal anterior portion of the pharynx, which is about: one-third as wide as the front of the head and contains the strongly cutinized apex of the massive dorsal onchium, which has, passing backward from it, distinct refractive extensions that gradually merge into the structures of the oesophagus. The narrow portion of the pharynx merges gradually into the lumen of the oesophagus. The pharyngeal swelling contains special muscles for the operation of the lips and dorsal onchium; these are much more strongly developed on the dorsal side, and the pharyngeal swelling presents the peculiarity of being widest near its anterior part, and tapering gradually nearly half way to the nervering, where the diameter has been so reduced as to be one-third that of the corresponding portion of the neck. The oesophagus continues to have this width until after it passes through the nerve-ring, where it enlarges rather gradually for a short distance, and then more rapidly to form the clavate, or elongatedpyriform, strongly developed cardiac bulb, which occupies the posterior threesevenths of the neck, and becomes three-fourths as wide as the base of the neck. This bulb is divided into two approximately equal parts by a transverse division of the musculature and its valvular apparatus is correspondingly divided. This latter consists essentially in a plain expansion of the lining of the oesophagus, which is in its widest part, about one-third as wide as the bulb. There is no distinct cardia. The intestine joins the middle of the posterior surface of the cardiac bulb, and at this point is only one-fifth as wide as the neck. It gradually widens out so as to become half as wide as the body and then diminishes again to give place to the enormously developed renette cell, behind which it again becomes about half as wide as the body. The intestine is rather thin-walled, its cross-section presenting about four to six cells containing scattered yellowish granules of variable size, the largest of which have a diameter nearly as great as the width of one of the adjacent annules of the cuticle. From the slightly raised anus, the rectum extends inward a distance equal to the anal body diameter. The lateral fields are fully one-third as wide as the body. The renette cell, twothirds to three-fourths as long as the neck, is clavate, and at its widest part half 
as wide as the body. It contains a single nucleus, which is swung centrally in the protoplasmic network. The renette cell has two spherical ellipsoidal companion cells in the rear. The excretory pore lies at the base of the lips, and is connected with the elongated, fusiform ampulla by means of a duct which extends from the base of the lips to nearly opposite the base of the pharynx. Sometimes the ampulla is much elongated, and one-fourth as wide as the corresponding portion of the neck, reaching nearly to the nerve-ring. The tail begins to taper from far in front of the anus, and tapers at about the same rate for some little distance behind the anus, then tapers more rapidly for a short distance, and then finally tapers gradually to the terminus. The caudal glands are located in the base of the tail. The vulva is massive and yet does not project much beyond the main ventral contour. It is prominent on account of a depression which surrounds it. From the vulva the large vagina leads inward half way across the body. The tapering, reflexed ovaries reach half way back to the vulva and contain upwards of ten ova arranged single file. The prolate eggs are nearly as long as the body is wide and have been seen in the uterus one at a time. The distance between the wings on the cuticle, near the anus of the male, is about equal to the width of two of the annules. The tapering, yellowish spicula are more strongly arcuate in their proximal halves than elsewhere. At their widest part they are about one-sixth as wide as the corresponding portion of the body. Their proximal ends, when viewed laterally, appear to lie opposite the bodyaxis. An indistinct refractive line passes from the proximal ends of the spicula to the distal ends, indicating that the spicula may be wider than would be judged by a consideration of only the main portion of their framework. The accessory pieces are one-half as long as the spicula and in the main parallel to them and are arranged in comparatively close contact. Near the anus, however, the framework of the accessory pieces bends away from the spicula. Each accessory piece ends in an acute protrusile point, which is rather suddenly and rather markedly bent forward. The spicula present the same arcuate appearances at their tips as do the accessory pieces. Both the ejaculatory duct and vas deferens are about one-third as wide as the corresponding portion of the body.

Habitat: Larat, East Indies. Marine; apparently very common. Sublimate to balsam. Fig. 116, p. 337. An examination of sloughed cuticle of the head gave equally satisfactory evidence of the existence of joints in the setae.

117. Actinonema pachydermatum n. sp. Cuticle naked, very thick, occupying one-half the radius as measured at the base of the neck. Annules alike except

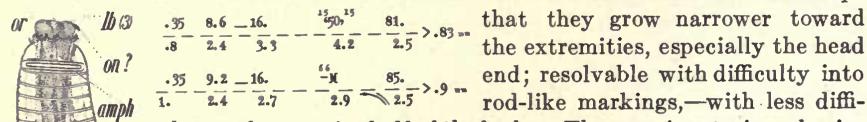
culty on the anterior half of the body. The prominent wings, begin-

spn ning on the neck, end near the middle of the tail. As there are deep, $\times 750$ narrow grooves between the annules, the contour of the body is irregularly crenate. Head set off by contraction and a deep constriction, subtruncate; mouth more or less depressed. The lip-region is one-half as high as wide, and appears to be composed of three fairly well developed, rounded, subdistinct lips, which are set off by constriction. This lip-region appears as if protruding from within the thick cuticle, and stains in carmine while the cuticle remains quite colorless. On the lips there are no obvious signs of a cuticle. The 
pharynx is very small and inconspicuous, or even absent apparently. There seems to exist, however, a minute, more or less arcuate dorsal onchium, to be seen in balsam specimens only when exserted. The neck is conoid, the head convexconoid. Amphids nearly straight transverse slits, four-fifths as long as the corresponding diameter of the head, and apparently eight times as long as wide. One of the foremost annules appears to be more or less dislocated;- that is to say, the regular succession of the fore-most annules is somewhat disturbed by $\$ p$ the presence of the amphids, which are otherwise almost
invisible. Oesophagus more or less conoid, but decidedly
clavate posteriorly. Near the head it is two-fifths, near
the nerve-ring about one-fourth, and finally one-half, as
wide as the corresponding portion of the neck. There is
no distinct cardia. The rather thin-walled intestine, which
becomes at once two-fifths as wide as the body, has a dis-
tinct lumen. Its cross-section presents four to six cells.
Cardiac collum one-fifth as wide as the body. From the cells of the intestine contain numerous, very fine, colorless granules. The conoid, subarcuate tail tapers from in front of the anus to the simple, unarmed, convex-conoid, rather blunt spinneret. The ellipsoidal caudal glands are small and inconspicuous, more or less unsymmetrical, and apparently lie in a close tandem opposite the anus. The elongated to fusiform, granular renette cell lies behind the neck a distance equal to one and one-half body-diameters; it is as long as the body is wide and one-fifth as wide as long. Female sexual organs double. The tapering ovaries are of moderate size and extend half way back to the vulva. They contain few ova arranged for the most part single file. The simple, strong, tapering, rather stout spicula are at their widest part about onesixth as wide as long. Their proximal ends appear to lie more or less opposite the body-axis. The simple, rather frail, slender accessory piece is about twothirds as long as the spicula. There are no supplements or special papillae.

Habitat: "Seagrass," shoal, two miles off Key West, Florida, U. S. A. Fig. 117.

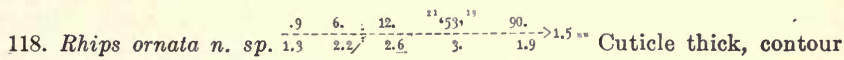
more or less crenate. Striae resolvable into rod-like markings, set(ll) pd(6) changing so as to produce a basketwork-like effect on the neck. On the posterior half of the body on each lateral field each on annule presents two forward-pointing, "V"-shaped marks of unequal size, the ventral being the larger; these are inconspicuous, but are less so posteriorly. These markings occur also on $d t$ the anterior half of the body, but are there reversed. On a par amp with the celphalic setae, and just in front of the ends of the amphids, there are four sublateral, special scales, or short, $\times 750$ amphids, there are four sublateral, special scales, or short, What function is associated with these peculiar appendages is unknown. Lips very minute, apparently six and double. The neck has the peculiarity of diminishing more rapidly from the middle forward for a short distance. The dorsal onchium is opposed by one or two very minute ventrally submedian ones. The oesophagus is conoid, though there is a more or less pronounced clavate cardiac swelling. At the nerve-ring, the oesophagus is one-half, and finally three-fourths, 
as wide as the corresponding portion of the neck. The rather prominent lining of the oesophagus finds expression in about three parallel refractive lines, occupying a space equal to two-fifths its width. The fine musculature is more or less pigmented. There is a decided thickening of the lining in the cardiac swelling. There is no cardia. The thin-walled intestine presents a faint lumen, and becomes at once three-fifths as wide as the body. Cardiac collum one-fourth as wide as the neck. From the more or less continuous anus, the inconspicuous rectum leads inward a distance one and one-fourth times as great as the anal body-diameter. The postanal region has the appearance of lacking one of the regular striae. Colorless granules of variable size are scattered in the intestinal cells. The largest of them are about half as wide as one of the annules. The conoid, subarcuate tail tapers from in front of the anus to the convex-conoid spinneret. Apparently, the caudal glands lie in the anterior third of the tail. Though the renette has not been clearly seen, it appears to lie two body-widths behind the neck, to be elongated and twice as long as the body is wide. The large, elevated, conspicuous vulva, occupying twenty-five to thirty annules

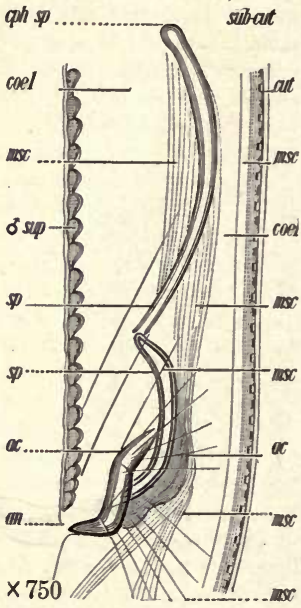
of the cuticle, leads to a medium-sized vagina. The female sexual organs are double and symmetrically reflexed. The eggs are probably rather large, since an ovum which has not yet entered the uterus, but appears to be mature, is three times as long as the body is wide. Spherical, granular spermatozoa,

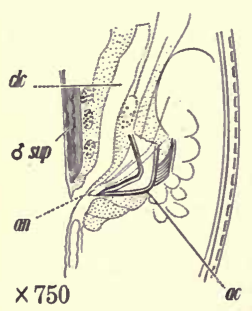
1.2. uterus. Spicula two-jointed, as shown in the illustration. A mature specimen was seen in which the spicula were lacking, suggesting that possibly they are lost at the time of copulation. See adjacent illustration. Accessory pieces, two in front of the spicula and two behind, with an additional median one behind,that is to say, five in all. The ventral, longitudinal optical section, about twenty-five annules in front of the anus, is such that the subcuticle appears more or less as if retrorsely serrate. This alteration in the annules is co-extensive with the oblique copulatory muscles, so that no doubt these structures must be regarded as indicating the presence of supplementary organs.

Habitat: Marine mud, Ocean Beach, Florida, U. S. A. Fig. 118a, p. 339; Figs. $118 b$ and $c$. 


\section{ABBREVIATIONS USED IN ILLUSTRATIONS}

Frequently the abbreviations are combined, thus: sct trm, seta terminalis, terminal hair; set cph axt subl, seta cephalica.dextra sublateralis, right sublateral cephalic seta.

A c, accessory
al, wing

$a m p$, ampulla

amph, amphid

an, anus, anal

anak, anakinesis, anaphase

ann, annule

ant, anterior

anus, anus

apic, apiculum

apnd, appendage

$a p p h$, apophysis

ar, area, field

$a s p$, aspect, view

ast, aster

axil, axil, axillary

$a x$, axis, axis

\section{Bas, base}

B birefr, birefringent

$b l b$, bulb, bulbous

brs, bursa, bursal

Cal, lime, calcareous

C cav, cavity

$c d, c d l$, tail, caudal

cer, ceratin, ceratinous

cerv, cervical

chrm, color, chromatio

chrmt, chromatin

chrsm, chromosome

chrt, chromatin

cir, cirrus, cirri

$c l$, cell, cellular

clc, cloaca, cloacal

$c l v$, cleavage, cleft

cntr, centrosome

col, collum, constriction

com, commissure

comp, companion

constr, constriction

cop, copulatory

$\operatorname{cor} p$, body, thing, corpus

cost, costa, rib, costal

$c p h$, cephalum, cephalio

$c p l$, hair, capillary

crd, cardia, cardiac

crib, sieve, mesh

crp, corpus, body, thing

crv, neck, cervical

cryst, crystal, crystalline

cst, costa, rib, costal

cut, cuticle, cuticular

cutn, cutin, cutinous

cytp, cytoplasm

Det, duct

1 dep, deposit, deposited diast, diaster

dir, directrix, guide

dnt, tooth, dental

dntcl, denticle, little tooth

$d s c$, disc, discoid

$d s l$, dorsal, dorsad

dst, distal, far, farther

$d x t$, right, right-hand

\section{T $f$, efferent}

Hi elast, elastic spring, springy

elev, elevation

elmt, element, component

$e m b$, embryo, embryonio

$e q$, equator, equatorial

$e x$, excreta, excretory

ex $p$, excretory pore

extn, extension, extended

extnl, external

extr, exterior, outer

F $a b, f b$, framework

fasc, fascicle

fibr, fibre, fibrous

$f i x$, flexure $f r$, front, frontal

furc, furcation, forked

Gam, gamete

$\checkmark \mathrm{gl}$, gland, glandular

glot, glottis, glottoid

oub, gubernaculum, guide, accessory piece

and, gonad

gng, ganglion

grn, granule, granular

T $m$, immature

ing, ingeste

int, intestine, intestinal

intnl, internal, inner

intr, interior

$\boldsymbol{J}^{n c \text {, junction, junctional }}$

Kar, karyokinetio figure

T. line

14 lac, lacuna, pit, depression lam, lamina, plate, laminate lat, lateral, laterad

$l b$, lip, labial

lns, lens (of eye)

lob, lobe, lobate

longt, longitudina

lro, larva, larval

lngt sec, longitudinal section

lum, lumen, luminal

Maj, major, the larger

mat, mature

marg, margin, edge, marginal

md, middle, median

mesok, mesokinesis, mesophase

metak, metakinesis, metaphase

micrph, microphyte

micrz, microzoon

$\min$, minor, the lesser

mit, mitosis, mitosis-figure

mnd, mandible, jaw, mandibula

monast, monaster

msc, muscle, muscular

mur, wall, mural

N nerve

I ncl, nucleus, nuclear

nema, nema, nematode

$n r$, nerve-ring

nro, nerve

nud, naked

C, eye, ocular

o, oesophagus, oesophageal

on, onchium, tooth, spear

onch, onchium, spear, onchisl

öcyt, öocyte

or, mouth, oral

org, organ

org?, organ in doubt, doubtful

os, ostium

ov, ovum, egg

ovr, ovary, ovarian

P por, pore

par, parasite, parasitic

pct, pectoral

pclr, peculiar, remarkable

pgm, pigment, pigmented

$p h$, pharynx, pharyngeal

$p h s$, phase, state, condition

pigm, pigment, pigmented

$p l p$, palp

$p l r$, polar

pnct, point, dot, punctate

por, poro post, posterior

$p p, p p l$, papilla, papillate

prerct, prerectum

prm, primary, first

prok, prokinesis, prophase

prtph, protophyte

prtz, protozoon

prx, proxima, proximal

pseudon, pseudonchium

punct, point, bead, dot

pyl, pylorus, pylorio

Rach, rachis, axial strand

1 rcpt, receptacle, sack

rct, rectum, rectal

red, reduction, reduced

reg, region

rem, lost, discarded

ren, renette, ventral gland

res, reso, reservoir

ret, reticulum, reticular

rot, rotifer

$r t r$, retrorse, reversed

rtrct, retractor, retracting

rud, rudiment, rudimentary

Sal, saliva, salivary

sc, scale sca

sec, section, cross-section

secnd, second, secondary

seg, segment, segmented

sem, semen, seminal

set, seta, setaceous

snst, left, left-hand

som, soma, body, somatio

$s p$, spiculum, spicula

sphnc, sphincter

spir, spireme, spiral

spm, sperm, spermatozos

spmct, spermatocyte

spmtd, spermatid

spn, spinneret

spndl, spindle

spthc, spermatheca

$s q$, scale, squamule

st, stoma, mouth, pore

str, stria, striated

$s u b$. sub, nearly, almost

subd, subdorsal

subl, sublateral

subm, submedian

subst, substance, matte

subv, subventral

sup, supplement, supplementary

sut, suture

$T^{b}$, tube, tubular

I teg, shell, tegument

telek, telekinesis, telephase

tesl, tessellation

trans, transverse

trm, end, blind end, terminal

ttrd, tetrad, four-fold

tum, tumor, swelling, swollen

$\mathbf{U}^{\ell}$, uterus, uterine

V vessel

vag, vagina, vaginal

valv, valve, valvular

vas, vessel

tas def, $v$ def, vas deferens

vesic, vesicle, vesicular

vlv, vulva

vnt, ventral, ventrad

$v s t$, vestige, vestigial

vstbl, vestibule, vestibular

vstg, vestige, vestigia

Zon, zone, zonal 


\section{INDEX}

\begin{tabular}{|c|c|c|}
\hline nchus & cephalats & page \\
\hline A viviparus. & Pseudolella.............. 268 & $\ldots \ldots 281$ \\
\hline $\begin{array}{l}\text { canthurum } \\
\text { Zanems.... }\end{array}$ & Xennella.............. 233 & flexile \\
\hline Zanems............... 283 & cephalatum & Cyartonema. \\
\hline $\begin{array}{l}\text { ctinonems } \\
\text { pachydermatum......... } 338\end{array}$ & $\begin{array}{l}\text { Micromicron............ } 324 \\
\text { Cephalobellus }\end{array}$ & $\begin{array}{l}\text { Rhadinema......... } \\
\text { fontinalis }\end{array}$ \\
\hline $\begin{array}{l}\text { cuminatus } \\
\text { Catalaimus............... }\end{array}$ & papilliger........... & Colpurella. \\
\hline $\begin{array}{l}\text { Catalaimus. } \\
\text { cuts }\end{array}$ & Cephalobium & halum \\
\hline $\begin{array}{l}\text { cuts } \\
\text { Isonemella. }\end{array}$ & microbivorum.......... 270 & llonema. . \\
\hline $\begin{array}{l}\text { Isonemella.......... } \\
\text { equale } \\
\text { Litinium. . . . . . . . . }\end{array}$ & $\begin{array}{l}\text { eramonema } \\
\text { attenustum..... }\end{array}$ & Bnema \\
\hline Litinium........... & Chambersiella & ferox............. \\
\hline $\begin{array}{l}\text { aequalis } \\
\text { Dactylaimus. }\end{array}$ & $\begin{array}{l}\text { rodens............ } \\
\text { Choronema }\end{array}$ & $\begin{array}{l}\text { labra } \\
\text { Asymmetrella...... }\end{array}$ \\
\hline Alsimells & simplex........ & Gonionchus \\
\hline 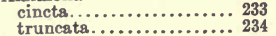 & cincta & $8 \ldots$. \\
\hline $\begin{array}{l}\text { truncata............... } \\
\text { Alaimonema }\end{array}$ & 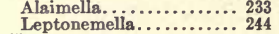 & Pseudolella....... . \\
\hline multicinctum.......... 327 & Cinctonems & $\operatorname{granu}$ \\
\hline $\begin{array}{l}\text { americanum } \\
\text { Rhsbdocoma........... }\end{array}$ & tenue. & ...... \\
\hline Rhabdocoma........... 252 & $\begin{array}{l}\text { inctum } \\
\text { Croconems. }\end{array}$ & Trilepta.. \\
\hline rotundicephala........... 324 & Rhynchonems............ & gutts \\
\hline $\begin{array}{l}\text { mplicolle } \\
\text { Axonchium............ } 305\end{array}$ & ema & plaimus. . . . . . . . \\
\hline $\begin{array}{l}\text { Axonchium. } \\
\text { Anaxonchium }\end{array}$ & $\begin{array}{l}\text { punctatum.. } \\
\text { Colpurells }\end{array}$ & alsi \\
\hline litorium ............... 319 & fontinalis.............. & rurus...... \\
\hline $\begin{array}{l}\text { Anoncbolaimus } \\
\text { mobilis................ } 312\end{array}$ & $\begin{array}{l}\text { Cophonchus } \\
\text { ocellatus.............. }\end{array}$ & im.. \\
\hline $\begin{array}{l}\text { Anticyathus } \\
\text { tenuicaudatus........... } 245\end{array}$ & $\begin{array}{l}\text { Croconema } \\
\text { cinctum }\end{array}$ & Myolsimus. \\
\hline $\begin{array}{l}\text { tenuicaudatus............ } 245 \\
\text { Anticyclus }\end{array}$ & $\begin{array}{l}\text { cinctum....... } \\
\text { Crystallonema }\end{array}$ & Hyalaimus \\
\hline exilis........ & fuscacepbalum......... 282 & ollis... \\
\hline $\begin{array}{l}\text { Antomicron } \\
\text { pellucidum,............241 }\end{array}$ & $\begin{array}{l}\text { simile................. } 283 \\
\text { Cyartonema }\end{array}$ & llium \\
\hline pellucidum............ 241 & $\begin{array}{l}\text { Cyartonema } \\
\text { flexile....... }\end{array}$ & e...... \\
\hline mollis................. 301 & ricolle & (............. \\
\hline $\begin{array}{l}\text { Apodontium } \\
\text { pacificum } \ldots \ldots \ldots \ldots \ldots .277\end{array}$ & hium...$\ldots \ldots \ldots$ & ineq \\
\hline $\begin{array}{l}\text { pacificum.. } \\
\text { Aponchium }\end{array}$ & icollis & ylaimus... \\
\hline cylindricolle...... & dorus............. & $\begin{array}{l}\text { lonema } \\
\text { ocellatum. . }\end{array}$ \\
\hline Asymmetrella & lonchium......... 300 & Iotsdorus \\
\hline $\begin{array}{l}\text { glabra.................. } \\
\text { attenustum }\end{array}$ & $\begin{array}{l}\text { Cynurs } \\
\text { uniformis. . . . . . . . . . . . }\end{array}$ & ulatus............ 336 \\
\hline Ceramonema........... 264 & Cytolaimium & uns.................... \\
\hline aureus & exile.............. & Iotonchium \\
\hline $\begin{array}{l}\text { Protrellus. } \\
\text { xonchium }\end{array}$ & $\mathrm{mu}$ & (Bütschli). 302 \\
\hline $\begin{array}{l}\text { axonchium } \\
\text { amplicolle ..... }\end{array}$ & AII8. & Iro \\
\hline & Dapt & olaima........... \\
\hline attophils & $\begin{array}{l}\text { fimbriatum } \ldots \ldots \ldots \ldots \ldots \ldots \\
\text { fissidens................ }\end{array}$ & atum...........258 \\
\hline $\begin{array}{l}\text { D sphaerolaima.......... } 271 \\
\text { Bolbella }\end{array}$ & Dasynema & $\begin{array}{l}\text { Isonemella } \\
\text { acuts................. }\end{array}$ \\
\hline $\begin{array}{l}\text { Bolbella } \\
\text { tenuidens... }\end{array}$ & eatum...........253 & \\
\hline $\begin{array}{l}\text { Bolbinium } \\
\text { brevicolle. }\end{array}$ & $\begin{array}{l}\text { demonstrans } \\
\text { Campydora. }\end{array}$ & $\begin{array}{l}\text { llo } \\
\text { ggicauda. . }\end{array}$ \\
\hline 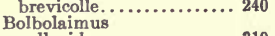 & Didelta & $\begin{array}{l}\text { L longicauda } \\
\text { Laxonema }\end{array}$ \\
\hline pellucidus.............. 319 & $\ldots \ldots \ldots \ldots 252$ & maj \\
\hline us............... 321 & $\begin{array}{l}\text { chus } \\
\text { mis. . . . . . . . . . . }\end{array}$ & $\begin{array}{l}\text { Leptogastrells } \\
\text { pellucida............. }\end{array}$ \\
\hline ...... 264 & imus Dujardin & Leptonchus \\
\hline braziliense & longicollis............. 306 & osus........... \\
\hline 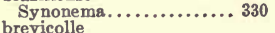 & $\begin{array}{l}\text { Doryllium } \\
\text { uniforme. }\end{array}$ & $\begin{array}{l}\text { mella } \\
\ldots \ldots \ldots \ldots \ldots\end{array}$ \\
\hline $\operatorname{m} \ldots \ldots \ldots \ldots \ldots, 240$ & $\ldots \ldots \ldots \ldots$ & linests \\
\hline Bolbonema............... 264 & exile & lora... \\
\hline Stilbonema............ 24 & $\begin{array}{l}\text { tanema } \\
\text { tolaimi }\end{array}$ & moella \\
\hline $\begin{array}{l}\text { revicollis } \\
\text { Hyalaimus. . }\end{array}$ & $m \ldots \ldots \ldots \ldots 2$ & exilis..... \\
\hline imus.. & m............. & le........... \\
\hline & exilis & 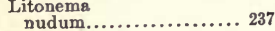 \\
\hline .. 307 & Antic & lito \\
\hline Camp & oella...........2 & onchiu \\
\hline inequalis......... & Schistodera........... 2 & \\
\hline $\begin{array}{l}\text { carcinicolum (Baylis) } \\
\text { Tripylium............ } 288\end{array}$ & & Litot \\
\hline Tripylium............ 288 & Syndum & 23 \\
\hline ellus....... & Synodonti & lon \\
\hline Catal & fero: & atus \\
\hline acuminatu & Thoönchus & $\begin{array}{l}\text { adatus } \\
\text { ulus...... }\end{array}$ \\
\hline ........ & fimbriatum & long \\
\hline & ... & )uj \\
\hline
\end{tabular}




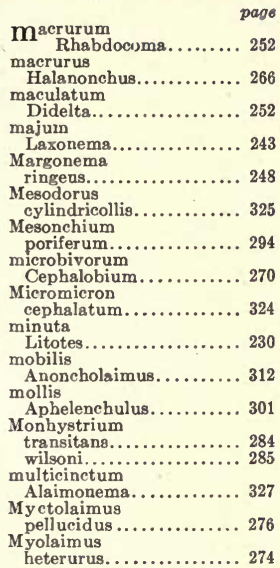

\section{$N^{\text {annolaimus }}$}

guttatus.............. 255

Nemanema

simplex.............. 230

Nemella

ocellata, .............. 236

Neurella

simplex.............. 246

nuda

Xanthodora ........... 317

Nudora

lineata................ 334

nudum

Litonema.............. 237

Zalonema............... 272

$\mathrm{O}^{\text {besum }}$

Xenonema........... 323

obtusum

Synonchium........... 290

oceanus

Trissonchulus........... 297

ocellata

Nemella .............., 236

Onchulella............., 306

ocellatum

Ionems............... 235

Onchium.................... 303

ocellatus

Cophonchus............. 328

Omicronema

litorium $\ldots \ldots \ldots \ldots \ldots .265$

Onchium

ocellatum.............. 303

Onchulella

ocellata................. 306

Onchulus

longicaudatus.......... 313

ornata

Rhips............... 339

pachydermata

Tycnodora............. 231

pachydermatum pacificum page

Apodontiu

papillatum

Isolaimium............ 258

papilliger

Cephalobellus............245

pellucida

Leptogastrella........... 249

pellucidum

Antomicron........... 241

Pepsonema............... 295

pellucidus

Bolbolaimus........... 319

Myctolaimus................ 276

Pepsonema

pellucidum............. 295

perfectum

Xinema............... 263

Polylaimium

exile.................. 274

Polysigma

uniforme............. 326

poriferum

Mesonchium ............ 294

Porocoma
striata................ 236

striata................ 236

prismatolaima

Ironella................ 277

Pratrellus

sureus. . . ............. 256

Pseudolella

cephalata.............. 268

granulifera.............. 268

Pseudonchus

rotundicephalus......... 273

Ptycholaim

carinatus............... 337

punctatum

Coinonema............. 259

punctatus

Bolbolaimus............ 321

punctulatus

Iotadorus.............. 336

Pyenolaimus

pygmaeus............. 258

pygmaeus

Pycnolaimus........... 258

retrorsum

Rhinema.

Rhabdocoma

americanum 252

macrurum .............. 252

Rhsdinems

flexile............... 256

Rhinema

retrorsum............. 335

Rhips

ornata.................. 339

Rhynchonema

cinctum................ 260

ringens

Margonema........... 248

rodens

Chambersiella........... 278

rotundicephala

Amphispira............. 324

rotundicephalus

Pseudonchus........... 273

Schistodera

231

sexalineatum

Dasynema. . ........... 253

simile

Crystallonema. ......... 283

aimplex

Choronema............ 238

Nemanema............... 230

Neurella.................. 246

sphaerolaima

Blattophila............ 271 page

Halinema............. 267

Stilbonema

brevicolle.............. 242 striata

Porocoma............... 236

Xyala....................... 289

Zygonemella...............247

striatus

Iotalaimus. . . . . . . . . 239

Xenolaimus.............. 250

Synodontium

fecundum............. 280

Synonchium

obtusum.............. 290

Synonema

braziliense.............. 330

$t^{\text {enue }}$

Cinctonema............244

tenuicaudatus

Anticyathus........... 245

tenuidens

Bolbella................ 308

Thoönchus

ferox.................. 310

transitans

Monhystrium.......... 284

Trilepta

guttata................ 312

Triplonchium

cylindricum ........... 300

Tripylium

carcinicolum (Baylis).... 288

Trissonchulus

oceanus. ............... 297

Trogolaimus

uniformis.............. 293

truncata

Alaimella............. 234

Tyenodors

pachydermats.......... 231

$u^{\text {niforme }}$

Doryllium............. 303

Polysigma............., 326

uniformis

Cynura................ 262

Digitonchus.............. 314

Trogolaimus........... 293

Villosus

Gonionchus......... 315

viviparus

Acanthonchus.......... 321

$W^{\text {ilsoni }}$

Monhystrium......285

Xanthodora

Xennella

cephalata.

Xenolaimus

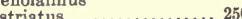

Xenonems

obesum................ 323

Xinema

perfectum.............. 263

Xyala

289

Ypsilon

314

$Z^{\text {alonema }}$

nudum ......... 272

Zanema

acanthurum............ 283

Zygonemella

striata... 



\section{HOWARDULA BENIGNA}

A nemic parasite of the Cucumber-beetle, (Diabrotica)

\section{Contributions to a Science of Nematology $\mathrm{X}$}

\section{By N. A. Совв}

Howardula Cobb, '21. Characters of Tylenchus Bastian, 1865, but without oesophageal bulb and with a non-bulbous onchium and much reflexed ovary. Female finally a flaccid, cylindroid sack, without distinct alimentary canal, and otherwise very much deteriorated. Amphigonic; male, freé-living. Howardula is probably related to Bradynema zur Strassen 1892. The latter however is anonchial, and even lacks a mouth opening.

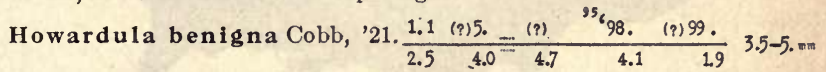

Anus none or vestigial; vulva sometimes terminal; uterus nearly filling the body-cavity, posteriorly packed with larvae and anteriorly with segmenting eggs, near the head in the vicinity of the small spermatheca narrowed and reflexed to the middle of the body, whence the narrow ovary turns forward and ends blind near the head; onchium of adult obscure but the minute mouth opening still persisting. Inert, viviparous, usually all of about the same stage of development in any individual host-insect, each when mature containing about two thousand embryos and segmenting eggs; the larvae, of two kinds, sometimes ten to twenty thousand of them, proceeding from the mother nemas into the body-cavity, and thence into the sexual apparatus, of the host, and so becoming deposited with the eggs of the latter. See Figs. 3, 6 and 7.

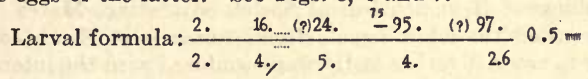
These are the measurements of the larvae as they issue from the vulva. Those within the mother nema and in the anterior part of the uterus are considerably shorter. Anus none or vestigial; tail conoid, straight, broadly rounded or subtruncate at the terminus. After deposition along with the beetle eggs, the young nemas moult with little increase in size; after mating, the female

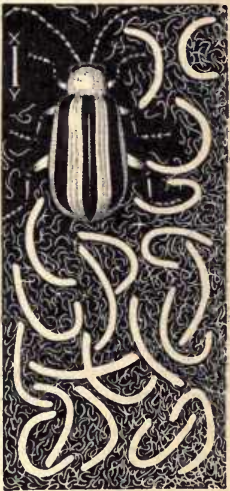

Fig. 1. Cucumber-beetle, and its nemic parasites mature and young; to show relative volume of host and parasites. The line $\mathbf{X Y}$
shows the actual length of the beetle. drills into the body-cavity of even very young beetle larvae of both sexes, sometimes to the number of thirty, but more often five or six. The following

Waverly Press, Baltimore, Md., June 9, 1928. Revised and continued from Nematology X, Aug. 8. 1921, Waverly Press. See Science, Dec. 30, 1921. 
are the dimensions and other details of these young but already spermatized individuals, as found both in the soil and in very young beetle-larvae, which in the body-cavity of the host reach the above seven to ten times longer, mature

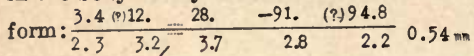

Fig. 2. Map showing distribution of the nema Howardula benrona as disclosed by examinations in 1921 The aim was to examine at least 100 cucumberbeetles from each locality. This aim was achieved beetles from each locality. This aim was achieved
in most cases. The map-figures give the percentage of beetles found infested by Howardula. The figures for different localities a few miles apart in any given region usually were in substantial agreement, but the figures for different regions varied much. Where the percentage of infestation was highest, the nematism percentage of infestation was highest, the nematism exclude other internal parasites, such as other insects and gregarines. Only one other nemic parasite was observed, represented by a single Nova Scotian specimen About $1500 \mathrm{D}$. vittata were examined. Below are the addresses of those who kindly contributed insects for examination.

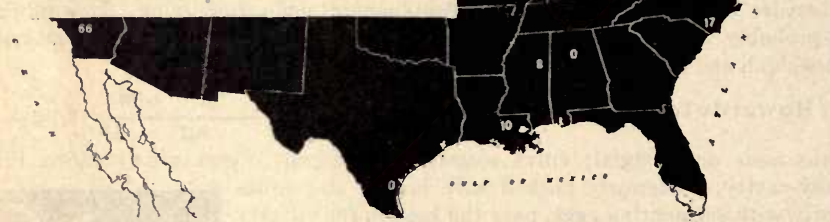

Balduf, W. V. Marietta, O. Hall, Dr. M. C. Chevy Chase, Md. Ross, W. A. Vineland Sta., Ont. Campbell, R. Alhambra, Cal. Harned, R. W. Agr. College, Miss. Smith, C. E. Baton Rouge, La. Cobb, Dr. F. Ann Arbor, Mich. High, M. M. Kingsville, Texas. Thomas, W. A. Chadbourn, N.C. Cobb, V. Whitman, Mass. Kelsall, A. Annapolis Royal, N. S. Walters, M. J. New Iondon, Ct. Gentner, L. Lansing, Mich. Raps, E. M. Oakton, Va.

Walters, M. J. New Iondon, C
Watson, J. R. Birmingham, Al.

Habitat: Common in the body-cavity (abdomen, thorax and even head) of all stages of Diabrotica vittata, trivittata, and 12-punctata, especially the two former, infesting the two sexes about equally.

My attention was called to this nema by Mr. W. V. Balduf, Assistant Entomologist, Ohio Agricultural Experiment Station, Marietta, Ohio, where he discovered the larvae in the course of experiments on Diabrotica. Owing to the economic aspect of the subject, beetles sent me by Mr. Balduf were exhibited, dissected, at the Washington Helminthological Society's meeting, March 17, 1921. Examination revealed the adult female form, which is so flaccid and otherwise deceptive as to cause it rather easily to be confused with the internal organs of the host by one not already versed in both insect and nemic anatomy.

With the aid of Dr. F. H. Chittenden and colleagues, Federal Bureau of Entomology, and others, the geographical distribution of the nema was studied with results shown on the accompanying map, which indicates that the distribution in the spring and summer of 1921 was probably nearly coextensive with that of the main hosts, Diabrotica vittata Fab. and trivittata Mann. The nematism is often high and affects on the average about $20 \%(0 \%-70 \%)$ of the insects. Beetles from a locality where they are not nematized are larger, brighter, more vigorous. Thus twenty-five beetles from an uninfested lot were much 
larger and averaged seventy per cent heavier than a similarly chosen twenty-five from a fifty per cent infested lot. Anatomical evidence shows the infested female beetles to be less fertile than the non-infested, doubt as to diminished fecundity vanishing where the female host harbors a dozen or more adult nemas. In such cases the mere relative volume of the parasites is convincing evidence of handicap. See Fig. 1. Mr. Balduf in a letter speaks of beetles, many of which "died of nemas." I have no rigid proof of such deaths, but believe them very probable and at times numerous. Among the grubs the mortality may be heavy.

In none of the numerous lots of beetles examined was the rate of infestation by any other zoö parasite as high as by Howardula, with the single exception of a forty-three per cent dipterous infestation; but no note was made of degrees of phyto-infestation (cucumber-wilt organism, etc.).

As many as thirteen thousand nemic larvae, by count, have been removed from the body-cavity of a single Diabrotica vittata, and no doubt the number may go much higher. On several occasions twenty or more adult Howardulas were taken from a single beetle. Theoretically these should produce some forty thousand larvae or more. The older female beetles, when nematized, deposit from a few to upwards of fifty nemic larvae with each egg. See Fig. 3. These soon mature on the eggs or in the soil (where they can live several weeks), moult and copulate, the female developing a more perfect spear, and by its aid drilling into the body-cavity of the beetle grubs soon after the latter hatch out. See Figs. 3 and 4. That it is most improbable the nemas enter the host by way of the mouth and alimentary canal is well illustrated in Fig. 8 . The active young beetle larvae are armed with sharp-toothed, well developed mandibles. That the fragile young nemas could, in any considerable numbers, pass so relatively small a throat and mouth, armed as the latter is, one hesitates to believe.

In plant-infesting triplonchs I have shown the development of the so-called salivary glands to be greatest in species noted for their efficiency in destroying the tissues of the host, e.g., Tylenchus dipsaci, Caconema radicicola, and suggested that these glands aid in dissolving the host tissues and thus supplement the mechanical action of the spear or onchium, which therefore should then act also as a spewing channel. In light of this, it may not be without significance that the salivary glands of Howardula benigna, at the time of entering its host, appear better developed than in some of its nearest known relatives. Conceivably this secretion is also antiseptic. Nemas of very many kinds make their way through the tissues of their hosts without causing fatal infections. For instance, I have observed the most important abdomi-

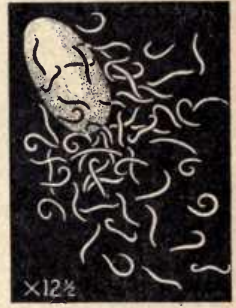

Fig. 3. Egg of a cucumber-beetle and the charge of nemic larvae deposited with it. nal and thoracic organs, heart excepted, of Dasyurus literally sewn through and through by long and slender nemas without apparent infection. The existence of an antiseptic nemic secretion or excretion might explain this. In the case of Diabrotica, there is no known trace left of the relatively large breach made by the parasite (see Fig. 4), a benignant result perhaps facilitated by the parasite itself in the way indicated. 
The Soll-inhabiting Male and Female Howardula, Fig. 4.

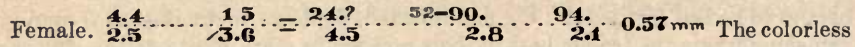
cuticle is traversed by plain transverse striae, all alike, 1.3 microns apart, somewhat difficult of resolution except with the highest powers. Very slightly oblique $15 . \cdots a^{5}$ longitudinal striae, due to the attachment of the musculature, are visible in most regions of the body. No series of pores have been seen in the cuticle. The lips are amalgamated; the lip region has no apparent framework. No labial papillae have been seen. Behind the pharynx the œsophagus is about one-fourth, at the nervering about one-fifth, as wide as the corresponding part of the neck.

The beginning of the intestine is somewhat indefinite; but probably is indicated by the anterior limits of the minute, elongate birefringents, grn bif, Fig. 4. A dorsal œsophageal gland empties through a duct and ampulla near the base of the spear. Two large coarser glands empty a trifle farther back. These glands, Fig. 4,

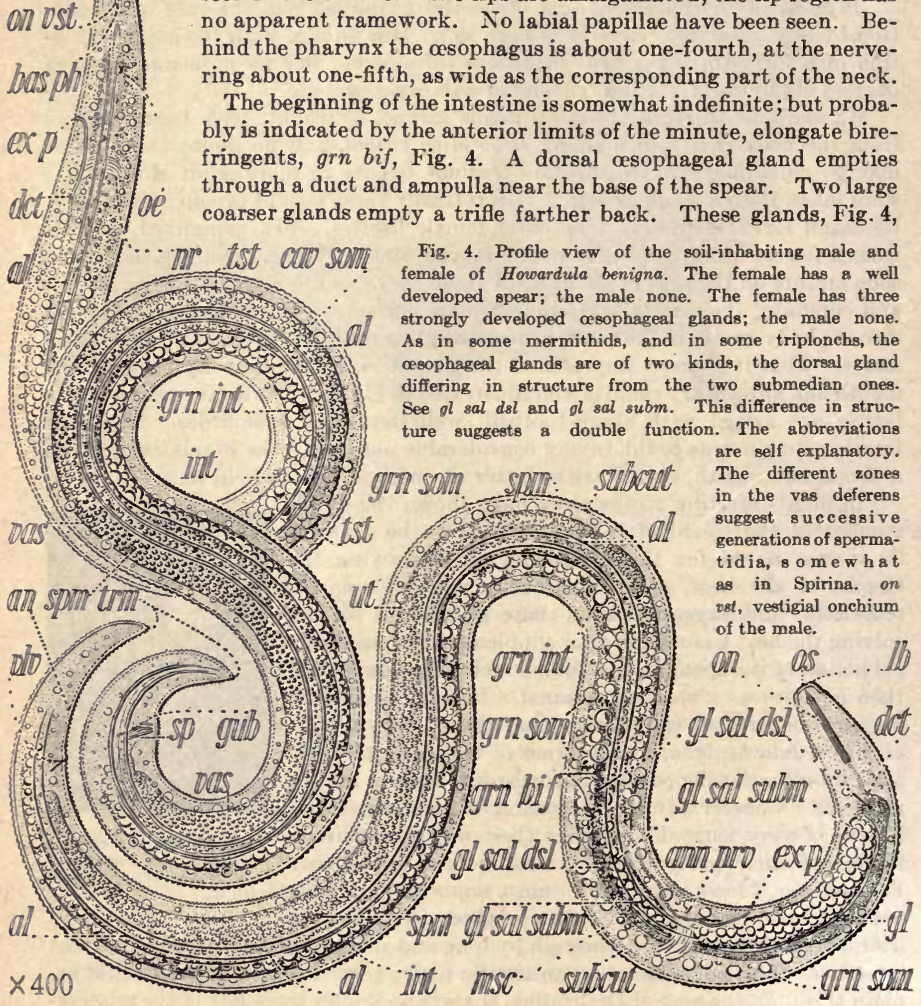

are reminiscent of those in the neck of mermithidae (e.g. Agamermis larvae) and of Tylenchus and Heterodera. The striking difference in the composition of the 
glandular secretions suggests two functions; e.g., (1) solvent action on the chitin of the host at the time the nema drills into the host, and (2) an aseptic effect to aid in healing the wound thus inflicted on the host. Small, irregularly polyhedral birefringents occur among the spherical granules of the intestine. Very small amphids are believed to occur. There are no eyespots. Sometimes the terminus of the female is marked by the presence of one or two small, backward-pointing, short, truncate, cylindroid mucrones, about one micron long and about equally wide. The blind end of the gonad, half as wide as the body, lies near the middle of the nema and is directed forward. Passing backward two body widths, the gonad becomes one-third as wide as the body; five body widths back it is only about one-fifth as wide as the body. It then expands a little, and is separated from the large sperm receptacle comprising the rest of the gonad, by a rather distinct constriction. Beginning narrow near the head, the lateral chords widen regularly until they reach their full width,-about one-third that of the body, -a little behind the nervering. Here, at intervals equalling the body diameter, the lateral chords carry broad ellipsoid cells about one-fourth as wide as the body, containing

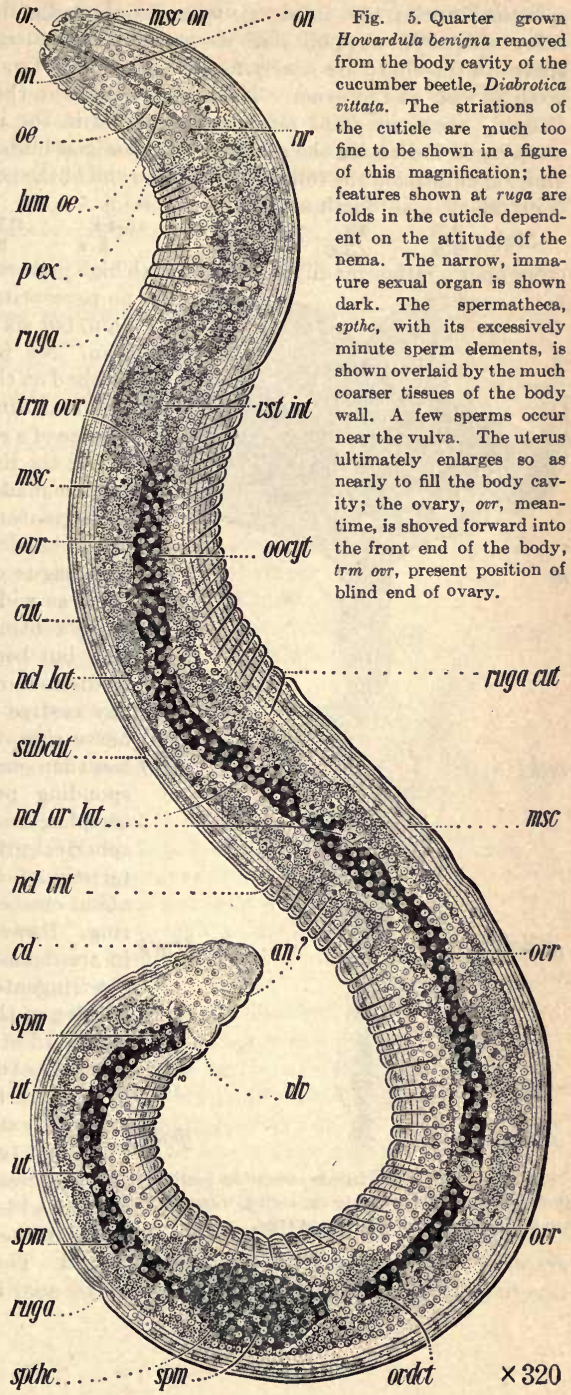


spherical nuclei two-thirds as wide as the cells themselves; and these nuclei carry spherical nucleoli half as wide as the nuclei themselves. The interspaces in the chord are nearly filled by granules of variable size, the largest onesixth as wide as the nema. The chords narrow in the tail to near the terminus. Possibly there are faint refractive elements in the inconspicuous lining of the œsophagus indicating the presence of a vestigial bulbous body. In the specimen under examination the cells near the blind end of the gonad have considerable size, being about one-fourth as wide as the body.

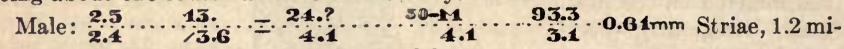
crons apart, rather readily resolvable with high powers. As in the female, there is

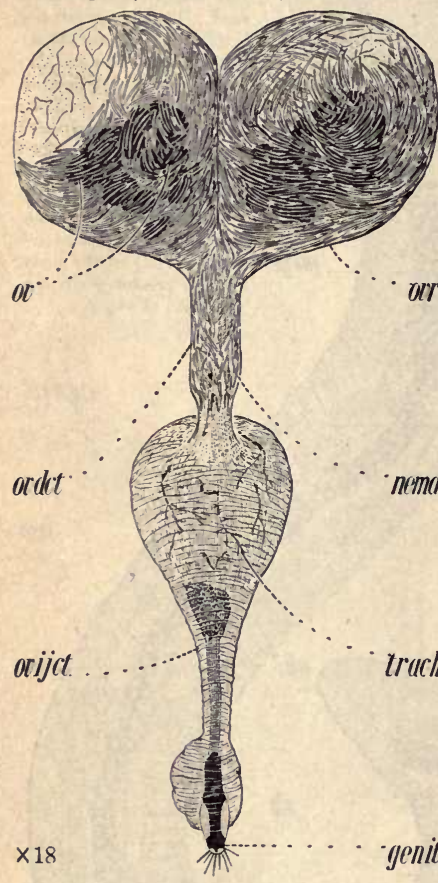

Fig. 6. Gonads of the female cucumber beetle, Diabrotica rittata, previous to egg-laying, when the nemic larvae swarm into the ovaries. no perceptible framework to the lip region, but six very flat lips can be faintly seen. No papillae have been distinguished on the lips. The tail presents a terminal dimple. There is very little vestige of a spear in the pharynx (Fig. 4) but the limits of the pharynx usually can be made out, -its length being a little greater than the width of the base of the head. Behind the pharynx, the

orr œesophagus can be seen to be about onethird as wide as the base of the head, and it continues at first more or less normal, but becomes indefinite. There is no distinct œsophageal swelling, nor has any vestige of such been seen. At the nerve-ring, the osophagus is probably

nema less than one-third as wide as the corresponding portion of the neck. The œsophagus is indefinite behind, but spherical granules, such as are characteristic of the intestinal cells, occur about one body width behind the nervering. However, it seems likely that, as in the female, the first appearance of birefringents probably indicates the beginning of the vestigial intestine. The blind end of the testis, about one-third as wide as the body, is indistinct, and a little more than twice as far from the anterior extremity as the nerve-ring. For about two to three body widths the testis increases in diameter, becoming half as wide as the body, this portion being rather coarsely and irregularly granular. The anal region is very slightly raised. There are two, nearly straight, tapering, blunt spicula, nearly as long as the anal body diameter. These are 
vaguely cephalated, and at their cephalated ends are a little wider than anywhere else. They taper rather regularly to the blunt distal ends. There is a short, simple, narrow, refractive gubernaculum, about one-third as long as the spicula. No definite ribs or special papillae have been seen on the tail, or in front of the anus. The wings project sufficiently so that the cross section of the nema in the vicinity of the anus must be thrown out of dorso-ventral symmetry considerably. The posterior extremity of the male is more or less helical, and, opposite the anus, the somewhat raised wing occupies nearly onefourth the corresponding width of the body. The contour of the wing is almost imperceptibly and very finely crenate, the corresponding annules of the cuticle here averaging about one micron.

Habitat: Garden soils near cucurbits, and especially near eggs and very young larvae of $D$. vittata; widespread. The spherical and refractive intestinal granules hinder an examination of other anatomical and histological features.

\section{Possible transfer of nemas by beetles in copula.}

Males and females of $D$. vittata were gathered and examined for their nematization with $H$. benigna. Of 15 females, 7 were infested; 8 not infested. Of 29 males, 17 were infested; 12 not infested. Roughly speaking, therefore, there was a 50 per cent nematization, of which each sex had about half its individuals nematized. This seemed a suitable case to scrutinize with regard to the transfer of larval Howardulas from the male beetle to his mate.

Of 19 infested males, 7 had nemas in the genitalia,- the number of nemas varying from 1 to 14 . The nemas seemed to tend to gather in the distal end of the genitalia, though found in all parts.

Following up this observation, beetles in copula were gathered and the females examined. If the female was infested in the body cavity, she was not further examined; if not infested in the body cavity, then her more external genitalia were scrutinized for larval nemas, which, if found, could be assumed to have been placed there by the males with which these females were known to have copulated.

Examination of 13 such non-nematized females disclosed no

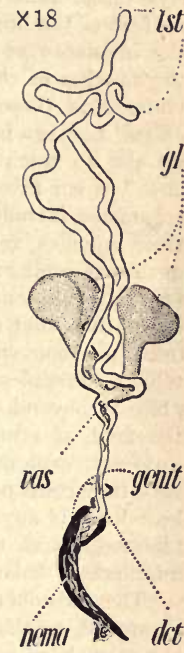

Fig. 7. Gonads of male cucumberbeetle. $t s t$, testis; $g l$, glands. Note nemas in cavity of genitalia, genit (white), and vas (black). Nemas seem less abundant in male genitalia.

larvae of Howardula in the distal genitalia. This might seem to indicate that such transfer of Howardula larvae from male beetle to female was improbable. On the other hand, no sperms were found in these females, although they were present in all the males examined. The fact that no sperms were transferred to the females seems to indicate that the copulation was a mere gesture, which did not result in fertilization. The females were not yet at the egg-laying stage, as was indicated by the condition of their ovaries. In only one female examined during the whole of these examinations was an egg found lower down than the ovaries. This egg was in an infested female and had two or three nemas on its surface; the egg was in the oviduct. This experiment should be repeated. 
The present investigations suggest how far we are from appreciating the abundance and importance of insect parasites and how backward in attempting their control. Howardula is, beyond any reasonable question, ages old, for on no other supposition can the remarkable relationship of host and parasite be explained. It is only one of a considerable number of parasites of the same destructive insect that have much to do with the welfare of the host. Intelligently increasing the incidence of the parasite will decrease the ravages of the host. When we come to understand these relationships, these "balances" between host and parasites, doubtless we can do much toward inclining the "balance" in our favor. We hear more or less of organisms introduced to new areas without their enemies and parasites, and in consequence becoming frightful pests; and we have, very painfully and slowly it seems to some of us, learned that searching for and introducing these same enemies and parasites affords relief. Marked successes of this kind at last place it beyond doubt that this portion of the field of economic parasitology will be carefully explored. But there is another very important part of the field of which we

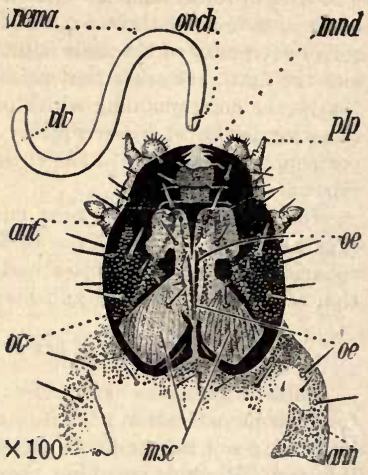

Fig. 8. Head of newly hatched cucumberbeetle grub and young nemia at stage of entering the grub. The sharp active mandibles, mnd, would seem to be rather impassable to the nema. hear little if anything, and that is the comprehension and watchful control of what may be termed indigenous or longestablished "balances."

The cucumber-beetle affords good enough example of these latter to justify an appeal, on the basis of it, to economic biologists to scrutinize more carefully the ever changing "balances" between pests and their parasites and other enemies, including pests of long standing, with a view to keeping the "balance" always inclined in our favor. I believe any well trained, experienced and thoughtful biologist will agree that such a course is bound finally to result in notable economies. A case in point is the existence of localities, among those here tested, in which the total zoö-parasitism of the beetles reached only about two per cent. At the same time not very far away there was a nemic infestation exceeding fifty per cent and a dipterous infestation exceeding forty per cent. The investigation showed that the transference by post of the two parasites mentioned from highly infested areas to low or non-infested areas was easily feasible at small cost. Beetles simply posted in a ventilated box containing cucurbit leaves survived a two to four days' journey; turned loose at night they lived. 


\section{MARIONELLA ${ }^{1}$}

(Eurystoma Marion, 1870);

an emendation, with notes on a new birefringent substance, marionellin, found in the intestinal cells.

Contributions to a Science of Nematology XI

By N. A. Совв

United States Department of Agriculture

Marionella, ${ }^{2}$ nom. nov.

Eurystoma Marion, Ann. Sci. Nat. Zool. V. 13: 19. 1870. Not Eurystoma Raf. 1818.

Cuticle and setae.-The thin layers of the transparent, colorless cuticle are traversed by transverse striae so exceedingly fine that they are very difficult to see even with highest powers of the microscope. Occasionally oblique striae also are to be seen in the cuticle of the lips, running in the direction of a left-handed screw. There are no lateral wings to the cuticle, and the striae are not altered on the lateral fields. There are no longitudinal striae in the cuticle itself, but the attachments of the somatic muscles give rise to longitudinal markings of a character somewhat resembling true striae. The contour of the body is always plain.

The cephalic setae, which are one-third to two-thirds as long as the lipregion is wide, are six or ten in number. Even when at first sight there appear to be only six, it is frequently found that in reality there are ten,each of the four submedian setae having a very short and very inconspicuous companion hugging its base. The setae, two lateral and four or eight submedian, are usually slightly curved, of medium size to very slender, tapering, and somewhat acute, and have innervations that are most clearly visible near their bases; they are of medium stiffness to flexible, and spread outward from the lateral surface of the head opposite the apex of the onchium. Occasionally the larger setae can be seen to be about three-jointed. There are no special subcephalic setae. On the anterior portion of the neck in the vicinity of the head there are always to be found a few small, slender setae, one-sixth to one-eighth as long as the corresponding body diameter; these usually project at right angles to the neck or incline forward at an angle of sometimes as much as forty-five degrees. Not infrequently among the cervical setae there is one, a dorsal one, two to three times as far back as

1 This paper was published in the Journal of the Washington Academy of Sciences, vol. 11 , no. 21 , pp. 504-509 (issued January 9, 1922). It is reprinted with changed pagination but without change of page content.

2 The name Eurystoma, applied by Marion in 1870 to a new genus of free living marine nemas discovered by him near Marseilles, France, was preempted, having been applied by Rafinesque in 1818 to a genus of molluscs. Naturally, investigations made during the last half century enable one, at the present time, to emend Marion's original description. Having examined as many more new, and as yet unpublished, species of the genus as have been already published, I venture to make this emendation, based on a study of about twenty-five species, and to propose for the genus the new name Marionella, in commemoration of its original author. 
the base of the head, which is more prominent than the others. The somatic setae appear always to be reduced to innervations. There are no conspicuous pores on the surface of the body, but the ellipsoidal glandular cells of considerable size invariably found in the lateral fields are connected with the exterior by means of very small and inconspicuous pores.

Head and pharynx.-The somewhat rounded to subtruncate head is usually continuous with the neck, but is sometimes set off by a slight contraction, or by a very slight, broad, shallow constriction. The lip-region, on the other hand, is nearly always set off by a very slight, narrow and shallow, but usually distinctly visible constriction. The membranous lips are themselves thin and confluent, and apparently close by virtue of their elasticity. Their margins are very finely striated and possibly sometimes fimbriate. The lips are supplied externally with six forward-pointing papillae (or six sets of papillae), arranged in a single circlet having a diameter about onehalf as great as that of the lip-region. These papillae are so exceedingly small as usually to escape observation. Their innervations are also exceedingly inconspicuous. Usually it is only when the papillae happen to project forward a little in exact profile that they come into view.

At first sight the pharynx appears to have the form of that of Oncholaimus. It is, however, nearly always divided into two distinct chambers of more or less equal length, the anterior of which is somewhat the wider and the more symmetrical. This regular anterior chamber is nearly always a napiform cavity one-half to two-thirds or even three-fourths as wide as the corresponding portion of the head. The posterior chamber is of smaller size, usually only about three-fourths as wide as the anterior chamber, and more or less irregularly spheroidal in form. The refractive walls of the pharynx are well-developed, but not very thick. The pharynx is usually armed with a single well-developed, forward-pointing onchium, but there may be one or two additional smaller onchia in exceptional cases. The apex of the main, and usually only, onchium, always occupying the right ventral submedian position, extends forward sometimes as far as the lips. The convex-conoid, enlarged base of the onchium fills the posterior chamber of the pharynx comparatively full. This enlarged portion of the onchium is continued in the front chamber by a narrower, more slowly tapering, convex-conoid, more or less acute summit. This organ is perforated and so serves as the outlet of a large oesophageal gland. The posterior pharyngeal chamber is separated from the anterior by a constriction, marked in its most pronounced form by the presence of narrow and refractive, transverse, arcuate elements or thickenings, placed end to end in a circle. Adjacent to this circle, usually in front of it and close together, there are one to three transverse rows of denticles, each consisting of either about thirty-two, or about sixty-four to eighty, somewhat forward-pointing units. These more or less inward pointing denticles are rather acute, cylindroid-conoid, uniform in size, and are usually so minute as to be barely resolvable with high powers of the microscope.

When viewed from in front, the pharynx is seen to be very nearly round. The margin of the lip-region is so thin and filmy that oftentimes it is difficult to delimit. An exact count of the denticles in a specimen of an unpublished but typical species, gave the following figures:- anterior series of denticles 62 ; second series 62 ; back series of larger denticles 20 ; - this decreased number in the back row being due not only to the fact that the denticles are larger 
and farther apart, but also to the fact that one-third of the circumference is destitute of denticles, namely that part of the circumference opposite the large onchium. In this particular region, however, the interior walls of the pharynx are very finely longitudinally striated. The back row of denticles, it should be pointed out, is on the wall of the posterior half of the pharynx.

The neck is conoid, or occasionally subcylindroid, and ends in a cylindroid or, more often, a rather decidedly convex-conoid head.

Amphids and eye-spots.-Though the amphids are well-developed, they are rarely plainly to be seen. Their exterior expression consists of two dorsally sub-lateral concavities two to three times as wide as long, impinging on the bases of the lateral setae. Though the peripheries of the amphids may seem to be closed, they are, in fact, nearly always found to be open on the posterior margin near the lateral fields. As a rule their contours are almost invisible, so that their form and extent are mainly indicated by the apparent absence in them of the fine structural elements to be seen elsewhere in the cuticle of the head. When clearly defined, their contours are found to be reniform with the convex side forward. They are located on or near the base of the lip-region, and more or less opposite to the rows of denticles. They are usually one-third to two-fifths as wide as the corresponding diameter of the lip-region and two to three times as wide as long.

More often than not two eye-spots are present. 'These take the form of spheroidal, compact collections of about one hundred brownish granules, each collection lateral in position and lying between the oesophagus and bodywall,-being about one-fourth as wide as the corresponding portion of the neck, and removed from the anterior extremity by a distance two to four times as great as the width of the head. Anteriorly, these ocelli often present a spherical cavity in which there is at least the suggestion of a spherical lens.

Oesophagus.-The simple conoid oesophagus is destitute of bulbs and receives the base of the pharynx in its anterior extremity, where it is usually about half as wide as the base of the head. Near the nerve-ring it is usually about one-half, and posteriorly usually about three-fifths, as wide as the corresponding part of the neck. It is always separated from the intestine by a distinct cardiac collum about one-third as wide as the base of the neck. While not conspicuous, the lining of the oesophagus is a distinct feature throughout its length. Though the oesophageal musculature is usually fine, the structure is occasionally coarse in the posterior part. Rarely, yellowish spherical granules are found in the tissues of the oesophagus. The oesophageal glands are well-developed, or at least one of them is; as before stated, they empty into the pharynx through pores in the onchia. The right submedian gland is without exception the largest. There is usually a well-developed conoid or hemispherical cardia one-third to one-half as wide as the corresponding portion of the neck.

Intestine.-The intestine becomes at once one-half to two-thirds as wide as the body, and is made up of cells of such a size that few are required to build a circumference. Its walls are thick, and its lumen faint. Its cells invariably contain fine spherical granules of more or less variable size, the largest of them being one-fortieth to one-twentieth as wide as the body. The granules are scattered, or sometimes numerous, in the cells, and may be so arranged as to give rise to a faint tessellated effect, though this is unusual. 
Doubly refractive granules (Marionellin) in the intestinal cells. - In the single layer of cells composing the intestine, an undescribed species of Marionella presented about twenty scattered special cells, more numerous and closer together anteriorly, each packed with doubly refractive granules (marionellin) mostly of very small size. Marionellin occurs in other species of Marionella.

These special intestinal cells were not distributed along a definite longitudinal line as in Ironus, where there is a decided dorso-ventral symmetry to the intestine due to the dorsal cells having a different character from the ventral. The interspaces between these special cells in this species of Marionella increased rather regularly from front to rear.

The discovery of these special intestinal cells is an additional observation indicating differentiation among the cells of the nema intestine. Such differentiated cells are now known to the writer in the following genera, among others: Enoplus, Bathylaimus, Ironus, Mononchus, Eurystoma (all carnivorous). It seems very reasonable to suppose that these differentiated cells may have functions similar to those of the glands accessory to the intestine of other and larger animals. Assuming that digestion in nemas has a general similarity to that of the higher animals, it would seem that gastric, hepatic, renal and other functions must exist in some form in the nema; thus far, however, very few of these functions can be assigned to special organs, as few or no such special organs exist. Instead of each cell of the intestine carrying out all of these distinct functions, in view of the above observations there is now morphological evidence of "division of labor," and when these differentiated cells have been adequately investigated, we shall probably be able to assign to them definite functions, and, for illustration, be able to apply to them some such terms as "hepatic cells," "renal cells," "splenic cells," etc.

Tail.-The tail in Marionella takes on one of two distinct forms, according as there is or is not a spinneret present. If there is no spinneret the tail is conoid from the anus to the acute terminus, sometimes however tapering a little more rapidly in the anterior portion than elsewhere. In species possessing this form of tail, usually the tail of the male is the shorter, and the narrow conoid posterior part may appear rather as an appendage to the short but bulky anterior part. Most of the species, however, possess three well-developed unicellular caudal glands and a blunt, conoid, unarmed, and symmetrical terminal spinneret about one-fourth as wide as the base of the tail. If any setae occur on the tail, they are exceedingly small and very inconspicuous. The three quite separate ducts of the caudal glands are plainly visible in the tail and end posteriorly in three separate ampullae. The unicellular glands themselves are arranged in a loose tandem in front of the anus, the foremost being removed a distance from the anus several times as great as the corresponding body diameter.

Lateral fields: glandular cells.-The lateral fields are usually about half as wide as the body, and contain large, granular, ellipsoidal, glandular cells ahout one-third as wide as the body and emptying on the surface of the cuticle by means of exceedingly minute pores. These large glandular cells are situated from point to point throughout the length of the body, the distance between them being from one to four times as great as the width of the body

Renette.-The renette cell is invariably situated behind the neck and empties by means of a long, narrow, faintly visible duct. The narrow in- 
conspicuous ampulla is situated nearly opposite the base of the pharynx. The obscure excretory pore is invariably located in the lip-region opposite the row of cephalic setae. The presence of the duct and ampulla usually causes the pharynx as well as the portion of the oesophagus near the head to be a little nearer to the dorsal side of the body than to the ventral.

Nerve-ring. - The nerve-ring is always a rather conspicuous feature. As a distinct collar it surrounds the oesophagus a trifle obliquely and is of medium size, and has arranged both in front of it and behind it numerous large nuclei, whose grouping, however, does not appear to be very orderly.

Female organs. - The female sexual organs are invariably double and reflexed (' $\mathrm{f}$ '). The vulva, though large, is more or less continuous and not very conspicuous. The well-developed vagina leads inward at right angles to the ventral surface about two-fifths of the way across the body, and, though fairly muscular, is not very amply cutinized.

The two straight uteri are of such a size as to contain two or more eggs at a time, arranged tandem; these latter are thin-shelled, smooth, usually ellipsoidal or somewhat elongated, and are deposited before segmentation begins. The reflexed ovaries are broad, or of medium width, taper more or less, and extend one-half to two-thirds the distance back to the vulva. The ova in them are arranged single file except near the blind end, where they are arranged irregularly.

Male organs. - The tail of the male is like that of the female except that it is usually shorter and more pronounced in its features, especially in species lacking a spinneret. In all the species that have been carefully examined in this respect, namely in the majority of the species, there are two outstretched testes extending in opposite directions, the anterior one ending a neck-length or more behind the cardia, the other near the beginning of the posterior fourth of the body. The two equal spicula are invariably arcuate, and occasionally strongly so. At their widest part they are one-sixth to oneeighth as wide as the corresponding portion of the body. They are from one and one-fourth to two times as long as the anal body diameter, and when viewed in profile their proximal ends appear to lie opposite to or slightly dorsad from the body axis,-very rarely ventrad. The proximal ends are almost always very slightly cephalated by expansion, but they are sometimes faintly cephalated by constriction or by contraction. They are somewhat slender, of rather uniform width, and rather blunt at the free end, where they sometimes terminate in a simple or denticulate crochet. The gubernaculum, placed at right angles to the distal parts in the spicula, though sometimes of uniform width, usually tapers internally to a blunt or acute point, which lies opposite to or dorsad from the body diameter. From this apophysis muscles lead fore and aft to the dorsal body wall. The portion of the gubernaculum applied to the spicula is one-sixth to one-eighth as long as these latter.

Supplementary organs. - Invariably two large, ventral, pre-anal supplementary organs are present, though in a few species they are more or less restigial. They are placed in front of the anus in such fashion that the posterior one is about as far in front of the anus as the spinneret is behind it, and the anterior one about as far in front of the posterior as this latter is in front of the anus. There is, however, some variation in the situation of this pair of supplementary organs in the different species. Nearly always the posterior supplement is a little smaller than the anterior, some- 
times markedly so. When well-developed, these organs consist of highly refractive elements, both external and internal, which are very striking in their appearance. To a considerable extent these supplements can be protruded and withdrawn. When protruded they are very prominent. When withdrawn they may leave the ventral contour comparatively even; and yet, even when withdrawn, they are hardly less conspicuous than when protruded, owing to their highly refractive character. The most striking internal elements are two in number to each supplement, extending, one forward and the other backward, and may appropriately be termed "levers." These levers are somewhat finger-shaped pieces of cutinized material that serve for the attachment of muscles. They usually taper but little, and their internal extremities are invariably blunt. The two levers of a given supplement are usually practically equal in size. When the supplement is at rest, the levers lie near the ventral side of the body and parallel to it. In such circumstances the exterior portion of the organ protrudes only slightly; but when the free inner ends of the levers are drawn inward so that they lie at an angle with the ventral surface, sometimes as great an angle as fortyfive degrees, the external portions of the supplements are protruded. The external portion of each organ has the form of a laterally compressed cup, or trough, whose profile is exteriorly flat, or more often slightly concave, and interiorly more or less semi-circular. While the depth of the organ may sometimes equal its width, often it is less, and sometimes only one-half or one-third as great. Those species showing the maximum development of the supplementary organs present cases where the depth of the organ is one-fourth as great as the corresponding diameter of the body. In one species the supplementary organs are asymmetrical, the anterior lever or anchor having become vestigial, and the anterior portion of the cup or trough having diminished relatively in size, so that the contour of the longitudinal section of the organ is triangular rather than semicircular. By means of a duct each supplementary organ is connected internally and forward with a large glandular cell, as in Bolbella.

Setae on the male.-Just in front of the anus on the male there are usually to be found a few minute setae, either ventral or subventral in position. They are very short and very inconspicuous. There may be a single one at the anus; more often there are one or two subventral ones on each side Occasionally there are two rows extending to near the posterior supplement. These setae are arcuate, acute, and when two are present on each side of the anus, one of the pair is usually located immediately behind the other and is of smaller size. No other papillae or setae have been observed on the tail end of the male. There is no bursa.

Habitat.-The genus Marionella has hitherto been supposed to be of rather small size. It is in reality large, and is widespread in the various oceans. While the individuals of a given species may not be numerous, or very widespread, the number of specific forms observed is yearly augmenting. The two sexes are about equally common.

The genus is most nearly related structurally to Bolbella, Symplocostoma, Thoönchus and Catalaimus.

Marionella spectabilis (Marion) is still retained as the type species. 




\section{GREEFFIELLA}

(Trichoderma Greeff, 1869; not Trichoderma Steph. 1835)

Contributions to a Science of Nematology, XII

By N. A. Совв

United States Department of Agriculture

In 1869 , Greeff described an externally peculiar and very interesting small animal form under the name Trichoderma. Though it proves in the end to be internally a typical nema, it is only after many years that the fact becomes fully established. The minute size of the species and the fact that the setose cuticle obscures the internal organs, taken together, have delayed a fuller understanding of the internal anatomy.

Opportunity has occurred to reexamine a species of this genus in a living condition, and the results are presented herewith. They serve to establish the view that the genus comprises typical nemas presenting striking relationships to Desmoscolex. Greeff's original discovery is commemorated by renaming the genus Greeffiella. $G$. oxycaudata (Greeff) is retained as the type species.

\section{Greeffiella, nom. nov.}

Trichoderma Greeff, Arch. f. Naturg., Berlin, v. 35, bd. 1, 1869 . Not Trichoderma Steph., 1835, or Swains., 1839.

Greeffiella dasyura n. sp. $\frac{1.5}{3.4} \frac{10.4}{9.6,13 .}-\frac{56 .}{15 .}-\frac{70 .}{9.8}>0.31$. of the transparent, colorless, hairy cuticle are traversed by about fifty-six plain transverse annules, easy of resolution, which are not materially altered on the lateral fields. The number of annules corresponds with the number of encircling rows of somatic setae. While there are no wings opposite the lateral fields, wing spaces are faintly indicated by a slight spareness, or absence, of setae near the lateral lines; this however is a faint feature extending only from the neck to the anus, and is perhaps more pronounced on the female than on the male. The contour of the body is crenate, especially toward the head. There appear to be toward thirty small unequal cephalic setae on the front of the head, disposed, apparently, in two closely approximated circlets. These setae average to be about as long as the head is wide and are apparently too numerous and crowded to permit of any exact order; however, about twelve of the anterior ones are spread outward and forward while all the others spread out more or less backward. These somewhat curved, rather slender, tapering acute, somewhat stiff cephalic setae are of the same

1 This paper was published in the Journal of the Washington Academy of Sciences, vol. 12 , no. 13 , pp. 229-303 (issued July 19, 1922). It is printed with changed pagination but without other material alteration. 
character as the great bulk of the somatic setae. Among the somatic setae however are a few relatively large, hollow, open bristles of another character, resembling the locomotor bristles found on Draconema, Desmoscolex, etc. For in-

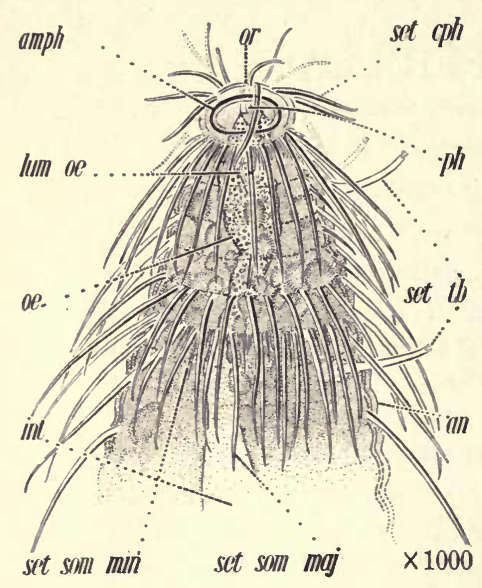

Fig. 1.-Head end of Greeffiella dasyura. The setae on several annules immediately behind the head have been omitted so as to show internal details more clearly. amph, amphid; an, annule; int, location of the beginning of the intestine (see also Fig. 2.); lum oe, lumen of the oesophagus; oe, oesophagus; or, mouth opening; ph, pharynx; set $c p h$, cephalic setae,-a number of which are omitted; set som maj, one of the larger somatic setae; set som min, one of the smaller somatic setae; set $t b$, tubular setae. flattish hemispheroidal head, set off by a narrow, deep and distinct constriction. The lips are amalgamated and fixed. Nothing is known concerning the labial papillae. The pharynx is exceedingly minute and easily overlooked, but is, in fact, a minute, simple, obscure, straight, regular, tubular, closed, unarmed region about one-sixth as wide as the head and twice as long as wide; these measurements include its enclosing pharyngeal tissue. Under ordinary circumstances there is to be seen here only a closed lumen. Passing backward from the pharynx, the oesophagus for a distance two and one-half times as great as the width of the head, is cylindroid; however, it widens slightly, so that it becomes as wide as the head, or one-half as wide as the corresponding portion of the neck, that is to say that portion of the neck marked by the fifth circlet of cervical setae. At this point there is a rather faint diminution of the oesophagus, which continues thence a little narrower, afterward widening out, and then soon coming to contain granules like those found in the cells of the intestine. This latter appears to begin about opposite the tenth row of setae. There are two narrow ducts, one emptying into the posterior part of 
each amphid; these ducts can be followed backward to near the pigmented bodies soon to be mentioned, and possibly may be connected with them. The external expressions of the amphids, each of which is symmetrical to two lines, are of unequal diameter, without central markings, and are located toward the front of the head; they are about as wide as the corresponding portion of the head, each being about twice as wide as long. The two greenish pigmented bodies mentioned above (org?, Fig. 2), are olive green in color and present a nucleus in the midst of a colorless spherical cell (?) as wide as one of the cuticular annules in the immediate vicinity. These bodies are naturally rather difficult to observe on account of the hairy nature of the cuticle through which they are viewed; they are located well outside the intestine, one on each side of the body, somewhat behind the base of the neck. The broad cardiac constriction lies opposite the eighth to tenth rows of setae, and is about as wide as the distance between these rows. The thick-walled intestine presents a faint lumen and is composed of cells of such a size that about twelve occur in each cross section. In the male, at least, the intestine gradually becomes one-half as wide as the body. There is no pre-rectum. From the minute anus, whose anterior lip is somewhat elevated, the inconspicuous rectum extends inward at right angles to the ventral surface half way across the body; the intestine itself extends past the anus. No anal muscles are to be seen. There are two kinds of colorless granules of variable size to be seen in the cells of the intestine; the largest of these have a diameter equal to the distance between the rows of somatic setae; the finest of the granules are exceedingly fine. The granules are not so arranged as to give rise to a tessel-

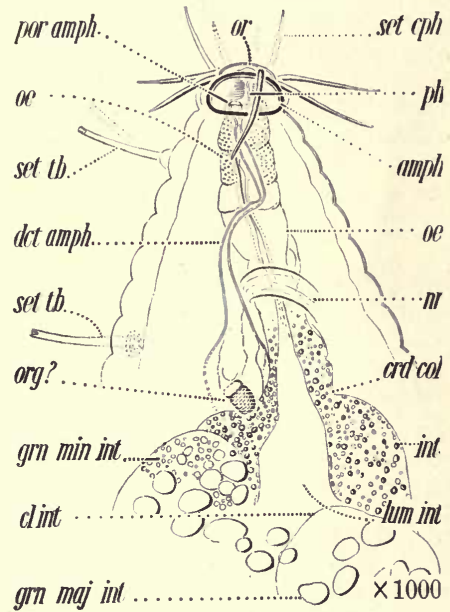

Fig. 2.-Internal anatomy of the head end of Greeffiella dasyura. Lettering as in Fig. 1. $\mathrm{cl}$ int, one of the cells of the intestine; $c r d$ col, cardiac collum; dct amph, duct connecting with the amphidial pore; grn maj int, one of the larger intestinal granules; grn min int, smaller intestinal granules; int, intestine; lum int, lumen of the intestine; $n r$, nerve ring; org ?, organ of doubtful significance; set $t b$, tubular seta.

lated effect. The more or less convex-conoid tail tapers from in front of the anus to the tubular spinneret, which comprises two-sevenths of the whole tail. This tubular spinneret is about as wide as one of the spicules of the male; it is a simple truncate affair which tapers but very little. A marked peculiarity of the posterior extremity of the nema is the existence of numerous minute setae; for a distance equal to the length of the spinneret the setae on the portion of the tail immediately in front of the spinneret are very much reduced and more numerous. The spherical caudal glands are located behind the anus in the anterior fourth of the tail and empty through separate ducts; each is about one-fifth as wide as the corresponding portion of the tail, or as wide as one of the somatic annules opposite. Only two nuclei were seen in 
connection with these glands, and these were located in the vicinity of the anus, their number indicating that the number of caudal glands may be less than the usual three. The excretory pore lies near the nerve-ring opposite the sixth annule in the male and opposite the seventh in the female; its spherical ampulla is one-fourth as wide as the corresponding portion of the neck. The nervering surrounds the oesophagus somewhat obliquely where it first diminishes in diameter somewhat behind the middle. In the dorsal side of the neck, opposite the $9-14$ rows of setae there are some relatively large organs, probably two or more finely granular cells. From the somewhat inconspicuous, small, elevated vulva, which is surrounded by minute setae, the small, weak, non-cutinized, tubular vagina leads inward at right angles to the ventral surface about one-third the distance across the body. Little is known concerning the double symmetrically reflexed female sexual organs.

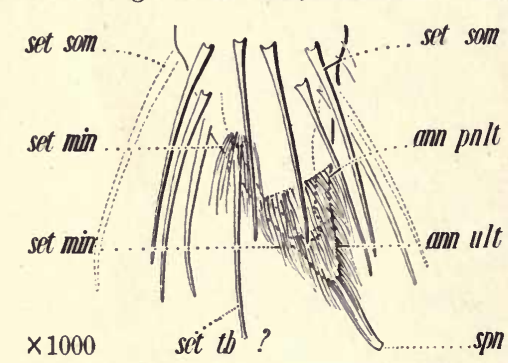

Fig. 3.-Tail end of Greeffiella dasyura. Lettering as in Figs. 1 and 2. ann pnlt, penultimate annule; ann ult, ultimate annule; $s p n$, spinneret. $\frac{1.5}{3.2} \frac{6.6-10.6}{7.5 \%} \frac{1 \mathrm{M}}{18 .}-\frac{76 .}{11 .}>0.34 .$.

The slightly arcuate, irregularly conoid, faintly sigmoid tail of the male, on the whole, rather resembles that of his mate. The two equal, straight, very slender, uniform, acute, colorless spicula, which are slightly cephalated by expansion, are about one and onefourth times as long as the anal body diameter. If swung around behind the anus, they would just about reach to the base of the spinneret; they are about twice as wide as the bases of the somatic setae, are a little larger distally than elsewhere, and are perhaps winged. No gubernaculum has been seen. On the fifth and eleventh annules in front of the anus occur ventrally submedian papilla-like organs, indicated by the presence of minute setae arranged in a cluster about a nerve ending (?),-about ten setae on the fifth annule and a much smaller number on the eleventh. There are also similar ventrally sublateral bunches of setae on the annules preceding the large (duplex?) conical one bearing the spinneret. On the lateral field near the middle of the male, a bunch of minute setae was observed like those on the fifth annule in front of the anus. The nature of these special organs, for such they must be, remains in doubt. It seems quite possible that some of them are male supplementary organs. The wide cylindrical testis is one-half as wide as the body and is reflexed to near the proximal ends of the spicula.

Habitat: Found in sponges, Biscayne Bay, Florida, U. S. A., March, 1916. Male examined and measured in a living condition; female fixed in Flemming's solution and soon after examined and measured in water. The form of the pharynx and oesophagus; the presence of special tubular setae; the structure of the spinneret, and the relatively small number of annules, seem to indica te a closer relationship of Greeffiella with Desmoscolex than has been hitherto imagined. Perhaps Greeffiella should be placed in the same family with Desmoscolex, Tricoma, etc. 


\title{
An Amendation
}

of

\section{HOPLOLAIMUS DADAY 1905 nec auctores}

\author{
Contributions to a Science of Nematology, XIII
}

By N. A. Совв

In 1905 Daday proposed the new genus Hoplolaimus on the basis of a single female nema from soil in Paraguay.

Hoplolaimus was so imperfectly characterized that numerous subsequent authors have referred to it a variety of species that seem certain not to belong to it, in the light of recent discoveries now to be described.

Hoplolaimus Daday 1905 amend.

Coarsely annuled typical tylenchidae with a prominently set off, lobed lip-region composed of several annules, and an onchium with more or less lobed basal bulbs. -f- and -m. Males with lobed bursa encompassing the tail.

H. coronatus n. $\mathrm{sp} . \quad \frac{3.4}{2 .} \frac{4}{4}-\frac{7.8}{2.7}=\frac{12 .}{3 .}-\frac{23}{-56} \cdot \frac{26}{3.3}-\frac{98.4}{2.4} 1.6 \mathrm{~m}$ The transparent colorless layers of the naked cuticle are traversed by plain, transverse striae, all alike, about three microns apart and easy of resolution, which are not further resolvable, and which are altered materially on the lateral fields by the presence of three longitudinal wings, occupying a space, measured midray on the midway on the nema, one-third as great as the width Hoplolaimus at lat. 86, and in of the body. The optical expression of these wings the right lateral chord of the consists of four parallel lines, of which the two outer interesting unparred spheroiare rather distinctly crenate, while the two inner, dal amphid-like organ oneoccupying a little less than a third of the wing space, having connections fore and connect with refractive cuticular alterations of the aft, and opening outward on striae, which thus give rise to a rather distinct more ${ }_{\text {microns }}^{\text {acessed neas-circle, } 8} 8$ or less quadrangular network on the lateral fields.

On the neck the wings become reduced to two. The cuticle, about two and one-half microns thick, is striated internally as well as externally. As usual, the annules close to the lip region are somewhat narrower than those farther, back. From somewhat behind the anus the final striae on the tail of the female make a gradually smaller angle with the lateral line and finally eneirole. the terminus in the lateral plane. Very slightly oblique longitudinal striae; due to the attachment of the musculature, are visible in most regions of the body. There are no dermal appendages and no series of pores has $/$ boen! seen in the cuticle, but there is an unpaired lateral organ on both the female and male. (See Fig. 1.) The cylindroid neck lbeconies convex-conoid at the rounded head, which is continuous and presents a central rnouth opening only very slightly depressed. The dip negion however is a flat, bluntish cone, about twenty microns broad by eight mierons high; it is set apart by a very idistinct 


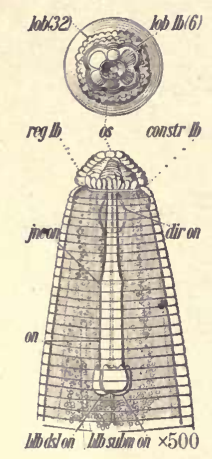

Fre. 2. An oblique dorso-ventral view of bead of $H$. coronatus $\mathrm{n}$. sp. The quadrangular nature of the cap is shown in the front view, above.

constriction so that it constitutes a sort of cap on the front of the head. This somewhat quadrate cap is longitudinally faintly six-lobed and each lobe is again longitudinally as well as transversely subdivided. See Fig. 2. The lip region of the male is like that of the female except that it is more nearly hemispheroid, that is, relatively higher and slightly larger. There is a rather robust six-ribbed, yellowish, dome-like structure as the framework of the lipregion, through it is obscured by the nature of the cuticular covering. This framework extends a little back of the labial constriction and its yellow color becomes more evident here. See Fig. 3. Needless to say, therefore, the amalgamated lips are fixed, and shut closely around the anterior extremity of the onchium. Whether the lip region is innervated remains unknown, but no innervations have been seen. The tylenchoid pharynx is of a very robust nature and reminiscent of that of Nemonchus. The base of the onchium, or spear, thirteen microns wide by ten high, is very distinctly three bulbed and is about one-fifth as wide as the corresponding portion of the head. Each of the three bulbs is anteriorly somewhat "lobed," presenting sometimes two and sometimes three rather distinct forward pointing knobs. Owing to its index of refraction this lobed base of the onchium, as well as the "hilt," are almost totally invisible in balsam mounts; while the acute tapering anterior part remains distinctly visible,-another evidence of the two-fold character (and origin) of the tylenchoid onchium. The posterior attachment of the musculature comprising the ellipsoidal pharyngeal bulb, which is one-half as wide as the head, is not only to the front portion of the bulbs of the onchium but also to their posterior surfaces. The hilt is about half as wide as the bulbous base; the anterior end of the spear is blunt, and the lumen relatively unusually narrow; - all which makes the spear an unusually substantial structure, capable of puncturing tissues offering considerable resistance. In harmony with this, the ellipsoidal to obpyriform spear guide is of strong construction, consisting in part of six outwardly bowed elastic elements surrounding the anterior third of the spear and springing backward from the base of the cutinized lip region. (Figs. 2 and 3.) This six-fold spear guide has a variable length and width, its form changing with the attitude of the spear; - when at rest, with a length of fourteen microns, its width is about eight microns, - that is, it is about one-third as wide as the corresponding portion of the head. In addition to this spear guide the cutinized lipregion fits closely around the anterior portion of the spear for a considerable distance. Both the spear and the spear guide appear to present traces of transverse striation corresponding in fineness with the minute subdivisions of the annules sometimes visible in the subcuticle. The two parts of the spear are rather distinctly set off from each other by a very fine transverse junction mark, as in many Tylenchi. No amphids, deirids or phasmids have been seen. There are no eyespots. The oesophagus is tylenchoid, presenting however, as already indicated, a rather distinct pharyngeal bulb, something rather uncommon in the Tylenchidae. The spherical or oblate median oesophageal bulb is half as wide as the corresponding portion of the neck, and is set off both fore and aft from the remaining narrow portions of the oesophagus,very abruptly behind and rather abruptly in front. Behind the median bulb the narrow oesophagus gradually enlarges to form a rather obscure posterior 
long-clavate swelling which at its widest part is one-fourth as wide as the base of the neck. It is however natural to imagine the swollen salivary glands to be joined with this inconspicuous posterior portion of the oesophagus and thus at first to get an idea that the posterior part of the oesophagus comprises a wide clavate swelling, three-fourths as wide as the base of the neck. We may say therefore that the oesophagus behind the pharynx is about one-seventh, at the nerve-ring also about one-seventh, and finally about one-fourth as wide as the corresponding portion of the neck. The lining of the oesophagus is a distinct feature arteriorly and consists of a narrow highly refractive tube; posteriorly the lining is inconspicuous. The musculature is fine and colorless. There are three salivary glands clustered opposite the posterior two-fifths of the oesophagus. One of these cells comprises the anterior portion of the cluster, while the other two may lie more or less opposite each other as the posterior part. The salivary glands are very well developed, and two of them, submedian, empty through ducts into the base of the valve of the median bulb, while the third, the dorsal, passes forward and empties into the dorsal side of the oesophageal lumen not far behind the base of the onchium. At the mouth of each duct a faint ampulla is usually visible. The median oesophageal bulb presents a spheroidal, simple, strongly refractive valve one-sixth as wide as the bulb itself. There is no distinct cardia. The thick-walled intestine, which presents a distinct refractive lumen, is not set off from the oesophagus by a cardiac collum, the change from oesophagus to intestine being gradual. The intestine has its cells closely packed with granules of variable size, the largest being one-twelfth to one-sixteenth as wide as the body. These colorless, non-birefringent, spherical granules gradually decrease in number in the cardiac region and cease altogether opposite the posterior portion of the salivary glands; they are sometimes so arranged as to give rise to a tessellated effect. The intestine somewhat gradually becomes four-fifths as wide as the body, and is composed of eells of such a size that two to three are presented in each cross section. The cells of the intestine are so closely packed with granules as to make it difficult to examine successfully in living specimens details

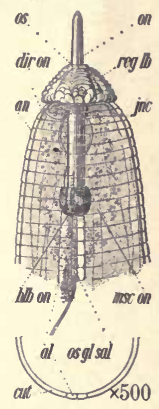

Fig. 3. Nearly lateral view of $H$. coronatus n. sp. Below, a semi-contour of the body in the same region, showing the double wing. of the anatomy of adjacent organs. The exceedingly small anus and the rectum are very inconspicuous. There is no pre-rectum. The tail is of an elongate hemispheroid form, very broad and rounded at the extremity, and symmetrical. There is no spinneret and there are no caudal glands. Measured at the latitude of the nerve-ring, the lateral chords are as wide as the cuticle is thick, or wider; somewhat farther back, they are about one-third as wide as the body. They contain scattered colorless refractive spherical granules of variable size, considerably smaller than those of the intestine. Behind the base of the neck, at a distance about equal to the diameter of the body, there is a cell which presses the intestine well to one side. This cell is about as long as wide but not spheroidal. It is finely granular, one-half as wide as the body, and seems very probably to be connected with the renette. The excretory pore, which is opposite the base of the neck, is rather distinctly to be seen, though of small size. It lies between two annules, and the nearer striation may bend a little to one side for it. The renette duct leads inward and to the right alorg the right lateral chord. From the somewhat depressed, rather large and rather conspicuous vulva, which is a transverse slit two-fifths as long 
as the body is wide, the strongly cutinized medium sized vagina extends inward at right angles to the ventral surface half the way across the body. From toward the ends of the valvular opening four muscles pass obliquely to

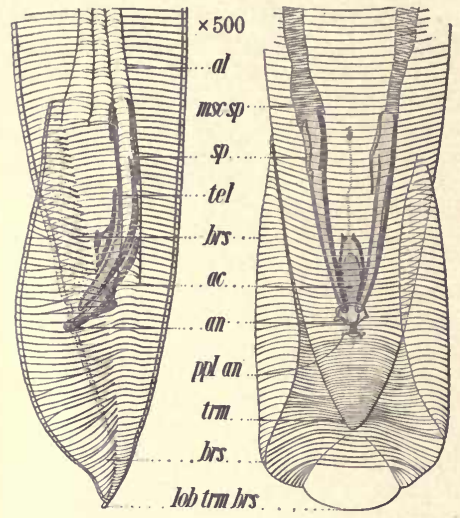

Fra. 4. Lateral and ventral views of the tail end of the male of Hoplolaimus coronatus n. sp. Treated with potassium hydrate to obliterate non-cutinized structures. the ventrally submedian regions oi the body, two forward and two backward, - two to the right and two to the left. Furthermore there are two transverse valvular muscles attached near the ends of the opening and fanning out to the lateral parts of the body wall, one right and the other left, each partially encircling the intestine. Each of the two outstretched uteri, about two bodywidths long and about one-fourth as wide as the body, at its distal extremity presents a spermatheca three-fourths as wide as the body, sometimes containing what appear to be toward one hundred sausage shaped sperm cells each sometimes having a bunch of chromosomes at orte end. These spermatheca are located at a distance from the vulva two or three times as great as the diameter of the body. In the late autumn they are a very uniform feature of the adult females which have deposited no, or very few, eggs. As thus far seen, the slender outstretched ovaries are about one-fourth as wide as the body; both lie on the left side. They are narrow and somewhat cylindroid and contain one hundred or more ova arranged somewhat irregularly.

The male is like the female in form. The spicula are colorless. A portion of the $\frac{3.4}{2.4}-\frac{8.9}{2.8}=\frac{13}{3.2}-2-\frac{M}{3.5}=\frac{97.9}{2.2} 1.4 \ldots$
gubernaculum lies ventrad. - (telamon of Hall). See Fig. 4. There are no preanal ventral supplementary male organs, and no ribs occur in the bursa. The striae of the bursa on the ventral side are less distinct near the ventral line. The terminal loke of the bursa appears destitute of striation; if any striae are present they must be exceedingly fine. The vas deferens appears to be about one-half as wide as the body. The narrow cylindroid testis tapers a little, and at the blind end is only one-fourth as wide as the body. The granular sperm cells seen in the vas deferens are about one-tenth as wide as the body; the spermatocytes, farther forward, one-eighth.

Habitat: Found in soil immediately about a Mermis "nest," (Agamermis decaudata), Four Mile Run, Falls Church, Va., U.S.A. Nov., 1922.

The movements of this nema are very slow. The limber body readily takes on sharp sigmoid curves and is sometimes seen coiled; in fact the males can coil rather closely. From this amended characterization it seems evident the Hoplolaimus Daday (not of other authors) is a rather clearly marked genus. A close relative of Hoplolaimus is Dolichodorus Cobb 1914. 


\title{
NOTES ON PARATYLENCHUS, A GENUS OF NEMAS
}

\author{
By N. A. Совв
}

Contributions to a Siience of Nematology, XIV.

The following paragraphs contain new information with regard to the lip region, vestibule, spear guide, structure of the spear, median oesophageal bulb, salivary glands, deirids (cervical papillae), renette, eggs and their deposition, and gonism of Paratylenchus Micoletsky. 8.2. 18. $24 . \quad 33-82 . \quad 95.8$

Paratylenchus nanus n. sp. 3.7 4.3, $4.5-4.2^{-} 2^{-0.41 \mathrm{~mm}}$ The transparent, colorless, naked cuticle, about 1.5 microns thick, is traversed by plain, transverse striae, 2.0 microns apart except near the extremities, all alike and fairly easy of resolution, which are materially altered on the lateral fields by the presence of wing regions, about one-seventh as wide as the body, beginning on the neck and ending on the tail. The optical expression of the wings on living specimens usually consists in four parallel longitudinal lines on each lateral field, the two outer of which are fainter than the two inner. Very slightly oblique longitudinal striae of the subcuticle, all alike, due to the attachment of the musculature, are rather easily to be seen in nearly all regions of the body. The contour of the body is crenate or very faintly serratecrenate. There are no dermal appendages and there are no series of pores to be seen in the cuticle. On the neck opposite the excretory pore, lat. 22.2,* there is a papilla on each lateral line, and, leading inward, ventrad and slightly backward from the middle of each papilla is an obscurely sinuous element connecting with the nervous system. These organs are believed to be deirids.

The neck, which is cylindroid posteriorly, and to a considerable extent also anteriorly, becomes decidedly convex-conoid farther forward, and ends in a rounded or subtruncate, continuous head compassing about thirty annules of the cuticle, which presents a somewhat depressed, very minute, central mouth opening, closely surrounded by six equal, exceedingly minute lips. The truncation of the head occurs at the lip region, which has at this point, that is at the anterior extremity of the nema, a width of about two microns. The lip

*The Word "Latitude" in Descriptive Nematology. I have lately come to use the word "latitude" in a conventional sense in dealing with nema anatomy, and find it so useful as to lead to this attempt more accurately to define the word as thus used.

The meaning of latitude in this connection arises from geographical usage, but in nematology the term applies to a transverse plane or section of the organism, and not to a circle on the surface only, as in geography, and it has not seemed desirable to have two sorts of latitude, such as north and south.

One hundred degrees of latitude is assumed, with zero at the anterior extremity of the organism. Thus an element of the organism in latitude 50 would be at the middle; and in latitude 100 at the end of the tail. The terms can be abbreviated. Thus: lat. 60.

In the case of nemas, which are so nearly round in cross section, a similar use of the word "longitude" sometimes becomes useful, the ventral line being taken as zero.

The conventional use of the words latitude and longitude in this way is more or less "logical", and very easily acquired, and, according to my experience, is a decided saving in time and space, and has the merit of definiteness, as well as brevity. 
region is supported by a faintly visible six-ribbed, refractive, somewhat domeshaped, cuticular framework, six to seven microns across at the base, and about two-thirds as "high" as it is wide. The more or less immobile lips are usually closed.

There is a small combined vestibule and spear guide, about as wide as the lip region and some ten microns long, more or less visible on account of the refractive nature of its elements. This portion of the labial structure has for one of its main functions the guidance of the spear when in action. The vestibular part is about four microns deep and varies somewhat in diameter according to the attitude of the lips and spear. Leading backward from the base of the vestibule there is a symmetrical set of outwardly bowed, somewhat flexible, rather slender, longitudinal elements constituting the main portion of the spear guide. The relatively very robust spear is about twice as long as the base of the head is wide. It ends posteriorly in a distinctly three-lobed expansion toward one-third as wide as the base of the head, the dorsal lobe being slightly farthest back, and, sometimes at least, presenting a dorso-posterior condyle. It is somewhat behind, and in a line with, the axil of the dorsal lobe that the dorsal salivary gland empties into the oesophageal lumen. The spear often tapers more or less regularly throughout its length; nevertheless there is a distinct basal part, comprising about two-fifths of the whole, set off by a minute but distinct junction mark, and averaging about one-sixth as wide as the corresponding portion of the head. At its distal end the spear is exceedingly finely pointed. Well developed muscles for the protrusion of the spear are readily seen and often lie rather close to the spear,-not forming any very marked swelling when at rest. Anteriorly there are six of these muscles,-one passing to each sector of the labial framework.

No amphids have been seen. There are no eyespots.

The oesophagus is tylenchoid and presents a very definite, somewhat pineapple-shaped, non-muscular, valveless cardiac swelling, half as wide as the base of the neck. The very long, large, rather ob-clavate, median swelling, which is two-thirds as wide as the middle of the neck, is set off abruptly behind, but is decurrent in front and reaches to, and somewhat includes, the base of the onchium; in its posterior part it presents a well-developed, elongated-fusiform, triplex valve, occupying one-third of the diameter, to which are attached the usual radial muscles for the opening of the valve in the act of swallowing. An interesting peculiarity of the median swelling is that the contained robust tubular oesophageal lining, which is disposed in a single loop or coil when at rest, takes on this attitude without much disturbance to the evenness of the contour of the swelling itself, thus showing the "clavate swelling" to be a distinctly two-fold affair,-partly (posteriorly) muscular, and partly (dorsally throughout) glandular, and with the two tissues so little connected that the glandular part is comparatively separate and responds but little to the movements of the tubular lining. Ordinarily one would expect the anterior narrower part of such a long median swelling to curve or coil along with the lining. Though the limits of the true median bulb (not the clavate swelling but the included median bulb more properly speaking), are often somewhat indefinite anteriorly, it may properly be described as ellipsoidal, two-thirds as wide as the neck and two and one-half times as long as wide; in other words the entity of the median muscular bulb is not entirely lost. Behind the pharynx the oesophagus is onesixth, at the nerve ring only about one-tenth, in front of the cardiac swelling about one-eighth, and finally one-half, as wide as the corresponding portion of the neck. The lining of the oesophagus is tubular and narrow, and distinct except in the posterior glandular bulb, - most distinct in the clavate swelling.

There are well developed salivary glands. The nucleus of one of these organs may be seen in the dorsal sector of the cardiac swelling, as already described, - 
dorsad and occupying the major part of it,-and emptying into the oesophageal lumen near the onchium. It is doubtful if salivary secretion passes also into the base of the fusiform median valve, though there seem to be two subordinate nuclei in the cardiac swelling.

There is no cardia. The thick walled intestine, set off by a rather faint cardiac collum one-half as wide as the base of the neck, presents a faint, though fairly capacious lumen. It is composed of cells of such a size that probably only about two are presented in each cross section. It becomes at once two-thirds as wide as the body. From the very inconspicuous, continuous anus, the rectum, which is also very inconspicuous, extends inward and forward. There is no distinct pre-rectum. The numerous, colorless granules found in the cells of the intestine, the largest of which are about onetenth as wide as the body, namely about two microns in diameter, are not so arranged as to give rise to a tessellated effect. Sometimes the cells throughout the intestine are uniformly filled with granules; more often the granules are absent here and there, so as to create a "segmented" effect.

The tail, which compasses about twenty annules of the cuticle, is conoid, subarcuate, and tapers from in front of the anus to the rather blunt, or sometimes subacute, unarmed, symmetrical terminus. There is no spinneret. There are no caudal glands and there are no caudal setae.

Apparently the lateral chords are about onethird as wide as the body. The rather prominent excretory pore is located just behind the nerve ring and the excretory duct can be followed inward and backward along the right lateral chord at least as far as the middle of the body.

The nerve ring is oblique, of medium size and accompanied, fore and aft, by numerous nerve cells, some of which lie as far forward as opposite the middle of the median oesophageal swelling.

The single female sexual organ is outstretched forward. From the unusually large, depressed and very conspicuous vulva, the vagina, which is large, extends inward obliquely forward, three-fourths the distance across the body. Its walls are rather strongly cutinized. The larger anterior lip of the vulva may be slightly elevated. The body of the nema decreases a little in diameter rather suddenly at the vulva and tapers more rapidly thence backward. The thin-shelled, smooth, elongated egg is nearly thrice as long as the body is wide and measures about $60 \times 20$ microns. Only one egg occurs in the uterus at a time. A prolate compact mass of sperm cells, often comprising some two to five hundred minute, spherical, refractive elements, occurs regularly in the uterus of newly adult females; this sperm mass is often two-thirds to three-fourths as wide as the body. From the formation and size of the sperm cells it is concluded that the species is syngonic. No males have been seen among about fifty females, many gravid, from two North American regions. The medium sized ovary is usually cylindroid posteriorly, and tapers anteriorly; it averages to be about one-third as wide as the body. Toward fifty ova, arranged for the most part single file, are to be seen in the ovary. There is prac-

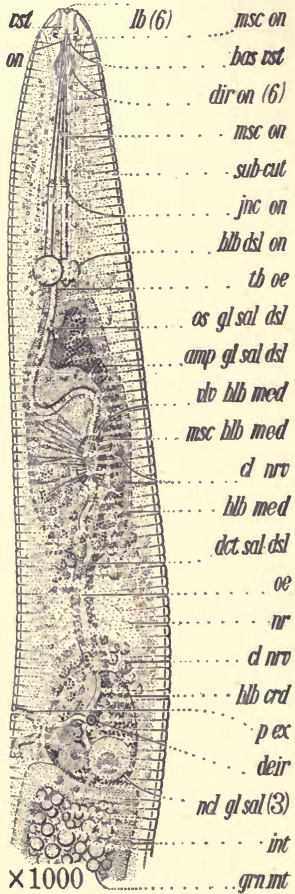

Fig. 1. Anterior part of a female Paratylenchus nanus. tically no post-vulvar rudiment of a sexual organ. 
There are two or three somewhat ellipsoidal organs, half as wide as the body, about two-thirds as wide as long, located just behind the base of the neck, and closely associated with the beginning of the intestine. These regularly darken in Flemming's solution and are as yet of unknown significance.

Habitat: Found in soil about the roots of grasses, Devil's Lake, North Dakota, April, 1915; and Four Mile Run, Falls Church, Va., August, 1922. Flemming's solution to glycerine jelly. In many respects this species closely resembles Tylenchus macrophallus de Man, but differs in the following particulars; - the spear is somewhat longer and possibly somewhat more robust; the striation is coarser; the body is wider; the tail of nanus compasses twenty annules while that of macrophallus appears to compass about fifty; opposite the spear in nanus there are about twenty-five to thirty annules, while in macrophallus there appear to be about forty. Should opportunity occur it would perhaps be advisable to re-examine the median oesophageal region of macrophallus. For the present at least it seems best, unless the undiscovered male of nanus should prove to be extraordinarily like the male of macrophallus, to regard the two species as distinct. Paratylenchus is related to the very welldefined genus Iota, a genus whose numerous representatives typically are minute, very short, very broad, coarsely annuled, rather inflexible nemas found in acid soils, and having the single outstretched female sexual organ emptying through a vulva located very close to the minute, inconspicuous anus and often possessing external coarse retrorse cuticular elements,--ridges, scales, spines, fringes, etc., according to the species. There is a number of as yet unpublished species of which it is not easy to make a satisfactory assignment as between Iota and Paratylenchus. The unknown males of nanus, if such exist, may be expected to throw more light on the generic relationships. $P$. nanus may be synonymous with $P$. bukowinensis Micoletzky, 1922.

10. 26. 31. $\quad 33-83$. 93.

$4.8 \quad 5.1,5.6-5.1-5.10 .36 \mathrm{~m}$ are the measurements of a living specimen of $P$. nanus under slight pressure and therefore a little flattened, and furthermore showing a neck-length unaltered by fixation and preservation.

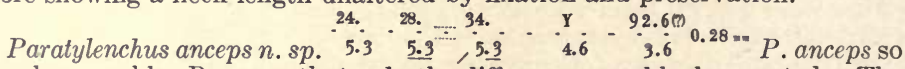
closely resembles $P$. nanus that only the differences need be here noted. The striae are one micron apart. The optical expression of the wings is a pair of refractive parallel lines whose distance apart is about equal to the width of two annules of the cuticle. The conoid neck becomes convex-conoid at the head, at the front of which the lip region is about four microns wide. The spear guide is six microns long, and the spear about half as long as the neck, the long slender anterior part comprising three-fourths or four-fifths of the whole. The three-lobed, flattish basal bulb of the spear is about one-fourth as wide as the corresponding portion of the neck, that is about four microns wide. The somewhat elongated-pyriform or pineapple-shaped posterior bulb is threefifths as wide as the base of the neck. The deirids are near the base of the neck. The tail is slightly conoid to the broad, rounded terminus, which is half as wide as the base of the tail. The vulva was about to appear at the same relative position as in $P$. nanus. In all other respects almost precisely as in $P$. nanus.

Habitat: Roots of Umbellularia californica, Riverside, California, 1912. 


\section{BIOLOGICAL RELATIONSHIPS OF THE MATHEMATI- CAL SERIES 1, 2, 4, ETC.}

With a Description of a New Nema, Tylenchus Cancellatus

Contributions to a Science of Nematology. $X V$

By N. A. Совв

The behavior of the components of matter, e.g., in chemical reactions, appears to compel discontinuous variation in the evolution of organisms. Organic evolution has been thought continuous, but mutation now suggests that it is discontinuous. Must it not necessarily be discontinuous ${ }^{1}$ from the very nature of the composition of matter? Morphological changes in organisms originate in chemical changes in the matter of which they are composed. Now, a chemical change is one that either takes place or does not take place; nothing intermediate is known. Hence it seems that the fundamental changes in the evolution of organisms, so far as we can conceive at present, i.e., chemical changes, must be saltatory. But we cannot conceive of the greater and obvious (visible) changes, except as summations of these minute changes. The visible changes then must per force be considered of the same character as that of their components, i.e., all visible evolutionary changes in organisms must be of a saltatory nature.

The mathematics of the morphology of organic evolution may therefore be considered as, at least mainly, discontinuous, - arithmetical.

The material basis of life is discontinuous, but is the only known form of matter so organized as to grow and multiply by assimilation; in this lies the fundamental difference between living objects and all others; - not a mathematical difference.

Matter is dual, or, less abstractly, there exists in matter an exceedingly widespread, probably universal, "bipolarity", exemplified, therefore, in organisms. The universality of "bipolarity" is more or less understood and generally admitted. Its universality might be assumed to prove, and at least very strongly suggests, its necessity. Assuming its necessity, this bipolarity determines that cells, as well as many of their components, multiplying, do so by binary division in a bipolar manner. ${ }^{2}$

${ }^{1}$ Mathematics. Arithmetic and its derivatives arose through everyday problems connected with matter, which is discontinuous. The Calculus, mathematics of continuity, arose through problems like those of astronomy, where the continuity of space and time impress us most vividly.

Quantity. It may be said we cannot conceive of anything so small that it cannot be divided, or so large that nothing can be added to it; but as the two opposite statements seem just as true, we find ourselves within two limits at each of which we confront something that must be so, but can't be so. Between these two irrationalities lie quantities we can handle rationally by mathematics.

2 Thence "fore-and-aftness" and bilateral symmetry in organisms arose (doubtless modified by gravity). Bilateral symmetry seems the invariable result of the growth of what we may call, for lack of a better term, "untrammeled protoplasm." When protoplasm is "hampered," say by inorganic materials tending to produce other forms of symmetry - as, for instance, through the laws of crystallization-then bilaterality may be more or less masked; otherwise it is manifest. We readily recognize it in nearly all animals and plants. 


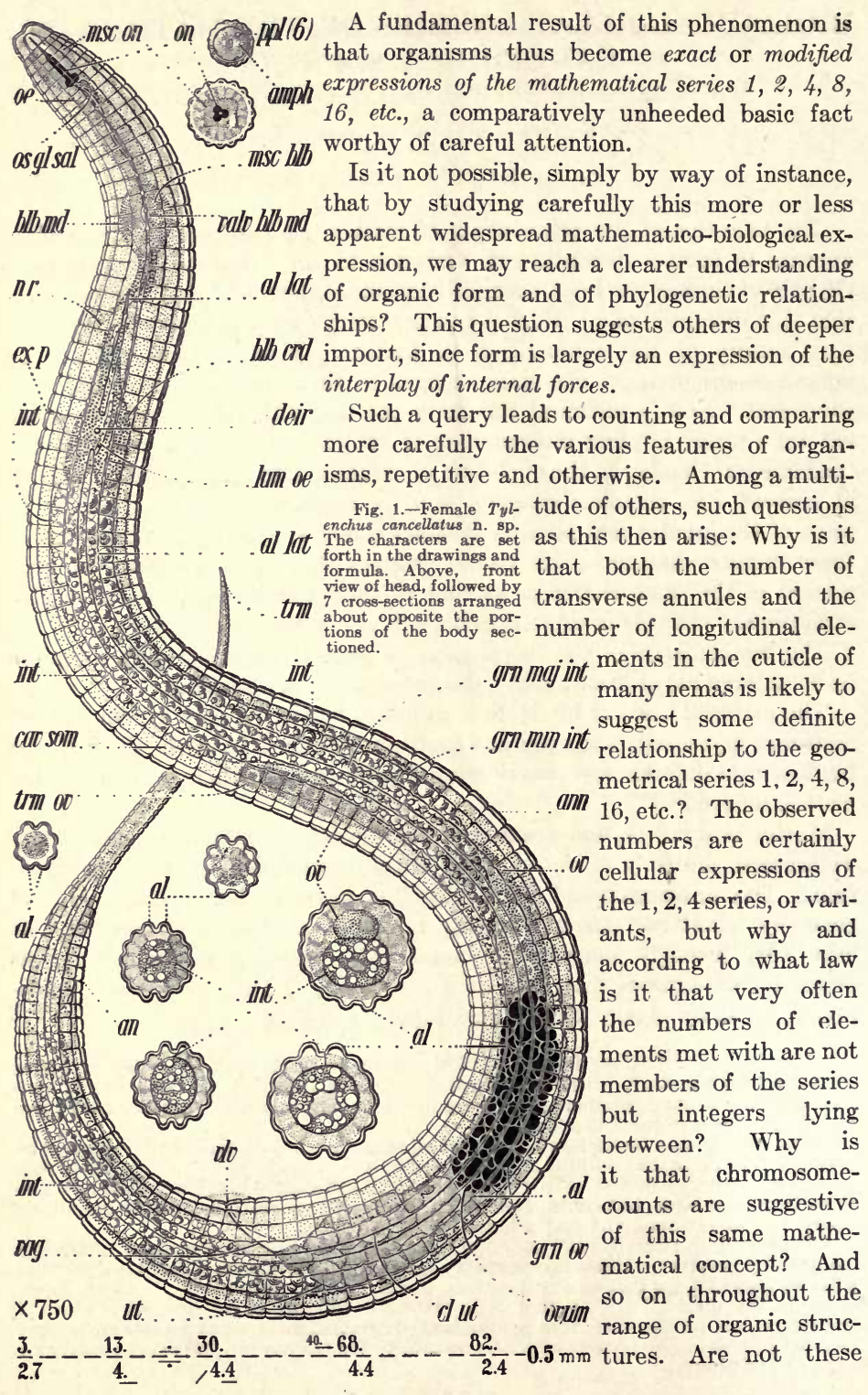


numbers not only necessarily and definitely, but perhaps somewhat simply, modified mathematical expressions of the fundamental mathematico-biological phenomena inevitably arising from the fact that cells (as well as some of their components) divide in accordance with the 1,2, 4 series?

Variations of the 1, 2, 4 series, as expressed in cell multiplication, say in a segmenting egg, can be readily diagrammed. (See Fig. 3.) If in such a celldivision diagram any particular multiplying cell or cells be pictured as halted, while the others continue to divide, the next step will bring about a variation from the geometrical series. If the reader will draw a few simple diagrams, he will find it easy, by such variations, graphically to represent, as existing at successive early stages in the imagined ontogeny, numbers of cells, say, from 1 to 10 inclusive, and will see that conceivably this could go on indefinitely, and that therefore any number whatever is a possible biological variation of the 1, 2, 4 series. But this broadening of the possibilities must not be allowed to obscure the basic fact that the numbers are nevertheless definite mathematical variations of the 1,2, 4 series due to the binary division of cells and their components; - which in turn seems compulsory owing to the nature of matter itself. Our problem seems to be: Which of these numerous variations are the more significant, and what are their mathematical and biological relationships?

A new triplonch, Tylenchus cancellatus n. sp. (Figs. 1 and 2), infesting the roots of peonies, will serve, in a very limited way, to illustrate the foregoing remarks. The figures (Fig. 1) show the existence, near the head, of sixteen external longitudinal grooves. Near the middle of the neck this number changes to eighteen by the splitting, on each side of the nema, of one of the lateral, or sublateral, elements of the series, so that most of the body presents 18 grooves. Posteriorly this number reduces to 14,10 , then 8 . (Fig. 1.)

This emphasizes the value of pondering the variants of the $1,2,4$ series. If the numbers of the various elements were confined to the 1,2, 4 series, they would be less significant, hence less useful; - - e.g., in the interpretation of relationships. But variations abound, and are, as yet, for the most part unexplained; probably often highly complex. It is certain, however, that if these variations $\underset{\text { Peony }}{\text { Fig. }}{ }_{\text {root }}$. can be envisaged and understood, they will serve as basic data.

There seems at present no way of stating exactly the upper cinalis), nat. limit of the numbers representing these variations of the $1,2,4 \begin{gathered}(1,2,2 \text { and } 3) \\ \text { contain } T y l-\end{gathered}$

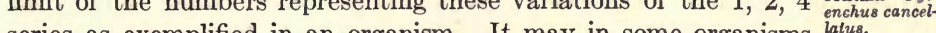
series as exemplified in an organism. It may in some organisms latus. reach twenty figures, and therefore the discovery and interpretation of some of the highest members of this modified geometrical series, as exemplified in organisms, may be beyond our present compass. Nevertheless, does it not seem likely that relationships traced in this manner may at least be set upon a firmer basis than is the case when data of other sorts are used,-or even upon an entirely new basis? 
In a 1, 2, 4 series, let $P_{N}$ be the final product and $N$ its series number, then $\mathrm{P}_{\mathrm{N}}=2^{\mathrm{N}-1}$; thus, $16=2^{5-1}$.

Similarly in a simple organism, at any particular instant in its growth, let $\mathrm{P}_{\mathrm{N}}$ have a corresponding value,- that is to say, be the number of cells that either actually exist or would have arisen by the uniform and continuous dichotomous division of the single primal cell. Such simple and easily understood organisms occur among the lower forms, and in the early embryonic stages of the higher forms, but are rare among the adult stages of the higher forms, because in these latter some cells lag or cease in their dichotomy, and because of losses of cells from various causes. Hence, the number of cells actually present in an organism at any particular instant is likely to be $P_{N}$ minus a certain number of cells, $(X)$, due to delay or failure in some part or parts of the dichotomy, or to loss. In this discussion account is taken of all the cells that have been produced during the growth, whether present in the organism at the proposed instant or not. This is in order to allow for worn out or wasted cells; these, possibly vanished, cells are included in $P_{N}$

The general 1, 2, 4 equation of an organism thus becomes $P_{N}=2^{N-1}-X$,

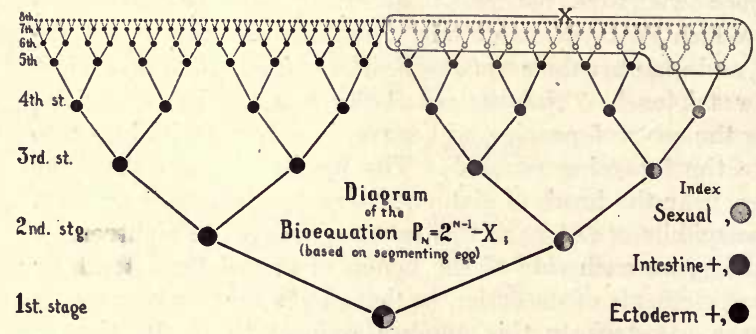

Fig. 3.-Diagram of 8 generations of cells produced by dichotomous divisions;-as, for instance, in a segmenting egg. Three general characters of tissue are shown: (1) Sexual, (2) intestinal and related tissues, (3) ectoderm and related tissues. The sexual and intestinal tissues are shown to have lagged behind those of the ectoderm, so that $\mathrm{P}_{\mathrm{N}}$ in this instance equals 71 .

in which $X$ is a whole number and a function of one or more " $p$ 's" of a lower order, i.e., of the $1,2,4$ character, or $\mathrm{p}=2^{\mathrm{n}-1}$ character, in which, of course $\mathrm{p}$ is smaller than $\mathrm{P}$ and $\mathrm{n}$ is smaller than $\mathrm{N}$. These smaller (ascertainable) groups are 1, 2, 4 groups of cells due to the lag or failure of "earlier" generations than $N$. (See the loop (X) in Fig. 3.)

$\mathrm{P}_{\mathrm{N}}=2^{\mathrm{N}-1}-\mathrm{X}$ is a general equation, which, when $\mathrm{X}=0$, represents a strictly uniform and continuous mathematical dichotomy, found only in the lower organisms or in the early embryonic stages of the higher ones.

The various " $p$ 's" from $\mathrm{P}_{\mathrm{N}}$ down to $\mathrm{P}=1$, (the primal number) become, therefore, historical insignia, indicating particular generations of cells, and may be made the basis of a definite and fundamental mathematico-biological nomenclature applicable to the generations of cells in an organism, and hence to the organism itself. Applications of the equation are endless. 


\section{NEMIC SPERMATOGENESIS}

With a suggested discussion of simple organisms,-Litobionts

\section{Contributions to a Science of Nematology XVI}

\section{By N. A. Совв}

Definitions. Spermatidium: one of a plurality of cells derived from a spermatid by subdivision; a secondary, tertiary, or quaternary, etc., spermatid. Spermule: an individual spermatidium which, after growth and transformation, is capable of activating or fertilizing an egg,--being not a metamorphosed spermatid, but a descendant of a spermatid, one or more cell-generations removed.

The definitions will be better understood by at once consulting the illustrations, especially spmtd and spml Figs. 2 and 12.

Spermatogenesis. At the blind end of the single testis of the nema, Spirina parasitifera (Bastian '65) Filipjev (Figs. 1 and 2), - a free living marine species, common an inch or two deep in sand and among small stones between the tide marks of protected coasts on both sides of the North Atlantic through a wide range of latitude,- the primordial gonic elements give rise by 14-chromosome mitotic division to numerous twin cells (Figs. 2, 14), which arrange themselves tandem in the testis (Fig. 3) where each

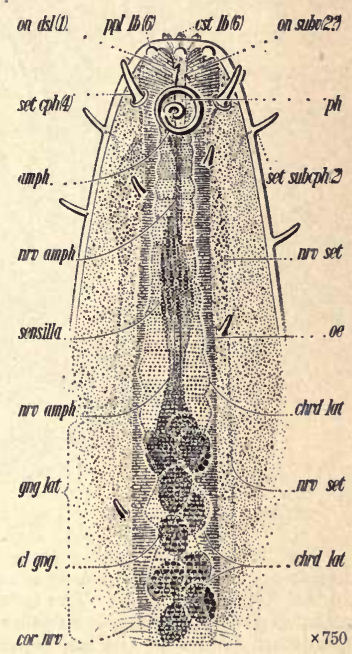

Fig. 1.-Lateral view of the head of Spirina parasitifera. The amphidial nerve, nrvamph, expands into a sensilla, then again into a 10-12 celled ganglion (seen through the lateral chord, chrd. lat.) joining the nerve-ring, cor nrv. 


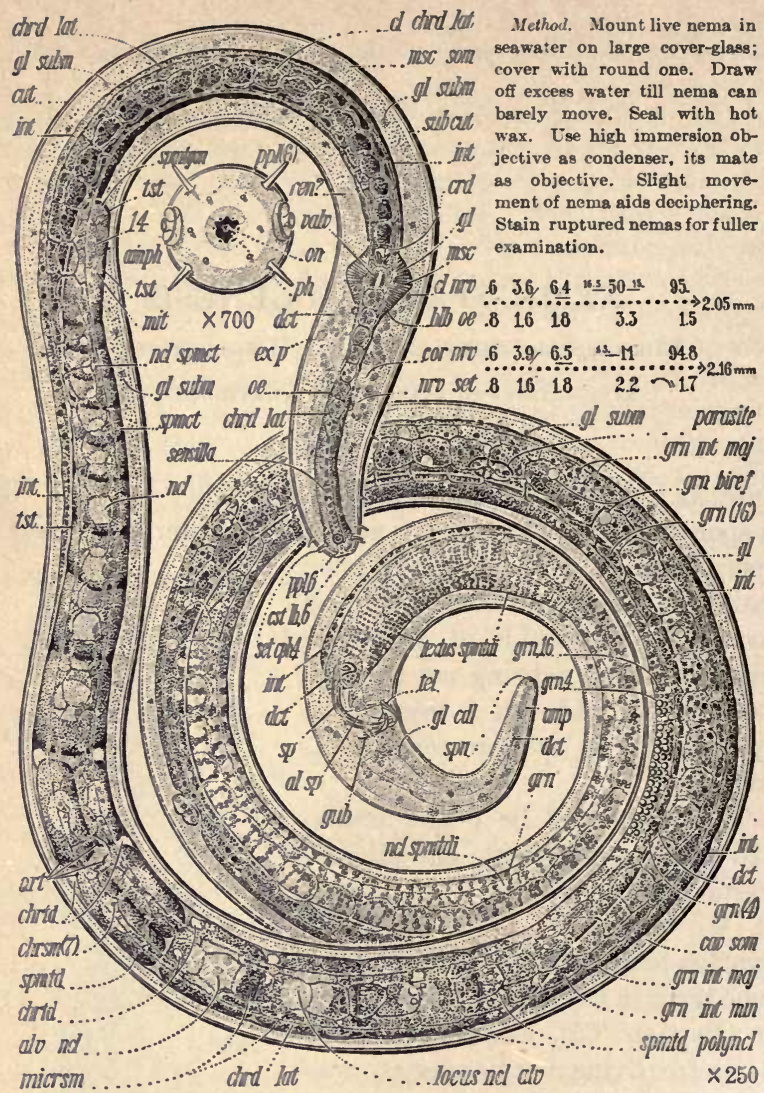

Fig. 2.-The male of $S$. parasitifera drawn from life. The tinting of drawing modified in accordance with study of stained specimens. Nearly all details shown were seen in the living specimen. The front view of head, however, is from a decapitated specimen. In life the chromosomes have not been seen definitely enough to admit of accurate counting. Most of the subsequent camera lucida drawings were obtained from fixed and stained material. In nearly all cases the fixing and staining were done simultaneously by means of acetic acid methyl green. Just to the right are placed, in the form of the decimal formula, the average measurements of specimens used. Material collected at Woods Hole, Mass., U. S. A.

The self-explanatory abbreviations are the same throughout the various figures, and are of necessary Latin anatomical terms; thus, chrd lat, chorda lateralis, lateral chord; qrt, quartet of spermatids; chrtd, chromatoids; spmtd, spermatid; alv ncl, alveoli of nuclear space; micrsm, microsomes, of spermatid; 14, a 14-chromosome spermatogonial mitosis; mit, mitotic figure; grn, a cell of primary spermatidian tissue containing four granules; grn 16, cell of spermatidian tissue containing sixteen granules; locus $n c l$ alv, locus of the diminishing alveolated nuclear space; spmtd polyncl, polynucleate spermatid in process of becoming a 64-celled tissue; textus spmtdi, spermatidian tissue. 
cell, growing, forms a primary spermatocyte. At the end of the growth period the primary spermatocytes, one after another, divide transversely, i.e., at right angles to the nema's body axis, and then, sometimes almost simultaneously, longitudinally, to produce four similar, juxtaposed spermatids (Fig. 2, qrt), each soon packed with several thousand very slightly elongate microsomes, nearly all of which are located outside the large central, faintly alveolated, diminishing nuclear space. (Fig. 2, micrsm and alv ncl; and Fig. 14.)

The microsomes seem to arise toward the center of the spermatid and migrate outward till the entire cell is colonized by them. Seen toward the center of the spermatid they usually have their long axes arranged radially, as if moving outward, end on. Some 810,000 uniform microsomes come thus into existence in each spermatid. The arrangement of these around 64 centers is the first indication of the formation of the first generation of 64 spermatidia. In the living spermatids the microsomes are the only objects seen visibly connected with such a division of the cell contents as is necessarily postulated to account for the phenomena of heredity.

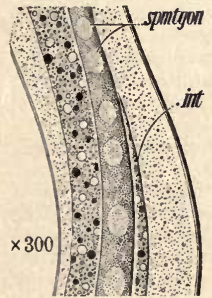

Fig. 3

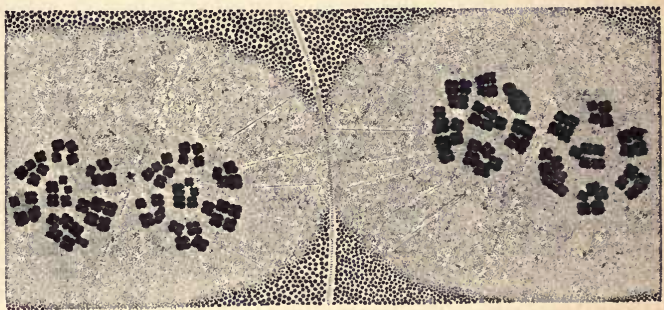

Fig. 4

Fig. 3.-View near blind end of testis of S. parasitifera, showing pairs of cells resulting from division of primordial nuclei. This testis had broken open and become partially evacuated so that these pairs of cells in tandem could readily be distinguished as such. Normally these nuclei are so packed that the mass effect obscures the fact that they are twins.

Fig. 4.-Second stage of reduction division of a spermatocyte of S. parasitifera, which will result in 4 similar juxtaposed spermatids, as at $q r t$, Fig. 2, each having 7 chromosomes. The compound chromosomes present considerable individuality. Between the 2 double groups of chromosomes is seen portion of the new cell wall. $\times 1200$.

In the first of these two divisions the chromosome number is reduced to seven. Probably the smallest one of the seven chromosomes of the secondary spermatocytes differs slightly in relative size in the two cells. Thus far the spermatogenesis presents nothing very new or striking, but the amount of growth,-from 3 to 60 microns (tst, Fig. 2),-is worthy of note, and, connected with reduction, there is 
a more or less orderly (e.g., more or less definitely oriented) extrusion from the spermatids of structureless looking chromatoid substance, (chrtd Fig. 2 and Fig. 14), barely possibly by a very "degenerate" mitosis; these chromatoid masses are soon absorbed.

"Normally," the four cells just described would develop into four sperms, but here the spermatogenesis proceeds as follows: Moving along the testis with soldier-like precision, the two caudad members

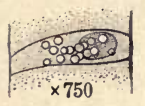

Fig. 6

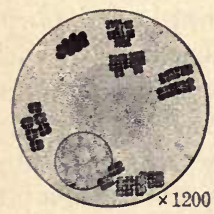

Fig. 5

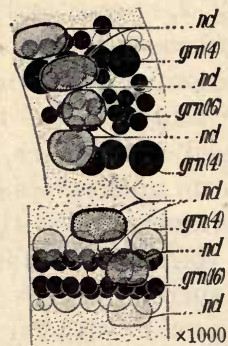

Fig. 7

Fig. 5.-Nucleus of full grown spermatocyte seen in synapsis. The nuclear membrane is still intact and the spherical nucleolus is still visible. The chromosomes are in seven pairs. It was possible to resolve the chromosomes into numerous components, suggesting a possible explanation of the difference in chromosome counts in certain nemas, e.g., in Ascaris; i.e., differences between counts at this stage and counts in later somatic divisions; for, should such loosely organized chromosomes later break apart, the count would be much increased.

Fig. 6.-A single spermatidium of first generation with its nucleus and sixteen granules. From life. In this case the cell wall is shown.

Fig. 7.-Above, camera lucida drawing of nuclei and granules in spermatidia of S. parasitifera at the point grn (4), Fig. 2. Below a diagram of four spermatidia. The diagram is derived from drawing above, and shows more clearly the numerical relationships of nuclei and granules. The boundaries of the spermatidia are almost invisible and are not shown. The granules are shown black, white or, when seen through the nucleus, gray. The larger ellipsoidal objects are nuclei. In the drawing, at top and on the hither side, a nucleus with its accompanying four granules, the nucleus being this side of granules. In the drawing, on the farther side, again at top, a spermatidium three of whose granules have already given rise to four smaller granules each. In the drawing and below, a spermatidium none of whose four granules have divided, one of them shown behind the nucleus. grn (16) shows a spermatidium with a nucleus and 16 granules. The lower figure is only somewhat schematised. Very rarely are spermatidian cells so systematically arranged as to disclose so clearly the relationships of granules and nuclei. In this diagrammatic lower figure the far spermatidium is shown in an intermediate state. Illustration derived from material stained with methyl green.

of the quartet form a tandem, followed by the other two, also in tandem; i.e., the quartet falls into single file. These spermatids in file grow, and one after another divide internally without evidence of mitosis into 64 uninucleate elements which proceed to surround themselves with walls and form a tissue of 64 cells. (See lowest part of Fig. 2.) As this tissue leaves the testis and enters the duct it 
elongates (2-4 nuclei abreast), and each of its 64 cells in turn, following on the disappearance of the microsomes, acquires four equal, refractive, spherical granules ( 4 microns in diameter), and the tissues thus take on a granulated appearance,- the nuclei and cell-walls being almost completely hidden by the closely packed granules. By the time the cephalad part of each tissue enters the duct the caudad part has undergone a further change, in that the four granules, each dividing endogenously into four similar but smaller spherical granules, populate each cell with 16 granules (grn 4 and grn 16, Fig. 2).

This very interesting behavior of the granules (Figs. 7 and 9) more than suggests a different order of mechanism from that typical

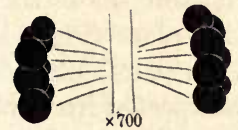

Fig. 8

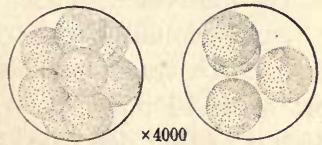

Fig. 9

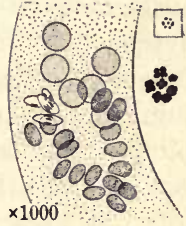

Fig. 11

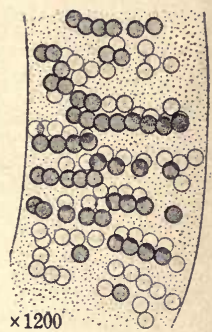

Fig. 10

Fig. 8.-Reduction division. Sublimate-acid carmine toto preparation. The smallest chromosome differed somewhat in size in the two sets. Fixation less delicate than with acid methyl green.

Fig. 9.-Two granules from the spermatidia of $S$. parasitifera;-one showing 4 smaller granules formed endogenously, the other 8 . The right hand granule is from near grn 4, Fig. 2. The left hand granule, taken from farther back in the testis, where microscopic details are so fine that exact relationship of granules and their descendants has not as yet been fully deciphered.

Fig. 10.-Spermatidia each containing sixteen refractive spherical granules. From life. The cell walls and nuclei of this tissue are nearly invisible in life.

Fig. 11.-Nuclear spindles in later mitoses of spermatidia taking place in vas deferens. Polar views of spindles show 7 chromosomes; see small figures to right, from another part of the same specimen.

of cell division, but since irritability, ingestion, transportation, transformation and so forth, all seem involved, it appears necessary to base the concept on what is known of cell physiology and mechanics; the changes, however, are carried out on a smaller scale and doubtless with a more limited variety of molecules forming a different kind of plasm-litoplasm. In short, the facts indicate a distinctly lower order of "organism." Many of what now are often called louer organisms might better be regarded simply as less multiplicate. Thus certain ciliates are smaller and less multiplicate, rather than "lower," as compared with nemas for instance. This matter is 


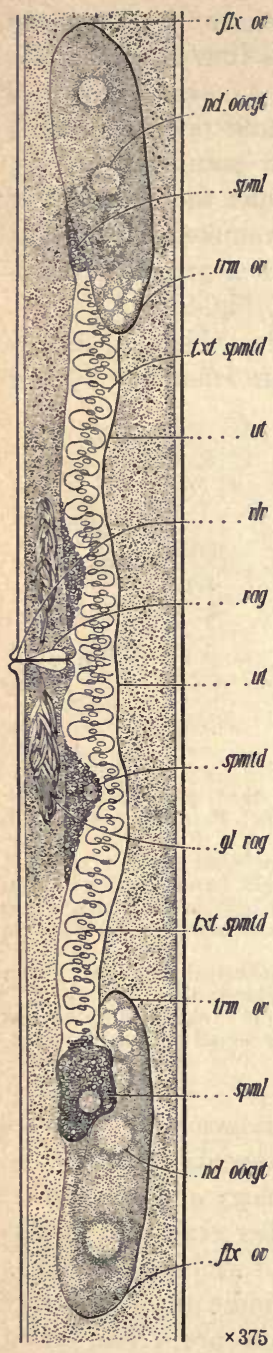

briefly discussed on a later page,-under the heading, Size and Number as related to Organisms.

As the tissue proceeds along the duct, the cells containing 16 granules undergo a further diminution in the size of their granules, and a change in the number and nature of the granules, so that the cells become more transparent; at the same time the nuclei divide mitotically, (7 chromosomes), giving rise to a tissue of 128 cells. The evidence that this increase is by mitosis is as follows: 1 . At the part of the duct where this change is taking place (Fig. 11) the sizes, form and position (in pairs) of the new nuclei are what would be expected from mitotic division. 2. The new smaller nuclei,-posterior to the larger, as yet undivided, nuclei,stain more strongly. 3. Occasionally 7-element spindles can be seen. 4. No trace has been seen of any other sort of division.

Two or more such tissues as that described fill the duct of the male nema, the number of tissues varying with the age of the nema and with the copulatory history. The tissues seem to be of two styles, and, if so, perhaps correspond to the two styles of chromosomes in the secondary spermatocytes (textus spmtdi, Fig. 2).

Fertili:ation and Syngamy. The two sexes of $S$. parasitifera seem about equally common. During copulation the male passes the spermatidian tissues on intact to the female, and afterward they may be seen in the uteri,

Fig. 12.-Carefully proportioned free-hand sketch of gonads of female $S$. parasitifera after impregnation. The two uteri, outstretched in opposite directions, are filled with spermatidian tissue. The young ovaries are just beginning to function and the ova next the flexures, flex ov, are about to enter the uterus. The spermatidia adjacent to the ova about to enter the uteri have metamorphosed into spermules, spml, and have taken on the form characteristic of nemic sperms as hitherto described. In this case two other cells of the spermatidian tissue nearer the vulva have also begun to metamorphose. trm, blind end of ovary; flex ov, flexure of ovary; txt spmtd, spermatidian tissue; $g l v a g$, vaginal gland, for which see also Fig. 16. 
often jumbled, sometimes extended along the length of the two uteri.

Fertilization is preceded by increase in size of that cell of the spermatidian tissue adjacent to the ovum next to be fertilized and its transformation into a cell, spermule, having the form, and discharging the functions, of a nemic sperm as hitherto understood;a transformation involving a growth of about 50 per cent in diameter together with a greater growth longitudinally, and a marked change in the granulation of the cytoplasm (spml, Fig. 12). These transformed cells, detached one by one, fertilize the eggs in what seems a normal manner. The polocytes seem normal. The female gamete has seven chromosomes (Fig. 13).

The spermatidian tissues,-tissues whose history seems to justify us in regarding them as spermatophores, may be removed from either the male or the female nema and then separately stained and examined, and this has been repeatedly done; in which case the details of their structure can of course be seen with greater ease and distinctness than is the case with such living preparations as are shown in figures 1 and 12. The enumeration of the elements of the 128-cell stage of the spermatidian tissue was found

Fig. 13.-Sketch of one end of ovum of $S$. parasitifera, in synapsis. Above, o $0^{x}$ zygote. Below, o nucleus in synapsis; one set of chromosomes shown behind the other and individuality of chromosomes thus obscured by their position.

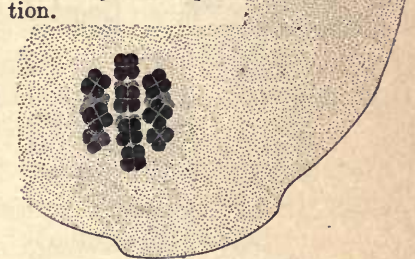
possible in this way, as well as in balsam specimens fixed in corrosive sublimate and stained in Mayer's acid carmine.

This method of spermatogenesis is normal to nemas. A large number of species belonging to numerous and varied genera are known to the writer in which the general appearances in the gonad of the male so closely resemble those of Spirina parasitifera as to leave him no doubt that the details of their spermatogenesis will show the features here described, or something similar. The formation of the spermatidian tissue is not an essential feature; in others of the above species the spermatidia may remain separate.

Current postulates must be modified in order to account for hereditary transmission in this and similar animal species. The factors usually believed to reside wholly, or in part, in the chromosomes must here, in order to accord with the usual theories of heredity, 


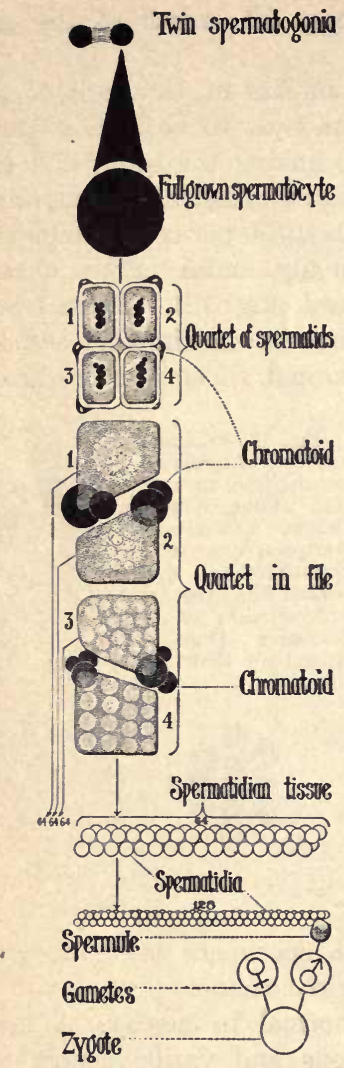

Fig. 14

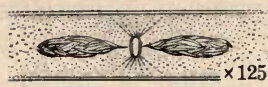

Fig. 16

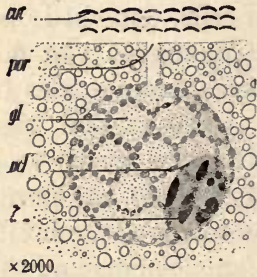

Fig. 17

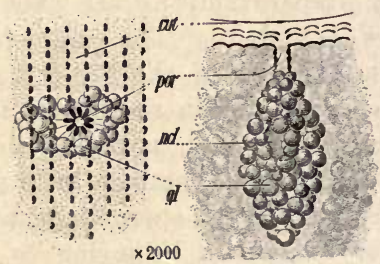

Fig. 15

Fig. 14.-Boverian diagram of spermatogenesis of Spirina. Spermatids are formed in the "conventional" way, -four from a spermatocyte. Instead of metamorphosing into ciliated spermatozoa, the spermatids undergo further changes and divisions, which give rise to a spermatidian tissue of 64 cells from each spermatid. These by mitotic division produce 128-celled tissues. One by one the spermatidia, when transferred to the uteri of a female, grow into spermules, capable of activating an egg and initiating normal development. Whether every one of the 128 metamorphose in this way is as yet undetermined.

Fig. 15.-Two views of one of the lateral glands of $S$. parasitifera. At the left only the pore and distal portions of gland are shown. The gland is uninucleate and consists mainly of spherical granules. Spent glands contain fewer granules than that shown.

Fig. 16.-Ventral view of vulva and vaginal glands of $S$. parasitifera. See also Fig. 12.

Fig. 17.-Lateral view of one of the lateral glands of $S$. parasitifera. The gland in a different state, or stage of development, from that shown in Fig. 4. 
be "divisible" in the spermatid into numerous parts such that when they appear in the spermule they are capable of bringing about "normal" syngamy.

It will be interesting to discover how factors or genes, concepts essential to clear thinking on the subject of heredity, can be imagined to "carry on" through the mazes of the division that, extending throughout the spermatid, gives rise without mitosis to 64 apparently equivalent elements in the spermatidian tissue (see spmtd polyncl, Fig. 2). The spermatidian tissues (aggregates of haploid cells,gametophores-Fig. 12) seem more clearly reminiscent of the alternation of generations in plants than any animal structure hitherto made known.

Subjoined is an alteration of the Boverian diagram illustrating the spermatogenesis here described. It will be seen that in this Boverian diagram (Fig. 14) the proportions of the camera lucida drawing (Fig. 2) are to a large extent adhered to. The microsomes and the alveolated nuclear spaces are shown with no very great departure from nature. The number and size of the microsomes is approximately correct and the new arrangement of the microsomes around 64 centers as shown in the diagram is not violently schematized. The same is true of the size, color and disposition of the chromatoid bodies. For simplicity the spermatidian tissues are reduced in the diagram to masses of 64 and 128 nuclei respectively.

The features accompanying and following the oöcytic synapsis seem at least a gesture toward the path followed in the spermatogenesis, but they have not yet been carefully studied.

Occasion for staining the gonads of Spirina parasitifera offered an opportunity for a more careful study of the unicellular glands of this species that "empty" through minute pores in the cuticle of most regions of the body, but particularly along the lateral fields. Unicellular structures of this character are known to be widespread among nemas, having been recorded for a great variety of free-living genera and a few parasitic genera. It is not known whether the various unicellular organs of this character hitherto recorded are homologous or whether they are connected with a variety of functions. The fact that they are well developed on aquatic forms that experiment proved to be in urgent need of oxygen has led the writer to suggest the possibility that these "glands" or some of them, may be connected in some way with respiration. This would seem in accord with the failure hitherto to observe any such organs in the 
vast majority of the parasitic species,-whose "respiration" it would seem natural to explain in other ways.

In Spirina parasitifera these organs are very small, and it therefore seems not unlikely that the present methods, when applied to more suitable material, may give results much more detailed and intelligible. The structure of one of these glands of $S$. parasitifera, so far as determined, is shown in Figs. 15 and 16. The great difference in the size of the organs in the two cases, as well as the difference in structure and space relationships, suggests the probability that the shape of the organ changes materially, perhaps rapidly, under various conditions. To this surmise it may be added that the method of collecting the spirinas, and the varying length of time between their existence under natural conditions and the time of examination, would result in a very material alteration in the environment; and it is believed that the longer this time became the less free oxygen would exist in the sea water in which the specimens were kept. This length of time varied widely.

\section{SIZE AND NUMBER AS RELATED TO ORGANISMS}

The interesting behavior of the spermatidian granules as described on pages 38 to 41 has suggested the following sketchy discussion of the relationship of organisms to size and number.

Why not vertebrates a mile long and a thousand feet high? Why not vertebrates only a quarter of an inch long? The known facts clearly indicate limits in both directions.

Among the reasons for the existence of the upper limit are, circulation difficulties due to friction in the blood vessels; accumulation of an excess of excreta in the blood during the long journey to the distant extremities and back; the difficulty of maintaining the requisite temperature at the extremities; limits set by the strength of materials,-bone could not be strong enough or muscles efficient enough properly to support and move so large an organism; food supply difficulties; space limitations connected with protecting such an organism from the elements, etc., etc.

Reasons for the non-existence of exceedingly small vertebrates also come readily to mind. The complicated vertebrate mechanism would be in the way in an organism of such small size. Why an elaborate pumping system to pump blood for a distance through which it might diffuse without such a system? So with "centralized" respiration. An internal skeleton plus the necessary protective 
cuticle become incompatible in this range of sizes. The competition of such imaginary small vertebrates with other organisms, say insects, of simpler structure better adapted to such small sizes would be a hopeless struggle.

Why not insects as large as moles or as small as microbes? Here again the mechanical relations of the organism to the menstrua furnish numerous reasons for the known size limits.

Generalizing, why not multicellular organisms beyond certain maximum and minimum limits? A little thought shows that limits are set by the relationships of particular mechanisms to the sizes and distances involved; and as size, in such cases, is a function of the number of cooperating cells, the limits are set in numerical terms. This becomes clearer when we consider our ability to represent a cellular organism by a strictly numerical expression, the bioequation, ${ }^{2}$ and all the more certainly true when, continuing the same line of thought, we consider the size limits of cells.

Why do we not have cells a meter long; and why not typical cells below the limits of a micron or two? Again, among other reasons, in this range of still smaller sizes the mechanism of the typical cell becomes so complex as to "be in its own way" when the distances involved become sufficiently small and the number of properties to be transmitted sufficiently few, as will be indicated in a moment.

Size limits in these various cases are set by a fundamental necessity, having its "final" source in the size of the electronic combinations.

Particular attention is called to the fact that, usually, the size limits of "adjacent" higher and lower groups of organisms reciprocally overlap (e.g. Vertebrates and Insects); as well as to the fact that individuals of certain species of unicellular organisms are larger than some of the multicellulars; or, to emphasize by reversing, many multicellulars are smaller than some of the larger unicellulars. There is a distinct lapping of the size limits of one on to the size limits of the other.

Organisms of greater size; "social organisms."-Developing a more complex nervous system, the higher organisms have evolved "mental pictures" of distant and invisible things and events, and have invented means for transmitting through various media signs that represent these mental pictures. Along this path the social organisms evolved. When we speak of a social organism it is usually assumed

2Biological Relationships of the Mathematical Series 1, 2, 4, etc." Contributions to a Science of Nematology XV. 
that we are using analogy, but an interesting formulation might be made out for homology. Are not the interactions between relatively widely separated intellectual individuals, existing in the sea of air surrounding the earth, in many ways actually homologous with the passage of stimuli, etc., through more viscous fluid media between cells? As, for instance, when two small organisms live in symbiosis; or, where cells exist together as they do in blood; or, between cells even more intimately organized.

The concept of organisms of this higher or social grade suggests the possibility of there being also lower orders of organisms at the other end of the accepted series. This idea is not new, for their existence was specifically asserted by acute observers and adventurous thinkers in the plainest of language at least half a century ago; but at that time the supporting evidence was so meagre that the idea did not rise to the dignity of an acceptable working hypothesis. Now it is quite different. Today what we know about certain small living elements, both inside and outside of cells, compels such a working hypothesis, -if mayhap we are not already beyond the hypothetical stage.

Here again, size seems a prime determining element. When a cell (really a relatively complex and large organism) transmits its exceedingly numerous properties to its "descendants," nothing short of an elaborate mobilization and census is adequate to the coming transmigration. Hence follow mitosis and its complications.

We are perhaps prone to forget that every cell has, in a great degree, to care for itself; and so must have many of the multitudinous properties characteristic of the groups of cells constituting higher organisms. It must nourish itself. "You can take the horse to food (or vice versa), but you cannot make him eat;-he must do that himself," seems to summarize the situation. If the cell assimilates ("eats"), and is to continue, then it must have mechanism adequate to select, transport, digest, excrete, etc., and at least to take some part in reproducing itself. All this complexity is because of the number of its characteristics, and because of the size, i.e., the distances involved. But what if all these be a hundredfold or more reduced, and the system be at the same time "isolated" or individualized? Plainly, the requirements would call for a simpler mechanism; cell-mechanism would be so complicated as to be in the way. Under such conditions simpler organisms, organisms simpler than cells, seem a logical necessity. 
Litobionts.-I have ventured to suggest a general or inclusive name, Litobionts, for the organisms which my observations lead me to believe to exist, these very organisms of lower grade;-( $\lambda_{\text {cros}}$, simple), simple-organisms. The Litobionts have distinctive characters, such as small size, and simplicity of composition, but nevertheless, live, assimilate, grow, multiply;-not only segmenting somewhat after the manner of some higher, more or less filamentous organisms, but multiplying by endogenous division, this latter being one of the present observations, the endogenous process being exemplified in the "granules" of the spermatidia of Spirina. (See p. 41.)

Yet it is possible to over-emphasize the smallness of Litobionts. It seems likely that we have been looking at Litobionts a long time,Litobionts of the larger size,-without recognizing their nature, just as observers previous to the time of Schleiden and Schwann had been looking at cells without recognizing their nature.

Just as the multicellular and unicellular organisms overlap each other in the matter of size, so the unicellular organisms (having the characteristic properties of cells as now defined) overlap the Litobionts. There are unicellular organisms smaller than some Litobionts. Or, in reverse, some Litobionts larger than some unicellular organisms.

That the Litobionts are much simpler than cells, is indicated by a number of considerations. Their effects on light indicate that in the main, they are composed of a smaller number of kinds of molecules of a more orderly arrangement,- what may perhaps be thought of as forming a simpler plasm, Litoplasm. The fact that some of them are soluble in certain chemical reagents (e.g., acetic acid), is another indication of relative simplicity. In a word, we must conceive of the Litobionts as made up of a smaller number of kinds of simpler molecules manipulated through very much smaller distances, and therefore necessarily (a matter of "economy," "least resistance") by simpler mechanism. It is quite conceivable that some Litobionts may be smaller than some of the largest molecules. Not needing these large and complex molecules, the mass of the Litobiont may even be smaller than that of some such molecules.

The duality characteristic of all matter must lead, however, to an arrangement of the parts in Litobionts such that we can only think of them at present largely in terms of what we know of cell physiology and mechanics; simply because knowledge progresses exclusively through the known to the unknown. Our knowledge of cells must be one of the main sources of our Litobiont concepts. 


\section{THE SCREW NEMAS}

(Ascarophis van Beneden, 1871)

\section{PARASITES OF CODFISH, HADDOCK AND OTHER FISHES}

\section{Contributions to a Science of Nematology XVII}

By N. A. Совв

The screw-nemas, as it is here proposed to call them, have yet to be adequately studied. Not very much has been added to van Beneden's

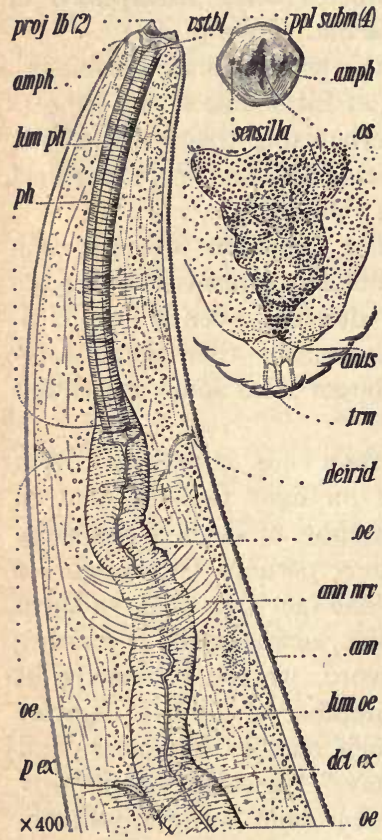

Fig. 1.-Head and tail end of Ascarophis helix n. sp. Above, to the right, front view of the lip region. The head end is nearly a ventral view, but slightly oblique. The tail end is a dorsal view, and the anus, being on the far side, is but indistinctly shown. nous ways in which this comes about may well give pause to any who, original description. Almost nothing is known about their life history and habits. However, the present communication adds considerably to our knowledge of their morphology. The suggested explanation of the remarkable screw form, and its probable mode of evolution, present the nemic cuticle in a new rôle. (See Figs. 5, 6, and 7.)

Considering the number of screwnemas thus far seen, it is remarkable that no males have been discovered. Nicoll records screw-nemas as "extremely numerous" in haddock. Van Beneden found them originally in the codfish; Nicoll, in the codfish and haddock, and in the fishes Hippoglossus vulgaris and Cotus bubalis; MacCallum now finds a species in the stingray.

Nemas so widespread and numerous probably have economic significance. This probability can not be dismissed by citing the absence of definite evidence to the contrary, for, at rather frequent intervals nowadays, nematologists are showing that nemas long known and lightly regarded, are not only of some importance in their relationship to mankind but sometimes of great importance; and the multitudiWaverly Press, Baltimore, Md., June 9, 1928. From Jour. Wash. Acad. Sc., Feb. 19, 1928. Repaged without material alterations. 
basing their views on past records and much current opinion, see in the presence of such parasites merely an interesting phenomenon.

For example, it is now found that the presence of nemic parasites not infrequently has a profound effect upon the reproductive organs of the host, a limited number of the parasites even producing complete sterility in a host otherwise apparently normal. That such cases in their most definite form have thus far been found mainly in the invertebrate phyla does not invalidate the application of the idea to vertebrates, even were such cases wholly unknown in the vertebrata,-which they are not. Considering the well known universal specificity of certain chemical reagents, - chloroform for instance, a "universal" anaesthetic,-we should be prepared to accept without very much surprise some such universal specificity in the action of some hormones, particularly sexual hormones, whose origin traces back to comparatively simple, but fundamental, ancestral cell phenomena.

Again, there is abundant evidence of high infant mortality in a great variety of animals and plants, due to nematism. This, coupled with our ignorance of the early life histories and food habits of fishes, even common ones, makes it unwise to ignore the possible economic importance of the nemic parasites of fishes.

Many other examples could be cited of the multitudinous and unexpected ways in which nemas are being shown beneficial or injurious to mankind.

Ascarophis helix n. sp.

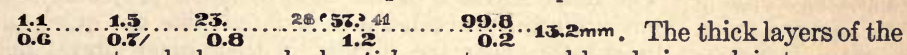
transparent, colorless, naked cuticle are traversed by obvious plain transverse striae, which vary markedly in different portions of the body. On the head, however, the transverse striae are hard to resolve; yet critical examination of the striae immediately on and behind the lip region even resolves them into rows of dot-like elements. In this region the crenations of the contour seem duplex, four double crenations a short distance behind the head occupying 10 microns, so that each crenation encompasses about 1.25 microns. In the latitude of the nerve-ring the striae are 1.7 microns apart; thence backward they are gradually coarser and more distinct, each striation becoming a double line. Furthermore, it is soon apparent that the striations pass around the body in the form of right-handed helices-coarser and coarser, and more oblique, with increasing latitude, so that at the base of the long neck the coils are about 8 microns apart and lie at an angle of about $23^{\circ}$ with a transverse plane. This obliquity increases until, near the middle of the body, it reaches a maximum of about $30^{\circ}$ (Fig. 2). Thence onward, however, the obliquity diminishes. Somewhat behind the middle of the body, certain coils of the helix fade, so that the other, now more prominent, striae are as much as 20 microns apart, while their width is nearly two microns, - namely the distance apart of the double "lines" representing the striae. This "dropping out," or fading, of course, is evidence of the existence of a plurality of helicoid "striae." In this way the body of the nema takes 
on the external form of an ordinary multiple-threaded screw. Here, in the middle, the contour of the body has become very coarsely and very pronouncedly compound-crenate. The more pronounced striae come to subtend twelve minor ones (Fig. 3). Finally near the tail end, the more pronounced striae subtend six minor ones (Fig. 3); this is near where the body is bluntly rounded off, in a hemispherical-conoid manner, in the course of a distance equal to about one and one-half body widths (Fig. 1). At first sight the deceptive appearance of the cuticle toward the posterior end of the nema suggests moulting, and consequent wrinkling of the cuticle. Longitudinal "striations,"

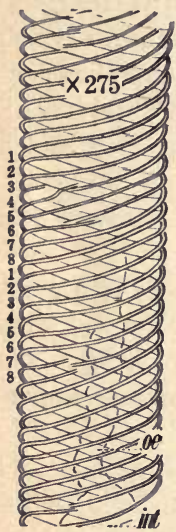

Fig. 2.-Camera lucida drawing of an oblique view of the eight-fold helicoid striae of $A_{s-}$ carophis helix, at lat. $23^{\circ}$, near the beginning of the intestine. The anastomosing occurs opposite the lateral chords. about 2 microns apart, due to the attachment of the musculature, are visible in most regions of the body. Posteriorly these longitudinal "striae" are still slightly oblique, and this slight obliquity extends practically to the terminus. There are no cuticular wings. With the nema in profile the lateral chords appear about one-seventh as wide as the body.

The groove-like unarmed "vestibule" is very simple and shallow,-about as deep as the height of the two prominent, lateral, forward-pointing, conical labial projections (Fig. 1, proj $l b)$. The vestibule leads through the slit-like mouth opening into a long, uniform, tubular pharynx, extending more than halfway to the nerve-ring. The pharynx is a marked feature of the front end, though it is so transparent and dimly refractive that it might, perhaps, under some circumstances, rather easily be overlooked (Fig. 1). Van Beneden seems to have figured the pharynx; Nicoll not, or at least not definitely. The mouth seems to lead into a minute pharyngeal or vestibular cavity, not very much wider than the amphids, perhaps six to eight microns wide,-a little longer dorso-ventrally than transversely. The median axil between the two lips is not sharp and distinct. The inner surfaces of the conical labial projections are not uniformly rounded and striated, like the outer surfaces,-for, near the middle, in their inner lateral lines or fields there are refractive longitudinal elements extending from the tips back to the mouth opening. It seems quite certain that there is an axial element extending to the apex of each of these conical projections, and when this is viewed in optical section, as one focuses from front to back, the appearances give rise to the opinion that there is a single innervation to each conical projection. One sees no evidence of radial musculature round the vestibule. There are no eyespots; and there is no pigment near the head, or elsewhere in the nema.

Returning now to the profile and dorsal views of the head;-four to five microns behind the tips of the two cephalic projections, exceedingly minute openings in the lateral region indicate the external amphids. As viewed dorso-ventrally, the anterior part of the walls of the pharynx, without diminishing much in thickness, bend together and nearly meet near the base of the vestibule, thus giving rise to the narrow mouth opening; in this anterior portion of the pharynx, the transverse striation is less apparent.

Behind the pharynx the oesophagus is a little less than one-third, at the nerve-ring about one-fourth, twice as far back as the nerve-ring a little less 
than one-third, and then again soon-rather suddenly increasing - a little more than one-third, and finally is one-half, as wide as the corresponding portion of the neck. The lining of the oesophagus is a rather distinct feature throughout its length, and finds its main optical expression as a somewhat sinuous axial element. The musculature of the oesophagus is rather fine. Behind where the above-mentioned enlargement takes place there is a considerable amount of granular matter in the oesophageal tissues.

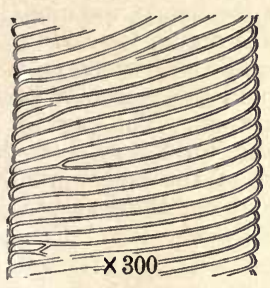

Fig. 3

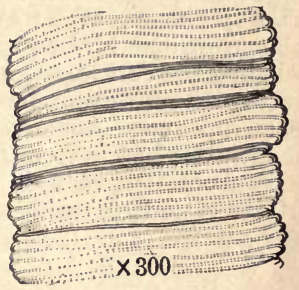

Fig. 4

Fig. 3.-Helicoid striae of Ascarophis helix considerably behind the middle of the body.

Fig. 4.-Camera lucida drawing-of the contour of Ascarophis helix near the tail end of a female.

In front of this region the radial fibers are of a finer nature, closer together, and the granulation much less apparent, if present at all; in other words, there is a distinct change in the structure of the eosophagus at a point twice as far back as the nerve-ring. The intestine becomes almost at once twothirds as wide as the body; it is separated from the oesophagus by a distinct cardiac collum somewhat less than half as wide as the body.
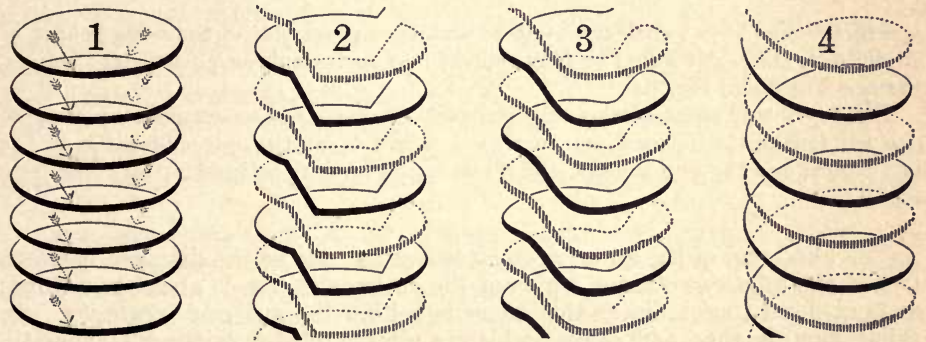

Fig. 5.-Diagrams illustrating a theory of the mode of origin of helicoid striae through anastomosing of the ordinary transverse striae of the nemic cuticle. Let 1 represent seven ordinary annules of a nemic cuticle, and suppose the anastomosing to take place on opposite sides of the nema at the places indicated by the arrows; 2 represents the anastomosing as having taken place, precisely as indicated by the arrows in 1 ; while 3 and 4 show the further theoretical transition to perfect helices. It will be observed that two helices are formed. Bilaterally symmetrical growth would necessarily lead to helices of even number, as exemplified in Ascarophis. See also Figs. 6 and 7. 
The wall of the intestine, while not very thick, is somewhat irregular in thickness, the lumen appearing zigzag. At places the wall of the intestine is one-fourth as thick as the intestine is wide; at other places nearby its thickness may diminish by two-thirds. There is a distinct lining to the intestine, apparently made up of "columnar" elements vertical to the inner surface, though these have not been very clearly seen (Fig. 8). The granules contained in the intestinal cells are rather uniform in size, but their histological characters can not be made out on account of the state of preservation of the specimen. Well forward, near the blind end of the ovary, the intestine is not over one-third as wide as the body; and in this region the body wall, including the cuticle, occupies about one-fourth the radius, of which amount the vaguely retrorse cuticle occupies eight microns and the muscular tissue fifteen microns. There seems to be a very short rectum. The portion of the intestine just in front of the rectum is saccate, and, for a very short distance about half as wide as the corresponding part of the body; whereas in front of this enlargement the intestine is only about one-third as wide as the body.

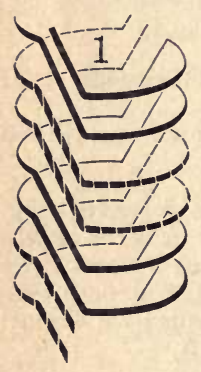

Fig. 6

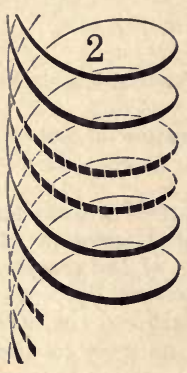

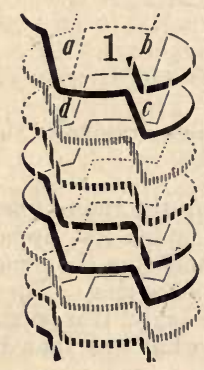

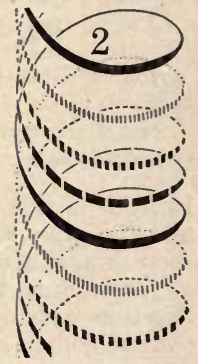

Fig. 7

Fig. 6. - Should two ordinary adjacent annules on each side of the nema behave as shown in 1 , the result would be four helices; four such would originate eight helices. See also Fig. 7 and Fig. 5.

Fig. 7.-Should anastomosing take place simultaneously in successive annules opposite any four of the longitudinal chords $a, b, c$ and $d$, say the four submedian, or the two lateral and the two median, the result would be four helicoid striae. See also Fig. 6.

The blind end of the anterior ovary, about as wide as the distance between two of the adjacent oblique winds of the cuticular helix, is about two-thirds as far behind the cardia as this latter is behind the anterior extremity. In this region, in the body cavity, which is relatively of considerable capacity, there are "floating" organs made up of ellipsoidal or subspherical, fine granules, the largest of which are about eight microns in diameter (Fig. 8, org fluit). These "loose" organs are reminiscent of those known and figured in some of the ascarids, - e.g., Ascaris kükenthalii. The ovaries lie in elongate coils, and at first contain oöcytes about four microns across, which soon increase and become packed in the ovaries in the form of polyhedrons whose optical contour is often hexagonal, and which are 10 to 12 microns across where the ovary is one-third as wide as the body. The stretched-out ovary would be about 
twenty times as long as the body of the nema is wide, and at its greatest width about one-third as wide as the nema. Sperms have not been seen, nor has the extent and nature of the oviduct been observed. The two uteri are filled with six to eight hundred ellipsoidal eggs about one-third as long as the body is wide and averaging $40 \times 24$ microns. For a short distance near their equator the eggs are practically cylindrical. The shells are thick - a little over 2 microns - and structureless looking; are of uniform thickness throughout; and, as seen in the uteri, are without any surface markings or appendages. No indications were seen of "two flagellae at one pole," as noted by van Beneden and Nicoll. It is possible that appendages might arise later, e.g., from some vaginal secretion coagulated during deposition. The eggs, before deposition, contain fairly well developed larvae. There is a single ovijector of considerable length passing inward from the vulva; apparently the ovijector is several times as long as the body is wide, - say at least three times. Its walls are thick and muscular; viewed in optical section it is nearly one-third as wide as the body, being somewhat flattened when collapsed, and so, in cross-section, a little more than half as wide as long. Its lining is thin and strongly refractive; the wall, when seen in optical section, is glassy internally and fibrous externally. The vulva is a transverse ellipsoidal affair near the middle of the body, about one-fifth as wide as the corresponding portion of the body and interrupting two to three of the spirals. It is about twice as wide as long, is distinctly marked, and presents a double refractive contour, especially posteriorly. The excretory pore is an opening of considerable size, taking up the space of about three annules of the cuticle. For a short distance the tube is strongly refractive, then suddenly becomes almost invisible. In the specimen under

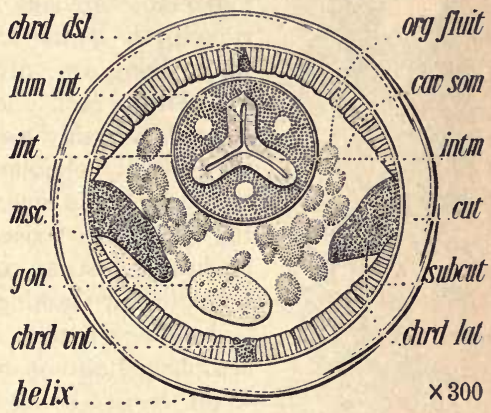

Fig. 8. - Somewhat schematized drawing of a cross-section of Ascarophis helix, taken not far behind the neck. Eight helices are cut, as at helix.

examination it is impossible to follow it far enough to say whether in its course it becomes double and symmetrical or remains single and asymmetrical (Fig. 1, $p$ ex.)

Diagnosis: Ascarophis having a length of $13 \mathrm{~mm}$.; striae helicoid, the sub-cephalic ones very fine and not retrorse, the posterior ones very coarse and compound, their maximum obliquity, - behind the nerve-ring, $-30^{\circ}$; the two labial projections broadly conoid; pharynx tubular, $1.1 \%$; tail convex, and rather symmetrically short-conoid, $0.2 \%$; eggs without polar filaments.

Habitat: Gills of the fish, Dasyatis centrura,-Sting-ray. G. A. MacCallum, Woods Hole, Mass., August, 1927. Hitherto Ascarophis has been found only in the intestinal canal of fishes. The plurality of helices has probably evolved through anastomosis; this anastomosis, if increased in extent and systematized as shown in the diagrams (Figs. 5, 6 and 7) could give rise to helical striae. The anastomoses in $A$. helix, as far as seen, are lateral. 


\section{UNGELLA SECTA}

n.gen. n. sp.

A nemic parasite of the Burmese Oligochaete (earthworm),

Eutyphoeus rarus.

Contributions to a Science of Nematology XVIII

By N. A. Совв

\section{Ungella secta $n$. gen. $n$. sp.}

The transparent colorless cuticle is traversed by transverse striae, about one micron apart, hard to resolve even with high powers, at least in alcoholic

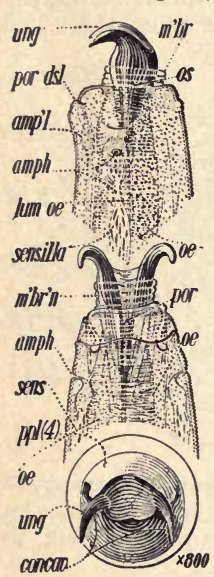

Fig. 1.-Ungella secta. Side, dorsal and end views of the same head. $M^{\prime} b r$, the intussusception membrane. specimens. In certain stages of the nema the striae are much more lobvious and double lin size. Though interrupted, the striae are not altered, on the lateral fields, where there are only faint single wings-non-existent or faint on the neck and anterior portion of the body, but somewhat readily seen along the middle of the body. The very slightly oblique longitudinal striae, due to the attachment of the musculature, are more readily visible than the transverse striae. (Fig. 3, str longt) Between the longitudinal striae are faint rows of dots, reminiscent of the cuticular markings of Diplogaster.

And here it may be said that, though valveless, the oesophagus also is reminiscent of Diplogaster; and that of all the free-living genera, Diplogaster is that to which Ungella seems most closely related. It is readily conceivable that the submedian duplex onchium (Fig. 1), could have been evolved from an armature such as characterizes one of the types of diplogastric pharynx.

Onchium. The duplex onchium of Ungella has its amalgamated roots movably imbedded in the head end of the nema backward for a distance equal to two-thirds the width of the head or more; it is assumed therefore that this represents the depth of the otherwise unarmed pharynx. The onchium, which can be exserted for the greater part of its length, is a strong refractive organ, colorless except distally, where it is yellowish; it is a conspicuousfeature of the head, especially when protruded. The two equal claws of the onchium are joined rigidly in such a way as to make it impossible for them to be juxtaposed, and their internal structure makes plain that they represent the two ventrally submedian sectors of the oesophagus. Thus the onchium and its 
"hilt" have the general form of the ultimate two-clawed segment of a beetle's tarsus. (See Fig. 1.) Rather weakly developed retractor muscles are attached to the amalgamated onchial apophyses. It seems not unlikely that the caudal "suckers" may also aid-as a base of resistance-in the use of the onchium, the object of which must be to claw; it must wound by clawing, hence the specific name secta. When the onchium is withdrawn and at rest, as in the female of Figure 2, the outer or distal parts of the two claws rest in special lateral depressions on the outside of the front of the head (see concav Fig. 1) and to that extent are not withdrawn into the head.

Oesophageal glands. The median dorsad pore in the front of the head, por $d s l$, is the exit of a large well developed special cervical gland, $g l \mathrm{crv}$. The excretory pore of the renette, $p$ $e x$, is farther back and ventral. There is an almost imperceptible short alteration in the oesophageal lining between the fore and after parts of the oesophagus, - probably the vestige of a median bulb. The indistinctly clavate, posterior,"non-valvate, oesophageal swelling contains a single, bright, refractive, three-micron nucleus near the base in the dorsal sector, proving the presence of an oesophageal gland. Radial oesophageal muscles are only faintly to be seen.

Intestine. A cross-section of the intestine cuts through only about two relatively large cells. The refractive lining of the intestine of ten is distinctly to be seen. In the front portion of the body the wall of the intestine is hardly as thick as that of the body; here the lumen of the intestine often is more

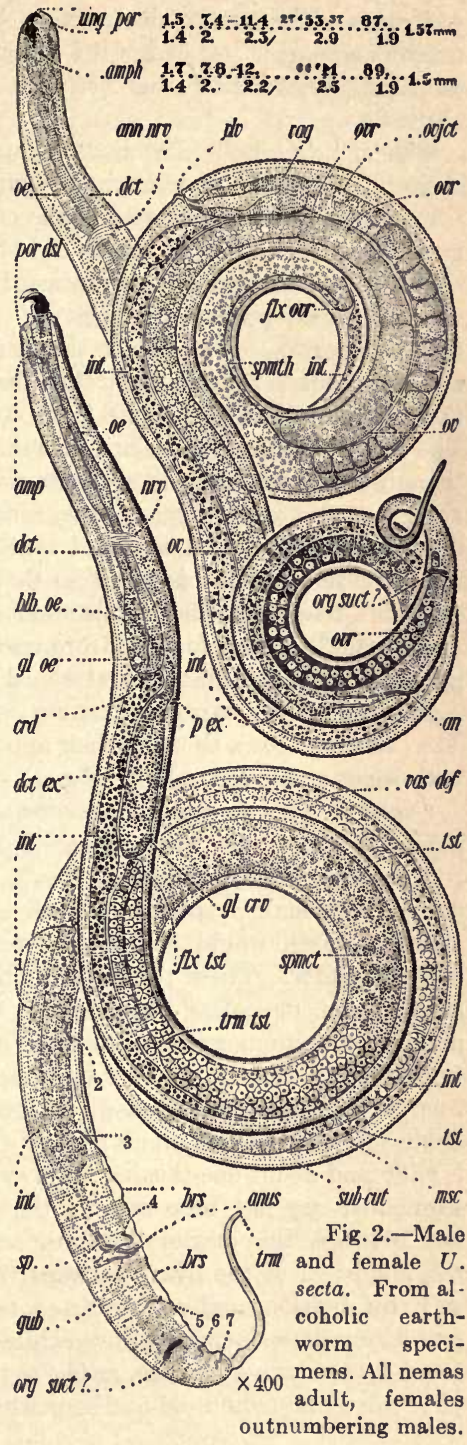
than twice as wide as the thickness of the intestinal wall. 
Renette. The renette duct, dct ex, is distinctly refractive and nearly two microns across; it passes inward at right angles to the ventral surface and then turns backward on the left side and becomes narrower and apparently bifurcate.

Caudal "Suckers." The tissue composing the mouths of the two lateral caudal "suckers" is comparatively structureless looking, and externally partakes of the general character of the cuticle. These two large lateral openings, found on the tail of adults of both sexes, when viewed dorso-ventrally are seen to lead inward and forward into two well developed pockets or "suckers," so massive that this portion of the tail, in the median aspect appears about 50 per cent wider than the portion of the tail immediately behind (Fig. 3, org suct). The cavity of each organ is lined with thick striated tissue whose most obvious elements are arranged at right angles to its inner surface, which presents a very definite internal sectional contour, due to the refractiveness of this tissue; so that the whole organ is a relatively conspicuous affair. A strand (contractile?) leading forward from each "sucker" into the corresponding lateral chord is at first rather wide, then narrows (text org suct, Fig. 3). The "suckers" seem to make their appearance on both sexes at the last moult (Fig. 4).

Gonads. The elevated transverse vulva apparently is not very wide. Near the flexure the gonad presents a spermatheca, spmth, containing numerous spherical sperms of such a size that about a dozen would be required to span the body diameter. These possess refractive, faintly lobed nuclei, indicating the presence of a small number of chromosomes-probably about five. Contained in the uterus of adult females, as a rule, is a single thin-shelled, smooth egg, ov, about one and one-half times as long as the body is wide and about one-third as wide as long. No

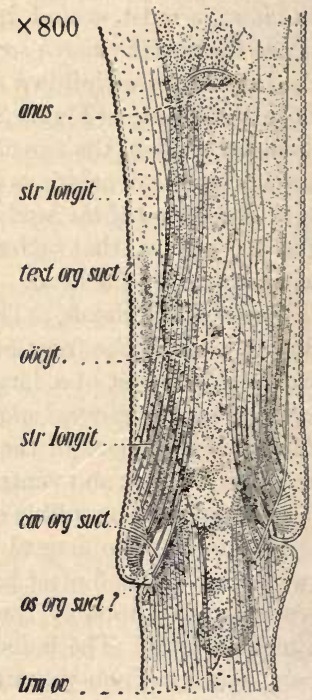

Fig. 3.-Ventral view of the post-anal region of $U n$ gella secta, showing in the lower part of the figure the two lateral pockets or suckers, cav org suct. segmented egg has been seen in the uterus. The blind end of the ovary lies between the caudal "suckers" or somewhat farther forward. From the blind end of the ovary forward the oöcytes very soon become smaller, as if by division, and not far from the anus are arranged several abreast, and so continue, increasing meanwhile in size, for a good fraction of the distance to the vulva; thence, owing to increased size, they are arranged single file, each ovum cylindroid and somewhat longer than wide. 
The male. The relatively large gubernaculum, gub, is single, rather shallow, nearly straight, and extends inward nearly at right angles to the ventral surface, so that its proximal end is considerably dorsad of the body axis. It appears quite as massive as the two equal spicula, and has the form of a relatively very broad, shallow, somewhat boat-like trough, deepest amidships,

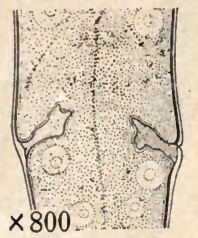

Fig. 4.-Ventral view of the suckers of Ungella secta just before the last moult. its depth being about one-third its length. The long narrow bursa, brs, presents seven whiplash-like ribs (1-7, Fig. 2) extending into each of the colorless, thin, glassylooking, ventrally submedian, bursal expansions of the cuticle.

Since the suckers are common to both sexes, they can hardly be considered secondary sexual organs. Figure 4, derived from one of the few immature specimens thus far seen, seems to indicate that these interesting organs come into existence at the last moult, for, just previous to the last moult, they appear immature or "embryonic." It seems hardly possible that these organs can be homologous with the phasmids; nor does it seem possible to link them with such ventral suckers as occur for instance on male Heterachids. In short, further observation is needed fully to determine their function.

Habitat: Body cavity and muscles of the earthworm, Eutyphoeus rarus; fide Mr. G. E. Gates, to whom the discovery of the nema is due. Locality, Prome, Burma, India.

\section{Ungella, n. gen.}

Genus Diagnosis: Amphigonic onchia hooked protrusile, dorsally arcuate; and special cervical gland; oesophagus degenerate-diplogastroid; adults with posterior lateral pockets or "suckers;" 'm and 'f; males with two equal spicula and a gubernaculum, and an elongate pre- and post-anally ribbed bursa. Parasitic in earthworms. Proposed as type species is:

Species Diagnosis: Flexible-tailed ungellas, dimensioned as in the formulae and illustrations, with two practically submedian, amalgamated onchia (ungellae), having the form of the final joint of a beetle's tarsus; cervical gland just behind the cardia, its outlet dorsad on the lip region; pockets or suckers not far in front of the middle of the tail; external amphids more or less circular and opposite the base of the pharynx; oviparous; males with three pre- and four post-anal slender ribs to the bursa, as shown in Figure 2; posterior part of the tail cylindroid, fine yet blunt,-in the male distinctly set off.

Only a more careful study of the nemas thus far described as parasitic in earthworms can determine the nature and limits of most of the genera and species that have been proposed for their reception.

For literature consulted see the list of Pierantoni (Boll. Soc. Nat. Napoli, 1915, p. 150-3) and Baylis \& Daubney (Synopsis, 1926). 


\section{THE NEMIC GENUS SYRINGOLAIMUS}

With a note on the fossorium of nemas

Contributions to a Science of Nematology XIX

By N. A. СовB

The writer's collection of Syringolaims shows them to live on temperate and tropical sea coasts in many parts of the world. Among other places, his Syringolaims (1888-1927) represent the East Indies (Larat), Polynesia (Noumea, Hawaii), the Atlantic and Pacific Coasts of Panama, the Atlantic Coast of the United States, and the English Channel. The manuscript record of these collections contains full descriptions of a number of new but unpublished closely related species.

Our knowledge of this genus has increased but little since de Man described the type species, his $S$. striatocaudatus. The present publication adds information concerning (1) the labial papillae, (2) the amphids, (3) the phasmids (?), (4) the fossorium, (5) the intestine, (6) the male gone, (7) the food habits, and (8) the geographic distribution.

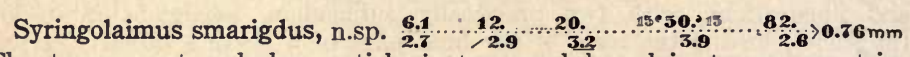
The transparent, colorless cuticle is traversed by plain transverse striae very difficult, or almost impossible, to resolve, which are not altered on the lateral fields. Faint traces of wings occur, beginning near the head and ending on the tail. Longitudinal "striae," due to the attachment of the musculature, are visible in nearly all regions of the body. No series of pores have been seen in the cuticle. Of the highly mobile lips there probably are three, but they are no more than sub-distinct, and are small and somewhat rounded. The pharynx is armed in front with three duplex (somewhat lobster-clawlike), in profile somewhat inverted-comma-shaped, subacute odontia (Fig. $1, m n d$ ) having an outward throw of about $180^{\circ}$, a movement seen on more

Waverly Press, Baltimore, Md., June 9, 1928. From Jour. Wash. Acad. Sc., May 4, 1928. Repaged without material alterations. 


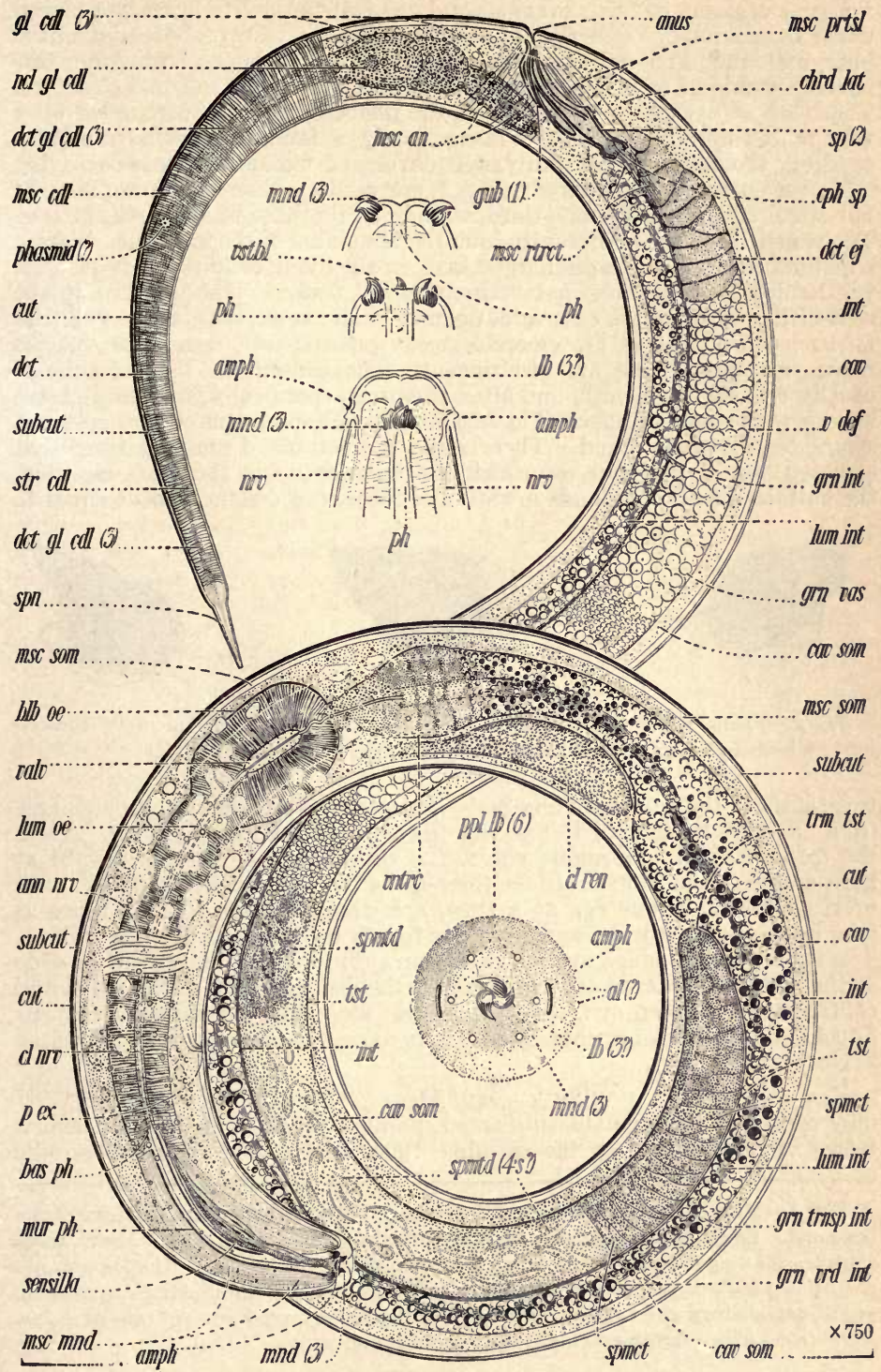

Fig. 1 
than one occasion to occur every second or so when the live nema was placed under the microscope under slight pressure (Fig. 1). The cross-section of the pharynx is round-triquetrous, almost circular, with faint subordinate markings in the middle of each side, indicating on the whole a hexagonal structure. There are no eyespots. The base of the pharynx may be surrounded by a very faint ellipsoidal swelling. There is only a faint pharyngeal muscular swelling, though there are fairly well developed mandibular muscles, lying along the outside of the pharynx (Fig. 1, msc mnd). There is a rather distinct but small conoid cardia, one-third as wide as the base of the neck, or less. The ventriculus stains differently from the remainder of the intestine, showing a distinct function to be discharged here; in the living condition however the ventriculus appears somewhat "structureless" (vntrc). The granules in the cells of the intestine are of several distinct kinds: some of them are colorless (grn trnsp int), others are emerald-green ( $g r n$ vrd int)-hence the specific name smarigdus; none are birefringent. The content of the intestine is usually reddish or greenish, and often is derived specifically from an alga belonging to the family Ralfsiae (Fig. 3), among which specimens of Syringolaimus smarigdus are often found. There is no prerectum. From the somewhat elevated lips of the anus, of which the anterior lip is the more elevated, the cutinized rectum extends inward and forward a distance about equal to
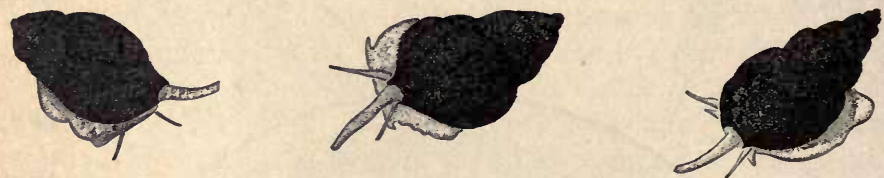

Fig. 2.-Snails, natural size, covered with a very dark green "pile" or "felt" consisting of microscopic algae. The nature of this growth is illustrated in Fig. 3.

two-thirds of the corresponding body diameter. The lateral chords enlarge from one-fifth (terminad) to one-half (mediad) as wide as the body. From the medium-sized continuous vulva, the cutinized vagina leads inward at right angles to the ventral surface three-fifths the way across the body. The uteri contain only one egg at a time, are straight, three to four times as long as the body is wide, and from one-fourth to one-sixth as wide as long. The two opposite, equal, symmetrically arranged ovaries, about half as wide as the body, are reflexed about two-thirds the distance back to the vulva and contain ten to fifteen ova, mostly in single file. The elongate egg may be 3 to 4 body-widths long, appears relatively narrow, and seems to be deposited before segmentation.

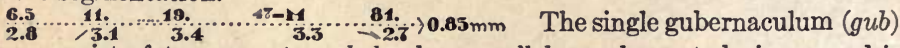
may consist of two arcuate, subslender, parallel, amalgamated pieces, and is rather closely applied to the spicula. Phasmids(?) (Fig. 1) occur on the

Fig. 1.-Male of Syringolaimus smarigdus, together with four different views of the head end. Below, a diagrammatic drawing of the front view of the head. Above, three sketches showing different attitudes of the "mandibles" or fossores. The fossores are also shown in both the other illustrations. The lettering of the illustrations consists of self-explanatory abbreviations arranged in the Latin order; grn vrd int-granulum viride intestinalis, - green granule of the intestine; etc. 
lateral lines near the middle of the tail on both sexes. While there is no distinct bursa, the cuticle is faintly thickened in the submedian region near the anus, possibly a very rudimentary bursa.

Habitat: Common among minute filamentous algae on the surface of marine mollusks, especially the snail Alectrion obsoleta (Fig. 2). Also found in sand on beaches, and in sand in several feet of water off shore. Woods Hole region, 1916 to 1927 . There is good reason to suppose it ranges both north and south from Woods Hole along the Atlantic Coast. It occurs in beach sand from near Falmouth; and in clear white sand in five feet of water in a cove at the entrance to Buzzard's Bay; also at Waquoit, Mass., among algae on the shell of the living snail, Alectrion obsoleta (Nassa); and on the shells of live snails from the Eel Pond at Woods Hole. Its food seems always to be vegetable matter, and in many cases consists entirely of the contents of the cells of a microscopic alga belonging to the genus Ralfsia (?).

\section{Outward Acting Nemic "Mandibles"}

The writer's study of the attitudes in which the mouth parts became fixed led to the conclusion that in Axonolaimus and its relatives, as well as in a large number of other nemas, the onchia (and odontia) had an outward throw. If so, it was an obvious deduction that these organs were digging organs, for which the word fossor ${ }^{2}$ seems indicated. This deduction led the writer long ago to introduce into generic names of such nemas root words indicating a digging function on the part of the mouth organs, as for instance in the genus names Scaptrella, Diploscapter.

It is, however, difficult to observe these organs in operation, and hence of interest to record that such organs have been seen in action in a Syringolaim (Fig. 1), and furthermore that S. smarigdus has been observed under conditions constituting strong additional circumstantial evidence that these organs are verily digging organs. $S$.

${ }^{2}$ Fossor (plural, fossores; collective, fossorium); a tool or organ used for digging, usually existing in a plurality and acting symmetrically outward from a plane or axis. Related to "fossorial"-said of animals that dig. 
smarigdus is found in algal "incrustations" of the family Ralfsiae, and probably genus Ralfsia. ${ }^{3}$ It is very apparent that the nema feeds upon the Ralfsia, for the color and structure of the contents of the cells of this alga are strikingly characteristic, and the intestinal content of the associated Syringolaims not only has exactly the same color, but frequently is otherwise of such a character that it could be derived only from the interior of the Ralfsia cells. Often, however, there are scattered foreign birefringent particles (carbonate of lime) mixed with the ingested food; but these birefringent particles are similar to those found among the filaments of the Ralfsia, and, taking into account the relative size of the mouth parts of the Syringolaim, it is very natural to suppose that some of this foreign matter would be taken in with the food.

No one had previously explained the precise nature of the mouth organs of Syringolaimus. They consist of three small, arcuate, more or less acute odontia with a spirally outward throw, well adapted to boring and digging (Fig. 1). Now, it so happens that the location and structure of the incrustation formed by the Ralfsia would require digging on the part of the nema in order to obtain food from it, for the Ralfsia incrustation on the snail shells (Alectrion) is usually overgrown with a thick comparatively impenetrable felt of filamentous green algae (Fig. 3); hence the Ralfsia can be reached by the Syringolaimus only by digging.

The snail, Alectrion obsoleta (Nassa), lives between tide-marks and hence twice daily is exposed to the air, and on each such occasion any algal growth on it naturally dries up more or less. Here then is an additional complication in the environment of the Syringolaim-a highly and rapidly variable temperature and salinity. It is reasonable to suppose these unusual circumstances might give rise to a peculiar nemic form adapted to the environment. Thus a clue is found to the marked peculiarities of form and structure noted in Syringolaimus.

${ }^{3}$ Ralfsia; fide Dr. I. F. Lewis. 


\title{
THE CHROMATROPISM OF MERMIS SUBNIGRESCENS
}

\author{
A Nemic Parasite of Grasshoppers
}

Contributions to a Science of Nematology $\mathrm{XX}^{1}$

Bx N. A. СовB

The adult female Mermis subnigrescens, when ripe for ovijection, has a way of moving her head in more or less horizontal curves;--her head, directed skyward, is waved in "circles," now clockwise, now the reverse. This seemingly purposeful behavior occurs when she emerges from the soil and while she is ascending the herbage to deposit her eggs. Inasmuch as the head of the egg-laying female,-unlike that of the young female as well as that of the male (neither of which ever quits the subterranean darkness), - contains reddish transparent pigment rather definitely distributed with reference to certain cephalic nerves, the question arose whether we do not have here a phototrope ${ }^{2}$ and an

${ }^{1}$ Reprinted from the Journal of The Wasmington Academy of Sciences, Vol. 19, No. 8, April 19, 1929.

2 It is suggested that the mechanisms through whose activation the responses of organisms termed tropisms find expression be called "tropes;"- "tropism" to be taken in almost any of its more or less well accepted meanings.

These meanings (interpretations of various investigators,- - see Mast, 1915) vary all the way from (1) "an inherent tendency to respond" (Standard Dictionary), to (2) an "irresistible" or "predictable" orientation as definite and mechanical as that of a magnetic needle; but whatever the accepted interpretation, the reaction-mechanism must always be present, and be a system of intimately connected elements or organs, as is the digestive system, for instance, or the excretory system. Since we have for this system of intimately connected elements no inclusive single descriptive term, and since it is found highly convenient, or even necessary, for purposes of thought and discussion mentally to "isolate," and separately to denominate, the digestive system or enteron, and other systems, it is suggested that in behavior studies a like situation be met by a similar,

Warerly Press, Inc., Baltimore, Maryland, June, 1980 
affirmative answer was forecast, for the obvious reason, among others, that, as the pigment must absorb certain light frequencies and transmit others, the absorption might well result in some such changes of energy as characterize vision. Very suggestive also is the fact that the transparent, colorless parts of the head immediately in front of, and alongside, the suspected phototrope condense light rays upon it. (See Fig. 1.)

Previous experiment showed the spectrum frequencies concerned in bringing about ovijection in this nema probably to be some of those in the light-blue and low violet region of the spectrum, together with red (also infra-?). ${ }^{3}$ It would therefore be natural to suspect, under all the circumstances, that the cephalic pigment characteristic of the adult female absorbs, and "makes use of," the frequencies present just previous to and during ovijection. Hence a wish, (1), to determine what frequencies are present during and just previous to natural ovijection; and, (2) to determine what frequencies are absorbed by the cephalic pigment.

In this field comparatively little seems to have been published, though somewhat pertinent papers by Crozier, Mast, and others exist. Investigators have been mainly occupied with the optics of the various colorless, transparent, organic elements; the relative location and probable function of certain pigments, usually dark or black (opaque); and the "migration" and other changes of pigment, such as visual purple, due to the action of light; and, of course, with the associated nervous and contractile elements. Little has been published with regard to tropism definitely due to the absorption of rays of a particular frequency solely by transparent, colored pigment (other than visual

but if possible better (to wit monosyllabic), terminology. The advantages of a monosyllable from which short, convenient adjectives, verbs, adverbs and other nouns can readily be derived, are almost too obvious to need mention,-tropic, tropically, to trope, troping, etc., etc. Most help\{ul, perhaps, will be its use as a component, e. g., in "chromatrope."

Primarily "trope" denotes action,-action that is in progress rather than completed. By metonymy the word denoting an action (here, what is called a reaction) may be applied to the (re)action-mechanism, i.e., in the present case, to the responding system of intimately connected organs.

Following this suggestion, we may speak of a reaction mechanism that aids or causes an organism to face toward or away from light, as a phototrope; a reaction mechanism used in orientation with reference to gravity, a geotrope, etc., etc., etc., etc.; thus the statocystic mechanism of crustaceans is a species of geotrope.

Accordingly, certain cephalic apparatus of Mermis subnigrescens is here spoken of as a phototrope; or, better, because more specific, as a chromatrope,-inasmuch as its reactions apparently are to definite frequencies (colors) of the solar spectrum. Glaucotrope $\gamma \lambda \alpha v k o \sigma=$ blue) may prove to be even more precise.

Species of Mermis. Journ. Parasitology, 8:66. 1926. 
purple) located definitely with reference to nerves, these nerves themselves so located as possibly to be sensory.

SPECTROSCOPIC TEST OF THE PRESUMPTIVE CEPHALIC CHROMATROPE OF Mermis subnigrescens

One-third of a millimeter of the front end of an adult female Mermis subnigrescens, including the head, was ligated and cut off, and then mounted on a microscope slide in water. The head was examined under a 1.5 $\mathrm{mm}$. apochromatic microscope objective, having a similar objective as a condenser, in such a way that the image would fill as much as possible of the microscope field with the color of the pigment. (See Fig. 1.) The microscope was fitted with a spectroscopic eyepiece.

Sunlight was taken from a flat planished aluminum reflector placed so that a maximum of sunlight was reflected through both instruments,-i.e., the above apochromatic-micro-spectroscope and a comparison spectroscope. The pigmented tissue was brought into focus, and then the iris diaphragm of the microscope thrown open, so as to admit a "flood" of light. This produced a spectrum fairly readily seen in a darkened room, notwithstanding the very high magnification.

Much of the violet end of the spectrum (well into

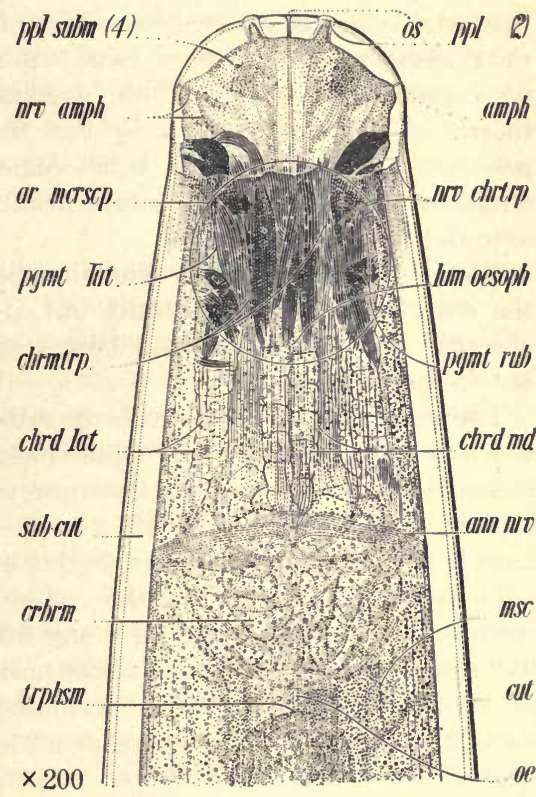

Figure 1. Head end of Mermis subnigrescens, showing the chromatrope. os, mouth; $p p l$ (2), the two lateral so-called "papillae;" amph, amphid; nrv chrtrp, nerves of the chromatrope; lum oesoph, lumen of the oesophagus; pgmt rub, reddish pigment of the chromatrope; chrd md, median chord; ann nrv, nerve-ring; $m s c$, body-wall muscles; cut, cuticle; oe, oesophagus; $p p l \mathrm{subm}$ (4), the four submedian papillae; nrv amph, nerve of the amphid; ar mcrscp, shows the circular area of the microscopic field that was tested spectroscopically; pgmt lut, orange-colored pigment of the chromatrope; chrmirp, chromatrope; chrd lat, lateral chord; sub cut, the thick subcuticle; crbrm, the cerebrum; trphsm, anterior end of the trophosome. 
the blue) was absorbed to a very considerable degree by the living cephalic pigment of the Mermis subnigrescens. The microscope field was never completely and uniformly filled with the color of the pigment. There were streaks at each margin of the field, and another through the middle, that showed little color (see Fig. 1), so that doubtless the absorption was not so pronounced as would be obtained by dissolved pigment of equal density filling the entire field of the microscope.

A satisfactory demonstration was made by placing the object under the microscope so as to produce absorption, and after the spectra had been arranged for comparison, suddenly removing the pigmented tissue. The effect produced by this instantaneous change was very pronounced. The blue and violet region of the spectrum, hitherto obscured by absorption, of course instantly assumed the same co'ors as in the comparison spectrum.

There seemed also to be some absorption in the outermost part of the red of the visible spectrum, but, if so, it was very slight. No absorption was noticed in the orange, yellow and green, and little if any in the bluish green.

The experiment was particularly satisfactory in that the pigment was in a living condition. True, the head had been ligated and cut off immediately before the spectroscopic examination, but from much experience it is known that such a head continues to "live" and move anywhere from a few hours to a day, or even more.

Phototropes of somewhat the character here described probably occur in the cervical region of many other nemas, particularly freeliving ones, - the outer tissues of the neck serving as a cylindrical lens for condensing light upon the pigmented tissues. Aquatic nemas so situated as to utilize light rays penetrating water, not infrequently present structures that may be suspected to be phototropes,--now that we have a clue to the nature of such structures in this phylum. These primitive phototropes may well have been the forerunners of the more highly developed phototropes, ocellate systems, of certain nemas (e.g. Enchelidium).

A general review of the nature and relationships of localized transparent pigments in organisms in the light of the foregoing experiment, may, perhaps, suggest new ideas and experiments with regard to their functions both in animals and plants. 


\section{NATURAL CONDITIONS UNDER WHICH Mermis subnigrescens DEPOSITS ITS EGGS}

At Woods Hole, Mass., U. S. A., on July 28, 1928, from long before daylight up to 10 o'clock A.M. the weather was warm and showery. The showers were gentle but subcontinuous, with light-intervals between; i.e., during two or three brief intervals the sunlight actually came through the fog and clouds rather clearly.

Two full-grown female specimens of $M$. subnigrescens, very much alike, were found depositing eggs naturally on grass etc. in an experiment field. As each nema still contained a good many eggs, both were taken to the laboratory in cold tap-water, and both subjected to radiant heat of low frequency, emanating from hot steel. The results of three trials on one of the nemas and four on the other were quite consistent.

An ordinary steel file about nine inches long and three-fourths of an inch wide was heated until hot, though not red. As near as could be judged the temperature of the file during the trials was from $400^{\circ}-500^{\circ}$ C. The hot steel was held within an inch and a half to two inches of the nemas. Held at this distance from one's cheek, it caused an agreeable warm sensation;-no disagreeable sensation of heat, however,-no suggestion of scorching.

When the nemas were brought into the laboratory, both were still slowly depositing eggs; one, however, very slowly,--putting out only one or two eggs semi-occasionally. When one of these ten-centimeter nemas in this laboring condition was removed from water and stretched out on a broad-leaf plantain, Plantago major, and the hot file brought near, she immediately responded by increased rapidity of movement, and in from ten to twenty seconds became coiled, sometimes rather closely, so that the entire space occupied by her would not be over fifteen to twenty millimeters across. Barely enough water was used on the plantain leaf so that only at her points of contact with the leaf was she in contact also with water. Doubtless the warmth may have caused the water to evaporate a trifle more rapidly, notwithstanding the saturated condition of the atmosphere, and one cannot say that this change in rate of evaporation may not have had some effect on the behavior, but the inevitable inference is that the "radiant heat" caused the change in behavior, corroborating, in a reverse way, experiments of previous years with direct sunlight and sunlight passed through heat-diminishing screens (both green glass and living foliage). 
After the nemas had been rayed and returned to water in a watchglass, and after they had resumed their former less active somewhat outstretched state, they were tested again and again with the infrared rays. Two observers, noting the nemas before they were removed from the watchglass of water and after they had been rayed, declared the ovijection to be stimulated, and in one case the stimulation to be very marked indeed. In this case, at the time when the nema was removed from the glass, oviposition was diminishing to almost nil, only now and then an egg being deposited,- at intervals of half a minute or thereabouts; however, after she had been rayed and returned to the water where her behavior could be observed more accurately, deposition was going on vigorously, - batches of something like twenty eggs were being ejected at intervals of five to ten seconds. It should be remembered that these two females already had their egg-laying capacity partially exhausted and were therefore probably less favorable specimens for experiment than if they had just issued from the ground.

The conclusions drawn from the experiments were that, without doubt, the radiant heat from the hot steel met uith instant response by the nema and that the response was very definite and that the egg deposition was very markedly stimulated by the rays. ${ }^{4}$

Apart from ultra-violet, apparently very little is known about the relative amounts of various light frequencies that are passed through different quantities of fog and watery vapor in the atmosphere. It is known that fog and vapor are more or less impervious to ultra-violet, but pervious to many other frequencies, among them blue and a certain amount of red and infra-red. However, nobody appears to have devised a method or instrument by which the amount of any particular one of these various other frequencies penetrating under various atmospheric conditions can be satisfactorily measured, although there is reason to hope that such data can be established.

It is very evident, however, that during the morning under consideration, which was showery with light rain much of the time, the weather varying all the way from thick fog to almost sunny,-fog so thick that the fog-horns were blowing, and yet at times the sky toward the east such that the sunlight came through rather clearly,-it is very evident that the amount of any given spectrum frequency reaching the experiment field probably would vary during the morning nearly through the entire daylight scale, or at least much of it.

\footnotetext{
But whatever stimulus, if any, the nema received from the sky was not sufficient by itself to cause any marked oviposition.
} 


\section{APPLICATION TO THE OVIJECTION OF Mermis subnigrescens.}

The observations to date seem to shut out the possibility that ultraviolet has much of anything to do with ovijection taking place naturally in the open. The present observations seem again to make it exceedingly probable that radiant heat must have much to do with it. Recalling that the early morning light is relatively rich in red and infra-red, and that as moisture (dew, rain) is essential, or at any rate highly favorable, to the oviposition of $M$. subnigrescens, then obviously early morning and forenoon would be a favorable time of day for the oviposition. It is certain, from spectroscopic tests made during the morning in question, that all the time after sunrise a good deal of blue light was being passed through the atmosphere; and it therefore might have been a behavior stimulus, and no doubt was so.

All this harmonizes with previous experiments on the ovijection of this species, - an account of which is already published,- and explains the motive for the tests described above.

Thus we have a fairly complete theory of the above-ground egglaying activities of Mermis subnigrescens. When the nema is ripe for labor, she moves from her pitch dark, subterranean "domicile" to the surface of the ground. Her movements during this trip no doubt exemplify apogeo-, hydro-, thermo-, rheo-, thigmo-, and finally, just before she reaches the surface, photo-tropism.

Once her head is free of the surface of the ground, her chromatrope comes into full play, "detecting" the direction and amount of light from the sky, particularly, perhaps only, blue light. The structure of the chromatrope is particularly adapted to the reception of light from above or from any side, for the light will be concentrated in the chromatrope by the transparent front tissues of the head acting as a hemispherical lens, and the side tissues acting as a cylindrical lens.

As she clambers higher and higher on the herbage, she responds to such blue sky light as is not intercepted by the green blades of grass and other foliage above and around her. Led by the blue light and the urge to deposit, she will at last reach an elevation on the herbage subject to a more direct action of the sun's rays, when the ovijector and uterine muscles will be affected by "red" rays and ovijection will begin; and this place in many instances would be at the altitude of grazing grasshoppers, the definitive hosts.

This would be a new and special parallel to the ordinary sequence of events in ovijection and parturition. In other words the "voluntary" nervous system comes first into play, bringing the organism into con- 
ditions favorable to the events about to follow. Thereafter the behavior is more or less "involuntary," as has been shown in the present instance by the fact that ovijection continues under the stimulus of sunlight, even if the head, including the chromatrope and central nervous system, be removed,- - seared off.

\section{CIRCUMSTANTIAL EVIDENCE FOR THE CHROMATROPISM OF}

\section{Mermis subnigrescens}

1. The commonly infested grasshoppers graze mostly within certain limits above the ground; harmoniously, the eggs of the mermithid parasite are found to occur preponderantly within these limits, suggesting highly developed egg-laying instincts on the part of the nema that might well presuppose tropism.

2. A definite mechanism, believably a phototrope [includes chromatrope, (includes glaucotrope)] embodying what are believably receptors, transmitters, and effectors, is present;-a mechanism not otherwise readily explicable. The only mermithid individuals known to possess such a mechanism fully developed are those whose blackish eggs are deposited in the way characteristic of Mermis subnigrescens.

3. The putatively-chromatropic pigment absorbs,-i.e., can be sensitive to,--blue rays.

4. Only adult, chromatroped, egg-laying females clamber as described. Males and young females having no power, or occasion, to deposit eggs are not chromatroped.

5. The clambering of the nemas ripe for oviposition is skyward; i.e., toward blue sky, rather than vertical (distinction from negative geotropism). Beams of blue light from the sky, often oblique, and coming from many widely different directions, are those most certain promiscuously to penetrate the depths of the herbage, and thus reach to near the ground. The nema's lens-like tissues concentrating light upon the chromatrope, accept it from above and from all sides; this harmonizes with the distribution of blue sky light.

6. Oviposition is stopped, or very much slowed, by green screens (including living foliage) that absorb red and infra-red; indicating a necessity for the nema to escape from exclusively green light before oviposition can take place. In the grasshopper habitats, blue light (sky-light) is the most diffused and most likely to be useful in leading to the known consummation, should chromatropism come into play at all. In nature, clambering skyward ("blue-ward") from out the green, brings the nema soonest under the incidence of the longer wave lengths so stimulative to the ovijectors. 


\title{
INITIAL STRATIGRAPHIC SURVEY OF NEMAS
}

In the upper $20 \mathrm{~mm}$. of

\section{MARINE BEACH SAND, NEAR LOW TIDE MARK'}

\author{
Contributions to a Science of Nematology XXI
}

By N. A. Совв

In 1916 studies at the laboratory of the Bureau of Fisheries, Woods Hole, Massachusetts, showed that sandy beaches, far from being utterly barren, carry a comparatively rich fauna of microscopic organisms. What biological and economic rôle these organisms may play is little known. Conceivably they may be more important than would at first be suspected. Recall that many larger aquatic organisms, at one period or another, may pass a certain amount of time on the bottom. During this sojourn their relationship to the microscopic inhabitants of the sand, mud, etc. constituting the bottom, is a matter about which we know very little, but it is easy to imagine that sometimes the relationship may be important. Again, the sand- and mudinhabiting organisms themselves may be temporary stages (eggs, larvae) in a varied life history.

Such thoughts led to a stratigraphic examination of marine beach sand at Woods Hole, August, 1928. A small sheet-metal box, 10 centimeters square, carrying a series of thin metal slides was devised, such that by its aid layers of sand $5 \mathrm{~mm}$. thick, lying one above another in series, could be collected (Figs. 1 and 2). In each of two collections four such successive horizontal layers were examined for their nemas. The results are indicated in the following tables:

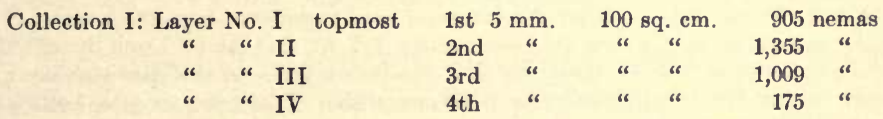

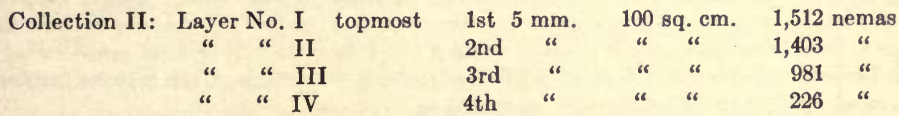

Only two collections were made, as the census is a very tedious one. It will be seen that the two collections, taken a few yards apart, give

1 Reprinted from the Journal of The Washington Academy of Scifnces, Vol. 19, No. 10, May 19, 1929. 
rather consistent stratigraphic numbers; so that we may say, as a result of these two examinations, that probably the number of nemas in each successive layer decreases, rather gradually at first, but rapidly below the third $5 \mathrm{~mm}$. layer; i.e., to a depth of about $15 \mathrm{~mm}$. the sand is thickly populated with nemas; below that level the number falls off rapidly. The deeper layers contain fewer nemas, and fewer species.
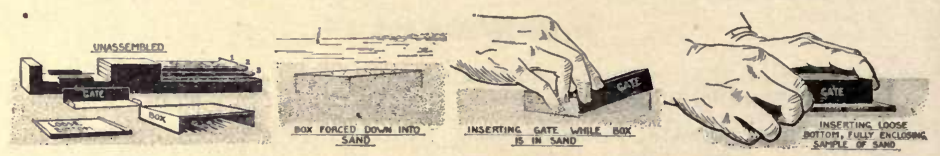

Fig. 1. See also Fig. 2. Apparatus for collecting layers of sand and mud on beaches, mud flats, and at the bottom of ponds, lakes and seas. The unassembled apparatus is shown at the left; its use in collecting is explained in the successive illustrations in Figs. 1 and 2 , reading from left to right. All the operations except the last must be performed at the time of collecting. The last operation may take place in the laboratory. The rectangular collecting box, with two sides missing, is forced into the sand or mud. The two missing sides (gate and loose bottom) are then inserted, as shown above. To insert the loose bottom the adjacent sand is pawed away.
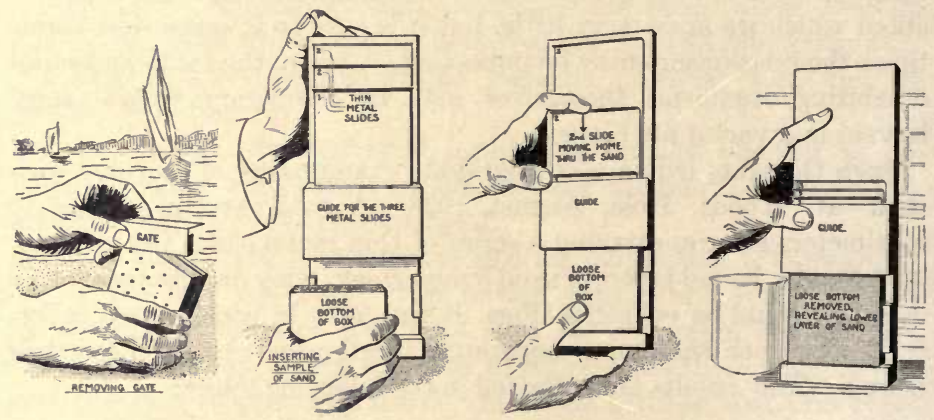

Fig. 2. See also Fig. 1. After the sample of sand is removed from the bottom, it is placed in a holder having three thin metal slides $(1,2,3)$, that can be forced through the sand parallel to each other, thus cutting it into four layers;-in this case each layer is $5 \mathrm{~mm}$. thick. After the slides are forced home, as shown at the right, the loose bottom of the box is removed, disclosing the lowest layer of sand, $-5 \mathrm{~mm}$. thick. This is washed into a beaker and treated as desired. The top of the box has fine perforations in order to allow the supernatant water to escape without disturbing the top layer of sand or mud. To prevent the possible escape of small organisms, this perforated top may be further covered with the finest bolting silk, stretched on.

Averaging the figures from the two collections mentioned above, it will be seen that, if they are typical collections, the beach sand examined carried about $3,742,000,000$ nemas per hectare in the top $20 \mathrm{~mm}$., or about 1,500,000,000 per acre. In 1916, but earlier in the summer, an examination of the same beach showed at least $1,040,000$,000 per acre in the topmost inch of sand. 


\section{THE AMBULATORY TUBES AND OTHER FEATURES}

of the nema

\section{DRACONEMA CEPHALATUM}

\section{Contributions to a Science of Nematology XXII ${ }^{1}$}

By N. A. Совв

There is no adequate published description of the locomotion of Draconema and its numerous marine relatives; very few persons have ever witnessed one of these nemas perambulating its natural substratum.

Draconema moves much after the manner of the ordinary inchworm, or measuring worm. The caterpillar called the "inchworm" has two bunches of feet, a bunch near each extremity. Standing on the bunch of hind feet, it stretches forward and takes hold with the front bunch. Then, releasing the hind bunch, it draws the body forward into a loop so that the posterior bunch may attach itself near the front one. Loosening the front bunch, the caterpillar again stretches forward, etc.

It is convenient to speak of the two attachment organs of the adult Draconema as "soles." The method of attachment of these soles to the substratum in Draconema is very different from that of the inchworm. The sole of Draconema is armed with projecting hollow setae connected with internal glands supplying a sticky and, presumably, non-water-soluble secretion, and it is by the aid of this secretion emerging from ends of the hollow setae that the sole is attached.

1 Reprinted from the Journal of The Washington Academy of Sciences, Vol. 19, No. 12, June 19, 1929. 

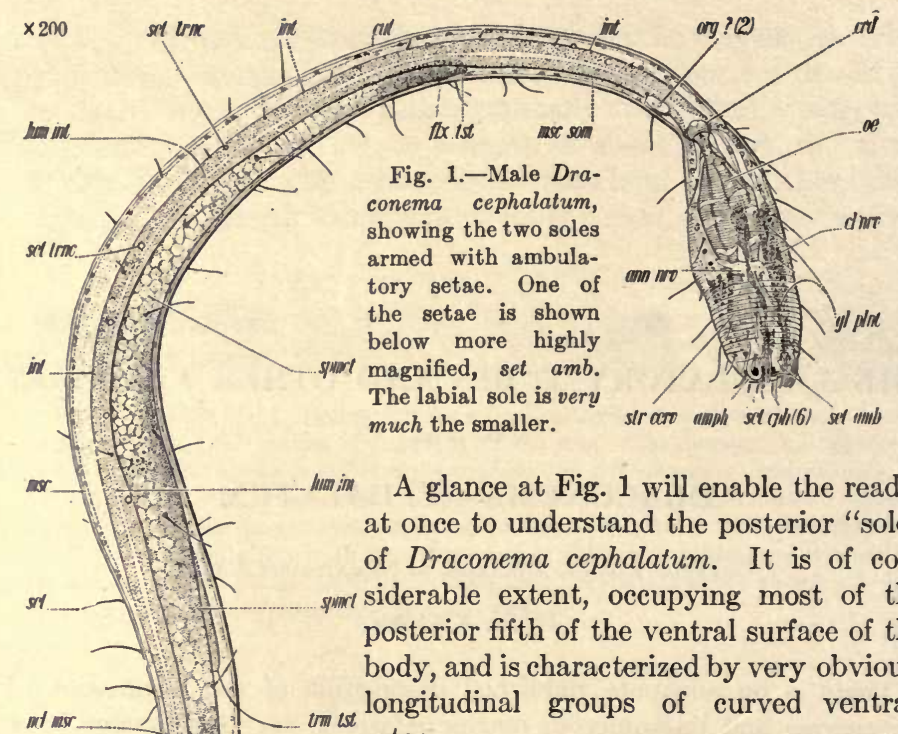

A glance at Fig. 1 will enable the reader at once to understand the posterior "sole" of Draconema cephalatum. It is of considerable extent, occupying most of the posterior fifth of the ventral surface of the body, and is characterized by very obvious, longitudinal groups of curved ventrad setae.

The much smaller anterior sole is very near the front of the head on the dorsal

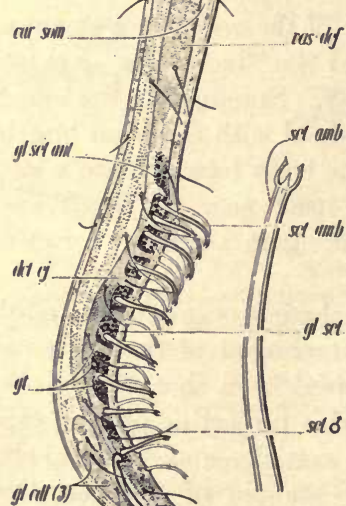
side, and is characterized by setae, smaller than, but similar to, those of the other sole.

The main sole.-Favorably stained specimens exhibit the structure of the ambulatory adhesion tubes, as well as of the associated multicellular ventral glands arranged in a long ventral group opposite to If st and a little in front of the major sole. Each adhesion tube connects, at its base, with a duct that leads to a separate gland. The anterior pair may be taken as typical of any group-member of this compact composite series of glands (see Figs. 1 and 2 ). This anterior pair is sometimes more distinctly separated from the general mass of the glands than are any of the subsequent 
pairs or groups; and each of these two glands proves to have three nuclei. (See Fig. 2.) The cells of each gland, as well as their spheroidal nuclei, increase in size from front to back, the anterior, i.e., distal, cell having less than one-eighth the volume of the posterior; the nucleus in the small anterior cell is also less conspicuous. This group (pair) of glands is more or less clavate in form and is nearly as long as the corresponding body diameter. Posteriorly, each gland diminishes suddenly in diameter to form a duct about half as wide as one of the adjacent annules of the cuticle. Near the gland the wall of the duct contains somewhat elongated nuclei of considerably smaller size than the nuclei of the glands. The duct also lacks the granular character of the glandular cells themselves. The two ducts, at first ventral, diverge backward to the two foremost adhesion tubes, and are one to two times as long as the glands. Near where a duct enters the somewhat swollen base of an adhesion tube, there is a small duplex enlargement or ampulla. In the specimens under examination, only in the very basal portion of the adhesion tube is there any indication of the staining action of the acid carmine.

The numerous glands composing this ventral series are so closely packed together that, as a rule, it is difficult to distinguish the exact number of groups, but it is evident that throughout the series the glands are arranged in groups side by side, apparently mostly in pairs or quartets, the number of glands being commensurate with the number of adhesion tubes. On occasions when the entire group of glands is slightly separated from the body wall, and therefore from the bases of the adhesion tubes, the ducts leading to the tubes are distinctly visible, and have the appearance, when viewed laterally, of a rather complicated plexus.

The minor, or cephalic, sole.-The dozen or so adhesion tubes and glands of the cephalic sole have the same general plan as the sublateral and subventral ones of the posterior sole just described. The glands connected with the cephalic tubes

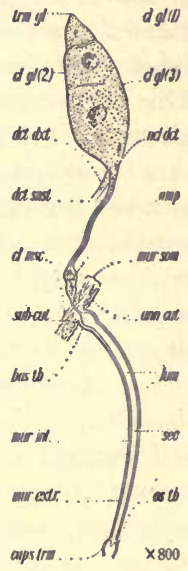

Fig. 2.-Anterior ambulatory seta of D. cephalatum with its 3-celled gland, its 3-celled mate concealed, though part of its duct shows,-dct snst.; som, body wall; $c l$ msc, somatic muscle; an cut, cuticular annules; mur ext and mur inl, outer and inner walls of ambulatory tube; os $t b$, secretory pore. (gl. $p \ln t)$ are located mainly dorsad in the anterior two-fifths of the neck between the oesophagus and the body-wall; there are two dorsally 
sublateral groups of glandular cells and four subdorsal groups, corresponding to a similar grouping of the six pairs of adhesion tubes. The glands of the cephalic sole are sometimes rather more strongly stained than those of the posterior sole in the same specimen, but present the same general anatomical and histological structure. Referring to the dorsally sublateral cervical glands as examples (see Fig. 1), these are distinctly granular in structure and apparently duplex, that is, have a well marked constriction near the middle, on opposite sides of which, fore and aft, is a considerable volume of nuclear (?) matter; the remaining portion of the cells is rather coarsely granular, the granules measuring nearly $1 \mu$ in diameter. This duplex glandular mass is half as long as the neck and anteriorly tapers to a tubular portion not much wider than one of the cervical annules. This narrow portion of the gland in turn tapers to a very narrow duct about $2 \mu$ in diameter, which swells to form a fusiform duplex ampulla as wide as one of the coarser cervical annules. The ampulla empties forward through a short narrow duct, 1 to $2 \mu$ wide, directly into the base of the corresponding adhesion tube, where a little stained matter may usually be seen. The adhesion tubes reach exactly to the lips, so that their outpour is practically terminal. All these details are shown on a small scale in Fig. 1,-ampullae reduced to two dots.

The distal extremity of the ambulatory tube is somewhat bellshaped, and the lumen of the tube is prolonged into the cavity of the bell through a short conoid extension. The significance of this bellshaped structure remains more or less problematical. No elements have been seen in the tube, or in the bell, that could be regarded as contractile, and one therefore seems obliged to assume that whatever changes of form are exhibited by this bell-shaped structure are due to such factors as its own elasticity, the pressure of the internal secretion and counter pressure of the external water. It would seem advantageous to the nema if the secretion which flows out through the bellshaped organ could be "cut off" at will, and it is conceivable that this bell-shaped affair in some way accomplishes that end. Again, it is conceivable that the bell may mechanically give to the end of the tube a greater adhesiveness, conceivably through suction,- - a suction that might be made to vary with the relationship of the distal end of the tube to the substratum, as in the familiar elastic concave rubber suction disc.

The facts that the setae of the inner rows are always the shorter, and that all the setae are incurved, suggest that a suitable substratum may be a microscopic filament, or a thin edge of something. This 
thought arises from the fact that the main sole appears "bow-legged," as it were. Such apparently suitable thin and narrow forms of substratum occur on certain marine algae and, as a matter of fact, at least two observers - Cobb and Chambers-have seen Draconema perambulating the surface of algae.

In balsam specimens from Hudson Bay the body-wall tissues surrounding the mouth-opening have a golden yellow color and take on the form of a six-parted, but twelve-pointed, star.

Probably the excretory pore is at the lips. I formerly referred to a cell just behind the cardia as possibly representing the renette; this suggestion may not be well founded. In this region I observe two of these cells lying side by side in subventral position (see Fig. 1), separated by a small space. In the same latitude, just behind the minute cardiac cavity, on each side of the intestine, there is a rather compact group of granular cells containing about a dozen nuclei. These groups are as long as the corresponding body diameter, and about half as wide as long. Between the two groups, on the dorsal side, there is a pair of subdorsal granular cells rather closely resembling the subventral ones just described. As to the function of these lateral groups of cells, it seems not impossible, - as they are free at their posterior ends and seem connected with the intestine anteriorly,-that they may be special glands associated with digestion. There is a similar pair of lateral groups of cells in front of the cardia, emptying (?) backward. I do not think the possibility is excluded that some of these cells are nerve cells.

The intestine is composed of cells of such a size that about six are required to complete a circumference. The thin refractive lining is hardly $1 \mu$ thick. The cardia, as usual, presents a distinct group of closely packed nuclei, indicating that it is composed of cells of rather small size, of which the number is probably about fifteen to twenty. Notable is the presence of scattered cells in the wall of the intestine which stain differently from their neighbors. These, no doubt, discharge some special function. Thus far they have been observed only in the anterior portion of the intestine.

The portion of the neck opposite, and adjacent to, the oesophageal constriction, except such portion as is occupied by the glands of the minor sole, is rather closely packed with nerve cells.

The lateral fields are one-third as wide as the body and contain a considerable number of nuclei rather irregularly arranged.

The cuticle in the posterior region of the tail is traversed by radial elements that give rise to a finely punctate appearance on the surface (see Fig. 1). 
An interesting observation is the apparent connection of the tandem group of three caudal glands with the dorsal field by means of a narrow strand running forward, possibly of a nervous character, as is suggested: 1 , by its form and position; 2 , by its size and structure.

The internal extremity of the gubernaculum lies near the body axis and is connected fore and aft with the ventral body-wall by slender strands of muscular tissue. The testis is now believed to be reflexed, not outstretched as formerly figured.

The broad, rapidly tapering ovaries,- the anterior lying to the right, the posterior to the left,- -are reflexed to nearly opposite the vulva and contain comparatively few ova, arranged in single file in the wider part, elsewhere irregularly. Hundreds of subspherical sperm cells may occur in the uterus. Usually there is one egg at a time in the uterus, thin shelled and smooth, and deposited before segmentation begins. The eggs are three-fourths as wide as long and about twothirds as long as the corresponding body diameter.

Some of the results of the present investigation have been more or less definitely forecast by various observers on several occasions, notably by Steiner and Irwin-Smith, both of whom from inadequate material, have, with admirable insight, suggested the probable connection of the adhesion tubes with internal structures and also the possibility of the glandular nature of these latter.

While no doubt now remains that both the major and minor soles are glandular and are organs of locomotion, it still seems to me quite doubtful whether we fully understand the bodily structure of Draconema. The form of the head and neck and other parts do not seem to be explained merely on the basis of the use of these two "sticky" soles in locomotion. There probably are other unknown factors playing a part in the activities of Draconema that will further explain its highly peculiar and interesting structure. ${ }^{2}$

${ }^{2}$ Nomenclature. The proposal of the genus Draconema in 1913 appears reasonable in view of the fact that at that time the published descriptions of the species belonging to its family, though in no case as complete as desirable, indicated the existence of two (or more) genera, the type species of one of which should be that originally named by Claparède Chaetosoma ophiocephalum 1863 (not Chaetosoma Westwood, 1851, Coleoptera). Draconema cephalatum, was then, and continues to be, thought generically different from Claparède's ophiocephalum. The name Chaetosoma, being preëmpted, should be replaced by its synonym Notochaetosoma Irwin-Smith 1917. Should the opinion prevail that all the described forms of the family belong to one genus, then the oldest synonym for Chaetosoma would be Draconema, and should be substituted. In that event, the family name would naturally become Draconematidae; in any case, it seems likely that Draconema is a better representative of the group than the only other genus so far proposed, Notochaetosoma.

Draconema cephalatum is cosmopolitan, and seems to have been described by several different authors under as many different specific names, e.g., annulatum Ditlev., haswelli Irwin-Smith, hibernicum Southern. 


\title{
OBSERVATIONS ON THE MORPHOLOGY AND PHYSIOLOGY OF NEMAS
}

Including notes on new species

\author{
Contributions to a Science of Nematology XXIII \\ By N. A. Совв
}

\section{A NEW SUBGENUS OF RHABDITIS}

There is a group of slender-tailed amphigonic rhabdites having lips and pharynx as shown in Fig. 1, the males of which have weakly developed bursas. Such rhabdites have been described from time to time but no author seems to have had adequate material for a completely satisfactory description. Having examined living specimens of both sexes of a new species of this group I took the occasion to prepare a fuller description of it, and propose it as the type of Rhabditella, a new subgenus of the genus Rhabditis Dujardin.

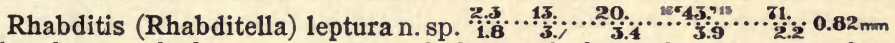
Thin layers of the transparent, colorless, naked cuticle are traversed by excessively fine, plain, transverse striae, resolvable only with the highest powers. Longitudinal striations, due to the attachment of the musculature, are visible in most regions of the body. No deirids have been observed. The neck is very slightly conoid. The cross section of the pharynx is roundish-triangular; yet the almost imperceptibly sigmoid pharynx is nearly equidiametral throughout, though anteriorly the walls are a trifle more strongly refractive. The glottis is a trifle oblique, but otherwise fairly typical. The oesophagus presents a median, fairly prominent, ellipsoidal swelling, or bulb, two-thirds as wide as the middle of the neck, and a somewhat ellipsoidal, or obscurely pyriform posterior bulb two-thirds as wide as the base of the neck,-both swellings of approximately the same diameter. The median swelling presents an elongated, obscure but rather large, valvular apparatus, while the cardiac bulb presents a rather strongly refractive, somewhat three-fold, striated valvular apparatus, located a little in front of the middle of the bulb. At the nerve-ring the oesophagus is one-third, and in front of the cardiac bulb about one-fourth, as wide as the corresponding portion of the neck. There is a distinct cardiac collum constituting a rather broad constriction, so that the anterior portion of the intestine through a distance nearly equal to one body-width enlarges from about one-third to five-sixths as wide as the body. This appearance, however, is somewhat variable. The nerve-ring surrounds the oesophagus obliquely. The intestine, the lining of which is somewhat refractive, is

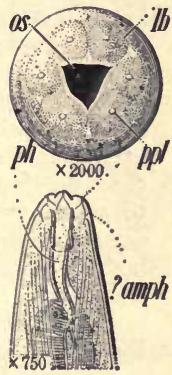
Fig. 1. Front and dorsal view of the head of Rhabditella leptura. The beginning of the œBophagus is shown in the made up of cells of such size that probably only about two lower illustration. are presented in each cross section; these cells contain granules of variable size, which are not strongly birefringent. With crossed nicols there is no suggestion of a St. Andrew's cross;-not at all like the strong birefringence of $R$. monohystera. The posterior lip of the anus is very slightly raised. The rectum, whose lining is only slightly refractive, is one and one-

1 Reprinted from the Journal of The Washington Academy of Sciences, Vol. 19, No. 13, July 19, 1929. 
third times as long as the anal body diameter. The vulva is slightly depressed, though its lips are slightly elevated. The ovaries extend two-thirds the distance back to the vulva, and are only about onesixth as wide as the body of the female. The smooth, thinshelled, ellipsoidal eggs, about as long as the body is wide and two-thirds as wide as long, have been scen in the uteri one at a time. Their yolk is made up of elosely packed, faintly refractive, spherical granules, scattered among which are a few exceedingly small granules less than one micron in diameter.

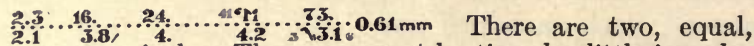
separate spicula. The nema must be tipped a little in order to bring the slightly developed obscure bursa into profile view. The foremost papilla, or bursal rib, is somewhat variable in position and is sometimes found nearly as far forward as the proximal ends of the spicula. Fig. 2.

The testis is reflexed at its free end for a distance equal to about two body diameters. This portion of the testis is only about one-fourth as wide as the body, though it is very slightly swollen at its extremity. Behind the flexure for some little distance the testis still remains narrow, - only a little wider than the reflexed portion lying alongside. Then, however, it rather suddenly enlarges and soon becomes half as wide as the body and so continues, enlarging slightly, however, for three or four body widths. In this portion of the testis the spermatocytes pass through their growth period; they appear as if in two rows and in pairs side by side, and one gets the impression that these pairs are twin cells. Their nuclei are large, becoming at last half as wide as the elongated spermatocytes themselves, i.e. one-fifth as wide as the nema About halfway from the blind end of the testis to the anus the full grown spermatocytes, here half as wide as the body and about as long as wide, apparently break successively into quartets, the resulting subspherical cells being a little more than one-fourth as wide as the body. For a distance equal to about two body diameters forward from the spicula the sexual organ is narrower,-about one-third as wide as the body. Whether the cells of the quartet divide further remains unknown.

Examination of one of the members of a quartet indicated the probable presence of about seven chromosomes.

Habitat: Decaying fruit of Iuffa acutangula from Tcla, Honduras, October, 1926. Sent by Horace S. Dean.

Diagnosis: Rhabditella subg. nov. Rhabdites having lips and pharynx as shown in Fig. 1, the males of which have weakly developed costate bursa and long slender tails.

Diagnosis: Rhabditis (Rhabditella) leptura n. sp. Rhabditellas dimensioned as shown in the formulae; male with two separate spicula, a simple inconspicuous gubernaculum, and with nine bursal ribs arranged as shown in Fig. 2 ; phasmids present.

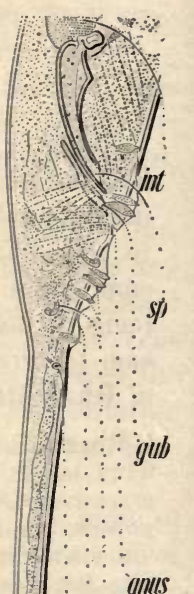

ilmils

rst lirs(t)

Jors

jplsm

t!m

$\times 750$

Fig. 2. Tail of male of Rhabditella leptura n. sp. 


\section{MYOLABIA ON A NEMIC PARASITE OF MILLIPEDS}

The front view of the lip region of Thelastoma attenuatum Leidy, a nema from the intestine of the milliped Sporobolus marginatus, gives the impression at first that there is a circlet of 10 "lobes" surrounding the usual three

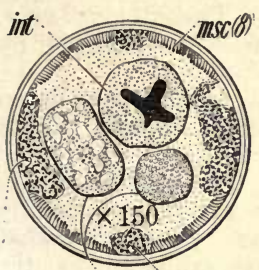

clird lat in chird unt
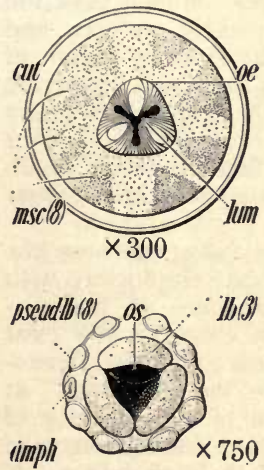

$\frac{0.5}{1.5} \frac{8}{4} \cdot \frac{18 .}{7.5}-\frac{266}{9.4}-\frac{78}{4.6} 2.8$ m

Fig. 3. Measurements, front view of the lip region and nearby cross-sections of Thelastome (Thelastoma) spicatum n. Bp.

These lips may be called pseudolabia; - or, because of their connection with the longitudinal muscular fields of the nema, myolabia. No cephalic papillae have as yet been seen on these myolabia.

Diagnosis. Thelastoma (Thelastoma) spicatum n. sp. Much like T. attenuatum Leidy, but smaller and with shorter spicate tail and more completely differentiated, though still rather vague, myolabia. Dimensioned as shown in the formula. Fig. 3. Attenuatum and spicatum may occur together in the intestine of the milliped, Sporobolus marginatus.

Diagnosis. Thelastomellum subg. nov. Thelastomas with 8 well-developed myolabia as in Fig. 5. Type species T. myolabiatum n. sp.

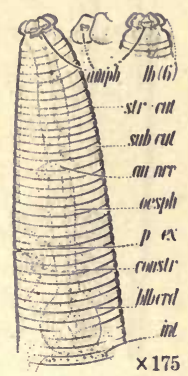

Fig. 4. Profile of head and neck of Thelastoma (Thelas tomellum) myolablatum.
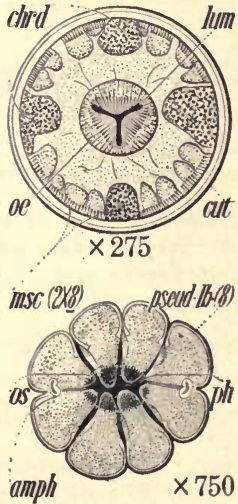

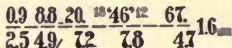

Fig. 5. Measurements, front view and cervical cross-section of Thelastoma (Thelastomellum) subgenus nov. myolabiatum spec. nov. 
Diagnosis. Thelastoma (Thelastomellum) myolabiatum n. sp. Dimensioned as shown in the formula, and with lips and amphids as in Fig. 5.

\section{SYNGONY IN A NEW NEMA FOUND IN MILLIPEDS}

Hitherto undescribed nemic parasites found in the intestine of the milliped Fontaria marginata Say and belonging to the genus Thelastoma Leidy,

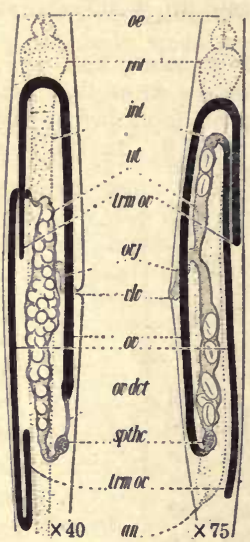

Fig. 6. Slightly diagrammatic drawings of the female gonads of (Thelastoma (left) and Thelastomellum (right). sp. the. spermatheca.

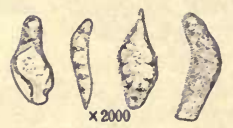

Fig. 7. Scoop shaped syngonic sperms of Thelastomellum. prove syngonic. Thelastoma would be assigned by most authors to the group Oxyuridae.

The discovery of syngony in this group opens up an interesting field for speculation and research. It has long been felt that the "Oxyuridae" present many resemblances to the rhabdites. The gonism of the rhabdites has been found very varied, particularly through the researches of Maupas. Query: To what extent, now, will the variations exhibited by the rhabdites be found to occur in the "Oxyuridae?"

Male "oxyurids" usually are less common than the corresponding females, quite frequently are rare, and in some cases are unknown. The striking nature of the recorded sex ratios has been explained by saying that the males, being very much smaller, are easily overlooked, and that possibly they die soon after copulation; and that these two factors, - their smallness and (assumed) relatively early death,-account for their supposed rarity.

In the rhabdites the evidence fully warrants the view that syngony has evolved from amphigony with a gradual diminution or disappearance of males;for many stages between the two extremes still exist as evidence of the possibility of such a change. Therefore the discovery of syngony in the "oxyurids" at once suggests a new explanation of the scarcity of "oxyurid" males, namely, the one now usually accepted in many cases for the rarity of male rhabdites. If this be true of the "oxyurids," it may have a considerable bearing on veterinary and medical questions connected with "oxyurid" parasites in man and domesticated animals, and in other hosts.

Species that have been assigned to the "Oxyuridae" are common parasites of insects and some other annulata. 


\title{
THE DEMANIAN VESSELS IN NEMAS OF THE GENUS ONCHOLAIMUS
}

\author{
With Notes on four new Oncholaims* \\ Contributions To a Science of Nematology XXIV ${ }^{1}$ \\ By N. A. CoвB
}

Continuing the work of deMan, 1884, and zur Strassen, 1896, observations have been made on Adoncholaimus fuscus (Bastian), Metoncholaimus pristiurus (zur Strassen) and other Oncholaims (listed on p. 425) with particular reference to the system of tubular organs discovered by deMan. Building on the foundation laid by these eminent observers, it has been possible to define the demanian system, and, within limits, assign it a function. The following definition and table of homologous terms, together with the aceompanying text appreciably advance our knowledge of this remarkable system of organs.

\section{DEFINITION}

Demanian Vessels:-In adult female nemas (Oneholaims) a complieated double system of efferent tubes; connecting, (1), with the middle or posterior part of the intestine through an osmosium (see p. 428), and (2), with the uterus (or uteri); these two efferents being confluent at a special glandular "gateway," the uvette (see p. 427), and emptying thence backward and outward, through one or two ducts having more or less moniliform affluent glands (see p. 426, Fig. 1). Normally, the ducts lead to exit pores in the body wall, usually laterad, one or more on each side, near the base of the tail.

In certain cases at least, apparently homologous tubular organs connect with the gonad of the male near the beginning of the vas deferens. For example, in Metoncholaimus pristiurus, Adoncholaimus fuscus and Oncho-

* Investigations carried on in part at the U. S. Fisheries Biological Station, Woods Hole, Mass. The abbreviations used are mostly self-explanatory; e.g. onch dsl, (onchium dorsale), dorsal tooth. Full list of abbreviations on p. 341 .

1 Reprinted from the Journal of The Washington Academy of Sciences, Vol. 20, No. 12, June 19, 1930. 
laimium appendiculatum I observe a tubular glandular vessel, outstretched forward and emptying backward into the vas deferens, that appears homologous with parts of the better known demanian system of the females. See Fig. 2.

In female nemas the functioning demanian vessels (e.g. pristiurus) elaborate a copious, elastic, sticky, non-water-soluble, nearly colorless secretion, possibly utilized ("spun"?) during agglomeration and copulation, and also presumably to protect and preserve the batches of eggs after deposition and during segmentation.

The demanian organs seem to prevail in mud-inhabiting, and sand-inhabiting oncholaims,-i.e. those of stagnant habitat; and to be absent or less prevalent in oncholaims living in more thoroughly oxygenated water,- on the surface of eelgrass, and among algae, e.g. in Prooncholaimus Micoletzky, 1924.

Origin. As to the primitive nemic tissue from which the demanian vessels may have originated, we seem driven to accept the primitive gonadic tissue as the probable source. The histology of the demanian system reminds one most strongly of the structure of nemic gonads; most of the histological elements known in the demanian system have homologues in the gonadic system of nemas; - while on the contrary there is no such tunic, and there are no such forms of nuclei, known in connection with the enteron.

\section{Equivalent terms of various authors}

\begin{tabular}{|c|c|c|}
\hline Author, de Man & Author, zur Strassen & Author, present \\
\hline $\begin{array}{l}\text { Röhrenförmiges Organ } \\
\text { Organe tubiform }\end{array}$ & Röhrenförmiges Organ & Demanian System \\
\hline $\begin{array}{l}\text { Hauptrohr (fuscus) } \\
\text { Canal principal (albidus) }\end{array}$ & Stammrohr & Enteric efferent \\
\hline $\begin{array}{l}\text { Verbindung zur Stütze } \\
\text { Blindes Vorderende }\end{array}$ & $\begin{array}{l}\text { Offene Verbindung } \\
\text { Mündungsorgan }\end{array}$ & $\begin{array}{l}\text { Osmosium, or Selective demanian } \\
\text { intake (enteric) }\end{array}$ \\
\hline $\begin{array}{l}\text { Verbindungsröhrchen zwischen } \\
\text { Warze und Uterus } \\
\text { Tube de communication }\end{array}$ & No mention & Uterine efferent \\
\hline Ausführungsgang in den Uterus & Blindgeschlosenen Sack & Demanian intake (uterine) \\
\hline $\begin{array}{l}\text { Warze } \\
\text { Papille ovulaire }\end{array}$ & Rosette & Uvette \\
\hline $\begin{array}{l}\text { Rothbraune drüsen (fuscus) } \\
\text { Tubes latereaux (albidus) }\end{array}$ & Endschlauch des Rohrorgans & Moniliform Glands \\
\hline
\end{tabular}

DIAGNOSES OF THE GENERA AND SPECIES MENTIONED HEREIN

ONCHOLAIMINAE Filipjev, 1918 and 1925

(but without Anoncholaimus, Pelagonema, Anoplostoma, Trilepta, Krampia. Filipjevia.) 


\section{ONCHOLAIMIUM, n. gen.}

Monodelphic Oncholaiminae with demanian system, whose males have a versatile, preanal, ventral appendicule. See Figs. 2 and 3.

Oncholaimium appendiculatum, n. sp. Oncholaimium with apparently deteriorated moniliform

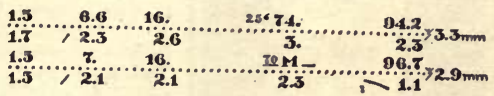
glands without exit pores, and with very simple ampulliform uvette. Appendicule "hinged" and mobile. Figs. 2, 3, 8, 9. Moniliform glands 24-fold, $(8+16)$. Exceptionally 32 -fold.

Oncholaimus nigrocephalatus n. sp. Oncholaimus with very slightly compound, non-refrac-

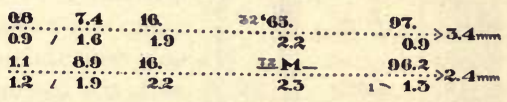
tive uvette, pigmented head, and hemispheroid, immobile, preanal, ventral male supplement; demanian system with two rather inconspicuous exit pores, each laterad; cells of the rouleaux (moniliform glands) oblique, little flattened.

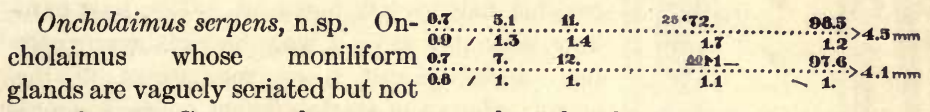
in rouleaux. Compound uvette not condensed and refractive.

Metoncholaimus pristiurus (z. Str.). Specimens from Woods Hole gave the opposite measure-

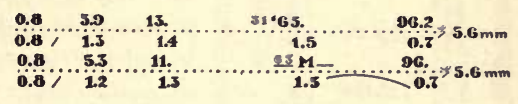
ments. Moniliform glands 64 -fold.

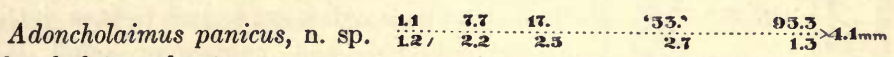
Adoncholaimus having a transverse row of seven demanian exit pores on each side. See Fig. 7.

Adoncholaimus fuscus (Bast.). Moniliform glands 8- or 16-fold,--see Fig. 1.

One soon appreciates the weight of zur Strassen's words where he says, in speaking of the demanian system of various oncholaims,- "In fact, the differences are such that, were they equally pronounced in any other system of organs, they would lead to the proposal of separate genera, or even families."

In this connection the present studies lead to the belief that the demanian system not only varies markedly in the different groups of oncholaims, but that in all probability the system is present but has been wholly overlooked in many of the forms described. Hence it seems premature to attempt a complete subdivision of the oncholaims into genera and subgenera. It may be doubtful whether the genera and subgenera so far proposed are natural ones. In particular, Oncholaimus, the group connected with the type species attenuatus, seems chaotic; yet no better course appears, at present, than to leave serpens and nigrocephalatas in this ill defined group: 


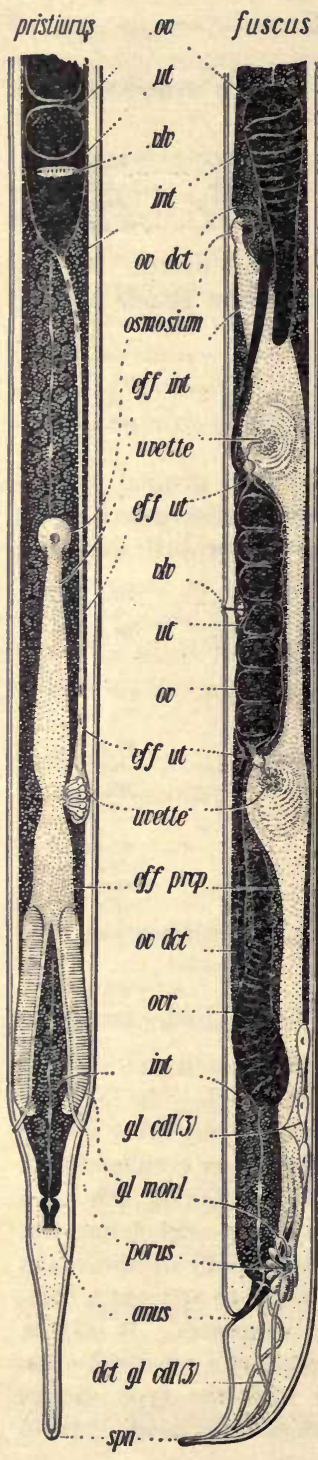

Fig. 1. Amended diagrams of the demanian system of Metoncholaimus pristiurus (dorsal view) and Adoncholaimus fuscus (side view), modified from the diagrams of zur Strassen and deMan respectively. eff int, enteric or intestinal efferent; eff $u t$, uterine efferent: $v l v$, vulva; $o v d c t$, oviduct; $g l c d l(3)$, the three caudal glands; $g l$ monl, the moniliform glands; porus, exit pores of the demanian system. Notice that in each case the uvette empties through a minute pore, the uvette pore. The moniliform glands in pristiurus are 64-fold; in fuscus 8- or 16-fold.

Uterine Efferent. An examination of Metoncholaimus pristiurus (zur Strassen) furnishes convincing evidence that the interesting female organ described by zur Strassen is connected not only with the intestine, as he discovered, but also with the uterus by means of a tube (see eff $u t$, Fig. 1) extending forward from the uvette,-i.e. from the "rosette" of zur Strassen. The evidenec is as follows: In many female specimens it is possible behind the vulva to follow backward from near the vulva a long, narrow, apparently (not really) vacant space, reminiscent of the uterine efferent of Oncholaimium appendiculatum (see Fig. 3) which on more careful examination proves to be a duct. This duct, however, is not so refractive or of such uniform diameter as in appendiculatum, and is even more difficult to sec. It varies slightly in diameter. Here and there throughout its length it can be seen to have a thin, double-contoured wall containing small but definite, much elongated nuclei. This tube is usually in a collapsed condition, more often presenting its edge toward the observer, but sometimes not. When it is presented edgewise, one may often detect in its thin wall the scattered elongated nuclei, especially in specimens fixed and stained in acetic acid methyl green; occasionally nuclei can be seen also in other views. Examining the requisite number of specimens leads to the conclusion that from the uterus near the vulva the duct arises dorsad as a broad tube, direeted backward, which narrows rapidly and extends along the right side of the nema,-approximately along the right lateral chord though not necessarily exactly opposite,- - and, expanding, joins and envelopes the uvette. See Fig. 1. 
Uvctte*. The "warze" of deMan,-i.e. the "rosette" of zur Strassen,-is the structure to which I apply what seems the more appropriate name "uvette." Comparisons show that, notwithstanding the very marked differences in form, the various organs herein ealled uvettes are homologous; the same is true of those ealled monil form glands.

The uvette of Adoncholaimus fuscus (Bastian) as illustrated by deMan probably presents 32 elements (Körnchen, deMan's Fig. 29) as does that of M. pristiurus; these elements have been outlined by deMan and figured somewhat more in detail by zur Strassen (deMan's Figs. 24, 29; zur Strassen's Figs. 13, 14).

In favorable specimens I have seen the uvette of fuscus to be a "radial" structure made up of about 32 elements surrounding a minute pore, somewhat as in the uvette of pristiurus, (See Fig. 1) but the elements here are far less refractive. Rarely ean one see the appearance illustrated by deMan in his figure 29; whereas the appearance he does not satisfactorily illustrate,-a very complicated one, by the way,-is the usual appearance; and when this appearance is more pronounced, commonly the minute refractive "Körnehen" that deMan figures are not to be seen, or only some of them faintly. DeMan's "Kugel," figured by him as if nearly round, I find seldom round or ball-shaped; frequently it is so "collapsed" (?) as to be difficult to see at all, and it is more likely to be elongate or ellipsoidal, or perhaps flattish-ellipsoidal, than to be equidiametral as shown in deMan's Fig. 29.

DeMan does not give a thoroughly satisfactory description or figure of his "Warze." In one of his figures (Fig. 29) I count 33 minute, cireular, dotlike elements where he makes his "Verbindungsröhrchen" join the "Warze." Oecasionally I also see this appearance, and with about the same number of elements (32?). It is difficult to say as yet what the exact function of the uvette is, but it seems a regular, doubtless glandular, eomponent of the demanian system. In Oncholaimium appendiculatum the uterine vessel, extending backward from the uterus, nearly as deseribed for pristiurus, fnally expands a trifle into a small, often rather indefinite, ampulliform uvette of the very simplest eharacter, which joins the right subdorsal of the two longitudinal series of cells, - the moniliform glands,-by means of a minute refractive pore,-the uvette pore. See $u v$, Fig. 3 . In Oncholaimus nigrocephalatus the uvette, which in 0 . appendiculatum appears as a simple ampulla, beeomes somewhat compound; that is to say, two additional or subordinate elements oceur, one on either side of the main "ampulla," so that the whole is rather obseurely triplex.

In another oncholaim, Oncholaimus serpens n. sp., the uterine tube extends backward just as definitely as in Oncholaimium appendiculatum and joins the rest of the demanian system in the form of an expanded and much larger

* Uvette; a diminutive cluster. From latin, uva, a cluster of grapes. 

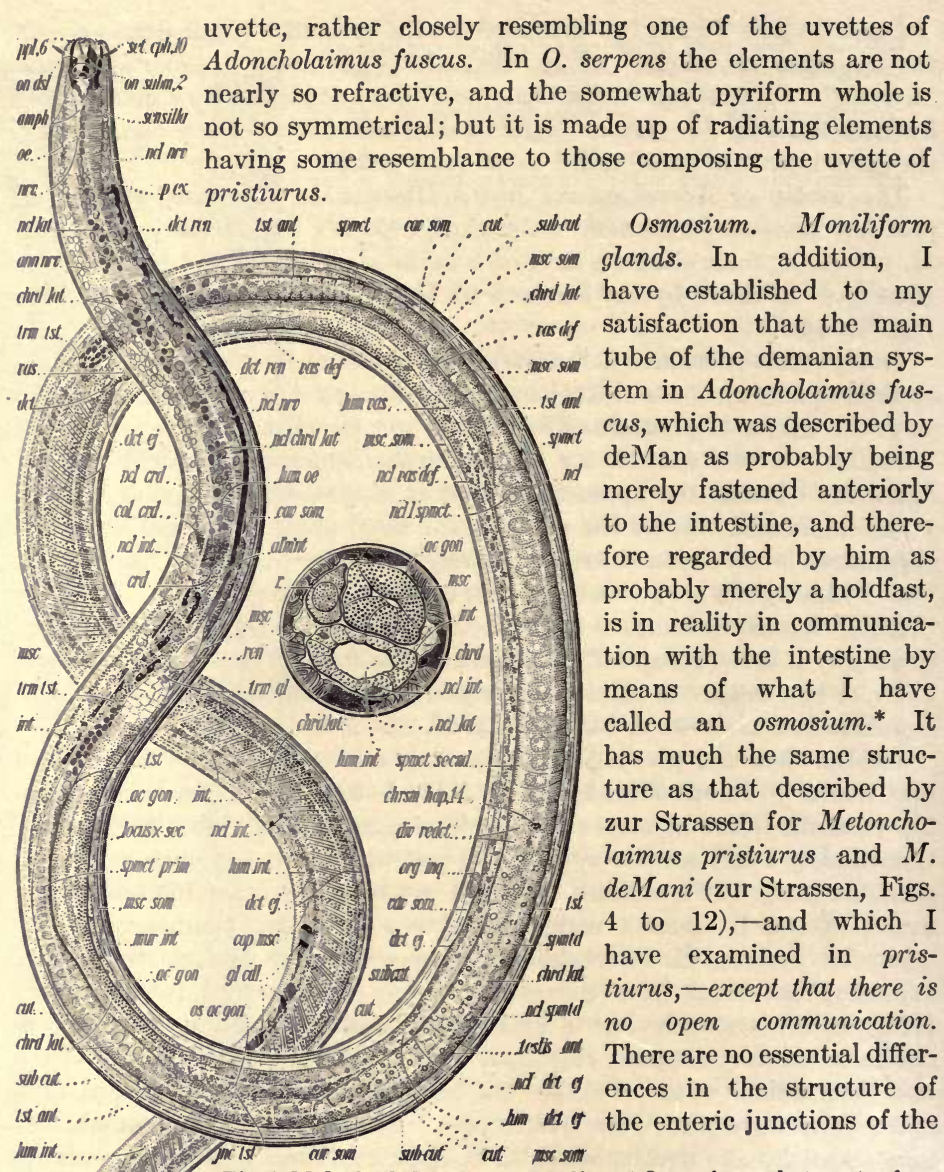

Osmosium. Moniliform glands. In addition, I have established to my satisfaction that the main tube of the demanian system in Adoncholaimus fuscus, which was described by deMan as probably being merely fastened anteriorly to the intestine, and therefore regarded by him as probably merely a holdfast, is in reality in communication with the intestine by means of what I have called an osmosium.* It has much the same structure as that described by zur Strassen for Metoncholaimus pristiurus and $M$. deMani (zur Strassen, Figs. 4 to 12), -and which I have examined in pristiurus,-except that there is no open communication. There are no essential differences in the structure of the enteric junctions of the

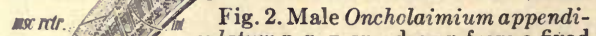

* Osmosium; that part of an culatum n.g.,n.sp., drawn from a fixed emunctorium or analogous ordt cel /..deg and stained balsam specimen. The gan through which, mainly by

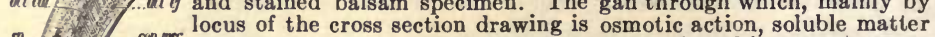
\$p. . . ....ap mown at locus $x$-sec. ac gon, accessory is transferred from one organ to aquall to gonad; al'm'nt, food material; another. The osmosium is .4. $1 . .$. appos haploid number of chromosomes; jnc still doubtful whether the os-

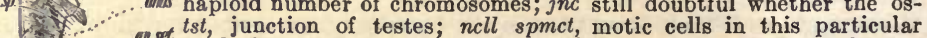
nucleolus; ncl spmtd, nucleus of case are of enteric or demanat ....... st sum 12 spermatid; org inq, organs of uncertain ian origin. While the staining dt.3 function; os ac gon, mouth of accessory of these cells seems to favor de$\times 175$ ant, - front testis $; r$, its cross-section. seems to favor enteric origin. 
various oncholaims I Fig. 3. Female of Oncholaimium appendiculatum n.g., n.sp., drawn have examined, except from living specimen under slight minor ones in the more pressure. The uterine efferent is or less, but very ob- at ut eff to where it joins the uterus scurely, radiating part moniliform gland at $u v$, the uvette:

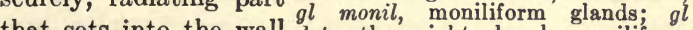
that sets into the wall $d x t$, the right hand moniliform of the intestine. This gland; $g l$ sns, left hand moniliform part forms motic" exit through the outer surface of the enteric motic exit through the enteric the intestinal wall in uterus; dctcdl, the three caudal ducts; the shape of special, chrd lat, borders of the right hand presumably metabolic lateral chord; amph extr, external and at least selective, of one of the sperms; trm ov, blind end glandular tissue of the the single ovary; ov tegmt, shell of osmosium.

In the species Onchothe nucleus of

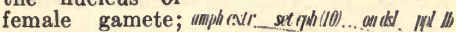
laimus nigrocephalatus there are external latplrcyt, polarcyte ov in dct, much st cro elongated egg sasilh passing through eral exit pores in the anal region much as in the oviduct from $\mathrm{nr}$ amph the ovary to the pristiurus but they are minute. Thus far, howuterus; pst, pus tules due to uri- $r$ /nr tis; grn bifr bireever, I have searched in vain for these pores in Oncholaimium in intestinal cells. diculatum.

In this latter species there are two moniliform subdorsal series of 24 cells each which I propose to call moniliform glands. These are rather close homologues of the 64-fold rouleaux of zur Strassen; less obviously, of the 8or 16-fold "Rothbraune drüsen" of deMan. The uterine vessel joins the right hand one of these moniliform glands, as is shown near the middle part of figure 3 on this page, at $u v$.

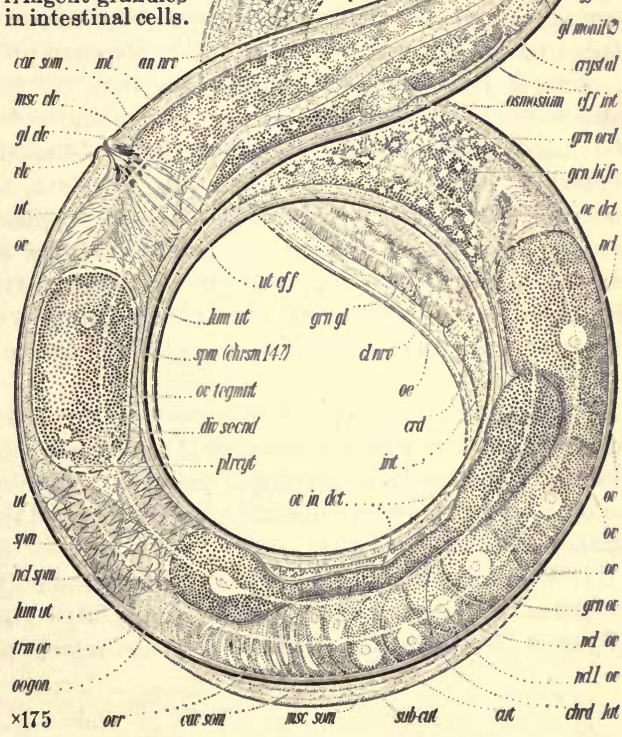


In 0 . appendiculatum at the posterior end of the two 24-cell moniliform glands, however, a number of the cells of each organ seem loosened from the series; opposite these I am unable to find any outlet whatever; - that is to say, the organs appear as if in a deteriorated condition. See $g l d x t, g l s n s t$, Fig. 3.

Perhaps causally connected with this is the extraordinary fact that the examination of hundreds of females of Oncholaimium appendiculatum over a number of years has failed to disclose a single healthy specimen. Every female is attacked by a disease that often results in necrosis of the posterior portion of the nema. (See section Uritis, p. 240). The disease (uritis) breaks out on the tail in the shape of minute pustules having an internal radiated, or linear and "parallel-fibered," strueture, pst, Fig. 3, sometimes extending forward for a considerable distance into the nema,-in extreme cases, as far forward as the vulva. There may be one, two, three, or even as many as a dozen of these pustules irregularly scattered on the posterior part of the female. The pustules are minute, exude, inter alia, an insoluble material, and frequently exhibit surface bacteria, though it seems doubtful if the bacteria thus far seen are connected with the disease. The uniform occurrence of this disease in females of Oncholaimium appendiculatum may perhaps be connected with the deteriorated (?) condition of the demanian system. On a later page attention will be called to the fact that other oncholaims possessing the demanian system have what appear to be similar diseases of the posterior extremity; but in none of them is there any such extraordinary condition as in $O$. appendiculatum, where examination has failed to disclose a single adult female free from uritis.

Enteric Efferent. Against the idea that in Adoncholaimus fuscus the anterior junction of the demanian system with the intestine is merely a holdfast, as suggested by deMan, it may be urged that of other elongated organs known to lie loose in the body cavity of nemas, none are secured in this particular way to the intestine. Why an exception in this case? If it is merely a matter of security, it would seem more in harmony with known nemic anatomy that the attachment be to the body wall rather than to the intestine, and especially that it be effected along a lateral chord. It is not unheard of for a nemic organ of this general form to be attached to a lateral chord.

From a mechanical point of view the idea that the connection of the demanian system with the intestine is merely a holdfast seems to have all the less to recommend it in the case of the monodelphic species, such as pristiurus and serpens, where this connection is so far caudad that such a holdfast seems rather needless.

DeMan's idea that his main tube is simply and only fastened to the intestine seems not borne out by facts; and his figure 25 , if I understand it, admits of a different interpretation. I find his "main vessel" anteriorly to be hollow to 
its very end,- - the "blind end" of deMan, - and that the freely moving contents of the tube are visible clear to what might be called the surface tissue of the intestine (tissue of the intestine altered, to be sure). DeMan's figure 25 seems easily to admit of this interpretation. I find the cells of the wall of the intestine (if they be really intestinal) are altered where the vessel is attached, and this fact suggests that we have here modified selective tissue,the osmosium,- - the function of which is to extract from the intestine and usher into the demanian system, presumably mainly by osmosis, a product utilized by the latter.

May not the evidence offered by zur Strassen for an open communication between the enteric efferent and the intestine in pristiurus,-i.e. the evidence of his microtome sections,-be capable of a different interpretation? Could zur Strassen's sections have been deceptive? The published figures of his "open connection" between the demanian system and the intestine are not satisfying, in that they appear to show a large portion of the cell walls missing. Now pristiurus ingests mud, and, in consequence, its intestine normally contains much fine grit. Is it not likely that this grit, acting as it naturally would during the sectioning, would damage, or even destroy, delicate cells that, before being broken, might have closed the aperture which zur Strassen shows and describes as an open connection? The suggestion is that this might occur, at the time the sections were cut, through the combined abrasive action of the grit and the coincident dulling of the microtome knife. All zur Strassen's figures show the intestinal lumen more or less open; but when the intestine is entirely empty and free of grit it is collapsed, not open, so that the lumen, in well made sections, is closed and difficult to see. May not this indicate that the vacant lumenal spaces shown in zur Strassen's illustrations probably did contain grit at the time of fixation, and hence, no doubt, at the time of sectioning?

Pristiurus, fuscus and some other mud-inhabiting Oncholaims can be kept alive in pure running sea water for days, or even weeks, and when so kept evacuate the intestine very completely. Sections may then be made without the interference of the grit normally present in the intestine. I have not found such sections to present the appearance figured by zur Strassen.

In an examination of very many specimens, alive and sectioned, I have never been able to convince myself of the existence of an open communication between the intestine and the demanian system.

Any such open connection would seem a grave menace to the well-being of the organism. For if the enteric intake were of the nature figured and described by zur Strassen, there would seem to be little or nothing to prevent the entrance into the demanian system of undigested detritus contained in the intestine, together with numerous living microorganisms which normally 
constitute a very appreciable part of the feces. No such detritus is ever seen in the demanian system.

Furthermore, on examining living pristiurus and fuscus, both of which I find to occur along Cape Cod, U. S. A., I find that when the food in the intestine is moving rapidly back and forth opposite zur Strassen's supposed open connection, no portion of it ever enters the enteric efferent. There is not even the slightest corresponding disturbance of the contents of the lumen of the enteric efferent close by, which, as zur Strassen also points out, can be seen in the end portion of the demanian tube where it joins the surface of the intestine.

Possibly the analogous connection with the uterus is hardly to be taken as a very distinctly open one. True, I have seen cases in pristiurus where, when the diseased uterus was filled with microorganisms (microorganisms causing the disease*), the continuous mass of them also filled the nearby part of the corresponding demanian vessel in such a way that there was a direct "tubular" connection between the uterus and the vessel. Normally, however, the conditions are as follows:-One traces the uterine demanian vessel directly forward to the uterus, where its lumen continues for a short distance into a glandular tissue in the posterior end of the uterus,--zur Strassen's so-called "blind end, behind the vulva," - and there ceases in the midst of a large number of uterine cells somewhat similar to many of those constituting the main portion of the wall, i.e. what seems to be a special collection of glandular uterine cells. In fuscus this same thing occurs where the oviducts join the proximal ends of the two uteri, not, as in pristiurus, at the posterior portion of the single uterus close to the vulva; the histology of this junction, however, is much the same in these two species. It is as if special uterine cells were devoted to secreting material to be delivered to the demanian system through the uterine efferent,- - the "tube de communication" of deMan.

In pristiurus the long tubular vessel connecting the uterus with the demanian system,- - the uterine efferent, - often is difficult to see, especially in its entirety. No better proof of this could be required than that it escaped so keen an observer as zur Strassen.

Even in Adoncholaimus fuscus, while the two short uterine efferents can sometimes be followed from the uteri to the main vessel of the demanian system, often it is practically impossible in a given specimen to follow them throughout their course. Knowing their locality and structure, one can usually determine how they lie and their probable limits, but that is about all. Of course, in a small minority of favorable soecimens quite the contrary is true; - the entire tube can be made out satisfactorily as was first done by deMan.

* This disease appears to have nothing to do with uritis (see p. 240); uritis seems an entirely distinct disease. 
Direction of Flow in the Demanian System. Evidently a considerable amount of matter is contributed by the intestine to the demanian system. Zur Strassen had no difficulty in assuming the entire amount to be so contributed in pristiurus (for he appears to have been unaware of the connection in pristiurus of the uterus with the uvette, and hence with the demanian system).

However, quite frequently in the contents of the enteric efferent of living Metoncholaimus pristiurus near and in front of the uvette pore, refractive, curved, wave-like effects are seen such as would be produced by the gradual mixing of two viscid fluids of unequal refractiveness, - an appearance that might readily be produced by the flowing of a liquid through the uvette pore from the uterine efferent into the enteric efferent in such quantity that some of it passed slightly forward,--perhaps through cover glass pressure.

On various occasions, I have seen a considerable quantity of matter in the main enteric vessel close to its junction with the intestine. While this is no proof that this matter was actually derived from the intestine, it is favorable to that conception. Such matter never contains intestinal debris,nor sperms (see F. H. Stewart, 1906), nor pseudo eggs,-- "balls of finely granular substance," (see zur Strassen.)

If the demanian system emptied into the intestine, it is to be expected that it would do so through an aperture, pore, similar to those of other affluent enteric glands,- those emptying into the oesophagus for instance. In nemas such pores are extremely small, have a definite refractive lining, and are adapted to check any "backwash" due to movement of the contents of the enteron,- e.g. just such a structure as occurs in the uvette of pristiurus. But no such pore has been seen in connection with any enteric demanian vessel.

Moreover, against the flow of any of the demanian fluids being toward the enteron, it may be urged that in pristiurus a special secretion is at times actually seen issuing rather copiously from the pores near the tail,- the external outlets of the demanian system,-and there is not the slightest reason to suppose that in this region the flow is ever anything but backward and outward. There is no evidence that the demanian system is, for instance, a water-vascular system; or that sea water is taken in through the antecaudal lateral pores.

Again, there is little if any reason to believe the demanian system accessory to digestion, because whatever digestive function would be advantageous to adult females would scem also to be advantageous to the young nemas; yet there are no such organs in young oncholaims, for they come into existence at the last moult. The same may be said of any supposable ordinary excretory function.

But if it be supposed that, for some unexplained reason, adult egg-producing females require to excrete (not secrete) matter peculiar to them, in other words that the demanian system, or some part of it, be a sort of temporary mal- 
pighian system,-a rather violent supposition,-it would seem that the excretion, as such, if poured into the intestine at all, should be poured in posteriorly. But in didelphs,-fuscus, panicus,-such a supposition would pour it in near the anterior end. Or, if it be supposed that the demanian system is simply an emunctorium accessory to the intestine and emptying outward and backward, then why the attachment to the fore part of the intestine as in fuscus?

If the demanian system is excretory, then it is necessary to assume that the necessities of adult females in the way of excretion are different from those of the male or the young female. No reason has been advanced for such an assumption.

Deduction by Elimination. In the demanian system of 0 . pristiurus three ducts come together at a single point, indicated by $\mathrm{X}$ in figure 4 , - ducts in each of which a fluid may conceivably flow in either direction; i.e., there are six different paths along which fluid may be conceived to flow. The assumption is, of course, that, when the organs are functioning normally, fluid passes constantly in one direction only in each of the three tubes, 1, 2, 3, Fig. 4 .

Indicating the six possible paths by arrows lettered $a, b, c$, $d, e, f$ (Fig. 4), mathematically, there are twenty possible combinations in groups of three as follows:

$a b c, a b e, a c d, a c f, a d f, \quad b d e, c d e, c e f, b e f, \quad b c f$. $a b d, a b f, a c e, a d e, b c d, b d f, \quad c d f$, aef, bce and def; this is according to the formula for combinations,

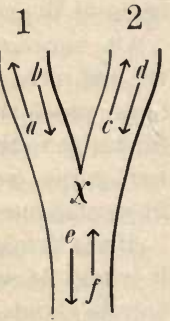

3 Fig. 4. Diagram of the six possible currents. $\frac{\mathrm{n}(\mathrm{n}-1)(\mathrm{n}-2) \ldots \ldots(\mathrm{n}-\mathrm{r}+1)}{\mathrm{r} !}=\frac{6 \times 5 \times 4}{3 \times 2 \times 1}=20$, when $\mathrm{n}=6$ and $\mathrm{r}=3$.

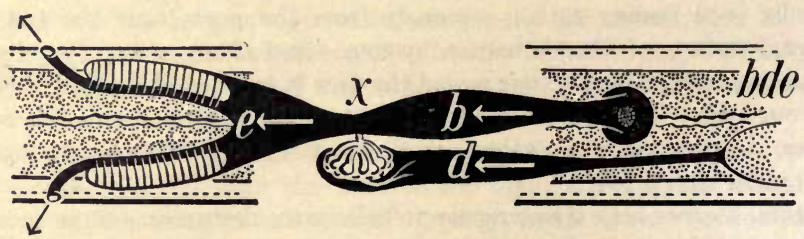

Fig. 5. Diagram showing the direction of flow of the fluid in the demanian system. The intestine and the posterior end of the uterus are shown near bde. The backward flow of the fluid in the enteric and uterine efferents is indicated at $b$ and $d$, and the backward flow of their combined products at $e$. The outward flow of the fully elaborated secretion after it has passed the moniliform glands is shown by the two oblique black arrows.

It is evident that a combination containing $a$ and $b$ represents a physical impossibility, i.e. represents opposite currents simultaneously in the same duct,- duct number 1; and so with combinations containing $c$ and $d$, and $e$ 
and $f$. (It is theoretically possible, of course, that the same tube might have a flow in one direction at one time and in the opposite direction at another time, but, physiologically speaking, this is an unusual occurrence, and practically an unheard of thing in a tubular organ "open" at both ends). We may therefore eliminate from the 20 possibilities, 12 of the combinations, leaving cight, - $a c f, a d f, b c f, a d e, b c e, b d e, b d f$ and ace.

But there are also two more combinations that obviously must be left out, as involving physical and physiological impossibility, namely ace and $b d f$, i.e., the cases where the three currents would simultaneously come to, or radiate from, the point $\mathrm{X} ; b d f,-$ (no outlet, or reservoir), and ace,-(no obvious source of supply). This leaves six combinations possibly worthy of discussion, acf, adf, bcf, ade, bce and bde. These six possibilities are diagrammed in figures 5 and 6 . Five of these possibilities (Fig. 6) are rendered

I. No exit pore for $a$; pore of uvette indieates reverse of $c ; f$ doubtful beeause entrance of sea water is possibly involved, while outflow of secretion is known from lateral pores, $p$.

II. No exit pore for $a$; $f$ doubtful as in I; moniliform glands are believed here to empty outward because of their form and loeation in $O$. fuscus; only outlet of $d$ and $f$ would be through $a$.

III. $c$ doubtful as in I; $f$ very doubtful as in I and II; the only outlet for $f$ and $b$ would be through the uvette and $c$,- -reverse of direction indicated by structure.

IV. No exit pore for $a$; the only source of $a$ would be $d$ and the uvette.

V. $c$ doubtful as in I; $c$ may also be reasoned against on the basis of homologous structures in $O$. nigrocephalatus and $O$. appendiculatum.

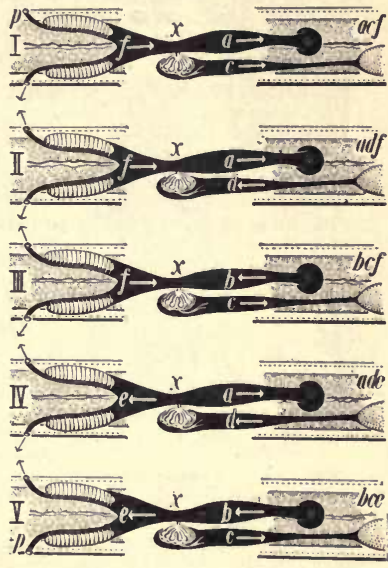

Fig. 6. Five diagrams of supposed currents in a demanian system. Objections to each supposition are listed opposite its diagram. Compare with Fig. 5 .

exceedingly improbable by the physiological and morphological considerations listed opposite their diagrams. We may therefore safely deduce, even from this single discussion, that the flow is almost certainly as shown in Fig.5.

The significance of seven exit pores on each side in panicus (sce Fig. 7) is an interesting subject for speculation. It can hardly be said that the existence of seven pores is for the purpose of furnishing a large outlet; it would secm much simpler to attain such a result by having a larger single pore. Nor 
does it seem that the multiple outlet would have anything to do with the quality of the secretion that is prepared. The most reasonable supposition is that, in use, the demanian secretion is rendered more effective through a multiple delivery, and it is not difficult to reason out why this might be so.

Take, as a basis of reasoning, the fact that spiders have multiple spinneret tubes. This plurality is an advantage in that if some tubes of the spinneret apparatus do not act, or are restrained from acting, the remaining ones may continue to act, an economy of a kind often seen in nature. Thus threads of varying size and composition can be "spun." It seems not unlikely that the multiple thread of the spider may have

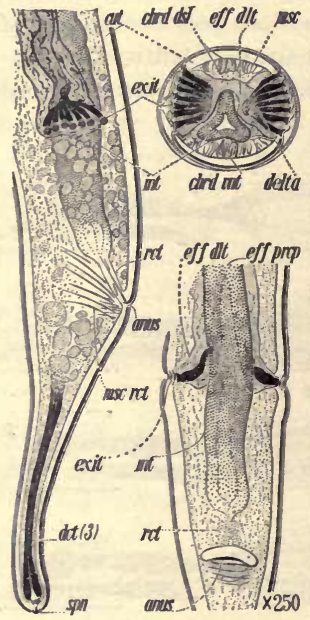

Fig. 7. Adoncholaimus panicus n.sp. Side, ventral and sectional views of the posterior portion of the same female. eff prcp, the principal efferent at the
point where it forks to produce the two deltic efferents, eff $d l t$; the seven-fold delta is shown at delta and the seven exit pores at exit. structural advantages; at any rate it is a fact that, in some cases at least, the thread can be artificially split into components harmonizing in number with the elements of the spinneret apparatus. If these be advantages, it is quite conceivable that they may apply in some way to the multiporous Adoncholaimus panicus, and this would harmonize with the previous conclusions concerning the function of the demanian system;-for presumably the secretion in panicus is like that of other oncholaims, i.e. a copious, sticky, non-watersoluble, elastic material; - at least these are its properties after it is delivered into sea water by pristiurus.

The location of the outlets of the demanian system is always well caudad, and the oncholaims having the system are agile and limber, all of which harmonizes with the belief that the system elaborates material used with some degree of "skill."

All oncholaims having the demanian system have a habit of coiling and uncoiling and can with the greatest ease place the exit pores of the system against any part of the body except the tail and its immediate vicinity, and this habit, no doubt, is correlated with the function of the secretion. Females of such oncholaims have relatively short tails, - as if longer ones would perhaps be in the way.

Conceivably, of course, the demanian secretion might have properties attractive to the other sex (odor, etc.), but the idea does not seem to appeal so strongly as that of having something to do with other matters.

In this connection it may be recalled that, opposite the demanian exit pores of Metoncholaimus albidus (Bastian), deMan described and figured a persistent girdle of left-over yellowish brown secretion. 
Oncholaims having the demanian system, at least most of them, have a way of collecting together in masses when artificially assembled in sea water. Conceivably this habit may have some connection with the demanian secretion, but it is not obvious why only adult females should secrete for this purpose alone.

The demanian system appears more distended when the uterus is full or nearly full of eggs. For instance, at this time the uterine efferent and portions of the uterus of pristiurus may contain an abundance of colorless, transparent, rather structureless-looking matter, resembling, under the microscope, partially, dissolved shavings of gelatin.

The question arises as to what becomes of the secretion of the accessory gland of the male, which is possibly or probably a homologue of the demanian system in the female. No reply to this question has occurred in connection with these investigations except that the "gum arabic-like" mixture seen in the uterus of pristiurus might possibly have been derived wholly or in part from the male. It should

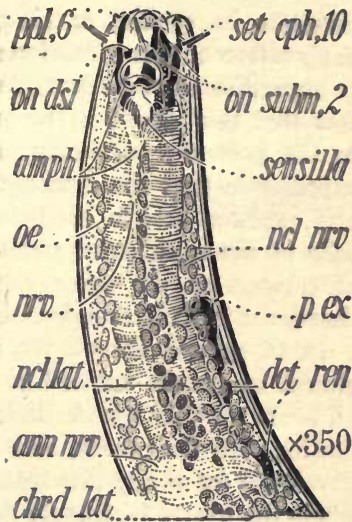

Fig. 8. Profile of head end of male Oncholaimium appendiculatum. The three onchia are shown; the left ventral submedian is the longest, - see on dsl and on subm (B). The sensilla and amphidial nerve are shown. Nuclei shown mostly central nervous system; the scattered darker ones are nuclei of the lateral chord, the width of which is pointed out at chrd lat. perhaps be mentioned that in the nemic

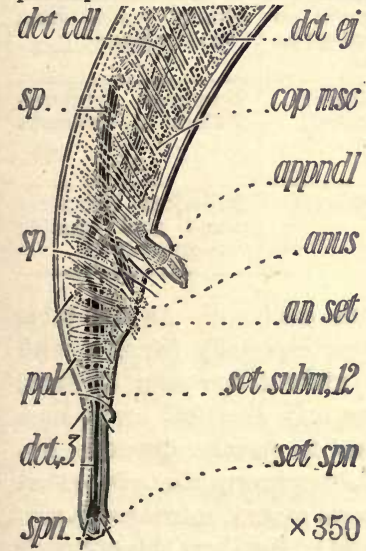

Fig. 9. Tail, male Oncholaimium appendiculatum. ppl, single ventral papilla; det (3), caudal ducts leading to spinneret; appndl, ventral, erectile appendicule; $a n$ set, anal setae; set subm (12), submedian setae on male only. genus Rhabditis, glands accessory to the male gonad are known that secrete a copulatory cement; but no such cement is yet known in connection with any oncholaim.

Of course, the mere presence of this material in the uterus and in the portion of the uterine efferent nearby does not of itself indicate the direction of the flow, but the structure of the organs distinctly suggests that the flow is caudad, i.e. from the uterus toward the external openings near the tail. Were the entire flow of the demanian system toward the uterus, it would seem strangely circuitous.

While the fact that no external exit pores have been discovered in Oncholaimium appendiculatum makes conceivable a flow from its enteric vessel and the moniliform glands through the uvette to the uterus, yet the structure of the uvette pore seems as distinctly adapted to a flow in the opposite 
direction, i.e. caudad, in this species as in the others. Moreover a different explanation of this exceptional case seems more plausible, namely, that in 0 . appendiculatum the demanian organs are in a deteriorated condition. The fact that this species is the only one that almost invariably presents disease in the posterior extremity of the adult females may harmonize with the supposition that the demanian system of this species is in a deteriorating and perhaps useless condition. The nonconsecutiveness of the posterior cells of the moniliform glands in 0 . appendiculatum, and the appearance of crystals on the outer surface of the enteric efferent, (Fig. 3) suggest decadence in this anomalous species.

In 0 . appendiculatum the uvette is reduced to a mere ampulla; and is almost as greatly reduced in nigrocephalatum. In neither of these is it at all likely that the uvette itself could produce any very appreciable secretion flowing into the uterus, and yet in both species the uterine tube is better developed (or at any rate more obvious) than it is, for instance, in pristiurus, where the uvette is strongly developed.

Uritis. It is interesting that the females of a number of oncholaims shown to possess demanian vessels seem unusually subject to disease.

Among such oncholaims, allusion is made to the following typical cases:

\begin{tabular}{|c|c|c|c|}
\hline Name & Location & Lesions & Regeneration \\
\hline$?$ & $\begin{array}{l}\text { Woods Hole, Mass., } \\
\text { U. S. A. }\end{array}$ & "tailless" & $\begin{array}{l}\text { Undoubtedly healed } \\
\text { over }\end{array}$ \\
\hline O. appendiculatum & Woods Hole, Mass. & $\begin{array}{l}\text { From tail end to half } \\
\text { of nema necrotic }\end{array}$ & $\begin{array}{l}\text { No signs of regenera- } \\
\text { tion }\end{array}$ \\
\hline M.pristiurus & Woods Hole, Mass. & $\begin{array}{l}\text { Tail end gone; no } \\
\text { anal opening; no } \\
\text { spinneret }\end{array}$ & $\begin{array}{l}\text { Merely healed over; } \\
\text { no openings }\end{array}$ \\
\hline A. fuscus & $\begin{array}{l}\text { 1. Cape Cod, Mass. } \\
\text { 2. Miss E. Horsman } \\
\text { Univ. College of } \\
\text { Wales }\end{array}$ & $\begin{array}{l}\text { Former uritis (?) } \\
\text { Former uritis (?) }\end{array}$ & $\begin{array}{l}\text { Terminus regener- } \\
\text { ated; no spinneret }\end{array}$ \\
\hline New Oncholaim & Florida, U. S. A. & Former uritis (?) & $\begin{array}{l}\text { Imperfect spinneret } \\
\text { and anal opening } \\
\text { regenerated }\end{array}$ \\
\hline
\end{tabular}

An interesting morphological problem is thus disclosed. As the table indicates, one not infrequently finds oncholaims, especially females, with highly peculiar caudal extremities, - sometimes without spinneret or anus, sometimes with these organs present but apparently abortive, or at least peculiar in form,-abnormalities probably due to specific disease. Apparently the disease is sometimes combated by the nemic organization, so that the posterior end of the nema heals over, and in some cases it seems as if a new anus is formed, and possibly even a new spinneret! Just how this occurs is not yet clear,*but I have seen both deformed anal openings and deformed spinnerets of female oncholaims that appeared to give evidence of having been imperfectly regenerated after some accident, or, more likely, after uritis.

* Regeneration seems to be uncommon in nemas.

Additional articles consulted-see zur Strassen's bibliographic list, 1896. 


\title{
METONCHOLAIMUS PRISTIURUS (ZUR STRASSEN)
}

A Nema Suitable for Use in Laboratory Courses in Zoology ${ }^{1}$

\author{
BY N. A. Совв
}

Contributions to a Science of Nematology $\mathrm{XXV}^{2}$

Zur Strassen, who first proposed the species Metoncholaimus pristiurus, alluded for the most part only to the organs whose forms served to distinguish it from its nearest allies among the oncholaims. The present attempt at a more complete understanding of its morphology adds to our knowledge in a number of ways, especially with regard to the remarkable demanian system.

At the same time the text and figures have been prepared with particular reference to requests of school, college and university instructors in invertebrate zoology, a course suggested by the fact that this species has been used with some promise of success in the invertebrate courses of a considerable number of universities.

Unfortunately few if any zoological textbooks treat nemas adequately. It is believed that any progressive and well equipped instructor who will study carefully the following descriptions, with the aid of good living as well as preserved specimens, will find himself all the better equipped to instruct students concerning the morphology of the important nemic phylum.

\section{METONCHOLAIMUS PRISTIURUS (Zur Strassen)}

[Meta, changed; Oncholaimus, tooth (in the) throat]

FEMALE. Fig. 1.

The cuticle and the body wall.

$0.8 \quad 5.9 .13 .13$ 3165. The contour of the nema is plain. The thin, transparent, colorless, nearly naked cuticle, 72,96 , about one micron thick, is traversed by plain transverse striae; but these are very difficult of resolution except with high powers of the microscope used skillfully under favorable conditions,- - ordinarily they will

1 Through the much appreciated courtesy of the United States Bureau of Fisheries a considerable part of these investigations was carried out at its Laboratories at Woods Hole, Mass. Received May 18, 1932.

${ }^{2}$ Reprinted from the Journal of the Washington Academy of Sciences, Vol. 22, No. 12, June 19, 1932.

Waverly Press, Inc., Baltimore, Maryland, December, 1932. 
not be seen. These striae are not altered on the lateral fields; there are no longitudinal wings. The subcuticle, 99, usually contains multitudinous pebbly,-i.e. roundish or slightly elongate,-yellowish pigment granules, 34, 82,95 , one to two microns across, "paved" in longitudinal bands of variable width;- - two broad lateral bands, one on each side of the body, about onethird as wide as the nema and having narrower submedian bands on each side; and three narrow ventral bands as well as even narrower dorsal bands. These bands are better seen after staining over night in seawater-methylene-blue, which may not only stain them but bring out the fact that the granules along the edges of the two main lateral bands are of a somewhat different nature from the rest. Longitudinal striations in the subcuticle, due to the attachment of the musculature, $4,16,77$, are faintly visible at high magnification in most regions of the body, especially the more translucent parts. The body wall, including the cuticle, is about six microns thick.

Ten widely spreading cephalic setae, 26 , are arranged on the lateral surface of the lip region in the usual way, i.e. a pair on each submedian line and one on each lateral line; the longest of these are one-fourth as long as the corresponding portion of the head is wide, the shorter member of each submedian pair being about three-fourths as long as the longer. The members of the submedian pairs grow so close together as sometimes to appear as one. These subcylindroid setae are nearly straight and are blunt at the end, where they seem more or less open, not closed, indicating, probably, that they may also be connected with some sense in addition to that of touch. There are a few scattered subcephalic setae near the head, of nearly the same length (ten to twelve microns) as the cephalic setae, but more slender. On the neck and on the body there are also a few scattered setae,- -very inconspicuous and seldom seen. There are also a few very short, very inconspicuous setae on the tail, especially toward its extremity and on the spinneret, 24,74 . There are no cuticular pores.

The neck and head. The head and neck occupy the anterior 11 to 13 per cent of the body, i.e. the part in front of the prominent constriction, 13, between the nearly colorless oesophagus, 12,36 , and the darker intestine, 83,94 . The slightly conoid neck ends in a subtruncate continuous head, the frontal mouth opening in which is not depressed. In front the pharynx, 31, 48, 57, is arched over by the six distinct and separate, flat and thin, fairly well developed, mobile lips, 28, 49, which are not set off by constriction or in any other way. As a rule the lips are not readily counted except when seen from in front. Toward the margin of the head there is a circlet of six, innervated, very minute and inconspicuous, forward-facing sensory papillae, 29, 45, one on each lip. This circlet is about two-thirds as wide as the front of the head. These papillae also are rather difficult to see except from in front, 45 . The rather simple subregular pharynx, $31,35,48,57$, about forty by twenty-three microns, is somewhat cylindroid anteriorly and vaguely conoid posteriorly. The posterior "chamber," 35, sixteen by nine microns, supports the three acute onchia, 25, 33, 53, the forward pointing projection of the largest of which is very readily seen. Taken as a whole the pharyngeal cavity might be described as somewhat convex-conoid. Its refractive, cuticularized wall is nearly two microns thick.

Its armature consists of three unequal, conoid, perforated, pointed onchia, one dorsal, 25 , two ventrally submedian, 33,53 . Of these the grooved left ventral submedian, 27,53 , is much the largest, and reaches two-thirds the distance to the lips. The other two, e.g. 33,25 , nearly equal in size, reach only about halfway to the lips. Each onchium is the outlet of a branched and 

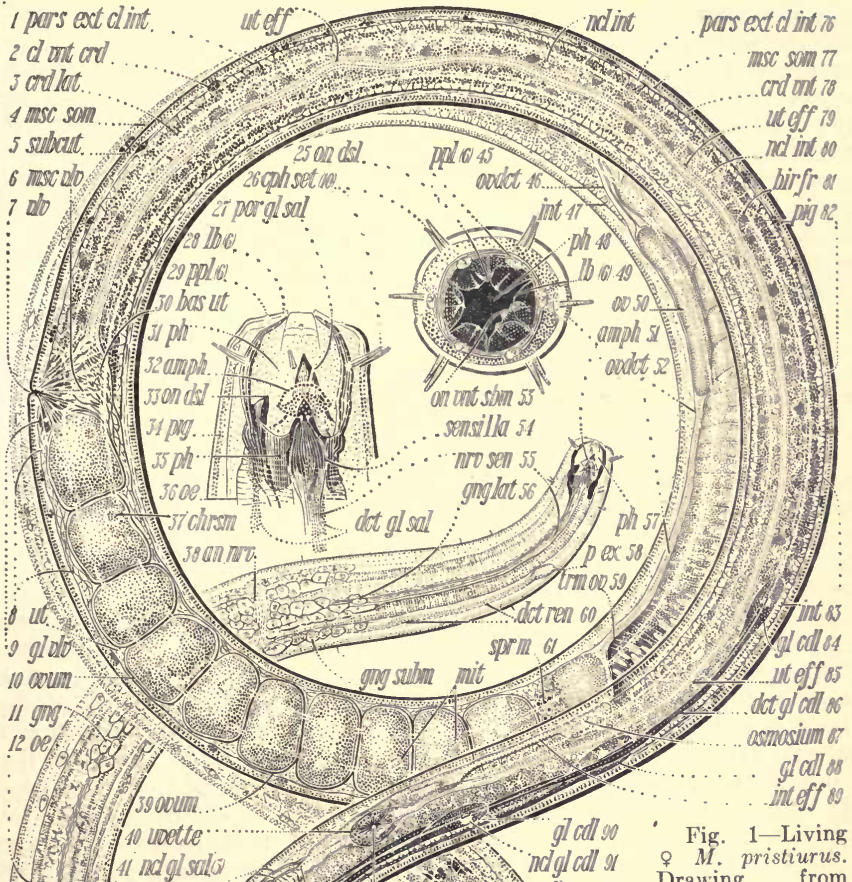

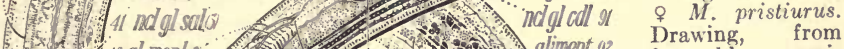

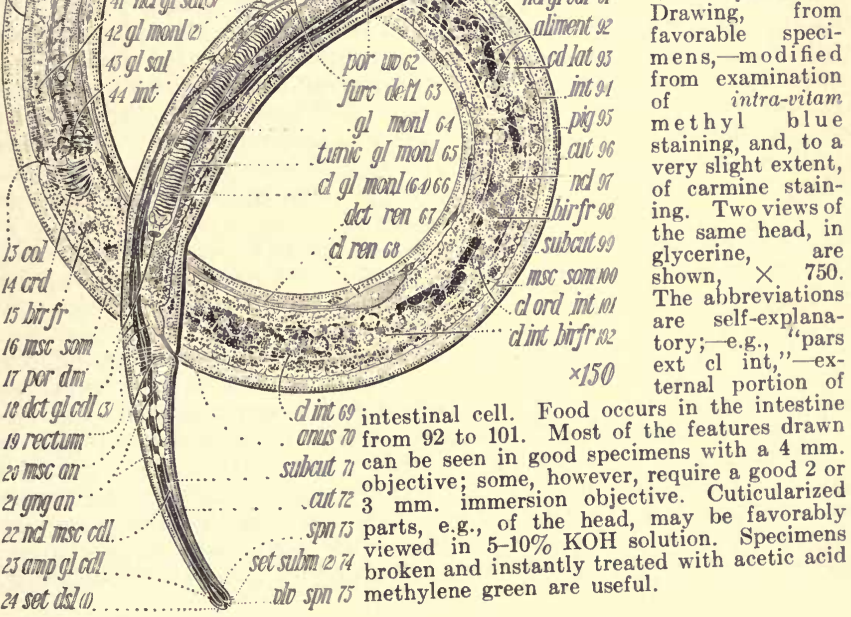


much elongated unicellular salivary gland, e.g. 43, and dct gl sal, located along the corresponding sector of the oesophagus and reaching back even to near the base of the neck, where the corresponding three nuclei, 41 , may be seen, about one body-width in front of the prominent constriction, 13, separating the oesophagus from the intestine. Each gland empties through a perforation, 27, in the corresponding onchium, by means of an inconspicuous ampulla and a very fine duct,-about one micron across. The distribution of the salivary glands among the radial contractile fibers of the oesophagus may be indicated by the granules, one micron or less in diameter, to be seen in various parts of the glands, $e . g$. at dct $g l s a l$. In favorable specimens the ducts of these glands, when filled with this granular secretion, can be followed throughout the length of the oesophagus, and the glands are then seen to have numerous short lateral branches, (see from 43 forward). The much larger, though inconspicuous pigment granules of the oesophagus are scattered throughout the organ.

The external amphids, 32, 51,-- one on each side of the head,--are somewhat escutcheon shaped, being symmetrical only to a longitudinal line, and are longer transversely than longitudinally. The anterior border of each amphid is removed from the anterior extremity of the nema a distance about equal to the radius of the head. They are much more obvious if looked at dorso-ventrally, when they are distinctly seen to be two pocket-like entrances to internal sensory organs, the internal amphids, located laterad in the back part of the head. Each of the external amphids is about one-fourth as wide as the corresponding portion of the head and about two-thirds as long as it is wide. Each outer amphid connects with a sensilla, 54 , or receptor, close behind, by means of an exceedingly narrow and very short (two and a half microns) but strongly refractive, duct, shown in the figure. The sensilla is one-fifth as wide as the head and lies opposite the basal part of the pharynx and is connected backward with the central nervous system by a lateral nerve, 55 , just beneath the body wall. The details of the sensilla, 54, are usually difficult to see except when specially stained. The amphids are held to be chemical sense organs.

The oesophagus, 12, 36, is cylindroid, enlarging very slightly posteriorly; behind the pharynx it is three-fifths, at the nerve-ring one-half, and finally two-thirds as wide as the corresponding portion of the neck. The refractive membranous "triquetrous" lining of the eosophagus, mainly about one micron thick, but two microns in the axial parts, is a distinct feature throughout the organ, and finds main optical expression in what appear as two or three closely approximated refractive, often slightly sinuous, axial elements, and, in the ordinary closed condition of the oesophagus, seeming to occupy about oneeighth of its width. The radial musculature of the eosophagus, to be seen throughout its length, consists of fine strands and is accompanied by only a slight amount of yellowish granular matter. There are no cuticularized valves in the oesophagus.

The intestine. The intestine, 83, 94, which becomes at once two-thirds as wide as the body, is thick-walled and is composed, as is usual in nemas, of a single layer of cells, 69 and vicinity, here of such a size that about twelve are required to complete the circumference. The walls of the cells are only faintly visible except sometimes in the outer colorless part, 1, 76. Usually the lumen of the intestine, (see just behind cardia, 14) can be seen only faintly, since the lining of the intestine is not refractive. As the nema bends back and forth, the food content of the intestine, e.g. at 92, may be seen to move backward and forward in the lumen. This nema appears to swallow mud 
rather indiscriminately, and to extract its nutriment from a variety of organic material contained in the mud. Large quantities of this food material in the intestine may interfere with microscopic examination; hence the advisability of keeping the nemas in clean cool seawater for a day or two before examination. The cardiac collum, or constriction, 13, between the oesophagus and intestine, is about two-fifths as wide as the base of the neck, making a very obvious demarcation between the oesophagus and the intestine. There is a conoid cardia, 14, about two-fifths as wide as the base of the neck; this is the very short extension of the oesophagus into the intestine, and is composed of numerous smaller cells of a distinct kind, having to do, among other things, with the prevention of regurgitation. Though small, the cardia is a very important part of the alimentary canal. The outer portion of the intestine, 1, 76, is usually more or less destitute of granules, but the inner and greater portion of each intestinal cell contains globular yellowish granules, 69, of variable size, the largest of which are about three microns in diameter, and the smallest less than one micron. These granules are varied and numerous, sometimes are even packed close together, and may be so arranged in the cells as to give rise to a faint, or sometimes a quite distinct, tessellated effect.

The intestine is made up of cells of different kinds,-discharging different functions. One of these various kinds is readily made out, especially with the aid of polarized light, namely the cells, as many as one hundred in number or even more, containing the exceedingly minute birefringent granules. These cells, 15, 81, 98, when examined by ordinary transmitted light, present a finer texture internally, and usually are more distinctly yellowish. If a suitable specimen be allowed to remain in a concentrated solution of seawatermethylene-blue a few minutes, a differential staining of the "birefringent" cells will often occur, but the effect does not last. The "birefringent" cells are everywhere less numerous than those that do not contain birefringents, and there are none of them at all in the posterior part of the intestine. We may therefore speak of two distinct intestinal regions, one fore, one aft. The "birefringent" cells occur in early ovic embryos.

The rather prominent short rectum, 19, the rear part of the intestine, is somewhat cuticularized, and is about as long as the anal body diameter; from the somewhat depressed anus, 70, it extends inward and forward at an angle of about forty-five degrees. Its structure in the female differs somewhat from that of the male, which appears "helical." The anterior and posterior lips of the anus are of about equal size. Small inconspicuous somewhat pear-shaped unicellular anal glands can sometimes be seen, lying alongside the rectum with their narrowed necks directed toward the anus.

Tail and spinneret. The slightly arcuate tail is first conoid, then cylindroid in the posterior fourth, where it ends in a somewhat blunt, almost imperceptibly swollen, rounded spinneret, 73 , armed only with three exceedingly inconspicuous setae, two ventrally submedian, 74, and a dorsal one, 24 . Though insignificant in appearance these sensory setae are important. The very nearly symmetrical spinneret displays internally the three very slightly swollen ampullae of the three caudal glands, 23 . The spinneret valve, or plug, 75, four microns across, almost at the very end of the tail, stains green with methyl blue ("intra vitam") while other nearby parts stain blue. This important valve is hemispherical posteriorly and tapers anteriorly to a fine contractile element, shown white in the drawing, fastened in the midst of the three ampullae (23). It is by the contraction of this minute fiber that the plug or valve is pulled away from its seat, so as to permit the sticky, non-water soluble, 
cement-like secretion of the three caudal glands to pour outward to be used in temporarily cementing the nema by the tail to the substratum in a versatile manner. The spinneret and associated glands are of vital importance to aquatic nemas; and this apparatus is all but universal among them. The three elongated ellipsoidal caudal glands, 84, 88, 90,- the remotest of them ten body-diameters in front of the anus,-are scattered in a loose tandem in the ventral part of the body cavity. Their ducts, 18,86 , leading to the spinneret, can be distinctly seen under some circumstances. Most of the caudal setae on the female are reduced and inconspicuous.

It is the sticky nature of the secretion of the caudal glands that enables these nemas to ensconce themselves so securely in the midst of the elements of the mud in which they live. By its aid they attach themselves to the substratum, especially in times of danger, and to each other. By means of this cement, they bind themselves together with mud etc. in almost inextricable tangles.

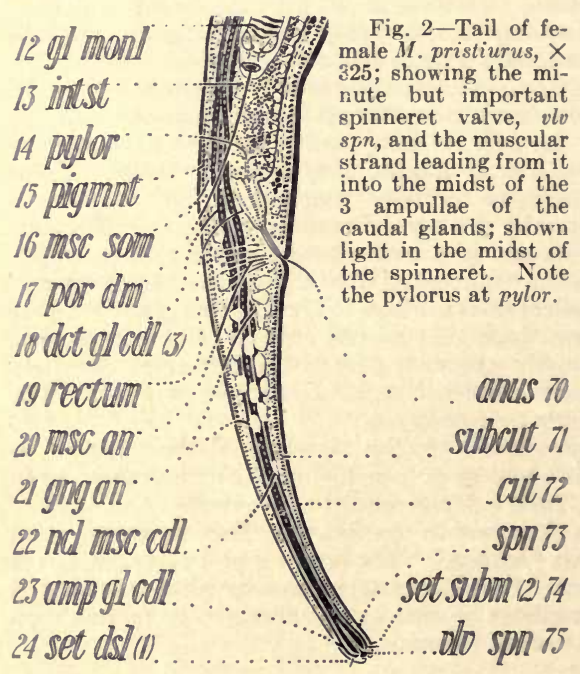

The two very thin ribbonlike lateral cords, 3, 93, of Metoncholaimus pristiurus, one on each side, immediately under the cuticle, are about half as wide as the body, each cord consisting of three regions, - a median region composed of a single broad row of quadrate cells, and a row, less than half as wide, on each side of it. In the anterior part of the body the quadrate cells are usually a little longer than they are broad, in the posterior part a little shorter than broad. As stated, these median cells are flanked by two much narrower longitudinal series of cells, having the same general composition, i.e. a very fine protoplasmic network (meshes two microns to five microns) in the intersections of which are roundish or somewhat ellipsoidal yellowish granules, usually not equidiametral. Even without staining, there are also to be seen, at least in each of the cells composing the central row of the lateral cord, faint indications of a nucleus; these indications in the living nema consist in an almost entire absence of the reticulation which is to be found elsewhere in the cell. These cells of the lateral cord are necessarily very flat; that is to say, their depth (radially to the nema) is much less than their diameter in either of the other two directions,-i.e. longitudinally to the nema or tangentially. The division line between the central row of cells and the narrower ones on the margin is an almost invisible, very thin, somewhat indirect cell-wall line. Around the outer margins of the two outer rows of cells the granules are slightly differentiated from the other granules; so that in 
seawater-methylene-blue the subcuticular pigment granules on the borders of the longitudinal bands, already described in connection with the cuticle, may stain green at a time when the rest of the granules stain blue. This appearance is similar to what is now being described for the unstained nema; so that the structure of the lateral cords is now shown to be in harmony with that of the longitudinal bands of subcuticular pigment. In other words, the arrangement of the pigment granules of the cuticle is doubtless in some way correlated with the arrangement of the cells in the lateral cords beneath. The protoplasmic network in the cells of the lateral cords is considerably finer than the protoplasmic network in the outer part of the cells composing the intestine, but nevertheless, has the same general appearance. The lacunae among the strands of the network are of variable size, more or less equidiametral, though never exactly so;--polygonal, but not regularly so. The lateral cords are wellsprings of the cuticle.

The granules of the subcuticle, $34,82,95$, differ from the yellowish granules contained in the network of the cells of the lateral cord; in the specimen under examination the granules in the subcuticle (a little under one micron) are more nearly colorless and are round, whereas those in the lateral cord are yellowish, and somewhat irregular in size and form.

Renette and excretory pore. The excretory pore, 58, is located about onefourth the distance to the nerve-ring on the ventral side of the neck. The nucleated single renette cell, 68, about four body-widths behind the neck, is a fusiform, granular, ventrad cell, about twice as long as wide, and nearly twothirds as long as the corresponding body diameter; the renette duct, 60,67 , leads from it, somewhat meanderingly, forward to the excretory pore, and is readily seen, as a rule,- or at least some of it is. It is a slender tube about one-twentieth as wide as the neck and ends anteriorly in a small ellipsoidal ampulla, near 58, nearly one-third as long as the neck is wide, emptying outward through the ventral excretory pore in the cuticle by means of an exceedingly narrow duct only three to four microns long. The excretory secretion of this gland, as seen in its duct, and ampulla, is granular, the uniform, spherical, colorless granules being about one micron in diameter. This entire apparatus, the renette, is regarded as excretory in function.

Nervous system. An important part of the central nervous system is the nerve-ring, 38, about ten microns wide, surrounding the oesophagus somewhat obliquely in front of the middle. It consists of a compact network, or skein, of exceedingly fine nerve fibers. Before and behind the nerve-ring are scores of distinct nucleated ganglion cells, 11, 56, etc., mostly bipolar, those in fror.t being arranged in eight obscure longitudinal groups,--two lateral, one ventral, one dorsal, and four submedian. The ganglion cells are variously connected with each other and with the nerve-ring. Placing the nema over night in seawater-methyl-blue discloses some of the elements of the ventral nerve leading from the nerve-ring along the ventral line to the tail. Usually about 128 fusiform elements in the ventral series may be disclosed (stained blue) in this way. These can be proved to be connected with each other. The same treatment is likely also to reveal the nerve elements entering the bases of setae, and papillae, especially in the tail of the male. See Fig. 4.

Female organs. From the slightly elevated vulva, 7 , which is a transverse ventral slit of mocierate size, the medium sized vagina leads inward and slightly forward about halfway across the body; the vagina is somewhat cuticularized and is accompanied by small and very inconspicuous vaginal glands, 9, fore and aft. About two dozen radiating muscles, 6, occur around the 
vulva, together with an associated complicated nerve plexus. This musculature is least developed behind the vulva.

The straight uterus, 30,8 , extends forward, and is of such capacity as to accommodate a maximum of about forty eggs, 10, 39, (i.e., many more than shown in this drawing) arranged approximately single file,-although this large number of eggs is rarely seen except toward autumn. Under such circumstances the oblate eggs, seeming to nearly fill the body cavity in this region, are more or less ellipsoidal in contour and half a body-width long, and twice as wide as long. When deposited, or when not crowded in the uterus, the eggs are ellipsoidal and longer than wide. The shells of the eggs, one and a half microns thick, are smooth, and the eggs are deposited before segmentation begins. Naturally, the length of the uterus varies according to the number of eggs it contains.

The broad reflexed ovary appears more or less cylindroid, and when there are, say, a dozen eggs in the uterus, the terminus of the ovary, 59, lies about halfway back to the vulva. The narrow oviduct, 46,52 , leading from the front end of the ovary back to the uterus, is usually nearly invisible, but when a ripe ovum, 50, having passed round the bend (flexure) near 46 , is being forced backward through it from the front part of the ovary back to the uterus, its presence is obvious. It is faintly visible at 52 . The ova are fertilized on first reaching the uterus, and soon after this it is not very uncommon to witness the early stages of the formation (mit, Fig. 1) of the polar bodies,-which appear later as small spherical bodies just under the eggshell. A small collection of sperms is seen in the spermathecal region at 61 .

The demanian system. In the adult female of Metoncholaimus pristiurus there is a complicated double system of efferent tubes, the demanian vessels, connecting, first, with the posterior part of the intestine through an osmosium, 87 , and second, with the posterior end of the uterus by means of a very long slender efferent duct, 79, 85. These two efferents join at a conspicuous thirty-two-merous, special glandular gateway, - the uvette, 40,-and empty, by way of the uvette pore, 62, thence backward and outward through two separate narrow lateral ducts, 42 , having attached to them, along their outer sides, relatively large and long conspicuous moniliform affuent glands, 64, seventeen microns wide, each consisting usually of sixy-four somewhat discoid elements, 66,- occasionally double (?) this number. These discoid cells of the two moniliform glands are three microns thick and packed with granules of the order of one micron; the flat ducts, along the inside of the moniliform glands, lead to two exit pores, the right hand one shown at 17 , five by seven microns, laterad in the body wall one-half tail-length in front of the anus. However, the caudad elements of the moniliform glands are "pyriform," as shown in the illustration, - not discoid. The demanian vessels elaborate a copious, elastic, sticky, non-water-soluble, nearly colorless secretion, possibly utilized during agglomeration and copulation, and also mayhap to protect and preserve the batches of eggs after deposition and during segmentation.

The uvette, 40 , is a very striking organ consisting of thirty-two concentrically arranged, highly refractive, flask-shaped, glandular elements, all concentric about a single minute central pore, 62 , leading into the large duct passing backward and dividing to form the two lateral efferent ducts each accompanied by a sixty-four-fold moniliform gland, as already described. The connection of the intestine with the demanian system at the osmosium is not an open one; the nature of the connection with the uterus, however, appears less certain. 
The osmosium, 87, of the enteric efferent is located about one-third the distance from the anus to the vulva, and may usually be seen on the dorsal side of the intestine,-being mainly visible on account of the somewhat greater transparency of its tissues. The narrow uterine efferent duct, ut. eff., 79, 85, is very difficult to follow throughout its length, and usually can be seen only in specially favorable specimens. Its connection with the posterior end of the uterus is sometimes easy to see,-near 30 . Its connection with the uvette is also nearly always easy to see, and it may be followed thence forward a short distance, but to trace it far is usually a matter of some difficulty.

The thirty-two flask-shaped elements of the uvette have their necks concentrated at the pore. The wall of the uterine duct, as previously described, spreads out over the uvette, and beneath it the thirty-two elements form a craterlike affair leading to the uvette pore. This pore opens into the somewhat duplex (but really monoluminal) corridor of the caudad part of the intestinal efferent. This latter efferent may show signs of forking at a distance in front of the uvette about equal to the corresponding body diameter, but is seldom, if ever, really bifurcate until behind the uvette.

Or,-following the demanian system from the rear toward the uvette:- Where the moniliform glands approach the uvette, they join to form a two-fold structure, and the pore of the uvette is placed between the two parts of this double structure. The structure of the tunic of the demanian system opposite the uvette presents two sets of exceedingly fine symmetrically arranged elements, - one sloping $45^{\circ}$ right, the other left,- which continue forward. This "spiral" structure can be seen throughout the duplex portion of the demanian system now being described, namely that portion in the vicinity of the uvette.

Sperms, 61, are to be seen at the cephalad end of the uterus i.e., the spermatheca, where the oviduct joins the uterus, sometimes in a mass comprising scores of sperms, each about one-tenth as wide as the corresponding portion of the body. They are rather difficult to see except when they are present in considerable numbers.

MALE. Fig. 3 .

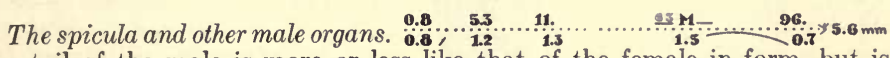
The tail of the male is more or less like that of the female in form, but is somewhat larger, more arcuate, and far more flexible, even prehensile, as Fig. 2 indicates. It diminishes a little more suddenly in size at the anus, and is armed with special setae and papillae. The two, equal, colorless, long and very slender, uniform spicula, 57, 58, seven times as long as the anal body diameter, are almost imperceptibly cephalated by expansion. They are simple and frail looking, their proximal ends lying more or less opposite the body axis. A long slender, duplex, nucleated retractor muscle, 16, extends forward from the proximal end of each spiculum to the body-wall in the corresponding subdorsal region, near 12; an antagonistic protrusor muscle, of about equal size ensheaths each spiculum. The small inconspicuous gubernaculum, 42, lying near the anus, is double and straight. Its two equal parts are somewhat frail and simple, but are expanded internally so as to be visible. They are only about half as long as the anal body diameter, and lie against the tips of the spicula in such a way that their swollen and more visible proximal ends, 42 , lie nearly opposite the axis of the base of the tail.

There is a single inconspicuous preanal ventral papilla, 22 , very close to the anus, 21 , but readily seen when searched for. There are about ten small 


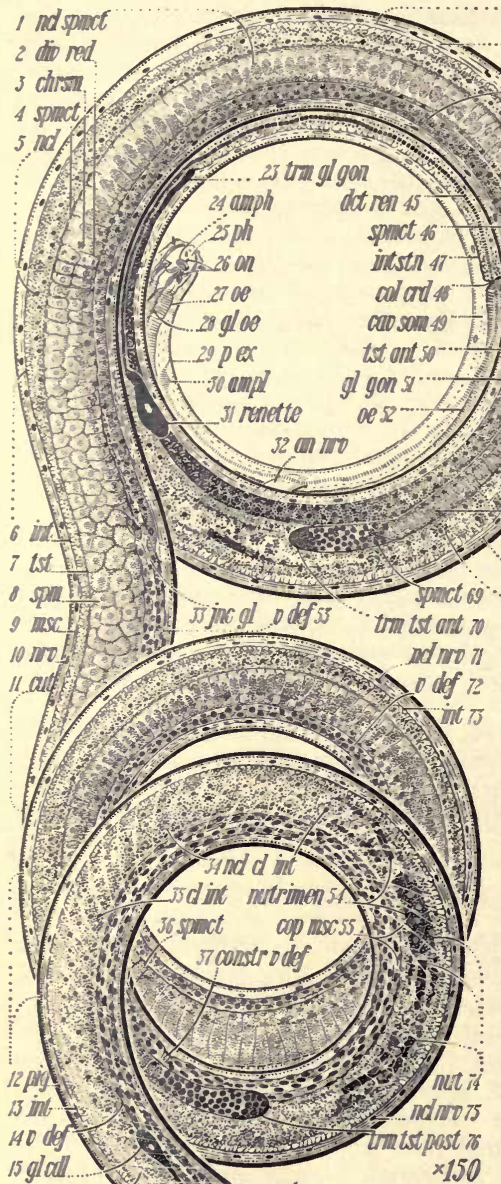

Fig. $3-\sigma^{7} M$. pristiurus,
60
from balsam COIO SOR GI stain, acid carmen. Comgl gon 62 pare with Fig. 1. Here the nuclei are brought out more distinctly. The renette and caudal glands may be followed throughout. Development of the sperms can be followed; reduction division is shown at 2 . The long gland accessory to the male gonads can be followed from 23 to 33 . One of the exceedingly slender spicula is shown, together with its long duplex, nucleated retractor muscle. The oblique copulatory muscles of the male extend forward to near the vicinity of 74 . The minute but important spinneret valve is shown at 20 . The oesophageal glands shown at 28 , may be profitably compared with the larger drawings in Fig. 1.

\section{silout os}

.cud d 67

...birfr 68

conical supplementary organs, 19 , on the ventral and subventral posterior two-fifths of the tail. These are arranged in a sort of ventral row, but the anterior ones are more or less staggered; they are somewhat unequally spaced, being wider apart posteriorly. They give a serrated appearance to the ventral contour of the posterior part of the tail, hence the specific name, pristiurus (sawtailed). There are also about thirty ventrally submedian short setae, 18 , on the front portion of the tail, about fifteen on each subventral line. These two rows extend forward to, and around, the anus, forming there a sort of circlet of inconspicuous character. These conical sup$\times 150$ plements and setae of the male are 16 matr. \% . constr56 special sensory organs; each is supplied with a

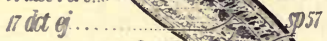

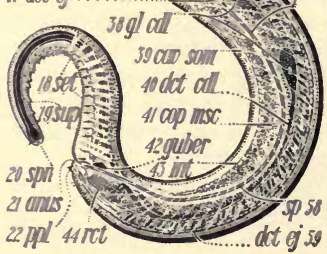
minute nerve readily demonstrable with seawatermethylene-blue (See Fig. 4).

The internal male organs. The two slender, straight testes, $7,63,70,76$, of about equal length, but the posterior somewhat the longer, are outstretched in opposite directions, and extend along the middle third of the body, each heing about sixteen body-widths long. The ejaculatory duct, 17,59 , toward the anus, is one-fifth; the vas deferens, 14, 53, next farther forward and set 
off from the ejaculatory duet by a distinct constriction, 56 , is onefourth; and the testes average one-fourth to one-half; - as wide as the body. There is a constriction midway in the vas deferens, 37. The blind end of the anterior testis, 70, directed forward, is about two neck lengths behind the cardia, while the blind end of the posterior testis, 76, directed backward, is about five tail-lengths in front of the anus.

Beginning between the renette cell and the cardia (at 23) there is a long, straight, tapering $a c$ cessory gland, 51, emptying backward into the beginning of the vas deferens, i.e., at the point where the two testes join it, 33 . This gland, accessory to the gonads, is, no doubt, a re-

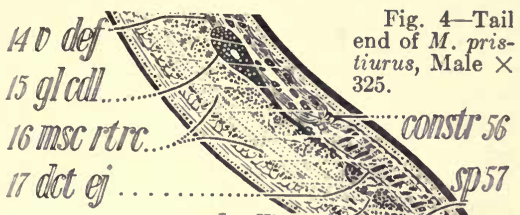
duced homologue of the demanian system of the female. A possible function is the production of cement (aseptic?) used in copulation.

The primary spermatocytes, 69, near the blind end of the testes are about forty microns in diameter. About twenty of them would be required to span the corresponding body diameter. Full grown spermatocytes, 4, 36, occur farther along the testes in rouleaux, and are two-fifths as wide as the body of the nema and one-third as long as wide. Nearly simultaneous synapses and reduction divisions of a full grown sperm are often in progress in one or the other testis, 2, 3, and the members of the resulting quartet, 2, of smaller cells, - that is the resulting spermatids, - are somewhat equidiametral and are about one-fifth as wide as the body.

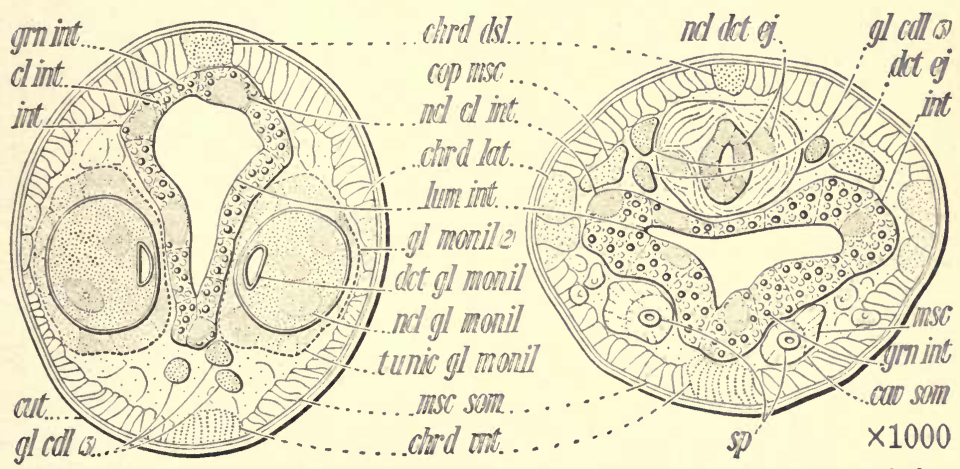

Fig. 5-Cross sections of Metoncholaimus pristiurus through the regions of the moniliform glands of the female and the spicula of the male respectively. 
The three caudal glands, 15,38 , and their three ducts, as at 40 , are shown more clearly when stained, as in the male specimen figured.

Habitat: Stagnant marine mud, below low tide, often where there is a slight overgrowth of eelgrass; harbor at Woods Hole, Massachusetts, U. S. A. at all seasons. It also occurs in the Mediterranean Sea, near Naples, Italy. This species is subject to autumnal (?) attacks of fungi and bacteria. The resulting diseases are of a very interesting character, and sometimes give rise to necrosis of the posterior part of the body. One of the common assailant cyanophytes(?) gives rise to an extensive aigrette-like appearance.

Examination of the living specimens may very profitably be supplemented by examination of temporary mounts in lactophenol, 5 per cent solution of potassium hydrate, and (broken open) in acetic acid-methylene green, as well as "intra-vitam" in seawater-methyl blue. 


\title{
A KEY TO THE GENERA OF FREE-LIVING NEMAS
}

\author{
CONTRIBUTIONS \\ TO A SCIENCE OF NEMATOLOGY \\ XXVI
}

BY

N. A. COBB

BALTIMORE 1935 



\title{
A KEY TO THE GENERA OF FREE-LIVING NEMAS
}

\author{
By N. A. Совв
}

Division of Nematology, U. S. Bureau of Plant Industry

Checked, revised and prepared for the press by Margaret V. Cobb and Corinne Cooper

Contributions to a Science of Nematology XXVI ${ }^{1}$

\section{PREFACE}

This key, which was built up and used by N. A. Cobb as a card catalog during forty years of work in nematology, had been reorganized in rough manuscript form during the last two years of his life. As Miss Cooper and I had previously worked with him on this draft, it seemed best for us to carry it to completion. In essentials and in general form it is his key, but we are responsible both for correctness of detail (an appreciable amount of the detailed work was incomplete, in need of revision, or in need of change because of addition or omission of genera), and for such decisions as have to be made in getting such a work printed. Our aim has been to follow his ideas wherever they were known to us, or where we could infer them, and to make as few changes as possible in his outline. Nearly a hundred entries have been omitted, chiefly his own new genera which it has not been possible to publish in advance of publication of the key. In some cases genera have been dropped as not being free-living. Index, glossary and list of abbreviations have been added. The bibliography has been prepared by Mrs. Rowena R. LeHew of the Division of Nematology. The definitions in the glossary are not intended to settle the meaning of terms for other workers in the field, but merely to indicate the terminology used in this key.

A few details in the form of the key stand in need of special explanation. Parentheses have been used around generic names in the key in two ways; one, to indicate that the genus is better placed elsewhere in the key, and two, with an equality sign, to indicate a synonym for the accepted name of a genus. An example of this second use is given by the first genus entry in the key. The symbols + for female, $o^{7}$, for

Received September 20, 1934.

${ }^{1}$ Reprinted from the Proceedings of the Helminthological Society of Washington, Vol. II, No. 1, January, 1935. Repaged without material alterations. 
male, and $\mathrm{J}$ for young or immature specimens are used throughout the key as a means of saving space; " $\sigma^{7}$ only," for instance, indicates that only the male form is known. The $\%$ sign is used to mean percent of body length, or, distant from anterior end, measured in percent of body length.

In general the intention has been to include all synonyms, together with the corresponding accepted generic names. The case of Dichromadora and Trichromadora, Kreis 1929, is an exception; these designations seemed to cut across the accepted classification in such a way as to make their inclusion impracticable.

Through the much appreciated courtesy of the United States Bureau of Fisheries, a part of the work on the key had been carried out at its Laboratories at Woods Hole, Mass. Needless to say, the work could not have been completed or completion even attempted by us without the resources and the expert advice of the Division of Nematology, of the Bureau of Plant Industry, U. S. Department of Agriculture, within which the work has been done. Dr. G. Steiner and Dr. J.R. Christie have given never-failing aid. Dr. Maurice Hall and Dr. B. Chitwood of the Division of Zoölogy have also given generously of their time, their knowledge and their experience.

Margaret V. Cobb.

\section{ABBREVIATIONS USED IN KEY}

abs, absent
am'g, among
alt, alternative
amph, amphid
ant, anterior
card, cardiac
caud, caudal
ceph, cephalic
cerv, cervical
circ, circular
conspic, conspicuous
constrict, constriction
cutic, cuticular
cylind, cylindroid
dent, denticles
devel, developed
diam, diameter
exc, except
excret, excretory
ext, external
gub, gubernaculum
gubernac, gubernaculum
inconspic, inconspicuous
indef, indefinite
intest, intestine
irreg, irregular
J, young, juvenile
junct, junction

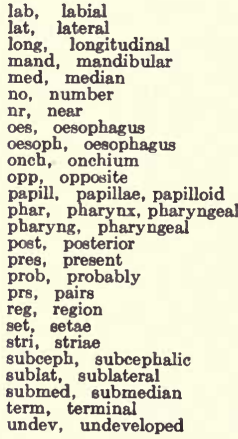




\section{GLOSSARY}

adhesion tubes, hollow tube-like setae by means of which some nemas "walk" along a surface ambulatory setae, setae, sometimes hollow and tube-like, by means of which some nemas "walk" along a surface amphid, a paired lateral sense organ the opening of which is often conspicuous, near the anterior end amphidial opening, the pore (or larger aperture) through the cuticle at which amphid opens exteriorly amphidial pocket, a chamber, outline often cup-shaped, sometimes present just behind external amphid ampulla, widening in canal, forming a reservoir annulated, having annules annules, definite transverse rings of the cuticle apophysate, having an apophysis

appendicule, a large single ventral pre-anal supplementary organ which is extensible bursa, flap-like extensions of the lateral cuticle of the tail end of the male calvarium, subcuticular cephalic framework

cardiac bulb, swelling of oesophageal wall, glandular or muscular, just anterior to beginning of intestine cardiac valve, thickening or complication of oesophageal lining in cardiac bulb, functioning as a valve caudal glands, 3 cells in or near tail, emptying by separate ducts into a common ampulla at spinneret cephalated, having the head or anterior end set off in some way cephalic setae, setae of the second or "outer" circlet around the mouth cephaloboid, resembling (that of) the genus Cephalobus cervical, on the neck

chromadoroid, resembling (that of) the genus Chromadora

cirri, elaborate cephalic appendages in front of the cephalic setae cuirasse, a helmet-like appearance of cuticle of head

cyatholaimoid, resembling (that of) the genus Cyatholaimus

demanian system, gonenteric system of vessels connecting intestine and uteri with each other, and posteriorly with the exterior

dentate, bearing teeth

denticles, minute teeth or "prickles," usually numerous denticulate, bearing denticles

diplogastroid, resembling (that of) the genus Diplogaster

dorylaimoid, resembling (that of) the genus Dorylaimus

enchelid, male form such as has been classified in the genus Enchelidium, male of Symplocostoma group eurystomoid, resembling (that of) the genus Eurystomina

excretory duct, canal leading from renette to ampulla near excretory pore

excretory pore, opening through cuticle (usually cervical or cephalic), at which the renette empties

external amphid, the cuticular manifestation of the amphid

glottoid apparatus, valvular structure at base of pharynx

gubernaculum, grooved cuticularized piece, sometimes paired, through which the spicula slide

head, portion of nema anterior to base of mouth cavity

jaws, cuticular framework around the mouth, for grasping and holding; fundamentally 3-parted

labial setae, setae of the "inner" circlet, on the lips or close to the mouth

male supplements, male organs, usually pre- or post-anal, usually a single ventral row, or paired, subventral

mandibles, hard, strong, biting or grasping organs around the mouth

mandibular, of the mandibles, resembling mandibles

median bulb, swelling of the oesophageal wall at or near the middle of its.length

monospiral, spiral of one wind or not much more

monospire, spiral, or a spiral, of one wind or not much more

mucron, a knob-like ending, shaped like that on the end of a lemon

multispiral, spiral of two or more winds

multispire, spiral, or a spiral, of two or more winds

neck, portion of nema anterior to base of oesophagus

odontium (odontia), labial tooth (teeth)

oesophagus, portion of alimentary tract between pharynx and intestine, sometimes surrounding pharynx onchium (onchia) pharyngeal tooth (teeth)

"palps," special labial appendages, apparently for touching, grasping, or getting material to mouth

percent, percent of body length, or distant from anterior end measured in percent of body length

pharyngeal bulb, muscular swelling of the oesophageal wall around the pharynx

pharynx, the mouth cavity and its walls

phasmids, a pair of cuticular pores on the tail

plectoid, resembling (that of) the genus Plectus

posterior bulb, cardiac bulb, q.v.

probolae, prominent and elaborate specialized appendages encircling the mouth

pseudonchs, structures in pharynx which from some points of view resemble onchia

renette, a cell or group of cells emptying through the excretory duct(s)

rhabditoid, resembling (that of) the genus $R$ habditis

sensory papilla, a structure in the amphidial area in which apparently nerves terminate

setae, hair-like structures on the cuticle

sole, the region of attachment of the special ambulatory setae or adhesion tubes

somatic setae, setae general on the surface of the body (opposed to cephalic, caudal, etc. setae)

spear. a long, rather slender onchium, in adult usually axial

spicula, male intromittent organs, often paired each an elongate cuticularized framework, extrusible through anus spicula, male intromittent organs, often paired each an elongate cuticularized framework,
spinneret, outlet, usually terminal, of the caudal glands, enabling nema to attach itself

striae, fine transverse lines in the cuticle

striate $(d)$, showing striae

stylet, a long slender spear

subcephalic setae, setae on the head but behind the cephalic circlet

supplementary organs, see male supplements

tooth, element of buccal armature,-onchium, odontium, spear etc.

tylenchoid, resembling (that of) the genus Tylenchus

uvette, rosette or group of cells between uterine afferent duct and efferent duct of demanian system

vestibule, entrance to the mouth cavity, sometimes a distinct chamber

wings, longitudinal structures in cuticle, or projecting from it, usually lateral, but sometimes numerous and evenly spaced around the nema 


\section{KEY}

PHARYNX ABSENT, or so obscure as easily to eacape notice (for alternative see page 458)

Oesophagus with median or posterior bulb or swelling, or both (for alternative see next page)

A mphid not known or obscure

Female known; wings 0

Ovary 1, anterior; ceph. set. 0; oesoph. with med. bulb; spinneret 0; bursa pres.; in soil. . Iotonchium Cobb 1920

Ovaries 2, reflexed

Spinneret none; amphid a pore at lips; cepn. set. 2 , minute; in beetle larvae.... (Neoa plectana Steiner 1929 ) Spinneret none; amphid a pore at lit median bulb; bursa none; marine

Spinneret present; oesoph. without median bulb; bursa none; marine

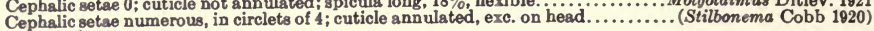

Female not known

Hemispherical cephalic cap suddenly wider than neck, edge set with dots.........Mitrephorus Linst. 1877

Hemispherical cephalic cap absent

Hemispherical cephalic setae 0; wings present; in soil; J..................... Litonema Cobb 1920

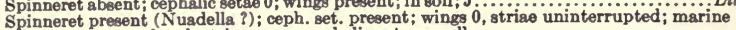

Cuticle not annulated, strise none; cephalic setae small

Neck $8 \%$; cardiac swelling slight, clavate $\ldots \ldots \ldots \ldots \ldots \ldots \ldots \ldots \ldots \ldots \ldots \ldots \ldots$. $\ldots \ldots \ldots$. $\ldots \ldots \ldots$ Allgen 1927

Neck under $4 \%$; card. bulb broad; cepb. set. in 3 circlets of 4 ; amph. small, near lips...(Catanema Cobb 1920$)$

Cuticle annulated, exc. on head; amphid near lips

Setae on head, labial 6 , ceph. 10 ; contour of nema \pm serrate; pores lateral....... (Leptonemella Cobb 1920$)$

Setae on head in circlets of 4 ; contour of nema crenate; pores 0 ; nema $10 \mathrm{~mm} . . . . . .$. (Laxonema Cobb 1920)

Amphid known

External amphid not spiral, circular nor elliptical

Head a hemispherical cap, suddenly wider than neck, edge set with dots...........Mitrephorus Linst. 1877

Head not suddenly wider nor cap-like

Habitat soil about roots

Spinneret present; lips 6, long, revolute, flower-like; amph. semicircular; wings 2; ' 8 ' Anthonema Cobb 1906

Spinneret absent; lips not long nor revolute; cephalic setse 0

Wings pres.;oesoph. \pm cephaloboid ;striae \pm coarse;amph. transverse, $\frac{1}{2}$ head-width; ' $\%$ Iotalaimus Cobb 1920

Wings absent, striae not interrupted

Ceph. papill.6, conspic.;amph.crescentic, head-width back;tail 3\%, rounded; o’ only Bolbinium Cobb 1920

Ceph. papill. tiny; amph. inconspic.,2 head-widths back;tail 6-12\%, slender,conoid Alaimus de Man 1880

Habitat marine; cephalic setae and spinneret present

Ambulatory \& body setae absent; nems not crooked

Wings pres.; amph. shepherd's crook; annules under 300, of tile-like elements; ' $\%$ ' Ceramonema Cobb 1920

Wings pres,; amph. shepherd's cretionema Cobb 1933)

Wings 0; amph. a tranverse slit, at lips; pores lateral; nema $\pm 2 \mathrm{~mm}$; ơ only...Leptonemella Cobb 1920 Ambulatory \& body setae pres.; amph. crook-shspe to spiral; nema \pm S-shaped; ' $?$ '

Oesoph. region hardly swollen; card. bulb faint; cerv. striae alike..... Notochaetosoma Irwin-Smith 1918

Oesoph. region swollen, ovoid; oesophageal bulbs 1 or 2; band of cervical striae accentuated, exc. Tristicochaeta falcatum

Ventral ambulatory adhesion tubes or setae in 3 or 4 rows............ Tristicochaeta Panceri 1878

Ventral ambulatory adhesion tubes or setae in 2 rows.................... Drepanonema Cobb 1913)

(=Chaetosoma Clap. 1863)

External amphid spiral, circular or elliptical

Female not known; J only, exc. Bolbinium

Annules \pm 90 , prominent; subdorsal setre 9 prs.; amph. 8accate; nema $10 \%+$ wide Eudesmoscolex Steiner 1916

Annules 0 or not prominent; subdorsal setae 0; amphid not saccate; nema under $5 \%$ wide

Spinneret absent; cephalis setae 0; in soil

Cephalic papillae 6, conspicuous, each on circular base........................Bolbinium Cobb 1920

Cephalic papillae 0 or inconspicuous; wings double; oesophagus \pm cephaloboid....... Litonema Cobb 1920 Spinneret present.

Wings single, projecting; amphid almost neck width, barely longer than broad...... Antomicron Cobb 1920

Wings 0 , striae not interrupted laterally

Pharynx with basal cuticularized ring; amphid monospire...............(Cricolaimus Southern 1914)

Pharynx without basal cuticularized ring; cephalic setae in circlets of 4

Amphid spiral, of 2 winds............................................ (aimonema Cobb 1920)

Amphid circular or elliptical

Head set off by constriction, spherical; cephalic setae 4 ; amphid on neck....... Cinctonema Cobb 1920

Head set off by lack of annules, if at all; ceph. set. many; amph. small, at lips; neck $2 \%$

Cuticle without annules; head not set off; pharnyx definite; nems 3-4 mm..... (Catanema Cobb 1920)

Cuticle with annules exc. on head; pharnyx obscure; nema $10 \mathrm{~mm} . . . . . . .$. . Laxonema Cobb 1920

Female known
Ovary 1; spinneret none; wings 0 ; cephalic setae 0 or papilloid

Gonad o posterior, reflexed; amphid tobscure; cardiac swelling slight; in soil. ...... (Alaimus de Man 1880)

Gonad \& anterior, outstretched

Habitat marine; anterior oesophagus fnsiform, cardiac bulb massive........... (Solenolaimus Cobb 1894)

Habitat soil; oesophagus with median bulb; bursa present..................... Iotonchium Cobb 1920

Ovaries 2

( $=$ Hemicycliophora de Man 1921)

Gonads \& outstretched; cephalic setae present; marine (for alternative see next page)

Spinneret doubtful or absent; striae very fine or seen with difficulty

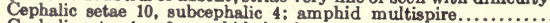

Cepbalic setae 4 or 8 ; amphid circular

Pharynx none; cardiac bulb broad, valvate; tail setaceous................ Terschellingia de Man 1888

Spinneret present

Length of oval monoepiral amphid twice its width, equalling head width.......Disconeme Fil. 1918

Gland ducts

Gland ducts cuticularized, pores ventrad, cerv. \& pre-anal; striae coarse Hala phanolaimus Southern 1914

Gland ducts and pores 0 ; striae fine, usually plain 
Oesophagus narrowed from middle; cephalic setae 4 ; amphid large; head narrowed Cyartonema Cobb 1920 Oesophagus not narrowed; cephalic setae present; wings 0

Setae on head 10,4 short specialized at tip; cardiac swelling slight............. (Linhomoella Cobb 1920) Setae on head 4-8, less than 1 head-width long, tip not specialized; amphid circular

Pharynx 0; nema small, tapered; card. bulb broad, valvate; tail setaceous..Terschellingia de Man 1888 Pharynx present though obscure

Ceph. set. 8-10; card. swelling none or clavate, non-valvate; nema not tapered Linhomoeus Bast. 1865

Ceph. set. 4 ; cardiac bulb pyriform, sometimes valvate; pharynx obconoid (Cry ptolaimus Cobb 1933) Gonads $\%$ reflexed; amphid circular, oval or monospiral (for alternative see preceding page)

Spinneret none; amphid a pore at lips; ceph. set. 2, minute; in beetle larvae... (Nea plectana Steiner 1929) Spinneret present; marine exc. Haliplectus

Ambulatory setae 0; nema not crooked; calvarium absent, or not truncate-conoid

Med. oes. bulb pres.; ceph. set. 0; pores in submed. rows; water \& brackish soil..(Haliplectus Cobb 1913) Med. oes. bulb absent; marine

Gland ducts cuticularized, pores cerv. \& pre-anal; striae coarse...(Halaphanolaimus Southern 1914) Gland ducts and pores none or not cuticularized

Head expanded opposite monospiral amphid; cephalic setae 4

Cephalic setae 0; wings 0; head narrowed from amphid forward....... Aegialoalaimus de Man 1907 Cephalic setae present; often in coastal sand

Amphid oval, 1 head-width long; annules fine; wing single; ceph. set. 4..Eutelolaimus de Man 1922 Amphid tequidiametral, less than $\frac{1}{3}$ corresponding neck width

Neck $21 \%$; striae duplex; wings 2; ceph. setae 4; pharynx narrow, inconspicuous $A$ plectus Cobb 1914

Neck $7 \%$ or less; striae not duplex; wings 0

Cuticle annulated; amph. small, at lips; set. on head many, in circles of 4..(Stilbonema Cobb 1920) Cuticle finely striated; amphid + to $\frac{1}{2}$ head-width, often monospiral

Pharynx straight,closed, with pharyng.8welling; nema 4-7 mm.; width 0.3-0.8\% Laxus Cobb 1894

Pharynx conoid, often with small onchium; nema 2-4 mm.; width $1.5-3.5 \%$ Spirina Fil. 1918 ( $=$ Spira Bast. 1865)

Ambulatory set. forming a ventral sole, midbody or pre-anal : nema crooked; calvarium unstriated
Nema narrowest in cardiac region, \pm S-shaped; ambulatory setae hollow, pre-anal; annules finer

Oesoph. region hardly swollen; card. bulb faint; cervical striae alike... Notochaetosoma Irwin-Smith 1918

Oesoph. region swollen, ovoid; oesophageal bulbs 1 or 2 ; band of cervical

striae accentuated, exc. Tristicochaeta falcatum
Ventral ambulatory adhesion tubes or setae in 3 or 4 rows.............. Tristicochaeta Panceri 1878

Ventral ambulatory adhesion tubes or setae in 2 rows................... ( Drepanonema Cobb 1913)

(=Chaetosoma Clap. 1863)

Nems narrowest midway, epsilon-shaped; ambulatory setae not open at end; annules coarser

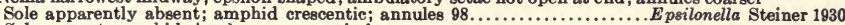
Sole present; amphid circular or monospiral

Oesophagus with median and cardiac bulbs.

Oesophagus with cardiac bulb only

Annules 80-112; ambulatory setae slender, falcate.

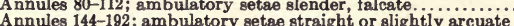

..Metepsilonema Steiner 1927

Annules 144-192; ambulatory setae straight or slightly arcuate

Body cylindroid, if crooked faintly so..........................Archepsilonema Steiner 1927

Body epsilon-like, i.e. body arches well developed

Cuticular annules homogeneous in structure..............Prochaetosoma Baylis \& Daubney 1926

Cuticular annules not homogeneous in structure

(=Rhabdogaster Metsch. 1867)

"Axial" part of the cuticular annules hollow ("frame rings")..........Epsilonema Steiner 1927

"Axial" part of the cuticular annules vacuolated, etc..................Epsilonoides Steiner 1931

Oesophagus plain, i.e. without median or posterior bulb or swelling (for alternative see preceding page)

Amphid not known or obscure (for alternative see next page)

Female not known

Habitat freshwater marshes; setre 0; spinneret none; neck 15-25\%; wings double Macroposthonia de Man 1880

Habitat marine

Neck $8 \%$, cylindroid; cephalic setae $4(?)$; tail cylindroid....................... vuadella Allgen 1927

Neck 16-43\%, \pm tapering; tail conoid

Spinneret absent; eyes 0 ; neck $\pm 40 \%$; cephalic setae 4 or 8 . . .

(Halalaimoides Cobb 1933)

Spinneret present; eyes with lenses; neck 16-25\%

Cephalic setae 4 ; head set off by cessation of very fine striation.

Nemella Cobb 1920

Cephalic setae 10; striae none; amphid and setae very large; adult ơ only...... Enchelidium Ehrenb. 1836

(=Lasiomitus Marion 1870 = Parasymplocostoma Schulz 1932)

Female known

Ovary 1, anterior exc. Antopus \& Thalassoalaimus (for alternative see next page)

Gonad o outstretched; setae 4, papilloid; amphid minute, open caudad; marine algae... (Litotes Cobb 1920)

Gonad o reflexed; spinneret none, exc. Antopus \& Thalassoalaimus

Cephalic setae 6; posterior ovary longer than anterior; marine

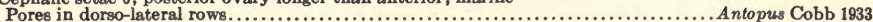

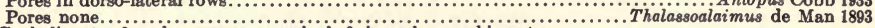
Cephalic setae 0 ; vulva near anus; spicula 2 ; in and around insects

Spear none or vestigial

Uterus not evaginated

Nema serpentine; oesophagus and intestine persisting; in beetles and weevils... Bradynema Strass, 1892

Nema serpentine; oeso phagus and intestine persisting; in beetles and weevils... Bradynema Strass. 1892
Nema saccate; organs degenerating exe. gonads............................ Allantonema Leuck. 1884

Uterus finally evaginated; oesophagus and intestine degenerating early

Evaginated uterus many times as large as nema........................... Sphaerularia Duf. 1837

Evaginated uterus not much larger than nema. ........................ Asconema Leuck. 1886

Spear present, at least in larva or $\$$; organs degenerating in adult

(=Atractonema Leuck. 1887)

Base of spear \pm bulbed; oes. fusiform, glands abs. in ơ ; bursa pre-and post-anal. . Scatonema Bovien 1932

Base of spear straight; spicula nearly straight

Vulva degenerating; o free living; bursa pre-anal; in Passalus. . Chondronema Christie \& Chitwood 1931 
Vulva remaining functional; adult \& parasitic; gubernaculum small

Anus none or vestigial; in cucumber beetle, Diabrotica........ Howardula Cobb 1921

Anus persisting; in frit-fly, Oscinella............................. T Tylenchinema Goodey 1930) Ovaries 2; spinneret present exc. Micoletzkyia (for alternative see preceding page)

Gonads of parallel, posterior; wings 10-12, segmenting the annules................. Porocoma Cobb 1920 Gonads $\%$ opposed

The ovaries outstretched; marine

Annules few, very coarse; amphid saccate; cephalic setae 4; nema wide

Number of annules \pm 17 , secreted annules prominent, separated; head small.... Desmoscolex Clap. 1863

Number of annules $32-91$, secreted annules absent; head concave-quadrate pyramidal..Tricoma Cobb 1894 (=Quadricoma Fil, 1922)

Annules and striae 0; amphid not saccate; cuticle thick................... Phanodermella Kreis 1928 The ovaries reflexed

Habitat freshwater; lips 3, thick; pharynx narrow, deep; onchium minute, deepset (Tripyla Bast. 1865)

Habitat marine; pharnyx and onchium absent

(=Pramonanchus Micol. 1923)

Annules \pm 17 ,secreted annules prominent;amphid saccate;ceph. set. 4 ;nema wide Desmoscolex Clap. 1863

Annules not few nor coarse; amphid not saccate; nema \pm slender

Odontia 6 ; lip region discoid; ceph. set. 4; gland ducts oft piercing cuticle.. Stephanolaimus Ditlev. 1918

Odontia 0 ; lip region not set off

Head set off by constrict.; ceph. set. 8; spicula long, slender; gub. complex Micoletzkyia Ditlev. 1926

Head not set off; cephalic setae 4 or 6

Setae on head 4; eyes with lenses; amphid transverse-oval, at lips; among algue.... (Ionema Cobb 1920)

Setae on head 6; eyes none

Spicula short, 1 anal body-diam.; pores 0; longest cerv. set. $\frac{1}{2}$ neck-width. .Paroxystomina Micol. 1924 Spicula long, several anal body-diameters

Cervical setae in 6 longitudinal rows; pores 0; ovaries equally long....... (Stenolaimus Marion 1870)

Cervical setae 0; pores in dorso-sublat. rows; ovary post., anterior a rudiment. . Antopus Cobb 1933

Amphid known (for alternative see preceding page)

External amphid not spiral, circular nor elliptical (for alternative see next page)

Female not known; marine

Neck $\pm 40 \%$;amph. narrow, several head-widths long;tail setaceous ; spinneret oft 0 Halalaimoides Cobb 1933

Neck not over $25 \%$; amphid not linear; spinneret present

Wings absent

Odontia 6 ;lip papill. 6 ;ceph. set. 10(?); subceph. 4, jointed; amph. oval, flat caudad A podontium Cobb 1920

Odontia 0; mouth \& phar. vestigial; eyes, amph. \& set. very large; adult ơ only Enchelidium Ehrenb. 1836 (=Lasiomitus Marion 1870

Wings conspicuous

$=$ Parasymplocostoma Schulz 1932)

Head mitreform, set off by constriction; striae 0; cephalic setae small, 4 or $6 . . \ldots \ldots \ldots$ Xennella Cobb 1920 Head not mitreform, set off by lack of annulation; annules of "tiles"; amph. crook-shape

Annules over 700; ceph. set. conspicuous, 8, in 2 circlets; onchia 0 ; nema $3 \mathrm{~mm}$. + Pristionema Cobb 1933

Annules under 300; cephalic setae 4; onchium present (?); nema under $1 \mathrm{~mm}$.......Pselionema Cobb 1933

(=Steineria Fil. 1922)

Female known

(cf. Ceramanema Cobb 1920)

Ovary 1

Gonad $ᄋ$ outstretched, anterior;ceph. papill. 4 ;amph. minute, bpen caudad;marine algae Litotes Cobb 1920

Gonad $\%$ reflexed

Spinneret present; ovary posterior, anterior branch short; wings 0; marine Thalassoalaimus de Man 1893

Spinneret absent; ovary anterior; in soil about roots

Wings present, striae \pm coarse; cephalic papillae 4; amphid 1 head-width back...Iotalaimus Cobb 1920

Wings 0 , striae 0; cephalic papillae minute; amphid 2 head-widths back........... Alaimus de Man 1880 Ovaries 2

Gonads $ᄋ$ outstretched; spinneret present; marine

Annules \pm 17 , secreted annules prominent; amphid saccate;coph. setae 4 ;nema wide Desmoscolex Clap. 1863

Annules not few nor coarse; amphid not saccate; nema tolender

Mouth ventrad; amphid huge, bent, dorsal limb the longer.

Mouth axial; amphid not huge

Amphid narrow, oft many head-widths long; eyes 0; cephalic setae 6, subceph. 4 Halalaimus de Man 1888 Amphid "folded," not over 1 head-width long; eyes 2, sometimes absent

Oesophagus and lumen widened between eyes and nerve ring; pharynx obvious (Coinonema Cobb 1920)

Oesophagus and lumen not widened; pharynx like oesoph. lining.... Araeolaimoides (de Man) Fil. 1918 Gonads o reflexed

Spinneret 0; tail 3\%; ceph. set. 10, cervical long, 1 group at amphid; marine..Platycamopsis Ditlev. 1926

Spinneret present

(=Dactylanema Fil. 1927)

Habitat freshwater; lips 3, thick; pharynx narrow, deep; onchium minute, deepset (Tripyla Bast. 1865)

Habitat marine; onchia absent exc. Actinonema

Amphid narrow, several head-widths long; head set off, with colorless cuticle

Cephalic \& subceph. setae \pm 1 head-width apart; cuticle thin (to 11 radius).... Halalaimus de Man 1888

Cephalic \& subceph. setae near together exc. Nuada isaitshikovi; cuticle thick, $\frac{1}{6}$ to $\frac{1}{2}$ radius

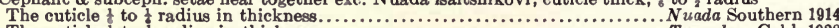

The cuticle $\frac{1}{8}$ to $\frac{1}{3}$ radius in thickness......................................... Tycnodora Cobb 1920

Amphid not narrow nor very long

Shape of amphid a shepherd's crook; each annule of tile-like elements.

Ceramonema Cobb 1920

Shape of amphid not a shepherd's crook; annules not of tile-like elements

(cf. Prelionema Cobb 1933)

Annules \pm 17 , secreted annules prominent;amph. saccate; ceph.set.4; nema wide Desmoscolex Clap. 1863

Annules not few nor coarse; amphid not saccate; nema \pm slender

Cuticle $\frac{1}{2}$ radius; striae coarse; amphid transverse, thead-width; setae 0.... (Actinanema Cobb 1920)

Cuticle not thick; amphid not a transverse slit; eyes with lenses; cephalic setae 4

Amphidial gland large, conspicuous; cephalic setae 4, $\frac{1}{2}$ head-width long ....... Ionema Cobb 1920

Amphidial gland not evident; external amph. Isemicircular, internal elongate Nemella Cobb 1920 
External amphid spiral, circular or elliptical (for alternative see preceding page)

Female not known; spinneret present

Habitat brackish earth; wings faint; $\sigma^{7}$ supplements on neck, ventral, papilloid Deontolaimus de Man 1880 Habitat marine

Annules \pm 90 , prominent;subdorsal setae 9 prs.; amph. saccate; nems 10\% + wide Eudesmoscolex Steiner 1916

Annules none or not prominent; subdorsal setae 0; amphid not sacsate; nema under $5 \%$ wide

Wing small, distinct; amphid almost neck width, joined dorsad............... (Antomicron Cobb 1920)

Wings absent

Mouth and digestive system vestigial; setae strongly developed

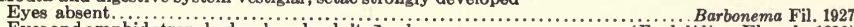

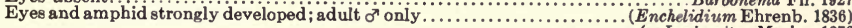

(=Lasiomitus Marion 1870

Mouth and digestive system functional

=Parasymplocostoma Schulz 1932)

Odontia 6, minute; cephalic setae 10(?), subcephalic 4, jointed.

Odontia 0 ; cephalic setae 0 or rather small

Annules rather coarse; setae minute; amphid transverse-oval.

Annules and striae none; amphid with internal pocket

Cephalic setae 0; amphid long-oval, internal pocket conspicuous............ Schistodera Cobb 1920

Cephalic setae small, 10; cervícal setae lateral; amphid small, round.....(Leptosomatides Fil. 1918) Female known

Ovary 1; amphid not multispire

Gonad \& outstretched

The ovary posterior; spinneret present

Amphid large, open caudad; spinneret sometimes doubtful; marine........... (Alaimella Cobb 1920)

Amphid round; anterior ovary rudimentary; ceph. set. 12 , in 2 circlets; in salt marsh Litinium Cobb 1920

The ovary anterior; marine

Pharynx absent; setae 4, papilloid; amph. small, open caudad; spinneret 0; among algae Litotes Cobb 1920

Pharynx present, of ten minute; setae not papilloid; amphid larger, circular; spinneret sometimes 0

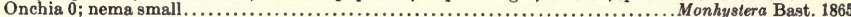

(= Tachyhodites Bast. 1865)

Onchium dorsal; nema often several millimetres long................... Linhomoevs Bast. 1865

Gonad $\%$ reflexed

$(=L$. obtusicaudatus de Man 1907)

Habitat soil or fresh water, or in insects; amphid circular

The ovary posterior; spinneret absent; in marshy soil..

The ovary anterior

Spinneret present; in fresh water. Alaimus de Man 1880

Spinneret absent; in beetle, Passalus.

Helalaimus de Cillis 1917

Habitat marine; ovary posterior; spinneret present (Alaimella ?)

Cephalic setae 0

Amphid monospire; in sand about algae.

Amphid long-oval, with posterior cuticularized pocket.

Nemanema Cobb 1920

Cephalic setae present

Schistodera Cobb 1920

Seta Setae 10 or 16,4 being subcephalio; striae 0 or fine

External amphid circular; cephalic setae 12, as long as head is wide.............Litinium Cobb 1920

External amphid oval, pocket cuticularized; cephalic setae 6 (4?)

Amphidial opening small transverse-oval, or circular.................. Nemanemella Fil. 1927

Amphidial opening large, long-oval ............................. Oxystomina Fil. 1921

Ovaries 2

$(=$ Oxystoma Bütsch. 1874)

Gonads \& outstretched; marine

Spinneret absent

Pharyngeal bulb definite; ceph. setae 6, stout, jointed; amphid circular; in sand (Cytolaimium Cobb 1920) Pharyngeal bulb absent

Tail 3\%, conoid; ceph. set. 10; cervical long, bunched; amphid transverse-oval.....Platycoma Cobb 1894

Tail over $12 \%$, conoid then cylindroid; ceph. set. minute, 18, in 3 circlets....... (Anticyathus Cobb 1920)

Spinneret present

Gland ducts cuticularized, pores cervical \& pre-anal; striae coarse...... (Halaphanolaimus Southern 1914)

Gland ducts and pores absent

Annules few, very coarse; amphid saccate; cephalic setae 4; nema wide

Number of annules \pm 17 , secreted annules prominent, separated; head small...Desmoscolex Clap. 1863

Number of annules 32-91, secreted annules none; head concave-quadrate pyramidal Tricoma Cobb 1894

Annules if present not coarse; amphid not saccate

(=Quadricoma Fil. 1922)

Ampbid multispire

Cephalic setae 10, shorter 4 specialized at tips; multispire faint; neck 5\% .... Linhomoella Cobb 1920

Cephalic setae without specialized tips; multispire distinct; neck $9-16 \%$

Male ventral pre-anal supplementary organs papilloid................Parasabatieria de Man 1907

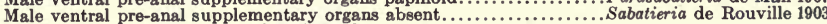

Amphid circular (Southernis slightly irregular)

Oesoph, \& lumen with ellipsoid enlargement behind eyes; eyes sometimes 0 Araeolaimus de Man 1888

Oesoph. without median enlargement; eyes 0

Cephalic setae 4 ; amphid slightly irregular............................Southernia Allgen 1929

Cephalic setae 6-10

Lips distinct; pharyngeal bulb pres.; ceph. set. 6, stout, jointed; papillae 6. .Cytolaimium Cobb 1920

Lips 0 or confluent

Setae on head 10, shorter 4 specialized at tips; multispire faint.......... (Linhomoella Cobb 1920)

Setae on head without specialized tips; amphid circular................ Linhomoeus Bast. 1865 Gonads $\%$ reflexed

Spinneret absent; amphid circular, elliptical or monospiral; wings 0 (for alternative see next page)

Habitat soil; internal amphid \pm conspicuous............................. Bastiania de Man 1876)

Habitat marine

Tail $40 \%$; vulva $33 \%$; ceph. set. 6, subceph. 6 ; internal amphid \pm conspicuous.... Trefusia de Man 1893 
Tail under $10 \%$; vulva $\pm 60 \%$; ceph. set. \pm 1 head-width long; internal amphid not conspicuous

Ceph. eet 10, cervical long, bunched, in o" 2 flat ones at amphid; tail $3 \%$, conoid.. Platycoma Cobb 1894

Ceph. set. 4 (6?), other setae 0; nema cephalated by contraction; tail slender, $9 \%$... Acoma Steiner 1916 Spinneret present (for alternative see preceding page)

Habitat not marine (Aphanolaimus ?); contour often crenate

Amphid obscure,small;pharynx narrow;onch.deepset;ceph.set.6-10,oft papilloid... (Tripyla Bast. 1865)

Amphid distinct, circular or spiral; pharynx none or closed; ceph. set. 6, rarely 4 or 0 $=$ Promononchus Micol. 1923)

Poition of amphid $3-2$ head-widths back.............................. A phanolaimus de Man 1880

Position of amphid $3-4$ head-widths back.................................. Bastiania de Man 1876

Habitat marine

Ambulatory tubes hollow; body setose, annulated

The amphid not saccate; ambulatory tubes ventral; setae not dense. . Notochaetosoma Irwin-Smith 1918

The amphid saccate; annules coarse, 17-91; nema broad

Body setae not dense; secondary annules secreted, prominent............. (Desmoscolex Clap. 1863)

Body setae dense, set along annules; junction oesoph. \& intestine indefinite.... (Greeffiella Cobb 1922) (=Trichoderma Greeff 1869)

Ambulatory tubes absent; body not densely setose; not annulated exc. Stephanolaimus

Wings \pm 50 ;nema broad, $\pm 16 \%$;neck "collared";amphid spiral;pharynx small... Richtersia Steiner 1916

Wings if present not numerous; nema slender, under $5 \%$

Odontia 6; ceph. set. 4, long; lip reg. discoid; gland ducts of t projecting. . Stephanolaimus Ditlev. 1918

Odontia 0; cephalic setae not longer than head is wide

Gland ducts cuticularized, pores cervical \& pre-anal; striae coarse .. Hala phanolaimus Southern 1914

Gland ducts and pores none, or not cuticularized

The amphid multispire; cephalic setao 16, \pm 1 head-width long; in sand .. Nannolaimus Cobb 1920

The amphid not multispire

Amphid at lips, transverse-oval, with large gland; eyes with lenses.............Ionema Cobb 1920

Amphid not at lips; eyes none exc. Leptosomatum

Anterior part of oesoph. the wider; cervical setae 0; amphid tirregular...Southernia Allgen 1929

Anterior part of oesoph. not wider; amphid with internal pocket

Oesophagus crenate posteriorly

Calvarium none: labial tubercles none.............. Stenolaimus Marion 1870

Calvarium in front of ceph. set., margin irregular; labial tubercles $3 . . . \ldots \ldots \ldots$ Klugea Fil. 1927

Oesophagus not crenate posteriorly

(=Phanodermopsis Ditlev. 1926, in part)

Cephalic setae 0; pores on anterior half of neck; tail rounded.........Leptosomatum Bast. 1865 Cephalic setae present; pores on neck absent

Setae on neck none; cephalic setae 10, \pm 1 head-width long............. Leptosomella Fil. 1927 Setae on neck in lateral series; amphid small

Gubernaculum present; $\sigma^{7}$ pre-anal ventral supplement tubular.........Anticoma Bast. 1865

Gubernaculum none; ơ pre-anal ventral supplement papilloid... (Anticomopsis Micol. 1930)

\section{PHARYNX PRESENT (for alternative see page 454)}

Wall of the Pharynx armed (for alternative see page 470)

Armature spear-like or apparently so (for alternative see page 461)

Spear bulbed; cephalic setae usually 0 ; amph. of t obscure; spinneret 0 ; not marine exc. Siphonolaimus (pg. 460)

Oesophagus plain, i.e. without median or posterior bulb or swelling; cephalic setae 0

Female not known;spear with retrorse points mid way, base 3-lobed;bursa lobed..Ecphyadophora de Man 1921

Female known; ovary 1

Gonad \& reflexed

Habit free-living; spear (dorso-ventral view) arrow-headed; ovary posterior. . Pharetrolaimus de Man 1921

Habit parasitic in beetles, etc.; ovary anterior

Nema saccate; mouth none, organs degenerated; vulva terminal............ (Allantonema Leuck. 1884)

Nema not saccate; anus none or vestigial

$(=$ Tylenchomorphus Fuchs 1914)

Spear none; parssitic in beetles, weevils, etc.

(Bradynema Strass. 1892)

Spear present in young \& only; in cucumber beetle, Diabrotica................ Howardula Cobb 1921

Gonsd $\&$ outstretched; mostly parasitic

Anus none or vestigial; spear Avestigial; in cucumber beetle, Diabrotica ....... (Hovoardula Cobb 1921)

Anus present; spear present, in A phelenchulus reduced; traces median bulb sometimes present

Junction oesoph. \& intest. definite; anterior oes. fusiform, isthmus at nerve ring; in or about plants

Habitat crop plants; head of 8 sectors; terminus of tail straight.............. Neotylenchus Steiner 1931

Habitat bladders of Fucus, or among marine algae; head of 6 sectors; tail hooked.. Halenchus Cobb 1933

Junction oesophagus and intestine indefinite

Spear with 6 basal lobes; oesoph. isthmus at nerve ring; in decaying vegetation.. Hexatylus Goodey 1926

Spear with tripartite base; renette \pm body length; parasitic in insects......... A phelenchulus Cobb 1920

Oesophagus with median or posterior bulb or swelling, or both

Female not known; spear tripartite at base

Cephalic setae 4, \pm 1 head-width long; oesoph. swellings med. \& card.; bursa striate.. Eutylenchus Cobb 1913

Cephalic setae 0 or papilloid

Spear with retrorse points midway, 3-lobed at base; bursa small, lobed .....Ecphyadophora de Man 1921

Spear without retrorse points midway; oesophagus without median bulb

Pharyngeal bulb 0,cardiac + neck length;ceph.set.0;nema $5 \%$ wide;bursa 0 . Tylencholaimellus Cobb 1915

Pharyngeal bulb pres.;ceph.set.6,papilloid;nema $9 \%$ wide;tail short,rounded...Brachynemella Cobb 1933

Female known

$(=$ Brachynema Cobb 1893)

Ovaries 2; cephalic setre 0; spear with tripartite base (for alternative see next page)

Gonads $\%$ outstretched (for alternative see next page)

Median oesophageal bulb absent; spear 3-pronged at base; in sandy soil... (Tylolaimophor us de Man 1880)

Median oesophageal bulb present; spear 3-lobed at base

Junction oesoph.\& intestine indefinite;annules plain;tail rounded,with bursa..Hoplolaimus Daday 1905

Junction oesoph. \& intestine dehnite, exc. sometimes Tylenchus

Spear-guide wide as head, forming cuirasse or helmet; bursa none; testes 2..Nemonchus Cobb 1913 
Spear-guide smaller, slighter, not forming ouirasse; bursa present

Nema cephalated by constriction, head \pm discoid; spear long; bursa lobate....Dolichodorus Cobb 1914 Nema cephalated by contraction or not at all; bursa not lobate

Anterior end extensible,narrow \& beak-like,inner framework cuticular., Tylenchorhynchus Cobb 1913

Anterior end never narrowed \& beak-like, without cuticular framework.......Tylenchus Bast. 1865

Gonads o reflexed (for alternative see preceding page)

Structure of pharynx obscure; oesophagus with cardiac swelling only

"Spear", 3 rods, ant. hinged to 2 U-shaped pieces, post. 2 stalked swellings. . (Tylopharynx de Man 1876)

"Spear" rods 2-3, base heavy, irreg.; setae 0 or papill.; ovary obscure... Diphtherophora de Man 1880 (=Chaolaimus Cobb 1893

$=$ Archionchus Cobb 1913)

Structure of pharynx obvious

Oesophagus without median bulb, cardiac bulb present

Cardiac swelling cylindroid (i.e. oesophagus dorylaimoid)

Oral spear large, over 5\%, base 3-bulbed but not trif urcate.................Xiphinema Cobb 1913

Oral spear smaller, under $5 \%$, base trif urcate....................... $(i y$ iencholaimus de Man 1876$)$

Cardiac swelling pyriform to clavate

Spear 3-bulbed at base, 士stout; ampbid protrusile, tube cuticularized.....Triplonchium Cobb 1920

Spear 3-pronged; amph. 3 head-width, transverse-oval; labial papillae 6 Tylolaimophorus de Man 1880

Oesophag us with median bulb, of ten with cardiac swelling also

Body 土thick, not serpentine; $\$$ and larval forms.

Heterodera Schmidt 1871

(=Meloidogyne Göldi 1887

= Caconema Cobb 1924)

Body \pm serpentine

Junction oesoph.\& intestine indef.;annules plain;tail rounded,with bursa.. (Hoplolaimus Daday 1905)

Junction oesoph. \& intestine definite; bursa none; $\sigma^{\prime}$ and larval forms

Spear really the apophyses of 3 movable onchia at base anterior pharynx....Tylenchodon Fuchs 1930

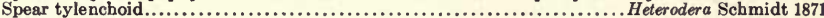

(=Meloidogyne Göldi 1887

=Caconema Cobb 1924)

Ovary 1 (for alternative see preceding page)

Gonad o reflexed; setae 0

Spear trifurcate half its length, base swollen; oesoph. with cardiac bulb only..Doryllium Cobb 1920

Spear furcate at base only; bulbs usually 3

Body thick, inert; parasitic o forms

Nema body swollen, head and neck narrow; vulva $90 \%$; in citrus roots ........ Tylenchulus Cobb 1913

Nema sausage-shaped;organs degenerated exc.gonads; vulva term.; in insects Allantonema Leuck. 1884

Body \pm slender or serpentine

(=Tylenchomorphus Fuchs 1914)

Oral spear tvestigial; median bulb \pm reduced; $\sigma^{\top}$ and larval forms

Habitat in citrus roots and soil................................... Tylenchulus Cobb 1913

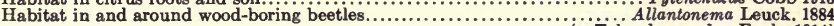

Oral spear well developed

( $=$ Tylenchomorphus Fuchs 1914)

Median oesophageal bulb elongate; vulva over $70 \%$, ovary anterior; bursa none

Spear 3-4\%; annules 0; median bulb treduced; anus not functional......... Tylenchulus Cobb 1913

Spear $8-23 \%$; annules under 150,retrorse;median bulb oft valvate;vulva $70-95 \%$. Ogma Southern 1914

Median oesophageal bulb absent

$(=$ Iota Cobb 1913)

"Spear" rods 2-3, base heavy, irreg.; setse 0 or papilloid; vulva $50 \%$... Diphtherophora de Man 1880 (=Chaolaimus Cobb 189

=Archionchus Cobb 1913)

"Spear" of usual structure, shaft distinct, base tripartite

Tip of spear tapering, posterior prongs little swollen; vulva 33-72\%... Tylencholaimus de Man 1876

Tip of spear (dorso-ventral view) arrow-headed; vulva $25 \%$, ovary post. Pharetrolaimus de Man 1921

Gonad of outstretched

Habitat marine;"spear" an evertible pharyng.lining;amph.round;neck 4\% Siphonolaimus de Man 1893

Habitat not marine; median oesophageal bulb present (Neotylenchus?)

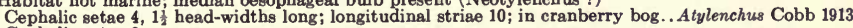

Cephalic setae 0

Dorsal oes.gland emptying at med.bulb;junct.oes.\&intest.indef.;bursa 0 Aphelenchoides Fischer 1894

(=Seinura Fuchs 193

$=$ Parasitaphelench $u$ Fuchs 1930

$=$ Pathoaphelenchus (Cobb) Steiner 1931)

Dorsal oes. gland emptying at base of spear; genera tylenchoid

Spear under $5 \%$; junction oesoph. \& intest. usually definite; nema under $5 \%$ wide; bursa present

Anterior oesoph, with median bulb; bead of 6 sectors; tail without mucron....Tylenchus Bast. 1865

Anterior oesoph. fusiform to isthmus; median bulb not definite; in or about plants

Habitat crop plants; head of 8 sectors; terminus of tail straight.......... Neotylenchus Steiner 1931

Habitat bladders of Fucus, \& am'g marine algae; head of 6 sectors; tail hooked Halenchus Cobb 1933

Spear 8-24\%; oesoph. lining flexible, looping; nema $5 \%+$ wide; bursa 0 ; vulva $72-94 \%$

Base of spear bulbed, not fluked; annules over 100, not retrorse; junct, oes. \& intest. definite

Oral area not raised; annulation fine........Paratylenchus Micol. 1922

Oral area raised, surrounded by "fossa"; annulation rather coarse...........Procriconema Micol. 1925

Base of spear anchor-shaped (fluked); annules under 150, retrorse; junct. oes. \& intest. indef.

Scales, prickles and fringes absent from annules.............. Criconema Hoffmänner \& Menzel 1914

Scales, prickles or fringes ornamenting the annules....................... Ogma Southern 191 
Spear plain, i.e. not bulbed at base (for alternative see page 458)

Oesophagus plain, i.e. without median or posterior bulb or swelling

Amphid known

External amph. reniform; dorsal onch. small, at spear base; ovary ant.; not marine Enoplocheilus Kreis 1932

External amphid circular, elliptical or monospiral

Spinneret absent; anus reduced; parasitic in beetles

Pharyng.bulb strong, head swollen; spear $1.5 \%$; junct.oesoph.\& intest. indef.; \& (Rhabdonchus Cobb 1933)

Pharyng. bulb none; spear vestigial; in beetle, Passalus; ' $\$$..... Chondronema Christie \& Chitwood 1931

Spinneret present; marine

(=Uracanthus Dies. 1861)

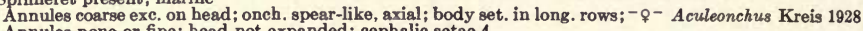

Annules none or fine; head not expanded; cephalic setae 4

Striae of dots, larger laterally; amph. multispire; spicula 2-jointed; - --.. Dorylaimopsis Ditlev. 1918

$(=$ Xinema Cobb 1920)

Striae 0; amph. monospire; spicula entire; junct. oes. \& intest. obscured by glands; eyes present

Spear $0.3 \%$, \& thickening of dorsal pharyngeal wall; ovaries 1 or 2 , reflexed..... (Onchium Cobb 1920)

Spear more obvious, $1.5 \% ;{ }^{\prime}{ }^{\prime}$ '............................................ Onchulella Cobb 1920 Amphid unknown or obscure

Female not known; spear a small prod at lips; marine.

Female known

Spinneret present; ovaries 2; marine

Calvarium subcuticular; spear long, slender; set. on bead long; nema $6 \mathrm{~mm}$. Thoracostomopsis Ditlev. 1918

Calvarium none; head not suddenly narrowed; spear short; ovaries reflexed..... (Onchulella Cobb 1920)

Spinneret absent; ovary 1; not marine

The female saccate, sausage-shaped; vulva terminal, ovary reflexed; in beetles Allantonema Leuck. 1884 (=Tylenchomorphus Fuchs 1914)

The female \pm elongate, usually not slender; vulva posterior, ovary anterior

Ovary outstretched; anus present; spear a mere prod, reduced in $\sigma^{3}$; wings 0 ... Iotonchium Cobb 1920

Ovary reflexed exc. Rhabdonchus; anus none or reduced; parasitic in' insects

Spear long, slender, in muscular bulb, head swollen; junct. oes. \& intest. indef. . Rhabdonchus Cobb 1933

Spear vestigial, minute or none; pharyngeal bulb absent

Mouth and spear absent; in beetles, weevils, etc.

Mouth and spear present in young o only; in beetles, frit fly, etc.

(=Uracanthus Dies. 1861)
(=Uristie \& Chitwood 1931

o parasitic; gubernaculum small

Anus vestigial; in cucumber beetle, Diabrotica.................... Howardula Cobb 192

Anus persisting; in frit-fy, Oscinella................................ (T ylenchinema Goodey 1930)

Oesophagus with median or posterior bulh or swelling, or both

Amphid not known or obscure

Spinneret present; cardiac swelling slight; spear a small prod at lips; $\mathrm{J}$. . .

Spinneret absent; cephalic setae 0

Nema parasitic, saccate female; vulva terminal; in beetles, etc.; ' $\%$............ (Allantonema Leuck. 1884)

Nema free-living

$(=$ Tylenchomorphus Fuchs 1914)

Armature a minute labial prod; expanding head suddenly truncate; vulva 88\%; - $\odot$ Iotonchium Cobb 1920 Armature an obvious spear

Spear closed, bent dorsad at tip when extruded; ' $O$.

Spear open, a hollow passage; not bent at tip

Lips petaloid, lip reg. expanded, discoid; oeso

Lips 0; median bulb valvate, with outlets of 3 glands; junction oesoph. \& intestine indefinite; - 8

Gubernaculum and bursa present................................. Aphelenchus Bast. 1865

(DIsonchus Cobb 1913)

Gubernaculum and bursa absent............................. phelenchoides Fischer 189

(= Seinura Fuchs 1931

$=$ Parasitaphelenchus Fuchs 1930

=Pathoaphelenchus (Cobb) Steiner 1931)

Amphid known

External amphid spiral, circular or elliptical (for alternative see next page)

Spinneret present; amphid labial, \pm spiral; "spear" filling posterior pharynx; marine

Width of nema $3.5 \%$; pharyngeal bulb definite; ‘ $\uparrow$ '............................. Onyx Cobb 1891

Width of nema $7 \%$; pharyngeal bulb faint; J................................... Öistolaimus Ditlev. 1921

Spinneret absent; amphid circular or elliptical

(ef. Onyx Cobb 1891)

Anus none;setae 0; pharyng.bulb strong; junct.oes.\&intest. indef.; in beetles; - $\odot$ Rhabdonchus Cobb 1933

Anus present; nema free-living

Cephalic setae 10, Siphonolaimus sometimes 0; amphid circular

Habitat marine; "spear" evertible phar.lining;nema 5-10 mm.;neck 4\%; - - Siphonolaimus de Man 1893

Habitat moist soil; spear tooth-like; nems $0.85 \mathrm{~mm}$.; neck $16 \% \ldots . . . . .$. Odontolaimus de Man 1880

Cephalic setae 0

$(=$ Neonchrs Cobb 1893)

Spear bent dorsad at tip when extruded; amphid small, oval, at lips; 'o ..... Campydora Cobb 1920

Spear not bent at tip

Med.oes.bulb 0;nema \pm dorylaimoid;lab.papillae 6, ceph.6;ovaries 2 (Tylolaimophorus de Man 1880)

Med. oes. bulb valvate; nema ttylenchoid; papillae 0; amphid oval, near lips

Junction of oesophagus and intestine \pm definite

Gubernaculum and bursa absent; tail of ten with mucron; - \&........Paraphelenchus Micol. 1925

Gubernaculum small; bursa without ribs; tail slender, long-conoid; $-Q-P$ silenchus de Man 1921

Junction oesoph. \& intestine indefinite; oes. gland outlets at med. bulb; tail short; - ?

Bursa and gubernaculum present......................................A phelenchus Bast. 1865

(= Isonchus Cobb 1913)

Bursa and gubernaculum absent (=Seinura Fuchs 193)

$=$ Parasitaphelenchus Fuchs 1930

=Pathoaphelenchus (Cobb) Steiner 1931) 
External amphid a transverse slit, oft at lip reg.; ceph. set. 0 ; fresh water or soil (from preceding page)

vary 1 , reflexed; spinneret 0 (Oionchus ?)

Spear a closed cutting or pricking organ, \pm tooth-like; vulva $55-60 \%$, ovary anterior

Spinneret (?) present; spear straight.................................... Oionchus Cobb 1913

Spinneret absent; tip of spear bent dorsad when extruded; amphid elliptical.....Campydora Cobb 1920

Spear open at end, a bollow passage, tip not bent; oesophagus dorylaimoid

Anterior ; of oesophagus narrow; cardiac swelling set off by constriction....... (Doryllium Cobb 1920)

Anterior $1-\frac{1}{2}$ of oesophagus narrow; both parts cylindroid

Narrow part of oesophagus set off behind by constriction..................Axonchium Cobb 1920

Narrow part of oesophagus \pm confluent with wider posterior part; $q^{\prime} ; \ldots . . . . .$. (Dorylaimus Duj. 1845)

Ovaries 2, reflexed; oesophagus \pm dorylaimoid

Spear a closed cutting or pricking organ, \pm tooth-like

The spear long, slender, flexible; cardiac swelling slight, basal, tclavate

Guide rings for spear absent; spear $30-40 \%$ of neck length................... Trichodorus Cobb 1913

Guide rings for spear present; spear $\pm 20 \%$ of neck length........................ Leptonchus Cobb 1920

The spear shorter, less slender, less flexible; cardiac swelling cylindroid, i i oesophagus

Tip of ventral spear oblique, ventrad when extruded; nema $6-10 \mathrm{~mm}$......... Sectonema Thorne 1930

Tip of submedian spear straight; nema under $4.5 \mathrm{~mm} . . \ldots \ldots \ldots \ldots \ldots \ldots \ldots$. Nygolaimus Cobb 1913

Spear open at end, a hollow passage

Pharynx with cup-shaped anterior portion

Cup-shaped portion with 6 ribs; spear with guiding ring.................Actinolaimus Cobb 1913

Cup-shaped portion without ribs; spear without guide ring; lip region discoid... Antholaimus Cobb 1913

Pharynx without cup-shaped anterior portion

Oesophagus with fusiform "bulb" behind spear......................... Dorylaimellus Cobb 1913

Oesophagus without swelling behind spear

Lip region discoid, much expanded..

Lip region usually not discoid, expanded little or not at a. ali

Labial papillae 0, cephalic 6; circumoral ridge present; vulva $34 \% . . . . .$. Chrysonema Thorne 1929

Labial papillae 6, cephalic 6 ; circumoral ridge absent .................... Dorylaimus Duj. 1845

Armature of one or more teeth (odontia or onchia) (for alternative see page 458)

Tooth (onchium) 1 (for alternative see page 465)

Oesophagus plain, i.e. without median or posterior bulb or swelling; amphid occasionally obsoure (pg. 463)

External amphid not spiral, circular nor elliptical (for alternative see next page)

Female not known

Habitat soil;spinneret 0;phar. $\frac{1}{2}$ neck-length;onch.outward-acting;oes.dorylaimoid. . Nanonema Cobb 1905

Habitat marine

$(=$ Cephalonema Cobb 1893)

Annules coarse, elements tile-like; amphid crook-shape; ceph. set. 4 ; nema 0.7 mm. Pselionema Cobb 1933

Annules not coarse; amphid not crook-shape

(=Steineria Fil. 1922)

(=Steineria Fil. 1922)

Onchium pharyngeal

Labial set. 6, cephalic 10; onchium dorsal; nema $0.5 \%$ wide; amphid transverse Trileptium Cobb 1933

Labial set. 0 , cephalic 6 ; onchium ventral; nema over $4 \%$ wide.a...

Female known.
(cf. Symplocostoma Bast. 1865)

Ovary 1; habitat not marine

Median onchium spear-like, dorsal onchium minute, basal...

Median spear-like onchium absent; ovary reflexed; Enoplidae

Phary nx tubular, depth 10 times width; tooth basal; cephalic setae 0.

Enoplocheilus Kreis 1932

Tooth minute, basal, deep-set; denticles 0 ; apinneret terminal..............Trischistoma Cobb 1913

Tooth large, subventral; denticles present; other onchia 2; spinneret ventrad(Mononchulus Cobb 1918)

Ovaries 2

Gonads $\&$ outstretched; spinneret present; striae plain; marine............Mononcholaimus Kreis 1924

Gonads o reflexed

Spinneret absent; amphidial opening oval or a transverse slit

Base of tooth without "flukes"; cephalic setae 6; onchium large, acute; in soil. .Onchulus Cobb 1920

Base of tooth anchor-shaped, "flukes" dorsad and ventrad............... Diphtherophora de Man 1880

(=Chaolaimus Cobb 1893

=Archionchus Cobb 1913)

Spinneret present, exc. sometimes Eurystominae

Habitat not marine; Trilobinae

Pharynx closed; lips 3; onchium minute, basal, deepset.

Pharynx open, domed, larger; lips 6 ; onchia often more than $1 . \ldots \ldots \ldots \ldots \ldots \ldots$ Trilobus Bast. 1865

Habitat marine

Striae conspicuous, resolvable into dots or rod-like elements; Chromadoridae

Amphid at lips, lenticular, oft obscure; cuticle of "basket-work" on neck Euchromadora de Man 1886

width conspiculus, reaching nearly across head

Cephalic setae 0; annules of obscure rod-like elements; spicula entire........Actinonema Cobb 1920

Cephalic setae 10; annules on neck of "basket-work"

Spicula entire; amphid a wide transverse oval......... Pareuchromadora Stekhoven \& Adams 1931

Spicula of 2 sections; amphid a narrow slit of even width...................Rhips Cobb 1820

Striae 0 , or plain and very tine

External amph.bent or crook-shaped,plate oval,1 head-width;"tooth" minute Diplopeltis Cobb 1905 (=Dipeltis Cobb 1891

External amphid not crook-8haped, plate absent; Enoplidae Discophora Villot 1875)

Head with elaborate, ornate calvarium.

Deontostoma Fil. 1916

Head without calvarium

Pharynx of single chamber; cephalic setae 10 
Mouth cavity \pm quadrate; onchium minute, close against wall; eyes 0 Anoncholaimus Cobb 1920 Mouth cavity twice as deep as wide; dorsal onch. bent in over pharynx Asymmetrella Cobb 1920 Pharynx of more than 1 chamber; large onchium subventral

Onchium conoid to base of short stylet; phar. Iquadrate; $\sigma^{7}$ supplements eurystomoid

Bulbs of oesophagus 5-8, serial, contiguous............................. Bolbella Cobb 1920 Bulbs of oesophagus none

Cervical setae long, about $30 ; \sigma^{7}$ supplements weakly developed............. Ledovtia Fil. 1927 Cervical setse 0 or minute; o' supplements well developed................ Eurystomina Fil. 1921 (=Euryatoma Marion 1870 $=$ Marionella Cobb 1922)

Onchium a long stylet; phar. much deeper than broad; $\sigma^{t}$ an enchelid

Anterior oesophagus (pharynx ?) narrower, lumen wide............... Calyptronema Marion 1870 (=Catalaimus Cobb 1920)

Anterior oesophagus not differentiated

Vestibule set off by row of "comma" markings; pharyng. rings 3 Symplocostomella Micol. 1930 Vestibule not set off by a ring or rows of denticles or markings... Symplocostoma Bast. 1865 External amphid spiral, circular or elliptical (for alternative see preceding page)

Fomale not known; marine

Amphid multispire, big ; phar.ribbed, complex; onch.3; ơ supplements chromadoroid Pomponema Cobb 1917 Amphid circular or oval (Rhinonema, Doryonchus?)

Annules of dots \& rods, altered laterally; ;amph.transverse-oval Pareuchromadora Stekhoven \& Adams 1931 Annules none or not ornamented; cephalic cuticle not thickened

Cephalic setae 6

Onchium a minute labial prod

Onchium ventral, pharyngeal, slender, lance-like.

Doryonchus Kreis 1932

Cephalic setae 8 or 10

(cf. Symplocostoma Bast. 1865)

Oesophagus surrounding phar.; onch. opposed by denticles; amph. long-oval A naxonchium Cobb 1920

Oesophagus ceasing at base of phary nx; denticles 0; amphid smaller, circular; Enoplidae

Pharynx of 1 chamber; onchium digitate............................... Cophonchus Cobb 1920

Pharynx of 2 chambers; onchium a slender, subventral stylet.................... Isonemella Cobb 1920

Female known

Ovary 1, anterior; onchium dorsal

Striae of dots; pharynx cyatholaimoid; ' 8 .

Strise none or plain; phary $\mathrm{nx}$ not cyatholaimoid

Pharynx sme, open; onchium large, apex far forward; amphid with pocket Pseudodilaimus Kreis 1928

Gonad 0 reflered, onchium basal or deepset, small

Goil...........Trischistoma Cobb 1913

Tons outstretched; onchum dorsal; amphid circular

The pharynx very small; oesophagus strongly developed................Prosphaerolaimus Fil. 1918 The pharynx 1 head-width, as deep as wide; onch. central; in warm salt springs...Anticyclus Cobb 1920 Ovaries 2

Gonads $\&$ outstretched; spinneret present; marine

Amphid not known; striae plain.

Mononcholaimus Kreis 1924

Amphid multispire; striae of dots

Head setose; striae not altered laterally; spicula long; apophysis absent......... Comesama Bast. 1865 Head less setose; spicula short; gubernaculum with apophysis

Male ventral pre-anal supplementary organs small, papilloid............Parasabatieria de Man 1907

Male supplementary organs absent............................. Sabatieria de Rouville 1903

Gonads $\&$ reflexed

Habitat soil or fresh water; cuticle without pores; wings 0

Amphid multispire; pharynx conoid; spinneret present; ơ supplements none Nannonchu Cobb 1913 Amphid elliptical, with internal pocket; pharynx not conoid

Cephalic setae 0; labial papillae in 2 circlets; spinneret usually present......... Mononehus Bast. 1865

Cephalic set. 6, jointed; labial papillae 1 circlet; spinneret 0; about roots..... Cyathonchus Cobb 1933

Habitat marine and brackish waters

Nema broad, $16 \%$; wings \pm 50 ; neck region "collared"; pharynx narrow...... (Richtersia Steiner 1916) Nems slender, not over $5 \%$; wings 0 exc. Rhinema 12

Amphid multispire; strise of dots; cuticular pores pres.; vestibule with 12 (6 double ?) ribs

Large o' supplement tubular, anterior to small supplements;nems viviparous Acanthonch us Cobb 1920

(=Seuratia Ditlev. 1918

= Seuratiella Ditlev. 1922)

Large of supplement absent, others if tubular small; nems usually oviparous

Phar. tubular behind onch.; gubernacula not serrate; o' papill. setose Paracyatholaimus Micol. 1922

Phar. cyathiform; gubernacula serrate distally; $\sigma^{7}$ setose papillae none

Dorsal onchium large, acute, projecting; striae altered laterally......Paracanthonchus Micol. 1924

Dorsal onchium 0 or not projecting; pharyngeal ribs extending to base

Tail setaceous; onchium small; ơ papillae present or not........... Longicyatholaimus Micol. 1924 Tail not setaceous

Dots larger laterally; o⿱ supplements tubular; gubernsc. oft joined Praeacanthonchus Micol. 1924

Dots not larger laterally; ơ tubular supplements 0; gubernacula joined Cyatholaimus Bast. 1865

Amphid circular, elliptical or monospiral

Wings 12, markings $V-s$ haped; pharyng. bulb stronger dorsad; amphid circular.... Rhinema Cobb 1920 Wings 0; striae plain

Dorsal pharyng. wall thick, tooth-like; amph. monospire at lips; ceph. set. 4; renette far back

Oesoph.glands obscuring junct.intest.; dorsal phar.element tuniform,slighter Onchium Cobb 1920

Oesoph. glands not prominent; dorsal pharyng. element not uniformly thick, but heavy

Tooth-like thickening parallel to axis................................ Camacolaimus de Man 1889 (= Digitonchus Cobb 1920 = Acontiolaimus Fil. 1918)

Tooth-like thickening bent outward from beginning of vestibule

Point of "onchium" single.......................................Acmaeolaimus Fil. 1918

Point of Y-shaped "onchium" duplex....................................... 
Dorsal pharyngeal wall not thickened; onchium a distinct projection (from preceding page)

Cephalic setae 20-30; cardiac glands conspicuous

Male supplementary organs complex; copulatory muscles conspicuous ....Xanthodora Cobb 1920

Male supplements simple, papilloid; copulatory muscles not conspic. Acanthopharynx Marion 1870 Cephalic setae not over 10

Head with elaborately ornate calvarium; striae 0; spinneret present....... Deontostoma Fil. 1916

Head without calvarium; cuticular pores absent

Plate round, with "bent" amph.;phar.small;ceph.set.4,cerv.oft long,many Diplopeltis Cobb 1905 (= Dipeltis Cobb 1891 $=$ Discophora Villot 1875)

Plate absent; pharynx large, complex, of more than 1 chamber; onchium large, sharp

Onchium conoid to base of short stylet; pharynx \pm quadrate; $\sigma^{7}$ supplements eurystomoid

Long cervical setae $\pm 30 ; \sigma^{7}$ supplements weakly developed.................. Ledovitia Fil. 1927 Long cervical setae 0

Tail rounded; spinneret oft present; $\sigma^{7}$ supplements well developed... Eurystomina Fil. 1921 (=Euryatoma Marion 1870 $=$ Marionella Cobb 1922)

Tail spicate; spinneret 0 ; $\sigma^{7}$ supplements weakly developed......Parersystomina Micol. 1930 Onchium a long stylet; phar. much deeper than wide; $\sigma^{7}$ an enchelid

Oesophageal bulbs 5-8, serial, contiguous.....................Polygastrophora de Man 1922 Oesophageal bulbs 0

Anterior oesophagus (pharynx ?) narrower, lumen wide

Calyptronema Marion 1870

(=Catalaimus Cobb 1920)

Anterior oesophagus not differentiated

Vestibule set off by row of "commas," pharyng. rings 3; eyes 0 Symplocostomella Micol. 1930 Vestibule set off by rows of denticles or markings, or by a ring

Pharyngeal chambers behind vestibule more than 2 ............. Symplocostoma Bast. 1865

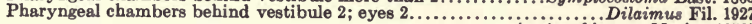
( $=$ Amphistenus Marion 1870)

- Oesophagus with median or posterior bulb or swelling, or both (for alternative see page 461)

- Amphid not known or obscure (for alternative see next page)

Female not known

Habitat soil;phar.tubular, $\frac{1}{2}$ neck;onch.outward-acting;oes.dorylaimoid;spinneret O Nanonema Cobb 1905 (=Cephalonema Cobb 1893)

Habitat marine; pharynx not over 10 neck-length; spinneret present (Rhinonema?)

Pharyngeal bulb stronger dorsad; onchium pharyngeal; cephalic setae small or 0...Iotadorus Cobb 1920

Pharyngeal bulb absent; onchium a mere prod at lips; cephalic setae $6 . . . . . .$. . (Rhinonema Allgen 1927)

- Female known

Ovary 1

Gonsd $\&$ outstretched;spinneret pres.;wings 10,marks V-shaped;ceph.set.4;marine (Nudora Cobb 1920)

Gonad o reflexed; spinneret absent; not marine

Onchium (spear) bent dorsad at tip; amphid small, oval, at lips; in soil ...... (Campydora Cobb 1920) Onchium not bent obliquely at tip

Cardiac bulb not valvate; median bulb strong

Tooth recurved outward; long. striae present; $\sigma^{7}$ supplements papilloid.. Diplogaster M. Schultze 1857

Tooth forward-pointing, deepset; long. striae none; ơ supplements setose..... Acrostichus Rahm 1928

Cardiac bulb valvate; median bulb absent, oesophagus cephaloboid

Posterior pharynx muscular, lumen closed; vulva $\pm 75 \%$; tail \pm blunt........Plectonchus Fuchs 1930

Posterior pharynx Iopen; vulva 53-65\%; tail conoid, usually slender

Vagina directed inward, not heavily muscular; not viviparous...........Macrolaimus Maupas 1900

Vagina directed forward, heavily muscular; viviparous or ovoviviparous...... Turbatrix Peters 1927

(=Anguillula auctores)

Ovaries 2

Gonads $\&$ outstretched; pharyngeal bulb \pm globular...................... (Bolbolaimus Cobb 1920)

- Gonads $\&$ reflexed, exc. perhaps Demaniella.

(=Bulbopharyngiella Allgen 1929)

Spinneret absent; oesophag us with muscular median bulb, usually valvate

Cardiac bulb valvate

Lip region not set off; odontia absent..............................Poikilolaimus Fuchs 1930

Lip region set off by constriction; curved odontia 2 or $4 \ldots \ldots \ldots \ldots \ldots \ldots \ldots \ldots \ldots$ Rhabditis Duj. 1845

Cardiac swelling not valvate, oesophagus diplogastroid

(=Diploscapteroides Rahm 1928)

Onchium small, anterior; median bulb wide as long, wider than oesophagus Neodiplogaster Cobb 192

Onchia larger, basal; "prod" at lips; median bulb not wider than oesophagus Demaniella Steiner 1914

(=Demania Steiner 1914)

- Spinneret present; oesophagus without median bulb; wings often present; marine exo. Punctodora

Lip reg. protrusile; ceph. set. 4 ; renette long; excretory pore opp. pharynx...Ptycholaimellus Cobb 1920

- Lip region not protrusile

Pharyngeal swelling stronger dorsad; $\sigma^{7}$ supplements chromadoroid exc. Odontonems

Striae altered laterally; onchium large, dorsal, apex ventral........... Hy podontolaimus de Man 1886 Striae not altered laterally

Onchium solid, inward-pointing.......

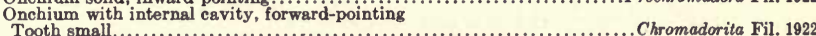

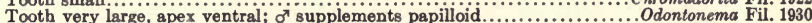

- Pharyngeal swelling not stronger dorsad

Cardiac bulb long, 2- or 3-parted; striae altered laterally; tooth large, forward-pointing

The bulb with dilated lumen......................................... Spilophorella Fil. 1918

The bulb with lumen not dilated; ơ supplements chromadoroid............Chromadorissa Fil. 1917

Cardiac bulb short, not divided

Striae altered laterally, several rows of dots larger.....................Chromadora Bast. 1865

Striae not altered laterally

Onchium solid, inward-pointing; $\sigma^{x}$ supplements chromadoroid...........Prochromadora Fil. 1922

Onchium with internal cavity, forward-pointing

Tooth small, sharp; $\sigma^{7}$ supplements chromadoroid..................... Chromadorita Fil. 1922 X

Tooth large, irregularly rectangular; cardiac bulb very large, simple........... Punctodora Fil. 1930 
Amphid known (for alternative see preceding page)

External amphid not spiral, circular nor elliptical, but a transverse slit

Female not known; amphid large

Pharyngeal bulb not stronger dorsad; ceph. set. 10; cutic. "basket-work" on neck Nygmatonchus Cobb 1933

Pharyngeal bulb stronger dorsad; striae of dots

Denticles in pharynx numerous; cephalic setae 4; in humus near sea coast......Denticulella Cobb 1933

Denticles 0 ; cephalic setae if present almost invisible; marine................. Iotadorus Cobb 1920 Female known

Ovary 1, reflexed; habitat soil about roots

Tooth short, closed, not bent at tip; ovary anterior, vulva 57\%; spinneret(?) present Oionchus Cobb 1913

Tooth (spear) bent obliquely dorsad at tip when extruded; spinneret absent ..... Campydora Cobb 1920

Ovaries 2, reflexed; spinneret present

Oesophageal bulbs 5-8, contiguous; amphid with pocket; $\sigma^{7}$ supplements eurystomoid Bolbella Cobb 1920

Oesophageal bulbs not more than 2 , not contiguous

Tooth small, near lips; setae 0; pharynx long, tubular; in fresh water...... Rhabdolaimus de Man 1880

Tooth not small; pharynx \pm 1 bead-width deep; amphid usually at lips

Onchium curved, solid, apparently mobile radially.....................Prochromadora Fil. 1922

Onchium forward-pointing or trectangular, motion apparently \pm parallei to axis

Lip reg. protrusile; ceph. set. 4; renette long; excretory pore opp. pharynx..Ptycholaimellus Cobb 1920

Lip region not protrusile

Neck with cuticular "basket-work"; 4 sublat. scales opp. phar.; spicula 2 sections Rhips Cobb 1920

Neck without "basket-work," or laterally only; scales 0 ; spiculs entire

Striae of rod-like elements, exc. towards extremities, changing to large dots laterally

Cardiac bulb broad, well set off, valvate.............................Punctodora Fil. 1930

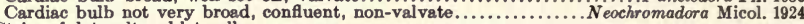

Striae of dots, altered laterally

Pharyngeal bulb stronger dorsad; onch. large; card. bulb 2-3-zoned . . Hypodontolaimus de Man 1886

Pharyngeal bulb if present not stronger dorsad; cardiac bulb not zoned. .. Chromadora Bast. 1865External amphid spiral, circular or elliptical

Female not known; marine or salt marsh (exc. Amphispira ?); wings none

Habitat soil (possibly marine); amphid spiral; cephalic setae 0; spinneret present Amphispira Cobb 1920

Habitat marine or salt marsh

Amphid multispire, large, on front curvature of head

Striae altered laterally .........................................Neotonchus Cobb 1933

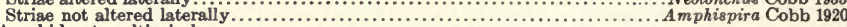

Amphid not multispiral

Onchium spear-like, free at tip.

Oistolaimus Ditlev. 1921

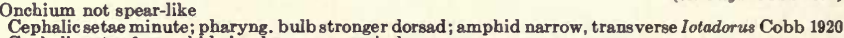

(cf. Onyx Cobb 1891)

Cephalic setae 0; amphid circular or monospiral

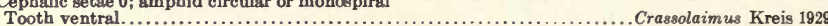

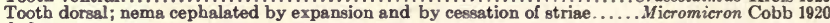

- Female known

Ovary 1

Gonad \& outstretched; amphid circular; cephalic setae 4; spinneret present; marine

Wings 10, of V-shaped marks; phar. bulb long, set off by constrict.; lab. papill. setose $N$ udora Cobb 1920

Wings 0 ; phar. bulb very slight; labial papillae 0 ; renette large, of \pm 6 cells...... Synonema Cobb 1920

Gonad o reflexed

Habitat marine; amphid round; spinneret pres.; wings 6-20, marks V-shaped Monoposthia de Man 1889

Habitat not marine; amphid round or oval; spinneret none exc. Udonchus

Median oesophageal bulb present, usually valvate; tail conoid, then setaceous; bursa none

Pharynx narrow, post. part 6 times width; ceph. set. 0; onch. small, basal Diplogasteroides de Man 1912

Pharynx not more than twice as deep as wide; cephalic setae usually present

Onchium anterior, recurved, outward-acting................. Diplogaster M. Schultze 1857

Onchium deepset, forward-pointing................................. Acrostichws Rahm 1928

Median oesophageal bulb absent; striae n̂ne, plain

Pharynx occupying $\frac{1}{2}$ neck-length; habitat decaying bulbs...........(Odontopharynx de Man 1912)

Pharynx occupying z neck-length or less

Tooth (spear) bent obliquely dorsad at lips when extruded; soil near roots.. (Campydora Cobb 1920)

Tooth not bent

Spinneret present; external amphid oval; pharyng. bulb absent; in fresh water Udonchus Cobb 1913 Spinneret absent; amphid circular

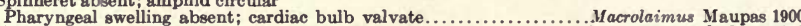

Pharyngeal swelling clavate; in moist soil ....................... Odontolaimus de Man 1880

Ovaries 2

Gonads o outstretched; spinneret present; habitat marine or brackish soil

Amphid not multispire; onchis several, often minute

Pharyngeal bulb none, or slight, confluent; ceph. setae 4; labial papillae 6...Microlaimus de Man 1880

Pharyngeal bulb almost globular; cephalic setae 6; labial setae 6............ (Bolbolaimus Cobb 1920)

Amphid multispire; striae finely dotted; pharyngeal bulb absent

Head setose; striae not altered laterally; spicula long; apophysis none........Comesoma Bast. 1865

Head less setose; spicula short; gubernaculum with apophysis

Male ventral pre-anal supplementary organs small, papilloid .........(Parasabatieria de Man 1907)

Male supplementary organs absent. (Sabatieria de Rouville 1903)

Male supplement

Spinneret absent (for alternative see next page)

Habitat marine; cephalic setae 4; pharyngeal bulb absent, pharynx small.......Spirina Fil. 1918

Habitat not marine; tail tsetaceous exc. Neodiplogaster

Cardiac bulb valvate..... Poikilolaimus Fuchs 1930

Cardiac bulb valvate....

Pharyngeal bulb clavate, fo-t neck-length;median bulb none;ceph.set.10 Odontolaimus de Man 1880

Pharyngeal bulb none or short, not tapering; oesoph. diplogastroid; onchium a projection 
Median oesophageal bulb not swollen; ceph. set. 0; striae plain....(Odontopharynx de Man 1912)

Median oesophagesl bulb swollen; cardiac swelling non-valvate

Pharynx $\frac{1}{2}$ wide as deep; ce, sh. set. present; tail setaceous; bursa 0 ....Diplogaster M. Schultze 1857

Pharynx narrow, posterior tubular portion not over $\$$ as wide as deep; ceph. set. 0

Onchium basal; tail conoid then setaceous; bursa none............. Diplogasteroides de Man 1912

Onchium anterior; striae of dots; tail conoid; bursa rhabditoid........... Neodiplogaster Cobb 1924 Spinneret present, exc. sometimes Spirina (for alternative see preceding page)

Oesophageal bulbs 5-8, serial, contiguous; $\sigma^{7}$ an enchelid; marine ...........Polygastrophora de Man 1922 Oesophageal bulb 1

Anterior oesophagus narrow, lumen wide; marine.........................

Anterior oesophagus not differentiated

(=Catalaimus Cobb 1920)

Wings present

Pharyngeal bulb set off by constriction; wings 12, bristles retrorse; marine... Rhinema Cobb 1920

Pharyngeal bulb none or confluent

Nema cephalated by cuticular change \& \pm by expansion; cardiac bulb short, not divided

Head "punctate"; wings 12-18; ceph. set. 6; denticles pres.; amph. spiral Desmodorella Cobb 1933

Head plain;wing 1;ceph.set.0;denticles 0;amph.round;caud.gl'nds pre-anal Xenonema Cobb 1920

Nema not cephalated; cardiac bulb long, 2- or 3-zoned; amphid spiral

Striae of dots; wings scalariform; o⿱ supplements 0.................. Spilophorium Cobb 1933

Striae plain

(=Spilophora Bast. 1865)

Cephalic setae many, jointed; $\sigma^{\top}$ pre-anal cuticle thickened

Cephalic setae 4, short; wing 1. "7 supplements post-anal .............

Wings 0; striae not altered laterally, exc. sometimes Desmodora

Habitat freshwater; minute subordinate onchia usually present

Pharynx behind onchia \pm parallel-sided; pharyngeal bulb definite...... Ethmolaimus de Man 1880

Pharynx behind onchia \pm conoid; pharyngeal bulb absent

Amphid spiral; cephalic setae usually 10; pharynx open, irregular..... Achromadora Cobb 1913

Amphid circular; cephalic setae 4; pharynx often obscure.............Prodesmodora Micol. 1923

Habitat marine

Dorsal pharyngeal wall thickened, onchium-like; amphid monospire at lips; cephalic setae 4 ; renette far back

Oesoph. glands obscuring junction of intest.; dorsal phar. element tuniform Onchium Cobb 1920

Oesoph. glands not prominent; dorsal pharyng. element not uniformly thick, but heavy

Tooth-like thickening parallel to axis.......................... Camacolaimus de Man 1889 (=Digitonchus Cobb 1920 = Acontiolaimus Fil. 1918)

Tooth-like thickening bent outward from beginning of vestibule

Point of "onchium" single..................................Acmaeolaimus Fil. 1918

Point of $Y$-shaped "onchium" duplex........................... Ypsilon Cobb 1920

Dorsal pharyngeal wall not thickened; onchium a distinct projection

Cephalic setae 12-26; cardiac bulb elongate; amphid spiral

Striae of dots, on head also; labial papillae setose; ceph. set. 12.... Bradylaimus Stekhoven 1931

Striae plain, absent on head; cephalic setae 24-26; amphid monospire

Pharynx denticulate; onch. sigmoid ventrally; ơ supplements sigmoid Sigmophora Cobb 1933

Pharynx not denticulate; onch. not sigmoid; $\sigma^{7}$ supplements papilloid $X$ anthodora Cobb 1920 Cephalic setae 4

Striae of dots

Dorsal onchium large, basal; pharyngeal bulb stronger dorsad......... Odontonema Fil. 1930

Dorsal onchium anterior; pharyngeal bulb \pm symmetrical............Chromadorina Fil. 1918 Striae plain

Head set off by lack of striae; pharynx \& onchium inconspic.; marine.. Desmodora de Man 1889 Head not set off; striae extending to lips

Pharyngeal bulb 0; cardiac bulb short; onchium minute; neck 6-7\% ......Spirina Fil. 1918 (=S pira Bast. 1865)

Pharyngeal bulb present; cardiac bulb long, massive, 2- or 3-zoned; neck over $12 \%$

Onchium spear-like; head rounded............................. Onyx Cobb 1891

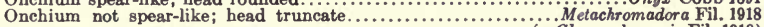
(=Chromadoropsis Fil. 1918)

Teeth (onchia or odontia) 2 or more (for alternative see page 461 )

Oesophagus with median or posterior bulb or swelling, or both (for alternative see page 467)

The teeth tequal in size, usually mobile radially (for alternative see next page)

Stroke of teeth outward; amphid often obscure (for alternative see next page)

Spinneret absent; " $\%$ or " $\%$

Cardiac bulb valvate; cuticle without longitudinal striae.

Cardiac bulb not valvate, median bulb valvate; cuticle with longitudinal striae

Posterior pharyngel

Posterior pharyngeal chamber absent; lips without radiate framework..... Diplogaster M. Schultze 1857

Spinneret present; median oesophageal bulb absent

Pharynx tubular, 4-6\% of body length

Amphid long, reaching base of pharynx or mid-body; odontia 6 , minute; - \&-.....Paeudolella Cobb 1920

Amphid small, at lips, with pocket; odontia 3, massive; tail oft striate; ‘ $\$$ '. . . . Syringolaimus de Man 1888

Pharynx conoid, not over $2 \%$ of body length

Striae plain

Odontia 0;onch. 3, at base shallow phar.;amph. round; card. swelling obvious;- $\%$ A ponchium Cobb 1920

Odontia 6 or 12 ;onch. 0 ;amph. bent or crook-shape;card. bulb 0 or weak; $q^{-}$Odontophora Bütsch. 1874 (=Trigonolaimus Ditlev. 1918 =Conolaimus Fil. 1918)

Striae of dots; cardiac swelling obvious

Amphid spiral;onch.3,curved; $\sigma^{7}$ supplements 0 or papilloid; gubernac. 0; $\sigma^{\top}$ only. . Statenia Allgen 1930 Amphid a transverse slit, near lips; "\%"

Lateral fields marked by longitudinal rows of larger dots ..............Chromadorella Fil. 1918

Lateral fields without longitudinal rows of larger dots...................... Prochromadorella Micol. 1924 
Stroke of teeth inward; amphid occasionally obscure (for alternative see preceding page)

Spinneret 0 ; phasmids present; odontia 6 ; oesophagus cephaloboid; ovaries reflexed

Nema usually cephalated; cirri 0 ; amphid round; soil \& fresh water; ' $\$$ ' $\ldots$.. (Teratocephalus de Man 1876)

Nema not cephalated; cirri 6; amphid oval; tail hooked; in bark, etc.; " 9 ....... (Chambersiella Cobb 1920)

Spinneret present

Mandibular jaws 2 or 3 ; pharyngeal and cardiac bulbs strong; marine

Jaws 3 ; amphid not known; ơ only.

(=Diastolaimus Rahm 1928)

Kreis 1928

Jaws 2, lateral, dentate; amphid multispire...........................Cheironchus Cobb 1917

Mandibular jaws absent

Amphid multispire; lips flat, confluent; pharynx rather narrow; marine

Striae very fine; wings 0; labial papillae not seen; ơ only.................. Alaimonema Cobb 1920)

Strias \pm coarse, of dots; wings pres.; labial papillae $12 ;-0 \div-$, or reflexed at tip (Mesonchium Cobb 1920 )

Amphid not multispire

Habitat soil;amph.monospire to crook-shape;phar. 6\%;nema small,0.2 mm. ;J (Pycnolaimus Cobb 1920)

Habitat marine; pharynx under $2 \%$, not tubular; ovaries not reflexed

Vulva 15\%; odontia 12, minute; amphid "folded"; $\$-\ldots \ldots \ldots \ldots \ldots \ldots \ldots$. Synodontium Cobb 1920

Vulva $81 \%$; odontia 0; onchia 3 ; pharyngeal swelling slight; - i................. (A ponchium Cobb 1920$)$

The teeth tunequal in size, usually not mobile radially; amph. usually not obscure (from preceding page)

External amphid not spiral, circular nor elliptical; ovaries 2; spinneret present

Gonads o outstretched;amph. long, reaching sometimes to mid-body;odontia 6; $\%-P$ seudolella Cobb 1920

Gonads o reflexed; amphid a transverse slit

Striae fine, plain; onchia dorsal and ventral........................... Deltanema Kreis 1929

Striae resolvable into elements; dorsal onchium usually the largest
Spicula of 2 joints; striae forming "basket-work" on neck; amphid opp. base pharynx Rhips Cobb 1920

(c.. Metalinhomoeus de Man 1907)

Spicula entire; striae of dots; amphid near lips....................... Chromadora Bast. 1865

External amphid spiral, circular or elliptical

Female not known; spinneret present; marine

Amphid multispire;head conoid;onch. minute;ceph. \& subceph. setae in sets of 4 Alaimonema Cobb 1920

Amphid circular, elliptical or monospiral

Pharynx surrounded at base by cuticularized ring................... Cricolaimus Southern 1914

Pharynx not surrounded by cuticularized ring

Striae of dots, interrupted laterally; pharynx cyatholaimoid, onchium large....Endolaimus Fil. 1922

Striae unaltered laterally; pharynx small; ơ supplements tubular, post-anal. ...... Catanema Cobb 1920

Female known

Ovary 1

Gonad \& outstretched;spinneret pres.; $\sigma^{\top}$ supplements numerous,complex;marine A ponchium Cobb 1920

Gonad $\$$ reflexed; amphid small, circular, obscure; spinneret 0 ; not marine

Cardiac bulb valvate, median bulb absent; bursa small, ribs stout............Myolaimus Cobb 1920

Cardiac bulb not valvate; oesophagus \pm diplogastroid

Oesophagus without (or with non-valvate, clavate) median swelling....... Odontopharynx de $\mathrm{Man} 1912$

Oesophagus with median valvate bulb....................... Diplogaster. M. Schultze 1857

Ovaries 2

(=Pristionchus Kreis 1932)

Gonads $\&$ outstretched, Mesonchium reflexed at tips; marine

Amphid spiral; dots larger laterally; onchis tequal; spinneret present.....(Mesonchium Cobb 1920)

Amphid circular; striae when present not altered laterally; onchia unequal Striae desmodoroid, coarse, plain; amph. behind pharynx, open caudad Paracothonolaimus Schula 1932

Pharyngeal bulb \pm globular, set off by constriction; spinneret present........Bolbolaimus Cobb 1920

Pharyngeal swelling slight, confluent; base of pharynx denticulate........... Linhomoeus Bast. 1865
( 1 Bulbopharyngilla Allgen 1929)

(i.e. subg. Eivlinhomoeus de Man 1907)

Gonads

Striae of dots; amphid opposite pharynx, of few winds

Subventral onchium small, dorsal acting out; dots unaltered laterally....Chromadorina Fil. 1918

Subventral \& dorsal onchis \pm equal, apices axial; dots larger laterally.............esonchium Cobb 1920

Striae none or plain

Head set off by cessation of coarse annulation; amphid usually multispire... Desmodora de Man 1889

Head not set off; striae none or very fine

Male ventral pre-anal supplements tubular, sigmoid, in 2 rows ...............Polysigma Cobb 1920

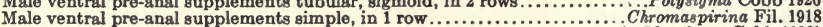

(= Mesodorus Cobb 1920)

Habitat not marine

Spinneret present; amphid spiral, behind pharynx; in fresh water.

Spinneret absent; phasmids present; amphid circular; usually in soil

Cardiac bulb valvate, oesophagus cephaloboid; onchia 4.

Cardiac bulb not valvate; oesophagus diplogastroid

Anterior oesophagus (corpus) without (or with clavate, non-valvate) swelling

Cephalic setae 6 or 10; dorsal onch. opposed by ventral onchium or ridge.... Butlerius Goodey 1929

Cephalic setae 6; dorsal onchium opposed by denticles................ Odontopharynx de Man 1912

Anterior oesophagus (corpus) ending in \pm valvate median bulb

Lips with radiate framework; post. pharyngeal chamber behind onchia... Mononchoides Rahm 1928

Lips without radiate framework; post. pharyngeal chamber none..... Diplogaster M. Schultze 1857

(=Pristionchus Kreis 1932) 
Oesophagus plain, i.e. without median or posterior bulb or swelling (for alternative see page 465)

The teeth tequal in size, usually mobile radially (for alternative see next page)

Stroke of teeth outward; amphid usually not obscure

External amphid spiral, circular or elliptical; spinneret present (Eleutherolaimus?)

Armature of 2 teeth;ceph. set. $4 ; \mathrm{amph}$. monospire,in front of setae; $\sigma^{7}$ only Diodontolaimus Southern 1914 Armature of 6 or 12 odontia

Odontia 3-jointed, heavy;phar. cylind.;onch. dorsal;amph. round-multispire; ${ }^{\circ}$ Scaptrella Cobb 1917 Odontia not jointed

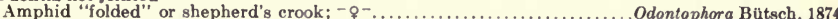

(= Trigonolaimus Ditlev. 1918

Amphid circular or elliptical, sometimes obscure; cephalic setae 4 =Conolaimus Fil. 1918)

Pharynx obscure; odontia small; lab. set. 6, setae long; striae coarse; 'o' Stephanolaimus Ditlev. 1918

Phary nx obvious; odontia larger; labial and subcephalic setae 0 ; $\sigma^{7}$ only

Striae of dots; amphid transverse, open caudad.......................A podontium Cobb 1920

Striae plain; amphid circular. .

External amphid not spiral, circular nor elliptical; spinneret pres. exc. Ironus and Parironus

Cardiac bulb slight; striae of dots; amphid a transverse slit near lips; ' $\%$ '

Dots of striae larger laterally, in longitudinal rows.

Dots of striae not altered laterally.

(Chromadorella Fil. 1918)

Cardiac bulb none; striae none, or plain and very fine

Odontia 6, sometimes dentate; amphid \pm "folded" or crook-shaped; -

Amphid opposite basal portion of pharynx; odontia less developed.

Odontophora Bütsch. 1874 (=Trigonolaimus Ditlev. 1918

Odontia or onchia 3 ; amphid transverse, with internal pocket; ovaries reflexed

Armature 3 awl-shaped onchia, protrusile forward; pharynx $0.5 \%$; $q$ '.

=Conolaimus Fil. 1918)

Armature 3 heavy odontia; phary nx tubular, 1-4\%; Ironinae

Lips expanded, denticulate, 6 ; tail $2 \%$; spinneret ventrad; \%

Lips not expanded; tail $5 \%$ or more; spinneret not ventrad; " $\%$ "

Spinneret none; pharynx 2-4\%; dorsal tooth duplex; in fresh water and soil......Ironus Bast. 1865

Spinneret present (in Parironus rudimentary or none); marine

Cephalic setae 0 or papilloid; odontia not duplex; pharyngeal swelling slight

Pharyngeal wall thin; pharynx $3 \%$; nema $3 \mathrm{~mm} . . . \ldots \ldots \ldots \ldots \ldots \ldots$. Dolicholaimus de Man 1888

Pharyngeal wall thick; pharynx $1.1 \%$; nema $2-7 \mathrm{~mm} . \ldots \ldots \ldots \ldots \ldots$. Thalassironus de Man 1889

Cephalic setae present

Odontia duplex; phar. $2.7 \%$, bulb set off by constriction; ceph. set.6, subceph.4..1ronella Cobb 1920

Odontia not duplex; pharynx 1-1.6\%, swelling slight; cephalic setae 10 ......Parironus Micol. 1930 Stroke of teeth inward; amphid known, in Enoplidae with internal pocket and of ten obscure

External amphid not spiral, circular nor elliptical; ovaries reflexed

Ovary 1, post.; phar. bulb strong; "palps" labial; onch. 3, at summit of apophyses Gammanema Cobb 1920 Ovaries 2; wings absent; amphid with internal pocket; Enoplidae

Calvarium a band with undulating margins; glands lateral; odontia large.........Fiacra Southern 1914

Calvarium absent

Onchia 3, awl-like, protrusile forward, like bristles around open mouth.........Fimbrilla Cobb 1905

Onchis not awl-like

Lips confluent, bearing 6 (or 3 duplex) odontia

Phary nx narrow; onchia 0 ; odontia probaby 6

(=Fimbria Cobb 1894)

Lips distinct, 3 ; armature beavy

The lips large, conoid, as high as broad; jaws 3

Mandibles 2-clawed; lips radially striate; spicula long, striate..........Enoploides Saveljev 1912

Mandibles absent; lips not striate; tooth midway on onchial framework... Enoplolaimus de Man 1893

The lips not conoid, nor as high as broad

Armature 3 short flattish acute onchia, doming the \pm small pharynx... Triodontolaimus de Man 1893

Armature of 3 jaws within the pharynx, anteriorly 2-pointed.................. Enoplus Duj. 1845

External amphid spiral, circular or elliptical

Female not known

A mphid monospire, transverse; lips 4 ; cephalic setae 4 ; $\sigma^{7}$ supplements $8-12$, tubular

Striae very fine; pharynx conoid; onchia 3; labial papillae 2.......... Dagda Soutbern 1914

Striae coarse; phar. cylind.; onch. 2, lateral or subventral; lab. papili. 4 Diodontolaimus Southern 1914

Amphid multispire; $0^{x}$ supplements \pm chromadoroid

Pharynx unarmed; lips 6, grasping organs, with longitudinal cuticular ribs

Pharynx armed, bulb strong; of ten nemativorous; $\sigma^{7}$ supplements chromadoroid

Mandibles 3, split exc. at tips, distal hooks 3, subordinates 2; phar. 1 chamber.. Synonchium Cobb 1920

Mandibles 0; phar. 2 chambers; onchia 3, midway, with backward apophyses...Trogolaimus Cobb 1920 Female known

Ovary 1; amphid circular or monospiral (for alternative see next page)

Gonad $\%$ post.; onch.3, on apophyses;phar.bulb strong;spinneret pres.;marine Gammanema Cobb 1920

Gonad \& anterior

Spinneret none; wings none; labial "palps" 6, small; marine; -

Amphid large, raised centrally; tail with thorn-like setae.................. (Zanema Cobb 1920)

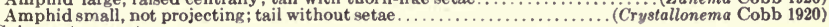

Spinneret present

Cephalic setae usually 0 or papilloid; ovoviviparous or viviparous; in gills of land crabs

Pharynx small, of 2 chambers; cephalic setae 10, often papilloid........ (Monhystrium Cobb 1920)

Pharynx large, open, of 1 chamber; cephalic setae 0 ; wings none..............Tripylium Cobb 1920

Cephalic setae present; ovary outstretched; marine

Lips thick, with 3 projecting finger-like "odontia"; wings up to 32 on head...... (Xyala Cobb 1920)
Lips 6, very thin, each with 3 parallel-sided cuticularized ribs............. (Daptonema Cobb 1920) 
()varies 2 (for alternative see preceding page)

Gonads o outstretched; spinneret present; marine

Amphid "folded" or crook-8haped; pharynx conoid; striae plain...... (Axonolaimus de Man 1889)

Amphid multispire; striae of dots, altered on lateral fields

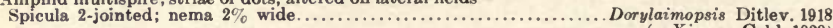

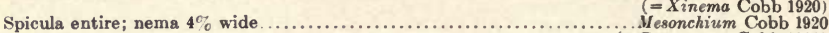

Spicula entire, nema 4 . Pepsonema Cobb 1920)

Gonads $\&$ reflexed; spinneret present

Amphid large, multispire; striae resolvable into elements; of ten nemativorous

Pharynx 2 chambers; mid-pharynx denticulate, apophyses forward \& backward; striae of dots

Circular muscles about anterior pharynx; phar. 8mall; denticles irregular..Cobbionema Fil. 1922

Circular muscles none; pharynx capacious; denticles regularly placed Halichoanolaimus de Man 1886

Pharynx not of 2 chambers; denticles absent (Demonema ?)

( $=$ Smalsundia Allgen 1929)

Mandibles none

Dots of striae not larger laterally; jaws with many horny teeth anteriorly.... Demonema Cobb 1894

Dots of striae larger laterally; onchia equal, apices axial................... Mesonchium Cobb 1920

Mandibles 2 or 3 ; Selachineminae

(=Pepsonema Cobb 1920)

Dorsal mandible a mere rod, developed mandibles 2.

Selachinema Cobb 1915

Dorsal mandible developed, making 3, \pm split; subordinate hooks pres.; cutic. pores pres.

Central mandibular prong 1, no. hooks odd; striae of dots; o' supplements 2 Synonchium Cobb 1920

Central mand.prongs 2,no.hooks even;stri.dots, dashes; $\sigma^{7}$ supplements 20 Synonchiella Cobb 1933

Amphid small, opening circular, obscure; lip8 3, large; Enoplinae

Lips not conoid, nor high as broad; labial papillae seldom setose; jaws 2-pointed. . Enoplus Duj. 1845

Lips large, conoid, often as high as broad; labial papillae 6 , setose

Mandibles 2-clawed; lips radially striate; spicula long. striate.....

Mandibles absent; lips not striate

Basal onchia large, reaching lips

Framework of jaws absent.

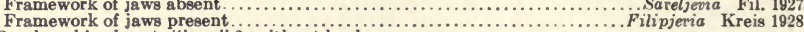

Basal onchia absent; "jaws" 3 , without hooks

Jaw framework bearing 3 equal mid-pharyngeal teeth.

Jaw framework without teeth ..........................

(=Enoplostoma Marion 1870)

Enoploides Saveljev 1912

tunequal in size, usually not mobile radially; amph., in

, in Enoplidae, oft obscure (from pg. 467)

Female not known

Cephalic setae 0; spiculum 1, gubernaculum absent.

Cephalic setse pres.; spicula 2, gubernac, pres. (Hyptiola................... Dioncholaimus Kreis 1932

Amphid multispire thead width; strise of dots, wings 2; labial "tacolaimus, Convexolaimus, J only)

Amphid circular, elliptical or monospiral

Pharynx with denticles, or many subordinate onchia; labial papill. 6, setose; amph. round or oval

Labial "palps"12, tips large; onch, dorsal, denticles not basal; wing scalariform Anaxonchium Cobb 1920

Labial "palps" 0; base of pharynx filled with subordinate onchia............Polydontus Schulz 1932

Pharynx without denticles or numerous subordinate onchia

Bulb around pharynx set off by sudden constriction.

Bulb around pharynx none or not set of by constriction

Striae coarse; onchia 2, lateral or subventral; labial papillae 4........ Diodontolaimus Southern 1914

Striae none or very fine; onchia 3

Lips 4,2 of them bearing papillae; cephalic setae 4; $\sigma^{*}$ supplements tubular...Dagda Southern 1914

Lips 6, papillae 6, setose; cephalic setae 10 , short; J

Head set off by thicker cuticle; wall of pharynx thin ...................Cacolaimus Kreis 1932

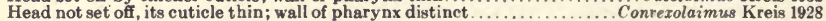
- Female known

- Ovary 1, anterior (Pseudoncholaimus?)

Median onchium spear-like, dorsal onchium minute, basal ...............Enoplocheilus Kreis 1932

- Median spear-like onchium absent

Gonad \& outstretched; amphid circular or monospiral

Onchia 2, like vertical plates, subventral; amphid monospire, longer than wide. Goniomehus Cobb 1920

Onchia not plate-like, basal, outlets of oesoph. glands; amph. round; tail 士setaceous Cobbia de Man 1907

- Gonad o reflexed

Spinneret absent; phasmids present

Cardiac bulb \pm glandular, not valvate; median swelling often present (Odontopharynx de Man 1912)

Card. bulb valvate, oes. cephaloboid; vagina directed forward, muscles heavy Tusbatrix Peters 1927

- Spinneret present; phasmids absent; amphid with internal pocket, usually small

= Anguillula auctores)

Cephalic setae 0 ; papillae in 2 circlets; in soil and fresh water

Pharynx large, open, tparallel-sided; spinneret terminal .....................Mononchus Bast. 1865

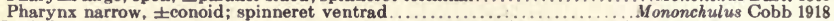

- Cephalic setae present; papillae 1 circlet; marine or in brackish waters; Oncholaiminae

- Demanian system absent

Ovary posterior; vulva 73-77\%; gubernaculum none.............. Pseudoncholaimus Kreis 1932

- Ovary ant.; vulva 70-75\%; gubernaculum pres.; spicula \pm tail length. Prooncholaimus Micol. 1924

Demanian system present

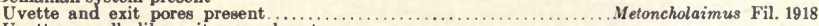

Uvette ampulla-like, exit pores absent

Male with pre-anal appendicule...

Ovaries 2

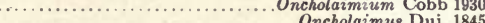

Gonads \& outstretched; striae of rods, exc. laterally; amph. a slit. . . Dicriconema Steiner \& Hoeppli 1926

Gonads \& reflexed

Habitat soil and fresh water (for alternative see next page)

Spinneret 0 ; anterior oesoph. (corpus) with or without non-valvate med. bulb; amph. round or oval

"Spear" none; dersal onch. opposed by ventral tooth or ridge; ceph, set. 6-10... Butlerius Goodey 1929 
"Spear" a minute prod between lips; cephalic setae 0

Demaniella Steiner 1914
$(=$ Demania Steiner 1914$)$

Spinneret present, exc. sometimes in Mononchus

Amphid spiral; pharynx tconoid.

Amphid circular or oval, of ten small, with internal pocket

. Nannonchus Cobb 1913

Pharyn irregularly conoi

Pharynx not conoid; one or more onchia large

Trilobus Bast. 1865

Lips thick; papillae 12, in 2 circlets; renette not known

Mononchus Bast. 1865

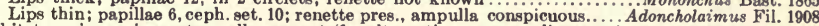

Habitat marine; spinneret usually present (for alternative see preceding page)

Cuticle annulated

Annules not resolvable into dots or rod-like elements

Head truncate; annules interrupted; ceph. set. 4; phar. ribs long., serrate. . Rhabdotoderma Marion 1870

Head rounded; annules plain; setae many, short, tirregular on calvarium.... Croconema Cobb 1920

Annules of rod-like marks; pharynx cyatholaimoid, vestibule ribbed; amphid a transverse slit

Ceph. set. 4, labial papillae setose; annules plain laterally..... Dicriconema Steiner \& Hoeppli 1926

Ceph. set. 10; annules with V-shaped marks laterally, "basket-work" on neck....Rhips Cobb 1920

Cuticle not annulated; striae if present very fine

Head with subcuticular calvarium; amphid with internal pocket; Enoplidae

Calvarium complex, ornate, long-conoid ................................. Th

man 8-17 mm.

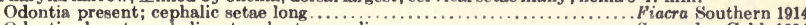

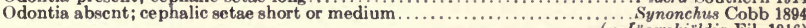

Head without calvarium

(=Jägerskioldia Fil. 1916)

Amphid spiral; pharynx small, irregularly conoid, vestibule ribbed

(Chromaspirina Fil. 1918)

(= Mesodorus Cobb 1920)

Amphid with internal pocket; pharynx large, open, squarish, ribbed vestibule absent

Pharynx not divided, walls tthick; amph. small; excretory pore of not at lips; Oncholaiminae

Demanian system present, with exit pores and developed uvette........Adoncholaimus Fil. 1918

Demanian system absent, or gonenteric duct only

Subventral onchia equal in size; amphidial opening longer than wide

Tail 33\%, filiform, spinneret none; gubernaculum none........... Filoncholaimus Fil. 1927 (=Pseudoparoncholaimus Kreis 1932)

Tail short, rounded; gubernaculum present. . . . . . . . . . . . . . . Pontonema Leidy 1855 =Paroncholaimus Fil. 1916)

Subventral onchia unequal in size; pharynx depth twice its width or more

Cuticle not viscous; phar. bulb clavate; lip reg. set off by constriction..Steineriella Allgen 1932

Cuticle viscous; pharyngeal bulb none; nema under $3 \mathrm{~mm}$.; tail 6-10\% (=Steineria Ditlev. 1928)

Pharynx very deep, \pm filled by subventral onch.; burss present Oncholaimellus de Man 1890

Phary nx not over twice as deep as wide, not filled by onchium; bursa none

Dorsal wall of pharynx well developed....................... Viscosia (de Man) Fil. 1918

Dorsal wall of pharynx rudimentary, an extension of dorsal tooth..... Meroviscosia Kreis 1932

Phary nx 2-5 chambers, wall thin; amph. large; excretory pore of at lips; large onch. subventral

Oesophageal bulbs $5-8$, serial, contiguous

Amphidial opening transverse, slit-like; $o^{7}$ supplements eurystomoid....... Bolbella Cobb 1920

Amphidial opening \pm circular; $\sigma^{7}$ an enchelid, supplements simple..Polygastrophora de Man 1922

Oesophageal bulbs none

External am phid transverse, slit-like; pharynx usually \pm square; caudal glands before anus

Denticles forming a broad mid-pharyngeal band; ơ supplements simple... Thoönchus Cobb 1920

Denticles in 1-3 rows; o" supplements complex, "fluked"...........Eurystomina Fil. 1921

(=Eurystoma Marion 1870

$=$ Marionella Cobb 1922)

External amphid round; pharynx deep; onch. spear-like, exc. Ditlevsenella; $\sigma^{\top}$ oft an enchelid

Anterior oesophagus narrower, lumen wide.................... Calyptronema Marion 1870

Anterior oesophagus not differentiated

Large onchia 3 .

(=Catalaimus Cobb 1920)

Fenestrolaimus Fil. 1927

Large onchium 1, other onchia 2

The large onchium conoid; nema 4-6 $\mathrm{mm}$

The large onchium long, slender, spear-like

Pharyngeal chambers behind vestibule 2 ; eyes 2

Ditlevsenella Fil. 1927

Pharyngeal chambers behind vestibule more than 2

Dilaimus Fil. 1927

Pharyngeal chambers behind vestibule more than 2

(=Amphistenus Marion 1870)

Symplocostoma Bast. 1865

Chambers (first 2) separated by "commas"; rings 3; eyes $\theta$..... Sym plocostomella Micol. 1930 
Wall of the Pharynx unarmed (for alternative see page 458)

Cavity not cylindroid nor prismoid, i.e. cavity conoid or irregular in long. section (for alt. see page 474)

Oesophagus with median or posterior bulb or swelling, or both (for alternative see next page)

Amphid not known or obscure

Female not known; spinneret present; cephalic setae present; nema $1 \%$ wide ; marine

Cuticle with distinct, anastomosing annules; amphid a transverse slit........... (Leptonemella Cobb 1920)

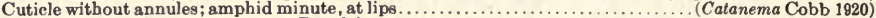

Female known; spinneret none, exc. Dasylaimus

Ovaries 2, reflexed; wings present

Spinneret pres.;phar.denticulate;oes.without med.bulb;post.ovary undev.;marine Dasylaimus Cobb 1933 Spinneret 0; post. pharynx concave-cylind.; oes. with med. bulb; in sheep dung Rhabditoides Goodey 1929 Ovary 1

Gonad o outstretched, anterior; cephalic setae 4

Gonad of reflexed; cephalic setae 0 or minute

Spinneret present; pharynx denticulate; striae of dots; post. ovary undev.; marine Dasylaimus Cobb 1933 Spinneret 0; pharynx not denticulate; oesophagus cephaloboid; not marine

Probolae present; amphid at lips; in soil

Cephalic appendages in 2 circlets.

Acrobeles Linst. 1877

Cephalic appendages in 1 circlet.

Cephalobus Bast, 1865

Probolae absent; cephalic appendages none or in 1 circle $(=A$ crobeloides (Cobb) Steiner \& Bührer 1933)

Pharyngeal wall a long. series of elements; onch. 0; vagina directed inward. Panagrolaimus Fuchs 1930 Pharyngeal wall not of serial elements; onch. oft pres.; vagina directed \pm forward; Turbatricinae

Posterior pharynx closed; vulva $\pm 75 \%$; vagina less muscular; tail \pm blunt.... Plectonchus Fucbs 1930

Posterior pharynx \pm open; vulva $\pm 65 \%$; vagina muscular; tail slender...... Turbatrix Peters 1927

(=Anguillula auctores)

Amphid known

External amphid not spiral, circular nor elliptical

Habitat not marine

Spinneret present; cephalic setae 4 ; in fresh water; 'O

Spinneret none; ovaries 2

Tail setaceous, $19 \%$;amph. semicirc.;oesoph. with med. bulb;vulva $21 \%$;' $^{\prime}$ ' (A ulolaimoides Micol. 1915)

Tail not setaceous, 9 -15\%; amphid inconspicuous, small or slit-like

Med. oesoph. bulb confluent in front; post. phar. closed; in decaying matter; ' \&' ... (Cheilobus Cobb 1924)

Med. oesoph. bulb none; pharynx ovoid; wings 0 ; amphid a slit; in soil; $-0^{-}$... Monhystera Bast. 1865

$(=M$. dintheriana de Man 1885)

Habitat marine; cephalic setae present; spinneret present

Ambulatory tubes forming ventral pre-anal sole; nema $\pm S$-shape, narrowed in card. reg.; marine; ' $q$ '

Oesoph. region hardly swollen; card. bulb faint; cerv. striae all alike.. Notochaetosoma Irwin-Smith 1918

Oesoph. region swollen, ovoid; cesophageal bulbs 1 or 2 ; band of cervical striae accentuated, exc. Tristicochaeta falcatum

Ventral ambulatory adhesion tubes or setae in 2 rows.

Drepanonema Cobb 1833 =Chaetosoma Clap. 1863)

Ventral ambulatory adhesion tubes or setae in 3 or 4 rows

Tristicochaeta Panceri 1878

(=Draconema Cobb 1913)

A mbulatory tubes absent; nema not crooked, not narrowed in cardiac region

Amphid transverse; pharynx minute; annules plain; ceph. set. 10, jointed; $\sigma^{7}$ only Leptonemella Cobb 1920

Amphid large, folded or shepherd's crook; pharynx obvious; ovaries outstretched

Pharynx 4-5\%, heavy,tubular;amphid 4-50\% body;ceph.set.4,subceph.12;- - $^{-}$(Pseudolella Cobb 1920)

Pharynx under $2 \%$, conoid, not heavy; cephalic setae large, long; odontia 6 or 12

Ovary 1 , posterior; cephalic setae 6 ; odontia 12 ; vulva $15 \%$

Ovaries 2; cephalic setae 4; odontia 6 ; vulva 45-67\%

Tail clavate; nema shorter, stouter; amphid larger

Tail conoid; nema long, slender; amphid smaller

(Synodontium Cobb 1920)

Axonolaimus de Man 1889

Ascolaimus Ditlev. 1919

External amphid spiral, circular or elliptical

Female not known

Habitat soil (possibly marine); spinneret present; amphid spiral; ceph, set. 0...(Amphispira Cobb 1920)

Habitat marine

Amphid multispire; wings 0 ; annules very fine

Pharyngeal bulb none; lip region not set off; head truncate-conoid; onch. 3 , small Alaimonema Cobb 1920

Pharyngeal bulb present

Head cylindroid; lip region discoid, set off by constriction . . . . . . . . (Choniolaimus Ditlev. 1918)

Head hemispherical; lip region not discoid ..........................

Amphid circular or monospire; spinneret present

Nema cepbalated, striae ceasing at constriction

Pharynx none; head \pm spherical; amphid behind head; nema slender, $1.4 \%$...Cinctonema Cobb 1920

Pharynx definite; head \pm hemispherical; amphid on head; nema wider, $3.8 \%$ (Micromicron Cobb 1920)

Nema not cephalated

Annules strong, each of 8 tile-like elements; oesphagus compound

Dasynemella Cobb 1933

Annules none; oesophagus not compound

Cephalic setae 8 or more; onchium minute; amphid minute, at lips....

(=Dasynema Cobb 1920)

Cephalic setae 4; onchia 0; amphid large, not at lips

Wings 0 ; amphids behind head, not joined

Base pharynx faintly cuticularized, without ring; amphid circular......Monhysteriella Kreis 1929

Base phary nx with cuticular ring, prongs 2, vertical; amphid monospire..Cricolaimus Southern 1914 Female known

Ovary 1 (for alternative sce next page)

Gonad $\$$ outstretched, anterior; amphid circular; striae 0 , or fine and plain; wings 0

Pharynx \pm quadrate; neck under $5 \%$; crystalline bodies lateral; marine...... (Crystallonema Cobb 1920)

Monhystera Bast. 1865)

Gonad \& reflexed; cepbalic setae 0 or minute

(i.e. subg. Monhystrella Cobb 1918) 
Spinneret present; striae of dots; phar. denticulate; post. ovary undev.; marine Dasylaimus Cobb 1933 Spinneret absent; pharynx not denticulate; not marine

Head with 6 biting odontia; amphid round; nema cephalated exc.

Head with entire or 3 -parted lip region; amphid small, at lips; nema not cephalated, exc. Acrobeles insubricus

Probolae absent; cephalic appendages 0 or in 1 circlet.

Probolae present; in soil

Cephalic appendages in 2 circlets.

Cephalic appendages in 1 circlet.

Teratocephalus de Man 1876

Panagrolaimus Fuchs 1930

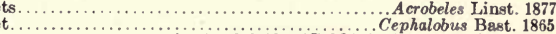

Ovaries 2 (for alternative see preceding page)

Gonads \& outstretched

Amphid multispire; striae fine; pharynx small, cup-shaped

Male ventral pre-anal supplementary organs small..................................

Amphid circular

Pharynx obconoid, cutic. rings 1 or more; spinneret pres.; brackish soil... (Desmolaimus de Man 1880)

Pharynx without transverse cuticularized rings; spinneret present or not

Habitat not marine; cephalic setae 0

Spinneret present; head with 4 circular "warts"; in fresh water. . . . . . Pseudochromadora Daday 1901

Spinneret absent; head without "warts" ; bursa present; in moss........... Himatidiophila Rahm 1925

Habitat marine; striae fine; cephalic setae present

The pharynx obconoid, with cylindroid vestibule

Base pharynx cuticularized; head tcylindroid; cardiac bulb valvate... Metalinhomoeus de Man 1907

(cf. Deltanema Kreis 1929)
Base pharynx not cuticularized; head tconoid; lips if everted knob-like Cryptolaimus Cobb 1933

The pharynx minute or none

Cardiac swelling not strongly developed; ceph. set. 8-10; nema not tapering Linhomoeus Bast. 1865

Cardiac bulb broad, valvate; ceph. set. 4; nema tapering, tail setaceous Terschellingia de Man 1888 Gonads \& reflexed

Habit parasitic, in insects; oesophagus without median bulb.......... Neoa plectana Steiner 1929

Habit free-living

Spinneret absent; cephalic setae 0 ; in soil and fresh water

Nema cephalated, exc. T. palustris; lip reg. lobed, with 6 biting odontia. Teratocephalus de Man 1876

Nema not cephalated; lip region not lobate; bursa present............... Himatidiophila Rahm 1925

Spinneret present

Cephalic setae 0; head with 4 circular "warts" ; in fresh water...........Pseudochromadora Daday 1901

Cephalic setae present; head without "warts"

Habitat fresh water or soil; amphid circular to monospiral

Habitat marine

Lips 2, mouth a dorso-ventral slit; "pseudonchs" dorso-ventral ridges. ... (Pseudonchus Cobb 1920)

Lips more than 2, mouth not a slit; "pseudonchs" absent

Amphid multispire

Pharynx irregular, with tooth-like projections

Pharynx simply conoid, regular, without tooth-like projections

Head set off by absence of striae...

Head not set off; striae extending to lips; neck 6-7\%; onchium minute...... Spirina Fil. 1918

Amphid circular or monospiral

The phary nx denticulate; post. ovary undev.; striae of dots; wings pres... (Dasylaimus Cobb 1933)

The pharynx not denticulate; both ovaries developed

Dorsal pharyngeal wall not thickened; amphid not at lips

Neck 14-21\%; wings present; amphid long-oval; head set off by groove at 4 cephalic setae

Length of neck 14-16\%; amphid large, $\frac{1}{2}$ head-width back..........Eutelolaimus de Man 1922

Length of neck $21 \%$; amphid 2 head-widths back..................... A plectus Cobb 1914

Neck under $7 \%$; wings 0 ; amphid spiral

Nema 4-7 mm; ; width 0.3-1\%; phar. straight, closed, with pharyng. swelling Laxus Cobb 189

Nema 2-4 mm.; width 1.5-4\%; pharynx conoid. of t with small onchium...... Spirina Fil. 1918 $(=$ Spira Bast. 1865)

Dorsal pharyng. wall thick, tooth-like; amph. monospire at lips; ceph. set. 4 ; renette far back

Oesoph. glands obscuring junct. of intest.; dorsal phar. element \pm uniform Onchium Cobb 1920

Oesoph. glands not prominent; dorsal pharyng. element not uniformly thick, but heavy

Tooth-like thickening parallel to axis.

Camacolaimus de Man 1889

(=Digitonchus Cobb 1920

= Acontialaimus Fil. 1918)

Tooth-like thickening bent outward from beginning of vestibule

Point of "onchium" single.

Point of Y-shaped "onchium" duplex

Acmaeolaimus Fil. 1918

Ypsilon Cobb 1920

Oesophagus plain, i.e. without median or posterior bulb or swelling (for alternative see preceding page)

Amphid not known or obscure (for alternative see next page)

Female not known

Habitat marine; lip region discoid; odontia 6 ; ceph. set.4; amphid small. . . . (Stephanolaimus Ditlev. 1918)

Habitat fresh water, marsh or sand

Hemispherical "cap" wider than neck, edge setose; phar. conoid; spinneret pres. Diplolaimus Linst. 1876

Hemispherical cap none; setae 0; spinneret 0 ; wings double; bursa striate.... Macroposthonia de Man 1880 Female known; spinneret present

Ovary 1; marine

Gonad 8 reflexed; ceph. set. 0 , phary nx denticulate; post. ovary undev.; wings pres. Dasylaimus Cobb 1933

Gonad \& outstretched; cephalic setae 4

The ovary anterior; nema $3 \mathrm{~mm}$; neck $12 \%$; vulva $75 \%$.

The ovary posterior; nena $1 \mathrm{~mm}$.; neck $19 \%$; vulva $35 \%$

Krampia Ditlev. 1922

Ovaries 2, reflexed

Habitat fresh water (for alternative see next page)

Lips 3, rounded; pharynx small, closed; onchium minute, deepset

Asymmetrica Kreis 1929 
Lips 6, thin; pharynx larger, domed; onchia in posterior chamber

(Trilobus Bast. 1865)

Habitat marine (for alternative see preceding page)

Wings conspic.; striae of dots; ceph. set. 0;pharynx denticulate; post.ovary undev.. Dasylaimus Cobb 1933 Wings 0 , striae plain, not altered laterally; cephalic setae present; Enoplidae

Calvarium elaborate, ornate; onchia often present............................ Deontostoma Fil. 1916

Calvarium not elaborate, sometimes abisent

Oesophagus crenate towards base; calvarium simple or reduced

Eyes 0; neck \pm tapering; calvarium reduced; cervical setae in several rows.... Stenolaimus Marion 1870 Eyes with lenses

Pharynx simple; calvarium reduced; neck tapering

Nasinema Fil. 1927

Pharynx with tooth-like processes; calvarium simple; neck \pm cylindroid..... Phanoderma Bast, 1865

Oesophagus not crenate; calvarium absent

(=Heterocephalus Marion 1870)

Jaws absent

The pharynx tapering into oesophagus; cephalic setae 6, cervical small. . Paroxystomina Micol. 1924

The phary nx broad, thin-walled,base truncate with mound;amph.with pocket Pelagonema Cobb 1894

Amphid known (for alternative see preceding page)

External amphid not spiral, circular nor elliptical; female known

Ovary 1 ; amphid not linear

Gonad \% outstretched; lips 6, thin, each with 3 parallel-sided cutic. ribs; marine Daptonema Cobb 1920

Gonad \& reflexed

The ovary posterior; spinneret absent; marine

(cf. Theristus Bast. 1865)

Ovaries 2

Gonads $\&$ outstretched; marine exc. Monhysters

Habitat soil among roots; amphid a transverse slit; pharynx ovoid.

Monhystera Bast. 1865

(=M. dintheriana de Man 1885)

Habitat marine

Amphid large, long-oval, on triangular plate, 土as wide as head; spinneret none... Didelta Cobb 1920

Amphid not on special plate

Mouth ventrad of axis; amphid large, long, "folded'.....................Campylaimus Cobb 1920

Mouth axial; amphid shorter

Odontis absent; amphid flattened, more or less reniform

Odontia, if present, 6 or 12 ; amphid crook-shaped or "folded"

Tail conoid; body slender; amphid smaller.................... Axonolaimus de Man 188

Gonads \& reflexed

Habitat freshwater

Pharynx larger, domed; lips 6, thin; onchia small, in posterior pharynx...... (Trilobus Bast. 1865)

Pharynx small, elosed; lips 3, rounded; onchium minute, deepset............... (Tripyla Bast. 1865)

Habitat marine

Wings present; amphid a transverse slit almost as wide as head.

(=Promononchus Micol. 1923)

ings absent; amphid with internal pocket (Rhabdocoma ?); Enoplidae (Rhabdocoma?)

Phaphagus crenate towards base

Pharynx with 2-3 forward-pointing tooth-like processes; calvarium pres... (Phanoderma Bast. 1865)

Oesophagus not crenate

(=Heterocephalus Marion 1870)

Calvarium present; onchis often 1-3

The calvarium elaborate, ornate, relatively short.

Deontostoma Fil. 1916

The calvarium a mere band margin wavy; external amphid transverse-oval Cylicolaimus de Man 1889

Calvarium absent

Lateral series of setae on neck absent; spinneret absent.

Lateral series of setae on neck of few members

Excretory pore on projection; pharynx definite, Iquadrate; testis 1...Paranticoma Micol. 1930

Excretory pore not raised; pharynx Iconoid, of ten obscure; testes 2

Gubernaculum present; $\sigma^{7}$ pre-anal ventral supplementary organ tubular Anticoma Bast. 1865

Gubernaculum none; $\sigma^{\text {T }}$ ventral pre-anal supplementary organ papilloid Anticomopsis Micol. 1930 External amphid spiral, circular or elliptical

Female not known; marine exc. Dintheria (for alternative see next page)

Spinneret none;ceph.set.4,long;amph.transverse-oval,

Spinneret present (Neurella, Bognenia ?)

Nema broad, tapering from head to tail; wings 12-24; amphid spiral...........Pteronium Cobb 1933

Nema slender, not widest at head; wings none or few

Amphid multispire

Wing single; cephalic setae 4; amphid broad, of few winds; spinneret(?)........ Neurella Cobb 1920

Wings absent; striae not altered laterally

Onchia 3,at tops apophyses;ceph.set.papilloid;pharynx complex,bulb strong Trogolaimus Cobb 1920

Onchia 0 ; cephalic setae longer; lips flap-like, at least distally

Lip supports thickened; phar. cyathiform; $\sigma^{\prime}$ supplements many, chromadoroid Dispira Cobb 1933

Lip supports none; pharynx spheroid, on front of head; o o' supplements 0. . Dispirella Cobb 1933

Amphid circular, elliptical or monospiral

Wings strong; amphid circular or monospiral

Oesoph.compound,wide anteriorly,card.bulb faint;amph.spiral;annules wide Dasynemella Cobb 1933

Oesoph. not compound; amphid circular

(=Dasynema Cobb 1920)

The wings.12, of spine-like markings...

Xenolaimus Cobb 1920

The wing single, with subordinate lines; amphids joined dorsally........ (Antomicron Cobb 1920)

Wings 0 ; strise not altered laterally

Onchium \pm spear-like; cephalic setae 10; cephalic cuticle thickened; eyes 2 (Cophonchus Cobb 1920)

Onchium none (Nudolaimus?) 
Nema 9-10 mm.; striae 0; pharyngeal walls heavily cuticularized; amphid small

Amphid labial, spiral; phar. large, processes basal; ceph. set. 4; eyes 0 ... (Nudolaimus Allgen 1929)

Amphid 1 head-width back, oval; phar. small; ceph. set. 10; eyes 2... Leptosomatides Fil. 1918 Nema under $3 \mathrm{~mm}$.; cephalic setse 4-6

Cephalic setae at least 2 head-widths long

Lip region discoid, set off; odontia 6; amphid small, oval............Stephanolaimus Ditlev, 1918

Lip region not discoid; odontia none; amphid large, circular.

Ceponolaimus Cobb 1933

Pharynx a wide tube; heed strongly conoid width of natia

Pharynx shallow; bead not strongly conoid

Cuticle annulated; amphid oval, near lips; lips 3, massive, low.......... Zygonemella Cobb 1920

Cuticle with fine striae or none; amph. circular, \pm 1 head-width back; lips \pm confluent

Ribs of pharynx 6, cuticularized, parallel to axis..................... Rhadinema Cobb 1920

Ribs of pharynx none; pharynx conoid............................... Bognenia Allgen 1932

Female known (for alternative see preceding page)

Ovary 1; amphid not multispire

Gonad \& reflexed; amphid circular or elliptical

The ovary posterior; marine

Spinneret none; cephalic setae 4; tail usually about $40 \%$

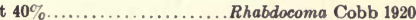

Spinneret present; cephalic setae 0 ; tail about $3 \% \ldots \ldots \ldots \ldots \ldots \ldots \ldots \ldots \ldots \ldots \ldots$ Nemanema Cobb 1920

The ovary anterior; cephalic setae present (in Monhystrium sometimes papilioid)

Habitat not marine; spinneret present

Pharynx deep; onch. small, basal; lips massive, with flaps; vulva $80 \%$; soil (Trischistoma Cobb 1913)

Pharynx less deep, vestibule definite; onchia 0 ; in gills of land crabs...... (Monhystrium Cobb 1920)

Habitat marine

Spinneret 0; ceph. set. 10; caudal setae thorn-like; amphid large, center raised (Zanema Cobb 1920)

Spinneret pres.; ceph. set. 4, small; thorn-like setae 0; phar. Icylind..(Conolaimella Allgen 1930)

Gonad of outstretched, anterior; usually marine

Spinneret none; wings 0; amphid circular; marine

Amphid large, with central mound; caudal setae thorn-like; nema $0.9 \%$ wide..(Zanema Cobb 1920)

Amphid not raised centrally; caudal setae if present not thorn-like

Labial palps 6 ; pharynx \pm quadrate; tail conoid, $4 \%$

Crystallonema Cobb 1920

Labial palps 0 ; pharynx conoid then spheroid; tail long, filiform...... (Diplolaimella Allgen 1929)

Spinneret present; marine exc. Monhystrium, and sometimes Monhystera and Theristus

Lips 3, each with finger-like odontium; striae coarse; wings 32, fewer towards tail $X$ yala Cobb 1920

Lips without finger-like processes; wings 0 or few

Mouth cavity large, complex, of several chambers, the first of radial elements; carnivorous

Base pharynx unsymmetrical, meeting oesoph.; ;middle chamber denticulate Sphaerolaimus Bast. 1865

Base pharynx symmetrical,oesoph.surrounding 2 chambers;dents.0 Parasphaerolaimus Ditlev. 1918

Mouth cavity smaller, of 1 or 2 chambers, walls relatively simple

Lips 6, conoid, twice high as broad, with hook-shaped set,; amph. monospire Dactylaimus Cobb 1920

Lips not as high as broad, without hook-shaped setae; mostly Monhysterinae

Caudal glands pre-anal, reaching vulva; excret. pore nr. phar.; ceph. set. 4 Conolaimella Allgen 1930

Caudal glands post-anal; excret. pore nr. nerve ring, when seen; ceph. set. oft many

Labial ribs 18, cuticularized, parallel-sided; testes $2 \ldots \ldots \ldots \ldots \ldots \ldots \ldots$. Daptonema Cobb 1920

(cf. Theristus Bast. 1865

Labial ribs absent or (Monhystrium) reduced;genera resembling Monhystera,exc.Sphaerocephalum

Margin of transverse-oval amphidial area not definite, sensory papilla at posterior margin

Intestine narrow, $\frac{1}{3}$ body-width; gubernaculum tubular............... Leptogastrella Cobb 1920

Intestine wider(?); gubernaculum not tubular(?)............ Paramonhystera (Steiner) Fil. 1918

Margin of amphidial area definite, sensory papilla not obvious

Habitat gills of land crabs; cephalic setse 10, sometimes papilloid. .....Monhystrium Cobb 1920

Habitat aquatic; marine exc. sometimes Monhystera and Theristus

Pharynx conoid, then spheroid; striae fine or 0; eyes oft present. . Diplolaimella Allgen 1929 Pharynx without spheroid chamber; amphid circular exc. Sphaerocephalum

Amphid monospire; neck 5-9\%; spinneret wide, plug striated...Sphaerocephalum Fil. 1918

Amphid circular; neck 10-27\%; spinneret without striated plug

Oesophagus wide in post, half; spicula hamate distally; gubernaculum 0 A ustronema Cobb 1914

Oesophagus not wider posteriorly; gubernaculum present

setae on head 8-fold; somatic set. oft long..Steineria (Micol.) Stekhoven \& de Coninck 1933

Setae on head not 8-fold; long paired somatic setae absent

Gubernaculum tubular, barbed, apophysis none............Penzancia (de Man) Fil. 1918

Gubernaculum not tubular

Spicula not much bent; gubernac. apophysate; mostly freshwater...Monhystera Bast. 1865

Spicula much bent or sickle-shape; gubernac. plate-like, dorsad ...... Theristus Bast. 1865)

(= Allomonhystera Micol. 1923)

(cf. Daptonema Cobb 1920)

Ovaries 2

Gonads o outstretched; marine (for alternative see next page)

Nema 12-16\% wide; neck "collared"; amphid spiral; wings many; spinneret present

Wings over 50; pharynx narrow; spicula 2, equal....................... Richtersia Steiner 1916

Wings 16-20, in pairs; pharynx capacious, open; spicula unequal, or but 1 .... (Richtersiella Kreis 1929)

Nema under $3 \%$ wide; neck not "collared"; wings if present not numerous

Spinneret absent; amphid circular exc. Didelta

Setao none; amphid \pm balf as wide as head.

Setae present; amphid small exc. Didelta

Amphid very large, long-oval, on triangular plate; cephalic setae 4.

Amphid small, not on plate; cephalic setae 6 or more

Pharyngeal bulb distinct; cephalic setae 6, stout, jointed............... Cytolaimium Cobb 1920

Pharyngeal bulb none; setae on head 18, small; amph. with faint multispire...Anticyathus Cobb 1920

Spinneret present; cephalic setae present exc. Margonema

External amphid multispire

Male ventral pre-anal supplementary organs papilloid................ Parasabatieria de Man 1907

Male supplementary organs none.

External amphid not multispire (Linhomoella faintly so) 
Amphid wider than long, \pm reniform; lips 3 , double, with papillae

Margonema Cobb 1920

Amphid wider than long, \pm ren
Amphid circular or monospiral

Lips distinct, with distal flaps; ceph. set. 6, stout, jointed; papillae 6... Cytolaimium Cobb 1920 Lips absent or confluent

Oesoph.\& lumen with oval enlargement behind eyes;eyessometimes none Araeolaimus de Man 1888

Oesoph. without median enlargement; eyes 0; cephalic setae 6-10

Setae on head 10, shorter 4 specialized at tip; amph. faint multispire. (Linhomoella Cobb 1920)

Setae on head 6-8, without specialized tips; amphid circular............. Linhomoers Bast. 1865 Gonads of reflexed (for alternative see preceding page)

Habitat not marine exc. sometimes A phanolaimus

Spinneret absent; amphid multispire; setae 0; striae of dots; in sand dunes. . Choanolaimus de Man 1880 Spinneret present

Wings present; contour crenate; glands lateral, serial; amphid monospire. A A phanolaimus de Man 1880

Wings 0, striae not altered laterally; contour not crenate; amphid circular or oval

Lips, papillae \& setae 0; amphid round; pharynx small, conoid, unarmed Diplohystera de Cillis 1917

Lips, papillae \& small setae pres.; amph small, oval; phar. closed; onch. basal Tripyla Bast. 1865

Habitat marine

Amphid spiral; spinneret present

Pharynx with apophyses forward and backward from mid-region

Mid-pharyngeal region not denticulate; wings numerous, markings V-shaped ....Nunema Cobb 1933

Mid-pharyngeal region denticulate ............................ Halichoanolaimus de Man 1886

Pharynx without forward apophyses; mid-pharyngeal region not denticulate

=Smalsundia Allgen 1929)

Wings numerous; neck region "collared"; nema 12-16\% wide

Spicula 2; wings over 50 ; pharynx narrow

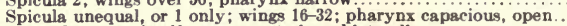

Wings absent, striae if present not altered laterally

Jaws 3, with backward apophyses and numerous horny teeth anteriorly

Richtersia Steiner 1916

Richtersiella Kreis 1929)

Jaws absent, pbarynx without apophyses

Pharynx 2 chambers; ceph. set. 10, oft jointed, tips specialized; striae fine Tripyloides de Man 1886

Pharynx 1 chamber; tips setae unspecialized; striae less fine; eyes present

Cuticular pores none; pharynx with definite bulb.

Cuticular pores present; pharynx without definite bulb ........ Nathoimus Bast. 1865

(=Necticonema Marion 1870)

Amphid circular, elliptical or monospiral

Spinneret absent; cephalic setae 4, stout; tail usually $\pm 40 \%$

Spinneret present

Oesophagus crenate towards base; calvarium reduced

Cervical setae in long. rows; pharynx \pm none; ext. amph. 土semi-circular (Stenolaimus Marion 1870)

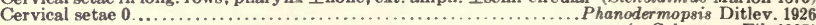

Oesophagus not crenate

(=Galeonema Fil. 1927)

Calvarium present; onchis often 1-3

The calvarium elaborate, ornate.

Deontostoma Fil. 1916

The calvarium a wavy band; often denticles at lips; amphid transverse. . Cylicolaimus de Man 1889

Calvarium absent

Jaws 3 , with simple supporting framework ......................... Parenoplus Fil. 1927 Jaws absent

Pharynx of 2 chambers

Chambers of pharynx tequal, small; cephalic setae 0

Chambers of pharym unequal, anterior large; setae 10 , often

Both

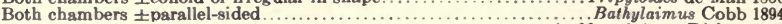

(= Macrolaimus Ditlev. 1918

=Cothonolaimus Ditlev. 1919)

Pharynx of 1 chamber; setae without specialized tips

Dorsal pharyng. wall thick, tooth-like; amph. monospire at lips; ceph. set. 4 ; renette far back

Oesoph. glands obscuring junct. of intest.; dorsal phar. element \pm uniform Onchium Cobb 1920

Oesoph. glands not prominent; dorsal pharyng. element not uniformly thick, but heavy

Tooth-like thickening parallel to axis........................ Camacolaimus de Man 1889

(=Digitonchus Cobb 1920

= Acontiolaimus Fil. 1918)

Tooth-like thickening bent outward from beginning of vestibule

Point of "onchium" single.

Point of Y-shaped "onchium" duplex.

Acmaeolaimus Fil. 1918 Ypsilon Cobb 1920

Dorsal pharyngeal wall not thickened; amphid not at lips; Enoplidae, exc. Aphanolaimus

The pharynx open, capacious, walls usually heavily cuticularized; amphid with pocket

External amphid circular; wall of pharynx irregularly thick....... (Pandolaimus Allgen 1929)

External amphid small,transverse;pharyng.wall thin.base with mounds Pelagonema Cobb 1894

The pharynx small or very narrow

Cervical setae absent; cephalic setae 4, in front of amphid........ A phanolaimus de Man 1880 Cervical setae absent; cephalic setae 4 , in front of amphid........ A phan lateral, in short longitudinal series; amphid with pocket

Gubernaculum present; $\sigma^{7}$ supplementary organ tubular.............. Anticoma Bast. 1865

Gubernaculum none; $\sigma^{7}$ supplementary organ papilloid............. Anticomopsis Micol. 1930

Cavity more or less cylindroid or prismoid, i.e. parallel-sided in long, section (for alt. see page 470)

Oesophagus with median or posterior bulb or swelling, or both (for alternative see page 477)

Amphid not known or obscure (for alternative see next page)

Female not known

Spinneret none;ceph.set.0;phar.closed;oesoph.cephaloboid;neck over 20\%;in swamp Litonema Cobb 1920

Spinneret present; setae on head numerous; neck under $5 \%$; amphid small, near lips; marine

Pharyngeal bulb present; striae none; length $3-4 \mathrm{~mm}$; onchia(?) 6 , minute.......(Catanema Cobb 1920 )

Pharyngeal bulb none; striae annulating the cuticle; length $10 \mathrm{~mm} \ldots \ldots \ldots \ldots \ldots$ (Laxonema Cobb 1920)

Female known; spinneret none exc. Rhabdolaimus; not marine; phasmids usually present 
Ovary 1, anterior; cephalic setae 0 or minute

Gonad \& outstretched

Pharynx long, tubular, base half way to nerve ring: spear none; median bulb 0 .. Gymnolaimus Cobb 1913 Pharynx base $\pm \frac{1}{s}$ distance to nerve ring; spear reduced; oes, bulb strong ( $A$ phelenchoides Fischer 1894)

(=Seinura Fuchs 1931

= Parasitaphelenchus Fuchs 1930

Gonad \& reflexed $=$ Pathoaphelenchus (Cobb) Steiner 1931)

Median oesophageal bulb present

Pharynx wide as long; cardiac bulb not valvate, median valvate; bursa none..Lycolaimus Rahm 1928

Pharynx much longer than wide; cardiac bulb valvate; burss present...........Rhabditis Duj. 1845 (=Diploscapteroides Rahm 1928 =Pseudorhabditis Kreis 1929)

Median oesophageal bulb absent; in soil and fresh water

Cardiac swelling long, non-valvate; pharyng. rods heavily cuticular.... Diphtherophora de Man 1880 (=Choolaimus Cobb 1893

$=$ Archionchus Cobb 1913)

Cardiac bulb valvate, preceded by isthmus, i.e. oesoph. cephaloboid; pharyng. rods none

Head end lobed by 6 large biting lips or odontia; carnivorous........... (Teratocephalus de Man 1876) Head end not lobed, exc. in Acrobeles insubricus

Pharynx wall simple,forming 2 chambers;onchium oft present;ceph.set.0-4 Macrolaimus Maupas 1900

Pharynx wall of a longitudinal series of elements, i.e. cephaloboid

Probolse on head none....................................Panagrolaimus Fuchs 1930

Probolae on head in 1 or 2 circlets

Circlets of probolae 2, elements of ten elaborate

Circlet of probolae single.

Acrobeles Linst. 1877

Cephalobus Bast. 1865

Ovaries 2; cephalic setae 0 , exc. in Teratocephalus minut

Gonads $\%$ outstretched; ceph. set. 0; phar. tubular, reaching oesoph. bulb; in soil A ulolaimus de Man 1880 Gonads $\%$ reflexed

Spinneret present; wings absent; in fresh water and moist soil. Spinneret absent

Cuticle with prominent tubercles; lip reg. set off by constrict.; ceph. set. with saccate bases

Tubercles encrusting dorsal surface; wings brosd, thin; in moss ........Craspedonema Richters 1908

Tubercles in longitudinal row (s), right lateral; oesophagus rhabditoid.......Bunonema Jägerskiöld 1905

Cuticle without tubercles

Pharynx a long straight tube, 1 neck length or more

Oesoph. short, a muscular bulb only; $\sigma^{7}$ supplements few, ventral, pre-anal Aulolaimus de Man 1880

Oesoph. long, of 2 parts; $\sigma^{7}$ supplements paired, post-anal, i.e. diplogastroid

Male papillae 9 pairs; gubernaculum sigmoid; labial papillae $16 \ldots . . . . . .$. Myctolaimus Cobb 1920

Male papillae 10 pairs; gubernaculum straight; bursal membrane narrow... Cylindrogaster Groodey 1927

Pharynx not a simple tube, much shorter

Oesophagus without median bulb

Cuticularized rods supporting pharynx; shed cuticle surrounding nema Diphtherophora de Man 1880 (=Chaolaimus Cobb 1893

= Archionchus Cobb 1913)

Cuticularized pharyngeal rods none

Head end lobed by 6 large biting lips or odontia; carnivorous........ (Teratocephalus de Man 1876)

Head end not lobed; bursa present.

Oesophagus with median and cardiac bulbs

Cardiac bulb valvate; median bulb well set off; glottoid apparatus present

Wall of pharynx cylindroid; bursa present; $\sigma^{7}$ tail rhabditoid................Rhabditis Duj. 1845

Wall of pharynx convex inward; bursa much reduced; $\sigma^{7}$ tail long, spicate $R$ habditoides Goodey 1929 $(=$ Rhabditella (Cobb) Chitwood 1933)

Cardiac bulb without valve; median bulb valvate; of tail setaceous, diplogastroid

Pharyng. rods 3, basal bulbs 2, stalked; tail 25-41\%; long. striae fine..... Tylopharynx de Man 1876

Pharyng. bulbed supporting-rods none; Diplogasterinae

Nema with evident longitudinal striation..................... Diplogaster M. Schultze 1857

Nema without longitudinal striation

Pharynx wide as deep, wall of several elements; ơ papillae 0(?).......Peronilaimus Rahm 1928

Pharynx tubular, wall entire; o⿱ papillae usually 5 pairs............... Rhabditidoides Rahm 1928

Amphid known (for alternative see preceding page)

External amphid not spiral, circular nor elliptical (for alternative see next page)

Habitat marine; cephalic setae and spinneret present; amphid crook-shaped, sometimes \pm spiral

Ambulatory \& somatic set. 0; nema not crooked, not narrowed in card. reg.; ventral arm amph. prolonged

Phar.wide, heavy,4-5\%;amphid long,4-50\% body;ceph.setae 4,subceph.12; - 9-...Pseudolella Cobb 1920

Phar.narrow, inconspic.;amph.under $2 \%$;ceph.set.6,subceph.4;annules tiled; 'O' Ceramonema Cobb 1920

(cf. Pselionema Cobb 1933)

Ambulatory set. forming ventral pre-anal sole; nema \pm S-shape, narrowed in card. reg.; marine; ' $O$ '

Oesoph. region hardly swollen; card. bulb faint; cerv. striae all alike.. Notochaetosoma Irwin-Smith 1918

Oesoph. region swollen, ovoid; oesophageal bulbs 1 or 2 ; band of cervical striae

accentuated, exc. Tristicochaeta falcatum

Ventral ambulatory adbesion tubes or setae in 2 rows.................. Drepanonema Cobb 1933 (=Chaetosoma Clap. 1863)

Ventral ambulatory adhesion tubes or setae in 3 or 4 rows............... Tristicochaeta Panceri 1878

Habitat not marine

Spinneret present; ' $\$$ ', exc. Chronogaster

Lips 6, distinct, revolute; pharynx 9\%; amphid semicircular; soil about cane roots Anthonema Cobb 1906 Lips not revolute; in fresh water

Cephalic setae 4; pharynx wider, under $1.5 \%$; ‘ㅇ

Cephalic setae 0 ; pharynx long-tubular; tail $25-30 \% \ldots \ldots \ldots \ldots \ldots \ldots \ldots \ldots \ldots \ldots$ Rhabdolaimus de Man 1880

Spinneret absent; cephalic setae 0 or papilloid

Tail setaceous, $19 \%$; oesoph. with 2 bulbs; amph. semicircular; vulva $21 \%$; 'o' A ulolaimnides Micol. 1915

Tail not setaceous, under $15 \%$; oesophagus without median bulb, exc. Cheilobıs 
Amphid transverse, curved, wide as head; phar. $7.6 \%$; tail $2 \%$; fresh water; ơ only Colpurella Cobb 1920 Amphid inconspicuous, small or slit-like; tail over $9 \%$; in soil or decaying material

Median oesoph. swelling before isthmus; cardiac bulb valvate; wings single; ' $O$ '. Cheilobus Cobb 1924

Median oesoph. bulb absent

(=Rhabditophanes Fuchs 1930)

Pharynx closed; amphid a pore, with internal cuticularized tube; wings 2; J. ... Litonema Cobb 1920 Pharynx open, or with anterior open chamber; wings $0 ; \sigma^{7}$ only

Tail 15\%, terminus setaceous; pharynx without cuticularized rods........ (Monhystera Bast. 1865)

$(=M$. dintheriana de Man 1885)

Tail 8\%, conoid; cuticularized rods in pharynx; shed cuticle retained...Diphtherophora de Man 1880 (= Chaolaimus Cobb 1893

External ampbid spiral, circular or elliptical (for alternative see preceding page)

Female not known; spinneret present

Habitat soil; ceph. set. 0; ceph. cuticle thick, flaps doming vestibule; amph. spiral Pycnolaimus Cobb 1920

Habitat marine; cephalic setae present

Ampbid multispire; annules not coarse; wings absent

Head cylindroid; lip reg. set off by constriction; pharyng. bulb present...(Choniolaimus Ditlev. 1918)

Head truncate-conoid;lip reg.not set off;pharyngeal bulb none;onch.3,minute Alaimonema Cobb 1920

Amphid circular, elliptical or monospiral

Wings 8; annules coarse; oesoph. to isthmus wide, muscular; card. swelling slight Dasynemella Cobb 1933

Wings 0

$(=$ Dasynema Cobb 1920)

Cutilarized pharyng. ring basal; ceph. set. 4; $\sigma^{7}$ supplements tubular, 14....Cricolaimus Southern 1914

Cuticularized pharyngeal ring none

Spiculum 1, gubernaculum absent; cephalic setae 10; amphid large, oval..Pelagonemella Kreis 1932

Spicula 2, gubernaculum present (Chloronemella?); cephalic setae in circlets of 4

Striae coarse; amphid minute; length $10 \mathrm{~mm}$; width $0.8 \% \ldots \ldots \ldots \ldots \ldots \ldots \ldots$.............

Striae fine; length under $4 \mathrm{~mm}$.

Pharyngeal bulb present; amphid at lips; onchia 6 , minute...............Catanema Cobb 1920 Female known

Pharyngeal bulb none; amphid behind pharynx; onchia $0 \ldots \ldots \ldots \ldots \ldots \ldots$ Chloronemella Allgen 1929

Ovary 1

Gonad $\&$ outstretched, anterior; amphid circular; wings absent

Spinneret present; pharynx long-conoid; neck $17 \%$; in fresh water..............Monhystera Bast. 1865

Spinneret absent; neck not over $5 \%$; marine

(i.e. subg. Monhystrella Cobb 1918)

Pharynx wide as deep; cardisc swelling faint, elongate; intestine not dark. . Crystallonema Cobb 1920

Pharynx tubular; posterior $3-1$ oesoph. a massive bulb; intestine dark colored (Solenolaimus ?)

Cephalic setae minute; neck 1.4\%; anterior oesoph. fusiform; tail rounded..Solenolaimus Cobb 1894

Cephalic setae often as long as mitreform bead is wide; neck $2-5 \%$

Anterior oesoph. not fusiform; pharynx not evertible; o" supplements 0... Chromagaster Cobb 1894

Anterior oesoph.fusif orm;phar." "spear" evertible; $\sigma^{7}$ supplements pres.. Siphonolaimus de Man 1893

Gonad \& reflexed; wings of ten present; not marine exc. rarely Rhabditis

The ovary posterior,vulva $21 \%$;med.bulb not valvate;amph.semicirc.;mud. . A ulolaimoides Micol. 1915

The ovary anterior

Spinneret pres.; cepb. set. 4; card. bulb 0 or faint; amph. monospire; freshwater (A nonchus Cobb 1913)

Spinneret none; setae none, cephalic papillae sometimes setose

Oesophageal bulbs median and cardiac; pharynx simple; setae 0

Cardiac bulb valvate; amphid at lips, obscure; wings \& bursa pres.; ubiquitous Rhabditis Duj. 1845

Cardiac bulb not valvate, median valvate; amph. transverse-oval, not at lips; phar. cylindroid

Dorsal onchium none; bursa present; ectoparasitic on beetle borer.......Rhabditolaim us Fuchs 1915

Dorsal onch. small, deepset; burşa 0 ; in exudate chestnut blight.. (Diplogasteroides de Man 1912)

Oesophageal bulb cardiac only, preceded by isthmus; phar. of serial plates; bursa 0 or reduced

Head with 6 large odontia; amphid not obscure, of ten large

Nema mostly cephalated; cirri 0; amphid circular; soil \& fresh water.. Teratocephalus de Man 1876

Nema not cephalated; cirri 6 ; amph, transverse-oval; tail hooked; in bark Chambersiella Cobb 1920

(=Diastolaimus Rahm 1928)

Head entire or 3-parted at lips; nema not cephalated, exc. Acrobeles insubricus; amph. small

Probolae absent; onchium 0 , or 1 inward-pointing......................Macrolaimus Maupas 1900

Probolae on head in 1 or 2 circlets

Circlets of probolae 2, labial and cephalic; amphid a pore near lips..... (Acrobeles Linst. 1877)

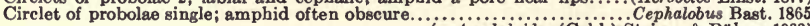
(=Acrobeloides (Cobb) Steiner \& Bührer 1933)

Ovaries 2

Gonads $\&$ outstretched; cephalic setae present; marine or in brackish soil

Amphid multispire; cephalic setae 10, unequal

Cardiac bulb ellipsoid; 6 of ceph. set. short; subcephalic setae 4; tail 30\%, fine. . Laimella Cobb 1920

Cardiac bulb slight; 4 of ceph. set. short, tips specialized; tail $7 \%$.......... (Linhomoella Cobb 1920)

Amphid circular or monospiral; tail not over $18 \%$, not setaceous

Gland ducts cuticularized, piercing cuticle, ventrad, cerv. \& pre-anal. . Halaphanolaimus Southern 1914

Gland ducts and pores absent; pharynx minute or none

Pharynx \pm none; ceph. set. 10, 4 short, tips specialized; tail 7\%............ (Linhomoella Cobb 1920)

Pharynx obconoid, when open $3-1$ as wide as head; spinneret sometimes present

Cuticularized rings in pharynx 1-3; neck 8-14\%; marine \& brackish soil (Desmolaimus de Man 1880)

Cuticularized pharyngeal rings absent; ventriculus present; marine

Base pharynx not cuticularized; cardiac bulb oft valvate; head tconoid Cryptolaimus Cobb 1933

Base pharynx cuticularized; cardiac bulb valvate; head Icylindroid Metalinhomoeus de Man 1907

Gonads $\&$ reflexed

(cf. Deltanema Kreis 1929)

Habitat not marine, exc. rarely Rhabditis and Haliplectus (for alternative see next page)

Oesophageal bulbs median and cardiac; cephalic setae 0; pharynx \pm tubular (for alt. see next page)

Vulva $21 \%$; anus $81 \%$; amph. semicirc., head-width; wings \& bursa none (A ulolaimoides Micol. 1915)

Vulva $40-94 \%$; in soil etc., in fresh water, and marine

Spinneret pres.;amph.monospire,protruding;wings \& bursa 0;pores lateral.. Haliplectus Cobb 1913 
Spinneret none; cardiac bulb valvate; amphid obscure; wings and bursa present Rhabditis Duj. 1845

Oesophageal bulb cardiac only (for alternative see preceding page)

Spinneret absent; ampbid circular, elliptical or monospiral

Head without odontia; nema not cephalated; setae 0; spear minute; in soil (Triplonchium Cobb 1920)

Head with 6 large odontia; bursa none or mucb reduced

Nema mostly cephalated; cirri 0 ; amph. circular; in soil \& fresh water...Teratocephalus de Man 1876

Nema not cephalated; cirri 6; amphid transverse-oval; tail booked; in bark Chambersiella Cobb 1920

Spinneret present

Pharynx simple; oesophagus not plectoid; neck 15-20\%

Cephalic setae 0; wings 0; pharynx long; amphid witb pocket; tail 27\% (Rhabdolaimus de Man 1880)

Cephalic setae 4; pharynx short; tail $15 \%$

Wings present; amphid monospire............................Paraphanolaimus Micol. 1923

Wings absent; amphid circular; oncbium minute, not at lips...............Prodesmodora Micol. 1923

Pharynx compound-elongate; oes. oft with isthmus to card. bulb (plectoid); wings pres.

Labial region not expanded; cephalic setae usually 4 , sometimes 0 or 6 ; neck $12-30 \%$

Cardiac bulb not valvate; pharynx long; neck 14-15\%; in fresh water \& mud Paraplectus Fil. 1930

Cardiac bulb valvate; amphid circular or transverse-oval..................... Plectus Bast. 1865

Labial region expanded, with complicated appendages; phary $\mathrm{nx} 4-9 \% ; \mathrm{neck} 27-31 \%$

Lips 6, distinct, revolute; striae fine; amphid semicircular; pharynx 9\% (Anthonema Cobb 1906)

Lips not revolute; bead with dorso-ventral bladders; striae coarse; amph. round; phar. 3-6\%

Cephalic appendages elaborately palmate.........................Wilsonema Cobb 1913

Cephalic appendages forming a closed, striated dome or cupola... Bitholinema de Coninck 1931

Habitat marine or brackish (for alternative see preceding page)

Ambulatory set. forming a ventral sole, midbody to pre-anal; nema crooked; calvarium unstriated

Nema narrowest in card. reg., \pm S-sbaped; ambulatory set. bollow, pre-anal; annules finer

Oesoph. region hardly swollen; card. bulb faint; cerv. striae alike Notochaetosoma Irwin-Smith 1918

Oesoph. region swollen, ovoid; oesopbageal bulbs 1 or 2 ; band of cervical striae accentuated, exc. Tristicochaeta falcatum

Ventral ambulatory adhesion tubes or setae in 2 rows.................. Drepanonema Cobb 1933 (=Chaetosoma Clap. 1863)

Ventral ambulatory adhesion tubes or setae in 3 or 4 rows............. Tristicochaeta Panceri 1878

(=Draconema Cobb 1913)

Nema narrowest midway, epsilon-shaped; ambulatory setae not open at end; annules coarser

Sole apparently absent; amphid cresentic; annules $98 . \ldots \ldots \ldots \ldots \ldots \ldots \ldots$. Epsilonella Steiner 1930

Sole present; amphid circular or monospiral

Oesophagus with median and cardiac bulbs.........................etepsilonema Steiner 1927

Oesophag us with cardiac bulb only

Annules 80-112; ambulatory setae slender, falcate...............Bathyepsilonema Steiner 1927

Annules 144-192; ambulatory setae straight or slightly arcuate

Body cylindroid, if crooked faintly so......................... Archepsilonema Steiner 1927

Body epsilon-like, i.e. body arches well developed

Cuticular annules bomogeneous in structure.............Prochaetosoma Baylis \& Daubney 1926

Cuticular annules not bomogeneous in structure (=Rhabdogaster Metsch. 1867)

"Axial" part of the cuticular annules hollow ("frame rings").........Epsilonema Steiner 1927

"Axial", part of the cuticular annules hollow " "frame rings")..........Epsilonema Steiner 1927 Ambulatory setae 0; nema not crooked; calvarium absent, or not truncate-conoid

Lips 2,moutb a slit;amph.faint multispire;" pseudonchs" dorso-ventral ridges Pseudonchus Cobb 1920 Lips not 2, mouth not a slit; "pseudonchs" absent

Amphid multispire; neck 9-13\%

Pharynx deeper than bead width, wall irregular, with projections; striae plain Zalonema Cobb 1920

Pharynx tubular, less deep, wall straight; striae of dots .................... Kreisia Allgen 1929

Amphid circular, elliptical or monospiral; neck $14-25 \%$, exc. Bolbonema $8 \%$

Wings present; anterior end set off by groove at 4 ceph. set. \& usually by lack of annulation

Neck $20-25 \%$

Species marine; striae duplex; wings 2; phar tubular, inconspic., prob. double A plectus Cobb 1914

Species in brackish soil \& marsh; lip reg. discoid; ceph. set. papilloid...Leptolaimus de Man 1876

Neck $14-16 \%$; marine

Gland ducts cuticularized, piercing cuticle, cerv. and pre-ana].. Hala phanolaimus Southern 1914

Gland ducts \& pores 0; pharynx of several long chambers; amphid round Polylaimium Cobb 1920

Wings absent

Median bulb present; ceph, set. 0 ; amph. protruding: neck $12 \%$; pores lateral Haliplectus Cobb 1913

Median bulb absent; pores none; marine

Nema cephalated by expansion opposite large monospiral amphid; neck 8\% Bolbonema Cobb 1920

Nema not cephalated by expansion

Setae on head 6-10, long, flexible; neck under 5\%: pharyngeel bulb present.. (Laxus Cobb 1894)

Setae on head $0-4$; neck $6 \%$ or over; pharyngeal bulb absent

Head constricted at cephalic setae, not at amphid; amphid 2 bead-widths back

Chambers of pharynx several, long; neck 10-11\%; cephalic setae 0(?) Polylaimium Cobb 1920

Chamber of pharynx single; neck 19\%; head conoid; cepbalic set. 4 Dermatolaimus Steiner 1916

Head not constricted at setae; ampbid near lips or not over 1 head-width back

Ceph.set.0;phar.a tube reaching card.bulb;amphid conspicuous.. Aegialoalaimus de Man 1907

Cepb. set 4; pharynx shorter

Amphid small,monospiral,at lips;neck 19\%; dorsal phar.wall thickened (Onchium Cobb 1920)

Amphid conspicuous, round,not at lips;neck 6\%; phar.wall not thick.. Linolaimus Cobb 1933

Oesophagus plain, i.e. without median or posterior bulb or swelling (for alternative see page 474)

Amphid not known or obscure (for alternative see next page)

Female not known; marine (for alternative see next page)

Neck over $40 \%$; amphid a long. slit; ceph. setae 4 or 8, fine; spinneret of none.. (Halalaimoides Cobb 1933)

Neck $10-30 \%$; amphid not longitudinal; spinneret present (Trileptium, Fimbriella ?)

Cephalic setae 0; onchia 3, awl-shaped, almost parallel to axis.................... (Fimbrilla Cobb 1905)

(=Fimbria Cobb 1894)

Cephalic setae 8 or more
Onchium dorsal,anterior,inward-pointing ;neck $12 \%$;nema $0.5 \%$ wide;amph. a slit (Trileptium Cobb 1923)

Onchium none; neck 22-30\%: nema 2-3\% wide; cephalic setae usually 8 , in 2 circlets $\quad(=$ Trilepta Cobb 1920) 
Calvarium present; setae 8 , near base of pharynx; eyes present....... Gullmarnia Allgen 1929

Calvarium absent; setae minute, around mouth; eyes 0; pharynx small, tubular.. Fimbriella Allgen 1929 Female known (for alternative see preceding page)

Ovary 1, anterior

Ceph. set. 4; pharynx large, open; spinneret present; spicula 2-jointed(?); marine...Filipjeva Ditlev. 1928

Ceph. set. 0 or papilloid; phary $\mathrm{nx}$ none or narrow; spinneret usually none; - ?

Pharynx a tube reaching halfway to nerve ring; in soil......................Gymnolaimus Cobb 1913

Pharynx closed, or marked by reduced spear, not over $\frac{1}{\text { distance to nerve ring }}$

Nema free-living, marine; spinneret doubtful; pharynx none or closed............... Litotes Cobb 1920

Nema parasitic in insects; spinneret 0 ; adult spear reduced, base 3-parted... (A phelenchulus Cobb 1920) Ovaries 2

Gonads $\&$ outstretched; spinneret present; marine

The ovaries extending backward; winge $10-12$; vulva $45 \%$

The ovaries opposed; wings 0

Annules none or not coarse; amphid not saccate, far back; nema not wide.... Anoplostoma Bütsch. 1874

Annules coarse; amphid saccate; nema wide; cephalic setae 4

Number annules \pm 17 , secreted annules prominent, separated; head small. . Desmoscolex Clap. 1863

Number annules 32-91, secreted annules 0; head concave-quadrate pyramidal. Tricoma Cobb 1894

Gonads \& reflexed

Spinneret none; wings 0; pharynx 2.5-3\%, tubular; tail rounded, under $2 \%$; in soil. . Isolaimium Cobb 1920 Spinneret present

Habitat freshwater; lips thick; pharynx closed; amphid small; onch. small, doepset (Tripyla Bast. 1865)

Habitat marine

Annules \pm 17 ,secreted annules prominent;amph.saccate;nema wide;ceph.set.4 . . Desmoscolex Clap. 1863

Annules none; amphid not saccate; nema less than $5 \%$ wide

Dorsal pharyngeal wall bent inward at lips,onchium-like;ceph.setae 10. . (Asymmetrella Cobb 1920)

Dorsal pharyngeal wall not onchium-like; cephalic setse 0.................Angustinema Cobb 1933 Amphid known (for alternative see preceding page)

External amphid not spiral, circular nor elliptical (for alternative see next page)

Female not known; Enoplidae (Trileptium?)

Head mitreform, set off by constriction; wings 6-8; spinneret doubtful; marine.... Xennella Cobb 1920

Head not mitreform; spinneret often absent

Ceph. set. 0; ext. amph.wide,curved;phar.tubular, halfway to nerve ring;freshwater Colpurella Cobb 1920

Ceph. set. present; amphid small, or long and narrow; marine

Amphid narrow,many head-widths long;neck $\pm 40 \%$;ceph.set.4-8;tail setaceous Halalaimoides Cobb 1933

Amphid small, transverse-oval; neck $25 \%$ or less

Neck $25 \%$;onchium none;ceph.setae 6,small,inconspicuous;spinneret present. . Vasculonema Kreis 1928

(cf. Pelagonema Cobb 1894)

Neck $12 \%$; onchium dorsal; cephalic setae 10, conspicuous; labial setae 6....Trileptium Cobb 1933

Female known

Ovary 1 , anterior

Gonad o outstretched;pharynx closed;ceph.set.papilloid spinneret doubtf ul;marine.. Litotes Cobb 1920

Gonad o reflexed; spinneret present; external amphid a transverse slit, not at lips; not marine

Onchia 0; amphid far behind the tquadrate pharynx; in fresh water...... Prismatolaimus de Man 1880 Onchium minute, deepset; amphid opposite pharynx; in soil about roots

Pharynx conoid when open; amphid opposite base of pharynx.............. (Trischistoma Cobb 1913)

Pharynx a long tube, amphid opposite its anterior end, with pocket............. Cryptonchus Cobb 1913

Ovaries 2; marine exc. Monhystera

Gonads $\&$ outstretched

Spinneret absent

Habitat marine; amphid long-oval, on large triangular plate.

............... Didelta Cobb 1920

(=M. dintheriana de Man 1885)

Spinneret present

Mouth opening 士ventrad;wing duplex;amph.big,bent,dorsal arm the longer (Campylaimus Cobb 1920)

Mouth opening axial, terminal; wings none exc. sometimes Pseudolella

Annules coarse; amphid saccate; nema wide; cephalic setae 4

Number of annules \pm 17 , secreted annules prominent, separated; head small. . Desmoscolex Clap. 1863

Number of annules 32-91, secreted annules 0; head concave-quadrate pyramidal.. Tricoma Cobb 1894

Annules none, striae fine; amphid not saccate; nema slender

Amphid small, opening transverse with internal pocket, far back. ...., (A noplostoma Bütsch. 1874)

Amphid shepherd's crook, dorsal arm the longer

Length of amphid extreme, sometimes exceeding neck; eyes $0 \ldots \ldots \ldots \ldots$....... (Peudolella Cobb 1920)

Length of amphid not over 1 head-width, loop-shaped; eyes 2, sometimes none

Oesophagus \& lumen widened between eyes and nerve ring;pharynx obvious Coinonema Cobb 1920

Oesophagus \& lumen not widened; pharynx like oesophagus... Araeolaimoides (de Man) Fil. 1918

Gonads \& reflexed

Spinneret absent; amphid with internal pocket

Cephalic setae 0; pharynx 2-3\%, tubular; tail rounded, under $2 \%$; in soil...... Isolaimium Cobb 1920

Cephalic setae 10, subcephalic many; marine.........................Platycomopsis Ditlev. 1926

Spinneret present

(=Dactylonema Fil. 1927)

Habitat freshwater; amphid usually small or inconspicuous

Pharynx wide,domed by thin lips;onch.0;amph.transverse slit,far back. . Prismatolaimus de Man 1880

Pharynx closed,lips thick;onch.minute,deepset;external amphid round or oval.. (Tripyla Bast. 1865)

Habitat marine

(=Promononchus Micol. 1923)

Cephalic setae none or papilloid; external amphid narrow, transverse (for alt. see next page)

Amphid head-width; cuticle $\frac{1}{2}$ radius, annules coarse, markings rod-like.. (Actinonema Cobb 1920)

Amphid head-width or less, with internal pocket; cuticle less thick; phar. with lumen; Enoplidae

Eyes 2; protrusile pharyngeal ribs none................................... Illium Cobb 1920 
Eyes 0; protrusile pharyngeal ribs 3, straight........................ Fimbrilla Cobb 1905

Cephalic setae present, not papilloid (for alternative see preceding page) (=Fimbria Cobb 1894) Amphid shepherd's crook

Annules not of tile-like elements...............................Parachromagaster Allgen 1929

Annules coarse, each of 8 tile-like elements.................................. Ceramonema Cobb 1920

Amphid not shepherd's crook

(cf. Prelionema Cobb 1933)

Nema wide;annules \pm 17 ,secreted annules obvious;amph .saccate;ceph.set.4. . Desmoscolex Clap. 1863

Nema slender, under $5 \%$; amphid not saccate; annules 0, striae fine or 0; Enoplidae

Onchium dorsal; labial setae 0; cephalic setae 10, near lips

Tooth vestigial, on wall; external amphid a small slit; eyes $0 \ldots . . .$. . (Anoncholaimus Cobb 1920)

Tooth a bending inward of dorsal pharyng.wall;amphid larger,faint.. (Asymmetrella Cobb 1920)

Onchia none; labial setae 6, obvious; cephalic setae 10 or more, length $1-5$ times head width

Pharynx narrow,closed;odontia (?) minute;ceph.set.3 times head width (Tubolaimella Cobb 1933)

Pharynx wide, open; odontia none

Lips 3, deep-cleft, with framework; ceph. set. 2-5 times head width........Chaetonema Fil. 1927

Lips without framework; ceph, set. 1 head-width; excret. pore raised...(Paranticoma Micol. 1930)

External amphid spiral, circular or elliptical (for alternative see preceding page)

Female not known; spinneret present; marine

Nema wide, 14-32\%, widest at lip region, tapering to terminus; wings numerous...Pteronium Cobb 1933

Nema slender, not over $5 \%$ wide, widest near middle

Amphid multispire; wings 0; cephalic \& labial setae 18; labial framework present.... Dispira Cobb 1933

Amphid circular, elliptical or monospiral

Odontia 2, large, lateral or subventral.

Odontia absent(in Stephanolaimus 6 minute ?

Onchium absent (Nudolaimus?)

Calvarium reduced to submed plates;phar large, wall strong "onchig" bosal. Nudolaimus Allgen 1020

Calvarium absent; cephalic setae present

Wings present; cephalic setae 6 or 10

Ceph. set. 6; wings 8; annules coarse, elements tile-like; oesoph. compound..Dasynemella Cobb 1933

(= Dasynema Cobb 1920)

Ceph. set. 10; wings 2; oesophagus simple; pharynx \pm quadrate........Pulchranemella Cobb 1933

Wings absent; cephalic setre 4-8

Lip region discoid, set off by constriction; odontia(?) 6..............Stephanolaimus Ditlev. 1918

Lip region not discoid; cephalic setre 4 or 6

Pharyng. vestibule with rod-like supports; head \pm cylindroid; striae 0; ceph. set. 4

Rods 6, straight, parallel to body axis; cephalic setre 4, jointed......... Rhadinema Cobb 1920

Rods more than 6 , curved, meeting axially ..................... Eleutherolaimus Fil, 1922

Pharyng. vestibule without supports; striae pres. exc. Vasculonema \& Bathylaimella

Amphid transverse-oval, near lips; head slightly conoid; setae small

Setae labial, cephalic and subcephalic, minute...................... Zygonemella Cobb 1920

Setae cephalic only, 6, small, inconspicuous..................... Vasculonema Kreis 1928

(cf. Pelagonema Cobb 1894)

Amphid circular, near base of wide-tubular pharynx

Head cylindroid; setae on head 16, longest 4 times head width.........Cenolaimus Cobb 1933

Head conoid, narrowing $50 \%$ from base; setae on head 4 , short

Cuticle striated; width (compressed ?) $6 \%$........................ Tubolaimus Allgen 1929

Female known

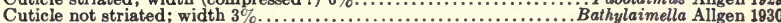

Ovary 1 (for alternative see next page)

Gonad o reflexed

Amphid multispire; ovary posterior; pharynx linear; spinneret doubtful; marine Alaimella Cabb 1920

Amphid not multispire; ovary anterior

Spinneret none; amph. round, with central mound; caudal setae thorn-like, 2 rows (Zanema Cobb 1920)

Spinneret present; tail without thorn-like setae

Habitat marine; pharynx tubular; cephalic setae 4; nema $\pm 2 \%$ wide...... (Conolaimella Allgen 1930)

Habitat not marine; pharynx open, not small nor narrow; nema $2-5 \%$ wide

Cephalic setae 4; pharynx with supporting pieces; amph. monospire; fresh-water A nonch us Cobb 1913

Cephalic setae 6 or 10, papilloid in Tripylium

A mphid monospire; in gills of land crabs.

Amphid small, circular, transverse-oval or slit-like

.(Tripylium Cobb 1920)

Onchium 0; pharynx \pm quadrate; amphid far back; in fresh water.... Prismatolaimus de Man 1880

Onchium minute, deepset; pharynx conoid; amphid opp. pharynx; in soil (Trischistoma Cobb 1920)

Gonad o outstretched

Habitat not marine

Spear minute; spinneret none; bursa present; parasitic in beetle.......... (A phelenchulus Cobb 1920)

Spear absent; bursa absent; in fresh water........................... Cylindrolaimus de Man 1880

Habitat marine

The ovary posterior

External amphid multispire; pharynx linear; tail $\pm 10 \%$; spinneret doubtful (Alaimella Cobb 1920 )

External amphid circular, pocket elongate; tail $34 \%$; spinneret none....... Halananchus Cobb 1920

The ovary anterior

Cephalic setae 4, papilloid; pharynx obscure, linear; amphid faint, open caudad.... Litotes Cobb 1920 Cephalic setae not papilloid

Spinneret absent; labial appendages ("palps") present; amphid circular

Tail with 2 rows of thorn-like setae; amphid with distinct central mound......Zanema Cobb 1920

Tail without thorn-like setae; amphid without mound; cardiac bulb faint Crystallonema Cobb 1920

Spinneret present; amphid circular or monospire

Pharynx 7\%, tubular, \pm hall way to nerve ring; head narrow ; amphid far back $R$ hynconema Cobb 1920

Pharynx less than $2 \%$, not a long tube

Depth of pharynx much greater than width, pharynx small

Oesophagus almost surrounding conoid pharynx.................Conolaimella Allgen 1930

Oesophagus meeting base of obconoid pharynx..................... Sphaerocephalum Fil. 1918

Depth of pharynx not much greater than width, pharynx large, open 
Amphid \pm 1 head-width; vulva $59 \%$; caudal set. stiff, 8 prs.; post. ovary small Halinema Cobb 1920

Amphid $\pm \frac{1}{1}$ bead-width; vulva $80-85 \%$; caudal set. 0 ; lips 6 , conoid, separate

Labial setae 6, hook-shaped, on tips of lips; amphid spiral; in mud...... (Dactylaimus Cobb 1920)

Labial setae 6, not hook-shaped nor at tips; amphid round; ceph. set. 18 . . Omicronema Cobb 1920

Ovaries 2 (for alternative see preceding page)

Gonads o outstretched: usually marine

Spinneret absent; marine exc. Pseudoaulolaimus

Habitat mud, rice fields; wings 16-32, fusing to 4 on 4-pronged tail....Pseudoaulolaimus Imamura 1931 Habitat marine

Amphid long oval, on wide triangular plate, almost as long as head is wide.

Didelta Cobb 1920

Amphid smaller, not on triangular plate

Cervical setae bunched; 2 flat setae in front of transverse-oval amphid..... (Platycoma Cobb 1894)

Cervical setae if present not bunched; amphid \pm circular

Pharynx long, simple, tubular; cephalic setae 6.................... Southerniella Allgen 1932

Pharynx minute; ceph. set. 18, minute, in 2 circlets; amphid faint multispire Anticyathus Cobb 1920

Spinneret present; marine exc. Cylindrolaimus

Habitat fresh water; ceph. set. 4; ovaries usually unequal; pharynx tubular Cylindrolaimus de Man 1880

Habitat marine

Annules few, coarse; amphid saccate; cephalic setae 4; nema wide

Number annules \pm 17 , secreted annules prominent, separated; head small. . Desmoscolex Clap. 1863

Number annules 32-91, secreted annules none; head concave-quadrate pyramidal Tricoma Cobb 1894

Annules if present not few nor cosrse; amphid not saccate

Gland ducts cuticularized. pores cervical \& pre-snal; striae coarse..... Halaphanolaimus Southern 1914

Gland ducts and pores absent

Cephalic setae 10 or more

Pharynx with transverse cuticularized ring

Cavity of pharynx simple, capacious, \pm cylindroid, t head-width....Eumorpholaimus Sehulz 1932

Cavity of pharynx small, cup-shaped, rings sometimes 2 or more......... (Linhomoeus Bast. 1865) (i.e. subg. Paralinhomoeus de Man 1907

= Paradesmolaimus Schulz 1932)

Pharynx without transverse cuticularized rings

Longest ceph. set. 4, total 16, tips simple;phar. quadrate;amph. Ineck-width Halinema Cobb 1920

Longest ceph. set. 6, 4 short specialized at tip; phar. 0(?); multispire faint Linhomoella Cobb 1920

Cephalic setae 4

Nema wide, 10-16\%; wings numerous; neck region "collared"; amphid spiral; Richtersiinae

Wings \pm 50 ; pharynx narrow; spiculs 2, equal. ........................

Wings 16-32; pharynx capacious, open; spiculs unequal, or 1 only........Richtersiella Kreis 1929

Nems slender, under $5 \%$; wings if present not numerous

Eyes absent; amphid circular; pharynx truncate at base............ Bathylaimella Allgen 1930

Eyes often present; setae behind head usually long, numerous, conspicuous

Oesophagus lumen not enlarged; amphid loop-shaped......... (Araeolaimoides (de Man) Fil. 1918)

Oesophagus and lumen widened between eyes and nerve ring; pharynx obviour

Shape of amphid loop-like ("folded") .............................(Coinonema Cobb 1920)

Shape of amphid circular or monospiral; eyes sometimes absent(?).... Araeolaimus de Man 1888

Gonads of reflexed

Spinneret absent; amphid circular, elliptical or monospiral

Habitat not marine; pharynx long, tubular, thalf as long as oesophagus

Wings 16-32, conspic., fusing to 4 on 4-pronged tail; in rice fields......Preudoaulolaimus Imamura 1931

Wings 0 ; end of tail not 4-parted; in soil............................... Isolaimium Cobb 1920

Habitat marine; pharynx short

Pharynx narrow; amphid \pm flattened, preceded by 2 flat setae...............Platycoma Cobb 1894

Pharynx 0 or linear; amphid transverse-oval, with pocket, 4 set. posterior.. Platycomopsis Ditlev, 1926

Spinneret present

Habitat freshwater

Mouth cavity absent or obscure, closed

Amphid monospire; gland cells lateral; o' supplements protrusile tubes. . A phanolaimus de Man 1880

A mphid circular or elliptical; $\sigma^{7}$ supplements not protrusile............. Tripyla Bast. 1865

Mouth cavity definite, open, obvious

(=Promononchus Micol. 1923)

Pharynx narrow, compound; amphid monospire, $\frac{1}{3}$ head-width; cephalic setae 4 Paraplectus Fil. 1930

Pharynx simple, i.e. of 1 chamber

The amphid small, transverse-oval or a slit; cephalic setae 6 or 10...Prismatolaimus de Man 1880

The amphid monospire, opp. pharynx; ceph. setae 4; gland cells lateral Paraphanolaimus Micol. 1923

Habitat marine

Nems wide, over $10 \%$

Annules few, coarse; amphid saccate

Body setse not dense; secreted annules prominent, separate; head small.. Desmoscolex Clap. 1863

Body setse dense, set along annules; junct. oesoph. \& intestine indefinite... Greeffiella Cobb 1922

Body setse dense, set along annules; junct. oesoph. \& intestine indefinite...Greeffiella Cobb 1922
(=Trichoderma Greeff 1869)

Annules if present many; longitudinal ridges or wings 16-50; neck "collared"; Richtersinae

Wings \pm 50 ; pharynx narrow; spicula 2, equal ............................Richtersia Steiner 1916

Wings 16-32; pharynx wide, capacious; spicula unequal, or 1 only.........Richtergiella Kreis 1929

Nema slender, not over $5 \%$

Calvarium present as a band or plates

Oesophagus not crenate; cal varium of plates; gland cells lateral. .......... Cylicolaimus de Man 1889

Oesophagus crenate; calvarium a band............................ (Phanodermopsis Ditlev. 1926)

Calvarium absent

(=Galeonema Fil. 1927)

Gland duets cuticularized, pores cervical \& pre-anal; striae coarse. . (Hala phanolaimus Southern 1914) Gland ducts and pores none, or not cuticularized

Denticles mid-phar., apophyses forward \& back; amph. multispire (Halichoanolaimus de Man 1886)

Denticles none: amphid not multispire 
Pharynx open, wide, \pm capacious

Amphid round; anterior pharyng. chamber large, posterior small; setae 10 Bathylaimus Cobb 1894 (= Macrolaimus Ditlev, 1918

Amphid small, with internal pocket (Pandolaimus ?); pharynx of 1 chamber =Cothonolaimus Ditlev. 1919)

Depth pharynx \pm equalling width; dorsal onch. small, ant., appressed Anoncholaimus Cobb 1920 Depth pharynx nearly twice width

Wall of pharynx irregularly thick; amphid apparently circular.......Pandolaimus Allgen 1929

Wall of pharynx thin; amphid small, transverse-oval, with pocket.... Pelagonema Cobb 1894

Pharynx narrow, often tubular or with closed lumen

(ef. Vasculonema Kreis 1928)

The amphid behind level of cephalic setae

Cephalic setae 0; pharynx $\frac{1}{3}$ head-width, with 3 refractive elements...Angustinema Cobb 1933 Cephalic setae present

Nema over $3 \mathrm{~mm}$. long..................................... Cynura Cobb 1920

Nema $0.6 \mathrm{~mm}$. long.......... Aplectus Cobb 1914

The amphid in front of level of cephalic setae; cephalic setae 4

Dorsal pharyng. wall not thicker; ampbidial glands large, conspic.; eyes 2 . ..Ionema Cobb 1920

Dorsal pharyng. wall thick, tooth-like; amph. monospire at lips; renette far back

Oesoph. glands obscuring junct. of intest.; dorsal phar. element uniform... Onchium Cobb 1920 Oesoph. glands not prominent; dorsal pharyng. element not uniformly thick, but heavy

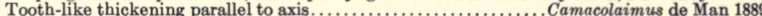
(=Digitonchus Cobb 1920 = Acontiolaimus Fil. 1918)

Tooth-like thickening bent outward from beginning of vestibule

Point of "onchium" single.................................Acmaeolaimus Fil 1918

Point of Y-shaped "onchium" duplex.............................. Y psilon Cobb 1920 


\section{LITERATURE CITED}

Allgen, C. Freilebende marine Nematoden von den Campbell-und Staten-Inseln. Nyt. Mag. Naturvidenskaberne. 66: 305-306. 1927.

- Südschwedische marine Nematoden. Göteborgs K. Vetensk. O. Vetterhets = Samhälles. Handl. Ser. B 1: 29. 1929.

- Neue freilebende marine Nematoden von der Westküste Schwedens. Zool. Jahrb. Abt. System. Ökol. u. Geogr. Tiere. 57: 433-434, 436-437, 445, 454, 461, 466, 467, 481, 491, 492, 493. 1929.

- Conolaimella, ein neues Genus mariner Nematoden von der Westküste Schwedens.

Zool. Anz. 88: 138. 1930.

- Freilebende marine Nematoden von der Stateninsel (Feuerland-Archipel.) I.

Zool. Anz. 89: 257. 1930.

- Freilebende marine Nematoden von der Stateninsel (Feuerland-Archipel.) II. Zool. Anz. 90: 28. 1930.

- Weitere Beiträge aur Kenntnis der marinen Nematodenfauna der Campbellinsel. Nyt. Mag. Naturv. 70: 186. 1932.

- Ueber einige freilebende marine Nematoden aus der Umgebung der Biologischen Station auf der Insel Herdla (Norwegen) mit Anhang; zur Richtigstellung alterer und neuerer mariner Nematodengenera I. Arch. Naturgesch. 1: 424, 432.1932.

Bastian, H. C. Monograph on the Anguillulidae, or free nematodes, marine, land, and fresh water; with descriptions of 100 new species. Trans. Linn. Soc. London 25:97, 100-101, 103-104, 115, 118, 121-122, 124, 125-126, 132, 141, 142-143, 144, 145, 154-155, $156,157,158,159,162-163,165,167,178.1865$.

Baylis, H. A. ANd Datbney, R. A synopsis of the families and genera of Nematoda. London. Pp. 107, 112. 1926.

Bovien, P. On a new nematode, Scatonema wülkeri gen. et sp. $n$. parasitic in the bodycavity of Scatopse fuscipes Meig. (Diptera nematocera). Vidensk. Meddel. Dansk Naturhist. For. Kjøbenhavn. 94: 15-31. 1932.

BüTschLI, O. Zur Kenntniss der freilebenden Nematoden. Abhandl. Senckenb. Naturf. Gesell. Frankfurt a. M. 9: 270-273, 285. 1874.

CARUs, J. V. Icones zootomicae. Erste Hälfte: Die wirbellosen Thiere. Leipzig. Pl.8, Fig. 1. 1857.

Chiтwood, B. G. Helminthological Society Proceedings. The Journal of Parasitology. 19: 243.1933.

Christie, J. R. and Chitwood, B. G. Chondronema passali (Leidy, 1852) n.g. (Nematoda), with notes on its life history. Jour. Wash. Acad. Sci. 21: 357. 1931.

Claparède, E. Beobachtungen über Anatomie und Entwicklungsgeschichte wirbelloser Thiere an der Küste von Normandie angestellt. Pp. 88-90. 1863.

Совв, M. V. Some fresh-water nematodes of the Douglas Lake Region of Michigan, U. S. A. Amer. Micros. Soc. Trans. 34: 28-29. 1915.

- (edited by) N.A. Cobb: New nemic genera and species, with taxonomic notes. Jour. Parasitol. 20: 81-94. 1933.

Совв, N. A. Onyx and Dipeltis: New nematode genera, with a note on Dorylaimus. Linn. Soc. N. S. Wales, Proc. 6: 146-153, 155. 1891.

- Plant diseases and their remedies. (III. Nematode worms found attacking sugarcane.) Agric. Gaz. N. S. Wales. Pp. 811, 819-820, 821, 825. 1893.

- Tricoma and other new nematode genera. Linn. Soc. N. S. Wales, Proc. (1893)

(s. 2). 8: 389-390, 391, 392-393, 399-401, 409-410, 411-413, 413-414, 416, 419, 420421. 1894 .

- Australian free-living marine nematodes. Linn. Soc. N. S. Wales, Proc. (s. 1). 23: 406-407. 1898. 
Совв, N. A. 1905. See Stiles and Hassall, 1905.

Fungus maladies of the sugar cane, with notes on associated insects and nematodes. Bull. (5), Hawaii Sugar Planters' Assoc. Expt. Sta., Div. Path. \& Physiol., 2nd ed., pp. 187-189. 1906.

- Draconema: A remarkable genus of marine free-living nematodes. Jour. Wash. Acad. Sci. 3: 146-149. 1913.

Notes on Mononchus and Tylenchulus. Jour. Wash. Acad. Sci. 3: 287. 1913. New nematode genera found inhabiting fresh water and non-brackish soils. Jour.

Wash. Acad. Sci. 3: 436-444. 1913.

- Antarctic marine free-living nematodes of the Shackleton Expedition. Contrib. Sci. Nematol. I, pp. 12, 16. 1914.

- North American free-living fresh-water nematodes. Amer. Micros. Soc. Trans. 33: 92-94. 1914.

- Selachinema a new nematode genus with remarkable mandibles. Contrib. Sci. Nematol. IV, p. 113.1915.

- Notes on nemas. Contrib. Sci. Nematol. V, pp. 118, 119. 1917.

- Filter-bed nemas: Nematodes of the slow sand filter beds of American cities (including new genera and species) with notes on hermaphroditism and parthenogenesis. Contrib. Sci. Nematol. VII, pp. 195-196, 203. 1918.

- One hundred new nemas (type species of 100 new genera). Contrib. Sci. Nematol. IX, pp. 230-340. 1920.

- Howardula benigna, a nema parasite of the cucumber-beetle (Diabrotica). Contrib. Sci. Nematol. X, pp. 1-2. 1921.

- Marionella (Eurystoma Marion, 1870); an emendation, with notes on a new birefringent substance, marionellin, found in the intestinal cells. Jour. Wash. Acad.

Sci. 11: 504-509. 1922.

—_ Greeffiella. Jour. Wash. Acad. Sci. 12: 299-303. 1922.

The Helminthological Society of Washington. Jour. Parasitol. 11: 105, 116, 120. 1924 .

Marine free-living nemas. Australas. Antarc. Exped. 1911-14. Scient. Rep., Sydney, Ser. C. Zool. \& Bot. 6(7): 22-23. 1930.

- The demanian vessels in nemas of the genus Oncholaimus; with notes on four new oncholaims. Jour. Wash. Acad. Sci. 20: 227. 1930.

Daday, E. von. Mikroskopische Süsswasserthiere aus Deutsch-Neu-Guinea. Természet. Füzetek. 24: 7. 1901.

- Untersuchungen über die Süsswasser-Mikrofauna Paraguays. Mit einem Anhang von W. Michaelsen. Zool. 18(44): p. 62.1905.

De Coninck, L. Sur trois espèces nouvelles de nématodes libres trouvés en Belgique. Bull. Mus. Roy. Hist. Nat. Belg. 7(11): 2-5. 1931.

Diesing, K. M. Revision der Nematoden. Sitzber. Akad. Wiss. Wien, Math. Naturw. Kl. (1860) 42: 728. 1861.

Ditlevsen, H. Marine free-living nematodes from Danish waters. Vidensk. Meddel. Dansk Naturhist. For. Kjøbenhavn. 70: 162-163, 168, 174-177, 177-178, 181-182, 183-184, 185, 188-189, 197, 299. Plate 7, figs. 3, 4 \& 6. Plate 8, figs. 2, 3 \& 8. 1918. Marine free-living nematodes from the Auckland and Campbell Islands. Vidensk. Meddel. Dansk Naturhist. For. Kjøbenhavn. 73: 2, 4. 1922. Advance separate apparently issued in 1921.

Nematological notes. Vidensk. Meddel. Dansk Naturhist. For. Kjøbenhavn.

74: $57-58,60.1922$.

Free-living nematodes. The Danish Ingolf-Expedition (1895-1896) Copenhagen. $4(6): 8,12,30-31.1926$. 
Ditlevsen, H. Free-living marine nematodes from Greenland waters. Meddel. Grønland 23 (supplement): 227, 230. 1928.

DUFOUR, L. Recherches sur quelques entozoaires et larves parasites des insects Orthoptères et Hyménoptères. Ann. Sci. Nat., Zool. 7(2): 9. 1837.

Dujardin, F. Histoire naturelle des helminthes ou vers intestinaux. Paris. Pp. 230, 233, 235-236, 239-240. 1845.

Ehrenberg, HrN. Über die Akalephen des Rothen Meeres und den Organismus der Medusen der Ostsee. Abhandl. Akad. Wiss. Berlin (1835). P. 219. 1837.

Filipjev, I. N. Les nématodes libres contenus dans les collections du Musée Zoologique de l'Académie Impériale des Sciences de Petrograd. Ann. Mus. Zool. Acad. Imp. Sci. 21: 65, 73-75, 83, 105-106. 1916.

- Novaia svobodnaia nematoda iz Kaspuskago Moria Chromadorissa gen. nov. (Chromadoridae, Chromadorina) (also French résumé). Russ. K. Zool. Zhurn. Moskoa. 2: 24-30. 1917.

- Free-living marine nematodes of the vicinity of Sebastopol. Part I. Trudy Osob. Zool. Lab. Sebastopol. Biol. Stantsii Russ. Akad. Nauk Petrograd. s. 2(4), pp. 50-51, 110, 122-123, 139-141, 186, 205, 206, 211, 218-219, 226-227, 229-230, 232233, 256-257, 259, 284, 303, 305, 323-324, 326, 328, 345. 1918.

- Free-living marine nematodes of the vicinity of Sebastopol. Part II. Spec. Zool. Lab. and Sebastopol Biol. Sta. Russ. Acad. Sci. s. 2(4). pp. 565,568. 1921.

- Encore sur les nématodes libres de la mer noire. Acta. Inst. Agron. Stauropolitani. (Zool.) 1: 114-115, 121-122, 133-134, 137, 138-139, 150, 173-174. 1922.

Les nématodes libres des mers septentrionales appartenant à la famille des Enoplidae. Arch. Naturgesch. (1925) Abt. A (6). 91: 65, 66, 79, 81-82, 105-106, 123, 130, 155-156, 160, 176-177, 180-182, 183, 188, 199, 203. 1927.

Les nématodes libres de la baie de la Neva et de l'extrémité orientale du Golfe de Finlande. Deuxième partie. Arch. Hydrobiol. 21: 12-13, 29, 35-36. 1930.

Fischer, M. Ueber eine Clematis-krankheit. Ber. Physiol. Lab. u. Versuchsanst. Landw. Inst. Halle. 3(11): 1-11. 1894.

Fuchs, G. Über Parasiten und andere biologisch an die Borkenkäfer gebundene Nematoden. Verhandl. Gesell. Deut. Naturf. u. Ärzte. 2nd Part, 1 Hälfte. P. 690. 1914.

- Die Naturgeschichte der Nematoden und einiger anderer Parasiten. Zool. Jahrb. Abt. System., Geogr. u. Biol. Tiere. 38: 158-160. 1915.

- Neue an Borken-und Rüsselkäfer gebundene Nematoden, halbparasitische und Wohnungseinmieter. Zool. Jahrb., Abt. System., OOkol. u. Geogr. Tiere. 59: 540544, 560-561, 565-566, 574-576, 586-588, 609-612, 616-618. 1930.

- Seinura gen. nov. Zool. Anz. 94: 226-227. 1931.

GöLd, E. A. Relacao dos estudos tendentes a elucidar a molestia do cafeeiro na provincia do Rio-Resultados dos tres primeiros mezes de estudos. (34 pp.) 1886.

Relatorio sobre a molestia do cafeeiro na provincia do Rio de Janeiro (1887). Archivos do Museu Nacional Rio de Janeiro. 8: 68. 1892.

Goodex, T. Hexatylus viviparus gen. et $s p$. nov., a nematode found in a diseased potato tuber. Jour. Helminthol. 4: 28, 30. 1926.

- On some new and little-known free-living nematodes. Jour. Helminthol. 7: 2730, 41-45. 1929.

- Cylindrogaster coprophaga gen. et sp. nov. a nematode found in a culture of faeces from a wild brown rat. Jour. Helminthol. 5: 26-31. 1927.

- On a remarkable new nematode, Tylenchinema oscinellae gen. et sp. $n$., parasitic in the frit-fly, Oscinella frit L., attacking oats. Roy. Soc. Proc., Ser. B. (London). 218: 338-339. 1930. 
Greeff, R. Untersuchungen über einige merkwürdige Formen des Arthropoden- und Wurm-typus. Arch. Naturgesch. 35. J., 1: 115. 1869.

Hofmänner, B. And Menzed, R. Neue Arten freilebender Nematoden aus der Schweiz. Zool. Anz. 44: 88.1914.

Imamura, S. Nematodes in the paddy field, with notes on their population before and after irrigation. Jour. Col. Agr., Imp. Univ., Tokyo. 11: 210-211. 1931.

Inwin-Smith, V. A. On the Chaetosomatidae, with descriptions of new species, and a new genus from the coast of New South Wales. Linn. Soc. N. S. Wales, Proc. (1917) (168). 42: 798. 1918.

J ÄGERSKIöLd, L. A. Bunonema richtersi n. g. n. sp. Ein eigentümlicher neuer Landnematode aus dem Schwarswald, von Kerguelen und Possession-Island (CrozetInseln). Zool. Anz. 28: 557-561. 1905.

KREIS, H. A. Zur Kenntnis der freilebenden marinen Nematoden. Schr. Süsswasser u. Meereskunde. 2(6): 167-168. 1924.

Weiterer Beitrag zur Kenntnis der freilebenden marinen Nematoden. Arch. Naturgesch. (1926). Abt. A(8). 92: 2-4, 10. 1928.

- Die freilebenden marinen Nematoden der Spitzbergen-Expedition von F. Roemer und F. Schaudinn im Jahr 1898. Mitt. Zool. Mus. Berlin. 14: 150-151, 158-159, 163-164, 171-172, 172-173, 177, 181-182. 1928.

- Freilebende terrestrische Nematoden aus der Umgebung von Peking (China). Zool. Anz. 84: 288.1929.

- Freilebende marine Nematoden von der Nordwest-Küste Frankreichs (Trébeurden: Côtes du Nord). Capita Zool. 2(7): 26, 44, 51, 54, 62, 67, 69. 1929.

- Fresh-water nematoda from the Paraguayan Chaco. Jour. Linn. Soc., Zool. 38(no. 257): 72. 1932.

- Beiträge aur Kenntnis pflanzenparasitischer Nematoden. Ztschr. Parasitenk. 5: 185-189. 1932.

- Freilebende marine Nematoden von den Sunda-Inseln. II. Oncholaiminae. Vidensk. Meddel. Dansk Naturhist. For. Kjøbenhavn. 93: 28-29, 30, 31, 38-39, 42, 52, 57, 67 . 1932-1933.

Leidy, J. Contributions towards a knowledge of the marine invertebrate fauna of the coasts of Rhode Island and New Jersey. Jour. Acad. Nat. Sci. Phila. 3(s.2): 144. 1855 .

Ledckart, R. Ueber einen neuen heterogenen Nematoden. Tagebl. d. 57. Versamml. Deutsch. Naturf. u. Aertze, Magdeb. (5). P. 320. 1884.

- Ein Sphaerularia-artiger neuer Nematode. Zool. Anz. 9: 743-746. 1886.

Neue Beiträge aur Kenntniss des Baues und der Lebensgeschichte der Nematoden. Abhandl. Math.-Phys. Cl: k. Sächs. Gesell. Wiss. 13: 678-703. 1887.

Linstow, O. von. Helminthologische beobachtungen. Arch. Naturgesch. 42.J., 1: 16-17. 1876.

- Helminthologica. Arch. Naturgesch. 43.J., 1: 2-3. 1877.

MAN, J. G., DE. Onderzoekingen over vrij in de aarde levende nematoden. Tijdschr. Nederl. Dierk. Vereen. 2: 116-117, 119, 120, 137-138, 168-169, 172.1876.

Die einheimischen, frei in der reinen Erde und im süssen Wasser lebenden Nematoden. Tijdschr. Nederl. Dierk. Vereen. 5: 2, 3-4, 5, 14, 15, 21, 28, 30-31, 34, 5864. 1880 .

Helminthologische beitraege. Tijdschr. Nederl. Dierk. Vereen. s.2, 1: 2-3. 1885.

Anatomische Untersuchungen über freilebende Nordsee-nematoden. Leipzig. Pp. 60-76. 1886.

Sur quelques nématodes libres de la mer du Nord nouveaux ou peu connus. Mém.

Soc. Zool. France. 1: 2-3, 11, 14-16, 31-32, 34-35. 1888. 
MAN, J. G., DE. Espèces et genres nouveaux de nématodes libres de la mer du Nord et de la manche.Mém. Soc. Zool. France. 2: 1, 3, 4, 8, 9-10. 1889.

Quatrième note sur les nématodes libres de la mer du Nord et de la manche. Mém.

Soc. Zool. France. 3: 189-190. 1890.

- Cinquième note sur les nématodes libres de la mer du Nord et de la manche. Mém.

Soc. Zool. France. 6: 81, 84-85, 99-100, 114-115, 118-119. 1893.

- Sur quelques espèces nouvelles ou peu connues de nématodes libres vivant sur les cótes de la Zélande. Tijdschr. Nederl. Dierk. Vereen. 10(2): 228, 232-233, 237, 239-240, 241-242. 1907.

- Helminthologische Beiträge. Zool. Jahrb. (Supplement 15). 1: 439-440. 1912.

Odontopharynx longicaudata n. g., n. sp. Eine neue Form von Anguilluliden.

Zool. Jahrb. Abt. System., Geogr. u. Biol. Tiere. 33: 637-638. 1912.

__ Nouvelles recherches sur les nématodes libres terricoles. Capita Zool. 1: 9, 14, $33,35,36,42-43.1921$.

_— Neue freilebende Nematoden aus der Zuidersee. Tijdschr. Nederl. Dierk. Vereen. 18: 126, 131. 1922.

MARION, A. F. Recherches zoologiques et anatomiques sur des nématodes non parasites, marins. Ann. Sci. Nat. Zool. (5) Art. 14. 13: 11-12, 14, 16, 18, 19, 25, 31, 32, 34. 1870.

MaUPas, E. Modes et formes de reproduction des nématodes. Arch. Zool. Expt. e. Gen. 8 (3): $578-582.1900$.

MeтschniкоғF, E. Beiträge zur Naturgeschichte der Würmer. Ztschr. Wiss. Zool. 17: 542.1867.

Micoletzky, H. Neue Süsswasser-Nematoden aus der Bukowina. Mitt. Naturw. Ver. Steiermark. (1914) 51: 447. 1915.

— Die freilebenden Erd-Nematoden. Arch. Naturgesch. (1921) 87: 119, 374-377, 606-607. 1922.

- Freilebende Nematoden der Wolga. Arb. Biol. Wolga-Sta. 7: 13, 14, 17-18, 21-24. 1923.

Weitere Beiträge aur Kenntnis freilebender Nematoden aus Suez. Sitzber. Akad. Wiss. Wien, Math. Naturw. KI. Abt. 1, 132: 228, 235, 242-244. 1924.

Letater Bericht über freilebende Nematoden aus Suez. Sitzber. Akad. Wiss.

Wien, Math. Naturw. Kl. Abt. 1, 133: 138-139, 140, 157-158, 164-165. 1924.

- Die freilebenden Süsswasser-und Moornematoden Dänemarks. Nebst. Anhang:

Über Amöbosporidien und andere Parasiten bei freilebenden Nematoden. K. Dansk.

Vidensk. Selsk. Skr. Naturv. Math. Afd. 10: 153, 248-249, 257-258. 1925.

- Freilebende marine Nematoden von den Sunda-Inseln. 1. Enoplidae. Vidensk.

Meddel. Dansk. Naturh. For. Kjøbenhavn. 87: 261, 266-267, 293, 312, 330-331. 1930.

Onorato de Cillis, M. I. Nuovi generi e nuove specie di nematodi liberi d'acqua dolce. Monit. Zool. Ital. 28: 58-59. 1917.

PANCERI, P. Osservazioni intorno a nuove forme di vermi nematodi marini. Atti. $\mathbf{r}$. Accad. Sci. Fis. e. Mat., Napoli. 7(10): 7-9. (1876) 1878.

Peters, B. G. On the nomenclature of the vinegar eelworm. Jour. Helminth. 5: 141142. 1927.

RAHM, G. Beitrag zur Kenntnis der Moostierwelt der preussischen Rheinlande. I. Systematisch beschreibender Teil. Arch. Naturgesch. (1924) Abt. A, 90: 176. 1925. Alguns nematodes parasitas e semi-parasitas das plantas culturaes do Brasil. Arch. Inst. Biol. I: 240, 241, 243-244, 246, 248, 250. 1928.

- Freilebende Nematoden Rotatorien und Tardigraden aus Südamerika (besonders aus Chile). Zool. Anz. 98: 96, 99, 106, 107. 1932. 
Richters, F. Moosfauna-Studien. Ber. Senckenb. Naturf. Gesell. Frankfurt a. M. (Part 2-Wiss. Mitt.) pp. 23-24. 1908.

Rouville, M. E., DE. Enumération des nématodes libres du canal des Bourdigues (Cette). Compt. Rend. Soc. Biol. (Paris). 55: 1529. 1903.

Saveljev, S. N. Zur Kenntnis der freilebenden Nematoden des Kolafjords und des Relictensee Mogilnoje. Trav. Soc. Nat. St. Petersb. 43: 113-114. 1912.

SchmidT, A. Ueber den Rüben-Nematoden (Heterodera schachtii A. S.). Ztschr. Ver. Rübenz-Indus. im Zollverein. 21: (n.F. v. 8) 1-18. 1871.

Schultze, M. See Carus, J. V. 1857.

Schulz, E. Beiträge zur Kenntnis mariner Nematoden aus der Kieler Bucht. Zool. Jahrb. Abt. Syst. Ökol. u. Geograph. Tiere. 62: 355, 358, 366, 407. 1932.

Southern, R. Clare Island survey part 54 Nemathelmia, Kinorhyncha, and Chaetognatha. Roy. Irish Acad. Proc. 31: 9-10, 11, 20, 29, 31, 34-35, 41-42, 66-67. 1914.

Steniner, G. Freilebende Nematoden aus der Schweiz. Arch. Hydrobiol. u. Planktonkunde. 9: 426, 427.1914.

- Freilebende Nematoden aus der Barentssee. Zool. Jahrb. Abt. System., Geogr. u. Biol. Tiere. 39: 579-580, 583, 603-604, 652. 1916.

Helminthological Society of Washington Proceedings. Jour. Parasitol. 14: 66. 1927.

Neoaplectana glaseri, n. g., n. sp. (Oxyuridae), a new nemic parasite of the Japanese beetle (Popillia japonica Newm.). Jour. Wash. Acad. Sci. 19: 437-438. 1929. - Die Nematoden der Deutschen Südpolar-Expedition 1901-1903. I. Teil. Deut. Südpolar Expedition. Zool. 20: 207. 1930.

- Die Nematoden der Deutschen Südpolar-Expedition 1901-1903. II. Teil. Deut. Südpolar Expedition 1901-1903. Zool. 20: 356. 1931.

- Neotylenchus abulbosus $n . g ., n . s p$. (Tylenchidae, Nematoda) the causal agent of a new nematosis of various crop plants. Jour. Wash. Acad. Sci. 21: 538. 1931.

- On the status of the nemic genera Aphelenchus Bastian (sic), Pathoaphelenchus Cobb, Paraphelenchus Micoletzky, Parasitaphelenchus Fuchs, Isonchus Cobb, and Seinura Fuchs. Jour. Wash. Acad. Sci. 21: 468-475. 1931.

- ANd Buhrer, E. M. The nematode Tylenchus similis Cobb, as a parasite of the tea plant (Thea sinensis, Linn.), its sexual dimorphism, and its nemic associates in the same host. Ztschr. Parasitenk. 5: 419. 1933.

- ANd Hoeppl, R. Studies on the exoskeleton of some Japanese marine nemas. Arch. Schiffs-u. Tropenhyg. 30: 573. 1926.

Steknoven, J. H. SchuUrmans, Jr. Ökologische und morphologische notizen über Zuiderseenematoden. I. Die westliche Hälfte der Zuidersee. Ztschr. Morph. u. Ökol. Tiere. Abt. A. 20: 648-649. 1931.

- AND Adam, W. The freeliving marine nemas of the Belgian coast. Mém. (49) Mus. Roy. Hist. Nat. Belg., pp. 42-44. 1931.

and Coninck, L. DE. Diagnoses of new Belgian marine nemas. Bul. Mus. Roy. Hist. Nat. Belg. 9: 9-10. 1933.

Stiles, C. W. AND Hassald, A. The determination of generic types and a list of roundworm genera with their original and type species. U. S. Dept. Agr., Bur. Anim. Indus., Bull. No. 79, pp. 100, 101, 107, 122. 1905.

Thorne, G. Nematodes from the summit of Long's Peak, Colorado. Amer. Micros. Soc. Trans. 48: 189-190. 1929.

- Predacious nemas of the genus Nygolaimus and a new genus, Sectonema. Jour. Agr. Research (U. S.). 41: 464.1930.

VinLot, A. Recherches sur les helminthes libres ou parasites des côtes de la Bretagne. Arch. Zool. Expt. et Gén. 4: 463. 1875.

Zur Strassen, O. Bradynema rigidum v. Sieb. Ztschr. Wiss. Zool. 54: 655-742. 1892. 


\section{INDEX}

page

A canthonchus ........ 462 Acanthopharynx............463 Achromadora .............465, 466 Acmaeolaimus......462, 465, 471 .

474,481

Acoms Acontiolaimus.......462, 465,471 474,48

Acrobeles........470, 471, 475, 476 Acrobeloides.....470, 471, 475, 476 Acrostichus...........463, 464 Actinolaimus....................461 Actinonema.......456, 46i, 472, 478 Aculeonchus...............4460 Adoncholaimus..........469,469 Aegialoalaimus. 455, 477 Alaimella.......457, 457, 479, 479 Alaimonema....454,466,466, 470

Alaimus.......454,454, 456, 457 Allantonems.... 455, 458, 459, 459 460,460

Allomonhystera............ 473 Amphispira......464, 464, 470, 470 Amphistenus.............463, 469 Anaxonchium............462, 468 Anguillula...........463, 468, 470 Angustinems.............478, 481 Anoncholaimus.......462,479, 481 Anonchus................476, 479 Anoplostoma...........478, 478 Antholgimus.............460, 461 Anthonema............454,475, 477 Anthonema........454, 475, 477
Anticoma..........458, 472, 474 Anticomopsis.........458, 472, 474 Anticyathus........457, 473, 480 Anticyclus.................. 462 Antomicron. ......454, 457, 470, 472 Antopus...............455, 456 Aphanolaimus ....458, 474, 474, 480 Aphelenchoides.....459,460,460

Aphelenchulus. $458,478,479$ A phelenchus............460, 460 Aplectus........455, 471, 477, 481 A podontium........456, 457, 467 Aponchium..........465, 466, 466 Araeolaimoides........456, 478, 480 Araeolaimus..........457, 474, 480 Archepsilonema...........455, 477 Archionchus....459, 459, 461, 475 475,476 Ascolaimus.............470, 47 Asconems................... 455 Asymmetrella........462, 478, 479 Asymmetrica............... 471 Atractonema ................ 455 Atylenchus............... 459 Aulolaimoides....470, 475, 476, 476 A ulolaimus..............475, 475 A ustronema.................. 473 Aronchium.............. 46 Axonolaim us.....467, 468, 470, 472

Barbonems...............457 B Bastiania.............457, 458 Batbyepsilonema............455, 477 Bathylaimella...........479, 480 Bathylaimus Cobb........474, 481 Bathylaimus Ditlevsen...470, 472 Bitbolinema..............477 Bognenia.................. 473

Bolbella...........462,464,469 page

Bolbinium ............454, 45

Bolbolaim us..........463, 464, 466 Bolbonema..............455, 477 Brachynema.............. 458 Brachynemella............. 458 Bradylaim us................... 465 Bradynema...........455, 458, 460 Bulbopharyngiella...463, 464, 466 Bunonema............... 475

Butlerius.................466, 468

Cacolaimus 468 Caconems... $\ldots \ldots \ldots 459,459$ Caly ptronema ...462, 463, 465, 469 474,481 Campydora.....460,460,461, 463 464,464

Campylaimus. ......456, 472, 478 Catalaimus .....462,463,465, 469 Catanema......454,454, 466, 470 $470,474,476$ Cenolaimus...........473, 479 Cephalobus......470,47i, 475, 476 Cephalonema..........461, 463 Ceramonema....454, 456, 456, 475

Chaetonema............ 479 Chaetonema........ Chambersiella.......466, 476, 477 Chaolaimus....459,459, 461, 475 475,476 Cheilobus 470,476 Cheironchus.................. 466 Chloronemella,............... 476 Choonolaimus................ 474 Chondronema............455, 460 Choniolaimus .......... 470, 476 Chromadora..........463, 464, 466 Chromadorella...........465, 467 Chromadorina............465, 466 Chromadorissa................ 463 Chromadorita............. 463, 463 Chromadoropsis............465, 465 Chromagaster ..............476 Chromaspirina...............466, 469 Chronogaster..............470, 475 Chrysonema............... 461 Cinctonems.................. 454,470

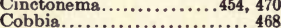
Cobbionema................... 468 Coinonems. 456, 478, 480 Colpurella...............476, 478 Comesoma.............462, 464 Conolaimella.....473, 473, 479, 479 Conolaimus..........465, 467, 467 Convexolaimus...................... 468 Cophonchus.............462, 472 Cothonolaimus...........474, 48 Craspedonema................ 475 Crassolaimus.................. 464 Cricolaimus......454,466, 470,476 Criconema................. 459 Croconems................... 469 Cryptolsimus.....454, 455, 47i, 476 Cryptonchus............461, 478 Crystallonema......467, 470, 473

476, 479 Cyartonema................. 455 Cyatholaimus............462, 474 Cylicolaimus.....469,472, 474, 480 Cylindrogaster............. 475 page

Cylindrolaimus.........479, 480 Cytolaimium ....457, $457,473,474$

Dactium Dactylaimus................ 478 Dactylonema.........456, 478, 48 Dagda...................467, 468 Daptonema.......467, $472,473,473$ Dasylaimus. .470, 470,471, 471, 471

Dasynema......470,472,476, 479 Dasynemella.....470, 472, 476, 479 Deltanema.........466, 471, 476 Demania Southern ...... 467 Demania Steiner.........463, 469 Demaniella..............463, 469 Demonema..............468, 47 Dentatonema................. Denticulella...............4 46

Deontolaim us................. 457

Deontostoma.....46i, $463, \mathbf{4} 72,472$

Dermatolaimus............. 477 Desmodora................465, 466

Desmodorella................ 465 Desmolaimus................ 471,476

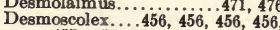
$457,458,478,478,478,479$

480,480

Diastolaim us.......466, 476, 47

Dicriconems............466, 476, 477

Didelta........472, $473,478,480$ Digitonchus....462, 465, 471, 474

Dignathoneme .a.c.......

Dilaimus...............463, 468

Dintheria................... 472 Diodontolaimus....467, 467, 468

Dioncholaimus............ 468

Dipeltis ................. 461,463

Diphtherophora....459, 459, 461

$475,475,476$

Diplogaster ....463, 464, 465,465 $466,466,475$

Diplogasteroides.....,464, 465, 476

Diplohystera.............. 474

Diplolaimella ............473, 473

Diplolaimus................. 47

Diplopeltis..............46i, 463

Diploscapter.............. 465

Diploscapteroides..........463, 475

Discolaimus.................. 461

Disconema................... 45

Discophora...............461, 463 Dispira............467, 472, 479 Dispirella.................. 479 Ditlevsenella................. 469 Ditlevsenella..............4 469 Dolichodorus................ 459 Dolicholaimus............. 467 Dorylaimellus.............. 461 Dorylaimopsis.............460, 468 Dorylaimus.............461, 461 Doryllium..............459, 46 Doryonchus............461, 462

Draconema . .454, 455, 470, 475, 477 Drepanonema...454,455, 470, 475

E Ehyadophora..........458, 458 
page

Enchelidium ......455, 456, 457

Endolaimus ............ 466 Enoplocheilus.........460,461, 468 Enoploides..............467, 468 Enoplolaimus...........467, 468

Enoplostoms...........467, 468 Enoplus...............467, 468 Epsilonella.............455, 477 Epsilonema...........455, 477 Ethmolaimus...............4 465

Euchromsdora.................. 461

Eudesmoscolex........... 454, 457 Eulinhomoeus (subgenus).... 466 Eumorpholaim us............ 480 Eurystoma. ........462, 463, 469 Eurystomina........462, 463, 469 Eutelolaimus.............455, 47 Eutylenchus................ 458

Fenestrolaimus. . . . . . . . . 469 Fiacra................467, 469 Filipjeva................. 478 Filipjevia................. 468 Filoncholaimus............ 469 Fimbria.......467, 467, 477, 479 Fimbriella.............. 478 Fimbrilla.......467, $467,477,479$ Fusonems...............466

Galeonema...........474, 480 G Gammanema..........467, 467 Gonionchus............... 468 Graphonema................... 461 Greeffiella................458, 480 Gullmarnia................478 Gymnolaimus...........475, 478

H alalaimoides. .455, 456, 477, 478 Halanonchus..............456, 456 Halaphanolaimus.......454, 455,457 , $458,476,477,480,480$

Halenchus. 458,459 Halichosnolaimus.............468, 474, 480 Halinems...............480, 480 Haliplectus.........455, 476, 477 Helalaimus............... 457 Hemicycliophora.........454, 454 Heterocephalus......... 472, 472 Heterodera..............459, 459 Heterodesmodora........... 471 Hexatylus. ................ 458 Himatidiophila........471, 471 Hoplolaimus...........458, 459 Howardula......456,458,458, 460 Hy podontolaimus.........463, 464 Hyptiolaimus............... 468

Tllium.................4 478 Ionems......456, 456, 458, 48 Iota...................459, 459 Iotadorus............463,464, 464 Iotalaimus.............454, 456 Iotonchium.....454,454,460,460 Ironella...................4 467 Ironus..................... 467 Isolaimium...........478, 478,480 Isonchus................460,460 Isonemella..............462, 479

Jägerskiöldia ............. 468

Klugea................458

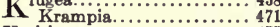

Kreisia...................47

T aimella.

454,476 1 Lasiomitus.........455,456,457 Laxonema.......454, 454, 474, 476 Laxus.............455, 471, 477 Ledovitia.................462, 463

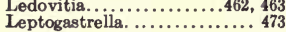

page

Leptolaimus...............477

Leptonchus...............46

Leptonemella....454, $454,470,470$

Leptosomatides..........457, 473

Leptosomatum............... 458

Leptosomella. .................. 458

Linhomoella.....45. $457,457,474$ $476,476,480$

Linhomoeus....455, 457, 457, 466 . $471,474,480$

L. obtusicaudatus......... 457 Linolaim us................. 47 Litinium................457, 457 Litonema.......454, 454, 474, 476 Litotes. . 455, 456, 457, 478, 478, 479

Longicyatholaimus......... 462

Lycolaimus.............. 462

$\mathrm{M}^{\text {acrolaimus Ditlevsen....474, }}$ Macrolaimus Maupas.....463, 464 Macroposthonia........ 475, 476 Margonema...............472, 47 Marionella............462,463,469 Meloidogyne.............459, 459 Meroviscosia.................... 469

Mesodorus.............466, 469 Mesonchium ....466, 466, 466, 468

Metachromadora........465, 465 Metalaimus................ 473 Metalinhomoeus.......466, 47i, 476 Metepsilonema..........455, 477

Metoncholaimus............ 468

Micoletzkyia................456

Microlaimus................. 464

Micromicron..............464, 470

Mitrephorus..............454,454

Molgolgimus...............45

Monhysters ......457, $470,470,472$ $473,476,476,478$

M. dintheriana. . $470,472,476,478$ Monhysteriella..............470

Monhystrella (subgenus). . $\mathbf{4 7 0}, 476$

Monhystrium.......467, 473, 473

Mononchoides...........465, 466

Mononcholaim us...........461, 462

Mononchulus ............461, 468

Mononchus.........462,468, 469

Monoposthis................. 464

Myctolaimus................. 475

Myolaimus...................466

Vannolaimus.........458, 474 Nannonchus.........4462, 469 Nanonema..............461, 463 Nasinema ................ 472

Necticonems...........462,474 Nemanema............457, 473 Nemanemella................ 457 Nemella.................455, 456 Nemonchus............... 458 Neoaplectans........454, 455, 471 Neochromadora............... 464 Neochromadora............... 464 Neonchus..............460, 464 Neonyx..................... 465 Neotonchus...............464 Neotylenchus.............458, 459 Neotylenchus............458, 459 Neurella............... $470,475,477$ Nuadells ................ 45 Nudella................454, 455 Nudolaim us ............473, 479 Nudora...................463, 464
Nunema.................474 Nygmatonchus ............... 464 Nygolaimus...............461

dontolaimus......460,464, 464 Odontonema.........463, 465 Odontopharynx......464,465,466 page

Odontophora........465, 467, 467 Ogma................459, 459 Oionchus ..............461, 464 Oistolaim us................460, 464 Omicronems............460, 464 Onicronema.................. $480,462,465,471$ Oncholgimellus... $\quad 474,477,481$ Oncholaimium........... 469

On

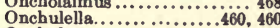
Onchulus.................... 461 Onyx ...........460, $460,404,465$

Oxystoma................ 457

Oxystomina..............457, 457

Panagrolaimus .....470,471, 475 Pandolaimus..........474, 481 Paracanthonchus............. 462 Parachromagaster..........479

Paracothonolaimus ...........466 Paracyatholaimus ..............4 462 Paracyatholaimus ............ 462 Paradesmolaimus............. 480 Paramonhystera........... 473 Paranticoma............472, 479 Paraphanolaimus.........477, 480 Paraphelenchus................ 460 Paraphelenchus............. 460 Parasabatieria ........457, 462, 464. $\quad 471,473$ .....459,460 460,475

Parasphaerolaim us...........473 Parasymplocostoma...455, 456, 457 Paratylenchus.............. 459 Parenoplus.........468, 472, 474 Pareuchromadora........441, 462 Pareurystomina..............463 Parironus.................... 467 Paroncholaimus .............. 469 Paroxystomins...........456, 472 Pathoaphelenchus........459, 460,

460,475

Pelagonema....472, 474, 478, 479 .

Pelagonemella............ 476 Penzancia.................473 Pepsonema..466, 466, 466, 468, 468 Peronilaimus.............. 475 Phanodermella................456 Phanodermopsis.......458, 474, 480 Pharetrolaimus...........458, 459 Platycoma......457, 458, 480, 480 Platycomopsis........456, 478, 480 Plectonchus.............463, 470 Plectus..................471, 477 Poikilolaimus...........463, 464 Polydontus ................. 468

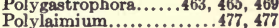
Polysigma..................466 Pomponema.............462, 468 Pontonema............... 469 Porocoms................456, 478 Praescanthonchus.......... 462 Prismatolaimus..478, 478, 479, 480 Pristionchus.........465, 466, 466 Pristionems.............. 456 Prochaetosoms........455, 477 Prochromadora.......463, 463, 464 Prochromadorella.........465, 467 Procriconema............... 459 Promononchus...456, 456, 458, 461. $471,472,474,478,478,480$ Proöncholaimus............ 468 Pselionema.....454, 456, 436,461 ,

Pseudoaulolaimus..... 475, 479 Pseudochromadora........471, 471 Pseudodilaimus............. 462 
page

Pseudolella. .465, 466, 470, 475, 478 Pseudoncholaimus........... 468 Preudonchus............471, 477 Pseudoparoncholaimus........ 469 Preudopelagonema...........474 Pseudorhabditis. .............. 475 Pailenchus...................460 Pteronium...............472, 479 Ptycholaimellus...........463, 464 Pulchranemells. 479 Punctodora...............463, 464 Punctodora.............463, 464

$Q^{\text {uadricoms ....456,457,478, } 478}$

R habditella..............475 R Rhabditidoides.............. 475 Rhabditis......463, $\mathbf{4 7 5}, \mathbf{4 7 5}, 475$ 476,477 ...470, 475 Rhabditolsimus. ............476 Rhabditophanes...........470, 476 Rhabdocoma .....472, 472, 473, 474 Rhabdodemsnia........... 467 Rhabdogaster...........455, 477 Rhabdolaimus...464, 475, 475, 477 Rhabdonchus........4 460,460,460 Rhabdotoderma............. 469 Rhadinems..............473, 479 Rhinema..............462, 465 Rhinonema ....460, 460,461, 462 .

Rhips.........461, 464, 466, 469 Rhodolaimus.............. 475 Rhynconema.............479 Richtersia.......458,462,473,474 480,480 Richtersiells.....473, 474, 480, 480

Sabatieria ...457, 462, 464, 471, 473 $\$$ Saveljevia..............4 468 Scaptrella...................... 467 Scatonema................. 455 Schistodera.............457, 457 Sectonema......... Seinura ........459,460,460, 475 Selachinema............... 468 Seleneella................. 466 Seuratia...................... 462 Seuratiella..................... 462 Sigmophora............... 465 page

Siphonolaimus.......459, 460, 476 Smalsundia...........468, 474, 480 Solenolaimus..............454, 476 Southernia.............457, 458 Southerniells.................. 480 Sphaerocephal um...........473, 479 Sphaerolaimus................ 473 Sphaerularia................... 455 Spilophora................ 465 Spilophorella...............463 Spilophorium ..................4 Spirs.......455, 464, $465,471,471$ Spirins......455,464, 465, 471, 471 Statenia. ................ 465 Steineria Filipjev..........456, 461 Steineris Ditlevsen............ 469 Steineria Stekhoven and de Coninck. .473 Steineriella. ........ 469 Stenolaimus..456, 458, 472, 472, 474 Stephanolaimus......456, 458, 467 $471,473,479$ Stilbonema...............454, 455
Symplocostoma.....461, 462, 462 Symplocostoma......461, 462, 462 ,

Symplocostomella. ...462, 463, 469 Synodontium...........466, 470 Synonchiella............... 468 Synonchium...............467, 468 Synonchus................. 469 Synonema................ 464 Syringolaimus................ 465

Tachyhodites...........457, 473 1 Teratocephalus ....466, 471, 471 , Terschellingia.........454, 455, 471 Thalassironus................. 467 Theristus........467, $472,473,473$ Thoönchus, ............. 469 Thoracostoms............. 469 Thoracostomopsis...............460 Trefusia, ................. 457 Trefusia.................... 457 Trichodorus.................. 461 Tricoma....456,457, 478,478, 480 Trigonolaimus.......465, 467, 467 Trilepta. ........461, 477, 478 Trilepta. ............461, 477,478
Trileptium.........461, 477,478 Trilobus..........461, 469, 472, 472 Triodontolaimus............467 page

Triplonchium..........459, 477 Tripyla.....456, 456, 458, 461, 47 $472,474,478,478,480$ Tripylium..........467, 479 Tripyloides.............474, 474 Trischistoma....461, 462, 472, 473 478,479 Trissonchulus.............. 46 Tristicochaeta........454,455,470 Trogolaimus...........4467, $\mathbf{4 7 2}$ Tubolaimella...............467, 479 Tubolaimus...............473, 479 Turbatrix...........463, 468, 470 Tycnodora......... 456 Tylenchinema.............456, 460 Tylenchodon..................459, 45 Tylencholaimellus............ 458 Tylencholaimus...........459, 459 Tylenchomorphus...455, 458, 459 ,

Tylenchorhy 460,460 Tylenchorhynchus........... 459 Tylenchulus..........459, 459, 459 Tylenchus................459, 459 Tylolaimophorus.....458, 459, 460 Tylopharynx...........459,475

Udonchus................ 464 Uracanthus...........455, 460 $\mathrm{V}$ asculonems......472, 474, 478, Viscosia..................469

Walcherenia.............. 470 Wilsonems. ....... 477

Yanthodora ...........463, 465 X Xennella...............456, 478 Xenolaimus............... 472 Xenonema................. 465 Xinema..............460,468

Xiphinema............... 45 Xyala ................ 467,473

$Y^{\text {psilon } \ldots .462,465,471,474,481}$

7alonema............471,477 Zanema..467, 473, 473, 479, 479 Zygonemella.........457, 473, 479 



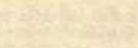




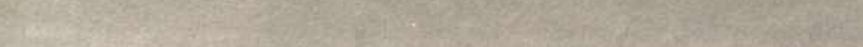

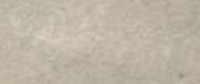


UNIVERSITY OF CALIFORNIA AT LOS ANGELES THE UNIVERSITY LIBRARY

This book is DUE on the last date stampedilangary 418.

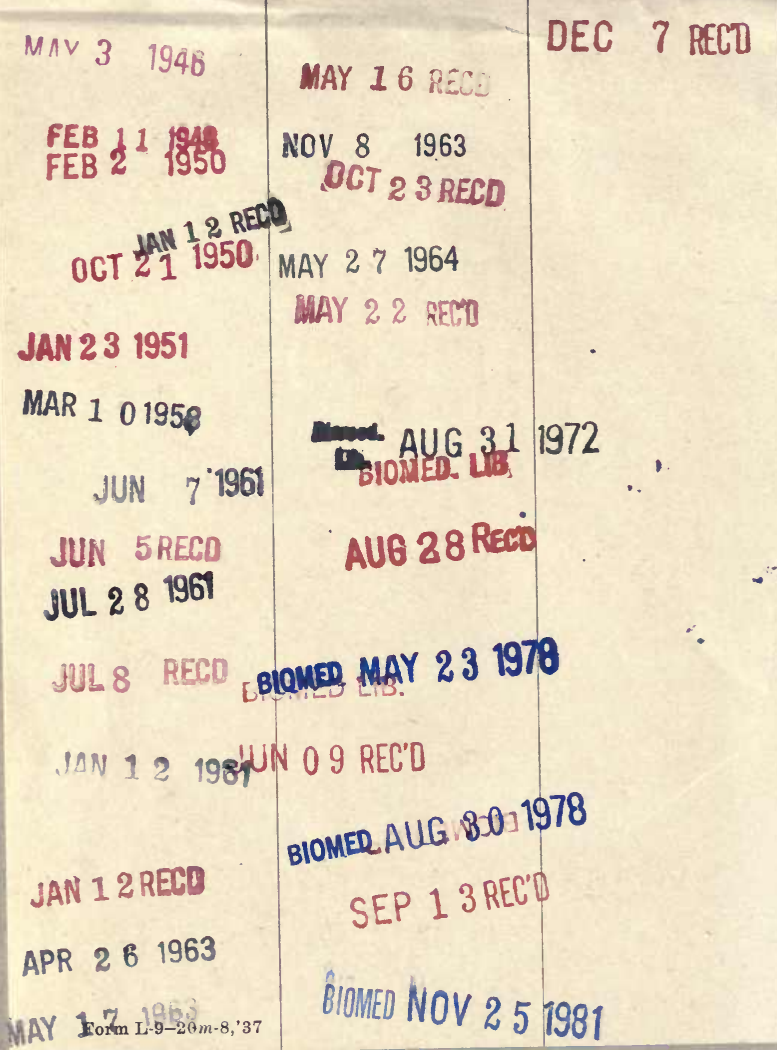


UC SOUTHERN REGIONAL LIBRARY FACILITY

AA 0000499327

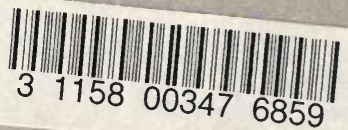


